\title{
Hyperarousal in the hospital and what to do about it
}

Citation for published version (APA):

Leue, C. (2017). Hyperarousal in the hospital and what to do about it: the MED-PSYCH-NET - a transitional network approach fostering personalized care in psychosomatic medicine. [Doctoral Thesis, Maastricht University]. Datawyse / Universitaire Pers Maastricht. https://doi.org/10.26481/dis.20171214cl

Document status and date:

Published: 01/01/2017

DOI:

$10.26481 /$ dis.20171214cl

Document Version:

Publisher's PDF, also known as Version of record

\section{Please check the document version of this publication:}

- A submitted manuscript is the version of the article upon submission and before peer-review. There can be important differences between the submitted version and the official published version of record.

People interested in the research are advised to contact the author for the final version of the publication, or visit the DOI to the publisher's website.

- The final author version and the galley proof are versions of the publication after peer review.

- The final published version features the final layout of the paper including the volume, issue and page numbers.

Link to publication

\footnotetext{
General rights rights.

- You may freely distribute the URL identifying the publication in the public portal. please follow below link for the End User Agreement:

www.umlib.nl/taverne-license

Take down policy

If you believe that this document breaches copyright please contact us at:

repository@maastrichtuniversity.nl

providing details and we will investigate your claim.
}

Copyright and moral rights for the publications made accessible in the public portal are retained by the authors and/or other copyright owners and it is a condition of accessing publications that users recognise and abide by the legal requirements associated with these

- Users may download and print one copy of any publication from the public portal for the purpose of private study or research.

- You may not further distribute the material or use it for any profit-making activity or commercial gain

If the publication is distributed under the terms of Article $25 \mathrm{fa}$ of the Dutch Copyright Act, indicated by the "Taverne" license above, 


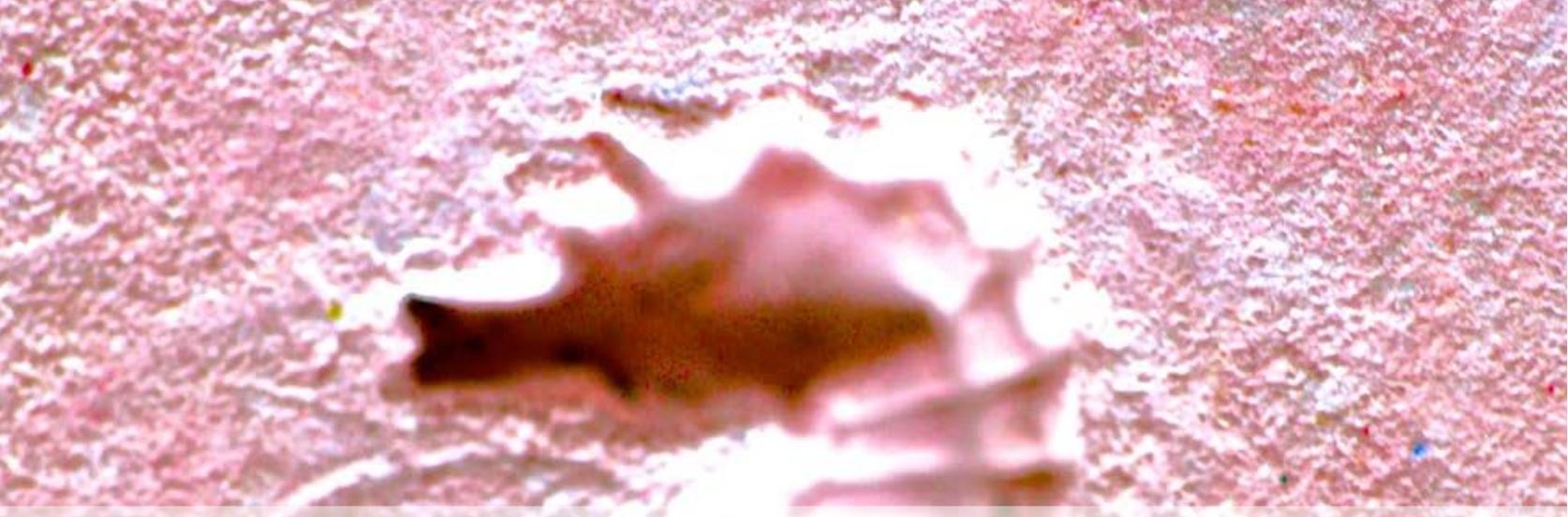

\section{Hyperarousal in the Hospital and what to do about it}

\section{The MED-PSYCH-NET - a transitional network approach fostering personalized care in psychosomatic medicine}

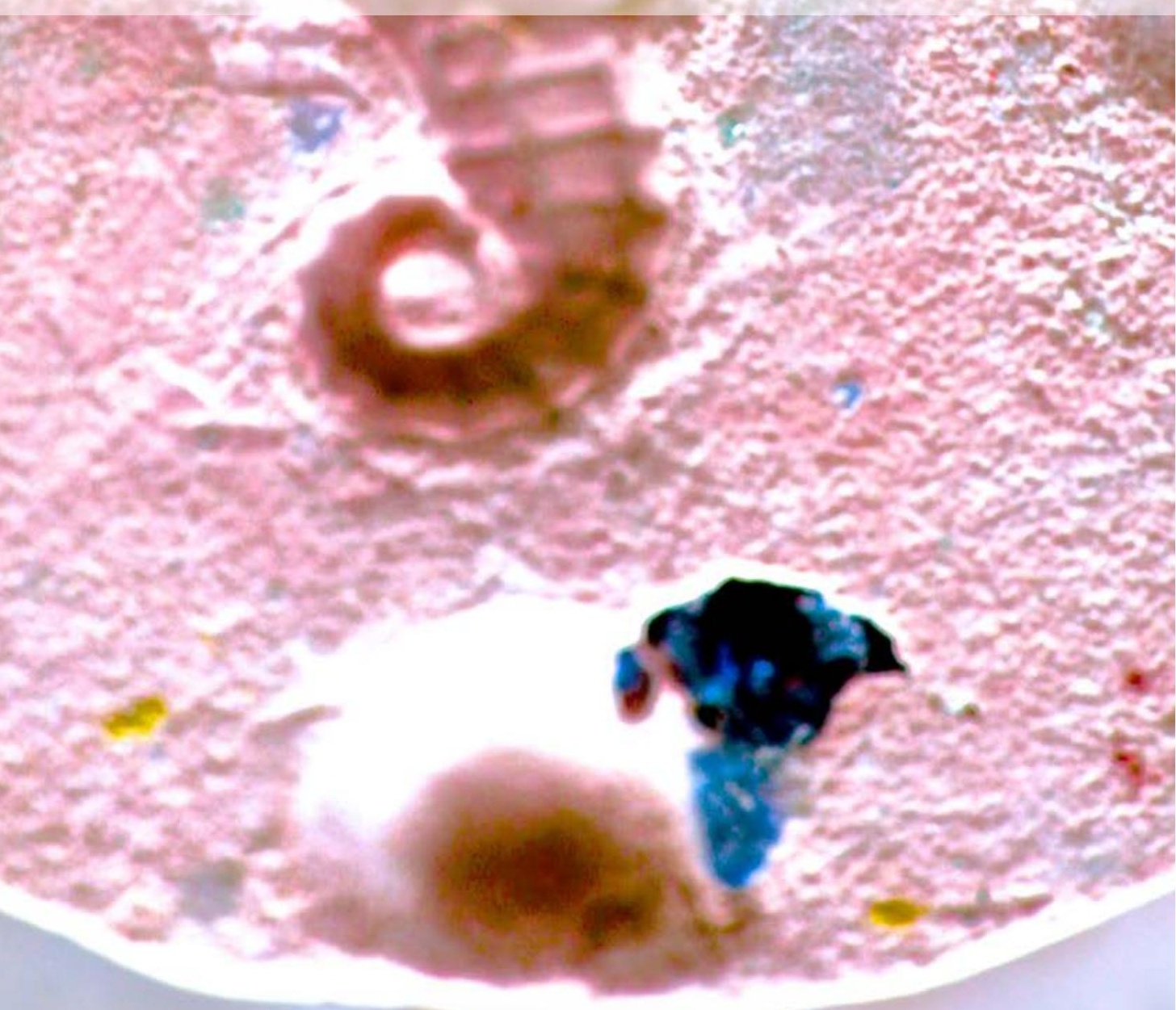


(C) Carsten Leue, Maastricht 2017

No part of this book may be reproduced or transmitted in any form or by any means, without prior permission in writing by the author, or when appropriate, by the publishers of the publications.

Layout: $\quad$ Tiny Wouters

Cover design: Marita Werner

Production: Datawyse University Press Maastricht, The Netherlands

ISBN:

9789461597700

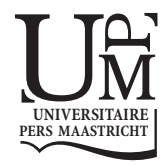

Printing and dissemination of this thesis was financially supported by Maastricht University. 


\section{Hyperarousal in the Hospital and what to do about it}

The MED-PSYCH-NET - a transitional network approach fostering personalized care in psychosomatic medicine

\section{PROEFSCHRIFT}

ter verkrijging van de graad van doctor aan de Universiteit Maastricht, op gezag van de Rector Magnificus, Prof. dr. Rianne M. Letschert volgens het besluit van het College van Decanen, in het openbaar te verdedigen op donderdag 14 december 2017 om 12.00 uur

door

Carsten Leue

geboren te Recklinghausen, Duitsland 


\section{Promotores}

Prof. dr. J.J. van Os

Prof. dr. A.A.M. Masclee

\section{Copromotores}

Dr. J.J.M.H. Strik

Dr. J. Kruimel

\section{Beoordelingscommissie}

Prof. dr. R.J. Stokroos (voorzitter)

Prof. dr. A. Honig, Vrije Universiteit Amsterdam

Prof. dr. R.W.H.M. Ponds

Prof. dr. R.J.E.M. Smeets

Prof. dr. W. Söllner, Paracelsus Medizinische Privatuniversität

Salzburg/Nürnberg; Österreich/Deutschland 
To Marita 


\section{Contents}

$\begin{array}{lll}\text { Chapter } 1 & \text { Introduction and outline } & 9\end{array}$

$\begin{array}{lll}\text { Part I Integrated care at the inpatient hospital setting } & 27\end{array}$

Chapter 2 Managing complex patients on a medical psychiatric unit: 29

An observational study of university hospital costs associated

with medical service use, length of stay, and psychiatric intervention

$\begin{array}{lll}\text { Part II Integrated care at the outpatient hospital setting } & 47\end{array}$

Chapter 3 Medically unexplained otorhinolaryngological symptoms: 49

Towards integrated psychiatric care

Chapter $4 \quad$ Integrated medical-psychiatric outpatient care in functional 61 gastrointestinal disorders improves outcome: A pilot study

Chapter 5 Prevalence of anxiety and affective symptoms and their 77 association with pelvic floor dysfunctions: A cross sectional cohort study at a Pelvic Care Centre

Part III L L Lack of integrated medical staff-guidance in psychosomatic primary care 91

Chapter 6 Observational evidence that urbanization and neighbourhood 93 deprivation are associated with escalation in chronic pharmacological pain treatment: a longitudinal population-based study in the Netherlands

$\begin{array}{lll}\text { Part IV Transitional care in psychosomatic medicine } & 115\end{array}$

Chapter 7 The MED-PSYCH-NET: Evidence that a psychosomatic 117 transitional network approach impacts costs in multi-conditional patients - a naturalistic pilot study

Part V Impact of integrated care on the understanding of psychosomatic aetiology

Chapter 8 The relationship between fiberoptic endoscopic evaluation of swallowing outcome and symptoms of anxiety and depression in dysphagic patients 
Chapter 9 Affective symptoms in patients with oropharyngeal

dysphagia: A systematic review

Chapter 10 Delirium: An unmet clinical need for integrated transitional care

[Organisch begründete psychische Erkrankungen - am Beispiel des Delirs]

Chapter 11 Chronic pain and psychiatry - an appeal for integrated care beyond the pain depression diade

[Chronische pijn en psychiatrie - een betoog voorbij de pijn Depressive diade]

Chapter 12 Affective symptoms and the overactive bladder: A systematic review

Chapter 13 Functional urological disorders: A sensitized defense response

in the bladder-gut-brain axis

Part VI Integrated care fostering momentary assessment

Chapter 14 The experience sampling method - a new digital tool for momentary symptom assessment in IBS: An exploratory study

Chapter 15 Demonstrating the reliability of transdiagnostic m-Health

Routine Outcome Monitoring in mental health services using

Experience Sampling Technology

Chapter 16 General discussion and valorisation

Appendix Summary

Samenvatting

Dankwoord

Publications

Curriculum vitae

403

Abbreviations 



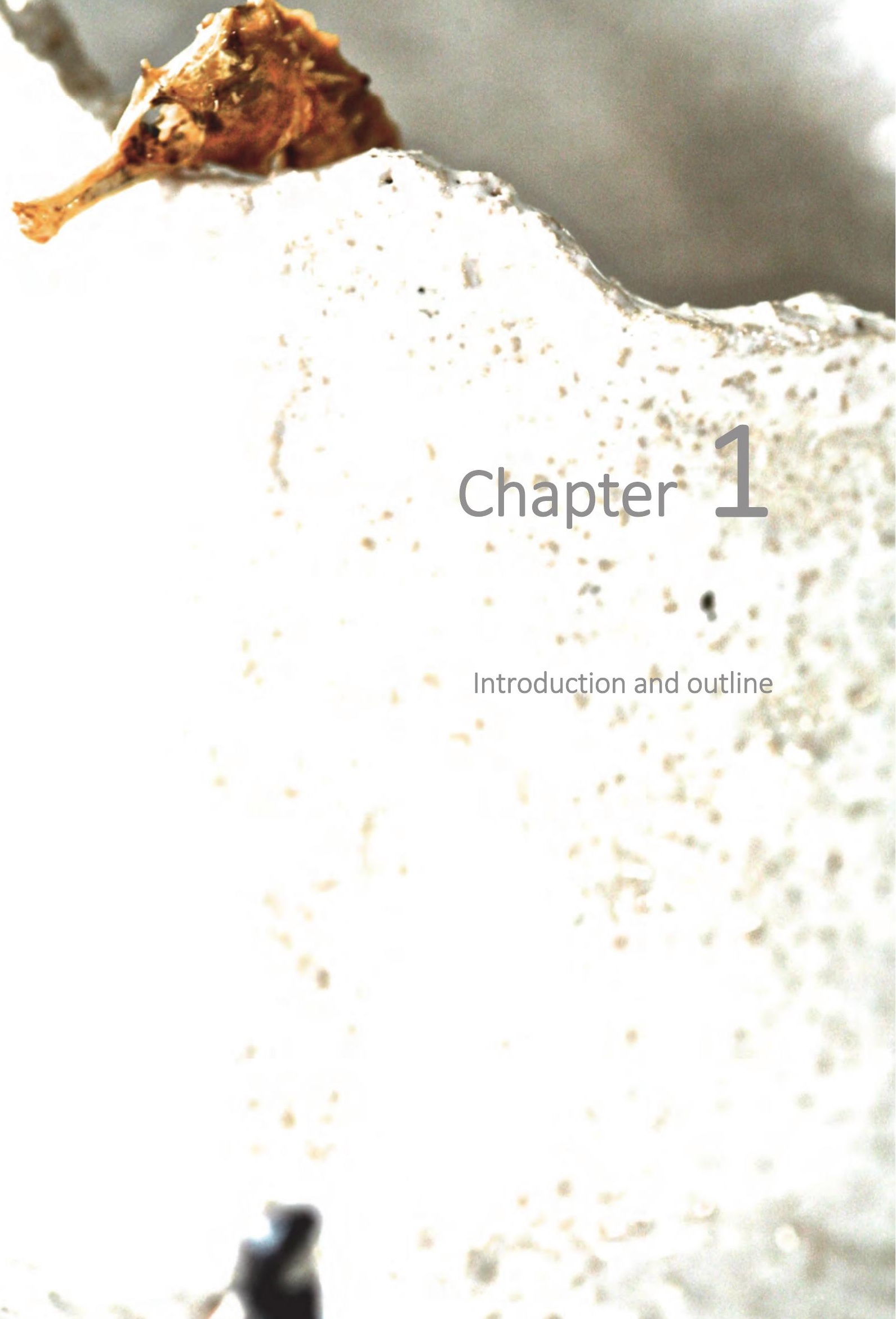





\section{Background}

Psychosomatic medicine is a multi-disciplinary arena, embracing both fundamental and clinical research, where different medical specialties explore the interrelationship between bodily processes and social, psychological and psychiatric factors. Clinical courses of mental processes affecting medical outcomes and, vice versa, somatic processes influencing mental well-being constitute the main field of interest. Attempts to distinguish between purely physical or solely mental disorders have become obsolete; almost all somatic diseases are known to be affected by mental factors concerning susceptibility, onset, clinical presentation and treatment adherence, and mental illness is associated with somatic pathways ${ }^{1,2,3}$. Diverse clinical disciplines, ranging from medical psychology to general hospital psychiatry, neurology, internal medicine, surgery, including various somatic subspecialties, are involved in the effort to understand the physiological pathways in psychosomatic conditions and, at least in an ideal world, to advocate multi-disciplinary management. Depending on the kind of problem, its acuity, its severity as well as its complexity, patients present at different medical settings. In order to gain a clinical overview of the complexity of psychosomatic conditions, an integrated approach to care is frequently warranted.

\section{Obstacles on the road to integrated psychosomatic care}

Integration of care (i.e., bringing together diverse specialty skills and expertise concerning health care in cases of multi-morbidity with comorbid psychiatric and somatic disorders) remains a difficult process in psychosomatic medicine. Whereas the aspiration to integrate medical and mental health care is widespread, integrated somatic and mental health care services are hard to find ${ }^{4,5}$. The obstacles that hinder integrated team-wise approaches are diverse, largely due to old-fashioned concepts of separate reimbursement. The current financial dichotomy creates competing interests in patient care where no distinction between mental and somatic health care should be made. Effective management of psychosomatic complexity is one of the most important healthrelated challenges facing patients, health professionals and society, since the cost of health care and the frequency of utilization will rise almost exponentially when additional comorbid conditions are present ${ }^{6,7}$. Although a robust body of evidence demonstrates the efficacy and cost-effectiveness of integrated care models, particularly for comorbid medical and affective conditions, cost-savings mostly accrue to the insurance companies alone instead of to the providers of primary or hospital-related care. In addition, financial incentives to implement evidence-based models for improving care should be offered to integrated care providers ${ }^{8}$. Till now, a "silo" structure of health care institutions has persisted, isolating clinical from scientific expertise. In this context, jointly submitted collaborative research grants may help overcome competing specialtyrelated research interests with regard to multi-conditional health care. 
Besides joint funding, organizational aspects of integrated care models should be taken into account in order to deliver care successfully. The presence of a multidisciplinary team is an indispensable condition. Furthermore, shared clinical protocols and a specialty-transcending information system (with electronically accessible patient files) have to be available. Colocated work would be the ideal arrangement for cooperating services. If not feasible, liaison services operating bi-directionally would be needed to approach the patient. In order to guarantee clinical expertise on a consultant level, navigators (medical staff) and 'steersmen' (care managers) should deliver guidance on the clinical course, either face-to-face with patients and/or caregivers or via digital information transferred to patients and their care managers. In addition, well-operating integrated teams should conduct multi-disciplinary research in order to evaluate their clinical practice. Last but not least, a multi-component strategy should include efforts to combat stigmatization ${ }^{9}$. The stigma attached to chronic conditions and especially to mental disorders has historically been one of the major obstacles to the realization of effective health care. The stigma-related obstacles range from attitudes of patients and caregivers that hamper their compliance, to treatment adherence and the willingness to conceptualize comorbidity issues in multi-conditional problems psychosomatically ${ }^{10,11}$. Moreover, stigmatization is probably the main reason for the persistence of rigidly separated systems of health care and reimbursement. For more than a century, the rationale for isolating mentally ill patients from the community has lain in the behavioural disturbances in psychiatric disorders, which have been relegated to the domain of psychiatric hospitals.

Against the background of today's obstacles with regard to psychosomatic care, health care organization at the medical-psychiatric interface is truly a clinical challenge. A major concern, and one that hinders the implementation of new care models, is that a tremendous investment of time, money and sustained caregivers' efforts is required to develop and establish changes in care over the long run ${ }^{8,12}$. On the other hand, given the innovations in psychosomatic medicine, integrated care in psychosomatic medicine delivered various solutions across a continuum of settings, from complex inpatient medical intervention to primary care.

\section{Med-Psych-Units: Re-inventing the past or fine-tuning the future?}

In a medical/surgical inpatient setting, psychiatric comorbidity is associated with excess health care costs derived from hospitalization frequency and duration of admissions ${ }^{13,14}$. The fact that this comorbidity is not generally recognized leads to medically unexplained physical symptoms with subsequent frequent care utilization ${ }^{14}$, which indicates a need for integration of clinical care at the inpatient consultation-liaison level and the level of integrated Med-Psych-Units (MPUs). The benefit of taking an integrated approach is the capacity to detect comorbidity and to initiate multi-disciplinary treatment ${ }^{15}$. In cases of multi-conditional patients with the most complex status who frequently utilize the facilities, it may be advisable to channel admissions through the MPUs instead of 
providing inpatient treatment at mono-disciplinary wards. There is preliminary evidence that MPUs improve a number of outcomes for medical inpatients with psychiatric conditions. This suggests that integrated inpatient psychosomatic treatment is conducive to a favourable course for symptoms of mental illness with a somatic component ${ }^{16-18}$. However, the development of integrated care at MPUs appears to be contextdependent. In rural areas of the US, for example, MPUs no longer have a significant impact on psychosomatic care. This observation makes sense; collaborative approaches are feasible in primary care for comorbid patients, even over long distances, with regard to the carers' communication ${ }^{19,20}$. Another reason why collaborative care (CC) deserved priority in the US has to do with the political circumstances behind the introduction of health care insurance for everyone. This political decision was implemented in the 'Affordable Care Act', which at first glance appears to encourage cheap and effective solutions ${ }^{8}$. A focus on integrated care at the primary level in psychiatric and somatic comorbidity makes sense for every industrialized country with exploding health care budgets. The fact is, more patients receive treatment for mental disorders by general practitioners (GPs) than in the mental health specialty setting ${ }^{21}$. This is also the case in the Netherlands (NL); at the level of primary care, a 'general module for somatic and psychiatric comorbidity' was initiated in co-operation with the Dutch associations of Internal Medicine, Psychiatry and General Practitioners, an initiative that has recently been finalized ${ }^{22}$. Additionally, unlike the situation in the US, the development of MPUrelated integrated care did not stop there. At the moment, 40 MPUs are operational in the Netherlands, starting with two MPUs in the late 1990s and still growing nationwide ${ }^{23}$. Moreover, 'MPU field standards' have been published and evaluated along with a 'general module for hospital psychiatry' ${ }^{23-25}$. There are various reasons why NL promotes integrated primary care- and hospital-based approaches. The Netherlands is a densely populated and highly urbanized country where complex multi-conditional patients in need of hospital care are clustered in cities, coinciding with access to 'high tech' care in general hospitals. Furthermore, the most complex severely ill patients and, therefore, those who frequently utilize health care facilities, may not be cured by primary care-based CC alone. Research suggests a lack of evidence or even an absence of efficacy in certain diseases regarding physical well-being ${ }^{26,27}$. Given the present barriers, including insufficient training or interest among primary care providers in managing comorbid mental disorders, it might be worthwhile to implement and develop integrated care models at every level of care. The effort could help close some gaps in research and clinical management concerning the most cost-intensive multi-conditional patients ${ }^{28-31}$.

\section{Pro-active integrated consultation-liaison services to compensate for short hospital stay}

Almost $50 \%$ of all inpatients at general hospitals have a diagnosable psychiatric disorder and are frequently in need of care ${ }^{32}$. The number of potentially referred inpatients 
would put far too much burden on integrated care in the MPU setting. This alone is a good reason for pro-active integrated consultation-liaison $(\mathrm{CL})$ solutions that guide patients from inpatient to outpatient settings or into primary care. Overall, CL services (CLS) can be cost-effective when involved early in the hospital stay and if referrers follow protocol-based recommendations ${ }^{33}$. However, there is a need for research on navigation and standardized guidance by and of the staff ${ }^{34,35}$. It is critical to initiate consultation in a timely manner, since the length of stay is correlated with the amount of time that elapses from admission to the moment of first contact with this service ${ }^{36}$. So, why are physicians at the hospital not referring their patients to psychosomatic CLS in time? Both patient and referrer play a role in the delay of referral, but there are systemic factors as well. One of these can improve the rate of timely referrals, namely a dedicated, encouraging and pro-active CLS. A referrer factor that decreases the number of referrals is discomfort with psychiatric or psychosomatic CLS, which makes a proactive and engaging communication with medical referrers a relevant issue ${ }^{36}$. Furthermore, given the short stay on medical wards, the lack of opportunity for behavioural observations must be compensated somehow. Bio-psychosocial health risks and the patient's unmet clinical needs should be assessed and integrated treatment should be planned right from the moment of admission. A practical tool for planning these steps is the INTERMED method $^{38}$. This instrument is available as a self-assessment tool. Because it quickly detects which inpatients have complex medical conditions, integrated case management can start as early as possible, whereas complexity risk factors are not typically uncovered during standard medical procedures ${ }^{38,39}$. An appropriate time and place to use the INTERMED instrument could be upon the first presentation of comorbid medicalpsychiatric patients at the hospital's emergency department (ED). Staff interviews revealed scenarios in which comorbid mental illnesses or related factors led to misdiagnosis, delayed treatment or even serious adverse events. Factors leading to misattribution in this regard include i) patients' complex presentation, ii) their challenging behaviour, iii) pressure due to the crowded ED environment, and last but not least, iv) the stigmatizing attitude of some staff members ${ }^{40}$. In that light, some recommendations are in order to optimize diagnosis and treatment of multi-conditional patients with medical and psychiatric comorbidity at the ED. In the first place, it is advisable to have a psychiatric $\mathrm{CL}$ team available 24 hours a day, which obviously does not fall under standard care in all general hospitals. Secondly, it is advisable to prepare detailed multi-disciplinary guidelines for the assessment and treatment of intoxicated patients by both the psychiatric $C L$ and the ED staff. Thirdly, the hallmark of integrated multi-disciplinary management would be to hold regular multi-disciplinary meetings with staff members of all departments involved ${ }^{41}$. With regard to the subsequent transition back to primary care at the end of a hospital stay, there is evidence that the GPs' concordance with CLS' recommendations may be enhanced by direct communication between the hospital and the primary care providers ${ }^{42}$. This evidence concurs with in- 
hospital findings that a more pro-active CLS involvement may prevent comorbid patients from going unrecognized ${ }^{43}$.

\section{Integrated day care and outpatient psychosomatic treatment}

Research into integrated psychosomatic care involving different medical specialties on a day hospital basis is scarce. In some countries psychosomatic hospitals provide multimodal psychotherapy programmes for inpatients whereas they focus on mental health care outcomes for the day care setting too. Although there are differences between inpatients and patients at the day care facility, in particular regarding their degree of physical impairment and severity of affective conditions, no differences are found for these treatment modalities when comparing their effectiveness, defined as a reduction of depressive symptomatology only ${ }^{44,45}$. This suggests that patients with milder somatic conditions may be equivalently treated for affective complaints in the day clinic setting compared to what they would receive as inpatients. Comparable results were found for depressed elderly persons, denoting a reduction in their overall health care utilization because the costs for day hospital treatment are lower than for inpatient care ${ }^{46}$. This indicates that day care treatment might also have a positive influence on somatic symptom burden or somatic treatment adherence and efficacy.

Psychosomatic medicine is increasingly practised in the outpatient setting too. In part that may be due to the long waiting lists for psychotherapy in private practices after completion of inpatient psychosomatic rehabilitation, at least in some countries ${ }^{47,48}$. The effects of outpatient-based psychotherapy (PT) generally show favourable pre-post differences in terms of the reduction of work-disability days, hospitalization days and overall inpatient costs a year after $\mathrm{PT}^{48,49}$. Hence, there is a need for instant linking between somatic and mental health care settings. Furthermore, there is also a need for co-ordinated participation of medical specialties according to their profile of medical expertise in psychosomatic outpatient care ${ }^{50}$. In that context, especially in cases of high clinical severity or an early indication of treatment-resistance, there is a role for tertiary care at a university hospital for psychosomatic outpatient care ${ }^{47,50,51}$. Given the pleiotropic situation in psychosomatic conditions, outpatient care should be organized as a multi-disciplinary team effort to prevent unsatisfactory outcomes or even treatment refractoriness ${ }^{52}$.

\section{Integrated care in the primary care setting}

The best evidence for the performance of psychosomatic integrated care comes from primary care. There are various models for jointly supported treatment trajectories involving GPs and medical consultants. One of these is the model of mental health care consultation for GPs delivered by mental health specialists (psychiatrists, psychologists, nurse practitioners) ${ }^{53}$. Despite the high prevalence of mental illness - almost $25 \%$ of the population will suffer from a psychiatric disorder at some point during the lifetime, and 
most of these people receive treatment for mental disorders in primary care - many patients remain undetected and consequently untreated ${ }^{31}$. Part of the explanation may lie in the somatic presentations of underlying disturbances in emotion regulation that are not instantly attributed to anxiety or mood disorders by general practitioners, for example ${ }^{52}$. In order to raise detection rates for mental illness, it is therefore essential to involve primary caregivers. In the model of mental health care consultation for primary caregivers, GPs play a pivotal role in care delivery, with the mental health care consultant supporting their position. This primary care-based consultation-liaison model improves patients' adherence to treatment and their satisfaction with it, and by extension the model improves mental health ${ }^{53}$.

On the other hand, there is some evidence that the consultation-liaison model is not as effective as primary care-based collaborative care, particularly when dealing with symptoms of psychiatric disorders and general health status ${ }^{31,53}$. Collaborative care (CC) as co-ordinated case management, which is delivered by a medically supervised nurse in co-operation with the patients' general practitioner and a navigating psychiatrist, improves affective and chronic disease states in multiple conditions ${ }^{19,20,31}$. Moving case managers (CMs) into the frontline of treatment rather than offering mental health consultation to GPs alone or providing care as usual may lead to advantages with regard to combined somatic and mental health care trajectories. CMs, who spend more time with the patient than GPs, may evoke better engagement, compliance and treatment adherence, a performance which eventually translates into a better outcome and favourable economic evaluations ${ }^{19,31,54}$. Comparable results of CC have been achieved in patients with mental and physical multi-morbidity outside the US, notably in European urbanized areas, with regard to decreased depressive symptoms and anxiety as well as improved self-management of chronic diseases ${ }^{55,56}$.

However, some uncertainty remains about the effectiveness of primary care-based interventions targeting multi-morbidity ${ }^{26,27,57}$. Especially in patients with severe somatic conditions, CC did not significantly reduce the occurrence of major adverse somatic events $^{27}$. Moreover, as multi-conditional or frequently utilizing patients present themselves at in- and outpatient hospital settings, there is a need to target integrated care, by means of CC, to address disease severity and complexity in the general hospital setting too. Given that collaborative care has successfully been applied in primary care and research settings, the initial steps have been taken to embed the CC model in the daily routine of outpatient medical settings ${ }^{58,59}$. For example, collaborative depression care for people with cancer has shown efficacy concerning their affective condition and has proved to be (cost-) effective ${ }^{59,60}$. As a collaborative care-based multi-component programme, trained cancer nurses and supervising psychiatrists collaborate with the oncology team and the general practitioner. So for depressed cancer patients, an outpatient-based expansion of CC towards specialty care settings has already taken place. 'Cancer nurses' established a therapeutic relationship with the patients and provided problem-solving therapy and behavioural activation under the supervision of 
psychiatrists at either the cancer centre or primary care clinics. Psychiatrists gave advice on pharmacotherapy to GPs and offered them direct consultations if their patients did not improve. Depressed cancer patients were enrolled in the programme from the cancer centres and associated primary care clinics, without stratification of the CC per setting. Thus, application of the CC model for depression in cancer patients provides some new evidence on the use of collaborative care in outpatient medical specialty settings ${ }^{59,60}$. Another example of the introduction of CC into somatic specialty care is a programme to improve the detection and treatment of affective disorders in patients admitted to cardiology inpatient units at a university medical centre. Patients received multi-component inpatient intervention. Besides education and treatment recommendation by specialists, it involved treatment initiation during the hospital stay followed by a phone-based evaluation including care co-ordination with the primary care physician. Post-discharge follow-up was of low intensity: each patient had three care manager (social worker) contacts under the supervision of a team psychiatrist. Overall, an improvement in mental health-related quality of life was achieved. Although there were no differences in overall costs compared to usual care, CC was associated with a significant increase in quality-adjusted life-years ${ }^{61,62}$. The question arises whether staffguided interventions by psychosomatically trained nurse practitioners or physician assistants would have yielded bigger effect sizes.

\section{Benefits and aspirations of integrated care in psychosomatic medicine}

As set forth above, studies providing evidence of integrated care in the hospital setting, including day and outpatient care, are sparse but encouraging. The research has mainly involved collaborative care in primary care or research settings 57,58 . Most CC studies focused predominantly on affective conditions as the primary outcome. In this context, $\mathrm{CC}$ is effective concerning the reduction of the bothersome mental ill-health burden, although the achieved improvements are small for some condtions ${ }^{63}$. In addition, CC tends to be at least cost-neutral or can even lead to modest cost-effectiveness ${ }^{58,62}$.

However, there are still some uncertainties about the effectiveness of primary carebased interventions targeting somatic aspects, especially with regard to complexity and severity in multi-conditional patients. These uncertainties remain in 'hard-core' somatic conditions (e.g., cancer, cardiovascular disease, etc.) with comorbid psychiatric disorders as well as in medically unexplained physical symptoms associated with affective or somatoform conditions ${ }^{26,63,64}$. Moreover, the current evidence does not support the use of 'frontline' primary caregivers in cases of functional somatic symptoms. The benefit may be confined to more intensive interventions with regard to change of functional outcome. Well-known bottlenecks in primary care are the limited consultation time, lack of skills, the need for a degree of diagnostic openness, and patient resistance towards psychosomatic attributions ${ }^{64}$. Hence, there is still a role for hospital-based integrated care approaches, since the momentum is building for a much wider collaboration than between primary care-based CC and hospital-based CL services alone. It is fed by the 
growing recognition of comorbid somatic and psychiatric disorders, their frequency, poorer treatment outcome and greater costs of care ${ }^{64,65,66}$. Realizing that the integration of care remains aspirational, the British King's Fund recently published a new report to address current shortcomings ${ }^{5,67}$. This report describes practical considerations, both the barriers to and the facilitators of integrated care ${ }^{67}$. It is noteworthy that integrated care goes beyond the colocation of services. In order to achieve an integration of medical and mental health care, cultural barriers which separate organizational and payment-related factors for physical and mental care have to be overcome ${ }^{5,67}$. Policy developments, like the Affordable Care Act in the US and the National Health Service's Five Year Forward View in the UK, prepared the ground in industrialized countries to facilitate integration of care ${ }^{8,68}$. However, leadership for change at both the clinical and board levels is still necessary to enable further innovations in the medical system and its participating organizations, namely medical hospitals, primary caregivers and mental health care services (MHCS).

Thus, there is a need for MHCS to be as well funded as medical health services and to provide care that is as good as physical care. In order to extend that level of care, it is necessary to deliver care as an integrated part of all health care services ${ }^{5}$. To support this integration, research is needed that focuses on the identification of multiconditional patients (including psychiatric comorbidity), on their unmet clinical needs, and on their outcome in relation to different integrated care approaches ${ }^{48,52.54}$. Multidisciplinary investigations should incorporate all settings where patients present themselves initially or repetitively, from primary care to various levels of medical hospitals ${ }^{5,52}$. In addition, research should facilitate the involvement of medical and paramedical disciplines in multi-morbidity care. Obviously such involvement would include staff-guided care managers, for instance physician assistants, nurse practitioners, psychologists, and if appropriate (i.e., for non-medical concerns) social workers $^{68,69}$. Moreover, integrated care and related scientific research could recognize and identify patients' natural organ-related symptom attribution but paradoxically also overcome treatment refractoriness related to multi-morbidity ${ }^{52}$. Furthermore, integrated care should respect clinical and economic interests of various specialty caregivers involved in psychosomatic medicine, which could paradoxically have the societal impact of reducing cost. Therefore, integrated care would best be construed as a virtual transitional network instead of colocated medical services. In light of the complexity and severity of medical conditions ${ }^{52}$, the introduction of a transitional medical-psychiatric-network (Med-Psych-Net) may contribute to the integration of psychosomatic care across different settings without violating the diverse and sometimes contradictory interests in the field. Starting from the medical hospital perspective, integrated care should focus predominantly on the most complex and/or severe multi-conditional patients, including psychiatric comorbidity, since comorbid affective conditions usually go unrecognized at medical services and somatoform conditions are not sustainably treated in primary care ${ }^{14,52,64}$. Notably, the affective multi- 
morbidity pattern is even present in functional somatic diseases with comorbid somatoform disorders which, if undetected and therefore untreated, eventually contribute to frequent utilization of care 22,70-72. $^{\text {. }}$

Although observational clinical data are not suitable for identifying causal inference in psychiatric and somatic comorbidity, integrated care that recognizes clinical complexity might help to understand and treat pathways of mental ill-health which may amplify somatic symptom formation ${ }^{52,70,73}$. Therefore, a clinical network approach based on etiologic medical concepts might help to establish integrated care by connecting specialty outpatient clinics in the hospital. It may thereby bridge the gap between the hospital and primary care too via medical staff-guided transitional care managers without compromising the quality of consultants' etiologic conceptualization of treatment.

\section{The Med-Psych-Net - a transitional network approach fostering personalized care in psychosomatic medicine}

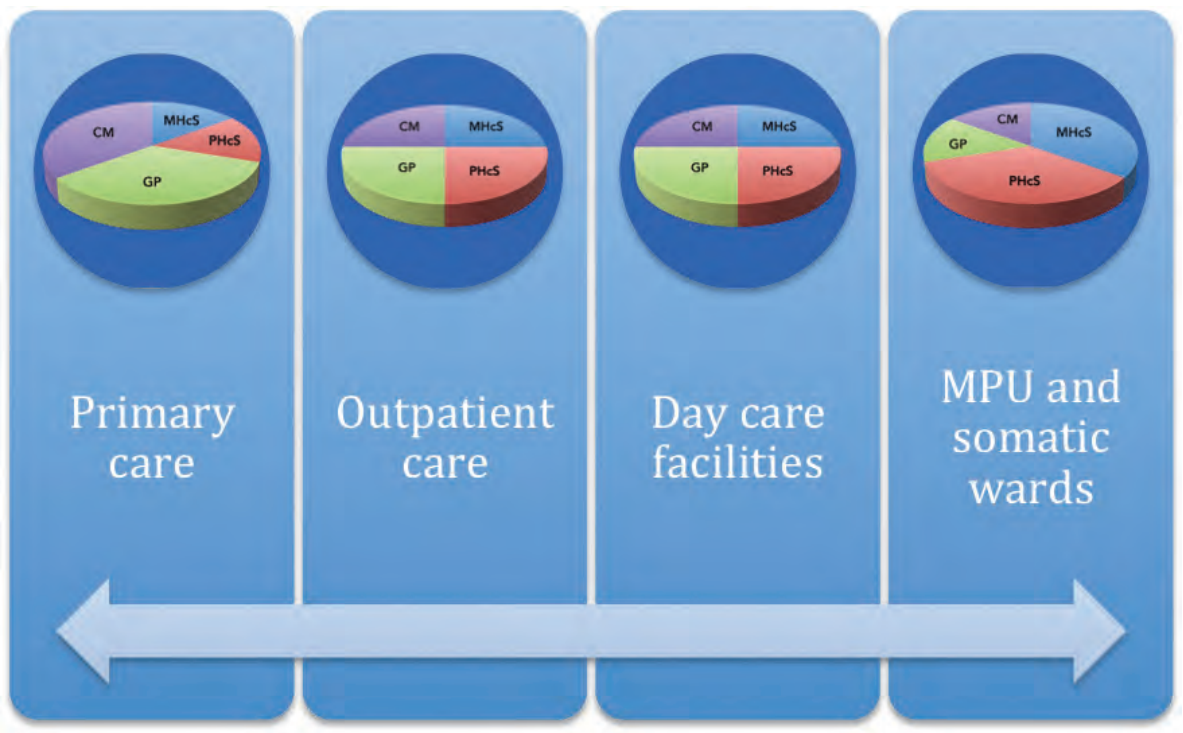

Legend: A Med-Psych-Net (MPN) is a virtual network of co-operating care givers who are treating patients with psychosomatic conditions, referring them from primary care to the hospital and vice versa. Care givers involved in this integrated approach provide treatment in different proportions depending on the severity and complexity of disease. Patients with the most severe conditions are admitted to the integrated hospital inpatient unit (Med-Psych-Unit (MPU)) or to somatic wards in co-operation with the mental health care consultation-liaison service (CLS). Patients with less impairment but whose medical and/or bio-psycho-social problems are still severe are followed on a day care or outpatient basis. The hospital-related integrated care, ranging from outpatient to inpatient interventions, is covered by the hospital-based Med-Psych-Centre (MPC). Transition of the patient back to primary care takes place at suitable moments when the roles of case manager $(\mathrm{CM})$ and general practitioner (GP) become more prominent. PHcS = Physical Health care Specialist; $\mathrm{MHcS}=$ Mental Health care Specialist. 


\section{Aims and outline of this PhD thesis}

From the perspective of a psychosomaticist (e.g., hospital psychiatrist, medical psychologist, GP, gastro-enterologist, urologist, ENT physician and other specialty consultants with an interest in multi-morbidity or functional complaints), the primary aim of this thesis is to gain deeper insight into meaningful possibilities for integrated care in psychosomatic medicine. Clinical research is conducted against a background of different hospital (i.e., inpatients and outpatients) and primary health care levels and related medical specialties. The secondary aims of this thesis are i) to introduce an overlapping and bridging model of integrated care across settings in order to connect medical specialties in multi-conditional patients and to avoid the disadvantages of mono-disciplinary care; ii) to contribute new insights into etiopathological pathways of multi-morbidity; and iii) to support real-life symptom assessment in psychosomatic medicine, which may help to identify how pleiotropic symptoms cluster to form complex syndromes.

The implications of our investigation may eventually lead us beyond the scope of this thesis and contribute to our conceptualization of multi-morbidity or syndrome complexity, turning our gaze away from symptom-based diagnostic constructs toward an etiopathogenetic concept of psychosomatic conditions ${ }^{52,74,75}$.

This thesis is divided in six parts, each of which contributes to the above-mentioned aims. The first part describes and investigates integrated psychosomatic care in the inpatient hospital setting. Chapter 2 explores the advantages and the costs of admission to a Med-Psych-Unit.

The second part of the thesis examines examples of integrated hospital outpatient care in psychosomatic medicine. Chapter 3 describes the possibility to merge with an integrated outpatient approach in dealing with medically unexplained otorhinolaryngological symptoms. Chapter 4 reports on integrated medical-psychiatric outpatient care in functional gastrointestinal disorders with regard to patient-related outcomes. Chapter 5 considers the prevalence of affective symptoms and their association with pelvic floor dysfunctions at an outpatient-based integrated Pelvic Care Centre.

The third part of the thesis is devoted to the primary care setting. Chapter 6 describes what happens in the absence of integrated guidance by medical staff in chronic pain treatment in the outpatient and primary care setting.

The fourth part of the thesis describes how shifting integrated care from the university hospital back to general practitioners may contribute to a successful transition of care. Chapter 7 investigates in a naturalistic pre-post comparison the positive impact of transitional integrated care on costs in multi-conditional patients.

The fifth part of the thesis focuses on possible influences that integrated care could have on the conceptual understanding of etiologic pathways in psychosomatic medicine. Chapter 8 describes how affective states may amplify the severity of patients' subjective experience in dysphagia. Chapter 9 reviews the evidence on affective symptoms in 
patients with oropharyngeal dysphagia. Chapter 10 demonstrates that integrated care may contribute to better treatment in severely disturbed delirious patients. Chapter 11 elucidates the interrelationship between chronic pain and affective disorders. Chapter 12 makes strides toward a clear definition of what is associated with overactive bladder from a mental health care perspective. Then Chapter 13 presents a sensitization and alarm-falsification concept of functional abdominal and urological disorders in the bladder-gut-brain axis.

The sixth part of the thesis points out how integrated care may contribute to a more informative approach to symptom assessment as a means to develop a better understanding of symptom formation and symptom clustering related to real-life contextual influences. Chapter 14 describes the experience sampling method (ESM) as a tool for momentary assessment in irritable bowel syndrome and how ESM may contribute to real-time symptom recording in psychosomatic medicine. Chapter 15 demonstrates the reliability of transdiagnostic $m$-health routine outcome monitoring in an outpatient-based psychosomatic mental health service using experience-sampling technology.

Finally, Chapter 16 provides a general discussion and a summary setting the stage on which to present an outlook on how symptoms related to psychosomatic conditions might better be conceptualized and therefore further investigated and treated. 


\section{References}

1. Levenson, J. L. Essentials of Psychosomatic Medicine. American Psychiatric Press Inc. ISBN 978-1-58562246-247 (2006).

2. Wise, T. N. Update on consultation-liaison psychiatry (psychosomatic medicine). Curr. Opin. Psychiatry 21,196-200 (2008).

3. Nusslock, R. \& Miller, G. E. Early-life adversity and physical and emotional health across the lifespan: A neuroimmune network hypothesis. Biol. Psychiatry Pii:S0006-3223(15)00466-7 (2015).

4. Huang, H. et al. What is integrated care? Int. Rev. Psychiatry 26, 620-628 (2014).

5. Sharpe, M. \& Naylor, C. Integration of mental and physical health care: From aspiration to practice. Lancet Psychiatry 3, 312-313 (2016).

6. Huyse, F. J. et al. Identifiers, or "red flags," of complexity and need for integrated care. Med. Clin. North Am. 90, 703-712 (2006).

7. McPhail, S. M. Multimorbidity in chronic disease: Impact on health care resources and costs. Risk Manag. Health Policy 9,143-56 (2016).

8. Katon, W. J. \& Unützer, J. Health reform and the Affordable Care Act: the importance of mental health treatment to achieving the triple aim. J. Psychosom. Res. 74, 533-537 (2013).

9. Rodgers, M. et al. Integrated care to address the physical health needs of people with severe mental illness: A rapid review. Southampton (UK): NIHR Journals Library (2016).

10. Taft, T. H. \& Keefer L. A systematic review of disease-related stigmatization in patients living with inflammatory bowel disease. Clin. Exp. Gastroenterol. 7, 49-58 (2016).

11. Committee on the Science of Changing Behavioral Health Social Norms; and National Academies of Sciences, Engineering, and Medicine. Ending Discrimination Against People with Mental and Substance Use Disorders: The Evidence for Stigma Change. Washington (DC): National Academies Press (US) (2016).

12. Janse, B. et al. Delivering integrated care for the frail elderly: The impact on professionlas' objective burden and job satisfaction. Int.J. Integr. Care 16:7 (2016).

13. Borckardt, J. J. et al. Excess health care service utilization and costs associated with underrecognition of psychiatric comorbidity in a medical/surgical inpatient setting. Qual. Manag. Health Care 20, 98-102 (2011).

14. Sharpe, M. et al. Is co-morbid depression adequately treated in patients repeatedly referred to specialist medical services with symptoms of a medical condition? J. Psychosom. Res. 72, 419-421 (2012).

15. Kishi, Y. \& Kathol, R. G. Integrating medical and psychiatric treatment in an inpatient medical setting. The type IV program. Psychosomatics 40, 345-355 (1999).

16. Hussain, M. \& Seitz, D. Integrated models of care for medical inpatients with psychiatric disorders: A systematic review. Psychosomatics 55, 315-325 (2014).

17. Honig, A. et al. A medical-psychiatric unit in a general hospital: Effective combined somatic and psychiatric care? Ned. Tijdschr. Geneeskd. 158, A6520 (2014).

18. Zastrow, A. et al. Outcome of simultaneous psychosomatic/internal-medicine inpatient care - a naturalistic follow-up study. Z. Psychosom. Med. Psychother. 55, 229-247 (2009).

19. Katon, W. J. et al. Collaborative care for patients with depression and chronic illnesses. N. Engl. J. Med. 363, 2611-2620 (2010).

20. Katon, W. et al. Cost-effectiveness of a multicondition collaborative care intervention: A randomized controlled trial. Arch. Gen. Psychiatry 69, 506-514 (2012).

21. Olfson, M. et al. Trends in office-based mental health care provide by psychiatrists and primary care physicians. J. Clin. Psychiatry 75, 247-253 (2014).

22. http://www.kwaliteitsontwikkelingggz.nl/project/generieke-module-comorbiditeit/ [General Module Somatic and Psychiatric Comorbidity]

23. van Schijndel, M. A. et al. Medical-psychiatric units in the Netherlands: An investigation into distribution an quality. Ned. Tijdschr. Geneeskd. 161, D890 (2017).

24. https://www.nvvp.net/stream/veldnormen-mpu-juni-2014 [MPU Field Standards]

25. http://www.kwaliteitsontwikkelingggz.nl/project/generieke-module-ziekenhuispsychiatrie/ [General Module Hospital Psychiatry] 
26. Archer, J. et al. Collaborative care for depression and anxiety problems. Cochrane Database Syst. Rev. 10:CD006525 (2012).

27. Tully, P. J. \& Baumeister, H. Collaborative care for comorbid depression and coronary heart disease: A systematic review and meta-analysis of randomised controlled trials. BMJ Open 5, e009128 (2015).

28. Kathol, R. G. et al. Barriers to physical and mental condition integrated service delivery. Psychosom. Med. 72, 511-518 (2010).

29. Kathol, R. G. et al. Psychiatrists for medically complex patients: Bringing value at the physical health and mental health/substance-use disorder interface. Psychosomatics. 50, 93-107.

30. Manderscheid, R. \& Kathol, R. G. Fostering sustainable integrated medical and behavioral health services in medical settings. Ann. Intern. Med. 160, 61-65 (2014).

31. Kroenke, K. \& Unützer, J. Closing the false divide: Sustainable approaches to integrating mental health services into primary care. J. Gen. Intern. Med. 32, 404-410 (2016).

32. Wise, M. G. \& Rundell, J. R. Clinical Manual of psychosomatic medicine - a guide to consultation-liaison psychiatry. American Psychiatric Press Inc. (2005).

33. Desan, P. H. et al. Proactive psychiatric consultation services reduce length of stay for admission to an inpatient medical team. Psychosomatics 52,513-520 (2011).

34. Wood, R. \& Wand, A. P. The effectiveness of consultation-liaison psychiatry in the general hospital setting: A systematic review. J. Psychosom. Res. 76, 175-192 (2014).

35. Wolf, M. et al. Psychiatric and psychosomatic consulationliaison. An overview. Nervenartz 84,639-47 (2013).

36. Wood, $\mathrm{R}$ et al. Relationship between timeliness of contact and length of stay in older and younger patients of a consultation-liaison psychiatry service. B. J. Psyc. Bull. 39, 128-133 (2015).

37. Chen, K. Y. et al. Why are hospital doctors not referring to Consultation-Liaison Psychiatry? A systemic review. BMC Pssychiatry 16: 390 (2016).

38. Stiefel, F. C. et al. Operationalizing integrated care on a clinical level: The INTERMED project. Med. Clin. North Am. 90, 713-758 (2006).

39. Lobo, E. et al. Identification of components of health complexity on internal medicine units by means of the INTERMED method. Int. J. Clin. Pract. 69, 1377-1386 (2015).

40. Shefer, G. et al. Diagnostic overshadowing and other challenges involved in the diagnostic process of patients with mental illness who present in emergency departments with physical symptoms - a qualitative study. PLoS One 9, e111682 (2014).

41. Shefer, G. et al. Improving the diagnosis of physical illness in patients with mental illness who present in emergency departments: consensus study. J. Psychosom. Res. 78, 346-351 (2015).

42. Burian, R. et al. Crossing the bridge - a prospective comparative study of the effect of communication between a hospital based consultation-liaison service and primary care on general practitioners' concordance with consultation-liaison psychiatrists' recommendations. J. Psychosom. Res. 86, 53-59 (2016).

43. Lücke, C. et al. A comparison of two psychiatric service approaches: findings from the Consultation vs. Liaison Psychiatry-Study. BMC Psychiatry 17: 8 (2017).

44. Zeeck, A. et al. Symptom course in inpatient and day clinic treatment of depression: Results from the INDDEP Study. J. Affect. Disord. 187, 35-44 (2015).

45. Dinger, U. et al. Day-clinic and Inpatient Psychotherapy of Depression (DIP-D) - Secondary Outcomes and Follow-up Results of a Randomized-Controlled Pilot Trial. Psychother. Psychosom. Med. Psychol. 65, 261267 (2015).

46. Wunner, C. et al. Effectiveness of a psychosomatic day hospital treatment for the elderly: A naturalistic longitudinal study with waiting time before treatment as control condition. J. Psychosom. Res. 76, 121126 (2014).

47. Rundell, J. R. et al. Toward defining the scope of psychosomatic medicine practice: Psychosomatic medicine in an outpatient, tertiary-care practice setting. Psychosomatics 49, 487-493 (2008).

48. Bürger, W. et al. Experiences of a working group about linking inpatient psychosomatic rehabilitation and outpatient psychotherapy. Psychother. Psychosom. Med. Psychol. 58, 280-283 (2008).

49. Altmann, U. et al. Outpatient Psychotherapy Reduces Health-Care Costs: A Study of 22,294 Insurants over 5 Years. Front. Psychiatry 7, 98 (2016). 
50. Kruse, J. et al. Psychosomatic and psychotherapeutic outpatient care in Germany - Capacity profile based on data from the National Association of Statutory Health Insurance Physicians. Z. Psychosom. Med. Psychother. 59, 254-272 (2013).

51. Rundell, J. R. et al. Pharmacogenomic testing in a tertiary care outpatient psychosomatic medicine practice. Psychosomatics 52, 141-146 (2011).

52. Leue, C. et al. Functional urological disorders: A sensitized defence response in the bladder-gut-brain axis. Nat. Rev. Urol. 14, 153-163 (2017).

53. Gillies, D. et al. Consultation liaison in primary care for people with mental disorders. Cochrane Database Syst. Rev. 9, CD007193 (2015).

54. Reed, S. J. et al. Effectiveness and value of integrating behavioral health into primary care. JAMA Intern. Med. 176, 691-692 (2016).

55. Coventry, P. et al. Integrated primary care for patients with mental and physical multimorbidity: cluster randomised controlled trial of collaborative care for patients with depression comorbid with diabetes or cardiovascular disease. BMJ 350:h638 (2015).

56. Zimmermann, T. et al. Collaborative nurse-led self-management support for primary care patients with anxiety, depressive or somatic symptoms: Cluster-randomised controlled trial (findings of the SMADS study). Int. J. Nurs. Stud. 63:101-111 (2016).

57. Smith, S. M. et al. Interventions for improving outcomes in patients with multimorbidity in primary care and community settings. Cochrane Database Syst. Rev. 3, CD006560 (2016).

58. Huffman, J. C. et al. Essential articles on collaborative care models for the treatment of psychiatric disorders in medical settings: A publication by the academy of psychosomatic medicine research and evidence-based practice committee. Psychosomatics 55, 109-122 (2014).

59. Sharpe, M. et al. Integrated collaborative care for comorbid major depression in patients with cancer (SMaRT Oncology-2): A multicentre randomised controlled effectiveness trial. Lancet 384, 1099-1108 (2014).

60. Duarte, A. et al. Cost-effectiveness of integrated collaborative care for comorbid major depression in patients with cancer. J. Psychosom. Res. 79, 465-470 (2015).

61. Huffman, J. C. et al. Collaborative care for depression and anxiety disorders in patients with recent cardiac events: The Management of Sadness and Anxiety in Cardiology (MOSAIC) randomized clinical trial. JAMA Intern. Med. 174, 927-935 (2014).

62. Celano, C.M. et al. Cost-effectiveness of a collaborative care depression and anxiety treatment program in patients with acute cardiac illness. Value Health 19, 185-191 (2016).

63. Sharpe, M. A collaborative care approach delivering treatment to patients with depression comorbid with diabetes or cardiovascular disease achieves significant but small improvements over usual care in depression and patient satisfaction. Comment in Evid. Based Ment. Health 18, 122 (2015).

64. Rosendal, M. et al. Enhanced care by generalists for functional somatic symptoms and disorders in primary care. Cochrane Database Syst. Rev. 10, CD008142 (2013).

65. Barnett, K. et al. Epidemiology of multimorbidity and implications for health care, research, and medical education: A cross-sectional study. Lancet 380, 37-43 (2012).

66. Naylor, C. et al. Long-term conditions and mental health: The cost of co-morbidities. London: The King's Fund (2012).

67. Naylor, C. et al. Bringing together mental and physical health: A new frontier for integrated care. London: The King's Fund (2016).

68. National Health Service: Five year forward view. London: National Health Service (2014).

69. Beach, S. R. et al. Implementing collaborative care programs for psychiatric disorders in medical settings: A practical guide. Gen. Hosp. Psychiatry 37, 522-527 (2015).

70. Leue, C. et al. Managing complex patients on a Medical Psychiatric Unit: An observational study of university hospital costs associated with medical service use, length of stay and psychiatric intervention. J. Psychosom. Res. 68, 295-302 (2010).

71. Häuser, W. et al. Conctruct validity and clinical utility of current research criteria of DSM-5 somatic symptom disorder diagnosis in patients with fibromyalgia syndrome. J. Psychosom. Res. 78, 546-552 (2015).

72. Brünahl, C. et al. Mental disorders in patients with chronic pelvic pain syndrome (CPPS). J. Psychosom. Res. 98, 19-26 (2017). 
73. Kendler, K. S. Causal inference in psychiatric epidemiology. JAMA Psychiatry published online May 3, E12 (2017).

74. Diagnostic and Statistical Manual of Mental Disorders. $5^{\text {th }}$ edition. Washington, DC: American Psychiatric Association (2013).

75. Kendler, K. S. David Skae and his nineteenth century etiologic psychiatric diagnostic system: Looking forward by looking back. Mol. Psychiatry 22, 802-807 (2017). 


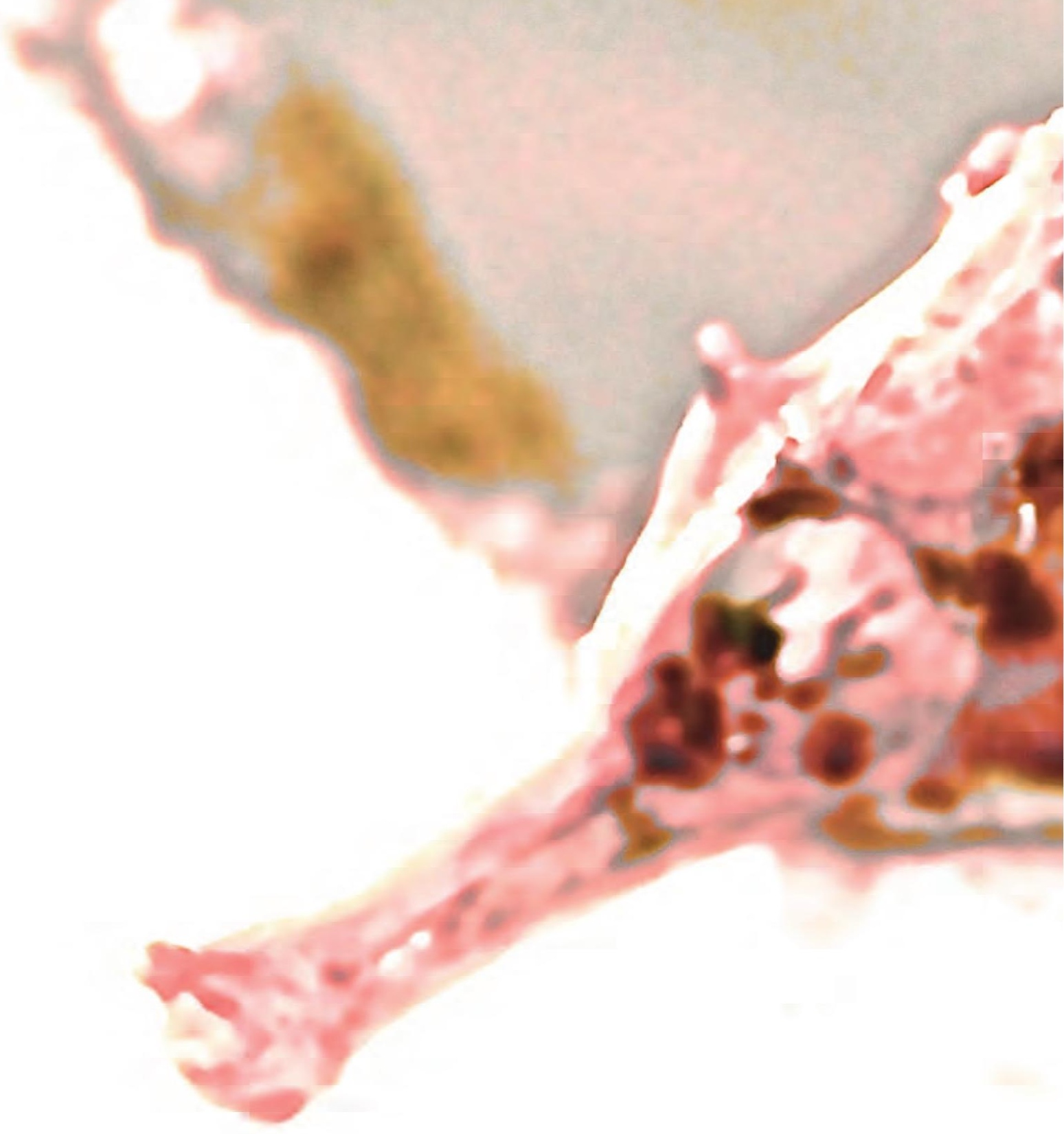




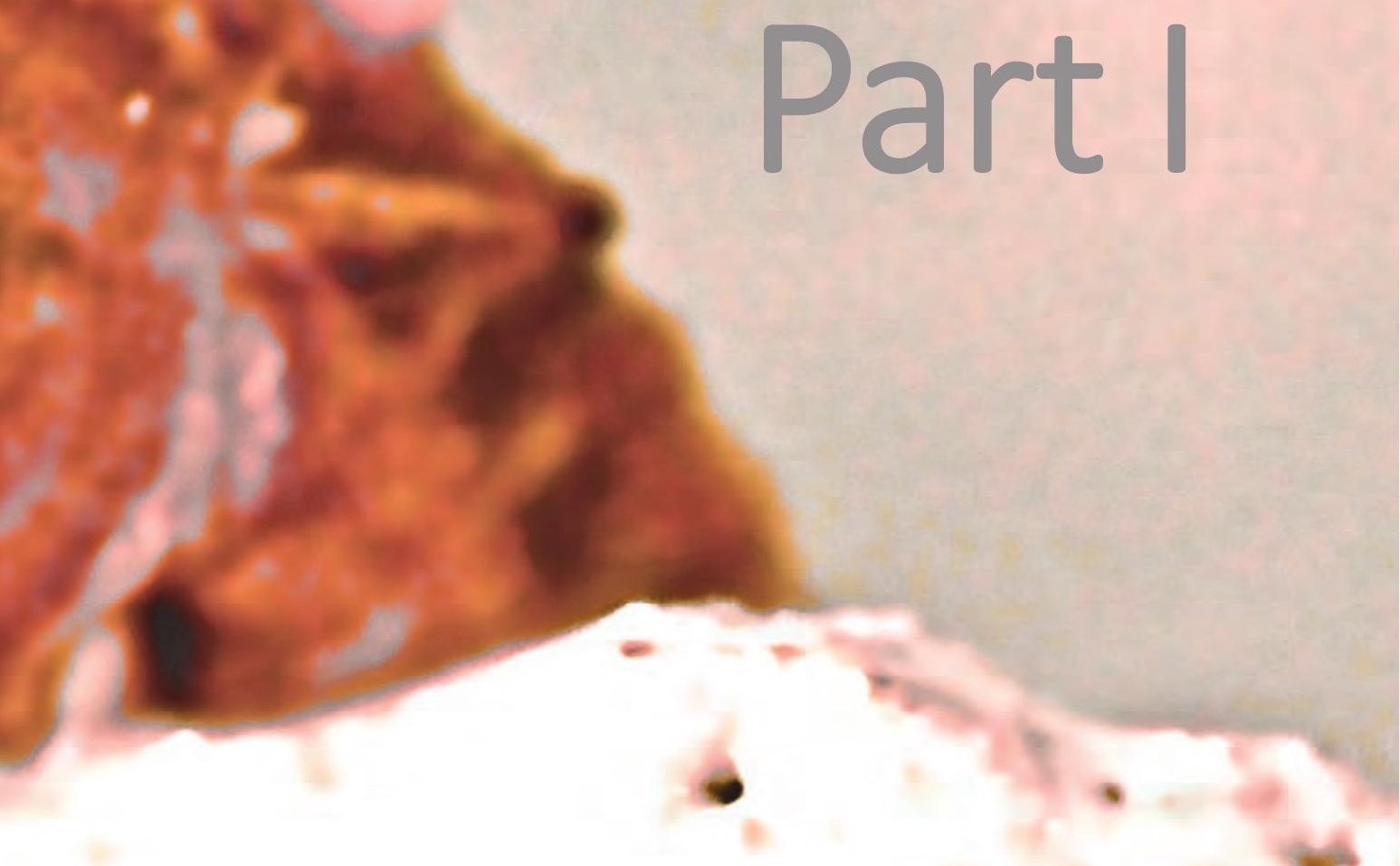

Integrated care at the inpatient

hospital setting 



\section{Abstract}

\section{Objective}

Although there is a suggestion that the Medical Psychiatric Unit (MPU) may reduce length of hospital stay (LOS), little is known about costs in terms of medical service use and psychiatric interventions in MPU care.

\section{Method}

A record linkage study was conducted, linking cost data of hospital medical service use, LOS and hospital psychiatric interventions to patients admitted to the MPU of the Maastricht University Medical Centre (MUMC) between 1998 and 2004. The data set was analysed to enable comparison between cost changes of the same complex patient population following either MPU index admission or index admissions to reference MUMC medical wards.

\section{Results}

Comparisons revealed lower costs of medical service use in favour of the MPU (-€104; $95 \% \mathrm{Cl}-€ 174$ to $-€ 35 ; \mathrm{p}$-value $<0.01)$. However, cost of psychiatric intervention and cost of LOS was higher after MPU admission (respectively: $+€ 165 ; 95 \% \mathrm{Cl}+€ 25$ to $+€ 305$; $\mathrm{p}$-value $<0.05$; and $+€ 202 ; 95 \% \mathrm{Cl}+€ 170$ to $+€ 235 ; \mathrm{p}$-value $<0.001)$. Total costs were higher after MPU admission compared to Medical Ward admission (+€263; 95\%+€68 to $+€ 458$; $p$-value $<0.05$ ). These differences were not moderated by somatic diagnosis or previous pattern of admissions.

\section{Conclusion}

The findings suggest that patients at the interface of psychiatric and somatic morbidity are diagnosed and treated adequately at the MPU, leading to a decrease in medical service use and an appropriate increase in exposure to psychiatric interventions. These results are specifically generalizable to MPUs with a focus on psychosomatic conditions, for instance, somatoform disorders or affective disorders with comorbid somatic diseases. However, failure to show cost savings in terms of LOS compared to medical wards outweighs cost-benefit derived from lower medical service use, suggesting that MPU activities may gain in cost-effectiveness if shifted more to outpatient psychosomatic care solutions. 


\section{Introduction}

The epidemiology of psychiatric disorders in medically ill patients illustrates the extent and origin of their comorbidity. Birth cohort data suggest that psychiatric disorders increase the odds of reporting physical complaints and that childhood mental ill-health predicts physical symptoms in adulthood ${ }^{1}$. In addition, somatic multi-morbidity and psychiatric ill-health are associated with behavioural features early in life, suggesting shared causes ${ }^{2}$. In particular, the personality trait neuroticism is associated with somatic and psychiatric ill-health and predicts later onset of psychiatric disorders as well as a spectrum of chronic somatic diseases ${ }^{2-4}$. Another feature associated with psychopathology and physical complaints is somatisation, which is associated with high levels of somatic service use in primary care, including higher use of somatic and psychotropic drugs 5,6 . Furthermore, patients with functional somatic symptoms are referred more often to somatic specialists, while showing more psychiatric morbidity ${ }^{7}$. Somatising patients also have more hospital admissions, eventually resulting in twice the annual medical care costs compared to non-somatisers ${ }^{8}$. In general hospitals, high rates of psychiatric and somatic comorbidity are typically found, not only in terms of somatoform disorders, but also with regard to affective disorders. The MICRO-CARES database showed that mood disorders rank first on prevalence lists of various specialties $^{9-11}$. Depression is increasingly recognised as a common comorbid disorder in patients with severe or chronic medical conditions ${ }^{12-14}$. Furthermore, depressive disorders may affect the prognosis of somatic conditions ${ }^{15,16}$ and the level of health care consumption, as evidenced by an increase in length of hospital stay (LOS) ${ }^{17,18}$. In patients admitted to an internal medicine ward, self-reported depressive symptoms are associated with physician's assessment of the severity of somatic conditions ${ }^{19}$. However, almost two-thirds of all patients with abnormal scores were not identified by the attending physician as suffering from psychiatric problems ${ }^{19,20}$. Similarly, physicians detect only one third of all cases in somatoform disorders ${ }^{21}$. Numerous investigations have demonstrated that patients with medical conditions and comorbid psychiatric disorders have greater morbidity associated with medical illness, increased health care use and worse functional outcome $\mathrm{e}^{22-24}$. Part of the excess morbidity may be the result of poorer preventive practices among psychiatric populations ${ }^{25-27}$.

Over the last two decades, emerging knowledge about the extent and impact of psychosomatic morbidity was a reason for sustained efforts in improving integrated multidisciplinary approaches from primary care to specialised psychiatric consultation liaison services ${ }^{28-30}$. In addition, since the late 1980s, the concept of the Medical Psychiatric Unit (MPU) has been influential in the development of psychosomatic health care in patients with most complex comorbidity ${ }^{31,32}$. 


\section{Medical Psychiatric Unit}

Worldwide, MPUs have been established to cater for the combination of somatic and psychiatric needs in hospital settings. A number of MPU descriptive studies have been published ${ }^{33-38}$. Only one investigation focussed indirectly on aspects of hospital costs by examining LOS. This study found that the MPU was able to decrease LOS in patients with combined medical and psychiatric diagnoses (total 358 patients) from more than 20 to less than 15 days, compared to admissions in other general hospital wards ${ }^{39}$. Thus, the scarce LOS data on MPU care suggest that MPUs may help patients with complex psychiatric and somatic pathology and may reduce LOS-related health care costs. However, the evidence concerning cost-effectiveness in MPUs is limited. In particular, evidence regarding cost of hospital stay remains to be replicated. This is a critical issue, given that LOS is the most important contributor to overall costs, accounting for approximately $80 \%$ of hospital costs, while the use of ancillary services can explain an additional $20 \%{ }^{40}$. Similarly, no controlled data have been presented regarding medical costs before and after MPU admission other than LOS. Arguably, data from a pre-post comparison, while acknowledging limitations related to possible confounding due to changing circumstances over the period of observation, represent an opportunity to inform the field further, in particular, if routine clinical data are available. The current study is the first to report a controlled MPU pre-post comparison in terms of university hospital costs regarding LOS, medical service use and psychiatric interventions, compared to medical wards.

\section{Aim}

The paper examines whether costs associated with medical service use [e.g., medical outpatient care and medical diagnostic and therapeutic procedures: laboratory tests, x-rays, haemodialysis, etc. (with the exception of pharmacy costs)] and LOS (i.e., bed costs) were lower after index admission to the MPU compared to index admissions of the same complex patient population to medical wards at Maastricht University Medical Center (MUMC). Furthermore, this study investigates whether costs associated with psychiatric interventions (e.g., MUMC psychiatric outpatient interventions and MUMC psychiatric day clinic interventions) of the same population were higher after MPU index admission compared to index admissions to medical wards of the same hospital.

Hypothesized moderators of cost changes are presence of somatic diagnosis and previous pattern of hospital service use ${ }^{41}$. Somatic diagnosis is a crucial factor with regard to costs. For instance, some MPUs cater for "functional" comorbidity and apply severe somatic illness as an exclusion criterion. Similarly, patients with a frequent pattern of hospital service use represent a subgroup with high impact on costs. Thus, a variable stage was constructed according to whether the patient was relatively new (first 
or second admission: initial) or whether there were more preceding admissions (third or later admission: advanced).

\section{Methods}

\section{Setting}

In the period from fall 1998 until winter 2004, 458 patients were admitted to the MPU at MUMC. This MPU was located on an internal medicine ward (gastroenterology (GE) section), characterised as a type III/IV unit, indicating that patients were admitted with a medium to high severity of psychiatric or somatic disorders ${ }^{42}$. Three consultants were working together on a regular basis: a gastroenterologist, a cardiologist and a psychiatrist. In addition, a psychologist, somatic and psychiatric nursing staff, and occupational therapists were members of the team.

Patients were referred by general practitioners (GP), hospital consultants or consultants from other health care facilities. A gate keeping function was provided by the psychiatrist and somatic specialists. Patients who did not need to be admitted were diagnosed and treated at the level of outpatient services. The patient population was characterised by complex psychiatric and somatic comorbidity, varying from chronic somatic diseases (e.g., inflammatory bowel disease, heart failure, etc.) and combined affective disorders to functional syndromes and somatoform disorders (e.g., irritable bowel disease, non-cardiac chest pain, etc.), or chronic severe mental illness combined with physical disorders (e.g., schizophrenia and diabetes mellitus, etc.). Patients suffering from severe somatic conditions with a comorbid delirium were not admitted. Instead, delirium care was provided at the medical ward in question. Furthermore, patients with high suicide risk were not admitted to the MPU. Depending on the medical or psychiatric problem, these patients were either admitted to the general psychiatric unit (GPU) or remained to be admitted at medical wards. During admission, somatic, psychiatric and psychological diagnostic procedures were initiated as needed, followed by somatic and psychiatric treatment as indicated ${ }^{43}$, often in combination with cognitive behavioural therapy (CBT)- based reattribution of unexplained somatic complaints to stress-related experiences ${ }^{44}$. After admission, patients were referred to the psychiatric day clinic, the MPU outpatient department, or back to the referring consultant, depending on the patient's diagnosis and region of residence.

\section{Data collection}

In the Netherlands, different sources of medical cost reimbursement exist: one for general hospital care and a national insurance covering mental health services. Consequently, two datasets from different sources were analysed, one containing data on somatic medical care and one on mental health care. Both datasets referred to the 
source population of a region in the south of the Netherlands with a medium-sized town (Maastricht, 120,000 inhabitants) and surrounding villages (90,000 inhabitants), for which the MUMC is the only hospital providing somatic care. Only patients originating from the source population were included. Patients from outside the catchment area were not taken into account, because follow-up of cost prices was not possible.

In order to identify MPU patients, data from the Psychiatric Case Register South Limburg (PCR) were used ${ }^{45}$. This register cumulatively collected data on mental health care contacts from the abovementioned area. Participating facilities were the general psychiatric hospital, the community mental health care centre, the psychiatric department of the MUMC, and others. Data from the same patient collected from different services were linked using a probability linkage procedure involving five variables: sex, date and place of birth, first three letters of (maiden) name and place of residence. In the limited subset of mental health care users, these variables were sufficient to identify patients without risk of violating privacy.

Data relevant for LOS, medical service use and psychiatric interventions were obtained from MUMC data concerning somatic and psychiatric health care. The medical administration provided full cost prices anonymously.

\section{Selection criterion}

Sample selection criteria were: (i) having been admitted at least once to the MPU and (ii) being inhabitant of the catchment area. The sampling frame consisted of all patients admitted to the MPU or to medical wards, over the period from 1998 to 2004, in combination with their somatic and psychiatric service use, both at the MPU and at other clinical wards of the MUMC. As many of these patients had been admitted not only to the MPU but also to medical wards, a comparison of costs was possible around index admission to the MPU in relation to costs around index admission of the same complex patient population to medical wards, as described in more detail below.

\section{Statistical analysis}

The aim of the study is to analyse costs of the same complex patient population regarding medical service use, LOS and psychiatric intervention before and after an index admission to the MPU or other MUMC wards. The longitudinal data set used in the present study consisted of multiple 28-day episodes (hereafter "observation episode") per patient during 1 year before and 1 year after index admission. Reference point was the first day of index admission: the last observation episode before index admission ended the day before the first admission day; the first observation episode after index admission started on the first day of admission. Thus, the overall observation period is a 1-year period before or a 1-year period after index admission (hereafter "observation period"). Costs were analysed in the year before index admission and the year following and including that first day of admission. Medical costs, bed costs and psychiatric costs 
were collected per day and summed per episode of 28 days, in order to examine trends in cost. All admissions were included, meaning that costs around one admission could count for different index admissions and different settings.

Given the fact that this procedure resulted in more than one observation period per individual (compromising statistical independence of observations), multilevel regression analyses using the STATA XTREG routine (multilevel random-effects model) were conducted to conservatively adjust standard errors and $P$ values for clustering within individuals ${ }^{46}$.

Costs after index admission were compared with costs before index admission using the variable phase ( $0=$ before; $1=$ after). The hypothesis that costs regarding medical service use and LOS would be lower and cost of psychiatric intervention would be higher in the MPU group than in the medical ward group (variable "group" denoting $0=$ medical ward; 1=MPU) was analysed in the main effect model by adding the interaction term phase*group.

In order to examine whether somatic diagnosis or stage moderated the phase*group interaction, additional three-way interactions were fitted with these two variables.

Patients who died so that subsequently it was impossible to complete observations were excluded from the index admission in question. Index admissions with 1-year observation periods outside the 1998-2004 sampling period were also excluded from analysis. Furthermore, patients who were admitted to non-MPU beds of the same GE ward were excluded, because overlap in working staff would have biased the outcome in one way or another by overlap in treatment attitude.

\section{Results}

\section{Sample and episodes}

One hundred eighty-three MPU patients could be linked to the PCR of the wider Maastricht area. Nine patients were excluded because they were only admitted outside the 1998-2004 sampling period. The remaining 174 patients were often admitted several times. For five patients, some index admissions (total 14, seven MPU and seven medical wards) were excluded from analysis because the patients died within the observation period following the first day of admission. For the same reason, two patients did not have any MPU index admission in the final analysis. For another five patients, there was no MPU index admission available because the 1-year observation period at the MPU started after 31 December 2003. Furthermore, 25 patients were excluded because they were not only admitted to the MPU but also to the GE section of the same ward.

Thirty-two patients were only admitted to the MPU; 117 patients were admitted to both MPU and medical wards. These 149 patients generated 624 observation episodes 
(160 MPU, 464 medical wards). There were 15 male and 17 female patients with index admission only to the MPU and 60 male and 57 female patients with index admissions to both settings. The mean age of the population was 52.8 years (S.D.=15.1). The mean LOS at the MPU was 24.1 days (S.D.=11.3), while the same complex patient population was admitted on average 8.2 days (S.D.=13.0) on medical wards (Table 2.1).

Table 2.1 Basic demographic characteristics of the study sample.

\begin{tabular}{lc}
\hline & $\begin{array}{c}149 \text { complex patients }=624 \text { observation periods } \\
\text { Number of admissions: }\end{array}$ \\
& Mean age of study population: 52.8 years (S.D. $=15.1$ ) \\
\hline 160 periods at MPU & 464 periods at medical wards \\
32 patients only admitted to MPU & 117 patients admitted to MPU \\
& and medical wards \\
Gender at MPU: & Gender at medical wards: \\
17 females/15 males & 57 females /60 males \\
Mean LOS at MPU: & Mean LOS at medical wards: \\
24.1 days (S.D.=11.3) & 8.2 days (S.D.=13.0) \\
\hline
\end{tabular}

Legend: ${ }^{a}$ The longitudinal data set used in the present study consisted of multiple 28-day episodes per patient during 1 year before and/or 1 year after index admission (i.e., observation period).

\section{Category and type of diagnosis}

The category of diagnosis at the MPU was psychiatric in $89 \%$ of the observations. Patients at the MPU received a somatic diagnosis in $44 \%$ of the observations. Furthermore, patients were diagnosed as suffering from a functional disorder (e.g., irritable bowel disorder, etc.) in $29 \%$ of the observations. The proportion of patients receiving a combined diagnosis was $41 \%$.

Only $6 \%$ of patients were diagnosed as suffering from a psychiatric disorder during admission on a medical ward, whereas the proportion receiving a somatic diagnosis was $69 \%$. In addition, $25 \%$ of patients were diagnosed as suffering from a functional disorder. Only $4 \%$ of patients received a combined diagnosis (Table $2.2 \mathrm{a}$ ).

Table 2.2a Distribution of diagnostic categories at the MPU and Medical Wards per observation period ${ }^{\mathrm{a}}$.

\begin{tabular}{|c|c|c|}
\hline Category of diagnosis $^{b}$ & MPU (160 periods) ${ }^{a}$ & Medical Wards (464 periods) \\
\hline Psychiatric diagnosis & $143(89 \%)$ & $28(6 \%)$ \\
\hline Somatic diagnosis & $70(44 \%)$ & 320 (69\%) \\
\hline Functional diagnosis ${ }^{c}$ & $46(29 \%)$ & 65 (41\%) \\
\hline Combined diagnosis $^{d}$ & $118(25 \%)$ & $18(4 \%)$ \\
\hline
\end{tabular}

Legend: ${ }^{a}$ The longitudinal data set used in the present study consisted of multiple 28-day episodes per patient during 1 year before and/or 1 year after index admission (i.e., observation period). ${ }^{b}$ Multiple diagnoses possible. ' Unexplained somatic symptoms, irritable bowel syndrome, fibromyalgia, etc. ${ }^{d}$ Somatic or functional, + psychiatric.

Regarding type of somatic diagnosis, minor somatic diseases (20\%), diagnosis of the circulatory system (15\%), and the digestive system (14\%) were most frequent at the 
MPU. Anxiety (54\%) and mood disorders (45\%) ranked first in psychiatric disorders (Table 2.2b).

Table 2.2b Type of diagnosis at the MPU ${ }^{a}$ during 160 observation periods ${ }^{b}$.

\begin{tabular}{lccccccc}
\hline Somatic & $\begin{array}{c}\text { Circulatory } \\
\text { diseases }\end{array}$ & $\begin{array}{c}\text { Respiratory } \\
\text { diseases }\end{array}$ & $\begin{array}{c}\text { Digestive } \\
\text { diseases }\end{array}$ & $\begin{array}{c}\text { Metabolic } \\
\text { diseases }\end{array}$ & $\begin{array}{c}\text { Genitourinary } \\
\text { diseases }\end{array}$ & $\begin{array}{c}\text { Other major } \\
\text { diseases }^{c}\end{array}$ & $\begin{array}{c}\text { Other minor } \\
\text { diseases }^{d}\end{array}$ \\
\hline Psychiatric & $\begin{array}{c}\text { Mood } \\
\text { disorders }\end{array}$ & $\begin{array}{c}\text { Anxiety } \\
\text { disorders }\end{array}$ & $\begin{array}{c}\text { Somatoform } \\
\text { disorders }\end{array}$ & $\begin{array}{c}\text { Psychotic } \\
\text { disorders }\end{array}$ & $\begin{array}{c}\text { Personality } \\
\text { disorders }\end{array}$ & Addiction & $\begin{array}{c}\text { Other } \\
\text { disorders }^{f}\end{array}$ \\
\hline & $72(45 \%)$ & $87(54 \%)$ & $58(36 \%)$ & $10(6 \%)$ & $37(23 \%)$ & $9(6 \%)$ & $11(7 \%)$ \\
\hline
\end{tabular}

Legend: ${ }^{a}$ Multiple diagnoses possible. ${ }^{b}$ The longitudinal data set used in the present study consisted of multiple 28-day episodes per patient during 1 year before and/or 1 year after index admission (i.e., observation period). ${ }^{c}$ e.g., cancer. ${ }^{d}$ e.g., cataract, otitis etc. ${ }^{e}$ No patient with delirium. ${ }^{f}$ e.g., cognitive disorder.

During medical ward admissions, minor somatic diseases (33\%) and diagnoses of the circulatory system (27\%) were the most frequent somatic diagnoses, taking into account that admissions to the GE ward were excluded. Mood disorders, anxiety disorders, and psychotic disorders ranked each $<5 \%$ at medical wards (Table 2.2c).

Table 2.2c Type of diagnosis at medical wards ${ }^{a}$ during 464 observation periods ${ }^{b}$.

\begin{tabular}{lccccccc}
\hline Somatic & Circulatory & Respiratory & Digestive & Metabolic & Genitourinary & Other major & Other minor \\
& diseases & diseases & diseases $^{c}$ & diseases & diseases $^{\text {diseases }^{d}}$ & diseases $^{{ }^{2}}$ \\
& $124(27 \%)$ & $25(5 \%)$ & $33(7 \%)$ & $16(3 \%)$ & $33(7 \%)$ & $29(6 \%)$ & $152(33 \%)$ \\
Psychiatric & Mood & Anxiety & Somatoform & Psychotic & Personality & Addiction & Other \\
& disorders & disorders & disorders & disorders & disorders & & disorders $^{f}$ \\
& $12(3 \%)$ & $10(2 \%)$ & $2(0 \%)$ & $3(1 \%)$ & $1(0 \%)$ & $5(1 \%)$ & $1(0 \%)$ \\
\hline
\end{tabular}

Legend: ${ }^{a}$ Multiple diagnoses possible. ${ }^{b}$ The longitudinal data set used in the present study consisted of multiple 28-day episodes per patient during 1 year before and/or 1 year after index admission (i.e., observation period). ' Admission to gastroenterology ward excluded (see Statistical Analysis). ${ }^{\text {d }}$ e.g., cancer. ${ }^{\text {e }}$ e.g., cataract, otitis etc. ${ }^{f}$ e.g. cognitive disorder.

\section{MPU vs. medical ward cost comparison}

A main pre-post cost reduction regarding cost of medical service use after MPU index admission compared to index admissions to medical wards was observed in favour of the MPU in the main model without moderators (-€104; 95\% $\mathrm{Cl}-€ 174$ to $-€ 35 ; P<0.01$ ). Psychiatric costs were higher in the pre-post comparison after MPU admission compared to medical wards $(+€ 202 ; 95 \% \mathrm{Cl}+€ 170$ to $+€ 235 ; P<0.001)$. Costs of LOS were also higher in MPU admission compared to medical wards $(+€ 165 ; 95 \% \mathrm{Cl}+€ 25$ to $+€ 305$; $P<0.05)$. Thus, total costs were higher after MPU admission compared to Medical Ward admission (+€263; 95\%+€68 to +€458; $P$-value <0.05) (Table 2,3). 
Table 2.3 University Hospital Costs in the medical ward vs. MPU model (costs after index-admission minus costs before index-admission).

\begin{tabular}{lcccc}
\hline Type of ward (Patients) & Medical Costs & Bed Costs & Psychiatric Costs & Total Costs \\
\hline Medical wards (117) vs. & $-€ 104$ & $+€ 165$ & $+€ 202$ & \\
MPU (142) & $(-€ 174 \text { to } €-35)^{* *}$ & $(+€ 25 \text { to }+€ 305)^{*}$ & (+€170 to +€235) $^{* * *}$ & $(+€ 68$ to +€458) \\
\hline
\end{tabular}

Legend: MPU vs. medical ward model refers to a comparison between medical ward and MPU as regards costs in medical service use, LOS (bed costs) and psychiatric intervention. Data are presented as beta weights (95\% $\mathrm{Cl}$ ) and show, for example, a decrease in costs of -€104 in favour of the MPU in the first column of "medical costs". 7 patients had no MPU index admission (see results - Sample and Episodes). ${ }^{*} P<0.05 .{ }^{* *} P<0.01$. ${ }^{* * *}$ $P<0.001$.

The phase*group interaction was not additionally moderated by either somatic diagnosis or stage - three way interactions with these variables were neither large nor significant.

\section{Discussion}

Admission of the same complex patient population to the MPU on one occasion or to medical wards on another led to a different distribution in categories of diagnosis. In the case of admission to the MPU, a psychiatric disorder was diagnosed in $89 \%$ of cases. However, if admission took place on a medical ward, a psychiatric diagnosis was confirmed in only $6 \%$. This is a remarkable difference in frequency of psychiatric diagnoses per setting. The reason for such a substantial difference in detected cases might be that patients admitted to medical wards were less psychiatrically and more physically ill compared to the period of admission to the MPU. However, better case detection at the MPU setting likely plays a major role, as psychiatric disorders at the MPU setting are frequently chronic or repetitive in course ${ }^{47}$. Thus, more likely and in accordance with the literature, psychiatric disorders were probably underrecognized in medical wards $^{18,19}$.

Regarding somatic diagnoses or somatic complaints, there was almost no difference (functional complaints) or only a small difference (somatic diagnoses) in case detection as a function of setting. This indicates that an integrated approach at the MPU may guarantee adequate psychiatric and somatic case finding. Furthermore, the distribution of somatic diagnoses per affected organ system did not differ much between the two settings, suggesting representative case detection of somatic illnesses at the MPU.

Concerning costs, medical service use data revealed a significant main difference in favour of the MPU. An explanation regarding reduction in cost of medical service use could be that the case mix at medical wards was more characterised by physical illness and required more diagnostic tests and treatment procedures. However, the results indicate that this may not be likely given the fact that the patient population did not differ much concerning frequency and type of somatic diagnosis at each setting. Furthermore, there was no evidence that cost differences were moderated by somatic 
diagnosis. Thus, the findings suggest that patients admitted to the MPU were not less complex compared to medical wards.

On the other hand, there was an increase in costs associated with psychiatric interventions compared to reference wards, reflecting the desired outcome of increased psychiatric treatment. The suggestion from an earlier investigation that MPUs may reduce LOS in complex patients could not be replicated ${ }^{39}$. Given the fact that LOS was much lower in medical wards, bed costs were significantly higher at the MPU. Thus, cost associated with extended hospitalisation and psychiatric treatment outweighs costbenefit derived from lower medical service use, at least in an MPU focusing on psychosomatic conditions (e.g., affective disorders with comorbid somatic diseases, somatoform disorders). The dramatically longer LOS was not related to the provision of care for delirium, or treatment for suicidal behaviour, since there was only one patient included with delirium (medical ward) and suicidal patients were only admitted to the GPU, or received treatment at medical wards in case of medical complications. The most likely explanation for the extended LOS may be that CBT-based reattribution of physically experienced symptoms to stress-related experiences mainly took place clinically. Thus, bed costs may be saved if psychiatric interventions are given in time. The question rises whether psychiatric interventions offered on an MPU inpatient basis may be provided as effectively as in an outpatient setting, given the low recognition of psychiatric comorbidity at medical wards. However, our data suggest that in order to organise care provided by MPUs more cost-effectively, a shift from inpatient to day-care and outpatient settings may be desirable. An exception may be formed by MPUs targeting delirium in severe somatic conditions and self-harm in the context of severe mental illness, as in these cases LOS may be shortened if a multidisciplinary team provides integrated care and treatment on an outpatient basis is not desirable ${ }^{48}$.

Over the last decennia, change in health care systems due to alterations in reimbursement policies and improvement of medical technology has profoundly affected the delivery of care. There has been a major shift away from inpatient to outpatient settings and LOS has become increasingly shorter ${ }^{49,50}$. The data suggest that services catering at the interface of medical and psychiatric comorbidity are also under pressure to adjust to these trends and should focus more on proactively operating liaison psychiatric services with extended day-care and outpatient settings. In addition, alliances with community mental health services can be formed to create collaborative services inside the hospital and at primary care settings, possibly resulting in cost reductions without compromising quality of care ${ }^{51,52}$. Thus, "Med-Psych-Centres" may more appropriately come to refer to a range of ambulatory solutions for a group of complex patients requiring integrated care, rather than a collection of hospital beds alone. A group of patients will continue to require MPU care and for these patients multidisciplinary inpatient care must be available. This, however, cannot be equated with the need to maintain a nonembedded unit without transitional integrated care solutions and high-added costs due to the increasing complexity of health care delivery 
over the last decade $e^{53-55}$. Within the distinction between case complexity and care complexity, this study contributed to the literature by adding university hospital cost data on medical service use, LOS and psychiatric interventions which are related to both patient characteristics and aspects of health care ${ }^{55}$.

\section{Methodological issues}

The results of this study should be seen in the light of several limitations. The use of routine data rather than a targeted data collection could have caused more random error resulting in type II error. Confounding may have played a role, as randomisation was not possible and pre-post designs are sensitive to effects of unmeasured changes affecting outcome measures over time. However, there is no reason to assume that this would have been different for MPU and medical ward admissions. Nevertheless, there were no data available regarding changes in physicians over time, with different diagnostic and treatment habits, which could have influenced the outcome. Furthermore, there was no possibility to control for evolving medical technology over time. Again, however, there is no reason to assume that technological innovations would have been applied differentially to the MPU or medical wards. Reimbursement developments over time may have changed selection criteria for admission, resulting in stricter gate keeping. This phenomenon would likely have influenced somatic wards as well.

Another limitation is the lack of outcomes other than hospital cost data. For instance, there were no estimates regarding primary care consumption or illness-related sick leave from work. Changes in more patient-related outcomes like illness severity, global functioning, quality of live and treatment satisfaction should be part of any prospective evaluation of MPU care. Indeed, a main limitation of the current report is the lack of data regarding health status outcome. There are indications that admission to a mixed psychiatric and somatic care unit can influence patient satisfaction positively ${ }^{56}$. Finally, this study only collected data over a twelve-month period before and after index admission. Evidence of collaborative depression treatment in medically ill patients at the primary care setting suggests that cost savings persist even in the second year after the initiation of treatment ${ }^{28}$.

\section{Recommendations}

Fragmentation of health care, separating medical and behavioural interventions, results in poor treatment outcomes, increased medical service use and impaired work performance ${ }^{57}$.

Defragmentation of health care is cost effective ${ }^{28}$. Defragmentation by applying clinically integrated health care for the most complex patients with severe somatic and psychiatric comorbidity at the MPU may contribute to cost-effectiveness but further evidence is necessary. LOS is a critical variable ${ }^{40}$. Thus, MPU care targeting patient 
groups that are sensitive to inpatient interventions should be embedded in psychosomatic hospital care programs (Med-Psych-Centres), offering ambulatory solutions where possible, in order to shorten hospital stay. Prospective, randomised controlled trials of sufficiently long duration analysing case and care complexity in cost developments around MPU care are required. 


\section{References}

1. Hotopf, M. et al. Temporal relationships between physical symptoms and psychiatric disorder. Results from a national birth cohort. Br. J. Psychiatry 173, 255-261 (1998).

2. Neeleman, J. et al. Propensity to psychiatric and somatic ill-health: Evidence from a birth cohort. Psychol. Med. 32, 793-803 (2002).

3. Goodwin, R. D. \& Gotlib, I. H. Sex differences in depression: The role of personality factors. Psychiatry Res. 126, 135-142 (2004).

4. Kroenke, K. \& Spitzer, R. L. Sex differences in the reporting of physical and somatoform symptoms. Psychosom.Med. 60, 150-155 (1998).

5. Lipowski, Z. J. Somatization: The concept and its clinical application. Am. J. Psychiatry 145, 1358-1368 (1988).

6. Hollifield, M. et al. Hypochondriasis, somatisation and perceived health and utilisation of health care services. Psychosomatics 40, 380-386 (1999).

7. olde Hartman, T. C. et al. Chronic functional somatic symptoms: A single syndrome? Br. J. Gen. Pract. 54, 922-927 (2004).

8. Barsky, A. J. et al. Orav EJ, Bates DW. Somatization increases medical utilization and costs independent of psychiatric and medical comorbidity. Arch. Gen. Psychiatry 62, 903-910 (2005).

9. Clarke, D. \& Smith, G. Consultation liaison psychiatry in general medical units. Aust. N. Z. J. Psychiatry 29, 424-432 (1995).

10. Kissane, D. W. \& Smith, G. C. Consultation-liaison psychiatry in an Australian oncology unit. Aust. N. Z. J. Psychiatry 30, 397-404 (1996).

11. Judd, F. K. et al. Liaison psychiatry in an HIV/AIDS unit. Aust. N. Z. J. Psychiatry 31, 391-397 (1997).

12. Frasure-Smith, N. et al. Depression and 18-month prognosis after myocardial infarction. Circulation 91, 999-1005 (1995).

13. Koenig, H. G. Depression in hospitalized older patients with congestive heart failure. Gen. Hosp. Psychiatry 20, 29-43 (1998).

14. Kimmel, P. L. et al. Psychiatric illness in patients with end-stage renal disease. Am. J. Med. 105, 214-221 (1998).

15. Penninx, B. W. et al. Depression and cardiac mortality: Results from a community-based longitudinal study. Arch. Gen. Psychiatry 58, 221-227 (2001).

16. Strik, J. J. et al. One year cumulative incidence of depression following myocardial infarction and impact on cardiac outcome. J. Psychosom. Res. 56, 59-66 (2004).

17. Sloan, D. et al. A five year study on the interactive effects of depression and physical illness on psychiatric unit length of stay. Psychosom. Med. 61, 21-25 (1999).

18. Saravay, S. M. \& Lavin, M. Psychiatric comorbidity and length of stay in the general hospital: A critical review of outcome studies. Psychosomatics 35, 233-252 (1994).

19. Meyer, T. et al. Depression but not anxiety is a significant predictor of physicians' assessments of medical status in physically ill patients. Psychother. Psychosom. 69, 147-154 (2000).

20. Kroenke, K. et al. Depressive and anxiety disorders in patients with physical complaints: Clinical predictors and outcome. Am. J. Med. 103, 339-347 (1997).

21. Fink, P. et al. The prevalence of somatoform disorders among internal medical patients. J. Psychosom. Res. 56, 413-418 (2004).

22. Druss, B. G. et al. Health and disability costs of depressive illness in a major US corporation. Am. J. Psychiatry 157, 1274-1278 (2000).

23. Kessler, R. C. et al. The epidemiology of major depressive disorder: Results from the national comorbidity survey replication (NCS-R). JAMA 289, 3095-3105 (2003).

24. Stewart, W. F. et al. Cost of lost productive work time among US workers with depression. JAMA 289, 3135-3144 (2003).

25. Brown, S. et al. The unhealthy lifestyle of people with schizophrenia. Psychol. Med. 29, 697-701 (1999).

26. Brown, S. et al. Causes of the excess mortality of schizophrenia. Br. J. Psychiatry 177, 212-217 (2000).

27. Davidson, S. et al. Cardiovascular risk factors for people with mental illness. Aust. N. Z. J. Psychiatry 35, 196-202 (2001). 
28. Simon, G. E. et al. Cost-effectiveness of systematic depression treatment among people with diabetes mellitus. Arch. Gen. Psychiatry 64, 65-72 (2007).

29. Huyse, F. J. et al. Operationalizing the biopsychosocial model: The INTERMED. Psychosomatics 42, 5-13 (2001).

30. de Jonge, P. et al. Medical inpatients at risk of extended hospital stay and poor discharge health status: Detection with COMPRI and INTERMED. Psychosom. Med. 65, 534-541 (2003).

31. Stoudemire, A. et al. The medical-psychiatric unit as a site for outcome research in dementia/depression syndromes. Psychiatr. Med. 9, 535-544 (1991).

32. Hall, R. \& Kathol, R. Developing a level III/IV medical psychiatric unit: Establishing a basis, design of the unit and pshysician responsibility. Psychosomatics 33, 368-375 (1992).

33. Campo, J. V. \& Raney, D. The pediatric medical-psychiatric unit in a psychiatric hospital. Psychosomatics 36, 438-444 (1995).

34. Nomura, S. et al. Evaluation of the first medical psychiatric unit in Japan. Psychiatry Clin. Neurosci. 50, 305-308 (1996).

35. Fennig, S. \& Fennig, S. Management of encopresis in early adolescence in a medical psychiatric unit. Gen. Hosp. Psychiatry 21, 360-367 (1999).

36. Kishi, Y. \& Kathol, R. Integrating medical and psychiatric treatment in an inpatient medical setting: The type IV program. Psychosomatics 40, 345-355 (1999).

37. van Waarde, J. et al. The medical psychiatric unit: Added value for patients, physicians and hospitals. Ned. Tijdschr. Geneeskd. 148, 209-211 (2004).

38. Hanna, S. J. et al. The coming of age of a joint elderly medicine-psychiatric ward: 18 years experience. Int. J. Clin. Pract. 62, 146-151 (2008).

39. Young, L. \& Harsch, H. H. Length of stay on a psychiatry medicine unit. Gen. Hosp. Psychiatry 11, 31-35 (1989).

40. Goldberg, R. \& Simundson, S. Managing Medicare reimbursement on medical psychiatry units. Gen. Hosp. Psychiatry 13, 313-318 (1991).

41. de Jonge P. et al. Case and care complexity in the medically ill. Med. Clin. N. Am. 90, 679-692 (2006).

42. Kathol, R. et al. Categorisation of types of medical psychiatry units based on level of acuity. Psychosomatics 33, 376-386 (1992).

43. Kuijpers, P. M. et al. Effect of treatment of panic disorder in patients with frequent ICD discharges: $A$ pilot study. Gen. Hosp. Psychiatry 24, 181-184 (2002).

44. Nezu, A. M. et al. Cognitive behavior therapy for medically unexplained symptoms: A critical review of the treatment literature. Behavior Therapy 32, 537-583 (2001).

45. Driessen, G. et al. Stroke an mental health care: A record linkage study. Soc. Psychiatry Psychiatr. Epidemiol. 36, 608-612 (2001).

46. StataCorp: Stata Statistical Software: Release 8.0. College Station, Tex, StataCorp, 2002.

47. Üstün, T. B. et al. Global burden of depressive disorder in the year 2000. Br. J. Psychiatry 184, 386-392 (2004).

48. Schieveld, J. N. et al. Pediatric illness severity measures predict delirium in a pediatric intensive care unit. Crit. Care Med. 36,1933-1936 (2008).

49. Gemmill, E. H. \& McCulloch, P. Systematic review of minimally invasive resection for gastro-oesophageal cancer. Br. J. Surg. 94, 1461-1467 (2007).

50. Kane, R. L. et al. The association of registered nurse staffing levels and patient outcomes: Systematic review and meta-analysis. Med. Care. 45, 1195-1204 (2007).

51. Kinder, L. S. et al. Improving depression care in patients with diabetes and multiple complications. J. Gen. Intern. Med. 21, 1036-1041 (2006).

52. Katon, W. J. et al. The Pathway Study: A randomized trial of collaborative care in patients with diabetes and depression. Arch. Gen. Psychiatry 61, 1042-1049 (2004).

53. Wilson, T \& Holt, T. Complexity and clinical care. BMJ 323, 685-688 (2001).

54. Stewart, M. Continuity, care and commitment: The course of patient-clinician relationships. Ann. Fam. Med. 2, 388-390 (2004).

55. de Jonge, P. et al. Care complexity in the general hospital: Results from a European study. Psychosomatics 42, 204-212 (2001). 
56. Eytan, A. et al. Patient's satisfaction with hospitalization in a mixed psychiatric and somatic care unit. Eur. Psychiatry 19, 499-501 (2004).

57. Kathol, R. et al. Epidemiologic trends and costs of fragmentation. Med. Clin. N. Am. 90, 549-572 (2006). 


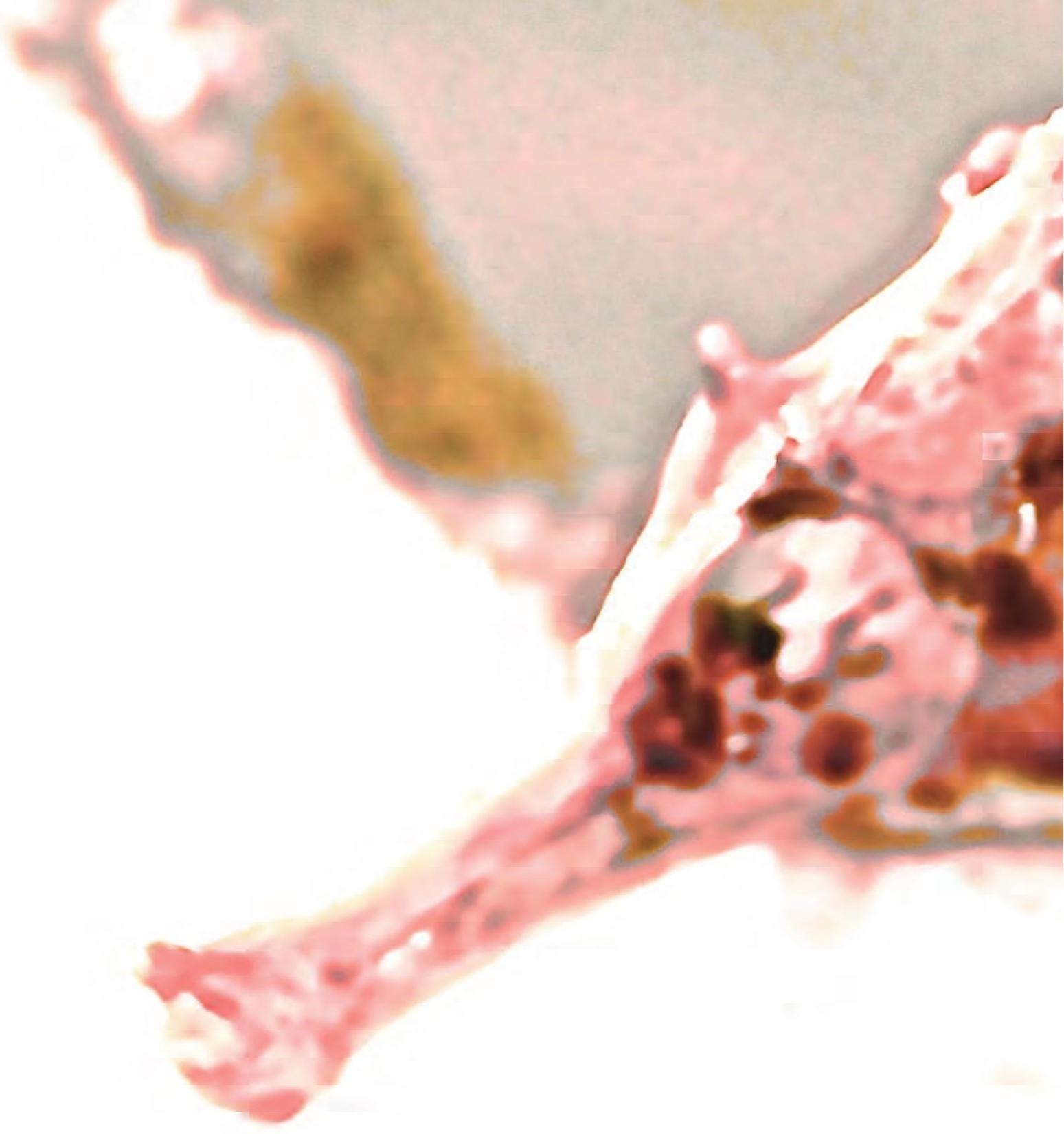




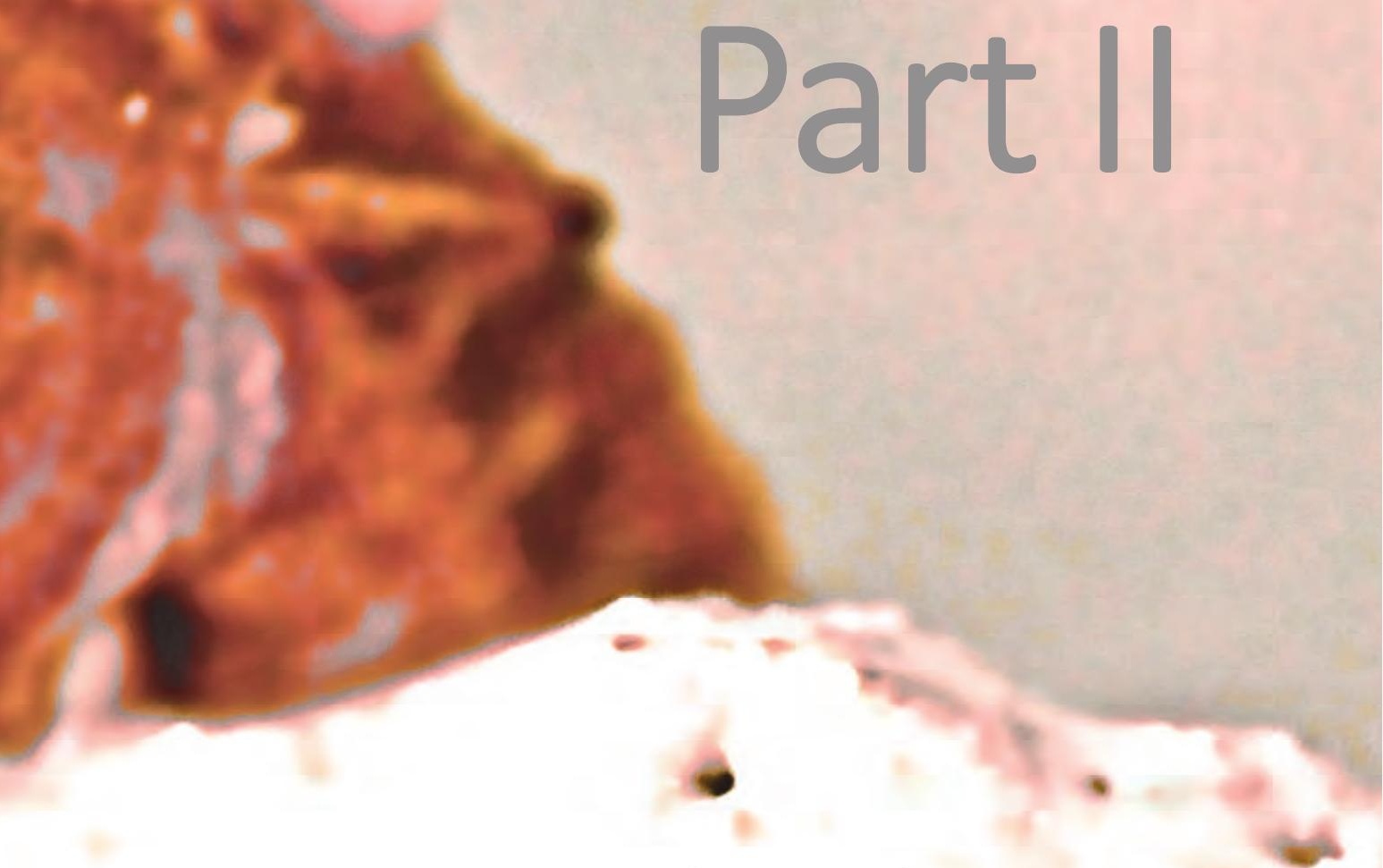

Integrated care at the outpatient hospital setting 



\section{Abstract}

\section{Objective}

To evaluate the presence of medically unexplained otorhinolaryngological symptoms in a patient cohort and propose an interdisciplinary approach for their care.

\section{Methods}

This prospective cohort study describes a population of patients presenting at the Department of Otorhinolaryngology at the Maastricht University Medical Center (MUMC). Patients with symptoms that did not meet clear "medical" criteria and were associated with psychological distress and high healthcare utilization were enrolled in the study by two experienced otorhinolaryngologists following informed consent. The aim of the study is 1) to specify the presence of medically unexplained otorhinolaryngological symptoms (MUORLS) and 2) to evaluate the integration of otorhinolaryngological and psychiatric treatment in an interdisciplinary approach in order to help otorhinolaryngologists improve patient care.

\section{Results}

Of the 102 patients included, $41 \%(\mathrm{~N}=42)$ did not have a proven somatic otorhinolaryngological diagnosis. For only $10.8 \%(\mathrm{~N}=4)$ of the latter, no psychiatric diagnosis had been established. Overall, $78 \%$ of the study population $(\mathrm{N}=80)$ was diagnosed with psychiatric morbidity/comorbidity, as defined in the Diagnostic and Statistical Manual of Mental Disorders, Fourth Edition (DSM-4-TR).

\section{Conclusion}

The preliminary data suggest that the majority of patients with these unexplained complaints may suffer from under- or undiagnosed psychiatric morbidity. Therefore, easy access to integrated interdisciplinary care (otorhinolaryngology and psychiatry) should be offered to patients with MUORLS after detailed information is made available to them about the pathogenesis of the complaints and the foreseen psychosomatic approach. 


\section{Introduction}

Medically unexplained otorhinolaryngological symptoms (MUORLS) are common in tertiary care settings, although exact prevalence rates are unknown ${ }^{1}$. In general, medically unexplained symptoms frequently accompany psychiatric disorders ${ }^{2}$. Nonetheless, psychiatric morbidity/co-morbidity is consistently underrecognized, which precludes effective treatment ${ }^{2}$. Given that MUORLS are associated with high impairment, healthcare costs, risk of iatrogenic damage, and frustration among both physician and patient, it is critical to improve early detection ${ }^{3,4}$. The first step is to identify the underlying disorder, which might be psychiatric, through a diagnostic process that could be lengthy due to the somatic focus of the physician and, therefore, delay successfully targeted interventions ${ }^{5,6}$.

For some otorhinolaryngological (ORL) subcategories, such as oropharyngeal dysphagia, benign paroxysmal positional vertigo, and tinnitus, affective symptomatology such as depression, phobia or anxiety has been reported ${ }^{7,8}$. However, the Dutch evidence-based guidelines on various ORL pathologies/complaints still do not help the physician to recognize and label MUORLS because these guidelines do not cover integrated care. Notably, consultation-liaison (CL) psychiatry and psychosomatics are not part of that recommendation ${ }^{9,10}$.

The current study investigates presentation with MUORLS in a patient cohort and, if present, proposes an integrated interdisciplinary approach (ORL and psychiatry) for their care. Ultimately, this could yield guidelines for multidisciplinary diagnosis to be followed by otorhinolaryngologists. Successful diagnosis may not only help control healthcare costs but may also improve patient care because MUORLS are associated with significant impairment, especially in patients with psychiatric comorbidity.

\section{Materials and methods}

\section{Data collection}

Participants. Patients with MUORLS were recruited from the Department of Otorhinolaryngology at the Maastricht University Medical Center (MUMC). Their complaints were heterogeneous: dizziness, postnasal drip, nasal airway obstruction, pharyngeal Globus sensation, upper airway distress, gagging while eating, tinnitus, and so forth. There were several exclusion criteria: not willing to cooperate with an integrated interdisciplinary approach; suffering from severe depression with suicidal behavior or already having a known psychiatric diagnosis or using antidepressant and/or anxiolytic drugs; not being able to understand the integrated interdisciplinary advice due to cognitive impairment; being under 18 years; and not adequately understanding the 
Dutch language. None of the patients was in a palliative state of disease. Informed consent was obtained from all patients.

\section{Study design and setting}

Patients with no clear "medical" explanation for their symptoms who had associated psychological distress and/or high healthcare utilization were enrolled in the study by two experienced otorhinolaryngologists (L.W.J. B and R. vd B) from December 2011 till December 2013. These somatic specialists served a gate-keeping function and as a last resort. Patients were referred by general practitioners (GP), ORL specialists from other hospitals, or medical practitioners from other healthcare facilities. By their very nature, unexplained symptoms are vague or difficult to characterize, which hampers differential diagnosis. Therefore, all patients underwent a structured interview, a standardized clinical ORL examination, and additional diagnostics (instrumental examinations, imaging, etc.), depending on the kind of symptoms and the Dutch evidence-based guidelines on the various ORL pathologies/complaints ${ }^{9,10}$. Examinations such as computed tomography scanning of paranasal sinuses, endoscopy of the upper aerodigestive tract, videofluoroscopy of swallowing, electronystagmography with caloric and rotatory testing, and magnetic resonance imaging scanning of the brain were performed to detect and/or rule out somatic causes of the complaints. These examinations were often performed before the patients met our two last-resort otorhinolaryngologists. The included patients had complaints in the ORL topographic region without a somatic substrate in the end-organ or with a somatic ORL diagnosis that did not fit the subjective complaints. This means that subjective complaints showed a discrepancy with the outcome of clinical and instrumental examinations. Patients were acquainted with the psychiatrist during a joint consultation in the ORL outpatient clinic. Following this first informative and motivational consultation, patients with a psychiatric diagnosis or lack of coping skills were invited to visit the outpatient clinic for hospital psychiatry and medical psychology at the MUMC in order to undergo further structured clinical psychiatric investigation. Depending on the diagnosis (anxiety disorder, depressive disorder, somatoform disorder, etc.) mental healthcare took place as indicated $^{11}$.

Given the patients' fear of stigmatization, the otorhinolaryngologists were not able to convince all patients with confirmed MUORLS of the benefits of an integrated interdisciplinary approach that draws upon an experienced psychiatrist at the same hospital. In patients diagnosed with psychiatric comorbidity and who consented to accept psychiatric treatment (i.e., pharmacological intervention and/or psychotherapy), follow-up took place either in the integrated ORL-psychiatry setting (in cases of somatic and psychiatric co-morbidity) or in the hospital psychiatry and medical psychology outpatient setting alone (in cases of medically unexplained symptoms and psychiatric morbidity). 


\section{Data analysis}

Variables of interest. Diverse variables were analyzed for the current study. These were selected to reflect the burden on the patient and the health care system. They also indirectly reflect the delay in care due to a difficult differential diagnosis and to multiple diagnostic examinations or visits to exclude somatic causes for the complaints. The variables include the number of visits to the ORL outpatient clinic or other departments, number of additional (instrumental) examinations, number of surgical interventions, and so forth (Table 3.1).

Table 3.1 Variables of interest associated with medically unexplained otorhinolaryngological symptoms.

\begin{tabular}{|c|c|c|}
\hline \multicolumn{2}{|c|}{ Variable } & \multirow{2}{*}{$\begin{array}{l}\text { Definition } \\
\text { Nature or topography of the subjective ORL complaint }\end{array}$} \\
\hline 1 & $\mathrm{ORL}^{\mathrm{a}}$ complaint & \\
\hline 2 & ORL somatic diagnosis (if present) & Established or proven somatic ORL diagnosis \\
\hline 3 & Other somatic diagnosis (if present) & Established or proven somatic diagnosis outside ORL \\
\hline 4 & Number of disciplines for ORL complaint & $\begin{array}{l}\text { Number of medical disciplines and health professions } \\
\text { involved in care for specific ORL complaint }\end{array}$ \\
\hline 5 & Number of disciplines for other complaints & $\begin{array}{l}\text { Number of medical disciplines and health professions } \\
\text { involved in care for other (non-ORL) complaints }\end{array}$ \\
\hline 6 & Number of visits to ORL outpatient clinic & $\begin{array}{l}\text { Number of visits to the ORL outpatient clinic for the } \\
\text { specific subjective ORL complaint }\end{array}$ \\
\hline 7 & Number of instrumental examinations & $\begin{array}{l}\text { Number of instrumental examinations for the specific } \\
\text { subjective ORL complaint }\end{array}$ \\
\hline 8 & Number of ORL surgical interventions & $\begin{array}{l}\text { Number of surgical interventions for the specific } \\
\text { subjective ORL complaint }\end{array}$ \\
\hline 9 & Number of other surgical interventions & $\begin{array}{l}\text { Number of surgical interventions for other (non-ORL) } \\
\text { complaints }\end{array}$ \\
\hline 10 & ORL treatment & ORL treatment for the specific subjective complaint \\
\hline 11 & Psychiatric diagnosis & $\begin{array}{l}\text { Final psychiatric diagnosis underlying the specific } \\
\text { subjective ORL complaint }\end{array}$ \\
\hline 12 & Psychiatric treatment & $\begin{array}{l}\text { Final psychiatric treatment to manage the specific } \\
\text { subjective ORL complaint }\end{array}$ \\
\hline
\end{tabular}

Legend: ${ }^{\mathrm{O} O R L}=$ otorhinolaryngological.

\section{Statistical analysis}

Descriptive statistical data are presented in Table 3.2, 3.3, 3.4, 3.5, 3.6, and 3.7.

\section{Results}

\section{Characteristics of participants}

One hundred and two patients with MUORLS were included (total N=102; 58 females and 44 males). They were receptive to an integrated multidisciplinary approach (ORL\& 
psychiatry) in order to screen for or diagnose psychiatric morbidity/co-morbidity. Their median age was 60 years (25th, 75th percentile: 49; 68 years).

\section{Results of the descriptive statistics}

Table 3.2 presents the frequency distribution of the various subjective ORL complaints for the study population. Fifty-three percent of the patients $(N=53)$ reported dizziness, and the second-largest group mentioned pharyngeal Globus sensation ( $N=14 ; 14.0 \%)$.

Table 3.2 Frequency distribution of the otorhinolaryngological complaints in the study population (N=102).

\begin{tabular}{lc}
\hline ORL $^{\text {a }}$ complaint & $\mathrm{N}(\%)$ \\
\hline Dizziness/vertigo & $53(53.0)$ \\
Pharyngeal globus & $14(14.0)$ \\
Dysphagia & $8(8.0)$ \\
Nasal obstruction & $6(6.0)$ \\
Dysphonia & $5(5.0)$ \\
Choking & $4(4.0)$ \\
Tinnitus & $4(4.0)$ \\
Discomfort tracheostomy & $2(2.0)$ \\
Stridor breathing sounds & $1(1.0)$ \\
Snoring with fatigue & $1(1.0)$ \\
Postnasal drip & $1(1.0)$ \\
Tubair catarrh sensation & $1(1.0)$ \\
Missing values & $2(2.0)$ \\
\hline
\end{tabular}

Legend: ${ }^{a}$ ORL= otorhinolaryngological.

Table 3.3 shows the frequency distribution of the ORL somatic diagnoses for the total study population. Forty-one percent of the patients $(\mathrm{N}=42)$ did not have a proven somatic ORL diagnosis. Out of this share, $10.8 \%(\mathrm{~N}=4)$ did not have an established psychiatric diagnosis.

Table 3.3 Frequency distribution of the otorhinolaryngological somatic diagnoses in the study population $(\mathrm{N}=102)$.

\begin{tabular}{lc}
\hline $\mathrm{ORL}^{\mathrm{a}}$ somatic diagnosis (if present) & $\mathrm{N}(\%)$ \\
\hline No somatic diagnosis & $42(41.2)$ \\
Vestibulopathy (vestibular migraine, Meniel̀re's disease, BPPV ${ }^{\mathrm{b}}$, utricular dysfunction etc.) & $34(33.3)$ \\
Otopathy (hearing loss, recurrent otitis, myringosclerosis etc.) & $7(6.9)$ \\
Head and neck cancer with various stages, locations etc. & $7(6.9)$ \\
Chronic rhinopathy (polyps, rhino sinusitis, maxillary sinus cyst etc.) & $5(4.9)$ \\
Obstructive sleep apnea syndrome & $4(3.9)$ \\
Benign vocal fold pathology (paralysis, polyps, cysts etc.) & $3(2.9)$ \\
Missing values & $0(0)$ \\
\hline
\end{tabular}

Legend: ${ }^{a}$ ORL= otorhinolaryngological. ${ }^{b} \mathrm{BPPV}=$ benign paroxysmal positional vertigo.

Table 3.4 shows the data on healthcare consumption in the present population. The number of disciplines involved, visits to the outpatient clinics, and (instrumental) 
examinations for the ORL complaint and other (non-ORL) complaints were reported. Furthermore, ORL surgery in the topographic area of complaint and interventions for other (non-ORL) disorders/complaints were registered (ventilation tubes, nasal septum correction, microlaryngeal surgery, etc.). The median number of visits to the ORL outpatient clinic was six, and the median number of additional (instrumental) examinations was four.

Table 3.4 Descriptive data of healthcare consumption for the study population $(N=102)$.

\begin{tabular}{|c|c|c|c|}
\hline \multicolumn{2}{|c|}{ Variable } & Median $\left(25^{t} h\right.$ perc ${ }^{1} ; 75$ th perc) & \multirow[t]{2}{*}{ Range (min-max) } \\
\hline 1 & Number of other somatic (non-ORL ${ }^{a}$ ) & & \\
\hline & diagnoses & $2(0 ; 4)$ & $0-13$ \\
\hline 2 & $\begin{array}{l}\text { Number of involved disciplines for the } \\
\text { specific subjective ORL complaint }\end{array}$ & $1(1 ; 1)$ & $0-3$ \\
\hline 3 & $\begin{array}{l}\text { Number of involved disciplines for other } \\
\text { (non-ORL) complaints }\end{array}$ & $0(0 ; 1)$ & $0-9$ \\
\hline 4 & $\begin{array}{l}\text { Number of visits to the ORL outpatient } \\
\text { clinic }\end{array}$ & $6(4 ; 10$ & -92 \\
\hline 5 & $\begin{array}{l}\text { Number of additional (instrumental } \\
\text { examinations }\end{array}$ & $4(2 ; 9)$ & $0-57$ \\
\hline 6 & Number of ORL surgical interventions & $0(0 ; 0)$ & $0-3$ \\
\hline 7 & Number of other surgical interventions & $0(0 ; 0)$ & $0-4$ \\
\hline
\end{tabular}

Legend: ${ }^{a} \mathrm{ORL}=$ otorhinolaryngological.

Table 3.5 shows the frequency distribution of the ORL treatment that was given to the patients for their unexplained complaints. More than half of the patients did not receive an $\mathrm{ORL}$ treatment ( $\mathrm{N}=68 ; 68.0 \%)$. Twenty-four patients $(24.0 \%)$ received a nonpsychotropic drug treatment for the complaints (topical nasal steroids, systemic steroids, antibiotics, etc.).

Table 3.5 Frequency distribution of otorhinolaryngological treatment in the study population ( $N=102)$.

\begin{tabular}{lc}
\hline Variable ORL $^{\text {a }}$ treatment & $\mathrm{N}(\%)$ \\
\hline No ORL treatment & $68(68.0)$ \\
Drug/pharmacological treatment & $24(24.0)$ \\
Surgical treatment & $5(5.0)$ \\
Outpatient follow-up & $3(3.0)$ \\
Missing values & $2(2.0)$ \\
\hline
\end{tabular}

Legend: ${ }^{a}$ ORL= otorhinolaryngological. ${ }^{b}$ Some patients underwent more than one surgical intervention (ventilation tubes in tympanic membrane, microlaryngoscopy for benign vocal fold lesions, nasal septum correction, rhinoplasty, etc.).

Table 3.6 represents the frequency distribution of the psychiatric diagnoses. Seventy-eight percent of the study population $(\mathrm{N}=80)$ was diagnosed with a psychiatric morbidity/co-morbidity based on the definitions of the Diagnostic and Statistical Manual of Mental Disorders, Fourth Edition (DSM-4-TR) ${ }^{12}$. The most frequent diagnosis was anxiety disorder ( $\mathrm{N}=31 ; 35.2 \%)$. Furthermore, depressive disorder and undifferentiated somatoform disorders were common, $\mathrm{N}=20$ (22.7\%) for both. Only $9.1 \%(\mathrm{~N}=8)$ of the 
patients were not diagnosed with a psychiatric disorder. Among the patients with an ORL somatic diagnosis $(\mathrm{N}=60)$ fifteen $(29.4 \%)$ were diagnosed with an anxiety disorder and sixteen (31.4\%) with an undifferentiated somatoform disorder.

Table 3.6 Frequency distribution of psychiatric diagnoses in the study population $(\mathrm{N}=102)$ and according to the presence or absence of an ORLa diagnosis.

\begin{tabular}{lccc}
\hline & \multicolumn{3}{c}{ ORL diagnosis } \\
\hline & Yes & No & Total \\
& $(\mathrm{N}=60)$ & $(\mathrm{N}=42)$ & $(\mathrm{N}=102)$ \\
Psychiatric diagnosis & $\mathrm{N}(\%)$ & $\mathrm{N}(\%)$ & $\mathrm{N}(\%)$ \\
\hline Anxiety disorder & $15(29.4)$ & $16(43.2)$ & $31(35.2)$ \\
Undifferentiated somatoform disorder & $16(31.4)$ & $4(10.8)$ & $20(22.7)$ \\
Depressive disorder & $11(21.6)$ & $9(24.3)$ & $20(22.7)$ \\
No psychiatric diagnosis & $4(7.8)$ & $4(10.8)$ & $8(9.1)$ \\
Adaptation disorder & $3(5.9)$ & $1(2.7)$ & $4(4.5)$ \\
Grief bereavement & $0(0.0)$ & $1(2.7)$ & $1(1.1)$ \\
PTSD & $0(0.0)$ & $1(2.7)$ & $1(1.1)$ \\
Mild cognitive impairment & $0(0.0)$ & $1(2.7)$ & $1(1.1)$ \\
Eating disorder & $1(2.0)$ & $0(0.0)$ & $1(1.1)$ \\
Bipolar disorder & $1(2.0)$ & $0(0.0)$ & $1(1.1)$ \\
Missing values & $9(15.0)$ & $5(12.0)$ & $14(13.7)$ \\
\hline
\end{tabular}

Legend: ${ }^{a}$ ORL= otorhinolaryngological. ${ }^{b}$ PTSD $=$ Posttraumatic Stress Disorder.

Table 3.7 gives the frequency distribution of the received psychiatric treatment (selective serotonin reuptake inhibitors, psychotherapy, etc.).

Table 3.7 Frequency distribution of psychiatric treatment in the study population $(\mathrm{N}=102)$.

\begin{tabular}{lc}
\hline Variable psychiatric treatment & $\mathrm{N}(\%)$ \\
\hline Psychotherapy & $38(42.7)$ \\
Psychotropic drugs & $27(30.3)$ \\
No treatment & $14(15.7)$ \\
Psychotropic drugs and psychotherapy & $6(6.7)$ \\
Outpatient follow-up (psychiatric management) & $4(4.4)$ \\
Missing values & $13(12.8)$ \\
\hline
\end{tabular}

Some observations included in Table 3.2, 3.5, 3.6, 3.7 had to be scored as missing values because of incomplete reporting of data in the patients' medical files.

\section{Discussion}

To our knowledge, this is the first study investigating MUORLS at an integrated ORLpsychiatry outpatient setting of a university medical center. MUORLS has become a topic of considerable interest, given the growing population of these patients and the increasing concern about quality of life, healthcare costs, risk of iatrogenic damage, and 
healthcare trajectories that are unsatisfactory to both the patients and their physicians ${ }^{3-}$ 6,13,14. An important outcome of this study is insight into the existence of this patient population and the detour they take to obtain appropriate treatment in daily clinical ORL practice. Among their medically unexplained physical symptoms, our patient cohort had a high prevalence of psychiatric comorbidity ${ }^{3,15}$. Ultimately, this investigation may inform efforts to develop an integrated interdisciplinary treatment plan and thus to decrease the risks and medical costs of MUORLS. Quite often a diagnosis of MUORLS is made by exclusion. Uncertainty as to the cause of the symptoms can make physicians uneasy; as they weigh the need to rule out serious illness and increasing chronicity against the cost, distress, and damage of extensive clinical testing ${ }^{16,17}$. It may be the fear of missing a "serious diagnosis" that underlies physicians' emphasis on the physical side of the symptoms. Queries about psychological distress are therefore subordinated to an examination of physical aspects or even forgotten during consultations ${ }^{5,16}$. The present study revealed that the majority of the patients suffering from MUORLS were diagnosed with a psychiatric disorder $(\mathrm{N}=80,78 \%)$. A final psychiatric diagnosis was often delayed by requiring additional (instrumental) examinations to exclude a somatic disorder or serious illness. Some of the patients visited the ORL outpatient clinic many times (up to 92) or received care in many different disciplines for diverse medical complaints, thereby raising both the costs of healthcare and the risk to the patient. Thirty-two percent of the patients had received some ORL treatment $(\mathrm{N}=32)$ without improvement of complaints before they visited our two ORL experts. Seventy-eight percent $(N=80)$ of the study population was diagnosed with a psychiatric morbidity/co-morbidity. However, the determination of a psychiatric diagnosis in MUORLS patients with an established somatic ORL diagnosis should not be disregarded ( $N=47$; $92 \%)$. The presence of a somatic ORL diagnosis has often delayed referral to the integrated ORL-psychiatry care setting. Among MUORLS patients without a somatic ORL diagnosis, 90\% ( $\mathrm{N}=38$ ) were diagnosed with a psychiatric comorbidity. Given the underdetection of psychiatric morbidity along a non-integrated trajectory, evidence-based treatment was implemented right from the start of our multidisciplinary outpatient clinic approach ${ }^{2}$. Thus, in patients with expected multi-morbidity, screening for psychiatric morbidity makes sense ${ }^{18}$. Consequently, an integrated approach is deemed necessary, as it gets patients motivated for and referred to psychosomatic care. Eventually, structured clinical diagnostics may lead to evidencebased treatment for psychiatric morbidity/co-morbidity.

\section{Limitations of the study}

This prospective investigation has methodological limitations. Although the study yielded some interesting preliminary data, the study population is too small to reveal significant group differences. The heterogeneous etiology of MUORLS and the small number of patients per complaint or somatic diagnostic group prevented the comparison of groups for significant differences. Furthermore, selection bias may have occurred due to patients' fear of stigmatization or other patient-related reasons (e.g., only willing 
patients were included, patients were referred to a tertiary university medical center), so it is impossible to generalize. Still, the enrollment reflected the theoretical population of patients with MUORLS who consult the otorhinolaryngologist for diagnosis and treatment at the university medical center. One potential drawback is the absence of controls, although finding and including patients with similar somatic ORL diagnosis without MUORLS would have introduced selection bias as well. Furthermore, although the study design was prospective, the search for patient data in the medical files may have been incomplete. It is possible that some information was missed despite extensive and careful searching, and patients may have visited other hospitals for the same complaints without reporting it. This may have led to an underestimation of the results in the current study; an overestimation is very unlikely. Finally, patients suffering from a known psychiatric disorder were excluded. Their inclusion would have led to a higher frequency of MUORLS. On the other hand, inclusion of patients treated with antidepressant and/or anxiolytic drugs would have led to lower frequencies of MUORLS and underestimation of its presence.

\section{Conclusion}

Findings from the present cohort study suggest that the majority of patients with MUORLS suffer from under- or undiagnosed psychiatric morbidity. Patients suffering from MUORLS deserve easy access to integrated interdisciplinary care (ORL and psychiatry) followed by detailed psycho-education about the pathogenesis of their complaints and the future psychosomatic approach. Further research should focus on the development of effective methods to ensure that patients with MUORLS will be identified and treated (in a multidisciplinary approach) accordingly. 


\section{References}

1. Van Dessel, N. et al. The PROSPECTS study: Design of a prospective cohort study on prognosis and perpetuating factors of medically unexplained physical symptoms (MUPS). J. Psychosom. Res. 76, 200206 (2014).

2. Leue, C. et al. Managing complex patients on a medical psychiatric unit: An observational study of university hospital costs associated with medical service use, length of stay, and psychiatric intervention. J. Psychosom. Res. 68, 295-302 (2010).

3. Fink, P. et al. Somatization in primary care. Prevalence, health care utilization, and general practitioner recognition. Psychosomatics 40, 330-338 (1999).

4. Hahn, S. R. et al. The difficult doctor-patient relationship: Somatization, personality and psychopathology. J. Clin. Epidemiol. 47, 647-657 (1994).

5. Smith, B. J. et al. The identification in primary care of patients who have been repeatedly referred to hospital for medically unexplained symptoms: a pilot study. J. Psychosom. Res. 67, 207-211 (2009).

6. Smith, R. C. \& Dwamena, F. C. Classification and diagnosis of patients with medically unexplained symptoms. J. Gen. Intern. Med. 22, 685-691 (2007).

7. Verdonschot RJ, Baijens LW, Serroyen JL, Leue C, Kremer B. Symptoms of anxiety and depression assessed with the hospital anxiety and depression scale in patients with oropharyngeal dysphagia. J. Psychosom. Res. 75, 451-455 (2013).

8. Ferrari, S. et al. Vertigo "in the pink": The impact of female gender on psychiatric-psychosomatic comorbidity in benign paroxysmal positional vertigo patients. Psychosomatics 55, 280-288 (2014).

9. http://www.cbo.nl

10. http://kims.orde.nl/het-kennisinstituut-voor-u/richtlijnen/

11. Landelijke Stuurgroep Multidisciplinaire Richtlijnontwikkeling in de GGZ. Multidisciplinaire Richtlijn Somatisch Onvoldoende Verklaarde Lichamelijke Klachten en Somatoforme Stoornissen (Multidisciplinary Guideline of MUPS and Somatoform Disorders). Utrecht, the Netherlands: Trimbos Instituut (2010).

12. American Psychiatric Association: Diagnostic and Statistical Manual of Mental Disorders, Fourth Edition (DSM-4-TR). Arlington, VA: American Psychiatric Publishing (2000).

13. Smith, G. R. et al. Patients with multiple unexplained symptoms: Their characteristics, functional health, and health care utilization. Arch. Intern. Med. 146, 69-72 (1986).

14. Kroenke, K. et al. Multisomatoform disorder. An alternative to undifferentiated somatoform disorder for the somatizing patient in primary care. Arch. Gen. Psychiatry 54, 352-358 (1997).

15. Fink, P. The use of hospitalizations by persistent somatizing patients. Psychol. Med. 22, 173-180 (1992).

16. Murray, A. M. et al. Barriers to the diagnosis of somatoform disorders in primary care: Protocol for a systematic review of the current status. Syst. Rev. 2, 99 (2013).

17. Reid, S. et al. Recognition of medically unexplained symptoms - do doctors agree? J. Psychosom. Res. 47, 483-485 (1999).

18. Zigmond, A. S. \& Snaith, R. P. The hospital anxiety and depression scale. Acta Psychiatr. Scand. 67, 361370 (1983). 



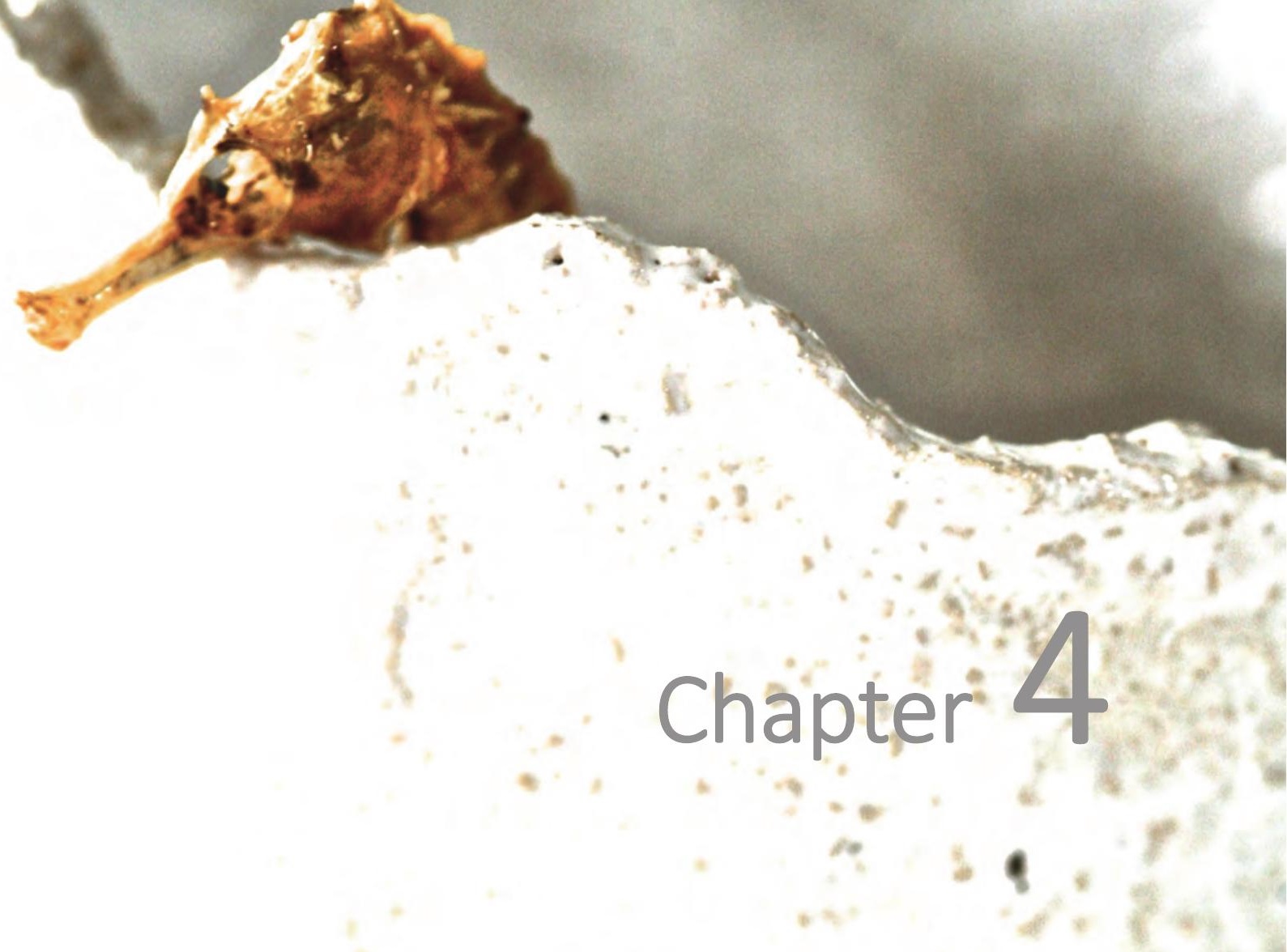

Integrated medical-psychiatric outpatient care in functional gastrointestinal disorders improves outcome: A pilot study

Leue $C^{a}$, Kruimel J ${ }^{a}$, Winkens B, Marcus D, Schoon S, Dellink R, van Os J, Masclee A ${ }^{a}$ These authors contributed equally to the present article

Eur. J. Gastroenterol. Hepatol. 27, 721-727 (2015) 


\section{Abstract}

\section{Background and objectives}

Functional gastrointestinal disorders have a multi-factorial aetiology, including somatic and psychosocial factors. We provide multidisciplinary outpatient consultations by a gastroenterologist and psychiatrist, using an integrated approach toward somatic and psychosocial factors in complex functional gastrointestinal disorders. The aim of this study was to determine the efficacy of this approach assessing gastrointestinal and psychiatric symptoms, and quality of life.

\section{Methods}

All patients with complex functional gastrointestinal disorders visiting for consultation were included and treated with antidepressants, psychotherapy, or both, or given advice for treatment in their own region. Questionnaires testing gastrointestinal and psychiatric symptoms, and quality of life at first visit and after 6 and 12 months were completed.

\section{Results}

A total of 124 patients were included (70\% women, mean age 48 years): $57 \%$ were diagnosed with irritable bowel syndrome and about $80 \%$ had a psychiatric diagnosis ( $50 \%$ anxiety disorder, $20 \%$ mood disorder). Of these patients. $57 \%$ were treated with antidepressants and psychotherapy, $6 \%$ with psychotherapy alone, and $38 \%$ received advice for treatment in their own region. After 1 year, patients showed a significant improvement on all questionnaires, with the exception of those testing gastrointestinal symptoms, although there were significant improvements in these at 6 months.

\section{Conclusions}

This is the first prospective study on the efficacy of an integrated medical-psychiatric outpatient care model in patients with complex functional gastrointestinal disorders, showing significant improvement of gastrointestinal and psychiatric symptoms, and quality of life after six months. With the exception of improvement in gastrointestinal symptoms, improvement persisted at the 1-year follow-up. This indicates that longer follow-up focusing on gastrointestinal symptoms may be needed. 


\section{Introduction}

Functional gastrointestinal disorders (FGIDs) comprise a group of disorders related to the gastrointestinal tract for which no structural causes have been identified. The most common and well-known FGIDs are irritable bowel syndrome (IBS) and functional dyspepsia. These FGIDs are diagnosed using symptom-based criteria formulated by the Rome III consensus ${ }^{1}$. In the case of IBS, subtypes are identified based on the predominant bowel habit such as diarrhoea (IBS-D), constipation (IBS-C) or both (IBS$M)^{2}$. FGIDs are highly relevant for health care, because of their high prevalence, their impact on daily functioning and quality of life, their socio-economic burden due to reduced work productivity, illness related sick leave, and high health care utilization ${ }^{3-5}$. IBS has been well studied in this respect. A European cohort study showed a prevalence of IBS ranging from 6 to $12 \%$ across different countries ${ }^{6}$. In this cohort, $90 \%$ of the IBS patients consulted at primary care centres and $17 \%$ consulted at a hospital. Moreover, $69 \%$ used medication for IBS symptoms ${ }^{6}$.

The pathophysiology of IBS is complex and remains elusive, but several mechanisms appear to play a role in symptom development, such as alterations in intestinal motility ${ }^{7}$, enhanced visceral sensitivity ${ }^{8}$, low grade inflammation, increased gut permeability ${ }^{9}$, and genetic susceptibility ${ }^{10}$. There is accumulating evidence that psychosocial factors, such as life stress, coping styles, and level of psychosocial support, play an important role by influencing symptom severity, illness behaviour, and, eventually, patient outcome ${ }^{11}$. IBS patients are more likely to have coexistent mood and anxiety disorders ${ }^{12}$, which may be in part related to the fact that the personality trait neuroticism is associated with both psychiatric as well as somatic ill-health ${ }^{13}$. The bi-directional interaction between somatic and psychological factors in the pathogenesis of gastrointestinal disorders is known as the 'brain-gut-axis', according to which it is proposed that FGIDs result from a disturbed regulation along this axis ${ }^{11}$.

However, in most complex patients with functional complaints there still exists underdetection of psychiatric comorbidity ${ }^{14}$. Reports and clinical experience have indicated that treatment of FGID patients with more severe symptoms by gastroenterologists only is moderately successful, resulting in patients consulting many specialists in various hospitals, often undergoing multiple medical and alternative treatments, and sometimes undergoing even unnecessary surgical interventions. At our department, FGID patients with moderate to severe gastrointestinal symptoms who do not show sufficient improvement by standard medical management, including dietary and life style advises, and are suspected of having psychiatric comorbidity are offered additional joined medical-psychiatric consultation. A gastroenterologist and a psychiatrist provided these multidisciplinary outpatient joint simultaneous consultations using an integrated approach toward the management of both somatic and psychosocial factors in complex FGIDs. The aim of the current study was to evaluate the results of our routine integrated outpatient care longitudinally. The main focus was on patient-related 
direct outcome in terms of gastrointestinal and psychiatric symptoms, and quality of life. We hypothesized that implementation of an integrated outpatient care model will result in a reduction of gastrointestinal and psychiatric symptoms, and improve the quality of life in patients with FGID.

\section{Patients and methods}

\section{Study Design}

This prospective open noncontrolled cohort study was carried at the Med-Psych-Centre (MPC) outpatient clinic for complex FGIDs at the Maastricht University Medical Centre (MUMC). The MPC represents a close collaboration between hospital psychiatry and several somatic specialties at the hospital, and it consists of a medical-psychiatric ward, day-treatment facilities, and an outpatient clinic. The MPC functions as a tertiary referral centre and focuses on patients with functional complaints such as FGIDs and psychiatric comorbidities, as well as patients with medical unexplained physical symptoms.

FGID patients referred to the MUMC undergo thorough gastrointestinal screening and treatment at the division of Gastroenterology-Hepatology. Patients with moderate to severe gastrointestinal symptoms who do not achieve sufficient remission with standard management and are suspected of having psychiatric co-morbidity are offered an additional multidisciplinary outpatient consultation by a gastroenterologist and a psychiatrist in a joint session. At these consultations, the gastroenterologist and the psychiatrist see patients at the same time, enabling evaluation and discussion of adequate diagnosis and treatment in an efficient manner using an integrated approach with focus on somatic and psychosocial factors and their interaction. In this manner, the widespread underdiagnosis of psychiatric comorbidities can be reduced and overcome ${ }^{14}$.

This cohort study enrolled all complex FGID patients referred to the MPC between September 2009 and January 2011 after gastroenterological work up. Patients were asked to fill in questionnaires at baseline after their first MPC outpatient clinic visit and after 6 and 12 months of treatment. Five validated questionnaires were used to assess various gastrointestinal and psychiatric symptoms, and quality of life. An intensive telephone call strategy was used to ascertain sufficiently high response rates. Nonresponders were contacted after 1 month by telephone, followed by re-sending of questionnaires if necessary. Patients not responding after the first reminder were called again on a second occasion. Nonresponders were defined as patients who had returned a baseline questionnaire, but did not respond to questionnaires after 6 and 12 months, even after repeated reminders. Participants were free to refuse participation at any time and had been given assurance that participation was in no way contingent on treatment. The Medical Ethics Committee of MUMC approved the study protocol. Patients provided written informed consent. 


\section{Study participants}

All consecutive FGID patients who visited the MPC outpatient clinic between September 2009 and January 2011 were included in the study. The diagnosis of FGID had been made on the basis of the Rome III criteria, after exclusion of organic causes of their abdominal symptoms by Gl analysis, including laboratory testing, imaging and/or endoscopy. There were no exclusion criteria. Patients were treated after their first MPCvisit at the medical-psychiatric ward with day-treatment facilities or at the outpatient clinic (local group of patients living in the Maastricht catchment area), or received treatment advice, to be implemented by the referring consultant or general practitioner in their own region (external group of patients living outside the Maastricht catchment area). Treatment options included the use of antidepressants, mostly serotonin selective reuptake inhibitors (SSRIs), psychotherapy or both. There was no control group. Patient characteristics such as age, sex, medication use, and gastrointestinal and psychiatric diagnosis were extracted from the hospital electronic patient database.

\section{Questionnaires}

To assess gastrointestinal symptoms, we used the gastrointestinal symptom rating scale $(G S R S)^{15}$ and the Cognitive Scale for Functional Bowel Disorders (CS-FBD) ${ }^{16}$ assessing symptoms related to cognition. To assess affective symptoms, patients were asked to complete the Hospital Anxiety and Depression Scale (HADS) ${ }^{17}$ and the Dutch version of the State-Trait Anxiety Inventory (STAI-DY) to measure levels of state anxiety (STAI-S) and trait anxiety (STAI-T) ${ }^{18}$. The validated Dutch version of the Short Form-36 (SF-36), the RAND-36, was used to measure generic health-related quality of life ${ }^{19}$. The RAND-36 consists of 8 different subscales: physical functioning, physical role functioning, bodily pain, general health, social functioning, emotional role functioning, mental health and vitality. From these 8 domains, a physical composite score consisting of physical functioning, physical role functioning, bodily pain, and general health, and a mental composite score consisting social functioning, emotional role functioning, mental health, and vitality can be derived ${ }^{20}$.

\section{Statistical analysis}

The primary goal in this study was to assess the efficacy of integrated outpatient MPC treatment, defined by a decrease in gastrointestinal and psychiatric symptom severity, and improvement of quality of life, as assessed by questionnaires after 6 months and 1 year of treatment, compared with baseline at the first MPC consultation. Data are presented for all 124 participants who completed the baseline questionnaires and received MPC-treatment or MPC advice for treatment in their own region of living. Data analyses were carried out and calculated using SPSS 21.0 (SPSS Inc., Chicago, Illinois, USA) ${ }^{21}$. Linear mixed models were used to assess the overall longitudinal trends and to compare these trends for gastrointestinal and psychiatric complaints, as well as quality 
of life, between the local group of patients living in the Maastricht catchment area and the external group of patients living outside the Maastricht catchment area. These models, based on restricted maximum likelihood and assuming an unstructured covariance structure, were used to account for the dependency in repeated measures within a patient and for missing data in the study cohort ${ }^{22}$. As for the comparison between local and external groups, the differences in outcome at 6 and 12 months were corrected for sex, IBS subtypes, and baseline differences. The estimated means, the differences between the means, corresponding standard errors (SEs), and the 95\% confidence intervals (Cls) obtained from these models were reported for each questionnaire. Two-sided $P$-values of up to 0.05 were considered statistically significant.

\section{Results}

\section{Patient disposition, demographics, baseline characteristics, treatment characteristics}

Between September 2009 and January 2011, 137 patients were considered candidates for the MPC consultation willing to proceed with the study. Of these, 124 received treatment and, therefore, were included. Of the 13 patients who were not included in the study, eight patients received no integrated advice for treatment during the first MPC visit: one patient was referred to a surgeon, two patients were referred to a gynaecologist, two patients were referred to a revalidation program, two patients were referred to an outpatient clinic for eating problems, and one patient was given an expectative advice. The other five patients were lost to follow up because they did not attend MPC treatment. Seventy-two patients returned questionnaires after 6 months of treatment (58\%) and 71 completed questionnaires after 12 months of treatment (57\%).

Out of the group of 124 patients, 77 patients were treated at the MPC in Maastricht (i.e. the local group) and 47 patients only received treatment advice to be implemented by the referring consultant or general practitioner in their own region, as they resided outside the Maastricht catchment area (i.e. the external group). Table 4.1 shows the demographics and baseline characteristics of the total group of 124 patients included in the study, as well as the local and external groups separately (Table 4.1). 
Table 4.1 Demographics and baseline patient characteristics for the entire group, as well as for the local and external groups separately.

\begin{tabular}{|c|c|c|c|}
\hline & $\begin{array}{c}\text { Total } \\
(n=124) \\
{[n(\%)]}\end{array}$ & $\begin{array}{l}\text { Local group } \\
\qquad \begin{array}{c}(n=77) \\
{[n(\%)]}\end{array}\end{array}$ & $\begin{array}{c}\text { External group } \\
(n=47) \\
{[n(\%)]}\end{array}$ \\
\hline Age, yrs. (SD) & $48(17.1)$ & $51(15.7)$ & $42(17.7)$ \\
\hline Female sex & $87(70.2)$ & $52(67.5)$ & $35(74.5)$ \\
\hline Opioids & $12(9.7)$ & $9(11.7)$ & $3(6.4)$ \\
\hline Antidepressants & $30(24.2)$ & $20(26.0)$ & $10(21.3)$ \\
\hline Gastrointestinal medication & $87(70.2)$ & $59(76.6)$ & $28(59.6)$ \\
\hline Smoking & $41(34.2)^{a}$ & $26(34.7)^{a}$ & $15(33.3)^{a}$ \\
\hline Alcohol & $56(45.5)^{b}$ & $28(36.8)^{b}$ & $28(59.6)^{b}$ \\
\hline Drugs & $6(5.0)^{c}$ & $2(2.7)^{c}$ & $4(8.7)^{c}$ \\
\hline Abdominal surgery in the past & $62(50.0)$ & 40 (51.9) & $22(46.8)$ \\
\hline $\mathrm{IBD}^{1}$ & $5(4.0)$ & $4(5.2)$ & $1(2.1)$ \\
\hline $\mathrm{GERD}^{2}$ & $20(16.1)$ & $13(16.9)$ & $7(14.9)$ \\
\hline Lactose intolerance & $15(12.1)$ & $7(9.1)$ & $8(17.0)$ \\
\hline Food allergy & $3(2.4)$ & $1(1.3)$ & $2(4.3)$ \\
\hline Slow transit constipation & $1(0.8)$ & $1(1.3)$ & $0(0.0)$ \\
\hline Endometriosis & $6(4.8)$ & $4(5.2)$ & $2(4.3)$ \\
\hline Diverticulosis & $13(10.5)$ & $9(11.7)$ & $4(8.5)$ \\
\hline Functional dyspepsia & $16(12.9)$ & $10(13.0)$ & $6(12.8)$ \\
\hline Irritable bowel syndrome & $70(56.5)$ & $42(54.5)$ & $28(59.6)$ \\
\hline IBS-diarrhea & $13(18.6)$ & $8(19.0)$ & $5(17.9)$ \\
\hline IBS-constipation & $28(40.0)$ & $16(38.1)$ & $12(42.9)$ \\
\hline IBS-mixed & $15(21.4)$ & $10(23.8)$ & $5(17.9)$ \\
\hline Other $^{d}$ & $14(20.0)$ & $8(19.0)$ & $6(21.4)$ \\
\hline Other functional disorders ${ }^{e}$ & $24(19.4)$ & $14(18.2)$ & $10(21.3)$ \\
\hline Anxiety disorder & $61(49.2)$ & $49(63.6)$ & $12(25.5)$ \\
\hline Mood disorder & $25(20.2)$ & $20(26.0)$ & $5(10.6)$ \\
\hline Pain disorder & $2(1.6)$ & $1(1.3)$ & $1(2.1)$ \\
\hline Eating disorder & $2(1.6)$ & $1(1.3)$ & $1(2.1)$ \\
\hline Adaptation disorder & $2(1.6)$ & $0(0.0)$ & $2(4.3)$ \\
\hline Addiction & $4(3.2)$ & $3(3.9)$ & $1(2.1)$ \\
\hline Personality disorder & $3(2.4)$ & $1(1.3)$ & $2(4.3)$ \\
\hline Psychotic disorder & $1(0.8)$ & $0(0.0)$ & $1(2.1)$ \\
\hline Other psychiatric disorders & $3(2.4)$ & $1(1.3)$ & $2(4.3)$ \\
\hline
\end{tabular}

Legend: ${ }^{1}$ inflammatory bowel disease; ${ }^{2}$ gastro-esophageal reflux disease. ${ }^{\text {a }}$ Smoking: four missing (two in the local group and two in the external group). ${ }^{b}$ Alcohol: one missing (one in the local group and none in the external group). ${ }^{c}$ Drugs: four missing (three in the local group and one in the external group). ${ }^{d}$ Includes IBS unspecified and post-infectious IBS. ${ }^{e}$ Other functional disorders such as fibromyalgia or chronic fatigue syndrome.

The majority of patients were women (70.2\%) and the mean age was 48 years. A total of 70 patients (56.5\%) were diagnosed with IBS. About $20 \%$ of IBS patients had a diarrhea predominant IBS subtype, $40 \%$ had a constipation predominant subtype, $20 \%$ had a mixed subtype, and $20 \%$ had an unspecified or postinfectious subtype. As expected, there was a high prevalence of psychiatric comorbidity in this population. About $80 \%$ of the patients were diagnosed with a comorbid psychiatric condition, of which anxiety disorder or mood disorder were the most prevalent (49\% and 20\% 
respectively). The most common nonfunctional concomitant somatic diagnosis was gastroesophageal reflux disease, which was present in $16 \%$ of patients. Almost $10 \%$ of the patients used opioids and 50\% had undergone abdominal surgery in the past. Treatment characteristics are shown in Table 4.2.

Table 4.2 Treatment characteristics.

\begin{tabular}{lc}
\hline Therapy & Total $(\mathrm{n}=124)$ \\
{$[\mathrm{n}(\%)]$}
\end{tabular}

Legend: CGT=group-based cognitive behavioural therapy; MBT= group-based mindfulness-based therapy.

Patients received antidepressants and psychotherapy, psychotherapy alone, or treatment advice to be implemented by the referring consultant or general practitioner in their own region, in case they lived outside the Maastricht catchment area. Antidepressants were prescribed to 70 patients (56.5\%), of whom 66 were treated with an SSRI, mostly citalopram, three with a tricyclic antidepressant, and one with a selective noradrenaline-serotonin reuptake inhibitor. Simultaneous psychotherapy was available as individual psychotherapy by a psychiatrist, day-treatment supportive therapy, groupbased mindfulness based therapy, group-based cognitive behavioural therapy, or supportive outpatient mental health care offered by a psychologist. Only seven patients (5.6\%) received psychotherapy alone. Forty-seven patients (37.9\%) were from outside the Maastricht catchment area and were only given advice for treatment in their home region, with regard to psychotherapy and medication, or psychotherapy alone.

\section{Abdominal and psychiatric symptoms, and quality of life}

Outcomes of the 124 patients are summarized in Tables 4.3 and 4.4. After 6 months of treatment, symptoms of anxiety (HADS-A) and depression (HADS-D) were significantly decreased compared to baseline, with mean differences of $2.0(95 \% \mathrm{Cl} 0.9-3.0, P<0.001)$ and $1.6(95 \% \mathrm{Cl} 0.5-2.7, P=0.004)$, respectively. This significant decrease in anxiety and depression scores was still present after 12 months of treatment compared with baseline: mean difference $2.7(95 \% \mathrm{Cl} 1.7-3.7, P<0.001)$ and $1.7(95 \% \mathrm{Cl} 0.6-2.9, P=0.003)$. 
The same decreasing trend in anxiety symptoms was observed on using the State-Trait Anxiety Inventory: a mean difference in STAI-T of $4.5(95 \% \mathrm{Cl} 2.0-7.0, P=0.001)$ and in STAI-S of $6.1(95 \% \mathrm{Cl} 3.0-9.2, p<0.001)$ after 6 months of treatment compared with baseline, and respectively $5.6(95 \% \mathrm{Cl} 3.0-8.1, P<0.001)$ and $5.7(95 \% \mathrm{Cl} 2.8-8.6, P<0.001)$ after 12 months of treatment. Mental quality of life, as measured with the SF-36 increased significantly after 6 months and 12 months of follow-up compared to baseline, with mean differences of $4.0(95 \% \mathrm{Cl} 0.4-7.5, p=0.029)$ and $4.4(95 \% \mathrm{Cl} 1.0-7.7, \mathrm{p}=0.012)$, respectively. Physical quality of life as measured with the SF-36, increased significantly after 6 months and 12 months of follow-up compared with baseline, with mean differences of $3.2(95 \% \mathrm{Cl} 0.8-5.5, P=0.009)$ and $3.3(95 \% \mathrm{Cl} 0.5-6.0, P=0.021)$, respectively. The mean difference in CS-FBD, indexing dysfunctional cognitions associated with gastrointestinal symptoms, decreased significantly during the first 6 months of the study, but not after 1 year, compared with baseline, with a mean difference of $11.9(95 \% \mathrm{Cl} 2.6-21.1, P=0.013)$ and $9.6(95 \% \mathrm{Cl}-0.1-19.2, P=0.053)$, respectively (Table 4.3).

Table 4.3 Outcome assessed by HADS-A, HADS-D, STAI-T, STAI-S, CS-FBD, SF-36 for the total group ( $n=124)$.

\begin{tabular}{|c|c|c|c|c|c|c|c|c|}
\hline & \multicolumn{2}{|c|}{ Baseline } & \multicolumn{2}{|c|}{6 months } & \multicolumn{2}{|c|}{12 months } & \multicolumn{2}{|c|}{$95 \% \mathrm{Cl}, P$-value } \\
\hline & Mean & SE & Mean & SE & Mean & SE & 0 vs. 6 & 0 vs. 12 \\
\hline HADS-A ${ }^{a}$ & 9.3 & 0.5 & 7.3 & 0.5 & 6.6 & 0.5 & $<0.001$ & $<0.001$ \\
\hline HADS-D ${ }^{b}$ & 7.4 & 0.5 & 5.8 & 0.6 & 5.7 & 0.6 & $=0.004$ & $=0.003$ \\
\hline STAI-T ${ }^{C}$ & 47.3 & 1.2 & 42.8 & 1.4 & 41.7 & 1.4 & $=0.001$ & $<0.001$ \\
\hline STAI-S ${ }^{d}$ & 45.9 & 1.3 & 39.8 & 1.6 & 40.2 & 1.5 & $<0.001$ & $<0.001$ \\
\hline CS-FBD ${ }^{e}$ & 103.1 & 4.4 & 91.2 & 4.8 & 93.5 & 5.4 & $=0.013$ & $=0.053$ \\
\hline \multicolumn{9}{|l|}{$S F-36^{f}$} \\
\hline $\mathrm{MCS}^{\mathrm{g}}$ & 41.4 & 1.4 & 45.3 & 1.6 & 45.7 & 1.5 & $=0.029$ & $=0.012$ \\
\hline $\mathrm{PCS}^{h}$ & 35.1 & 1.1 & 38.2 & 1.4 & 38.4 & 1.6 & $=0.009$ & $=0.021$ \\
\hline
\end{tabular}

Legend: Values represent estimated means, Standard Errors (SEs) and P-values for differences from baseline. aHADS-A: Hospital Anxiety and Depression Scale, subscore anxiety. ${ }^{\mathrm{b}}$ HADS-D: Hospital Anxiety and Depression Scale, subscore depression. 'STAI-T: State-Trait Anxiety Inventory, subscore trait anxiety. ${ }^{\text {' }}$ STAI-S: State-Trait Anxiety Inventory, subscore state anxiety. ${ }^{\mathrm{e} C S}$-FBD: Cognitive Scale for Functional Bowel Disorders. ${ }^{\text {S }}$ F-36: Short Form-36. ${ }^{\mathrm{g}} \mathrm{MCS}$ : Mental Composite Score. ${ }^{\mathrm{h}} \mathrm{PCS}$ : Physical Composite Score.

Gastrointestinal symptoms, as measured by the total score of the GSRS, improved significantly after 6 months, with a small mean difference of $0.4(95 \% \mathrm{Cl} 0.2-0.7$, $P<0.001$ ); however, this improvement was abolished after 1 year. Given a main interest in abdominal pain, diarrhea and constipation, the GSRS was subdivided post hoc into its five subdomains: abdominal pain, reflux syndrome, diarrhea syndrome, indigestion syndrome, and constipation syndrome. Only the change in the diarrhea syndrome subscore reached significance after 6 months ( $P=0.048)$, but not after 1 year (Table 4.4). 
Table 4.4 Outcome assessed by GSRS ${ }^{a}$ for the total group $(n=124)$ : total score and divided into five subscores.

\begin{tabular}{lcccccccc}
\hline GSRS & \multicolumn{2}{c}{ Baseline } & \multicolumn{2}{c}{6 months } & \multicolumn{2}{c}{12 months } & \multicolumn{2}{c}{$P$-value } \\
& Mean & SE & Mean & SE & Mean & SE & 0 vs. 6 & 0 vs. 12 \\
\hline Total & 3.2 & 0.1 & 2.8 & 0.1 & 3.1 & 0.1 & $<0.001$ & $=0.112$ \\
AP $^{\text {b }}$ & 3.5 & 0.1 & 3.3 & 0.1 & 3.5 & 0.2 & $=0.159$ & $=0.631$ \\
RS $^{c}$ & 2.5 & 0.1 & 2.4 & 0.2 & 2.4 & 0.2 & $=0.599$ & $=0.545$ \\
DS $^{\text {d }}$ & 3.2 & 0.2 & 2.8 & 0.2 & 3.0 & 0.2 & $=0.048$ & $=0.310$ \\
IS $^{\text {e }}$ & 4.0 & 0.1 & 3.7 & 0.1 & 3.7 & 0.2 & $=0.058$ & $=0.086$ \\
CS $^{f}$ & 3.0 & 0.1 & 3.3 & 0.2 & 3.1 & 0.2 & $=0.149$ & $=0.462$ \\
\hline
\end{tabular}

Legend: Values represent estimated means, Standard Errors (SEs) and P-values for differences from baseline. ${ }^{a}$ GSRS: Gastrointestinal Symptoms Rating Scale. ${ }^{b}$ AP: abdominal pain. ${ }^{c}$ RS: reflux syndrome. ${ }^{d}$ DS: diarrhea syndrome. ${ }^{\dagger}$ CS: constipation syndrome.

Finally, we assessed the difference between patients who were treated locally (in Maastricht) and patients who were treated externally (outside the Maastricht catchment area) after the first Med-Psych-Centre (MPC) consultation. The local group, 77 patients, was treated in the MPC-outpatient clinic and received medication and psychotherapy, or psychotherapy alone. The external group included 47 patients who received only advice for treatment in their own region. At baseline, the local group of patients had higher mean psychiatric symptom scores and lower mean mental and physical composite scores as measured with the SF-36, compared with the external group; the gastrointestinal symptom scores, as measured with the GSRS, did not differ significantly between the local and external group at baseline (Table 1 and 5). After 6 months and/or 1 year of follow-up, patients treated locally showed more improvement than patients treated externally, except for the CS-FBD, physical quality of life as measured with the SF-36, and the GSRS. STAI-T and STAI-S were significantly more improved in the local group compared to the external group after 6 months. After 1 year, no significant differences were apparent between both groups in STAI-T and STAI-S (Table 4.5).

\section{Discussion}

A prospective, open, non-controlled cohort study on the efficacy of an integrated medical-psychiatric outpatient care model in patients with complex FGIDs was conducted at a University Hospital, in a setting of standard clinical care. Applying an integrated approach, patients preliminarily diagnosed with, and treated for, a FGID by a gastroenterologist, were seen in a multidisciplinary outpatient consultation (MPC-visit) by a gastroenterologist and a psychiatrist at the same time, receiving further advice for standardized treatment. The results of the study showed a beneficial effect of additional integrated treatment on gastrointestinal and psychiatric symptoms, and quality of life, after 6 months. After one year, the beneficial effect was no longer present with regard 
to the gastrointestinal symptoms, but continued for the psychiatric symptoms and quality of life (Tables 4.3 and 4.4).

Table 4.5 Group effect (local vs. external) on questionnaires at 6 and 12 months.

\begin{tabular}{|c|c|c|c|c|c|c|c|c|}
\hline & \multicolumn{2}{|c|}{ Baseline } & \multicolumn{2}{|c|}{6 months } & \multicolumn{2}{|c|}{12 months } & \multicolumn{2}{|c|}{$\mathrm{p}$-value } \\
\hline & Mean & S.E. & Mean & S.E. & Mean & S.E. & 6 months & 12 months \\
\hline \multicolumn{9}{|l|}{$\overline{\text { GSRS }^{a}}$} \\
\hline Local & 3.4 & 0.1 & 2.9 & 0.2 & 3.2 & 0.2 & 0.851 & 0.664 \\
\hline External & 3.3 & 0.2 & 2.9 & 0.2 & 3.1 & 0.2 & & \\
\hline \multicolumn{9}{|l|}{ HADS-A } \\
\hline Local & 10.1 & 0.6 & 7.1 & 0.7 & 6.6 & 0.7 & 0.002 & 0.010 \\
\hline External & 7.9 & 0.8 & 8.3 & 0.9 & 7.1 & 0.9 & & \\
\hline HADS-D ${ }^{c}$ & & & & & & & 0.009 & 0.019 \\
\hline Local & 8.4 & 0.6 & 5.8 & 0.7 & 5.7 & 0.8 & & \\
\hline External & 6.4 & 0.8 & 6.9 & 1.0 & 6.7 & 1.0 & & \\
\hline STAI-T ${ }^{d}$ & & & & & & & 0.002 & 0.078 \\
\hline Local & 49.8 & 1.7 & 42.6 & 2.0 & 42.7 & 1.9 & & \\
\hline External & 44.2 & 2.1 & 45.1 & 2.5 & 41.8 & 2.5 & & \\
\hline STAI-S & & & & & & & 0.006 & 0.190 \\
\hline Local & 48.0 & 1.8 & 39.0 & 2.2 & 40.9 & 2.1 & & \\
\hline External & 42.9 & 2.3 & 42.6 & 2.8 & 39.9 & 2.8 & & \\
\hline$C S-F B D^{f}$ & & & & & & & 0.328 & 0.053 \\
\hline Local & 112.4 & 5.9 & 98.5 & 6.7 & 97.2 & 7.1 & & \\
\hline External & 103.2 & 7.5 & 98.9 & 8.7 & 107.8 & 9.5 & & \\
\hline \multicolumn{9}{|l|}{$\mathrm{SF}-36^{\mathrm{g}}$} \\
\hline $\mathrm{MCS}^{h}$ & & & & & & & 0.093 & 0.006 \\
\hline Local & 39.3 & 1.9 & 45.6 & 2.2 & 47.2 & 2.0 & & \\
\hline External & 43.0 & 2.4 & 43.2 & 2.8 & 41.3 & 2.7 & & \\
\hline $\mathrm{PCS}^{\mathrm{i}}$ & & & & & & & 0.987 & 0.672 \\
\hline Local & 35.5 & 1.5 & 38.8 & 1.9 & 39.3 & 2.1 & & \\
\hline External & 38.7 & 1.9 & 42.0 & 2.4 & 41.3 & 2.8 & & \\
\hline
\end{tabular}

Legend: Values represent estimated means, SEs and $P$-values for differences between local and external groups at 6 and 12 months, corrected for sex, IBS subtypes, and baseline differences in the outcome measure. aGSRS: Gastrointestinal Symptoms Rating Scale. ${ }^{b}$ HADS-A: Hospital Anxiety and Depression Scale, subscore anxiety. 'HADS-D: Hospital Anxiety and Depression Scale, subscore depression. ${ }^{d}$ STAI-T: State-Trait Anxiety Inventory, subscore trait anxiety. ${ }^{\text {e}}$ STAI-S: State-Trait Anxiety Inventory, subscore state anxiety. ${ }^{f}$ CS-FBD:

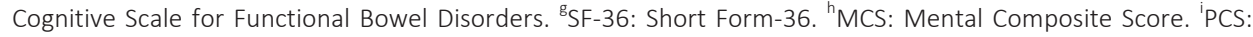
Physical Composite Score.

The most reasonable explanation for this finding is that in our daily practice, a gastroenterologist most often has intensively treated patients before the MPCconsultation. After the MPC-consultation by a gastroenterologist and a psychiatrist, the patient is followed up, particularly in the area of mental health, with a focus on hitherto undetected mental symptoms. With respect to the gastrointestinal complaints, after the MPC-consultation, patients were referred back to their general practitioner with advice on further management. The results indicate either that a prolonged integrated approach at the MUMC by a gastroenterologist and a psychiatrist is needed or that more collaboration with the general practitioner is required to optimize somatic symptom 
relief in FGID patients. The patient population included patients treated locally after their first MPC visit at the medical-psychiatric ward, at day-treatment facilities, or at an outpatient clinic, and patients only receiving treatment advice, to be implemented by the referring consultant or general practitioner in their own region, in case of living outside the Maastricht catchment area (external group). As shown in Table 4.1 and 4.5, more psychiatric symptoms and lower mean mental and physical quality of life, as measured with the SF-36, were observed at baseline in the local group compared with the external group, suggesting more psychiatric comorbidity among locally treated patients. The gastrointestinal symptoms, as measured with the GSRS, were equal at baseline in both groups. Patients treated locally showed more improvement than patients treated externally after 6 months and/or one year of follow-up, except for the CS-FBD, physical quality of life, as measured with the SF-36, and the GSRS. The greater improvement in psychiatric symptoms in the local Maastricht group could be the result of the more integrated expertise in our multidisciplinary approach or may be simply due to the higher symptom severity at baseline in the local group. This study was not designed to differentiate between both possibilities. Only for the local group are we adequately informed about initiated therapy and treatment related compliance.

The MPC for integrated outpatient care by a gastroenterologist and a psychiatrist in a multidisciplinary joint consultation is a tertiary centre for the referral of patients with complex FGIDs. Therefore, the results of this study cannot be extrapolated to a general hospital FGID population but are only applicable to the group of patients with complex FGIDs. Three-quarters of the patient population was women between 40 and 50 years of age (Table 4.1 ). In as much as $50 \%$ of the patients, a history of abdominal surgery was present, and $10 \%$ of the patients used opioids as pain medication. As expected, a high percentage of psychiatric comorbidity was observed, from as much as $80 \%$ in the entire population investigated to $90 \%$ in the locally treated group. The results point toward marked underdetection of psychiatric comorbidities among patients with complex FGIDs. Previously reported prevalence rates of psychiatric comorbidities were much lower in FGID patients, namely, 40-60\%, whereas psychiatric comorbidities were observed in less than $20 \%$ of healthy controls ${ }^{23}$.

It should be acknowledged that this study evaluates standard clinical care in a referral hospital without a control group. Thus, this study has some limitations. Our investigation is a pre-post comparison, which is characterized by an observational naturalistic design. Given that this study is explorative in nature, leading to new hypotheses, these hypotheses have to be evaluated by investigations of a deemed needed sophisticated prospective-controlled study design in the future. However, because this is a pilot investigation, there does not have to be a control condition initially, similar to explorative papers regarding neuromodulation under functional or chronic pain conditions or various functional somatic disorders ${ }^{24-26}$. Of course, there are shortcomings to an investigation of this kind, but we are convinced that this type of research deserves attention. There are several reasons why this study helps to inform 
the field further on unmet clinical needs in complex FGID patients with psychiatric comorbidities. First, there is almost no research of integrated care (gastroenterology and psychiatry) in the complex population of FGID patients. Most research that has been carried out is characterized by a monodisciplinary approach. Thus, the comorbidity of psychiatric disorders is frequently undetected ${ }^{14}$ and treatment outcome remains partially unsatisfactory due to unsolved heterogeneity issues in FGID populations ${ }^{27,28}$. Second, patients are referred to our integrated facility because of long-term suffering and unsolved diagnostic questions. It is almost unethical to include patients in a prospective case-control design if previously undetected psychiatric morbidity is present and evidence based treatment is available (e.g., IBS and panic disorder). Further, the inclusion of a control group of FGID patients with less complex disease characteristics would create an artificial situation as well. To estimate methodological influences on outcome, the external group representing advised treatment coordinated by the general practitioner or referring consultant could be seen as a 'kind of control condition'. Even though the two cohorts (local and external group) were not well matched, it is not likely that positive psychiatric and short term somatic outcomes of integrated care is simply a regression to the mean, as advised continuation of treatment coordinated externally did not influence outcome positively in FGID patients with psychiatric comorbidities. Third, from our experience, we know that it is quite difficult to include patients to a prospective randomized-controlled trial (RCT) if standard therapy for comorbid psychiatric disorders is available ${ }^{29}$. We are carrying out a standardized RCT on SSRIs for IBS and comorbid panic disorder at present, which has been prolonged a couple of times because of delays in inclusion. This is in accordance with RCT evidence in general, as almost $50 \%$ of surgical RCTs remain unpublished ${ }^{30}$. Thus, until publication of high-quality research in integrated care for FGID patients, other papers are necessary, even if quality is low form a methodological point of view. Moreover, this is justifiable since more than one third of RCTs have led to different conclusions at reanalysis ${ }^{31}$. It might be expected that RCTs directed by previous naturalistic clinical research may be more unerring. Fourth, the study population was heterogeneous and not well matched between the integrated local care group and the primary care-based external group. However, heterogeneity is not a convincing issue for withholding a paper from being published, especially in IBS research ${ }^{27,28}$.

Finally, this is a negative study of gastrointestinal symptom relief after 1 year of investigation. This finding may justify the assumption that something is lacking with respect to a complete bio-psychosocial approach. The question is, what is missing here? In terms of biological aspects, a lot has been done, as SSRIs are frequently prescribed in the majority of our patients in order to influence serotonergic transmission. However, frequent care utilizers with a history of a long-term monodisciplinary care trajectory may profit more from prolonged integrated care (gastroenterologist and psychiatrist), especially if a somatoform disorder is present, in order to reattribute somatization of 
complaints to a more stress depended context ${ }^{32}$. This reattribution might even be more consistent if a case manager transitionally accompanies the patient back to primary care.

\section{Conclusion}

The present prospective, open study on the efficacy of routine care applied through an integrated medical-psychiatric outpatient care model for complex FGID patients is the first study to report on a joint multidisciplinary approach for complex FGIDs with psychiatric comorbidity in an outpatient setting. We observed that as much as $80 \%$ of patients with complex FGIDs had not been diagnosed or treated for their psychiatric comorbid disorder up to the point of joint assessment. A beneficial effect of the integrated care approach was shown, with a significant improvement of gastrointestinal and psychiatric symptoms, and quality of life after 6 months. Except for the improvement in gastrointestinal symptoms, this improvement was sustained after 1 year, emphasizing the need of appropriate integrated follow-up management of gastrointestinal symptoms associated with psychiatric comorbidity. To confirm these results, further research is needed, focusing on specific treatment options, larger patient groups with a longer follow-up period as well as a control group to account for natural trends over time. Eventually, the results show that screening for psychiatric comorbidities in FGID patients is necessary, and that collaboration between gastroenterologists and psychiatrists may lead to a better treatment of complex FGID patients. This research indicates that a randomized controlled trial comparing the combined medical-psychiatric treatment with usual care is needed to further prove the advantages of an integrated medical-psychiatric outpatient approach. 


\section{References}

1. Drossman, D. A. The functional gastrointestinal disorders and the Rome III process. Gastroenterology 130, 1377-1390 (2006).

2. Longstreth, G. F. et al. Functional bowel disorders. Gastroenterology 130, 1480-1491 (2006).

3. Talley, N. J. Functional gastrointestinal disorders as a public health problem. Neurogastroenterol. Motil. 20 (Suppl 1), 121-129 (2008).

4. Fortea, J. \& Prior, M. Irritable bowel syndrome with constipation: A European-focused systematic literature review of disease burden. J. Med. Econ. 16, 329-41 (2013).

5. Agarwal, N. \& Spiegel, B. M. The effect of irritable bowel syndrome on health-related quality of life and health care expenditures. Gastroenterol. Clin. North Am. 40, 11-19 (2011).

6. Hungin, A. P. et al. The prevalence, patterns and impact of irritable bowel syndrome: An international survey of 40,000 subjects. Aliment. Pharmacol. Ther. 17, 643-650 (2003).

7. Stanghellini, V. et al. Dyspeptic symptoms and gastric emptying in the irritable bowel syndrome. Am. J. Gastroenterol. 97, 2738-2743 (2002).

8. Van der Veek, P. P. et al. Symptom severity but not psychopathology predicts visceral hypersensitivity in irritable bowel syndrome. Clin. Gastroenterol. Hepatol. 6, 321-328 (2008).

9. Camilleri, M. \& Gorman, H. Intestinal permeability and irritable bowel syndrome. Neurogastroenterol. Motil. 19, 545-552 (2007).

10. Villani, A. C. et al. Genetic risk factors for post-infectious irritable bowel syndrome following a waterborne outbreak of gastroenteritis. Gastroenterology 138, 1502-1513 (2010).

11. Drossman, D. A. et al. Psychosocial aspects of the functional gastrointestinal disorders. Gut 45, II25-30 (1999).

12. Mykletun, A. et al. Prevalence of mood and anxiety disorder in self-reported irritable bowel syndrome (IBS): An epidemiological population based study of women. BMC Gastroenterology 10, 88 (2010).

13. Neeleman, J. et al. The distribution of psychiatric and somatic ill health: Associations with personality and socioeconomic status. Psychosom. Med. 63, 239-247 (2001).

14. Leue, C. et al. Managing complex patients on a Medical Psychiatric Unit: An observational study of university hospital costs associated with medical service use, length of stay andpsychiatric intervention. J. Psychosom. Res. 68, 295-302 (2010).

15. Svedlund, J. et al. GSRS - a clinical rating scale for gastrointestinal symptoms in patients with irritable bowel syndrome and peptic ulcer disease. Dig. Dis. Sci. 33, 129-134 (1988).

16. Toner, B. B. et al. The development of a cognitive scale for functional bowel disorders. Psychosom. Med. 60, 492-497 (1998).

17. Bjelland, I. et al. The validity of the Hospital Anxiety and Depression Scale: An updated literature review. J. Psychosom. Res. 52, 69-77 (2002).

18. Van der Ploeg HM, Defares PB, Spielberger CD: Manual of the Self-Assessment Questionnaire. A Dutch adaptation of the Spielberger State-Trait Anxiety Inventory STAI-DY. Lisse, Swets \& Zeitlinger (1980).

19. Aaronson, N. K. et al. Translation, validation, and norming of the Dutch language version of the SF-36 Health Survey in community and chronic disease populations. J. Clin. Epidemiol. 51, 1055-1068 (1998).

20. Ware, J. E. et al. SF-36 Physical and Mental Health Summary Scales: A User's Manual. Boston, MA: The Health Institute (1994).

21. IBM Corp. Released 2012. IBM SPSS Statistics for Windows, Version 21.0. Armonk, NY: IBM Corp.

22. Cnaan, A. et al. Using the general linear mixed model to analyse unbalanced repeated measures and longitudinal data. Stat. Med. 16, 2349-2380 (1997).

23. Levy, R. L. et al. Psychosocial aspects of the functional gastrointestinal disorders. Gastroenterology 130, 1447-1458 (2006).

24. Stidd, D. A. et al. Spinal cord stimulation with implanted epidural paddle lead relieves chronic axial low back pain. J. Pain. Res. 7, 465-470 (2014).

25. Lihua, P. et al. Spinal cord stimulation for cancer related pain in adults. Cochrane Database Syst. Rev. 2, CD009389 (2013). 
26. Noblett, K. et al. Results of a prospectiev multicenter Study evaluating quality of life, safety, and efficacy of sacral neuromodulation at twelve months in subjects with symptoms of overactive bladder. Neurourol. Urodyn. 35, 246-251 (2016).

27. Ford, A. C. et al. Efficacy of antidepressants and psychological therapies in irritable bowle syndrome: Systematic review and meta-analysis. Gut 58, 367-378 (2009).

28. Ford, A. C. et al. Effect of antidepressants and psychological therapies, including hypnotherapy, in irritable bowel syndrome: Systematic review and met-analysis. Am. J. Gatsroenterol. 109, 1350-1365 (2014).

29. Clinical Trials.gov Protocol Registration System; Identifier:NCT01551225.

30. Chapman, S. J. et al. Discontinuation and non-publication of surgical randomised controlled trials: An observational study. BMJ 349, g6870 (2014).

31. Ebrahim, S. et al. Reanalyses of randomized clinical trial data. JAMA 312, 1024-1032 (2014).

32. Schweickhardt, A. et al. Short-term psychotherapeutic interventions for somatizing patients in the general hospital: A randomized controlled study. Psychother. Psychosom. 76, 339-346 (2007). 


\section{Abstract}

\section{Aims}

Pelvic floor dysfunctions (PFDs), like voiding complaints, urinary and fecal incontinence and prolapse, are prevalent and associated with decrease in quality of life. PFDs are often complex and multifactorial in origin showing interrelationships between different PFD and with affective conditions. The primary aim of this study is to describe the prevalence of affective complaints in a cohort of Pelvic Care Centre (PCC) patients. The secondary aim is to describe associations between PFDs and depression or anxiety.

\section{Methods}

A cross sectional cohort study at an University Hospital's PCC. First contact patients were included in a triage system and filled out questionnaires regarding pelvic floor complaints and the Hospital Anxiety and Depression Scale (HADS). Linear (dummy-) regression analysis of HADS sub-scores was performed to test the effects of relevant clinical predictors related, and not directly related, to pelvic floor problems, and demographic characteristics of the patients.

\section{Results}

From 1862 eligible first-contact PCC patients 1510 (mean age 57.1) had completed the questionnaire (352 missing, 18.9\%). The prevalence of anxiety and depression complaints was $30.9 \%$ and $20.3 \%$, respectively. The variance explained for depression score by PFDs was 0.12 and 0.074 for anxiety.

\section{Conclusion}

Anxiety and depression are prevalent (30.9\% and $20.3 \%$, respectively) in a cohort of PFDs. PFDs can explain variance within anxiety and depression complaints. Corrected for other contributing variables, $12 \%$ of depression and $7.4 \%$ of anxiety was directly related to PFDs. We advocate a multidisciplinary approach, containing psychometric assessment for PFDs in order to obtain better diagnostic results and personalized treatment options. 


\section{Introduction}

Pelvic floor dysfunctions (PFDs), such as voiding complaints, urinary and fecal incontinence (UI, FI), constipation, sexual problems and pelvic organ prolapse (POP) are prevalent and often distressing health problems in both sexes ${ }^{1}$. The incidence increases with age and PFDs are associated with a decrease in quality of life ${ }^{1}$. Life expectancy of people is rising, and therefore, the worldwide burden of PFDs is expected to increase significantly over time. Hence, future requirements for PFD-related healthcare is expected to rise by $50 \%$ over the next 30 years ${ }^{2}$. PFDs are complex and multi-factorial by origin, therefore a multidisciplinary approach is recommended ${ }^{3}$.

Recently, we reported about the complexity of PFDs presented at our Pelvic Care Centre (PCC), their multi-factorial character and frequent interactions between each other. We demonstrated that one out of four women and one out of seven male patients had more than one pelvic floor related health problem ${ }^{4}$. Overactive bladder $(O A B)$ is such a complex PFD, with a prevalence of around $17 \%^{5}$. OAB is a symptom complex of urgency (sudden compelling desire to pass urine, which is difficult to defer), usually with frequency (voiding 8 or more times per day) and nocturia (awakening at night to void), with (OAB wet) or without (OAB dry) urgency urinary incontinence ${ }^{6}$. OAB with or without incontinence is often associated with affective disorders ${ }^{7}$, and may lead to significant social and functional impairment ${ }^{8}$. OAB and affective disorders may even share common biological pathways comparable to functional bowel disorders ${ }^{9}$.

Affective disorders such as anxiety disorders or mood disorders, according to the DSM-V criteria $^{10,11}$ have a high lifetime prevalence ranging from $2-15 \%$ for depression ${ }^{11}$ and around $18 \%$ for anxiety disorders ${ }^{12}$. Anxiety disorders are globally the most common psychiatric disorders according to the World Health Organisation ${ }^{11,12}$. However, most physicians do not adequately identify affective conditions based on self-reported symptoms $^{12}$, similar to what has been reported for irritable bowel syndrome (IBS) ${ }^{13}$. For example, physicians miss the diagnosis of somatoform disorders in around two third of the cases, and psychiatric diagnoses remained undetected in even $95 \%$ of most complex patients with somatic and psychiatric comorbidity ${ }^{14,15}$.

Our aim is to describe, analyse, and interpret the relation between PFD symptoms and Hospital Anxiety and Depression Scale (HADS) scores in patients referred to our PCC.

\section{Methods}

The PCC where the study was conducted is a multidisciplinary outpatient clinic with participation of urologists, gynecologists, colorectal surgeons, gastro-enterologists, general hospital psychiatrists, pelvic physiotherapists, sexologists, and specially trained continence nurses. Since 2005, it is serving as a large secondary and tertiary referral centre, and more than 5500 patients with functional pelvic disorders have been treated 
yet. The PCC developed and used a digital triage system for patient selection, in order to measure subjective complaints during the preceding six months with regard to six different PFDs (i.e., voiding dysfunction, UI, POP, constipation, $\mathrm{FI}$, and sexual/pelvic pain problems). The triage has been described elsewhere ${ }^{4}$. In 2008, our PCC team started collaboration with the hospital psychiatrists. One of the reasons was that at the PCC many PFDs patients - especially those with frequent and recurrent visits - were seen with symptoms of psychological and psychiatric ill-health. Hence, it was decided to routinely screen PCC patients by using the HADS questionnaire ${ }^{16}$. The HADS is a short selfassessment tool to screen for the presence of a mood disorder in medically ill patients. It contains 14 items, each rated on a 4-point Likert scale, which are divided into two subscales that assess depression (HADS-D) and anxiety (HADS-A). The subscales are categorized as a four level ordinal variable: normal 0-7; mild 8-10, moderate 11-14 or severe mood disturbance 15-21. The HADS has a high internal consistency (Cronbach's alpha $=0.85)^{16}$. From 2008-2013, all first contact PCC patients with PFD were included in the present study.

\section{Statistics}

Statistics of categorical data are presented as frequencies and percentages. Deviations from normality of distribution in metric variables were tested by the Shapiro-Wilk test. If metric data were not-normally distributed minimum and maximum values are added to the mean and the standard deviation. In order to facilitate a parametric statistical analysis, it was decided to calculate the square root of the two outcome scores (the HADS anxiety and depression scales) and to use the transformation as separate dependent variables in regression analysis. A residual analysis was performed for each outcome to investigate whether the distribution of Studentized z-score residuals turns out to be not too dissimilar from a normal distribution. Linear (dummy-) regression analysis of HADS depression and HADS anxiety scales was done to test the effects of A: relevant clinical predictors related to pelvic floor problems, B: other relevant clinical predictors not directly related to pelvic floor problems; and C: demographic characteristics of the patients. Section A of the predictors was subdivided into six general factors measuring prevalence of problems in the pelvic region: urinary incontinence, voiding problems, prolapse, fecal incontinence, constipation, and sexual problems, and into 40 specific potential predictors measuring more detailed items from a questionnaire concerning pelvic problems. At first, explorative regression models both forward selection and backward elimination techniques with list wise deletion of missing values were used. It was decided that section $C$ predictors (age and gender) plus the six general prevalence predictors from section $\mathrm{A}$ always had to be included within the search for the best-fitting model, regardless whether they would have statistical significant effects upon the outcome or not. Next, the resulting direct-effects regression model was tested for all first-order interactions using forward selection only. Next to variance explained by the model, the additional variance explained by pelvic predictors is 
given. A p-value of $<0.05$ is considered to be statistically significant. All data-analysis was done with IBM SPSS-pc version 22.

\section{Results}

From the 1862 eligible first-contact PCC patients, 1510 satisfactorily completed the HADS questionnaire (352 missing, 18.9\%). Mean age of patients was 57.1, s.d. 16.9 (1494), and 1543 (82.9\%) were female patients, while 319 (17.1\%) were men. Table 5.1 shows - next to the prevalence of first contact pelvic floor complaints - the clinical background variables, which were also presumed to have an effect on depression and/or anxiety (Table 5.1).

Table 5.1 Demographic background variables and clinical characteristics specific for problems or complaints within the pelvic region in patients contacted for the first time at the PCC during 2008-2013 $(N=1862)$.

\begin{tabular}{|c|c|c|c|c|}
\hline Gender & & \multicolumn{2}{|c|}{ female: 1543 (82.9\%) } & male: 319 (17.1\%) \\
\hline Age & \multicolumn{3}{|c|}{56.1 years } & (s.d. 16.9; range: 14-94) \\
\hline \multicolumn{5}{|l|}{$\begin{array}{l}\text { Complaints of (now or during the } \\
\text { past year): }\end{array}$} \\
\hline Asthma & 223 & $(12.0 \%)$ & & \\
\hline High blood pressure & 414 & $(22.2 \%)$ & & \\
\hline Gall stones & 25 & $(1.3 \%)$ & & \\
\hline Kidney stones & 15 & $(0.8 \%)$ & & \\
\hline Diabetes & 135 & $(7.3 \%)$ & & \\
\hline $\begin{array}{l}\text { Headaches } \\
\text { (Prevalence of:) }\end{array}$ & \multicolumn{3}{|c|}{ (Prevalence of:) } & \\
\hline Urine incontinence & 726 & $(39.0 \%)$ & & \\
\hline Voiding problems & 1106 & $(59.4 \%)$ & & \\
\hline Prolapse & 501 & $(26.9 \%)$ & & \\
\hline Obstipation & 179 & $(9.6 \%)$ & & \\
\hline Fecal Incontinence & 261 & $(14.0 \%)$ & & \\
\hline Sexual problems & 61 & $(3.3 \%)$ & & \\
\hline \multirow[t]{2}{*}{ Uterus extirpation } & 315 & $(26.4 \%)$ & & \\
\hline & Sometimes & Often & Always & \\
\hline Urinary urgency & 169 (9.1\%) & $138(7.4 \%)$ & $180(9.7 \%)$ & \\
\hline Stress incontinence (coughing) & $241(12.9 \%)$ & $153(8.2 \%)$ & 99 (5.3\%) & \\
\hline Stress incontinence (coughing) & $241(12.9 \%)$ & $153(8.2 \%)$ & 99 (5.3\%) & \\
\hline Burning pain during voiding & 201(10.9\%) & $107(5.4 \%)$ & $25(1.3 \%)$ & \\
\hline Hematuria past 2 years & $77(4.1 \%)$ & 17 (0.9\%) & $9(0.5 \%)$ & \\
\hline Incomplete bladder emptying & $3(0.3 \%)$ & $327(27.9 \%)$ & $291(24.9 \%)$ & \\
\hline Abdominal pain past 6 months & $9(6.9 \%)$ & $57(4.0 \%)$ & $90 \quad(6.3 \%)$ & \\
\hline Fecal composition complaints & $125(6.7 \%)$ & $126(6.8 \%)$ & $230(12.4 \%)$ & \\
\hline Abdominal pain during defecation & $99(5.3 \%)$ & $57(3.1 \%)$ & $90(4.8 \%)$ & \\
\hline Anal pain during defecation & $94(6.6 \%)$ & $63(4.4 \%)$ & $69(4.8 \%)$ & \\
\hline Blood loss during defecation & $36(2.5 \%)$ & $14(1.0 \%)$ & $10(0.7 \%)$ & \\
\hline Visible prolapse during defecation & $50(3.5 \%)$ & $16(1.1 \%)$ & 22 (1.6\%) & \\
\hline Incomplete defecation & $102(7.2 \%)$ & $104(8.0 \%)$ & $190(13.3 \%)$ & \\
\hline
\end{tabular}


Table 5.1 (continued)

\begin{tabular}{|c|c|c|c|c|c|}
\hline & None/< once & Once & 2-3 times & 4-7 times & $>7$ times \\
\hline Frequency weekly urine loss & $1198(64.4 \%)$ & $32(1.7 \%)$ & 77 (4.1\%) & $187(10.0 \%)$ & $367(19.7 \%)$ \\
\hline \multirow[t]{2}{*}{ Frequency $24 \mathrm{hrs}$. urine loss } & $1219(65.5 \%)$ & $116(6.2 \%)$ & $267(14.3 \%)$ & $186(10.0 \%)$ & $73(3.9 \%)$ \\
\hline & drops & dashes & varies strongly & whole load & \\
\hline \multirow[t]{2}{*}{ Quantity of urine loss } & $170(9.1 \%)$ & $302(16.2 \%)$ & $177(9.5 \%)$ & $38(2.0 \%)$ & \\
\hline & 1-3 months & 4-12 months & 13-24 months & $>2$ years & \\
\hline \multirow[t]{2}{*}{ Ul duration } & $27(1.4 \%)$ & $170(9.1 \%)$ & $42(2.2 \%)$ & $448(24.1 \%)$ & \\
\hline & $<6$ & $6-10$ & $>10$ & & \\
\hline \multirow[t]{2}{*}{ Daytime urinary frequency } & $808(43.5 \%)$ & $759(40.9 \%)$ & $253(13.7 \%)$ & & \\
\hline & Never & $1-3$ & $>3$ & & \\
\hline Frequency of UTI past 12 months & $1308(70.6 \%)$ & $291(15.7 \%$ & $253(13.6 \%)$ & & \\
\hline
\end{tabular}

Legend: $U T \mathrm{TI}=$ urinary tract infection.

Table 5.2 presents the statistical characteristics of both main outcome variables: HADS anxiety and HADS depression scores concerning the four severity categories of both outcomes for the total group and for men and women separately. More than $30 \%$ of the patients had an abnormal anxiety score and more than $20 \%$ presented an abnormal depression score. Testing for normality of distribution, it was found that both parameters were non-normally distributed. The Shapiro-Wilk test results for HADSanxiety and HADS-depression respectively is $P<0.001$.

Table 5.2 Statistical characteristics of prevalence in HADS anxiety and depression scales in patients contacted for the first time at the PCC during 2008-2013 ( $N=1862)$.

\begin{tabular}{lcccccc}
\hline & \multicolumn{2}{c}{ Total } & \multicolumn{2}{c}{ Females } & \multicolumn{2}{c}{ Males } \\
\cline { 2 - 6 } & HADS-A & HADS-D & HADS-A & HADS-d & HADS-A & HADS-D \\
\hline $0-7$ & $1043(69.0 \%)$ & $1203(79.7 \%)$ & $872(68.8)$ & $1009(79.6)$ & $171(70.4)$ & $194(79.8)$ \\
$8-10$ & $203(13.4 \%)$ & $156(10.3 \%)$ & $173(13.7)$ & $137(10.8)$ & $30(12.3)$ & $10(7.8)$ \\
$11-14$ & $199(13.2 \%)$ & $118(7.8 \%)$ & $165(13.0)$ & $95(7.5)$ & $34(14.0)$ & $23(9.5)$ \\
$15-21$ & $65(4.3 \%)$ & $33(2.2 \%)$ & $57(4.5)$ & $26(2.1)$ & $8(3.3)$ & $7(2.9)$ \\
\hline Abnormal & $467(30.9 \%)$ & $307(20.3 \%)$ & $395(31.2)$ & $258(20.4)$ & $72(29.6)$ & $40(20.2)$ \\
Mean (SD) & $6.35(4.12)$ & $4.37(4.04)$ & $6.41(4.07)$ & $4.30(4.01)$ & $6.03(4.34)$ & $4.72(4.21)$ \\
Total & 1510 & 1510 & 1267 & 1267 & 243 & 243 \\
\hline
\end{tabular}

Legend: Frequencies (and percentages) of categorized HADS scores.

Within successive explorative regression models the final best-fitting model predicted the HADS depression and anxiety. A final regression model for each main outcome is presented in Tables 5.3 and 5.4. Table 5.3 shows the results of the final (dummy) regression model for HADS-depression. Eight direct regression effects and three first-order interactions had statistically significant effects upon HADS depression. Final results can be summarized as follows, interpreting the presented unstandardized regression coefficients. 1. (0.008) The higher the age of a patient, the higher the HADS depression scores are. 2. (0.220) If patients responded affirmatively on having urine incontinence, they showed higher scores on the HADS depression sub-score. 3. (0.460) If 
patients responded affirmatively on having sexual problems, they showed higher scores on HADS depression. 4. (0.066) If patients confirmed to have complaints about the composition of their faeces, they tended to have higher scores on the HADS depression sub-scale. 5. (0.156) If patients suffered from serious headaches, they had higher HADS depression sub-scores. 6. (0.049) If patients showed complaints on bladder emptying during the past 6 months, they had higher scores on the HADS depression sub-scale.

Table 5.3 Final model results from dummy-regression analysis on square-root-transformed scores of the HADS depression sub-scale as a dependent variable using clinical predictors and background demographics.

\begin{tabular}{|c|c|c|c|c|c|c|c|}
\hline \multirow[b]{2}{*}{ Predictors: } & \multicolumn{2}{|c|}{$\begin{array}{l}\text { Unstandardized } \\
\text { Coefficients }\end{array}$} & \multicolumn{3}{|c|}{$\begin{array}{l}\text { Standardized } \\
\text { Coefficients }\end{array}$} & \multicolumn{2}{|c|}{$\begin{array}{l}\text { 95\% Confidence } \\
\text { Interval for B }\end{array}$} \\
\hline & $B$ & Std. Error & Beta & $\mathrm{t}$ & $P$-value & Lower Bound & Upper Bound \\
\hline (Constant) & 0.891 & 0.118 & & 7.580 & $<0.001$ & 0.660 & 10.121 \\
\hline Age & 0.008 & 0.002 & 0.127 & 4.979 & $<0.001$ & 0.005 & 0.011 \\
\hline Gender(0=F,1=M) & 0.363 & 0.125 & 0.127 & 2.894 & 0.004 & 0.117 & 0.609 \\
\hline $\begin{array}{l}\text { Prevalence urine } \\
\text { incontinence }\end{array}$ & 0.220 & 0.067 & 0.102 & 3.263 & 0.001 & 0.088 & 0.352 \\
\hline $\begin{array}{l}\text { Prevalence voiding } \\
\text { problems }\end{array}$ & 0.021 & 0.067 & -0.010 & -0.306 & 0.760 & -0.152 & 0.111 \\
\hline $\begin{array}{l}\text { Prevalence prolapse } \\
\text { problems }\end{array}$ & 0.322 & 0.091 & 0.138 & 3.549 & $<0.001$ & -0.500 & -0.144 \\
\hline Prevalence obstipation & 0.122 & 0.090 & 0.036 & 1.353 & 0.176 & -0.055 & 0.300 \\
\hline $\begin{array}{l}\text { Prevalence fecal } \\
\text { incontinence }\end{array}$ & -0.001 & 0.086 & 0.000 & -0.007 & 0.994 & -0.169 & 0.168 \\
\hline $\begin{array}{l}\text { Prevalence sexual } \\
\text { problems }\end{array}$ & 0.460 & 0.149 & 0.077 & 3.080 & 0.002 & 0.167 & 0.752 \\
\hline $\begin{array}{l}\text { Complaints on fecal } \\
\text { composition }\end{array}$ & 0.066 & 0.020 & 0.098 & 3.230 & 0.001 & 0.026 & 0.106 \\
\hline $\begin{array}{l}\text { Having serious } \\
\text { headaches }\end{array}$ & 0.156 & 0.044 & 0.089 & 3,525 & $<0.001$ & 0.069 & 0.243 \\
\hline $\begin{array}{l}\text { Having bladder } \\
\text { problems(past } 6 \mathrm{~m} \text { ) }\end{array}$ & 0.049 & 0.024 & 0.058 & 2.093 & 0.037 & 0.003 & 0.095 \\
\hline $\begin{array}{l}\text { Freq. urine } \\
\text { incontinence(24hrs) }\end{array}$ & 0.084 & 0.028 & 0.104 & 3.052 & 0.002 & 0.030 & 0.139 \\
\hline $\begin{array}{l}\text { Having burning } \\
\text { sensation }\end{array}$ & 0.056 & 0.022 & 0.065 & 2.541 & 0.011 & 0.013 & 0.099 \\
\hline Diabetes mellitus & 0.101 & 0.047 & 0.054 & 2.131 & 0.033 & 0.008 & 0.193 \\
\hline $\begin{array}{l}\text { Prolapse-related } \\
\text { voiding problems }\end{array}$ & 0.284 & 0.118 & 0.099 & 2.413 & 0.016 & 0.053 & 0.515 \\
\hline $\begin{array}{l}\text { Gender-related } \\
\text { fecal incontinence* }\end{array}$ & 0.366 & 0.188 & 0.057 & 1.950 & 0.051 & -0.002 & 0.735 \\
\hline $\begin{array}{l}\text { Gender-related } \\
\text { freq. U.I.(24hrs)* }\end{array}$ & -0.145 & 0.055 & -0.111 & -2.608 & 0.009 & -2.54 & -0.036 \\
\hline
\end{tabular}

Legend: Variance explained: 0.120. $\mathrm{N}=1506$. ${ }^{*}$ Effects presented are for male patients. For female patients apply reverse sign on $\mathrm{B}$, Beta or $\mathrm{t}$ coefficient. 
7 (0.056) If patients said that they had a burning sensation or pain while urinating in the past 6 months, they were likely to score higher on HADS depression. 8. (0.101) If patients had diabetes mellitus, they also showed high scores on the HADS depression sub-scale. 9. (0.284) If patients confirmed that they had prolapse-related voiding problems, they showed high scores on depressive HADS symptoms. 10. (0.366) Faecal incontinence in men had higher effects on HADS depression scores than faecal incontinence in women. 11. (-0.145) The higher the involuntary loss of urine during 24 hours, the higher the score on HADS depression sub-score was. This effect is significantly higher in female patients compared to men. The explained variance for the final regression model in 1506 patients is 0.120 .

Table 5.4 shows the results of the final (dummy) regression model for HADSanxiety. Six direct regression effects and five first-order interaction effects have statistically significant effects upon HADS anxiety. Final results can be summarized as follows, interpreting the unstandardized regression coefficients. 1. (0.276) If patients responded affirmatively on having sexual problems, they showed higher scores on HADS anxiety sub-scores. 2. (0.135) If patients suffered from serious headaches, they had higher scores on HADS anxiety. 3. (0.053) If patients complained on bladder emptying during the past 6 months, they had higher scores on HADS anxiety. 4. (0.048) If patients said that they had a burning or pain while urinating in the past 6 months, they were likely to score high on HADS anxiety. 5. (0.211) If patients suffered from kidney stones, they also showed high scores on HADS anxiety. 6. (0.059) If patients complained about defecation during the past 6 months, they were likely to score high on HADS anxiety. 7. (0.208) If patients said that they had prolapse-related voiding problems, they had high scores on HADS anxiety sub-scores. 8. (0.006) Urine incontinence in senior patients had higher effects on HADS anxiety scores than urine incontinence in younger patients. 9. (0.371) The higher the obstipation problems, the higher the score on HADS anxiety was, but this effect is significantly higher in female patients than in men. 10. (0.069) If patients reported faecal incontinence, they generally had higher HADS anxiety scores, which is significantly higher in men. 11. (-0.008) Older women showed higher scores on the HADS anxiety sub-scale than older men. The explained variance for the final regression model in 1507 patients is 0.074 . 
Table 5.4 Final model results from dummy-regression analysis on square-root-transformed scores of the HADS anxiety sub-scale as a dependent variable using clinical predictors and background demographics.

\begin{tabular}{|c|c|c|c|c|c|c|c|}
\hline \multirow[b]{2}{*}{ Predictors: } & \multicolumn{2}{|c|}{$\begin{array}{l}\text { Unstandardized } \\
\text { Coefficients }\end{array}$} & $\begin{array}{l}\text { Standardized } \\
\text { Coefficients }\end{array}$ & \multicolumn{4}{|c|}{$\begin{array}{l}\text { 95\% Confidence } \\
\text { Interval for B }\end{array}$} \\
\hline & $\mathrm{B}$ & Std. Error & Beta & $\mathrm{t}$ & $P$-value & Lower Bound & Upper Bound \\
\hline (Constant) & 2.052 & 0.117 & & 17.605 & $<0.001$ & 10.823 & 20.280 \\
\hline Age & 0.002 & 0.002 & 0.044 & 1.308 & 0.191 & -0.001 & 0.006 \\
\hline Gender $(0=F, 1=M)$ & 0.239 & 0.229 & 0.098 & 1.041 & 0.298 & -0.211 & 0.689 \\
\hline $\begin{array}{l}\text { Prevalence urine } \\
\text { incontinence }\end{array}$ & -0.244 & 0.179 & -0.133 & -1.365 & 0.173 & -0.595 & 0.107 \\
\hline $\begin{array}{l}\text { Prevalence voiding } \\
\text { problems }\end{array}$ & -0.037 & 0.058 & -0.021 & -0.649 & 0.516 & -0.150 & 0.076 \\
\hline $\begin{array}{l}\text { Prevalence prolapse } \\
\text { problems }\end{array}$ & -0.209 & 0.079 & -0.105 & -2.656 & 0.008 & -0.363 & 0.055 \\
\hline Prevalence obstipation & 0.106 & 0.083 & 0.037 & 1.281 & 0.200 & -0.056 & 0.269 \\
\hline $\begin{array}{l}\text { Prevalence fecal } \\
\text { incontinence }\end{array}$ & 0.060 & 0.072 & 0.024 & 0.830 & 0.407 & -0.081 & 0.200 \\
\hline $\begin{array}{l}\text { Prevalence sexual } \\
\text { problems }\end{array}$ & 0.276 & 0.131 & 0.054 & 2.115 & 0.035 & 0.020 & 0.533 \\
\hline $\begin{array}{l}\text { Having serious } \\
\text { headaches }\end{array}$ & 0.135 & 0.038 & 0.090 & 3.527 & $<0.001$ & 0.060 & 0.211 \\
\hline $\begin{array}{l}\text { Having bladder } \\
\text { problems(past } 6 \mathrm{~m} \text { ) }\end{array}$ & 0.053 & 0.020 & 0.073 & 2.663 & 0.008 & 0.014 & 0.091 \\
\hline Having kidney stones & 0.211 & 0.073 & 0.073 & 2.889 & 0.004 & 0.068 & 0.354 \\
\hline $\begin{array}{l}\text { Having burning } \\
\text { sensation }\end{array}$ & 0.048 & 0.019 & 0.066 & 2.516 & 0.012 & 0.011 & 0.086 \\
\hline $\begin{array}{l}\text { Having slime in feces } \\
\text { (past } 6 \mathrm{~m} \text { ) }\end{array}$ & 0.059 & 0.029 & 0.054 & 2.040 & 0.041 & 0.002 & 0.115 \\
\hline $\begin{array}{l}\text { Prolapse-related } \\
\text { voiding problems }\end{array}$ & 0.208 & 0.103 & 0.085 & 2.024 & 0.043 & 0.006 & 0.409 \\
\hline Urine Incontinence age & 0.006 & 0.003 & 0.219 & 2.185 & 0.029 & 0.001 & 0.012 \\
\hline $\begin{array}{l}\text { Gender-related } \\
\text { obstipation }\end{array}$ & 0.371 & 0.191 & 0.057 & 1.941 & 0.052 & -0.004 & 0.747 \\
\hline $\begin{array}{l}\text { Gender-related } \\
\text { fecal incontinence }\end{array}$ & 0.377 & 0.167 & 0.069 & 2.261 & 0.024 & 0.050 & 0.704 \\
\hline Age ${ }^{*}$, Gender $^{*}$ & -0.008 & 0.004 & -0.194 & -2.051 & 0.040 & -0.015 & 0.000 \\
\hline
\end{tabular}

Legend: Variance explained: 0.074. N=1507. *Effects presented are for male patients. For female patients apply reverse sign on B, Beta or t coefficient.

\section{Discussion}

Although multidisciplinary care is advocated for $\mathrm{PFDs}^{17}$, up to now, psychological or psychiatric measurement is still not routinely incorporated into the assessment of PFDs. As in other multidisciplinary efforts ${ }^{18}$, our cohort data demonstrate that functional complaints are strongly associated with emotion regulation in PFDs. At our PCC, $30 \%$ of patients with PFDs had an abnormal anxiety score and $20 \%$ an abnormal depression score. Furthermore, corrected for confounding factors, PFDs may account for $7.4 \%$ of 
the variance in anxiety scores and $12 \%$ of the variance in depression scores. These findings are in accordance with the literature concerning psychiatric and somatic comorbidity in general and with regard to PFDs in particular. For example, in cancer patients, abnormal HADS anxiety scores were found in $30 \%$ of the patients and abnormal depression scores were seen in $24.1 \%^{19}$. In a cohort of patients with chronic obstructive pulmonary disease (COPD) hospitalized for an acute exacerbation, the prevalence of an abnormal HADS depression score was $44 \%$ at admittance, and $11 \%$ had an abnormal HADS anxiety score ${ }^{20}$. Moreover, the prevalence of affective disorders in patients with overactive bladder is considerable ${ }^{7}$. In the UK, HADS anxiety scores of 8 and higher were found in $30 \%$ and $47 \%$ of men with $O A B$ without and with bothersome complaints respectively, and in women this was $44 \%$ for $O A B$ without bother, up to almost $60 \%$ in $\mathrm{OAB}$ with bother ${ }^{21}$. Prevalence rates in Sweden were similar to our results with aberrant scores of $8 \%-19 \%$ in men and $18 \%-32 \%$ in women for OAB without and with bother, respectively ${ }^{21}$. Depression scores in our cohort are similar to scores reported by Swedish men and women with OAB, but lower compared to UK men and women ${ }^{21}$. Anxiety and depression scores, as measured by the HADS, were much higher for women with urgency incontinence (UUI). The majority of women with UUI reported anxiety (56\%) and over a third reported depression ${ }^{22}$. The prevalence of depression and anxiety in patients with overall PFDs is not well studied. In the United States, a prevalence of $22 \%$ depressed patients among women seeking treatment for advanced pelvic organ prolapse (POP) has been reported. This was five times higher in women with POP compared to those without ${ }^{23}$. Additionally, within the group of women with prolapse, women with symptoms of depression reported lower quality of life and worse prolapse related symptom scores compared to women without depressive symptoms ${ }^{23}$. Moreover, a qualitative study regarding the emotional burden experienced by women seeking treatment for prolapse revealed that women's psychological well-being is closely related to their pelvic floor symptoms ${ }^{24}$. In our cohort, there are many patients with multifactorial causes of PFDs or interacting PFDs. Patients with urinary incontinence, especially in case of higher severity and complexity due to other health problems (e.g., headache or diabetes mellitus) showed higher HADS depression and anxiety scores. This is in line with the findings of Di Gangi Herms et al. who found that patients with lowcomplexity incontinence problems had fewer depression or anxiety related symptoms, less distress, and better clinical outcome at follow-up, than patients with highcomplexity ${ }^{25}$.

There may be some reluctance in the field to consider a broader multidisciplinary approach as laid down in the NICE-urinary incontinence guideline, which may be related in part to low diagnostic sensitivity for comorbidity and complexity ${ }^{26}$. This is probably due to the practical difficulties related to medical specialists' experience in looking after patients with PFDs, since many will not have had sufficient training and will not schedule sufficient time and resources to implement complexity assessment. Nevertheless, psychometric questionnaires can detect mental co-morbidity quite easily in somatic 
patients. The HADS performs well in the screening for depression or anxiety, and detects cases of anxiety disorders and depression in somatic, psychiatric, and primary care patients as well as in the general population ${ }^{27}$. Psychiatric case detection is important, given that psychiatric disorders affect the prognosis of somatic conditions and the level of health care consumption ${ }^{28}$. Depression can adversely affect self-care and may increase the risk of new medical complaints, complications, and mortality ${ }^{28}$. Quite similar, anxiety can amplify symptoms of medical illnesses and may worsen clinical outcomes $^{29}$. In a longitudinal study, depression was, at baseline, associated with the persistence of urinary incontinence (UI) in women ${ }^{30}$, and anxiety and depression may intensify $\mathrm{UI}$ and decrease the benefit of treatment ${ }^{31}$. A large population based survey revealed an inverse relationship between depressive symptoms and health care seeking. Depressive symptoms may thus prevent patients from seeking treatment ${ }^{21}$. It is possible that there is a reinforcing cycle of increasing PFD symptoms, with increasing bother and increasing affective complaints that does not lead to consecutive treatment seeking.

However, combined consultations of a psychiatrist and an uro-gynaecologist, urologist or colorectal surgeon may not be feasible in many practice situations. Nevertheless, our PCC team increasingly experienced that especially patients with complicated and multifactorial PFDs appeared to have (hidden) symptoms of depression and/or anxiety, which is clearly supported by different observations in literature. Moreover, psychiatric disorders affect the prognosis of somatic conditions and the level of health care consumption in general ${ }^{28}$, and the present findings demonstrate the importance of an interdisciplinary approach in patients with PFDs, addressing physical, emotional, as well as social and relational aspects.

The results of our investigation should be seen in the light of limitations. Due to the cross-sectional design of our study, we were not able to investigate causality aspects. Hence, the question whether depression and anxiety are causing PFDs or PDFs are causing affective conditions remains unanswered. Hopefully, we are able to present a follow-up study of the effects of treatment of PFDs on the HADS score, and vice versa, in the near future.

\section{Conclusion}

In the present study, anxiety and depression, suggestive for affective conditions, are prevalent (30.9\% and $20.3 \%$, respectively) in a cohort of patients with PFDs. In our final regression model, the explained variance of depression and anxiety, measured with the HADS was $12 \%$ and $7.4 \%$, respectively, meaning that, corrected for other contributing variables/factors, $12 \%$ of depression, and $7.4 \%$ of anxiety was directly related to PFDs. Whether depression or anxiety may lead to PFDs was not part of this investigation. We advocate a multidisciplinary approach including psychological and psychiatric assessment, using the HADS questionnaire, at least in complex PFD patients. 


\section{References}

1. Milsom, I. et al. Epidemiology of urinary incontinence (IU) and other lower urinary tract symptoms (LUTS), pelvic orgen prolapse (POP) and anal incontinence. In: Abrams P, editor. Incontinence 5th ed, 15108 (2013).

2. Luber, K. M. et al. The demographics of pelvic floor disorders: Current observations and future projections. Am. J. Obstet. Gynecol. 184, 1496-1501 (2001).

3. Davis, K. J. et al. Pelvic floor dysfunction: A scoping study exploring current service provision in the UK, interprofessional collaboration and future management priorities. Int. J. Clin. Pract. 64, 1661-70 (2010).

4. Berghmans, B. et al. Prevalence and triage of first-contact complaints on pelvic floor dysfunctions in female patients at a Pelvic Care Centre. Neurourol. Urodyn. 35, 503-508 (2015).

5. Coyne, K. S. et al. National community prevalence of overactive bladder in the United States stratified by sex and age. Urology 77, 1081-1087 (2011).

6. Abrams, P. et al. The standardisation of terminology in lower urinary tract function: Report from the standardisation sub-committee of the International Continence Society. Urology 61, 37-49 (2003).

7. Vrijens, D. et al. Affective symptoms and the overactive bladder: A systematic review. J. Psychosom. Res. 78, 95-108 (2015).

8. Melville, J. L. et al. Incontinence severity and major depression in incontinent women. Obstet. Gynecol. 106, 585-592 (2005).

9. Leue, C. et al. Bidirectional associations between depression/anxiety and bowel disease in a population based cohort. J. Epidemiol. Community Health 59, 434 (2005).

10. Regier, D. A. et al. The DSM-5: Classification and criteria changes. World Psychiatry 12,92-98 (2013).

11. Wang, P. S. et al. Twelve-month use of mental health services in the United States: Results from the National Comorbidity Survey Replication. Arch. Gen. Psychiatry 62, 629-640 (2005).

12. Meyer, T. et al. Depression but not anxiety is a significant predictor of physicians' assessments of medical status in physically ill patients. Psychother. Psychosom. 69, 147-54 (2000).

13. Fukudo, S. et al. Evidence-based clinical practice guidelines for irritable bowel syndrome. J. Gastroenterol. 50, 11-30 (2015).

14. Fink, P. et al. The prevalence of somatoform disorders among internal medical inpatients. J. Psychosom. Res. 56, 413-418 (2004).

15. Leue, C. et al. Managing complex patients on a medical psychiatric unit: An observational study of university hospital costs associated with medical service use, length of stay, and psychiatric intervention. J. Psychosom. Res. 68, 295-302 (2010).

16. Zigmond, A. S. \& Snaith, R. P. The hospital anxiety and depression scale. Acta Psychiatr Scand. 67, 361370 (1983).

17. Chatoor, D. et al. Organising a clinical service for patients with pelvic floor disorders. Best Pract. Res. Clin. Gastroenterol. 23, 611-620 (2009).

18. Clemens, J. Q. et al. The MAPP research network: A novel study of urologic chronic pelvic pain syndromes. Bmc Urol. 14, doi: 10.1186/1471-2490-14-57 (2014).

19. Cardoso, G. et al. Depression and anxiety symptoms following cancer diagnosis: A cross-sectional study. Psychol. Health Med. 21, 562-570 (2016).

20. Ng, T. P. et al. Depressive symptoms and chronic obstructive pulmonary disease: Effect on mortality, hospital readmission, symptom burden, functional status, and quality of life. Arch. Intern. Med. 167, 6067 (2007).

21. Coyne, K. S. et al. The impact of overactive bladder on mental health, work productivity and healthrelated quality of life in the UK and Sweden: Results from EpiLUTS. BJU Int. 108,1459-1471 (2011).

22. Perry, S. et al. An investigation of the relationship between anxiety and depression and urge incontinence in women: Development of a psychological model. Br. J. Health Psychol. 11, 463-482 (2006) .

23. Ghetti, C. et al. Depressive symptoms in women seeking surgery for pelvic organ prolapse. Int. Urogynecol. J. 21, 855-860 (2010).

24. Ghetti, C. et al. The emotional burden of Pelvic Organ Prolapse in women seeking treatment: a qualitative study. Female Pelvic Med. Reconstr. Surg. 21, 332-338 (2015). 
25. Di Gangi Herms, A. M. et al. Assessing health care needs and clinical outcome with urological case complexity: A study using INTERMED. Psychosomatics 44,196-203 (2003).

26. Balachandran, A. et al. Management of female urinary incontinence: a survey of urogynaecologists' view on the NICE guideline. J. Obstet. Gynaecol. 36,487-491 (2016).

27. Bjelland, I. et al. The validity of the Hospital Anxiety and Depression Scale: An updated literature review. J. Psychosom. Res. 52, 69-77 (2002).

28. Penninx, B. W. et al. Depression and cardiac mortality: Results from a community-based longitudinal study. Arch. Gen. Psychiatry 2001 58, 221-227 (2001).

29. Roy-Byrne, P. P. et al. Anxiety disorders and comorbid medical illness. Gen. Hosp. Psychiatry 30, 208-225 (2008).

30. Maserejian, N. N. et al. Treatment status and risk factors for incidence and persistence of urinary incontinence in women. Int. Urogynecol. J. 25, 775-782 (2014).

31. Bogner, H. R. et al. Anxiety disorders and disability secondary to urinary incontinence among adults over age 50. Int. J. Psychiatry Med. 32, 141-154 (2002). 


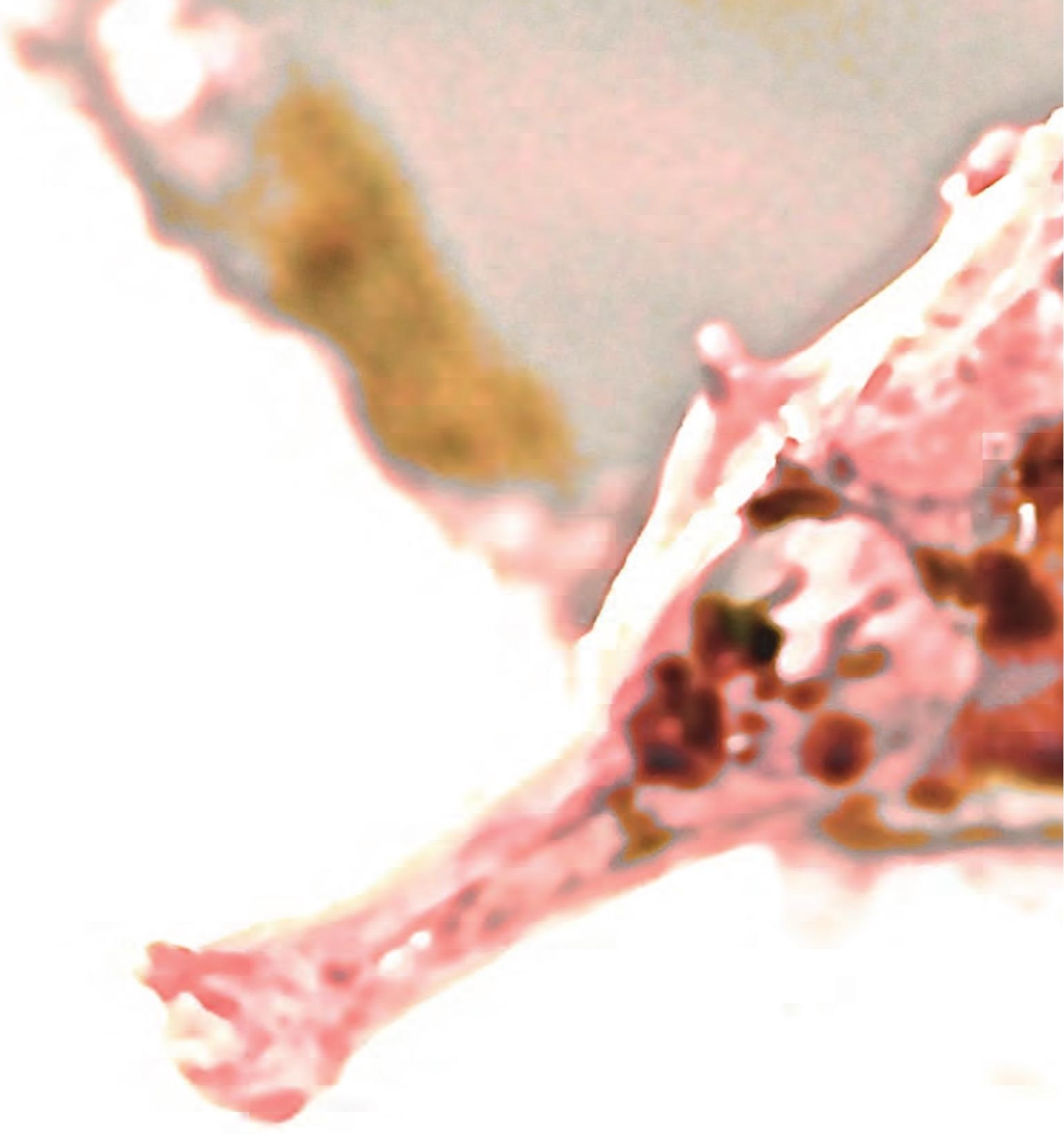




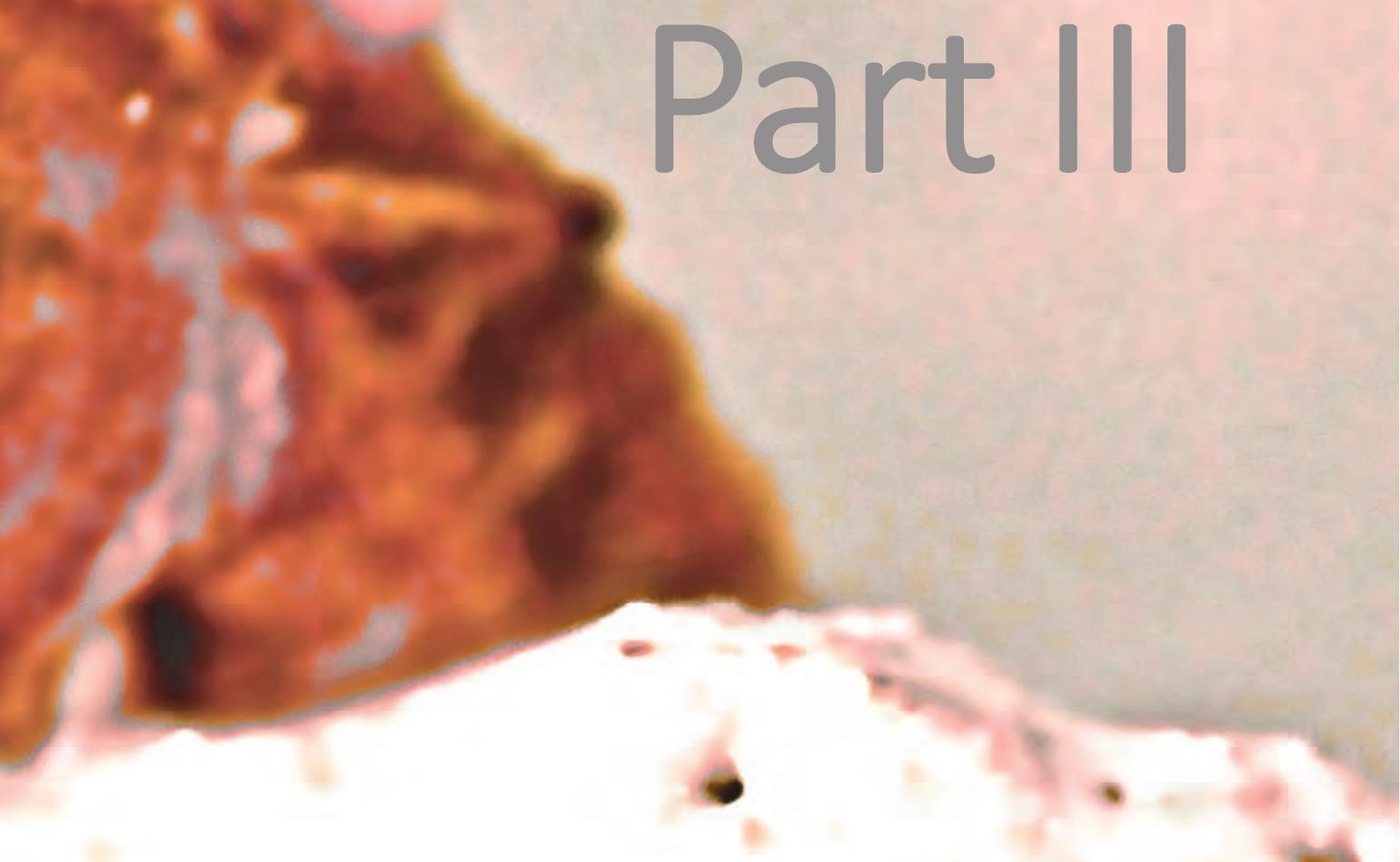

Lack of integrated medical staff-guidance in psychosomatic primary care 



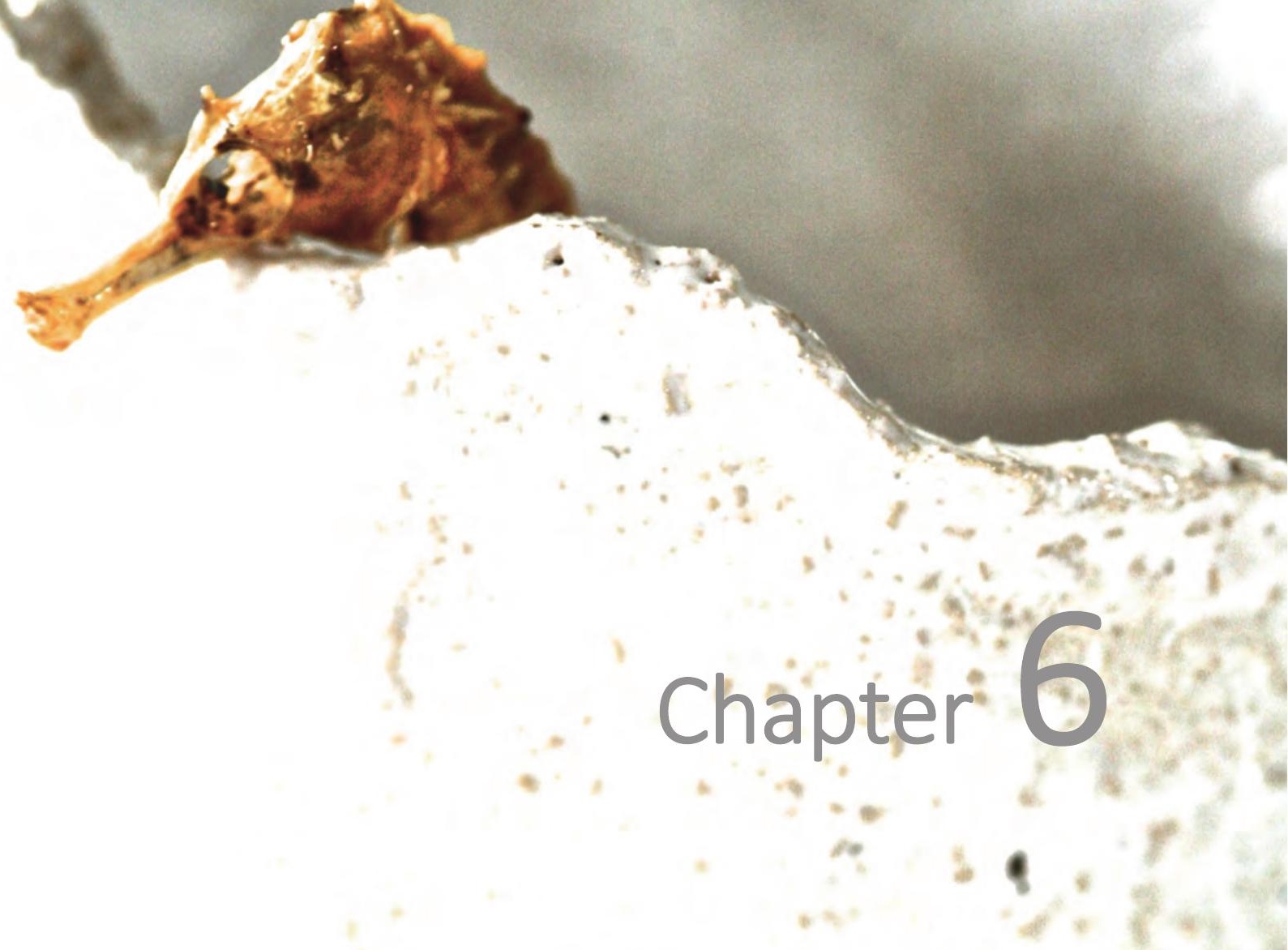

Observational evidence that urbanization and neighbourhood deprivation are associated with escalation in chronic pharmacological pain treatment: A longitudinal population-based study in the Netherlands

Leue C, Buijs S, Strik J, Lousberg R, Smit J, van Kleef M, van Os J

BMJ Open 2, e000731 (2012) 


\section{Abstract}

\section{Objective}

To examine, in the light of the association between urban environment and poor mental health, whether urbanisation and neighbourhood deprivation are associated with analgesic escalation in chronic pharmacological pain treatment and whether escalation is associated with prescriptions of psychotropic medication.

\section{Design}

Longitudinal analysis of a population-based routine dispensing database in the Netherlands.

\section{Setting}

Representative sample of pharmacies, covering $73 \%$ of the Dutch nationwide medication consumption in the primary care and hospital outpatient settings.

\section{Participants}

449410 patients aged 15-85 years were included, of whom 166374 were in the Starter group and 283036 in the Continuation group of chronic analgesic treatment.

\section{Main outcome measure}

Escalation of analgesics (i.e., change to a higher level of analgesic potency, classified across five levels) in association with urbanisation (five levels) and dichotomous neighbourhood deprivation was analysed over a 6-month observation period.

\section{Methods}

Ordered logistic multivariate model evaluating analgesic treatment.

\section{Results}

In both Starter and Continuation groups, escalation was positively associated with urbanisation in a dose-response fashion (Starter group: OR (urbanisation level 1 compared with level 5): 1.24; $95 \% \mathrm{Cl} 1.18$ to 1.30; Continuation group: OR $1.18 ; 95 \% \mathrm{Cl}$ 1.14 to 1.23$)$. An additional association was apparent with neighbourhood deprivation (Starter group: OR 1.07; 95\% Cl 1.02 to 1.11; Continuation group: OR 1.04; 95\% Cl 1.01 to 1.08). Use of somatic and particularly psychotropic co-medication was associated with escalation in both groups.

\section{Conclusion}

Escalation of chronic analgesic treatment is associated with urban and deprived environments and occurs in a context of adding psychotropic medication prescriptions. These findings suggest that pain outcomes and mental health outcomes share factors that increase risk and remedy suffering. 


\section{Introduction}

The validity of the well-known epidemiological association between urban environment and mental health ${ }^{1-3}$ is supported by work showing that urban living is associated with increased amygdala activity ${ }^{4}$, a key region in the regulation of stress, affective experience and pain ${ }^{5,6}$. Pain is the natural comorbid mental experience of somatic conditions ${ }^{7,8}$. In turn, pain is strongly influenced by comorbid common mental disorders particularly affective disorders ${ }^{9,10}$. Given evidence of urban impact on risk for common mental disorders ${ }^{11}$, including psychiatric medication prescriptions ${ }^{12}$, we hypothesised that pain outcomes, indexed through prescriptions, would be poorer in urban environments and disadvantaged urban neighbourhoods. Pain outcomes were examined at the level of primary care and specialist outpatient care and defined in two ways: (1) escalation of analgesic treatment (i.e., prescription of more potent analgesics) and (2) co-prescription of psychotropic medication in addition to analgesic treatment.

\section{Objective}

We examined the hypothesis that chronic pharmacological pain treatment of hospital outpatients and patients in primary care would show escalation of analgesics in association with the level of urbanisation and neighbourhood index of deprivation. It was predicted that the highest levels of urbanisation and neighbourhood deprivation would be associated with escalation of analgesic treatment to more potent pain medication (e.g., tramadol, morphine, methadone, etc.). Furthermore, we examined the hypothesis that prescriptions of psychotropic medication (e.g., antidepressants, antipsychotics, mood stabilizers, etc.) would be associated with escalation or de-escalation of analgesics in patients prescribed chronic analgesic treatment. Study hypotheses were specified before inspection of the data.

\section{Methods}

\section{Data collection}

The investigation was carried out by analysing records pertaining to Dutch routine general practice and hospital outpatient treatment settings. Data were obtained from the IMS Health's Iongitudinal prescription database (Lifelink, affiliate Capelle ad IJssel, The Netherlands) ${ }^{13}$. This data source consists of anonymous longitudinal prescription records from a representative sample of pharmacies and dispensing general practitioners, covering $73 \%$ of the Dutch nationwide medication consumption of outpatients and primary care patients. The computerized medication-dispensing 
histories contain data regarding dispensed medications, type of prescriber, dispensing date, dispensed amount of medication, prescribed dosage and length of prescription. Data for each patient were anonymously and independently sampled without linkage of prescriptions to the same patient across pharmacies, because patients in the Netherlands are usually loyal to a single pharmacy ${ }^{14}$. Furthermore, research from the Dutch Foundation for Pharmaceutical Statistics (SFK) revealed that in the Netherlands, almost all patients make use of a pharmacy located in their area of living. Eighty-two per cent of patients are living in a radius of 3 kilometres from their pharmacy ${ }^{15}$. Potential bias caused by patients getting hospitalised, moving to another address or dying was minimized by studying chronic pharmacological pain treatment because there were dispensing records for these patients during the whole study period.

\section{Patient groups}

Patient selection started with the identification of chronic users of analgesic medication during a 6-month prescription period (hereafter: observation period). Chronic use was defined as in receipt of analgesic pharmacotherapy during at least two distinct moments covering an interval of at least 2 months. In order to track medication for other therapeutic indications (i.e., psychotropic medication and pharmacotreatment for somatic disorders), patients were observed for a period of 6 months prior to initiation of analgesic treatment. Next, the cohort with chronic use of analgesics was divided into two groups. Starters were defined as patients who had not received any analgesics during the 6-month period prior to the observation period (hereafter: Starter group). Patients who continued with pain medication that was already prescribed in the 6 month before the observation period formed the second group (hereafter: Continuation group). The latter group consisted of all patients who had already received analgesics in the first month of the 6-month period prior to the observation period in order to define chronic analgesic treatment before observation. All data captured a calendar period from May 2008 to September 2009 (Figure 6.1).

Figure 6.1 Starter group and Continuation group of chronic analgesic treatment.

\begin{tabular}{|c|c|c|c|c|c|c|c|c|c|c|c|c|}
\hline & \multicolumn{12}{|c|}{ Time (months) } \\
\hline & \multicolumn{6}{|c|}{ period prior to observation } & \multicolumn{6}{|c|}{ observation period } \\
\hline & 1 & 2 & 3 & 4 & 5 & 6 & 7 & 8 & 9 & 10 & 11 & 12 \\
\hline starting patients & \multicolumn{6}{|c|}{ no $\mathrm{Rx}$ for analgesics } & \multicolumn{5}{|c|}{ first $P x-R x$} & last $\mathrm{Rx}$ \\
\hline continuing patients & \multicolumn{6}{|c|}{ first $\mathrm{Pa}$} & \multicolumn{5}{|c|}{$R x=R x$} & last $\mathrm{Rx}$ \\
\hline
\end{tabular}

Legend: Schedule of prescriptions ( $R x)$ in the Starter group (top) and the Continuation group (bottom) of chronic analgesic treatment covering a 12-month period. Months 7-12 are the observation period; months 1-6 are the pre-observation period. Patients in the Continuation group received the first prescription of analgesics in month 1 of the pre-observation period; there was no follow-up whether analgesics were continued over the entire 6-month interval prior to the observation period. The Starter group did not use any analgesics during the 6-month interval prior to the observation period. 
Data were obtained from the LRx database from month 1-12 as depicted in Figure 6.1. Use of other medications (e.g., psychotropic medication and medication for a broad spectrum of somatic conditions) was collected for all patients as well, covering the period of 12 months, consisting of (1) the pre-observation period (month 1-6) and (2) the observation period (month 7-12).

\section{Escalation of pharmacological pain treatment}

All individual prescriptions of analgesics were observed for each patient in both the Starter and the Continuation groups during the observation period and during the 6 months prior to the observation period. At each dispensing date, analgesics were classified a priori in five levels in order of analgesic potency (Figure 6.2). Five escalation levels were provided, based on a minor adaptation of the three-step WHO analgesic ladder $^{16}$. Level 5 and 4 are identical to WHO steps 3 (strong opioids) and 2 (weak opioids), respectively. WHO step 1 (non-opioid analgesics) was refined in order to enable further and clinically relevant differentiation between non-opioid analgesics (level 1: paracetamol, level 2: prostaglandin inhibitors, level 3: anticonvulsants) ${ }^{16-20}$. Furthermore, anti-epileptics were divided in anticonvulsants predominantly prescribed in pain conditions (level 3a: gabapentin and pregabalin) and anticonvulsants with best evidence for epilepsy treatment (level 3b: carbamazepine, valproic acid, lamotrigine $)^{19-21}$. In order to avoid prescription for indications of mood stabilisation or epilepsy, the latter group was classified at level $3 \mathrm{~b}$ only if prescribed in combination with analgesic medication at level 1 or 2 (i.e., pain indication) (Figure 6.2).

Figure 6.2 Five levels of analgesic potency, modified from the WHO analgesic ladder ${ }^{16}$.

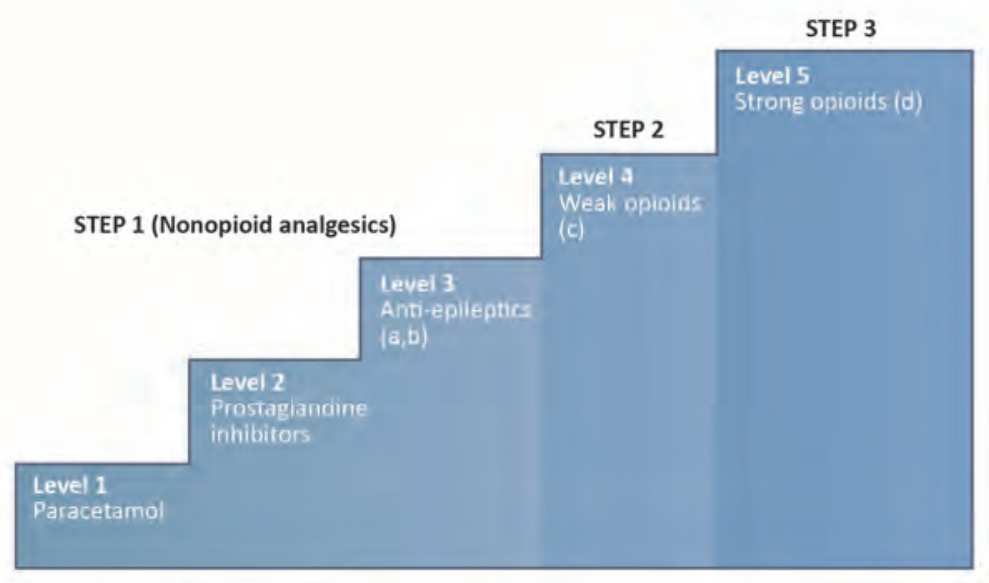

Legend: Level 1 (i.e., lowest potency) to level 5 (i.e., highest potency). (a) Gabapentin and pregabalin in the absence of other anti-epileptic drugs. (b) Carbamazepine, valproic acid, lamotrigine in combination with medication at level 1 or 2. (c) Tramadol, codeine. (d) Methadone, oxycodone, hydromorphone, morphine, buprenorphine, fentanyl, sufentanil, pethidine. 
Confirmation of escalation was based on the comparison of analgesic potency at the first dispensing day and the last dispensing day within the observation period.

The comparison of the first and the last prescription of analgesics resulted in the following categories of analgesic escalation: neutral (i.e., no change of analgesic potency), escalation in analgesic treatment (i.e., change to a higher level of analgesic potency), or de-escalation in pharmacological pain treatment (i.e., change to a lower analgesic potency) (Table 6.2).

If patients received several analgesics on the same day, both the highest and the second highest level of analgesic potency were included in the analyses in order to define escalation categories (e.g., a change from level 5 plus level 2 to level 5 plus level 3 indicating that escalation had occurred).

\section{Determinants of escalation in analgesic treatment}

Three groups of variables hypothesised to act as mediators or confounders were included in the analyses. The first group were patient characteristics such as sex $(0=$ male, $1=$ female), age (in years) and the location of patient's pharmacy (defined by postal code). The latter variable defined the level of urbanisation following the definition of the Dutch Central Bureau for Statistics (CBS). Conform previous work, and in line with the classification developed by CBS, level of urbanisation was defined as the number of addresses relative to area surface ${ }^{22}$. Level 1 (i.e. highest level of urbanisation) consists of more than 2500 addresses $/ \mathrm{km}^{2}$ (level $2=1500$ to 2500 addresses $/ \mathrm{km}^{2}$, level $3=1000$ to 1500 addresses $/ \mathrm{km}^{2}$, level $4=500$ to 1000 addresses $/ \mathrm{km}^{2}$ ). Level 5 (i.e., rural environment) consists of $<500$ addresses $/ \mathrm{km}^{2}$, described in more detail elsewhere ${ }^{23}$. More over, neighbourhood deprivation was defined dichotomously ( $0=$ no, $1=y e s)$. The dichotomous measure of neighbourhood deprivation was developed by the Netherlands Institute for Research in Healthcare (NIVEL), using socioeconomic indicators such as unemployment rate, average income, population density and ethnic variation. On the basis of empirical research in the Netherlands, NIVEL's neighbourhood deprivation index (NDI) is calculated as follows: $\mathrm{NDI}=((\mathrm{In}$ percentage unemployed people $3.0236) / 0.37706)$ - ((In average income - 2.8641)/0.14441) + ((In population density 7.0132)/1.06699) + ((In percentage people of 'non-western' ethnicity)/1.11147). NDIs were expressed continuously by NIVEL from low to high. Furthermore, NIVEL defined a dichotomous measure of deprivation at a cut-off of 5.5\% (i.e., 885000 people) in order to assess trends in the proportion of the Dutch population inhabiting an area with the highest NDI and for use in epidemiological research ${ }^{24}$. Healthcare professionals receive higher levels of funding for their services in these deprived areas ${ }^{25}$. Neighbourhood deprivation was associated with level of urbanisation: $86 \%$ of the sample living in deprived neighbourhoods lived in an area with the highest level of urbanisation. The other patients (14\%) living in deprived neighbourhoods lived in an area with the second highest level of urbanisation. The majority (76\%) of those living in an area of the highest level of urbanisation did not live in a deprived neighbourhood (Table 6.1). 
Table 6.1 Sample, stratified by Urbanisation and Neighbourhood Deprivation.

\begin{tabular}{llrrrrc}
\hline \multicolumn{7}{c}{ Deprived Neighbourhood } \\
\hline Type of patient & $\begin{array}{l}\text { Urbanisation } \\
\text { level }\end{array}$ & No (patients) & No (\%) & Yes (patients) & Yes (\%) & $\begin{array}{c}\text { \% Within deprived } \\
\text { neighbourhood }\end{array}$ \\
\hline Starter group & 1 & 34662 & 76.3 & 10796 & 23.7 & 86.5 \\
& 2 & 48673 & 96.6 & 1689 & 3.4 & 13.5 \\
& 3 & 31107 & 100.0 & 0 & 0.0 & - \\
& 4 & 28283 & 100.0 & 0 & 0.0 & - \\
& 5 & 11164 & 100.0 & 0 & 0.0 & - \\
\hline Continuation & 1 & 93889 & 92.5 & 12485 & 7.5 & 100.0 \\
\hline Group & Total & 59714 & 76.2 & 18644 & 23.8 & 85.5 \\
& 2 & 81406 & 96.3 & 3155 & 3.7 & 14.5 \\
& 3 & 50853 & 100.0 & 0 & 0.0 & - \\
& 4 & 48511 & 100.0 & 0 & 0.0 & - \\
& 5 & 20754 & 100.0 & 0 & 0.0 & - \\
\hline Total patients & Total & 261237 & 92.3 & 21799 & 7.7 & 100.0 \\
\hline
\end{tabular}

Legend: The sample is described in absolute numbers for the Starter and the Continuation groups, stratified by living in an urbanised area (level 1 - 5) and a dichotomous measure of neighbourhood deprivation. Furthermore, in the last column, tabulation is presented for living in a deprived neighbourhood as a function of level of urbanisation (e.g., in the Starter group, $86.5 \%$ of the sample living in deprived neighbourhoods lived in an area with urbanisation level 1).

Furthermore, psychotropic co-medication was classified into its different classes, and somatic co-medication was similarly grouped in 10 classes (ACE inhibitors and angiotensine II inhibitors; antidiabetics; $\beta$-blockers; calcium antagonists; functional bowel drugs; laxatives; migraine medication; respiratory medication; steroid antiphlogistics; stomach protectors) (Table 6.1-6.3). In the Starting group, occurrence of co-medication was time coded at three levels according to the day of first occurrence (i.e., co-medication prescription before start with analgesics, at the same day or after start of analgesic treatment) (Table 6.3). In the Continuation group, occurrence of comedication was recorded dichotomously (presence/absence) since it was impossible to distinguish occurrence of co-medication as before or at start of analgesic treatment (Table 6.4).

\section{Statistical analysis}

First, we analysed the pattern of (de-) escalation in analgesic treatment by means of an ordered logistic multivariable regression model with adjusted ORs (and 95\% Cl) using SAS V. $9^{26}$. Statistical significance for the model was defined at conventional $\alpha$ of 0.05 . The dependent variable in this model was the development of a patient's analgesic treatment (de-escalation, neutral, escalation). Independent variables, entered simultaneously in the model, were demographic characteristics, neighbourhood deprivation, and urbanisation, use of psychotropic medication and use of somatic medication. In the Starter group, we also included first occurrence of co-medication. The modelling strategy was to build, first, a fully saturated model (including all variables) in 
order to avoid missing relevant information by leaving out non-significant variables. Second, backward elimination was carried out to find the best model fit.

Models for Starter and Continuation groups were run separately, given different sample selection criteria. The ordered logistic multivariable regression model was chosen above the multinomial model, as the latter does not consider the natural order in our data regarding development of chronic pain treatment, ranging from deescalation to neutral to escalation. Proportional odds were assumed in the models of escalation and de-escalation of analgesic treatment and analyses inspected for violation of this assumption. Test on the proportional odds assumption showed significance, which gave us the confidence to use the ordered logistic model. If a determinant was positively associated with escalation of analgesics, absence of this variable was associated negatively with escalation or positively with de-escalation in analgesic treatment (and vice versa). This offered advantage compared with separate models for escalation and de-escalation (such as consistency of model estimates) and avoided double use of patients with a neutral development of analgesic treatment.

\section{Results}

Overall, 449410 patients were included, of which 166374 were in the Starter group and 283036 in the Continuation group. The baseline characteristics of both groups are shown in table 1 and 2 . About $7.6 \%$ of all patients were residing in a deprived neighbourhood, and approximately $27.6 \%$ were living in an area of the highest level of urbanisation (level 1) (Table 6.1). The majority were women, and there were more patients showing escalation $(15.4 \%)$ than de-escalation $(11.3 \%)$ of analgesic treatment. The majority of patients continued a neutral analgesic treatment regime (73.3\%). Most of the patients were treated at level 2 or level 4 of analgesic potency. Almost all patients were using other medications, regardless of the different categories (84.5\%). Around half were using psychotropic medication (45.2\%), most were using somatic co-medication (78.1\%) and more than a third were using both (38.8\%) (Table 6.2).

The Starter group mainly initiated an analgesic at level 2 (70.9\%) and level 4 (19.9\%), whereas only $2.6 \%$ directly initiated at level 5 . However, analgesic potency level 4 and 5 increased up to $20.5 \%$ respectively $5.2 \%$ by the time of the last prescription in the Starter group (Table 6.2).

In the Continuation group, patients already received analgesics at a higher level of potency at inclusion compared with the last observed level of medication potency in the Starter group. Level 4 and 5 was observed in $35.6 \%$ at the start of the observation period, increasing to $38.3 \%$ at the end of observation period (Table 6.2). 


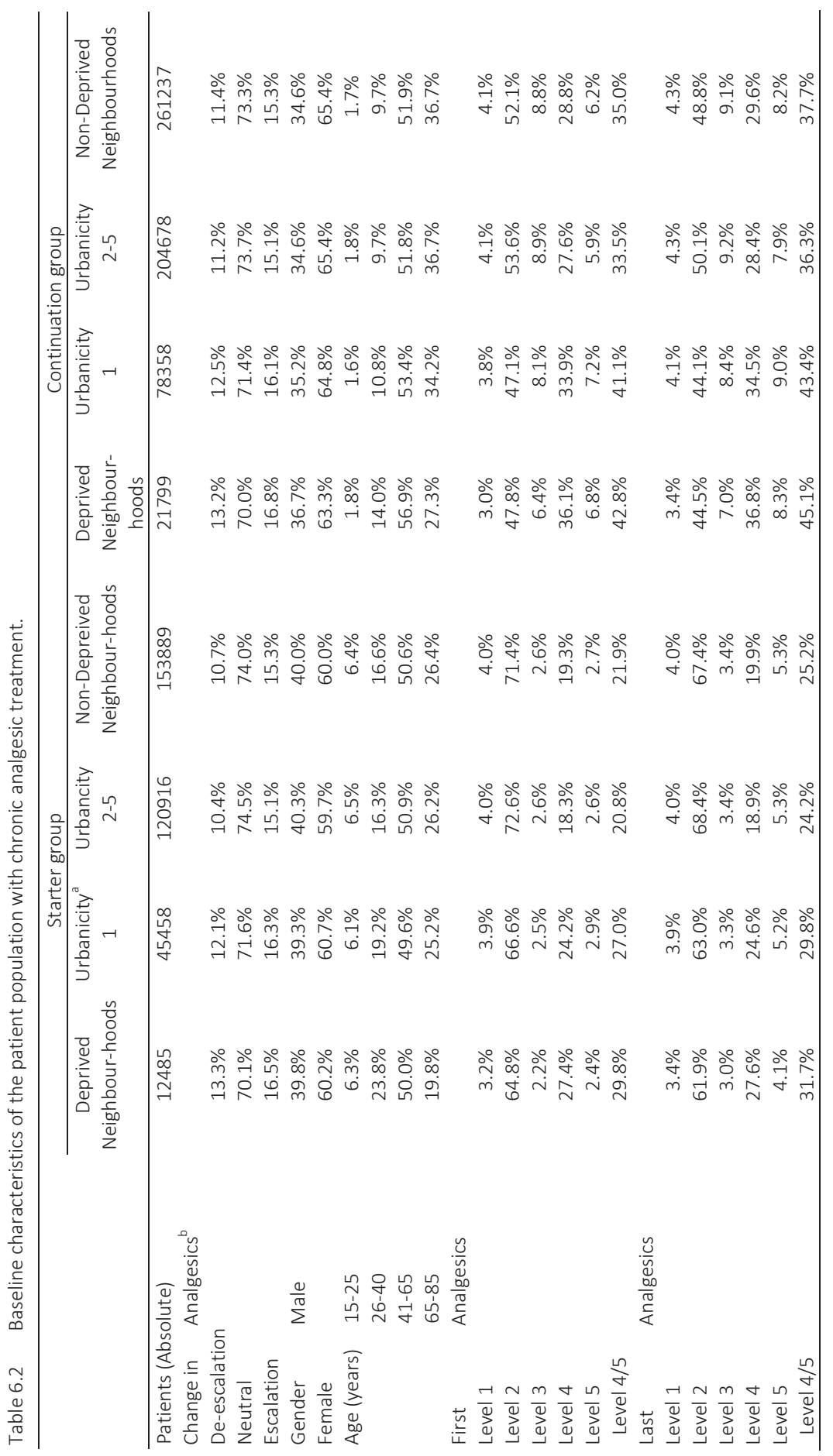




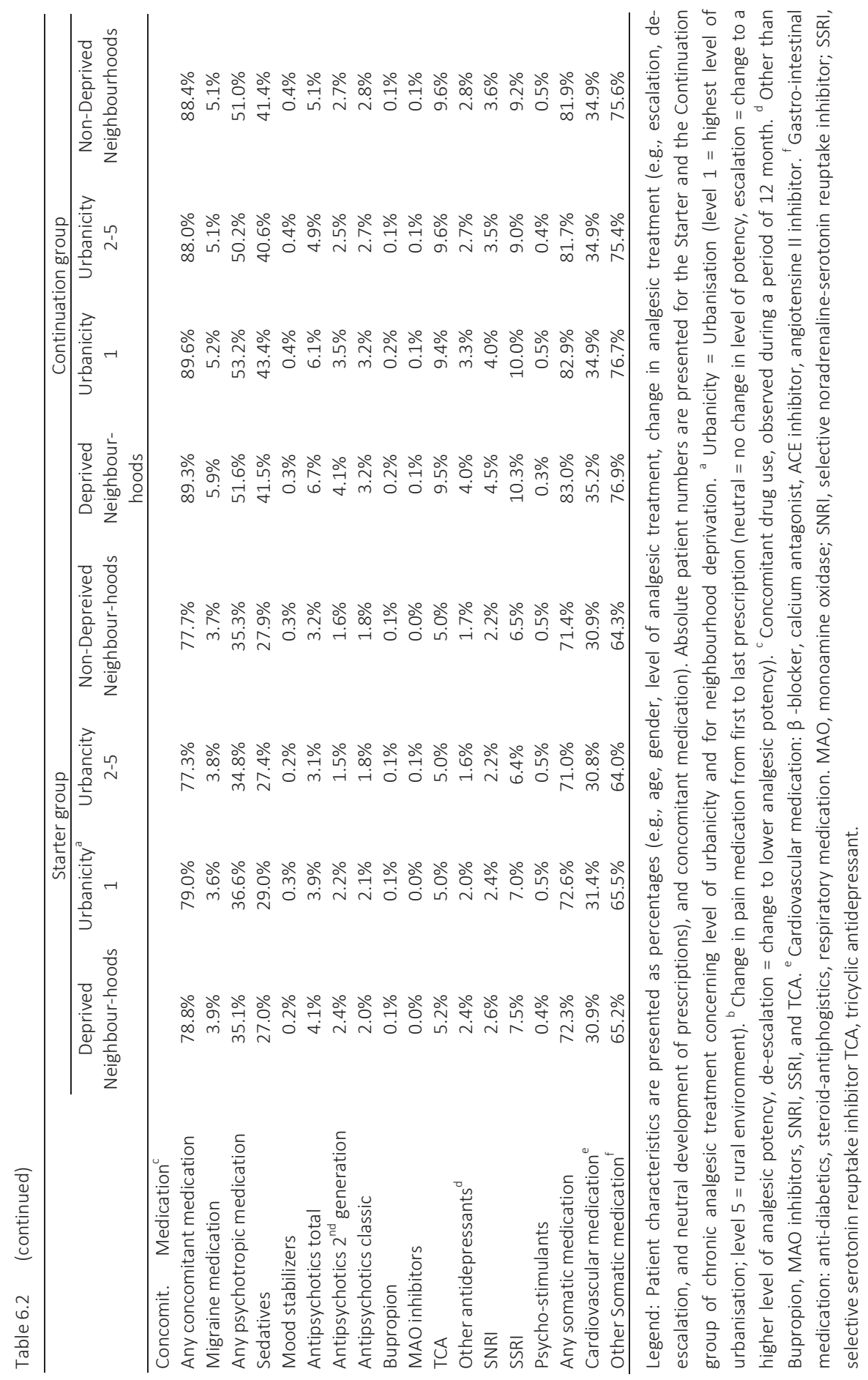


Escalation of analgesic treatment was observed more often in deprived neighbourhoods and in areas of the highest levels of urbanisation (16.8\% and $16.1 \%$ in the Continuation group, respectively $16.5 \%$ and $16.3 \%$ in the Starter group) compared to rural areas (15.1\%) and non-deprived neighbourhoods (15.3.\%). The proportion of patients with neutral development of analgesic treatment was lower in deprived neighbourhoods and areas with the highest degree of urbanisation compared with less densely populated areas (Table 6.2).

Table 6.3 Associations with escalation in pharmacological pain treatment for the Starter group of chronic analgesic treatment.

\begin{tabular}{|c|c|c|c|c|}
\hline \multirow[t]{2}{*}{ Exposure } & & \multirow[t]{2}{*}{ Adjusted OR } & \multicolumn{2}{|c|}{$95 \% \mathrm{Cl}^{\mathrm{a}}$} \\
\hline & & & Lower & Upper \\
\hline \multirow[t]{5}{*}{ Analgesics $^{b}$} & Level 1 & 58.23 & 53.60 & 63.27 \\
\hline & Level 2 & 17.92 & 16.75 & 19.16 \\
\hline & Level 3 & 4.66 & 4.23 & 5.14 \\
\hline & Level 4 & 1.36 & 1.27 & 1.45 \\
\hline & Level 5 & Reference & - & - \\
\hline \multirow[t]{2}{*}{ Gender } & Female & 0.97 & 0.95 & 0.99 \\
\hline & Male & Reference & 0.00 & 0.00 \\
\hline \multirow[t]{4}{*}{ Age (years) } & $15-25$ & 0.73 & 0.69 & 0.77 \\
\hline & $26-40$ & 0.81 & 0.78 & 0.84 \\
\hline & $41-65$ & 0.87 & 0.85 & 0.90 \\
\hline & $66-85$ & Reference & - & - \\
\hline \multirow[t]{5}{*}{ Urbanisation $^{\mathrm{c}}$} & 1 & 1.24 & 1.18 & 1.30 \\
\hline & 2 & 1.16 & 1.11 & 1.22 \\
\hline & 3 & 1.11 & 1.06 & 1.17 \\
\hline & 4 & 1.07 & 1.02 & 1.13 \\
\hline & 5 & Reference & - & - \\
\hline Deprived & Yes & 1.06 & 1.02 & 1.11 \\
\hline Neighbourhood & No & Reference & - & - \\
\hline \multirow[t]{4}{*}{ SNRI } & Before start of analgesics $^{d}$ & 1.05 & 0.96 & 1.14 \\
\hline & Same start date & 1.28 & 0.97 & 1.69 \\
\hline & After analgesics started & 1.26 & 1.09 & 1.45 \\
\hline & None & Reference & - & - \\
\hline \multirow[t]{4}{*}{ SSRI } & Before start of analgesics & 0.97 & 0.92 & 1.02 \\
\hline & Same start date & 0.97 & 0.83 & 1.15 \\
\hline & After analgesics started & 1.07 & 0.97 & 1.18 \\
\hline & None & Reference & - & - \\
\hline \multirow[t]{4}{*}{ TCA } & Before start of analgesics & 1.23 & 1.15 & 1.32 \\
\hline & Same start date & 1.32 & 1.12 & 1.54 \\
\hline & After analgesics started & 2.19 & 2.03 & 2.36 \\
\hline & None & Reference & - & - \\
\hline \multirow[t]{4}{*}{ Other AD } & Before start of analgesics & 1.03 & 0.93 & 1.15 \\
\hline & Same start date & 0.93 & 0.71 & 1.21 \\
\hline & After analgesics started & 1.22 & 1.06 & 1.42 \\
\hline & None & Reference & - & - \\
\hline \multirow[t]{4}{*}{ Antipsychotics } & Before start of analgesics & 0.92 & 0.85 & 1.01 \\
\hline & Same start date & 0.69 & 0.58 & 0.83 \\
\hline & After analgesics started & 2.42 & 2.18 & 2.67 \\
\hline & None & Reference & - & - \\
\hline
\end{tabular}


Table 6.3 (continued)

\begin{tabular}{|c|c|c|c|c|}
\hline \multirow[t]{4}{*}{ Mood stabilizers } & Before start of analgesics & 1.40 & 1.10 & 1.79 \\
\hline & Same start date & 0.91 & 0.43 & 1.89 \\
\hline & After analgesics started & 0.71 & 0.39 & 1.31 \\
\hline & None & Reference & - & - \\
\hline \multirow[t]{4}{*}{ Sedatives } & Before start of analgesics & 1.24 & 1.20 & 1.28 \\
\hline & Same start date & 1.25 & 1.18 & 1.33 \\
\hline & After analgesics started & 1.82 & 1.74 & 1.89 \\
\hline & None & Reference & - & - \\
\hline \multirow[t]{4}{*}{ Cardio-Vascular drugs } & Before start of analgesics & 1.16 & 1.13 & 1.19 \\
\hline & Same start date & 0.86 & 0.79 & 0.95 \\
\hline & After analgesics started & 1.35 & 1.26 & 1.45 \\
\hline & None & Reference & - & - \\
\hline \multirow[t]{4}{*}{ Other Somatic drugs } & Before start of analgesics & 1.25 & 1.22 & 1.29 \\
\hline & Same start date & 1.11 & 1.07 & 1.15 \\
\hline & After analgesics started & 1.19 & 1.15 & 1.23 \\
\hline & None & Reference & - & - \\
\hline \multirow[t]{4}{*}{ Migraine medication } & Before start of analgesics & 0.83 & 0.77 & 0.89 \\
\hline & Same start date & 0.95 & 0.78 & 1.17 \\
\hline & After analgesics started & 0.91 & 0.81 & 1.02 \\
\hline & None & Reference & - & - \\
\hline
\end{tabular}

Legend: TCA, tricyclic antidepressants; SNRI, selective noradrenaline-serotonin reuptake inhibitors; SSRI, selective serotonin reuptake inhibitors. ${ }^{a} 95 \% \mathrm{Cl}$ : confidence interval. ${ }^{b}$ Escalation = change to a higher level of analgesic potency, level 5 = highest level. ${ }^{c} 1$ highest level of urbanisation, $5=$ rural environment. ${ }^{d}$ Starting date of medication (before, at the same day or after start of analgesics).

In the Starter group, escalation was positively associated with lower level of first observed pain medication (highest adjusted Odds Ratio (OR) 58.23 at analgesic level 1; 95\% Confidence Interval (Cl) 53.60 to 63.27; lowest OR 1.36 at analgesic level 4; $95 \% \mathrm{Cl}$ 1.27 to 1.45; compared with reference level 5) (Table 6.3). Escalation was furthermore associated, in a dose-response fashion, with level of urbanisation (highest adjusted OR 1.24 at urbanisation level $1 ; 95 \% \mathrm{Cl} 1.18$ to 1.30; compared with reference level 5). Furthermore, a weak but additional association existed between escalation and neighbourhood deprivation (OR 1.06; 95\% Cl 1.02 to 1.11) (Table 6.3).

Use of tricyclic antidepressants (TCA), mood stabilizers (OR 1.40; 95\% Cl 1.10 to 1.79), sedatives, cardiovascular medication (OR 1.16; $95 \% \mathrm{Cl} 1.13$ to 1.19) and medications for other somatic conditions was associated with analgesics escalation, when prescribed before start of analgesics (Table 6.3). Similarly, in the Starter group, escalation of analgesic treatment was also associated with the use of selective noradrenalin serotonin reuptake inhibitors (SNRI), sedatives (OR 1.82; 95\% Cl 1.74 to 1.89), TCA (OR 2.19; $95 \% \mathrm{Cl} 2.03$ to 2.36), and antipsychotics (OR 2.42; 95\% Cl 2.18 to 2.67) when prescribed after start of analgesics (Table 6.3). Negative associations with escalation (i.e., positive association with de-escalation) were apparent for younger age, female sex, and pharmacological migraine treatment. Furthermore, use of antipsychotics was negatively associated with escalation if started simultaneously with analgesic treatment (OR 0.69; 95\% Cl 0.58 to 0.83) (Table 6.3). 
The use of selective serotonin reuptake inhibitors (SSRI), before, at or after start of analgesic treatment was not associated with escalation of analgesics in the Starter group (Table 6.3).

In the Starter group, the original fully saturated model and the model after backward elimination revealed the same variables associated significantly with escalation in chronic pharmacological pain treatment (Table 6.3).

In the Continuation group, escalation of analgesics was positively associated with lowest levels of first observed analgesics (highest adjusted OR 16.00 at analgesic level 1; $95 \% \mathrm{Cl} 15.20$ to 16.85 ; lowest OR 1.55 at analgesic level 4; 95\% Cl 1.50 to 1.61; compared with reference level 5) (Table 6.4). Furthermore, escalation was associated with level of urbanisation in a dose response fashion (highest adjusted OR 1.18 at level 1; $95 \% \mathrm{Cl} 1.14$ to 1.23; compared with reference level 5). There was also an association between escalation and deprived neighbourhoods, use of SSRI, SNRI, TCA, all antipsychotics, and sedatives (OR $1.31 ; 95 \% \mathrm{Cl} 1.29$ to 1.34 ) as well as use of somatic comedication (OR 1.12; $95 \% \mathrm{Cl} 1.10$ to 1.14 ) (Table 6.4). De-escalation was associated with female sex, younger age, treatment of migraine, and use of second-generation antipsychotics (OR 0.80; 95\% Cl 0.70 to 0.91) (Table 6.4).

The saturated model showed that 22 of 29 variables had significant associations. All these variables remained significant in the backward elimination approach. One additional variable displayed a significant association after backward elimination: 'TCA high dosage'. The OR's did not (16 variables) or only minimally (6 variables) differ between the fully saturated model and the backward elimination model. The only variable showing a degree of difference was 'TCA total' (fully saturated model: OR = 1.19 (Cl: $1.06-1.32)$; backward elimination model: $\mathrm{OR}=1.33$ (Cl: $1.29-1.36)$ ) (Table 6.4).

Over time, the escalation process continues even after the first 6 months of chronic analgesic treatment. In the Starter group, opioid-analgesics (level 4/5) were dispensed in $29.8 \%$ of patients living in a deprived neighbourhood. In contrast, $42.8 \%$ of patients in deprived neighbourhoods used opioids in the Continuation group, after one year of prescription. A similar, but attenuated development was seen at urbanisation level 1 and level 2-5 (Table 6.2). 
Table 6.4 Associations with escalation in pharmacological pain treatment for the Continuation group of chronic analgesic treatment.

\begin{tabular}{|c|c|c|c|c|}
\hline \multirow[t]{2}{*}{ Exposure } & & \multirow[t]{2}{*}{ Adjusted OR } & \multicolumn{2}{|c|}{$95 \% \mathrm{Cl}^{\mathrm{a}}$} \\
\hline & & & Lower & Upper \\
\hline \multirow[t]{5}{*}{ Analgesics $^{b}$} & Level 1 & 16.00 & 15.20 & 16.85 \\
\hline & Level 2 & 7.87 & 7.59 & 8.16 \\
\hline & Level 3 & 3.14 & 3.00 & 3.28 \\
\hline & Level 4 & 1.55 & 1.50 & 1.61 \\
\hline & Level 5 & Reference & - & - \\
\hline \multirow[t]{2}{*}{ Gender } & Female & 0.96 & 0.94 & 0.98 \\
\hline & Male & Reference & - & - \\
\hline \multirow[t]{4}{*}{ Age (years) } & $15-25$ & 0.91 & 0.85 & 0.97 \\
\hline & $26-40$ & 0.98 & 0.95 & 1.01 \\
\hline & $41-65$ & 0.99 & 0.97 & 1.01 \\
\hline & $66-85$ & Reference & - & - \\
\hline \multirow[t]{5}{*}{ Urbanisation $^{c}$} & 1 & 1.18 & 1.14 & 1.23 \\
\hline & 2 & 1.14 & 1.10 & 1.17 \\
\hline & 3 & 1.08 & 1.04 & 1.12 \\
\hline & 4 & 1.05 & 1.01 & 1.09 \\
\hline & 5 & Reference & - & - \\
\hline \multirow[t]{2}{*}{ Deprived Neighbourhood } & Yes & 1.04 & 1.01 & 1.08 \\
\hline & No & Reference & - & - \\
\hline SNRI & & 1.19 & 1.02 & 1.40 \\
\hline SSRI & & 1.03 & 1.004 & 1.07 \\
\hline $\mathrm{TCA}^{\mathrm{d}}$ & & 1.19 & 1.06 & 1.32 \\
\hline Other AD & & 1.08 & 1.03 & 1.14 \\
\hline \multirow[t]{3}{*}{ Antipsychotics } & Total & 1.24 & 1.08 & 1.43 \\
\hline & $1^{\text {st }}$ generation & 1.01 & 0.88 & 1.15 \\
\hline & $2^{\text {nd }}$ generation & 0.80 & 0.70 & 0.91 \\
\hline Mood stabilizers & & 0.97 & 0.85 & 1.10 \\
\hline Sedatives & & 1.31 & 1.29 & 1.34 \\
\hline Migraine & & 0.95 & 0.91 & 0.99 \\
\hline Cardio Vascular Drugs & & 1.12 & 1.10 & 1.14 \\
\hline Other Somatic Drug classes & & 1.12 & 1.10 & 1.14 \\
\hline
\end{tabular}

Legend: ${ }^{a} 95 \% \mathrm{Cl}$ : confidence interval. ${ }^{\mathrm{b}}$ Escalation = change to a higher level of analgesic potency, level $5=$ highest level. ${ }^{\mathrm{c}} 1$ = highest level of urbanisation, 5 = rural environment. ${ }^{\mathrm{d}}$ TCA total (fully saturated model: OR = $1.19(\mathrm{Cl}: 1.06-1.32)$; BWE model: OR = $1.33(\mathrm{Cl}: 1.29-1.36)$ ).

\section{Discussion}

Escalation of chronic analgesic treatment was observed more often in urban areas and deprived neighbourhoods within urban areas, suggesting pain outcomes either are associated with individual characteristics that are more prevalent in urban and deprived areas or subject to contextual influences, like area-level stress or social fragmentation, regardless of individual-level characteristics. One individual-level variable that may explain part of the association with urbanicity and deprivation is socio-economic status $^{27,28}$, which was not available for inclusion in the model. Nevertheless, the fact that the association with urbanicity remained with deprivation adjusted for in the same 
model suggests that urban effects may not be reducible entirely to individual-level socioeconomic status. Furthermore, although neighbourhood deprivation and urbanicity are correlated, additional association of deprivation with escalation of analgesics exists over and above urbanisation, indicating that other parameters than population density are involved too. However, the findings could also be attributed to reverse causation, that is, patients with worsening pain may move into more urban and deprived neighbourhoods as a consequence of being disabled due to ill health.

Regardless of the underlying mechanism, results clearly echo findings of unconfounded higher rates of poor mental health in areas of higher levels of urbanisation and greater neighbourhood deprivation ${ }^{11,29}$, and suggest that the outcome of mental disorder comorbidity associated with somatic disorders may show similar predictable variation. Functional pain syndromes and psychiatric disorders show high levels of interdependency ${ }^{30-35}$, and psychiatric conditions enhance severity of somatic symptoms $^{36}$. Thus, part of the mechanism underlying the association between pharmacological pain escalation and urban environment may be explained by urbanisation increasing the risk for mental ill health. This hypothesis is supported by the findings that, as in both the Starter and the Continuation groups, escalation of chronic analgesic treatment was associated not only with urban environment and neighbourhood deprivation, but also with prescription of various psychotropic medications prescribed in association. In general, the positive association of escalating analgesic treatment with psychotropic medication was as strong or even stronger than the association with prescribed somatic co-medication, with the exception of the observed de-escalating effect, in the Continuation group, of second-generation antipsychotics, which possess powerful analgesic properties ${ }^{37,38}$. This is accordance with the literature, given the fact that psychiatric conditions can enhance symptom severity in somatic patients ${ }^{36}$, which sometimes may impact even more than the somatic condition itself ${ }^{39}$.

Although the absolute difference between analgesic escalations in the urban versus less urban environment was small, this difference may be relevant from a public health perspective, given the high rate of painful conditions in the general population. Furthermore, prevention of persistent pain states is relevant with regard to $\operatorname{costs}^{40}$. Given the well-known increase of health care costs in complex patients with frequent utilization of health care, with or with out psychiatric comorbidity, only a small number of patients is required to cause relevant clinical cost changes ${ }^{41}$. A more effective treatment of persistent pain, including treatment of psychiatric comorbidity, may have a cost-saving effect. Targeting populations with painful conditions for early recognition and treatment of mental health problems may be cost-effective from a public health perspective and also represents an area of considerable unmet clinical need since opioid escalation is an inflationary development in the treatment of painful conditions ${ }^{42,43}$. Moreover, broadening the pain agenda to a better understanding of associated mental health problems could minimise failed surgery outcomes, for example, in patients with 
undetected mental disorders. For instance, new surgical procedures were found to be more common in chronic back pain patients with post-traumatic stress disorder (PTSD) compared with chronic back pain patients without PTSD ${ }^{44}$. Similarly, depression was demonstrated in $47.4 \%$ of patients with low back pain who had no surgery, in $50 \%$ of those with one surgical procedure and in $62.5 \%$ of those who had undergone surgery more than once ${ }^{45}$. Influencing central pain sensitisation by providing adequate antidepressant treatment in depressive conditions may help prevent surgical escalation.

ORs for escalation of analgesics in relation to original level of analgesics may represent ceiling effects in both the Starter and the Continuation groups - patients already at level 5 have nowhere stronger to go; treatment of patients at level 1 at baseline can escalate to stronger medication. Ceiling effects may reflect the pattern of prescribing analgesics in general practice. Given these, it has been suggested that the WHO analgesic ladder is in need of updating ${ }^{46}$. For example, Vargas-Schaffer is broadening the ladder with a $4^{\text {th }}$ surgical step; in the current article, however, we guide attention to treatment aspects related to underestimated mental disorder comorbidity in persistent pain states.

The speculative question remains to what degree escalation of analgesic treatment and its association with psychotropic medication reflects therapeutic paradigms to remedy pain, treatment of psychiatric comorbidity or a cause of psychopathology. For instance, in the Starter and the Continuation groups of chronic analgesic treatment, escalation of analgesics was consistently and positively associated with the use of TCA. This prescription habit may reflect routine off-label paradigms in the pharmacological treatment of pain syndromes ${ }^{10,47-49}$. However, given the evidence regarding TCA's efficacy in pain conditions, negative rather than positive associations with escalation of analgesics should have been expected. More likely, since the association with TCAs was as strong as the association of sedatives with analgesic escalation, it may be a reflection of affective or addictive comorbidity in persistent pain, for example, in vulnerable cases of opiate-induced sensitisation, tolerance and hyperalgesia ${ }^{50-56}$. Our data indicate that escalation of analgesics may represent an ongoing process after even months of treatment, which occurs not exclusively in the context of environmental deprivation. Since prescriptions of psychotropic and (attenuated) somatic medication show a similar pattern over time, escalation may also be driven to a degree by patient factors such as opioid tolerance, opioid-induced hyperalgesia ${ }^{53}$, or disease progression.

Given the literature on this topic ${ }^{47-51,57}$, negative associations of particularly antidepressants with escalation of chronic analgesic treatment would have been expected. Nevertheless, negative associations between escalation of chronic analgesic treatment were also found, for example, with migraine treatment (Tables 3 and 4). Moreover, the use of antipsychotics was negatively associated with analgesic escalation in the Starter group - if prescribed after start of analgesic treatment. In the Continuation group, de-escalation was specifically associated with the use of second-generation 
antipsychotics. This outcome is interesting and deserves further investigation, given that limited evidence for the efficacy of antipsychotics in pain conditions already exists ${ }^{37,38}$.

The results of the current study should be seen in the light of several limitations. The use of routine data rather than a targeted data collection could have caused more random error resulting in type II error. Unidentified confounding may have played a role, as randomisation was not possible and pre-post designs are sensitive to effects of unmeasured changes affecting outcome measures over time. Another limitation is the lack of outcomes other than urbanisation, psychotropic medication or somatic comedication. For instance, there were no estimates regarding care consumption or illness-related sick leave. Changes in patient-related outcomes like illness severity, global functioning, quality of life and treatment satisfaction should also form part of prospective evaluations. The type of data used is subject to the possibility of ecological fallacy: people whose pharmacy is in a deprived or urban neighbourhood do not necessarily experience that level of deprivation or urbanicity. Furthermore, this study only collected data over a twelve-month period. Affect and pain monitoring deserves longer evaluation. Finally, due to the study design, associations do not allow for causal inference. 


\section{References}

1. Lofors, J. \& Sundquist, K. Low-linking social capital as a predictor of mental disorders: A cohort study of 4.5 million Swedes. Soc. Sci. Med. 64, 21-34 (2007).

2. Thomas, H. et al. Mental health and quality of residential environment. Br. J. Psychiatry 191, 500-505 (2007).

3. Ivory, V. C. et al. When does neighbourhood matter? Multilevel relationships between neighbourhood social fragmentation and mental health. Soc. Sci, Med. 72, 1993-2002 (2011).

4. Lederbogen, F. et al. City living and urban upbringing affect neural stress processing in humans. Nature 474, 498-501 (2011).

5. Hubbard, C. et al. Corticotropin-releasing factor receptor 1 antagonist alters regional activation and effective connectivity in an emotional-arousal circuit during expectation of abdominal pain. J. Neurosci. 31, 12491-12500 (2011).

6. Murrough, J. W. et al. Reduced amygdala serotonin transporter binding in posttraumatic stress disorder. Biol. Psychiatry 70, 1033-1038 (2011).

7. Smith, H. S. et al. Fibromyalgia: an afferent processing disorder leading to a complex pain generalized syndrome. Pain Physician 14, E217-245 (2011).

8. Price, D. D, et al. Widespread hyperalgesia in irritable bowel syndrome is dynamically maintained by tonic visceral impulse input and placebo/nocebo factors: Evidence from human psychophysics, animal models and neuroimaging. Neuroimage 47, 995-1001 (2009).

9. Wise, T. N. et al. Painful physical symptoms in depression: A clinical challenge. Pain Med. 8 (Suppl 2), 7582 (2007).

10. Goldenberg DL. Pain/Depression dyad: A key to a better understanding and treatment of functional somatic syndromes. Am. J. Med. 123, 675-682 (2010).

11. Peen, J. et al. The current status of urban-rural differences in psychiatric disorders. Acta Psychiatr. Scand. 121, 84-93 (2010).

12. Crump, C. et al. Neighborhood deprivation and psychiatric medication prescription: A Swedish national multilevel study. Ann. Epidemiol. 21, 231-237.

13. Netelenbos, J. C. et al. Adherence and profile of non-persistence in patients treated for osteoporosis: A large-scale, long-term retrospective study in The Netherlands. Osteoporos. Int. 22, 1537-1546.

14. Buurma, H. et al. Prevalence and determinants of pharmacy shopping behaviour. J. Clin. Pharm. Ther. 33, 17-23 (2008).

15. http://www.sfk.nl/nieuws-publicaties/PW/2003/2003-15.htm.

16. World Health Organization. Traitement de la douleur cancéreuse. Geneva, Switzerland: World Health Organization; 1987.

17. Wiffen, P. J. et al. Carbamazepine for acute and chronic pain in adults. Cochrane Database Sys. Rev. 1, CD005451 (2011).

18. Wiffen, P. J. et al. Lamotrigine for acute and chronic pain. Cochrane Database Sys. Rev. 2, CD006044 (2011).

19. Moore, R. A. et al. Gabapentin for chronic neuropathic pain and fibromyalgia in adults. Cochrane Database Sys. Rev. 3, CD007938 (2011).

20. Moore, R. A. et al. Pregabalin for acute and chronic pain in adults. Cochrane Database Sys. Rev. 3, CD007076 (2009).

21. Perucca E, Tomson T. The pharmacological treatment of epilepsy in adults. Lancet Neurol. 10, 446-456 (2011).

22. Van Os, J. et al. Prevalence of psychotic disorder and community level of psychotic symptoms: An urbanrural comparison. Arch. Gen. Psychiatry 58, 663-668 (2001).

23. Central Bureau of Statistics. Bevolking der gemeenten van Nederland. The Hague, The Netherlands: CBS Publications 1993.

24. Wiegers TA DW. Herijking stedelijke achterstandsgebieden. Utrecht, The Netherlands: NIVEL publications 2008.

25. NZA, tariefbeschikking 5000-1900-09-3, 30 juni 2009. http://www.naz.nl/.

26. http://www.sas.com/. 
27. Drukker, M. \& van Os, J. Mediators of neighbourhood socioeconomic deprivation and quality of life. Soc. Psychiatry Psychiatr. Epidemiol. 38, 698-706 (2003).

28. Skapinakis, P. et al. Socio-economic position and common mental disorders: Longitudinal study in the general population in the UK. Br. J. Psychiatry 189, 109-117 (2006).

29. March, D. et al. Psychosis and place. Epidemiol. Rev. 30, 84-100 (2008).

30. Hotopf, M. et al. Temporal relationships between physical symptoms and psychiatric disorder: Results from a national birth cohort. Br. J. Psychiatry 173, 255-261 (1998).

31. Kroenke, K. et al. Depressive and anxiety disorders in patients presenting with physical complaints: Clinical predictors and outcome. Am. J. Med. 103, 339-347 (1997).

32. Barsky, A. J. et al. Somatization increases medical utilization and costs independent of psychiatric and medical comorbidity. Arch. Gen. Psychiatry 62, 903-910 (2005).

33. Fink, P. et al. The prevalence of somatoform disorders among internal medical inpatients. J. Psychosom. Res. 56, 413-418 (2004).

34. Levy, R. L. et al. Psychosocial aspects of the functional gastrointestinal disorders. Gastroenterology 130, 1447-1458 (2006).

35. Leue, C. et al. Managing complex patients on a medical psychiatric unit: An observational study of university hospital costs associated with medical service use, length of stay, and psychiatric intervention. J Psychosom. Res. 68, 295-302 (2010).

36. Meyer, T. et al. Depression but not anxiety is a significant predictor of physicians' assessments of medical status in physically ill patients. Psychother. Psychosom. 69, 147-54(2000) .

37. Freedenfeld, R. N. et al. Decreased pain and improved quality of life in fibromyalgia patients treated with olanzapine, an atypical neuroleptic. Pain Pract. 6, 112-118 (2006).

38. Seidel, S. et al. Antipsychotics for acute and chronic pain in adults. Cochrane database Syst. Rev. 4, CD004844 (2008).

39. Kim, K. W. et al. Association between comorbid depression and osteoarthritis symptom severity in patients with knee osteoarthritis. J. Bone Joint. Surg. Am. 93, 556-563 (2011).

40. Maniadakis, N. \& Gray, A. The economic burden of back pain in the UK. Pain 84, 95-103 (2000).

41. de Jonge, P. et al. Case and Care Complexity in the Medically III. Med. Clin. North Am. 90, 679-692 (2006).

42. Seal, K. H. et al. Association of mental health disorders with prescription opioids and high-risk opioid use in US veterans of Iraq and Afghanistan. JAMA 307, 940-947 (2012).

43. Bohnert, A. S. et al. Association between opioid prescribing patterns and opioid overdose-related deaths. JAMA 305, 1315-1321 (2011).

44. Dersh, J. et al. Chronic pain and psychopathology: Research findings and theoretical considerations. Psychosom. Med. 64, 773-786 (2002).

45. Edit, V. et al. Psychosocial, educational, and somatic factors in chronic non-specific low back pain. Rheumatol. Int. 33, 587-592(2013).

46. Vargas-Schaffer G. Is the WHO analgesic ladder still valid? Twenty-four years of experience. Can. Fam. Physician 56, 514-517 (2010).

47. Häuser, W. et al. Treatment of fibromyalgia syndrome with antidepressants: A meta-analysis. JAMA 301, 198-209 (2009).

48. Ford, A. C. et al. Efficacy of antidepressants and psychological therapies in irritable bowel syndrome: Systematic review and meta-analysis. Gut 58, 367-378 (2009).

49. Saarto, T. \& Wiffen, P. J. Antidepressants for neuropathic pain. Cochrane database Sys. Rev. 4, CD005454 (2007).

50. Krebs, E. E. et al. Treating the physical symptoms of depression with second-generation antidepressants: A systematic review and meta-analysis. Psychosomatics 49, 191-198 (2008).

51. Arnold, L. M. et al. Comparisons of the efficacy and safety of duloxetine for the treatment of fibromyalgia in patients with versus without major depressive disorder. Clin. J. Pain 25, 461-468 (2009).

52. Arnold, L. M. et al. Comorbidity of fibromyalgia and psychiatric disorders. J Clin. Psychiatry 67, 1219-1225 (2006).

53. Bekhit MH. Opioid-induced hyperalgesia and tolerance. Am. J. Ther. 17, 498-510 (2010).

54. Berton, O. et al. Essential role of BDNF in the mesolimbic dopamine pathway in social defeat stress. Science 311, 864-868 (2006). 
55. Upadhyay, J. et al. Alterations in brain structure and functional connectivity in prescription opioiddependent patients. Brain 133, 2098-2114 (2010).

56. Angst, M. S. \& Clark, J. D. Opioid-induced hyperalgesia: A qualitative systematic review. Anesthesiology 104, 570-587 (2006).

57. Rayner, L. et al. Antidepressants for depression in physically ill people. Cochrane database Sys Rev 3, CD007503 (2010). 


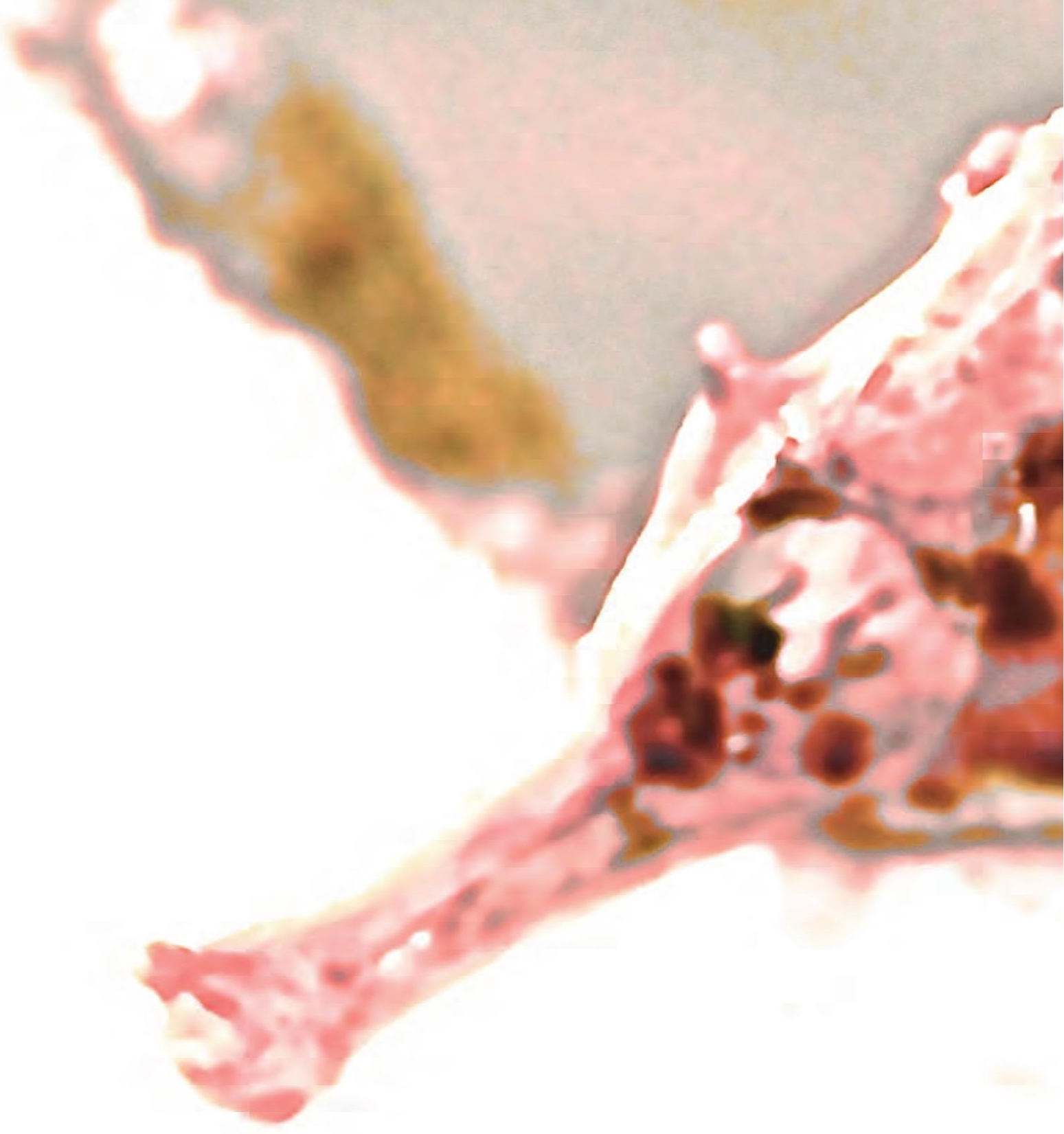



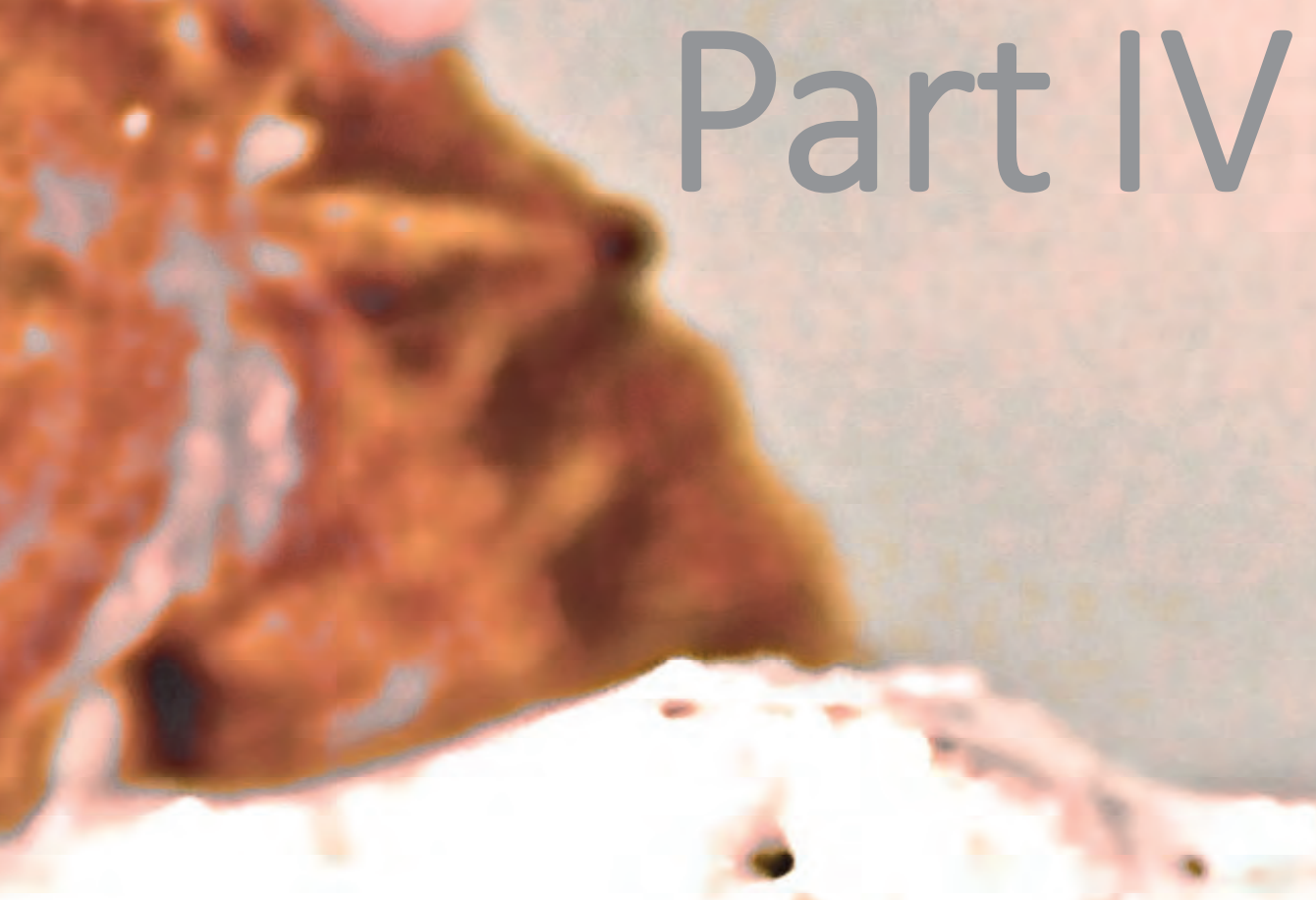

Transitional care in psychosomatic medicine 



\section{Abstract}

\section{Background}

Services providing care at the interface of medical and psychiatric comorbidity in the general hospital should be cost-effective. Previous work suggests that psychosomatic care may gain in this dimension by shifting from inpatient to outpatient and primary care solutions.

\section{Methods}

A transitional integrated care model, the Medical-Psychiatric-Network (MPN), was created between a tertiary University Medical Center and a primary Community Mental Health Service with a view to detect and treat somatic and psychiatric comorbidity initially inside, and if acceptable, eventually outside the hospital. A record linkage study was conducted, linking cost of hospital medical service use, length of stay (LOS) and (transitional) mental health care in patients referred to the MPN over a two-year period (2007-2008). Analyses quantified pre-post cost changes around MPN referral.

\section{Results}

Referral rates were highest for somatoform disorders or affective conditions in comorbid somatic diseases. Comparisons revealed lower cost of medical service use $(-€ 70.5 ; 95 \% \mathrm{Cl}$ $-€ 100.9$ to $-€ 40.1 ; P$-value $<0.001)$ and LOS $(-€ 53.4 ; 95 \% \mathrm{Cl}-€ 89.9$ to $-€ 17.0 ; P$-value $<0.01)$ after referral to the MPN. Conversely, cost of transitional psychiatric interventions was higher after MPN referral $(+€ 28.5 ; 95 \% \mathrm{Cl}+€ 21.2$ to $+€ 35.9 ; P$-value $<0.001)$. Overall, costs per patient and month were lower after MPN referral (-€84.5; $95 \% \mathrm{Cl}-€ 143.3$ to $-€ 25.8 ; P$-value $<0.01)$ and could not be explained by general hospital cost developments over the period of investigation.

\section{Conclusion}

Transitional psychosomatic care towards treatment of common somatoform disorders or affective conditions in comorbid somatic diseases, guiding patients from inside the hospital to accept primary mental health care, may be acceptable and cost-effective. 


\section{Introduction}

Admissions to the somatic hospital are increasingly brief, limiting the time that health care professionals can dedicate to psychosomatic consultation and intervention in complex patients with somatic and psychiatric multi-morbidity ${ }^{1,2}$. These changes favour a reduction in the rate of detection and treatment of psychiatric comorbidity in routine settings ${ }^{3}$. Given high rates of somatic and psychiatric multi-morbidity ${ }^{2}$, predicting longer hospital stay and greater hospital costs 3,4 , as well as poorer outcomes and increased subsequent health care use ${ }^{5}$, care alternatives are required. There is evidence that modern proactive inpatient integrated care approaches are effective, despite observed barriers to discharge ${ }^{6,7}$. However, although transitional communication between hospital consultants and primary care givers gathers a basis of evidence ${ }^{8}$, transition of care from insight the hospital back to general practitioners deserves further improvement ${ }^{9,10}$. Furthermore, although hospital-initiated transitional care improves outcome in some somatic conditions ${ }^{11}$, quality of discharge practice remains deficient in the academic setting $^{12}$. Transitional care may cut readmissions in patients with complex chronic conditions $^{13,14}$, however, research on cost developments in patients with psychosomatic multi-morbidity receiving transitional care is scarce. Moreover, the original care transitions intervention (CTI) specifically excluded patients with psychiatric comorbidity $^{13}$. Selection biases may operate; for example a state wide transitional care intervention was found to possibly have put patients with psychiatric morbidity unintentionally at a disadvantage by a selection to the care as usual condition ${ }^{14}$, whereas previous work suggests that psychosomatic care in particular may gain in costeffectiveness by shifting from inpatient to primary care solutions ${ }^{3}$. Primary care-based collaborative care (CC) represents a useful addition to clinical care in affective disorders. However, there is lack of evidence concerning re-admission rates and benefit of physical quality of life ${ }^{15,16}$. Thus, the trend for increasingly brief hospital stays needs to be offset by a transitional care organisation combining proactive liaison psychiatric services with extended integrated day-care, outpatient and integrated primary care settings ${ }^{2,3,17-22}$.

In the current article, transitional care refers to bridging the gap between specialty hospital care on the one hand and primary mental health care at the level of a Community Mental Health Service (CMHS) and general practitioners (GP), on the other. Application of this principle to somatic and psychiatric multi-morbidity may result in cost reduction without compromising quality of care. Furthermore, the term "Medical Psychiatric Network" (MPN) does not refer to a collection of hospital beds within the hospital alone ${ }^{3}$, but rather to a range of transitional outpatient care solutions for a group of complex patients requiring integrated care, guiding patients from inside the hospital to eventually accept integrated primary mental health care. 


\section{The Medical Psychiatric Network - an integrated transitional care approach in psychosomatic medicine}

In a previous report, it was shown that integrated hospital inpatient care in complex patients with somatic and psychiatric multi-morbidity, admitted to a multidisciplinary specialty ward, the Med-Psych-Unit (MPU), significantly reduced cost of medical service use compared to standalone medical wards ${ }^{3}$. Costs related to psychiatric interventions increased post-MPU admission, suggesting that patients with psychosomatic morbidity received adequate diagnosis and treatment ${ }^{3}$. Nevertheless, overall costs (including bed costs) were higher in the MPU setting, indicating an unfavourable balance in terms of cost-benefit ${ }^{3}$. Therefore, a more favourable balance of cost-effectiveness might be achieved by shifting activities, where possible, from inpatient treatment to integrated day care and outpatient treatment, and, if convenient and acceptable, to further integrated treatment outside the general hospital.

The Med-Psych-Net (MPN) is an umbrella term to denote a close collaboration to deliver integrated care, combining specialty medical and psychiatric hospital care and primary mental health care. The MPN of interest in the current investigation is not a physical centre but rather represents a virtual entity that is part of the Maastricht University Medical Centre (MUMC), in combination with MUMC's associated GPs. The MPN consists of psychiatry and medical psychology consultation liaison services, and a Community Mental Health Service, in collaboration with hospital based medical consultants and primary care based GPs, situated in a city of 120.000 inhabitants in the South of the Netherlands (Maastricht). The MPN started in 2006, providing a wide range of inpatient (including MPU beds) as well as transitional outpatient diagnostic and treatment options for a group of patients with somatic and psychiatric multi-morbidity. Key elements next to medical care are psychiatric diagnosis and management, psychotherapy and managed care, delivering a graded transition from integrated care starting inside the hospital back to primary care GPs or, if required, to the CMHS. At the level of hospital consultation-liaison-service (CLS) activities, psychosomatic care is embedded in the UMC's Med-Psych-Centre (MPC), which bundles psychosomatic care inside the University Hospital from inpatient (i.e., MPU and CLS) to outpatient care. In this regard, hospital medical psychologists and psychiatrists proactively carry out multidisciplinary psychiatric and psychological screening and case finding at medical wards or at outpatient clinics, for example at the multidisciplinary pain centre, the cancer centre, the centre for functional bowel disorders or the pelvic care centre $(P C C)^{23-25}$. A motivational procedure (towards accepting a transitional trajectory to primary mental health care) is administered prior or parallel to treatment at the medical ward and/or medical and psychiatric outpatient setting. This procedure is finally followed by a transition of care via CMHS professionals working initially inside the hospital and at a later moment of patients' treatment at the GPs practices, or, if a more lengthy or specialised mental health care treatment trajectory is deemed necessary, at 
the nearby CMHS. This care staff-guided care transition form the hospital to primary care completes our approach to the so-called Med-Psych-Net(-work). The specific MPN intervention is that care managers from the CMHS see patients for five to eight times at the MUMC's mental health care outpatient clinic and thus 'take over' treatment inside the hospital, in order to accompany the patient on his way back to primary care. This clearly distinguishes the MPN-approach from a stand alone in-hospital psychiatric CL service that has an established link with GPS or the CMHS, or from somatically focused CTI (one inpatient contact by a professional, followed by primary care based follow up) or primary care based CC (no initial inpatient face to face contact) ${ }^{13,15}$.

Given that the MPN physically combines professionals from both the medical hospital and the primary mental health care setting, it provides a unique opportunity to gain patients' acceptance for mental health explanations underlying somatic complaints. In addition, the MPN has the advantage of convenience and transitional continuity of care, which can be expected to impact positively on treatment adherence ${ }^{2,18,26}$. Another advantage of the transitional MPN is easy access for medical and mental health care (MHC) disciplines, and GPs to multidisciplinary consultations. Indeed, the MPN guarantees visibility and feasibility of psychosomatic care throughout different settings (Figure 7.1).

Figure 7.1 Organisation of a transitional Medical Psychiatric Network.

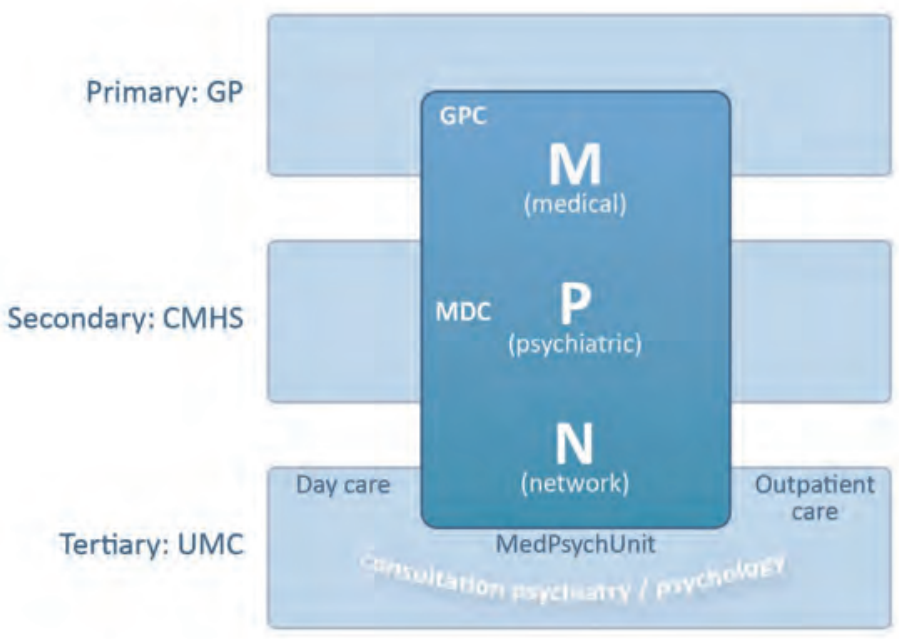

Legend: A Med-Psych-Net (MPN) is a virtual organization operating at different health care levels, from primary to tertiary care and vice versa. Different health care providers are participating: General Practitioners (GP), Community Mental Health Service (CMHS), and the Maastricht University Medical Centre (UMC) with its hospital based Med-Psych-Centre (MPC). This transitional care model provides treatment independent from setting; from tertiary clinical care to medical outpatient settings with multidisciplinary consultation (MDC) and general practitioner consultation (GPC). 
To our knowledge, no comparative data are available with regard to cost developments of transitional psychosomatic care following the introduction of a medical hospital based MPN. Recent psychosomatic research focuses on collaborative care management at the level of general practitioners, not on psychosomatically guided transition from inside the hospital to GPs or primary mental health care ${ }^{16,19-21}$. As far as hospital initiated transitional care is concerned, research focuses predominantly on somatic conditions only ${ }^{11,13}$. The present study is the first reporting a comparison, in the context of an MPN being introduced in an University Medical Hospital, of cost associated with general hospital stay, medical service use and mental health care.

\section{Aim}

To examine the hypothesis that costs associated with medical service use (e.g., medical consultation and outpatient care, medical diagnostic and therapeutic procedures: laboratory tests, X-rays, endoscopies, etc.), and bed costs associated with length of stay (LOS) (i.e., duration of hospital stay due to primary admissions and readmissions) would decrease as a result of treatment at the MPN, compared to the pre-treatment period; and that costs associated with mental health care (MHC) (e.g., medical hospital psychiatric/psychological consultation, general hospital day-care and outpatient interventions, and psychiatric/psychological interventions at GPs and the CMHS) would increase after MPN referral, compared to the pre-treatment period.

In order to "control" for general hospital cost developments over the period of investigation, cost developments in patients receiving integrated MPN care were compared with time trends of costs in a random MUMC-patient cohort receiving medical care alone over the same period.

\section{Method}

\section{Setting}

Three psychiatrists and five psychologists were working together at the MPN, most of them on a part-time basis. In addition, psychiatric case managers (CMs) were members of the team. Patients were referred by hospital consultants or by GPs, either in the context of routine hospital consultation at one of the hospital wards or through outpatient referral. Psychiatrists and psychologists provided a gate-keeping function regarding outpatient treatment options and subsequent patients' transition to primary health care by CMHS CMs. Hence, there was staff-guidance in order to indicate a suitable care transition and to guarantee transfer of medical knowledge in order to continue hospital-initiated treatment in a primary care context. 
The patient population was characterised by somatic and psychiatric multimorbidity, varying from chronic somatic diseases (e.g., inflammatory bowel disease, heart failure, cancer, etc.) with comorbid affective disorders to functional syndromes and somatoform disorders (e.g., irritable bowel syndrome, overactive bladder, noncardiac chest pain, somatisation disorder, etc.). Patients at high suicidal risk were not referred to the MPN but seen by the mental hospital crisis team. Patients with severe psychotic disorders received medical hospital based specialized somatic service as part of assertive community treatment, which was also provided by the mental hospital and not by the MPN ${ }^{27,28}$.

Patients seen by the psychiatric/psychological consultation-liaison service for the first time on hospital wards, at somatic outpatient clinics, or in the psychiatric/psychological outpatient department received psychiatric and psychological diagnostic procedures as needed, followed by psychological and psychiatric treatment as indicated. For patients receiving further treatment at the CMHS, cognitive behavioural therapy (CBT) or longer term case-management were common options ${ }^{29}$.

\section{Data collection}

At the time of the study, different sources of medical cost reimbursement existed in the Netherlands - one for general hospital care and a National Insurance covering mental health services. Datasets pertaining to these two different sources were analysed. Both datasets referred to the source population of a region in the South of the Netherlands with a medium sized town (Maastricht, 120.000 inhabitants) and surrounding villages (90.000 inhabitants), for which the MUMC is the only hospital providing somatic care. Patients from outside the catchment area were not taken into account, as register follow-up was not possible.

In order to quantify mental health care costs, data from the Psychiatric Case Register South Limburg (PCR) were used ${ }^{3,30}$. This register cumulatively collects data on mental health care contacts from the above-mentioned area. Participating facilities were, amongst others, the CMHS and the psychiatry/psychology department of the MUMC. Data from the same patient collected from different services were linked using a probability linkage procedure involving five variables, as described in more detail elsewhere ${ }^{3,30}$. In the limited subset of mental health care users, these variables are sufficient to accurately identify patients without the risk of violating privacy. Cost data for LOS and medical service use were obtained from the MUMC's registration department, providing full cost prices anonymously.

\section{Statistical analysis}

The aim of the study was to analyse costs of patients with somatic and psychiatric multimorbidity as regards: (i) medical service use (i.e., medical outpatient care and clinical consultation as well as medical diagnostic and therapeutic procedures: laboratory tests, 
X-rays, endoscopies, etc. - pharmacy costs excepted), (ii) LOS (i.e., duration of hospital stay per admission (primary admissions and readmissions) and (iii) MHC (MUMC's psychiatric/psychological consultation, day-care and outpatient interventions and psychological/psychiatric interventions at the CMHS) before and after index referral to the MPN. The longitudinal data set used in the present study consisted of multiple 28day episodes per patient covering one year before and one year after index referral. The first observation episode after referral started on the day of referral. Thus, the overall observation period is a one-year period before or a one-year period after referral (hereafter: "observation period"). Costs were analysed in the year before index referral and the year following and including that day of referral. Medical costs, bed costs and mental health care costs were collected per day and summed per episode of 28 days, in order to examine trends in cost. The observation period was restricted if the one-year period exceeded December the 31st 2008. If the patient died within the one-year period, the one-year observation period was truncated at the last full 28-day period available.

\section{Examination of general time trends concerning costs}

In order to estimate whether any cost differences might be caused by the integrated MPN approach or mainly by time trends in medical interventions alone, medical pre-post cost changes concerning bed costs and cost of medical service use in the MPN subcohort of those with a first medical consultation were compared with medical cost changes in a sample of randomly selected MUMC patients, with a first medical consultation not receiving any psychiatric or psychological intervention at any time during the same period (2007-2008). These analyses were carried out around first medical consultation in order to define a moment of reference and to avoid unduly weighting the sample to patients with either acute or most chronic somatic conditions. First medical consultation was defined as the very first moment that specialized medical consultation at the MUMC was required. Before that moment, cost related to medical service use (e.g., x-rays, laboratory tests, endoscopies, etc.) had been made in the context of treatment by general practitioners only.

To examine the hypothesis that medical cost development around first medical consultation would be equivalent in the MPN sub-cohort following first medical consultation (i.e., not yet receiving MPN integrated care) and a randomly selected cohort of MUMC patients after first medical consultation (i.e., never receiving MPN integrated care), medical costs (collected per day and summed per episode of 28 days) was analysed in both cohorts during the year before first medical consultation and the year following and including the day of first medical consultation (Figure 7.2).

In addition, the MPN sub-cohort following first medical consultation and the randomly selected MUMC cohort was analysed in both cohorts during the year before MPN referral (MPN sub-cohort; for the random MUMC cohort the time point was the year after first medical consultation - see Figure 7.2) and the year following and 
including the day of MPN referral (MPN sub-cohort; for random UMC cohort: second year after first medical consultation - see Figure 7.2), in order to distinguish estimates of cost developments related to integrated MPN-care from medical care alone in randomly selected MUMC patients (Figure 7.2).

Figure 7.2 Additional cost comparisons in a sub-cohort with first medical consultation (at T0) followed and not followed by later Medical Psychiatric Network (MPN) referral (at T1).

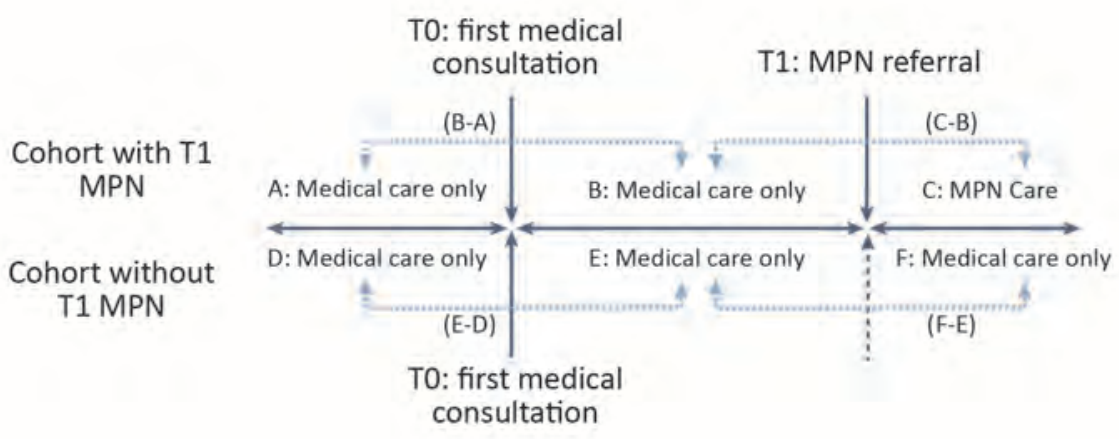

Legend: Pre-post analysis regarding medical cost (bed costs and cost of medical service use) in a randomly selected Maastricht University Medical Centre medical care cohort without integrated care (i.e., without T1 Med Psych Centre (MPC) (= Med Psych Net (MPN)) compared to a first medical consultation MPN sub-cohort (i.e., with T1 MPC (= MPN)). Costs comparisons consisted of a comparison of (i) medical costs around first medical consultation (T0), comparing the cohort with T1 MPC (= MPN) (cost difference period B-period A) with the cohort without T1 MPC (=MPN) (cost difference period E-period D), and (ii) medical costs around MPN referral (T1), comparing the cohort with T1 MPC (=MPN) (cost difference period C-period B) with the cohort without T1 MPC (=MPN) (cost difference period F-period E). The periods A, B, C, D, E and F are one-year periods.

Given the fact that these procedures resulted in more than one observation (of 28day episodes) per individual (violating the assumption of statistical independence of observations) multilevel random regression analysis using the STATA XTREG routine (multilevel random-effects model) was used to conservatively adjust standard errors and $p$-values for clustering within individuals ${ }^{31}$.

Costs after referral were compared with costs before referral using the variable phase $(0$ = before; 1 = after), testing the hypothesis that costs of medical service use and LOS would be lower, and cost of MHC would be higher in phase 1 compared to phase 0 . 


\section{Results}

\section{Sample}

In the year 2007 and 2008, 163 patients (62 men, 101 women) were referred (via MUMC'S MPC) to the transitional MPN. Mean age was 50.8 (SD=10.5) for men and 50.4 $(S D=12.6)$ for women. Seven patients died within the observation period. The patient sample of 163 yielded a total of 3702 observation periods.

\section{Category and type of diagnosis}

The category of diagnosis around MPN referral was psychiatric in $95 \%$ of the observations. The great majority of patients (80\%) additionally had a somatic diagnosis. Furthermore, $37 \%$ of patients were diagnosed as suffering from an isolated or comorbid functional somatic disorder (e.g., irritable bowel disorder, overactive bladder, etc.). Regarding type of somatic diagnosis, major somatic diseases (e.g., cancer) (28\%), diagnoses of the circulatory system (24\%), minor somatic diseases (e.g., cataract) (23\%), and conditions of the metabolic system (15\%) were most frequent (Table 7.1A).

Table 7.1A Type of somatic diagnosis around the time of referral to the Medical Psychiatric Network.

Type of somatic diagnosis ${ }^{*}$

\begin{tabular}{lc}
\hline Circulatory system & $39(24 \%)$ \\
Metabolic system & $24(15 \%)$ \\
Digestive system & $19(12 \%)$ \\
Respiratory system & $16(10 \%)$ \\
CNS & $14(9 \%)$ \\
Genito-urinary system $^{* *}$ & $4(2 \%)$ \\
Other major diseases $^{* * *}$ & $45(28 \%)$ \\
Other minor diseases $^{*}$ & $38(23 \%)$ \\
\hline
\end{tabular}

Legend: * Multiple diagnoses possible. ${ }^{* *}$ e.g., cancer. ${ }^{* * *}$ e.g., cataract, otitis, etc.

Referral rates per type of psychiatric diagnosis were highest for affective conditions or somatoform disorders in comorbid somatic diseases. Mood disorders (44\%), anxiety disorders (34\%), somatoform disorders (28\%), and adjustment disorders (22\%) were most prevalent (Table 7.1B). 
Table 7.1B Type of psychiatric diagnosis ${ }^{*}$ around the time of referral to the Medical Psychiatric Network.

\begin{tabular}{ll}
\hline Type of psychiatric diagnosis $^{* *}$ & Frequency $^{*}$ \\
\hline Mood disorder & $71(44 \%)$ \\
Anxiety disorder & $55(34 \%)$ \\
Somatoform disorder & $45(28 \%)$ \\
Adjustment disorder & $36(22 \%)$ \\
Personality disorder & $7(4 \%)$ \\
Addiction $^{\text {Eating disorder }}$ Psychotic disorder $^{+}$ & $5(3 \%)$ \\
Cognitive disorder $^{-}$ & $3(2 \%)$ \\
Other psychiatric disorder & $1(0 \%)$ \\
\hline
\end{tabular}

Legend: " According to DSM IV TR ${ }^{32}$. $^{* *}$ Multiple diagnoses possible. ${ }^{\dagger}$ Delirium excluded.

\section{Pre-post cost development around MPN referral}

Comparisons revealed lower cost of medical service use $(-€ 70.5 ; 95 \% \mathrm{Cl}-€ 100.9$ to $€ 40.1 ; P$-value $<0.001)$ and LOS $(-€ 53.4 ; 95 \% \mathrm{Cl}-€ 89.9$ to $-€ 17.0 ; P<0.01)$ after referral to the MPN. Conversely, cost of transitional MHC was higher after MPN referral $(+€ 28.5$; $95 \% \mathrm{Cl}+€ 21.2$ to $+€ 35.9 ; P<0.001$ for psychiatric interventions, and $+€ 50.9 ; 95 \% \mathrm{Cl}$ $+€ 37.9$ to $+€ 63.8 ; P<0.001)$ for psychological interventions). Overall, total costs were lower after MPN referral (-€84.5; 95\%Cl -€143.3 to -€25.8; $P<0.001)$ (Table 7.2).

Table 7.2 Pre-post comparisons (after Medical Psychiatric Network referral versus before) of university hospital costs (medical costs and bed costs), mental health care costs (transitional psychiatric or psychological care costs), and total costs.

\begin{tabular}{ccccc}
\hline Medical costs & Bed Costs (LOS) & $\begin{array}{c}\text { Transitional Costs } \\
\text { Psychiatry }\end{array}$ & $\begin{array}{c}\text { Transitional Costs } \\
\text { Psychology }\end{array}$ & Total Costs \\
\hline$-€ 70.5^{* *}$ & $-€ 53.4^{*}$ & $+€ 28.5^{* *}$ & $+€ 50.9 * *$ & $-€ 84.5^{* *}$ \\
$(-€ 100.9$ to & $(-€ 89.9$ to & $(€ 21.2$ to & $(€ 37.9$ to & $(-€ 143.3$ to \\
$-€ 40.1)$ & $-€ 17.0)$ & $€ 35.9)$ & $€ 63.8)$ & $-€ 25.8)$ \\
\hline
\end{tabular}

Legend: ${ }^{*} P \leq 0.01 ; * * P \leq 0.001$. Mean cost per patient and month in the year after MPN referral versus costs in the year before referral. Data are presented as beta weights $(95 \% \mathrm{Cl})$ and show, for instance, a decrease in costs of $53.4 €$ (Euros) after MPN referral in the second column of "bed costs".

\section{Medical cost developments of medical care in general in relation to MPN care}

Comparisons revealed equivalent cost developments around first medical consultation in the MPN sub-population and the random MUMC comparison cohort. For both populations, medical cost (bed costs and cost of medical service use) increased significantly in the year after first medical consultation: Medical Care population (+€70.5; $95 \% \mathrm{Cl}+€ 11.8$ to $+€ 129.1 ; P$-value $<0.05)$, MPN population $(+€ 111.1 ; 95 \% \mathrm{Cl}+€ 13.9$ to $+€ 208.2 ; P<0.05)$ (Table 7.3A) (Figure 7.2). 
Table 7.3A Pre-post comparisons (after first medical consultation versus before) of medical costs, comparing a university hospital medical care population with the university hospital Medical Psychiatric Network (MPN) population around time of first medical consultation.

\begin{tabular}{cc}
\hline $\begin{array}{c}\text { Medical Cost in Medical Care Population around } 1^{\text {st }} \\
\text { Medical Consultation }\end{array}$ & $\begin{array}{c}\text { Medical Cost in MPN Population around } 1^{\text {st }} \\
\text { Medical Consultation }\end{array}$ \\
\hline$+€ 70.5^{*}$ & $+€ 111.1^{*}$ \\
$(+€ 11.8$ to & $(+€ 13.9$ to \\
$+€ 129.1)$ & $+€ 208.2)$ \\
\hline
\end{tabular}

Legend: ${ }^{*} P \leq 0.05$. Mean medical cost per patient and month in the year after first medical consultation versus medical costs in the year before first medical consultation. Data are presented as beta weights $(95 \% \mathrm{Cl})$ and show, for example, a significant increase (+111.1€, Euros) in medical cost regarding the MPN population only receiving medical care (see also Figure 7.2).

In both populations (Medical Care and MPN-care), cost developments were further examined in the year following the time of MPN referral (Figure 2). Medical cost decreased significantly in the MPN population following the year of integrated care after MPN referral (-€226.0; 95\% $\mathrm{Cl}-€ 334.0$ to $-€ 117.9 ; P$-value <0.001). Medical cost remained unchanged during the same period (second year after first medical consultation) in the Medical Care population receiving medical care alone $(-€ 43.1 ; 95 \% \mathrm{Cl}$ -€91.5 to +€5.4; P>0.05) (Table 7.3B) (Figure 7.2).

Table 7.3B Pre-post comparisons (after Medical Psychiatric Network (MPN) referral versus before) of medical costs in a university hospital, comparing those receiving medical care alone with those receiving integrated care around the time of MPN referral.

\begin{tabular}{cc}
$\begin{array}{c}\text { Medical Cost in Medical Care Population around } \\
\text { time of MPN-referral } \\
\text { (Medical Care alone) }\end{array}$ & $\begin{array}{c}\text { Medical Cost in MPN Population around } \\
\text { time of MPN-referral } \\
\text { (Integrated Care) }\end{array}$ \\
\hline$-€ 43.1^{*}$ & $-€ 226.0^{* *}$ \\
$(-€ 91.5$ to & $(-€ 334.0$ to \\
$€ 5.4)$ & $-€ 117.9)$ \\
\hline
\end{tabular}

Legend: * $P>0.05 ; * * P \leq 0.001$. Mean medical costs per patient and month in the year after MPN referral versus medical costs in the year before MPN referral. Data are presented as beta weights $(95 \% \mathrm{Cl})$ and show, for example, a non-significant decrease $(-43.1 €$, Euros) in costs in the population receiving medical care only (see also Figure 7.2).

\section{Discussion}

The present study is the first to evaluate cost changes with regard to the referral of complex patients with somatic and psychiatric multi-morbidity to a transitional MedPsych-Net. This psychosomatic network consists of the University Hospital's psychosomatic inpatient (i.e, MPU and CLS) and outpatient care activities (e.g., PCC, Pain Centre, etc.), which are bundled in the hospital based Med-Psych-Centre, and the staff guided transitional care part that is delivered by CMHS's case managers bridging the gap 
between the MUMC and primary care. Costs concerning LOS, hospital related medical service use and transitional MHC were measured before and after referral to the MPN. A pre-post analysis was conducted in 163 patients over the period 2007 - 2008. In contrast to evidence presented in a previous publication regarding cost developments of an exclusively hospital-based $\mathrm{MPU}^{3}$, a reduction in costs, associated with medical service use and LOS, was hypothesised for the transitional collaborative MPN approach. Furthermore, it was hypothesized that the costs related to transitional MHC would be higher compared to the pre-MPN observation period but that, on balance, overall costs (i.e., costs of LOS, medical service use, and MHC) would still be lower after MPN referral. The results of the present study confirmed these hypotheses, in that medical service use data revealed a significant cost benefit after MPN referral. Similar results were obtained concerning LOS. In contrast, costs related to psychiatric or psychological interventions increased with MPN treatment. Given the fact that almost each referred patient (95\%) was diagnosed with a psychiatric disorder, while somatic conditions were less (but still high) prevalent (80\%), part of which was diagnosed as functional complaints (37\%), the increase in costs related to mental health care reflects a desirable and appropriate rise of $\mathrm{MHC}$ interventions in psychosomatic conditions. Even though cost associated with $\mathrm{MHC}$ increased, there was a decrease in overall costs of $84.5 €$ per patient and month, which is approximately estimated as 1000, - $€$ cost benefit per patient and year, associated with the integrated MPN-approach, as far as care related costs are concerned. These cost developments have emerged wihout changing of hospital psychiatry or medical psychology related staff-FTEs, since CMHS care givers started to join primary care during that period. Noteworthy, with regard to $\mathrm{MHC}$, these CMHS caregivers were only colocated in the MUMC and at GPS and not additionally employed. Thus, we engineered a care application that improves the affordability of hospital care via mechanisms, which enhanced outpatients' flow through the medical hospital without proportionately adding staff. Compared with primary care based collaborative care interventions, an amount of $1000,-€ / \$$ cost savings was realized already one-year after transition of care ${ }^{20,21}$. This cost reduction was due to hospital related cost savings alone and did not include further costs of social relevance (e.g., illness-related sick leaf, primary care consumption etc.).

The reduction in costs of medical service use and LOS after referral to the MPN may in part be due to successfully delivered medical treatment of somatic conditions, for example if medical consultants had admitted patients to the hospital for urgent (costly) treatment of a medical condition, followed by referral to the MPN for emergent psychiatric conditions after initial medical treatment. However, given the fact that the proportion of psychiatric and functional diagnoses in the MPN population was high, and somatic conditions were less prevalent, another explanation, in line with previous work, may be more likely - psychiatric disorders might be unrecognized in the medical setting ${ }^{3,17}$. Integrated care by an MPN facilitates detection and treatment of psychiatric disorders, preventing physicians from misunderstanding psychiatric disorders as a 
somatic complication in combination with prescribing costly diagnostic and therapeutic procedures ${ }^{3,17,33,34}$. Due to data management changes in the hospital (replacement of paper-based patient files by electronic patient files), a wel-matched comparison between the MPN and the randomly chosen control group condition was not possible. However, the fact that medical cost developments did not differ between an MPN subcohort and the random MUMC medical cohort after $1^{\text {st }}$ medical consultation, while a medical cost benefit was apparent in the second year after integrated MPN care in comparison to te $2^{\text {nd }}$ year of medical care alone, indicates that MPN care may truly add value in terms of cost savings. It is not likely that the cost reduction in the MPN condition simply reflects a regression to the mean, given that somatic morbidity was prevalent in the MPN cohort and that psychiatric comorbidity may also occur in chronic somatic diseases managed by a non-psychiatric medical interventions only ${ }^{35-39}$. Moreover, there is no reason to assume that costs require three years to decrease, especially not in patients with chronic or repetitively re-occurring somatic and psychiatric comorbidities $^{3,36,37,40,41}$ (Figure 7.3). Thus, transitional integrated care delivered by a Med-Psych-Net may fulfil unmet needs of multi-conditional patients concerning treatment as indicated, and may reduce waiting lists of medical consultants by accomplishing treatment transition back to GPs, hence, having societal impact due to cost-savings.

Figure 7.3 Mean Medical (MUMC) and Mental Health Care (MHC) costs - a description of cost developments 3 years before and 1 year after Med-Psych-Net (MPN) referral.

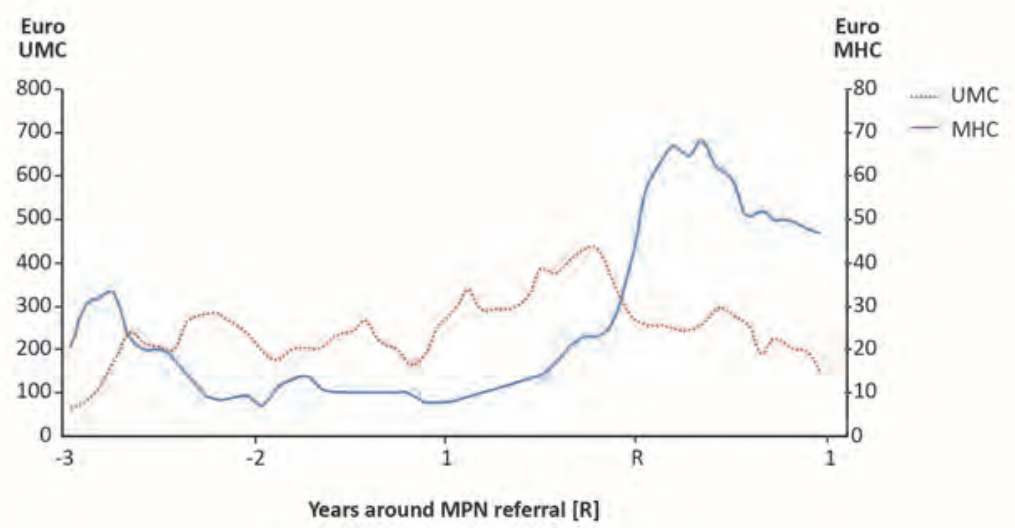

Legend: Figure 7.3 describes mean medical (Maastricht University Medical Centre (UMC)) and Mental Health Care (MHC) cost developments with regard to 163 investigated Med-Psych-Net (MPN = care transition starting at the MUMC's Med-Psych-Centre back to GPs in primary care) patients in three years before and one year after MPN-referral (i.e., index referral [R]). Thus, there is a gradual increase in UMC costs (costs of medical service use and bed costs) over the three years before MPN referral, with a fall in costs after MPN referral. Conversely, MHC cost related to psychiatric/psychological interventions increase after MPN referral. 
Figure 7.3 illustrates medical and psychiatric cost developments with regard to the 163 investigated MPN patients in the three years before and one year after MPN referral. There is an almost linear increase in medical cost (costs of medical service use and bed costs) over the three years preceding MPN referral, with a clear fall in costs after MPN referral. Conversely, costs due to psychiatric and psychological interventions increased after MPN referral (Figure 3). It is difficult to conceive of a decrease in medical cost after MPN referral being attributable to medical treatment. More likely, and in accordance with the literature, cost benefit may be caused by the integrated approach providing medical and psychiatric or psychological treatment that target patients as well as physicians $3,20,21,33-38,40-44$. While traditional integrated care is focussing on primary care or clinical care alone $e^{3,20,21}$, and transitional care in psychosomatic conditions is lacking, the MPN approach adds an organisational advantage - continuous psychiatric and psychological consultation and treatment in psychosomatic conditions independent from setting. Starting at the UMC based Med-Psych-Centre, multidisciplinary clinical, outpatient, and primary care-based round table conferences provide staff guided psychiatric and psychological case-management with consultation opportunities at different care levels ${ }^{42}$. As a result of integrated diagnosis and treatment, frequent utilizers of medical services may display better psychiatric/psychological treatment adherence, as evidenced by an increase in cost of transitional mental health care and a decrease in medical and overall costs. These cost savings were achieved by care delivery using pre-existing services. Neither the hospital consultation liaison services of psychiatry and medical psychology nor the CMHS had to hire extra staff. At both sides of the MPN (MUMC and CMHS), caregivers' fulltime equivalents (FTEs) remained the same, reflecting cost-effectiveness from a societal or health system perspective. Patients, if identified at all, would usually have been referred directly to CMHS. Due to the MPN, better case detection as well as medical staff guidance took place. Given the fact that patients adhered to treatment, and therefore, were not lost in the transition, hospital discharge was managed effectively without compromising FTEs ${ }^{44}$. Additionally, the MPN enabled the MUMC's psychiatry CLS to substantially increase the number of patient contacts on hospital wards and outpatient clinics since its introduction (data not shown). The MPN approach clearly closes gaps between different care providers in psychosomatic conditions, both inside and outside the medical hospital, and is translatable to every care situation with specialty care at different levels. It may also be used, as a blue print for transitional complexity interventions in multi-conditional patients even without psychiatric comorbidity, since the level of comorbidity care and the quality of illness management is relevant in avoiding every kind of hospital readmissions ${ }^{45-47}$. This consideration might indirectly be supported by hospital evaluations of our own MUMC, concerning wards still running without a transitional network approach, especially without routinely integrated psychosomatic care in patients with potential medical and psychiatric comorbidities ${ }^{48-50}$. From our experience, transitional care by case managers can be effectively realized by medical staff guided 
caregivers from outside the MUMC, as suggested by our investigation, or by care managers related to hospital based disease management programs. From our clinical perspective, patient empowerment due to a "warm handover" in between settings as well as medical skills guaranteed by integrated staff-guidance are essentials in care transition. Moreover, while primary care based collaborative care is very well suited to long distance communication in rural areas, the MPN approach may especially be productive in urban areas where complex patients are cumulating at the hospital level, and GPs as well as General Hospital related treatment options might fail, if not delivered transitionally ${ }^{12,50}$.

Hence, transitional integrated care adds value in complex patients' care $^{13,14}$, and the MPN delivers treatment results in psychosomatic complexity. It is important to emphasize that these findings can be generalized to psychosomatic conditions detected during University Hospital's clinical consultation-liaison and outpatient care. From this level, treatment was initiated and guided towards a collaborative primary mental health care service.

\section{Limitations}

Several limitations of the present study need further consideration. First of all, this study is not a case control study since the cost comparison with a randomly selected MUMC cohort is not well matched with regard to sex, age and diagnoses. The quasi comparison with a not transitionally treated UMC cohort only allows for an estimate of general time trends in cost developments due to general changes concerning hospital related diagnostic and treatment procedures. Thus, the present investigation is a non-controlled pre-post comparison concerning cost aspects. Nevertheless, as a naturalistic pilot study, the current investigation serves to generate a clinically relevant hypothesis that still has to be verified, namely, integrated transitional care may lead to relevant cost savings compared to care as usual. As in surgical randomized controlled trials (RCTs), prospective comparative research better has to be preceded by previous naturalistic clinical research since almost $50 \%$ of surgical RCTs remain unpublished and more than one-third of clinical RCTs have led to different conclusions at re-analysis ${ }^{51,52}$. Thus, prospective comparative controlled research concerning cost-effectiveness of integrated transitional care should be preceded by a study like this one.

Furthermore, this investigation is a single centre study in a tertiary academic hospital. The case mix discharged from the MUMC may differ from General Hospitals elsewhere. Since we conducted a pre-post analysis using routine data rather than a targeted data collection, we cannot rule out the possibility of confounding. For instance, as we know from our previous MPU investigation, there is a remarkable difference in frequency of detected psychiatric diagnoses per setting ${ }^{3}$. Thus, it cannot be excluded that a referral bias was operating, given the different distribution of referring medical specialties to the MPN. Additionally, the MPN patient population presented with mild to moderate psychosomatic complaints as seen in a consultation and outpatient setting, 
and was subsequently further treated in a primary care setting. Therefore, these findings cannot be extrapolated to a population with more severe psychiatric and/or somatic comorbidities in need of clinical MPU care.

There is a lack of outcomes other than cost data. For example, we did not address changes in patient-related outcomes, like illness severity, quality of life, treatment satisfaction or global functioning. Moreover, we lack estimates regarding illness-related sick leave from work, and primary care consumption. Finally, this study only collected data over a twelve-month period before and after an index referral. Evidence of collaborative depression treatment in medically ill patients at the primary care setting suggests that cost savings even persist in the second year after the initiation of treatment ${ }^{20,21}$. Hence, future research is needed, which should also focus on patientrelated health outcomes as well as more secondary cost developments. As the present study is observational in nature, controlled prospective data are deemed needed. 


\section{References}

1. Simon, G. E. \& Walker E. A. The primary care clinic. The American Psychiatric Publishing Textbook of Consultation-Liaison Psychiatry: Psychiatry in the Medically III, 2nd Edition. Edited by Wise, M. G. \& Rundell, J. R. Washington, DC, American Psychiatric Publishing, 917-925 (2002).

2. Hall, R. C. et al. Cost-effectiveness of the consultation-liaison service. The American Psychiatric Publishing Textbook of Consultation-Liaison Psychiatry: Psychiatry in the Medically III, 2nd Edition. Edited by Wise, M. G. \& Rundell, J. R. Washington, DC, American Psychiatric Publishing, 25-32 (2002).

3. Leue, C. et al. Managing complex patients on a Medical Psychiatric Unit: An observational study of university hospital costs associated with medical service use, length of stay and psychiatric intervention. J. Psychosom. Res. 68, 295-302 (2010).

4. Saravay, S. M. \& Lavin, M. Psychiatric comorbidity and length of stay in the general hospital. A critical review of outcome studies. Psychosomatics 35, 233-252 (1994).

5. Druss, B. G. \& Rosenheck, R. A. Patterns of health care costs associated with depression and substance abuse in a national sample. Psychiatr. Serv. 50, 214-218 (1999).

6. Desan, P. H. et al. Proactive psychiatric consultation services reduce length of stay for admission to an inpatient medical team. Psychosomatics 52,513-520 (2011).

7. Stiefel, F. et al. Effects of a multifaceted psychiatric intervention targeted for complex medically ill: A randomized controlled trial. Psychother. Psychosom. 77, 247-256 (2008).

8. Foy, R. et al. Meta-analysis: Effect of interactive communication between collaborating primary care physicians and specialists. Review. Ann. Intern. Med. 152, 247-258 (2010).

9. Holland, D. E. et al. Problems and unmet needs of patients discharged "home to self-care". Prof. Case Manag. 16, 240-250 (2011).

10. Mesteig, M. et al. Unwanted incidents during transition of geriatric patients from hospital to home: A prospective observational study. BMC Health Serv. Res. 10: doi: 10.1186/1472-6963-10-1 (2010).

11. Prvu Bettger, J. et al. Transitional care after hospitalization for acute stroke or myocardial infarction: A systematic review. Ann. Intern. Med. 157, 407-416 (2012).

12. Horwitz, L. I. et al. Quality of discharge practices and patient understanding at an academic medical center. JAMA Intern. Med. 173, 1715-1722 (2013).

13. Coleman, E. A. et al. The care transitions intervention: Results of a randomized controlled trial. Arch. Intern. Med. 166, 1822-1828 (2006).

14. Jackson, C. T. et al. Transitional care cut hospital readmissions for North Carolina Medicaid patients with complex chronic conditions. Health Aff. 32, 1407-1415 (2013).

15. Archer, J. et al. Collaborative care for depression and anxiety problems. Cochrane Database Syst. Rev. 10: doi 10.1002/14651858.CG006525.pub2. (2012).

16. Schaefert, R. et al. Specific collaborative group intervention for patients with medically unexplained symptoms in general practice: A cluster randomized controlled trial. Psychother. Psychosom. 82, 106-119 (2013).

17. Kathol, R. G. et al. Psychiatrists for medically complex patients: Bringing value at the physical health and mental health/substance-use disorder interface. Psychosomatics 50, 93-107 (2009).

18. Leentjens, A. F. et al. Can we increase adherence to treatment recommendations of the consultation psychiatrist working in a general hospital? A systematic review. J. Psychosom. Res. 68, 303-309 (2010).

19. Kinder, L. S. et al. Improving depression care in patients with diabetes and multiple complications. J. Gen. Intern. Med. 21, 1036-1041.

20. Katon, W. J. et al. The Pathway Study: A randomized trial of collaborative care in patients with diabetes and depression. Arch. Gen. Psychiatry 61, 1042-1049 (2004).

21. Unutzer, J. et al. Long-term cost effects of collaborative care for late-life depression. Am. J. Manag. Care. 14, 95-100 (2008).

22. Van der Feltz-Cornelis, C. M. et al. Effect of psychiatric consultation models in primary care. A systematic review and meta-analysis of randomized clinical trials. J. Psychosom. Res. 68, 521-533 (2010).

23. Kruimel, J. et al. Integrated medical-psychiatric outpatient care in functional gastrointestinal disorders improves outcome: A pilot study. Eur. J. Gastroenterol. Hepatol. 27, 721-727 (2015). 
24. Vrijens, D. et al. Prevalence of anxiety and depressive symptoms and their association with pelvic floor dysfunctions: A cross sectional cohort study at a Pelvic Care Centre. Neurourol. Urodyn. doi: 10.1002/nau:23186 (2017).

25. Baijens, L. W. et al. Medically unexplained otorhinolaryngological symptoms: Towards integrated psychiatric care. Laryngoscope 125, 1583-1587 (2015).

26. DiMatteo, M. R. et al. Depression is a risk factor for non-compliance with medical treatment: Metaanalysis of the effects of anxiety and depression on patient adherence. Arch. Intern. Med. 160, 2101-07 (2000).

27. Drukker, M. et al. A real-life observational study of effectiveness of FACT in a Dutch mental health region. BMC Psychiatry 8:93 (2008).

28. Drukker, M. et al. Systematic monitoring of needs for care and global outcomes in patients with severe mental illness. BMC Psychiatry 10:36 (2010).

29. Nezu A. M. et al. Cognitive behavior therapy for medically unexplained symptoms: A critical review of the treatment literature. Behavior. Therapy 32, 537-583 (2001).

30. Driessen, G. et al. Stroke and mental health care: A record linkage study. Soc. Psychiatry Psychiatr. Epidemiol. 36, 608-612 (2001).

31. StataCorp: Stata Statistical Software: Release 8.0. College Station, Tex, StataCorp. (2002).

32. American Psychiatric Association. Diagnostic and Statistical Manual of Mental Disorders (DSM-IV-TR). $4^{\text {th }}$ edition, text revision (2000).

33. Meyer, T. et al. Depression but not anxiety is a significant predictor of physicians' assessments of medical status in physically ill patients. Psychother. Psychosom. 69, 147-154 (2000).

34. Sharpe, M. et al. Is co-morbid depression adequately treated in patients repeatedly referred to specialist medical services with symptoms of a medical condition? J. Psychosom. Res. 72, 419-421 (2012).

35. Wells, K. B. et al. Psychiatric disorder in a sample of the general population with and without chronic medical conditions. Am. J. Psychiatry 145, 976-981 (1988).

36. Katon, W. J. Clinical and health services relationships between major depression, depressive symptoms, and general medical illness. Biol. Psychiatry 54, 216-226 (2003).

37. Katon, W. J. Epidemiology and treatment of depression in patients with chronic medical illness. Dialogues Clin. Neurosci. 13, 7-23 (2011).

38. Neeleman, J. et al. Propensity to psychiatric and somatic ill health: Evidence from a birth cohort. Psychol. Medicine 32, 793-803 (2002).

39. Härter, M. et al. Increased 12-month prevalence rates of mental disorders in patients with chronic somatic diseases. Psychother. Psychosom. 76, 354-360 (2007).

40. Konnopka, A. et al. Economics of medically unexplained symptoms: A systematic review of the literature. Psychother. Psychosom. 81, 265-275 (2012).

41. Cuijpers, P. et al. Economic costs of neuroticism: A population-based study. Arch. Gen. Psychiatry 67, 1086-1093 (2010).

42. Mitchell, A. J. Systematic review: Depression screening and management programmes with staff assistance in primary care increase response and remission rates, but programmes without staff assistance do not show benefits. Evid. Based Med. 15, 49-50 (2010).

43. Neeleman, J. et al. Neuroticism, a central link between somatic and psychiatric morbidity - path analysis of prospective data. Psychol. Med. 34, 521-531 (2004).

44. Coleman, E. A. \& Berenson, R. A. Lost in transition: Challenges and opportunities for improving the quality of transitional care. Ann. Intern. Med. 140, 533-536 (2004).

45. Donzé, J. et al. Causes and patterns of readmissions in patients with common comorbidities: Retrospective cohort study. BMJ 347, f7171 (2013).

46 Fabbian, F. et al. The crucial factor of hospital readmissions: A retrospective cohort study of patients evaluated in the emergency department and admitted to the department of medicine of a general hospital in Italy. Eur. J. Med. Res. 20, 6 (2015).

47. Scott, I. A. et al. Quality of care factors associated with unplanned readmissions of older medical patients: A case-control study. Intern. Med. J. 44, 161-170 (2014).

48. Magdelijns, F. J. et al. Unplanned readmissions in younger and older adult patients: The role of healthcare-related adverse events. Eur. J. Med. Res. 21, 35 (2016). 
49. Magdelijns, F. J. et al. Direct health care costs of hospital admissions due to adverse events in The Netherlands. Eur. J. Public Health. 24, 1028-1033 (2014).

50. Leue, C. et al. Observational evidence that urbanisation and neighbourhood deprivation are associated with escalation in pharmacological pain treatment - a longitudinal population-based study in the Netherlands. BMJ Open 2, pii: e000731 (2012).

51. Chapman, S. J. et al. Discontinuation and non-publication of surgical randomised controlled trials: Observational study. BMJ 349, g6870 (2014).

52. Ebrahim, S. et al. Reanalyses of randomized clinical trial data. JAMA 312, 1024-1032 (2014). 


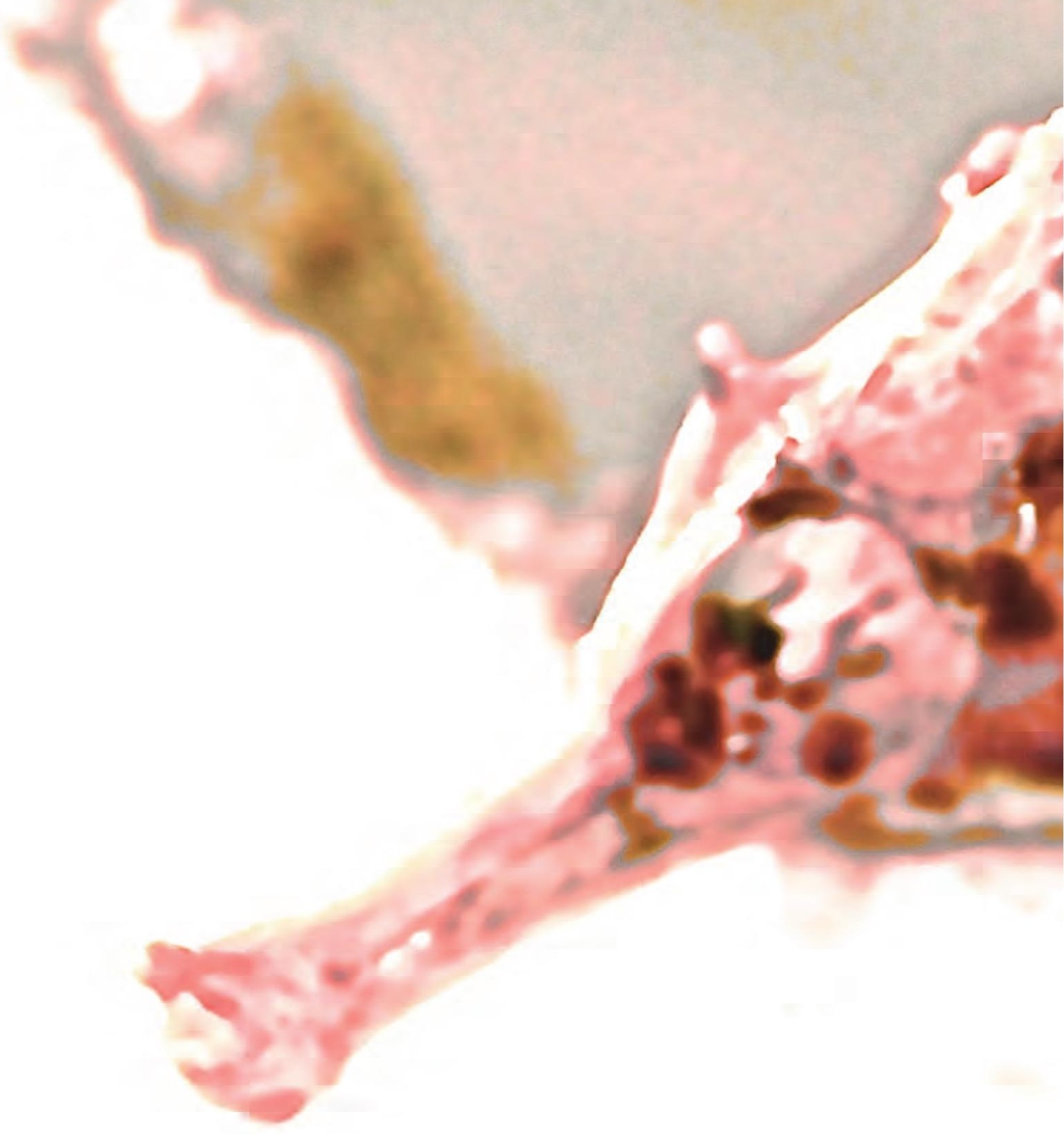




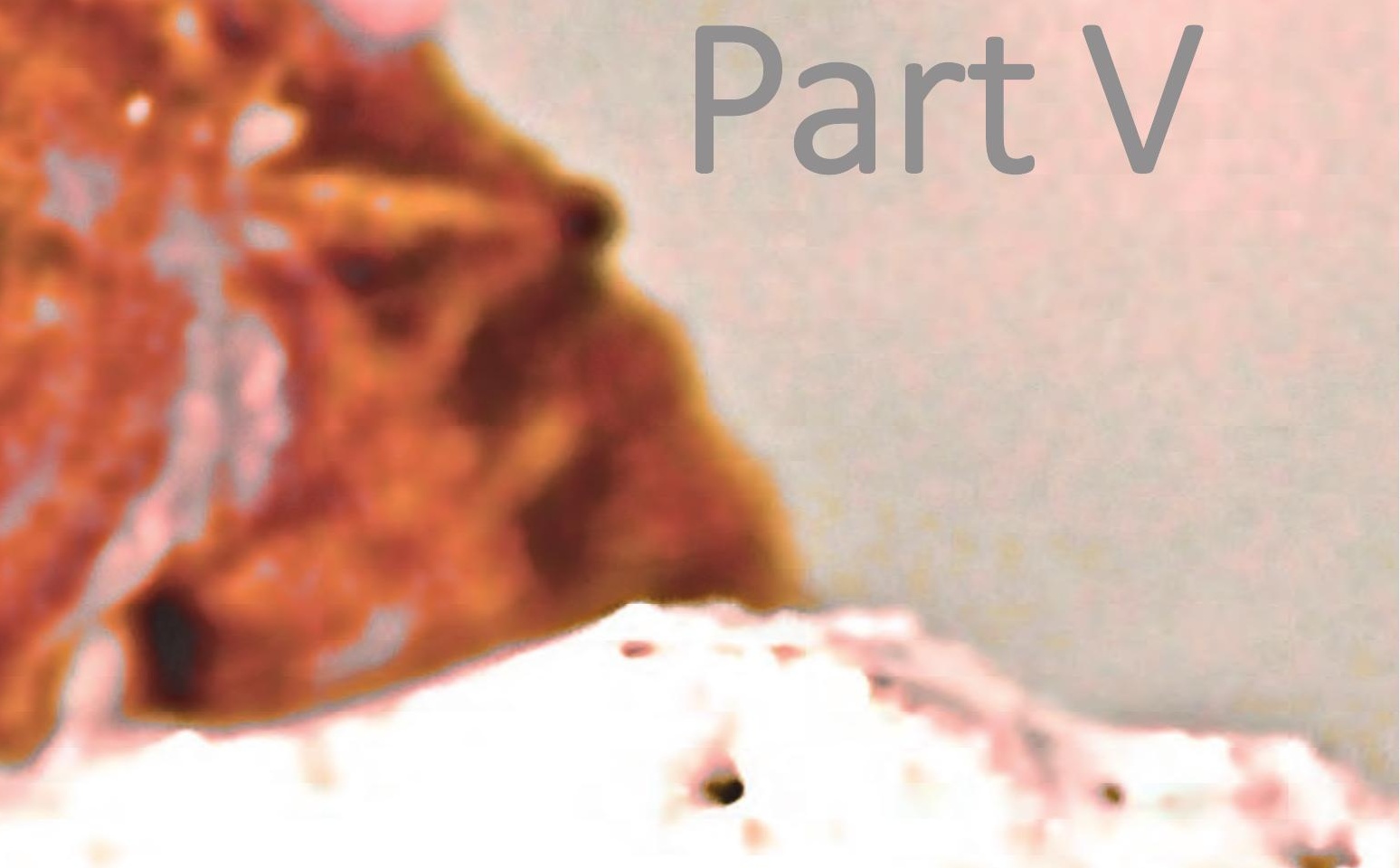

Impact of integrated care on the understanding of psychosomatic aetiology 



\section{Abstract}

\section{Objective}

Affective complaints are involved in bothersome oropharyngeal dysphagia (OD). The aim was to determine the relationship between the severity of OD and affective symptoms.

\section{Study design}

Prospective cohort study.

\section{Methods}

One hundred seven patients underwent a standardized examination protocol including the Hospital Anxiety and Depression Scale (HADS) and fiberoptic endoscopic evaluation of swallowing (FEES). Two observers independently assessed patient performance on four ordinal FEES-variables (for thin and thick liquid consistency, blindly assessed). The relationship between FEES outcome and the presence of clinically relevant symptoms of anxiety and depression was analysed using binary logistic regression.

\section{Results}

Significant associations were found between clinically relevant symptoms of anxiety and two variables: piecemeal deglutition (thin liquid consistency only) $(P=0.026)$ and postswallow vallecular pooling (thick liquid consistency only) $(P=0.015)$. The probability of presenting with anxiety symptoms decreased as the severity of piecemeal deglutition and postswallow vallecular pooling increased. No significant association was found between clinically relevant symptoms of depression and any specific FEES variable.

\section{Conclusion}

These data revealed few associations between anxiety symptoms and the measured FEES variables. However, the more severe the score on FEES variables, the less important the affective complaints were. Anxiety seems to play a role in $O D$, but no causal relationship was found, commensurate with a cross-sectional study design. The contribution of affective symptoms to the development and treatment of OD warrants longitudinal research. 


\section{Introduction}

Oropharyngeal dysphagia (OD) is commonly observed at the outpatient clinic for otorhinolaryngology. The problem can be caused by various conditions (e.g., head and neck cancer and its oncological treatment; neurological diseases such as Parkinson's disease, stroke, or multiple sclerosis; Zenker's diverticulum; cervical spine degeneration $)^{1-3}$. The epidemiology of $\mathrm{OD}$ is constantly changing as more diagnoses present with it. OD can have severe consequences such as aspiration pneumonia, and its possible complications can lead to malnutrition, dehydration, and sudden death ${ }^{3-6}$. Furthermore, OD can lessen one's quality of life $e^{6-10}$. Its psychosocial burden is a reason to investigate swallowing problems, as these are often associated with shame, diminished self-esteem, and embarrassment. Thereby, OD may contribute to a decreased quality of life and even social isolation ${ }^{4}$. In a previous study among patients who visited the outpatient clinic for OD, 37\% presented with clinically relevant symptoms of anxiety and $32.6 \%$ with depressive symptoms ${ }^{11}$.

The present study investigated the relationship between the severity of OD, by means of fiberoptic endoscopic evaluation of swallowing (FEES), and clinically relevant depressive and anxiety symptoms using the Hospital Anxiety and Depression Scale (HADS).

\section{Materials and methods}

\section{Participants}

One hundred and seven patients with OD complaints were recruited the outpatient clinic for otorhinolaryngology and divided into three main diagnostic groups (Table 1). Fifty-four patients suffered from neurological diseases (e.g., Parkinson's disease, stroke), 35 patients from miscellaneous or 'other' etiologies (e.g., Zenker's diverticulum, cervical spine degeneration), and 18 patients from head and neck oncological diseases. At the time of examination, all head and neck oncological patients had received curative oncological treatment at least 6 months prior to inclusion. All neurological patients were in a stable period of disease or on a stable medication program for at least 3 months. None of the included patients was in a stage of palliative care.

Exclusion criteria were: age older than 85 years (presbyphagia); a score below 23 on the Mini Mental State Examination $(M M S E)^{12}$; severe dyskinesia of head and neck (problems during swallow examinations); recent stroke (less than 3 months prior to investigation); previously diagnosed with depression or another psychiatric disease (use of antidepressant and/or anxiolytic drugs); having had speech therapy during the past 6 months (benefit of attention and rehabilitation); not knowing the Dutch language; suffering from illiteracy or blindness. 
Informed consent was obtained from all patients. The medical ethics committee approved the study protocol.

Table 8.1 Characteristics of the 107 patients with oropharyngeal dysphagia.

\begin{tabular}{lccc}
\hline Patient characteristics & $N$ & \% or Median (range) & Mean (SD)* \\
\hline Gender & 63 & $58.9 \%$ & \\
$\quad$ Male & 44 & $41.1 \%$ & $58.7(13.8)$ \\
$\quad$ Female & 107 & $60.0(21-82)$ & \\
Age & 101 & $25.8(13.7-36.5)$ & \\
BMI & & $50.5 \%$ & \\
Aetiology & 54 & $16.8 \%$ & \\
$\quad$ Neurology & 18 & $32.7 \%$ & \\
$\quad$ Oncology & 35 & $5.0(0-16)$ & \\
$\quad$ Other & 105 & $5.0(0-19)$ \\
HADS-A & 105 & $72(0-100)$ \\
HADS-D & 105 & \\
DSS & & & \\
\hline
\end{tabular}

Legend: Median and range are given for continuous variables while frequency and percentages are provided for nominal variables. *SD: standard deviation. BMI: Body Mass Index; HADS: Hospital Anxiety and Depression Scale (HADS-A: Anxiety subscale, HADS-D: Depression subscale); DSS: Dysphagia Severity Scale.

\section{Examination protocol}

All patients underwent a standardized examination as set forth in the protocol used in daily clinical practice at the outpatient clinic for otorhinolaryngology at the Maastricht University Medical Centre (MUMC). The same members of a multidisciplinary team performed all measurements. The protocol stipulated the following tests: a clinical examination by an experienced laryngologist, Body Mass Index (BMI) measurement, MMSE, FEES examination, the Dysphagia Severity Scale (DSS), and the HADS ${ }^{13}$. The DSS is a visual analogue scale (VAS) or psychometric response scale used to elicit patients' subjective evaluation of swallowing. Patients were asked to answer a question by indicating a position along a continuous line between two end-points. The question was, "How do you assess your swallowing function today?" ${ }^{6}$. The HADS questionnaire is a validated tool to assess symptoms of anxiety and depression, consisting of 14 items seven on anxiety and seven on depression. Every item is scored from 0 to 3, resulting in a range from 0 to 21 on each subscale. A score $\geq 8$ on one of the subscales points to the presence of clinically relevant symptoms of anxiety or depression, indicating mood disturbances or anxiety complaints ${ }^{13-15}$. All patients were given a standardized FEES examination ${ }^{16}$. First they had to perform three swallows of $10 \mathrm{~mL}$ thin liquid (water) and then three swallows of $10 \mathrm{~mL}$ standardized applesauce (hereinafter referred to as "thick liquid"). All liquids were dyed with $5 \%$ methylene blue $(10 \mathrm{mg} / \mathrm{ml})$. A flexible fiberoptic endoscope, Pentax FNL-10RP3 (Pentax Canada Inc., Mississauga, Ontario, Canada), was used with the tip in 'high position', just above the epiglottis. Thus positioned, the scope could not interfere with closure of the laryngeal vestibule ${ }^{16}$. The FEES videos were 
obtained with an Alphatron Stroboview ACLS camera, Alphatron Light Source, and IVACX computerized video archiving system (Alphatron Medical Systems, Rotterdam, Netherlands) and recorded on a DVD.

\section{FEES variables}

Four visuoperceptual ordinal variables (piecemeal deglutition, postswallow vallecular pooling, postswallow pyriform sinus pooling, and aspiration) (Table 8.2$)^{17}$ were scored for each FEES swallow at varying speed (slow motion, normal, up to frame-by-frame speed) using the software program Windows Movie Maker version 5.1 (Microsoft Corporation, Redmond, WA, USA). Before assessing the swallowing acts, two experts received consensus training for scoring these variables, as described previously ${ }^{18-20}$. The judges were blinded to the identity and medical history of the patients and to each other's scores (independent rating). To determine the level of intraobserver agreement, both judges were asked to blindly assess the four FEES variables in 27 (26\%) randomly selected patients (111 of the 428 videos) twice within a period of 2 weeks. To avoid observer fatigue, the measurement sessions were limited to a maximum duration of 2 hours.

\section{Statistical analysis}

Only the second swallow per consistency was taken into account in the statistical analysis. Intra- and interobserver agreement on the four FEES variables was determined with the linear weighted kappa coefficient. For each FEES variable, the assessments of the judge with the highest intraobserver agreement levels were then retained for the subsequent statistical analysis.

Results were expressed as the median (range) for continuous variables, while frequencies and proportions (\%) were used for ordinal FEES variables. Means for age between etiological groups were compared with a one-way analysis of variance analysis. The relationship between the presence of clinically relevant symptoms of anxiety and depression (HADS score $\geq 8$ ) and the severity of OD (assessed with FEES) was analyzed by means of the chi-square test for contingency tables and by multiple binary logistic regression analysis. Furthermore, multiple linear regression analysis was used to study the relationship between the DSS score and the severity of OD. The measured FEES variables were considered in separate regression models because of the high degree of association between them. Calculations were always carried out on the maximum amount of data available. Missing data were not replaced. Results were considered to be significant at the $5 \%$ critical level. Statistical analyses were conducted using IBM SPSS Statistics for Windows, version 21.0 (IBM Corp. Armonk, NY). 


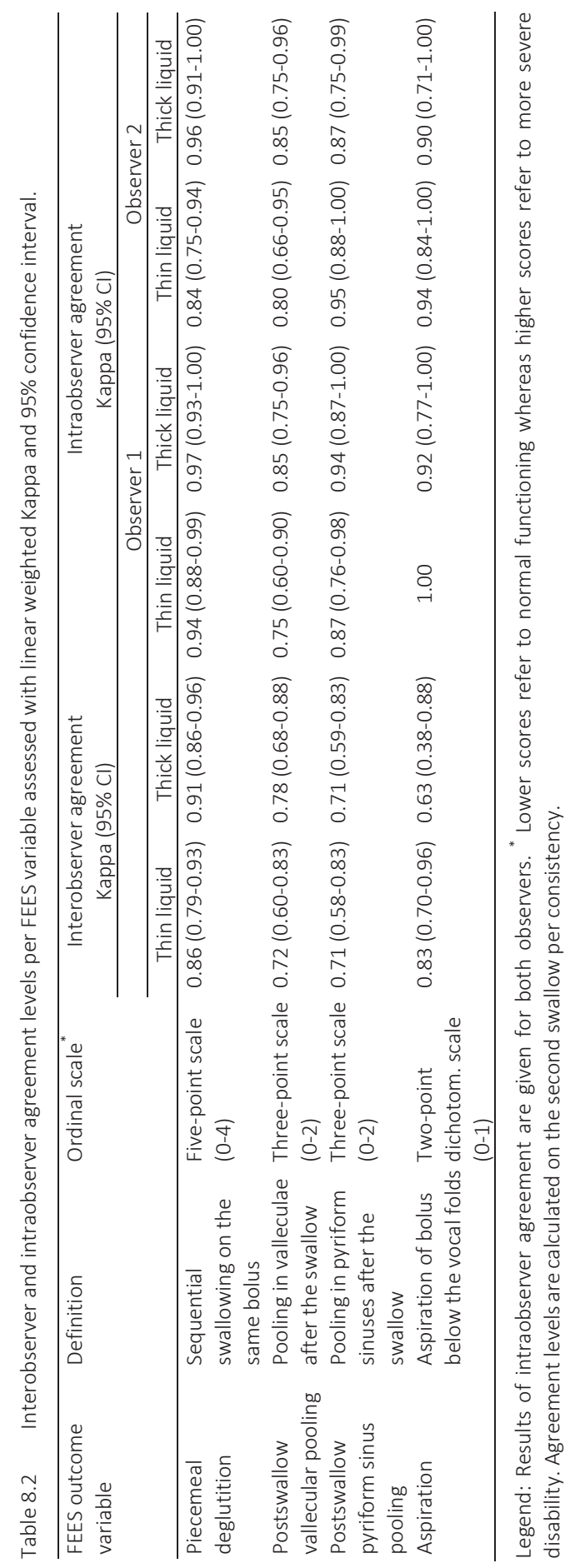




\section{Results}

\section{Participants}

A total of 107 patients who visited the outpatient clinic for OD were included. Their characteristics are described in Table 1. There were 44 (41.1\%) females and 63 (58.9\%) males with OD divided into three etiological groups, namely neurological (50.5\%), head and neck oncological (16.8\%), and 'other or miscellaneous' (32.7\%). Neurological aetiology encompasses stroke, Parkinson's disease, muscular dystrophies etc. Oncological aetiology refers to heterogeneity of tumor sites and stages in the head and neck topographical region. The category of 'other' covers Zenker's diverticulum, cervical spine degeneration, and dysfunction of the upper oesophageal sphincter. The median age in the study population was 60 years. The neurological patients were younger than the patients in the miscellaneous and oncological groups, with an average (standard deviation) of 53.4 (14.4) versus 64.1 (11.7) and $64.4(9.2)$, respectively $(F(2,104)=9.562$, $P<0.0005)$. The frequencies of patients per category of the different FEES variables are shown in Table 8.3, giving an indication of the average swallowing function of the study population.

Table 8.3 Frequency distribution of patients per category of the different FEES variables, given as absolute numbers $(\mathrm{N})$ and percentages (\%).

\begin{tabular}{lcc}
\hline FEES category frequencies & Thin liquid consistency & Thick liquid consistency \\
\hline Piecemeal deglutition & $\mathrm{N}(\%)$ & $\mathrm{N}(\%)$ \\
Category 0 & $23(21.5 \%)$ & $10(9.3 \%)$ \\
Category 1 & $35(32.7 \%)$ & $40(37.4 \%)$ \\
Category 2 & $30(28.0 \%)$ & $28(26.2 \%)$ \\
Category 3 & $8(7.5 \%)$ & $13(12.1 \%)$ \\
Category 4 & $7(6.5 \%)$ & $15(14.0 \%)$ \\
Postswallow vallecular pooling & & \\
Category 0 & $36(33.6 \%)$ & $21(19.6 \%)$ \\
Category 1 & $43(40.2 \%)$ & $37(34.6 \%)$ \\
Category 2 & $21(19.6 \%)$ & $42(39.3 \%)$ \\
Postswallow pyriform sinus pooling & & \\
Category 0 & $58(54.2 \%)$ & $58(54.2 \%)$ \\
Category 1 & $32(29.9 \%)$ & $17(15.9 \%)$ \\
Category 2 & $12(11.2 \%)$ & $21(19.6 \%)$ \\
Aspiration & & \\
Category 0 & & $92(86.0 \%)$ \\
Category 1 & $21(78.5 \%)$ & $12(11.2 \%)$ \\
\hline
\end{tabular}

\section{Observer agreement}

Table 8.2 displays the intra- and interobserver agreement levels on each FEES variable with 95\% confidence intervals (Cls) for thin and thick liquids separately. The agreement levels were sufficient for all measured FEES variables. The lowest intraobserver 
agreement level was 0.75 (95\% Cl: 0.60-0.90) for postswallow vallecular pooling in thin liquid consistency (observer 1 ), while the lowest interobserver agreement level was 0.63 (95\% Cl: 0.38-0.88) for aspiration of thick liquid.

\section{Descriptive data of the HADS questionnaire}

The HADS-Anxiety (A) and HADS-Depression (D) subscales were filled out completely by 98.1\% of the patients. The median score on HADS-A was 5.0 (range: 3.5-9.0); that on HADS-D was 5.0 (range: 2.0-8.0). Clinically relevant symptoms of anxiety or depression were present in $46(43.0 \%)$ patients. Thirty-seven (35\%) patients showed symptoms of anxiety, 31 (29\%) patients showed symptoms of depression. No floor or ceiling effect was observed because less than $5 \%$ of the patients had the lowest or highest possible score on the HADS questionnaire.

\section{HADS questionnaire and FEES}

Depression subscale - Results from the logistic regression analyses for the presence of clinically relevant depressive symptoms as a function of OD severity (category or severity level of the ordinal FEES variable) are given in Table 8.4. There was no significant association between clinically relevant symptoms of depression and any specific FEES variable (or level of its ordinal scale) for either consistency. However, a significant association was found between HADS-D and age, representing the probability of depressive symptoms rising with age (OR: 1.06, 95\% Cl: 1.01-1.10). A significant association was also found between the presence of clinically relevant depressive symptoms and OD aetiology, representing the probability of higher depressive symptoms in neurology and head and neck oncology patients as compared to patients in the 'miscellaneous' category. The odds ratios are 8.7 (95\% Cl: 2.08-36.4) for neurological patients and 7.6 (95\% Cl: 2.04-28.2) for oncological patients. The results of the chisquare test were consistent with the findings. Moreover, analysis of the third swallow per consistency led to the same results. 
Table 8.4 Logistic regression analyses of the presence of HADS-D clinical symptoms for thin (left) and thick (right) liquid consistency.

\begin{tabular}{|c|c|c|c|c|}
\hline \multirow[t]{2}{*}{ Liquid consistency: } & \multicolumn{2}{|c|}{ Thin } & \multicolumn{2}{|c|}{ Thick } \\
\hline & Estimate $(\mathrm{SE})^{*}$ & $P$-value & Estimate $(\mathrm{SE})^{*}$ & $P$-value \\
\hline \multicolumn{5}{|l|}{ Model 1} \\
\hline Intercept & $-3.07(1.31)$ & 0.019 & $-3.49(1.45)$ & 0.016 \\
\hline Piecemeal deglutition & & 0.14 & & 0.20 \\
\hline Category $1^{* *}$ & $-1.27(0.66)$ & 0.055 & $-0.60(0.81)$ & 0.46 \\
\hline Category 2 & $-0.79(0.65)$ & 0.23 & $-0.19(0.87)$ & 0.83 \\
\hline Category 3 & $0.37(0.94)$ & 0.70 & $1.06(0.96)$ & 0.27 \\
\hline Category 4 & $-1.91(1.28)$ & 0.14 & $-0.93(1.03)$ & 0.37 \\
\hline Age (years) & $0.06(0.02)$ & 0.010 & $0.06(0.02)$ & 0.013 \\
\hline Etiology & & $<0.0001$ & & $<0.0001$ \\
\hline Oncology & $0.31(0.68)$ & 0.65 & $0.34(0.66)$ & 0.61 \\
\hline Other & $-2.23(0.72)$ & 0.002 & $-2.11(0.70)$ & 0.003 \\
\hline \multicolumn{5}{|l|}{ Model 2} \\
\hline Intercept & $-3.71(1.28)$ & 0.004 & $-3.29(1.25)$ & 0.009 \\
\hline Postswallow vallecular pooling & & 0.77 & & 0.27 \\
\hline Category $1^{* *}$ & $0.15(0.53)$ & 0.78 & $0.51(0.62)$ & 0.41 \\
\hline Category 2 & $-0.33(0.66)$ & 0.62 & $-0.54(0.61)$ & 0.38 \\
\hline Age (years) & $0.06(0.02)$ & 0.007 & $0.05(0.02)$ & 0.016 \\
\hline Etiology & & 0.001 & & 0.001 \\
\hline Oncology & $0.12(0.60)$ & 0.85 & $0.13(0.62)$ & 0.83 \\
\hline Other & $-2.03(0.67)$ & 0.002 & $-2.14(0.70)$ & 0.002 \\
\hline \multicolumn{5}{|l|}{ Model 3} \\
\hline Intercept & $-3.54(1.22)$ & 0.004 & $-3.33(1.23)$ & 0.007 \\
\hline Postswallow pyriform sinus pooling & & 0.89 & & 0.19 \\
\hline Category $1^{* *}$ & $-0.20(0.55)$ & 0.72 & $-0.74(0.72)$ & 0.30 \\
\hline Category 2 & $-0.30(0.73)$ & 0.68 & $-1.04(0.65)$ & 0.11 \\
\hline Age (years) & $0.05(0.02)$ & 0.008 & $0.05(0.02)$ & 0.009 \\
\hline Etiology & & 0.001 & & $<0.0001$ \\
\hline Oncology & $0.14(0.61)$ & 0.82 & $0.10(0.63)$ & 0.87 \\
\hline Other & $-2.09(0.68)$ & 0.002 & $-2.18(0.69)$ & 0.001 \\
\hline \multicolumn{5}{|l|}{ Model 4} \\
\hline Intercept & $-3.48(1.23)$ & 0.005 & $-3.64(1.26)$ & 0.004 \\
\hline \multicolumn{5}{|l|}{ Aspiration } \\
\hline Category $1^{* *}$ & $-1.07(0.68)$ & 0.11 & $-1.52(0.91)$ & 0.095 \\
\hline Age (years) & $0.06(0.02)$ & 0.008 & $0.06(0.02)$ & 0.009 \\
\hline Etiology & & $<0.0001$ & & $<0.0001$ \\
\hline Oncology & $0.03(0.61)$ & 0.96 & $0.53(0.64)$ & 0.41 \\
\hline Other & $-2.23(0.69)$ & 0.001 & $-3.64(1.26)$ & 0.004 \\
\hline
\end{tabular}

Legend: Category 0 is the reference category for all FEES variables. Neurology is the reference category for aetiology. "SE: standard error; ${ }^{* *}$ Lower categories refer to normal functioning whereas higher categories refer to more severe disability.

Anxiety subscale - The results for clinically relevant anxiety symptoms are given in Table 8.5. For thin liquid consistency, there was a significant association between piecemeal deglutition and the presence of clinically relevant anxiety symptoms $(P=0.026)$. The probability of presenting with anxiety symptoms decreased with a rising severity of piecemeal deglutition. For thick liquid consistency, there was a significant 
association between postswallow vallecular pooling and the presence of clinically relevant anxiety symptoms ( $P=0.015)$. The probability of anxiety symptoms was higher in category 1 of postswallow vallecular pooling compared to category 0 (no pooling) and category 2 (severe pooling). All other results were not statistically significant. The results of the chi-square test were consistent with the findings. Moreover, analysis of the third swallow per consistency led to the same results.

Table 8.5 Logistic regression analyses of the presence of HADS-A clinical symptoms for thin (left) and thick (right) liquid consistency.

\begin{tabular}{|c|c|c|c|c|}
\hline \multirow[t]{2}{*}{ Liquid consistency: } & \multicolumn{2}{|l|}{ Thin } & \multicolumn{2}{|c|}{ Thick } \\
\hline & Estimate $(\mathrm{SE})^{*}$ & $P$-value & Estimate $(\mathrm{SE})^{*}$ & $P$-value \\
\hline \multicolumn{5}{|l|}{ Model 1} \\
\hline Intercept & $0.37(0.43)$ & 0.40 & $-0.98(0.68)$ & 0.15 \\
\hline Piecemeal deglutition & & 0.026 & & 0.35 \\
\hline Category $1^{* *}$ & $-1.15(0.57)$ & 0.043 & $0.51(0.75)$ & 0.50 \\
\hline Category 2 & $-1.17(0.59)$ & 0.048 & $0.55(0.78)$ & 0.48 \\
\hline Category $3+4$ & $-2.24(0.88)$ & 0.010 & & \\
\hline Category 3 & & & $0.82(0.88)$ & 0.35 \\
\hline Category 4 & & & $-0.81(1.02)$ & 0.43 \\
\hline \multicolumn{5}{|l|}{ Model 2} \\
\hline Intercept & $-0.49(0.32)$ & 0.14 & $-0.92(0.42)$ & 0.028 \\
\hline Postswallow vallecular pooling & & 0.88 & & 0.015 \\
\hline Category $1^{* *}$ & $-0.21(0.46)$ & 0.65 & $1.09(0.54)$ & 0.043 \\
\hline Category 2 & $-0.21(0.56)$ & 0.71 & $-0.25(0.55)$ & 0.66 \\
\hline \multicolumn{5}{|l|}{ Model 3} \\
\hline Intercept & $-0.64(0.27)$ & 0.017 & $-0.39(0.25)$ & 0.12 \\
\hline Postswallow pyriform sinus pooling & & 0.95 & & 0.32 \\
\hline Category $1^{* *}$ & $0.13(0.45)$ & 0.77 & $-0.48(0.59)$ & 0.41 \\
\hline Category 2 & $-0.05(0.67)$ & 0.94 & $-0.77(0.57)$ & 0.18 \\
\hline \multicolumn{5}{|l|}{ Model 4} \\
\hline Intercept & $-0.40(0.23)$ & 0.079 & $-0.64(0.22)$ & 0.004 \\
\hline \multicolumn{5}{|l|}{ Aspiration } \\
\hline Category $1^{* *}$ & $-1.05(0.60)$ & 0.079 & $-0.05(0.65)$ & 0.94 \\
\hline
\end{tabular}

Legend: Category 0 is the reference category for all FEES variables. "SE: standard error; ${ }^{* *}$ Lower categories refer to normal functioning whereas higher categories refer to more severe disability.

\section{DSS score and FEES}

Table 8.6 shows the results of linear regression analyses of the FEES variables, age, $\mathrm{BMI}$, and OD aetiology on the DSS score (patients' subjective evaluation of their own swallowing function). A significant association was found between DSS and postswallow pyriform sinus pooling for thick liquid consistency. The highest DSS scores (higher score = better swallowing perception) fall into the lower categories of the postswallow pyriform sinus pooling scale (lower category = better swallowing function). All other results were not statistically significant. 
Table 8.6 Results of the linear regression analysis for the Dysphagia Severity Scale for thin and thick liquid consistency.

\begin{tabular}{|c|c|c|c|c|}
\hline \multirow[t]{2}{*}{ Liquid consistency: } & \multicolumn{2}{|c|}{ DSS score (thin) } & \multicolumn{2}{|c|}{ DSS score (thick) } \\
\hline & Estimate $(\mathrm{SE})^{*}$ & $P$-value & Estimate $(\mathrm{SE})^{*}$ & $P$-value \\
\hline \multicolumn{5}{|l|}{ Model 1} \\
\hline Intercept & $80.5(12.4)$ & $<0.0001$ & $90.0(13.32)$ & $<0.0001$ \\
\hline Piecemeal deglutition & & 0.89 & & 0.061 \\
\hline Category $1^{* *}$ & $1.21(6.61)$ & 0.85 & $-14.4(8.40)$ & 0.089 \\
\hline Category 2 & $-4.12(6.92)$ & 0.55 & $-9.81(8.87)$ & 0.27 \\
\hline Category 3 & $-6.00(11.2)$ & 0.59 & $-30.9(10.6)$ & 0.004 \\
\hline Category 4 & $-4.92(11.3)$ & 0.66 & $-15.0(10.1)$ & 0.14 \\
\hline Age (years) & $-0.069(0.20)$ & 0.73 & $-0.043(0.20)$ & 0.83 \\
\hline Aetiology & & 0.011 & & 0.013 \\
\hline Oncology & $-23.6(7.84)$ & 0.003 & $-22.1(7.40)$ & 0.004 \\
\hline Other & $-11.2(6.11)$ & 0.069 & $-9.20(5.85)$ & 0.12 \\
\hline \multicolumn{5}{|l|}{ Model 2} \\
\hline Intercept & $83.1(11.5)$ & $<0.0001$ & $80.4(11.6)$ & $<0.0001$ \\
\hline Postswallow vallecular pooling & & 0.30 & & 0.56 \\
\hline Category $1^{* *}$ & $-8.36(5.53)$ & 0.13 & $-7.05(6.53)$ & 0.28 \\
\hline Category 2 & $-6.58(6.78)$ & 0.33 & $-4.72(6.30)$ & 0.46 \\
\hline Age (years) & $-0.056(0.20)$ & 0.78 & $-0.022(0.20)$ & 0.91 \\
\hline Aetiology & & 0.004 & & 0.006 \\
\hline Oncology & $-24.5(7.27)$ & 0.001 & $-23.8(7.30)$ & 0.002 \\
\hline Other & $-11.1(5.93)$ & 0.064 & $-10.5(5.98)$ & 0.081 \\
\hline \multicolumn{5}{|l|}{ Model 3} \\
\hline Intercept & $76.7(11.3)$ & $<0.0001$ & $73.5(10.8)$ & $<0.0001$ \\
\hline Postswallow pyriform sinus pooling & & 0.91 & & 0.046 \\
\hline Category $1^{* *}$ & $1.95(5.80)$ & 0.74 & $16.4(6.95)$ & 0.021 \\
\hline Category 2 & $-1.60(8.23)$ & 0.85 & $8.65(6.26)$ & 0.17 \\
\hline Age (years) & $-0.037(0.20)$ & 0.85 & $-0.062(0.19)$ & 0.75 \\
\hline Aetiology & & 0.007 & & 0.010 \\
\hline Oncology & $-23.7(7.40)$ & 0.002 & $-22.2(7.18)$ & 0.003 \\
\hline Other & $-10.8(6.19)$ & 0.083 & $-9.12(5.89)$ & 0.13 \\
\hline \multicolumn{5}{|l|}{ Model 4} \\
\hline Intercept & $76.7(11.2)$ & $<0.0001$ & $76.3(11.2)$ & $<0.0001$ \\
\hline \multicolumn{5}{|l|}{ Aspiration } \\
\hline Category $1^{* *}$ & $7.29(6.41)$ & 0.26 & 5.99 (7.99) & 0.46 \\
\hline Age (years) & $-0.056(0.20)$ & 0.78 & $-0.037(0.20)$ & 0.86 \\
\hline Etiology & & 0.006 & & 0.004 \\
\hline Oncology & $-23.7(7.27)$ & 0.002 & $-25.9(7.56)$ & 0.001 \\
\hline Other & -11.1 (6.09) & 0.072 & $-10.5(6.10)$ & 0.087 \\
\hline
\end{tabular}

Legend: Category 0 is the reference category for all FEES variables. Neurology is the reference category for aetiology.. "SE: standard error; ${ }^{* *}$ Lower categories refer to normal functioning whereas higher categories refer to more severe disability.

\section{Discussion}

The present study revealed a high prevalence (43\%) of clinically relevant affective symptoms in patients with OD. Little is known about the pathways by which psychiatric 
comorbidity may be involved in OD. This is the first study to investigate whether there is a relationship between the severity of affective complaints and the severity of OD as measured by FEES.

Our investigation did not demonstrate a significant association between clinically relevant depressive symptoms and the FEES outcome variables. This suggests that there may be no relationship between a depressive state and the severity of OD. However, our study did find a significant association between clinically relevant anxiety symptoms and the FEES variable piecemeal deglutition (for thin liquid consistency), with a higher probability of anxiety symptoms in the lower ordinal categories of the piecemeal deglutition scale. Furthermore, a significant association was found for clinically relevant symptoms of anxiety and the FEES variable postswallow vallecular pooling (for thick liquid consistency). The probability of anxiety symptoms was higher in the postswallow vallecular-pooling category 1 compared to category 0 and category 2. Apparently, severe OD did not result in a higher probability of having affective complaints. These findings may indicate that less severe or medically unexplained subjective OD complaints could be a manifestation of an anxious and somatically hyper-aroused state. Such a state is characterized by somatic anxiety equivalents due to either psychiatric disorders or early stages of somatic diseases ${ }^{21,22}$. Another possible explanation may be that patients with more severe chronic OD often had a long history of disease. They may have already adapted to their physical limitations, unlike patients with mild OD in the early stages or acute onset of disease. Regarding Parkinson's disease, for instance, the prevalence of depression peaks at the beginning of the disease and again in its latter stages; in between, the prevalence of affective complaints is lower ${ }^{23}$. Moreover, all head and neck oncological patients in this study were not in a palliative stage of care, meaning that they were cancer survivors. Head and neck oncological patients often show improvement in their psychological and psychiatric functioning during follow-up, while the severity of the disease or functional disability remains stable or even deteriorates. These patients successfully adjust to living with their physical problems; as time passes, their fear of recurrence and death is likely to decline ${ }^{24-26}$.

This study showed a relationship between the patients' subjective evaluation of their own swallowing as measured with a visual analogue scale (DSS), on the one hand, and the FEES variable postswallow pyriform sinus pooling (for thick liquid consistency) on the other. For the rest of the FEES variables, no significant association with the DSS was found. Given these results, no relation is apparent between the measured severity of $O D$ and the patients' subjective evaluation of their own swallowing. Some patients with a nearly normal swallowing function considered their swallowing to be very impaired, while some patients with severe OD considered it normal, though acknowledging that they were not always aware of their diminished swallowing function. For example 'silent aspiration', a severe swallowing abnormality, can occur without subjectively suffering from it ${ }^{27,28}$. Moreover, several patient groups (neurodegeneration, stroke, head and neck oncology postradiation and postsurgery) tend to have sensory 
deficits that may contribute to inaccurate judgment ${ }^{29-35}$. Inaccuracy of perceptual judgment (underestimation of the problem) may manifest itself in fewer subjective complaints $^{27}$. This could explain why the present study found very few significant associations between the DSS and the severity of OD as measured with FEES. Another explanation may be that higher anxiety levels influenced the patients' experience, in terms of suffering without severe OD. A previous study in the present patient population showed a significant association between DSS and symptoms of anxiety ${ }^{11}$. That finding indicated that a patient's subjective evaluation of swallowing probably is a better indicator of affective symptoms than the FEES outcome. These findings are in accordance with the literature on medically unexplained physical symptoms ${ }^{21,22}$. Recognizing that affective symptoms are common in OD patients ${ }^{11}$ and assuming that these problems are not solely dependent on the severity of OD could lead to new directions for future research. These insights could also serve as grounds for a clinically relevant implementation of affective symptom screening in dysphagia management. Such screening could extend diagnostic skills throughout the field while preventing underdetection of relevant psychiatric comorbidities ${ }^{11,36}$. Although OD intervention has already been organized as a multidisciplinary field in the Netherlands, psychiatric and psychological expertise has not yet been routinely integrated in the current approach $^{22,37}$.

\section{Limitations of the study}

Head and neck cancer and neurological diseases, regardless of OD, are associated with symptoms of anxiety and depression. In the current study it is difficult to define what affective symptoms can be purely attributed to OD. However, previous studies in posttreatment head and neck cancer patients and post stroke patients showed a lower prevalence of symptoms of anxiety and depression compared to our study population (15-24\% versus $29 \%$ for depression and $14-21 \%$ versus $35 \%$ for anxiety $)^{38-43}$. This may indicate a contributing effect of OD on affective symptoms. Moreover, only a few associations have been shown between the FEES outcome and symptoms of anxiety. The sample size was too small to yield additional significant results. Because of the heterogeneity of the study population and the small size of the aetiological subgroups, no further statistical analyses of group differences could be performed.

Furthermore, FEES was used as a diagnostic tool to measure the severity of swallowing dysfunction. The question remains whether other diagnostic tools (such as videofluoroscopy, manometry, or electromyography) would have provided different results. On the other hand, FEES is commonly used in the evaluation of OD worldwide. Finally, several disorders may be accompanied by cognitive impairment (e.g., Parkinson's disease, stroke). Several patients with severe OD were excluded from this study because of a MMSE below 23; their exclusion might have affected the frequencies of affective symptoms. 


\section{Conclusion}

Affective symptoms were observed in almost half of the patients with OD. Clinically relevant symptoms of anxiety were significantly associated with some FEES variables (piecemeal deglutition and postswallow vallecular pooling). However, the more severe the score on piecemeal deglutition and postswallow vallecular pooling, the less important the affective complaints were. Thus, anxiety seems to play a role in $O D$, particularly in the early stages of neurological or oncological disorders or in medically unexplained subjective OD complaints ${ }^{21,22}$. Given the cross-sectional study design, no causal relationship was found. Therefore, further investigation of the contribution of affective symptoms to the development and treatment of OD would warrant longitudinal research. 


\section{References}

1. Cichero, J. \& Murdoch, B. Dysphagia. Foundation, theory and practice. Chichester: John Wiley \& Sons, Ltd. 237-248 (2006).

2. Cook, I. J. \& Kahrilas, P. J. AGA Technical review on management of oropharyngeal dysphagia. Gastroenterology 116, 455-478 (1999).

3. Logemann, J. A. Evaluation and treatment of swallowing disorders. Second Edition. Texas: PRO-ED (1997).

4. Ekberg, O. et al. Social and psychological burden of dysphagia: Its impact on diagnosis and treatment. Dysphagia 17, 139-146 (2002).

5. Heijnen, B. J. et al. Neuromuscular electrical stimulation versus traditional therapy in patients with Parkinson's disease and oropharyngeal dysphagia: Effects on quality of life. Dysphagia 27, 336-345 (2012).

6. Speyer, R. et al. Quality of life in oncological patients with oropharyngeal dysphagia: Validity and reliability of the Dutch version of the MD Anderson Dysphagia Inventory and the Deglutition Handicap Index. Dysphagia 26:407-414 (2011).

7. Maclean, J. et al. Dysphagia following a total laryngectomy: The effect on quality of life, functioning, and psychological well-being. Dysphagia 24, 314-321 (2009).

8. Manor, Y. et al. Anxiety, depression and swallowing disorders in patients with Parkinson's disease. Parkinsonism. Relat. Disord. 15, 453-456 (2009).

9. Nguyen, N. P. et al. Impact of dysphagia on quality of life after treatment of head-and-neck cancer. Int. J. radiation Oncology Biol. Phys. 16, 772-778 (2005).

10. Eslick, G. D. \& Talley, N. J. Dysphagia: Epidemiology, risk factors and impact on quality of life - a population-based study. Aliment. Pharmacol. Ther. 27, 971-979 (2008).

11. Verdonschot, R. J. et al. Symptoms of anxiety and depression assessed with the hospital anxiety and depression scale in patients with oropharyngeal dysphagia. J. Psychosom. Res. 75, 451-455 (2013).

12. Folstein, M. F. et al. "Mini-mental state". A practical method for grading the cognitive state of patients for the clinician. J. Psychiatr. Res. 12, 189-198 (1975).

13. Zigmond, A. S. \& Snaith, R. P. The hospital anxiety and depression scale. Acta Psychiatr. Scand. 67, 361370 (1983).

14. Bjelland, I. et al. The validity of the hospital anxiety and depression scale. An updated literature review. J. Psychosom. Res. 52, 69-77 (2002).

15. Rush, A. J. et al. Handbook of Psychiatric Measures, First Edition. Washington DC: American Psychiatric Association 547-548 (2000).

16. Langmore, S. E. \& Aviv, J. E. Endoscopic evaluation and treatment of swallowing disorders. New York: Thieme; 2001.

17. Baijens, L. et al. Swallowing in Parkinson patients versus healthy controls: Reliability of measurements in videofluoroscopy. Gastroenterol. Res. Pract. 2011:380682 (2011).

18. Pilz, W. et al. Swallowing assessment in myotonic dystrophy type 1 using fiberoptic endoscopic evaluation of swallowing (FEES). Neuromuscul. Disord. 24, 1054-1062 (2014).

19. Baijens, L. W. et al. FEES Protocol Derived Estimates of Sensitivity: Aspiration in Dysphagic Patients. Dysphagia 29, 583-590 (2014).

20. Baijens, L. W. et al. Surface electrical stimulation in dysphagic Parkinson patients: A randomized clinical trial. Laryngoscope 123, E38-44 (2013).

21. Henningsen, P. et al. Medically unexplained physical symptoms, anxiety and depression: A meta-analytic review. Psychosom. Med. 65, 528-533 (2003).

22. Baijens, L. et al. Medically unexplained otorhinolaryngological symptoms: Towards integrated psychiatric care. Laryngoscope 125, 1583-1587 (2015).

23. Rickards H. Depression in neurological disorders: Parkinson's disease, multiple sclerosis, and stroke. J. Neurol. Neurosurg. Psychiatry 76, i48-i52 (2005).

24. Hammerlid, E. \& Taft, C. Health-related quality of life in long-term head and neck cancer survivors: A comparison with general population norms. Br. J. Cancer 84, 149-56 (2001). 
25. Ronis, D. L. et al. Changes in quality of life over 1 year in patients with head and neck cancer. Arch. Otolaryngol. Head Neck Surg. 134, 241-248 (2008).

26. De Graeff, A. et al. Long-term quality of life of patients with head and neck cancer. Laryngoscope 110, 98-106 (2000).

27. Rogus-Pulia, N. M. et al. Changes in swallowing physiology and patient perception of swallowing function following chemoradiation for head and neck cancer. Dysphagia 29, 223-233 (2014).

28. Troche, M. S. et al. Decreased cough sensitivity and aspiration in Parkinson's disease. Chest 146, 12941299 (2014).

29. Mu, L. et al. Parkinson disease affects peripheral sensory nerves in the pharynx. J. Neuropathol. Exp. Neurol. 72, 614-623 (2013).

30. Onofri, SM. et al. Correlation between laryngeal sensitivity and penetration/aspiration after stroke. Dysphagia 29, 256-261 (2014).

31. Manikantan K, Khode S, Sayed SI et al. Dysphagia in head and neck cancer. Complications of treatment. Cancer Treat. Rev. 35, 724-732 (2009).

32. Jäghagen, A. L. et al. Pharyngeal swallowing dysfunction following treatment for oral and pharyngeal cancer association with diminished intraoral sensation and discrimination ability. Head Neck 10, 13441351 (2008).

33. Ku, P. K. et al. Laryngopharyngeal sensory deficits and impaired pharyngeal motor function predict aspiration in patients irradiated for nasopharyngeal carcinoma. Laryngoscope 120, 223-228 (2009).

34. Martens, K. A. \& Almeida, Q. J. Dissociating between sensory and perceptual deficits in PD: More than simply a motor deficit. Move. Disord. 27, 387-392 (2012).

35. Pilz, W. et al. Oropharyngeal dysphagia in myotonic dystrophy type 1: A systematic review. Dysphagia 29, 319-331 (2014).

36. Leue, C. et al. Managing complex patients on a medical psychiatric unit: An observational study of university hospital costs associated with medical service use, length of stay, and psychiatric intervention. J. Psychosom. Res. 68, 295-302 (2010).

37. Baijens, LW. Multidisciplinaire polikliniek voor dysfagie. Dutch J. Ear Nose Throat 18, 5-6 (2012).

38. Hammerlid, E. et al. A prospective multi-centre study in Sweden and Norway of mental distress and psychiatric morbidity in head and neck cancer patients. Br. J. Cancer 80, 766-774 (1999).

39. Hammerlid, E. et al. Health-related quality of life three years after diagnosis of head and neck cancer - a longitudinal study. Head Neck 23, 113-125 (2001).

40. Elani, H. W. \& Allison P. J. Coping and psychological distress among head and neck cancer patients. Support.Care Cancer 19, 1735-1741 (2011).

41. Broomfield, N. M. et al. Depression and anxiety symptoms post-stroke/TIA: Prevalence and associations in cross-sectional data from a regional stroke registry. BMC Neurol. 14, 198 (2014).

42. Ihle-Hansen, $\mathrm{H}$. et al. Effect on anxiety and depression of a multifactorial risk factor intervention program after stroke and TIA: A randomized controlled trial. Aging Ment. Health 18, 540-546 (2014).

43. Tang, W. K. et al. Impact of anxiety on health-related quality of life after stroke: A cross-sectional study. Arch. Phys. Med. Rehabil. 94, 2535-2541 (2013). 


\section{Abstract}

\section{Objective}

Affective disorders are prevalent in different somatic conditions and influence somatic symptom bother and quality of life. Mood and anxiety disorders impact patients' compliance and adherence to treatment. This systematic review summarizes published studies on affective complaints in patients with oropharyngeal dysphagia (OD) in order to determine the quality of studies concerning any association of OD with symptoms of depression and/or anxiety.

\section{Methods}

A literature search was carried out using electronic databases Embase, Medline, Web-ofscience, PsycINFO, Cochrane Library, and Google scholar. Two reviewers made the preselecting cut by screening all articles on title and abstract and independently screened the full texts of this initial set of articles. Methodological quality of the studies that met the inclusion criteria was assessed independently.

\section{Results}

Twenty-six articles were included in the analysis after full-text screening and by applying the inclusion and exclusion criteria. All studies concluded that symptoms of depression were associated with impaired swallowing function, and 9 out of 12 studies concluded that symptoms of anxiety were associated with functional impairment of swallowing. The reviewers found heterogeneous outcomes and methodological limitations, which prevented data from pooling.

\section{Conclusion}

Although no meta-analytic conclusions can be drawn, it appears that symptoms of anxiety and depression are common in OD. Caregivers have to be aware of this in order to detect affective comorbidity. Given that affective conditions influence patients' treatment adherence and compliance, integrated care approaches should be advocated in case of comorbidity. Studies on treatment effect are lacking and well-designed prospective research is needed. 


\section{Introduction}

Oropharyngeal dysphagia (OD) is commonly seen in the otorhinolaryngology outpatient clinic, and is defined as 'difficulty to transport bolus from the mouth, via the pharynx, to the entrance of the esophagus' ${ }^{1}$. The etiology of OD is diverse: stroke; chronic neurological diseases (e.g., multiple sclerosis, Parkinson's disease, myasthenia gravis); head and neck cancer or its oncological treatment effects (e.g., surgery, radiation); inclusion body myositis; Zenker's diverticulum; cervical spine abnormalities (e.g., degenerative, osteophytes); rising age (presbyphagia), etc ${ }^{1-3}$. The prevalence of OD is high. For instance, it affects more than $30 \%$ of patients who had a stroke, and $52 \%-82 \%$ of patients with Parkinson's disease. Moreover, the prevalence of OD is increasing with age, and up to $40 \%$ of the elderly aged 65 years and older have OD. This frequency is even higher in institutionalized elderly ${ }^{4}$. It is estimated that OD affects 40 million people in Europe $e^{5}$. The complications of OD can be severe: aspiration pneumonia; dehydration; malnutrition; or even sudden death ${ }^{1,3,6-8}$. Furthermore, it affects health-related quality of life. Patients with OD often have feelings of shame, embarrassment, and social isolation ${ }^{7-}$ ${ }^{11}$. Because of the complexity of the etiology, and a high incidence of mental health related comorbidity, a multidisciplinary strategy including mental health care is essential $^{12,13}$. In general, psychiatric symptoms of anxiety and depression are common in patients with chronic conditions (chronic obstructive pulmonary disease (COPD), diabetes mellitus (DM), irritable bowel syndrome (IBS), overactive bladder (OAB), etc.), and $O D$ is also associated with clinically relevant affective complaints ${ }^{14-20}$. However, little is known about the strength and the direction of this association and investigations on the pathophysiology, risk factors, and treatment effect are scarce.

The aim of this systematic review is to summarize published studies on affective symptoms in patients with OD and to analyze the quality of the literature. The broader objective was to elucidate how affective comorbidities may impact on symptom bother and treatment compliance in order to advocate integrated or collaborative care approaches.

\section{Methods}

\section{Identification and selection of studies}

This review was conducted by following the Cochrane Collaboration criteria for systematic reviews ${ }^{21-23}$. One of the authors and an experienced university library information specialist carried out a literature search using the electronic biomedical databases Embase, Medline, Web-of-Science, PsycINFO, Cochrane Library, and Google Scholar. Search terms were related to dysphagia and to affective conditions (see Table 9.1 for the complete syntax). The entire search was performed on the $1^{\text {st }}$ of June 
2016. Two independent reviewers made the first preselecting cut by screening all articles on title and abstract. Then they independently screened full texts of this initial set of articles. Furthermore, the reference lists of the selected articles were screened for additional literature. If an article was not electronically available, the authors were contacted to obtain the full text. All studies that examined swallowing function and affective conditions were included. However, reviews, studies without use of validated assessment tools on affective symptoms, expert opinions, conference papers, studies with a sample size below 10, and studies in patients with eating disorders or other psychiatric disorders (not being affective conditions) were excluded.

Table 9.1 Literature search syntaxes.

\begin{tabular}{|c|c|}
\hline Embase & $\begin{array}{l}\text { (dysphagia/de OR (dysphag* OR ((swallow* OR deglutit*) NEAR/3 (disorder* OR } \\
\text { problem*))):ab,ti) AND(psychiatry/exp OR psychiatrist/de OR 'psychiatric diagnosis'/de OR } \\
\text { 'mood disorder'/exp OR 'anxiety disorder'/exp OR 'mental patient'/exp OR (psychiatr* OR } \\
\text { depressi* OR ((mood OR anxi* OR Affective) NEAR/3 disorder*) OR neuropsycho*):ab,ti) } \\
\text { NOT ([Conference Abstract]/lim OR [Letter]/lim OR [Note]/lim OR [Conference Paper]/lim } \\
\text { OR [Editorial]/lim) }\end{array}$ \\
\hline Medline & $\begin{array}{l}\text { (Deglutition Disorders/ OR (dysphag* OR ((swallow* OR deglutit*) ADJ3 (disorder* OR } \\
\text { problem*))).ab,ti.) AND (exp psychiatry/ OR exp mood disorders/ OR exp anxiety disorders/ } \\
\text { OR Mentally III Persons/ OR (psychiatr* OR depressi* OR ((mood OR anxi* OR Affective) } \\
\text { ADJ3 disorder*) OR neuropsycho*).ab,ti.) NOT (letter OR news OR comment OR editorial } \\
\text { OR congresses OR abstracts).pt. }\end{array}$ \\
\hline Web-of-science & $\begin{array}{l}\text { TS=(((dysphag* OR ((swallow* OR deglutit*) NEAR/3 (disorder* OR problem*)))) AND } \\
((\text { psychiatr* OR depressi* OR ((mood OR anxi* OR Affective) NEAR/3 disorder*) OR } \\
\text { neuropsycho*)) ) AND DT=(Article) }\end{array}$ \\
\hline PsyclNFO & $\begin{array}{l}\text { (Dysphagia/ OR (dysphag* OR ((swallow* OR deglutit*) ADJ3 (disorder* OR } \\
\text { problem*))).ab,ti.) AND (exp psychiatry/ OR exp Affective Disorders/ OR exp anxiety } \\
\text { disorders/ OR (psychiatr* OR depressi* OR ((mood OR anxi* OR Affective) ADJ3 disorder*) } \\
\text { OR neuropsycho*).ab,ti.) NOT (letter OR news OR comment OR editorial OR congresses OR } \\
\text { abstracts).pt. }\end{array}$ \\
\hline Cochrane & $\begin{array}{l}\text { ((dysphag* OR ((swallow* OR deglutit*) NEAR/3 (disorder* OR problem*))):ab,ti) AND } \\
\text { ((psychiatr* OR depressi* OR ((mood OR anxi* OR Affective) NEAR/3 disorder*) OR } \\
\text { neuropsycho*):ab,ti) }\end{array}$ \\
\hline Google scholar & $\begin{array}{l}\text { Dysphagia|"swallowing | deglutition disorder|disorders | problem | problems" } \\
\text { psychiatry|psychiatrist| psychiatric|"mood|affective|anxiety|disorders"| depression | depr } \\
\text { essive }\end{array}$ \\
\hline
\end{tabular}

\section{Data analysis and assessment of study quality}

The included studies were assessed for methodological quality using a 12-item critical appraisal tool derived from the Quality Assessment of Diagnostic Accuracy Studies (QUADAS) (Table 9.2). The QUADAS is a tool to assess the diagnostic accuracy of studies included in systematic reviews ${ }^{24}$. Two independent reviewers used its critical appraisal criteria to analyze all included articles, scoring each criterion with a 'yes', 'no', or 'unclear'. Internal validity was rated with items $3,4,5,6,9,10,11$, and 12 , and external validity with items $1,2,7$, and 8 . The reviewers resolved any differences between assigned scores through discussion. 
Table 9.2 Criteria for quality assessment according to QUADAS ${ }^{24^{*}}$

1. Was the spectrum of patients representative of the patients who will receive the test in practice?
2. Were the selection criteria clearly described?
3. Was the diagnostic swallowing tool (e.g. questionnaire, FEES) likely to correctly classify the
4. $\quad$ Wallowing condition?
5. Is the time period between the diagnostic swallowing test and the psychiatric screening test short
enough to be reasonably sure that the target conditions did not change between the two tests?
7. $\quad$ Wid all patients receive the same diagnostic tests?
8. $\quad$ test?
9. Was the execution of the psychiatric test described in sufficient detail to permit replication of the
10. Were the psychiatric test results interpreted without knowledge of the results of the swallowing test?
11. Were the swallowing test results interpreted without knowledge of the results of the psychiatric test?
12. Were withdrawals from the study explained?

Legend: * Quality Assessment of Diagnostic Accuracy Studies.

\section{Results}

A total of 3586 articles were identified, and 2528 remained after removing duplicates. All articles were screened for title and abstract, which left 60 potentially eligible for inclusion. The level of agreement between the two reviewers for this first selection was 91\%. The level of agreement between the two reviewers for eligibility after full text screening was 75\%. After discussion full consensus was achieved. Finally 24 articles were included in the analysis after full-text screening and by applying the inclusion and exclusion criteria ${ }^{9-11,20,25-44}$. All of the articles included were written in English (see Figure 9.1 for the flowchart of the literature search process).

Fifteen articles reported on cross-sectional studies $9,10,20,26-31,33-36,39,44,5$ on prospective cohort studies $^{25,32,40,42,43}, 2$ on retrospective cohort studies ${ }^{11,41}$, and 2 on case-control studies $^{37,38}$. The studies were too heterogeneous in outcome and not of sufficient quality to carry out a meta-analysis. Instead, a qualitative analysis was performed. Table 9.3 provides an overview of the included articles, summarizing study design, sample size, population characteristics, and measurement tools. The included articles are segregated by type of assessment tools used for OD (single swallowing questions, clinical evaluation of swallowing, swallowing-related questionnaires, videofluoroscopic swallowing studies and fiber-optic endoscopic evaluation of swallowing), and discussed below. 

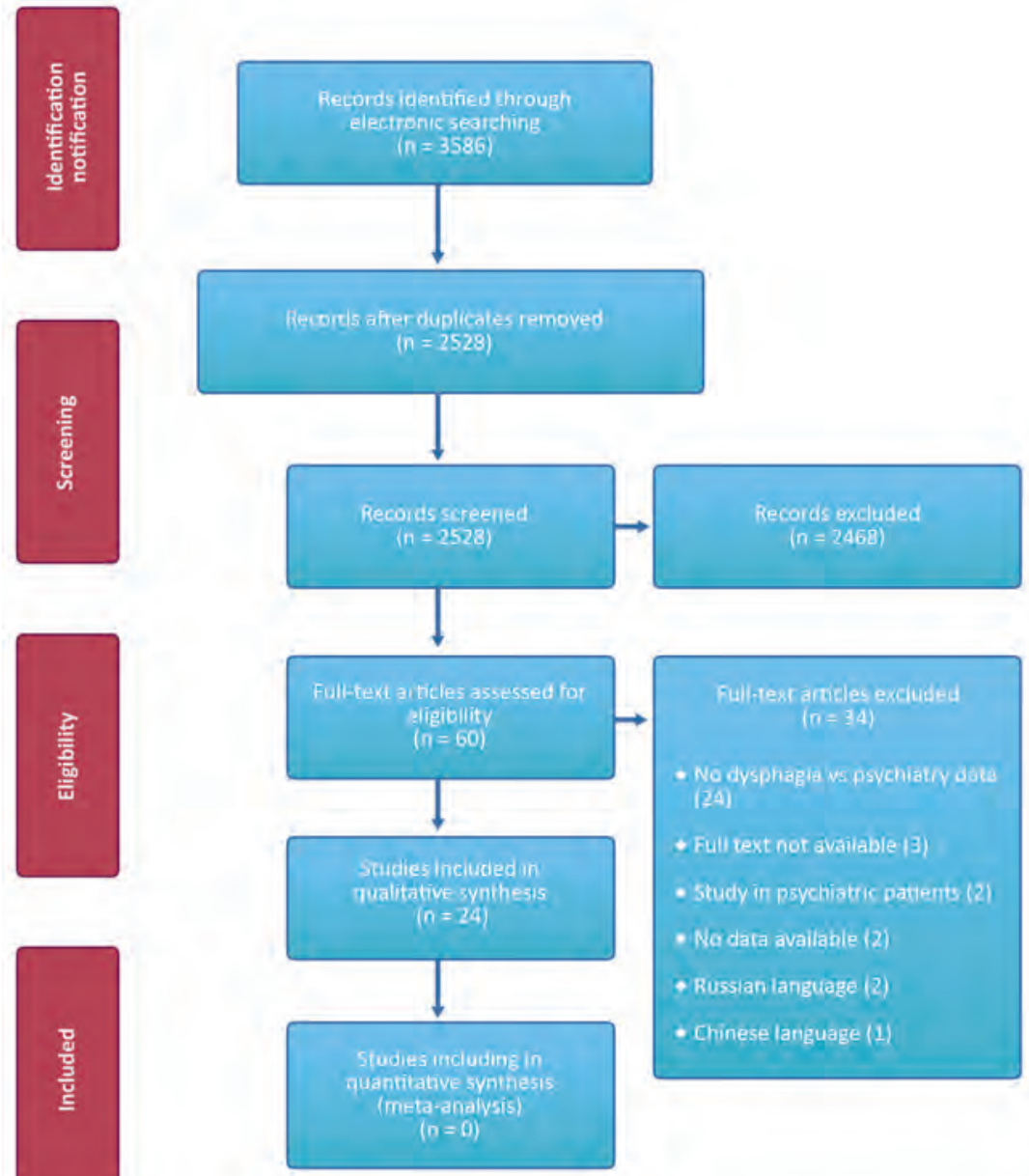

Fullatear ambles exciuded $(n=34)$

No dysphabla wa psvehiatry dote (24)

+ Fuil text not available (3)

- Study in psychiatric patternts (2)

- No data available (2)

* Russian language (2)

- Chinese language (1)

Figure 9.1 Flowchart of the literature review process.

\section{Quality of studies}

The quality assessment is presented in Table 9.4. The level of agreement between the two reviewers for quality assessment was $91.3 \%$ (263 of the 288 QUADAS items). After discussion full consensus was achieved. Thirteen of the included studies met all criteria for external validity $20,25,27,32-34,36,37,39,40,42,44$, whereas none met all criteria for internal validity. Eight studies fulfilled 6 out of 8 criteria for internal validity, $9,11,26,29,33,34,38,39$. Twenty-three studies were rated 'unclear' on items 9 and 10, as the articles did not reveal whether results of the swallowing test were interpreted without knowledge of the psychiatric assessment and vice versa ${ }^{9,10,20,25-44}$. 


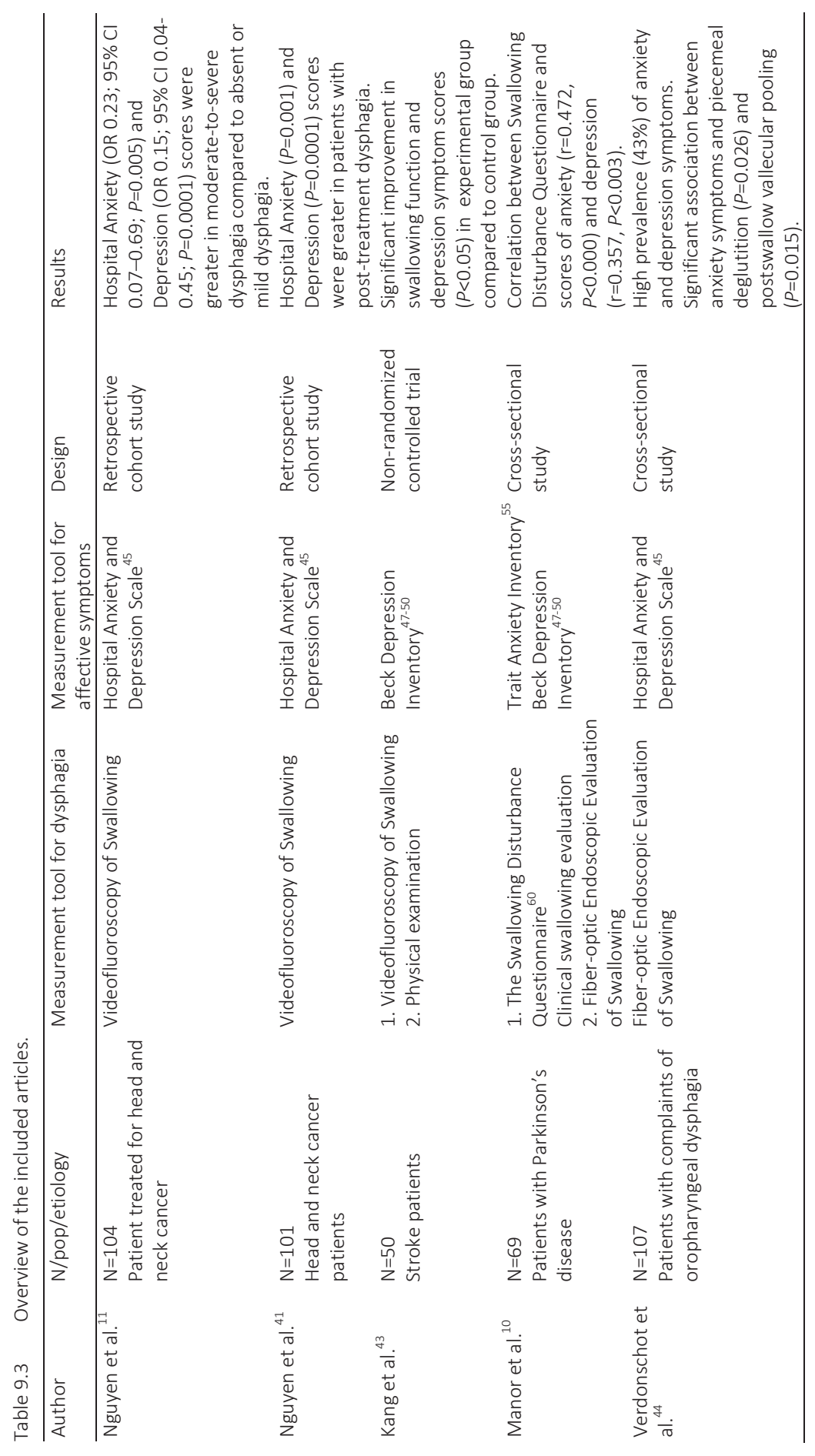




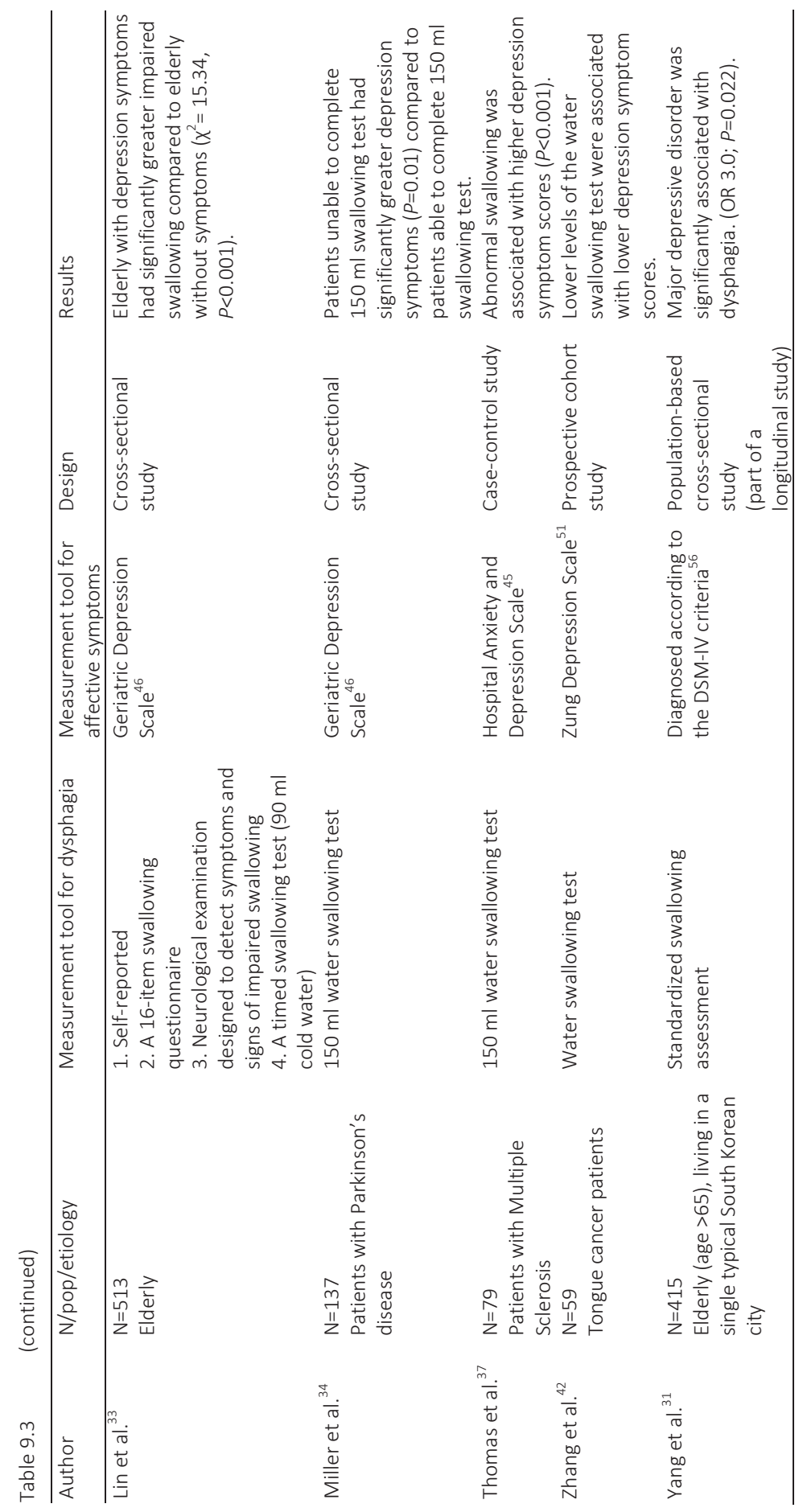




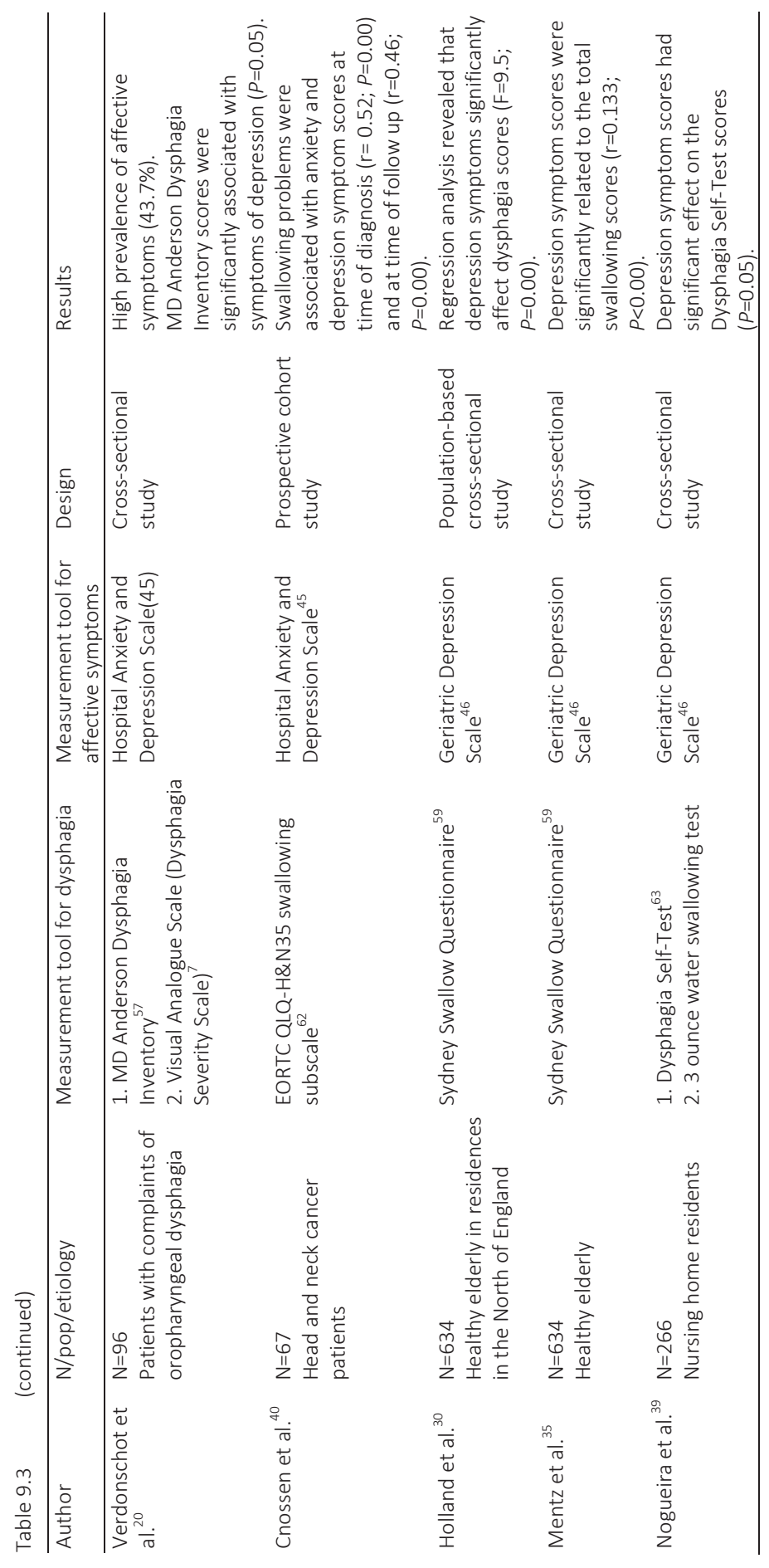




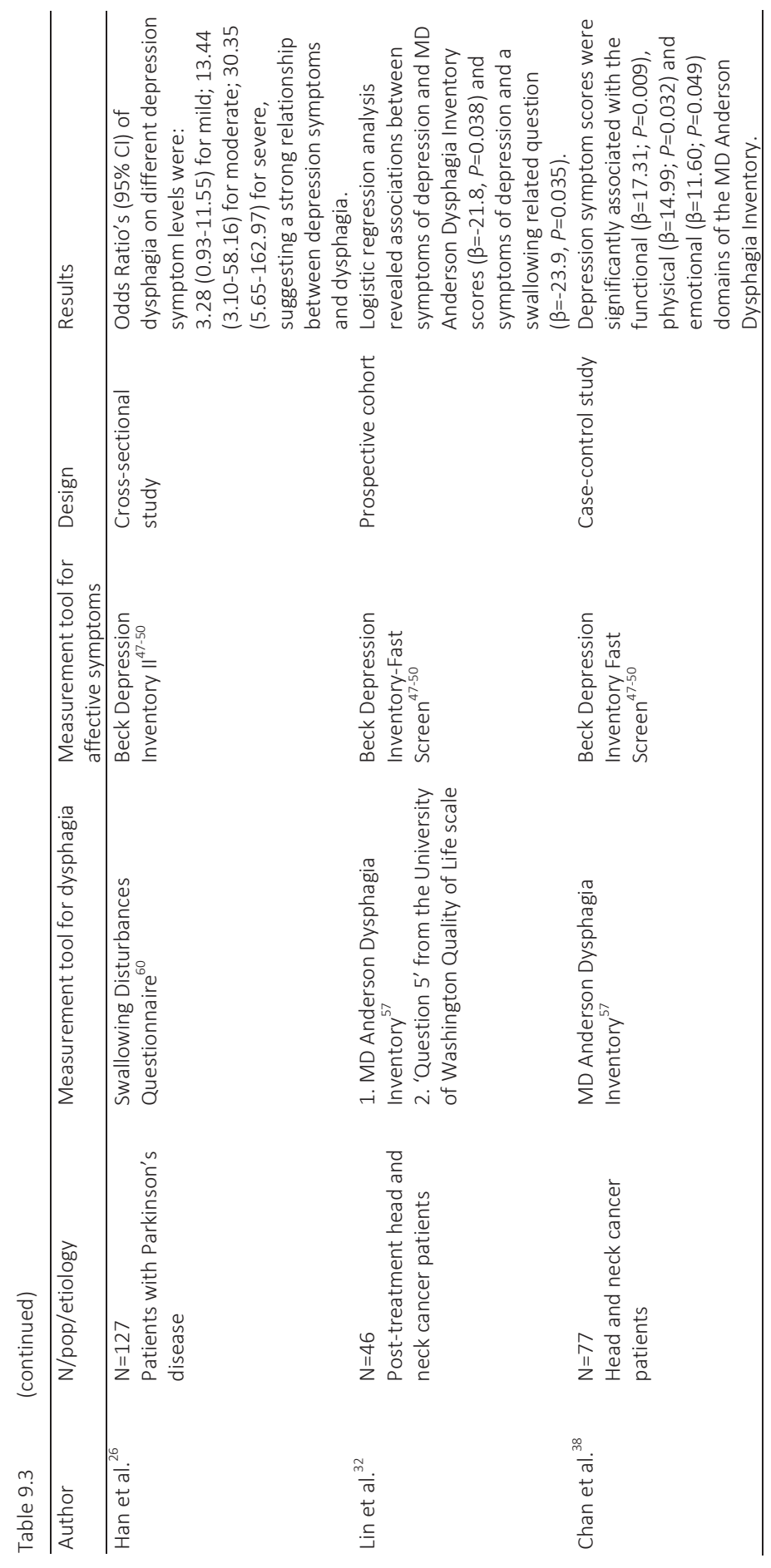




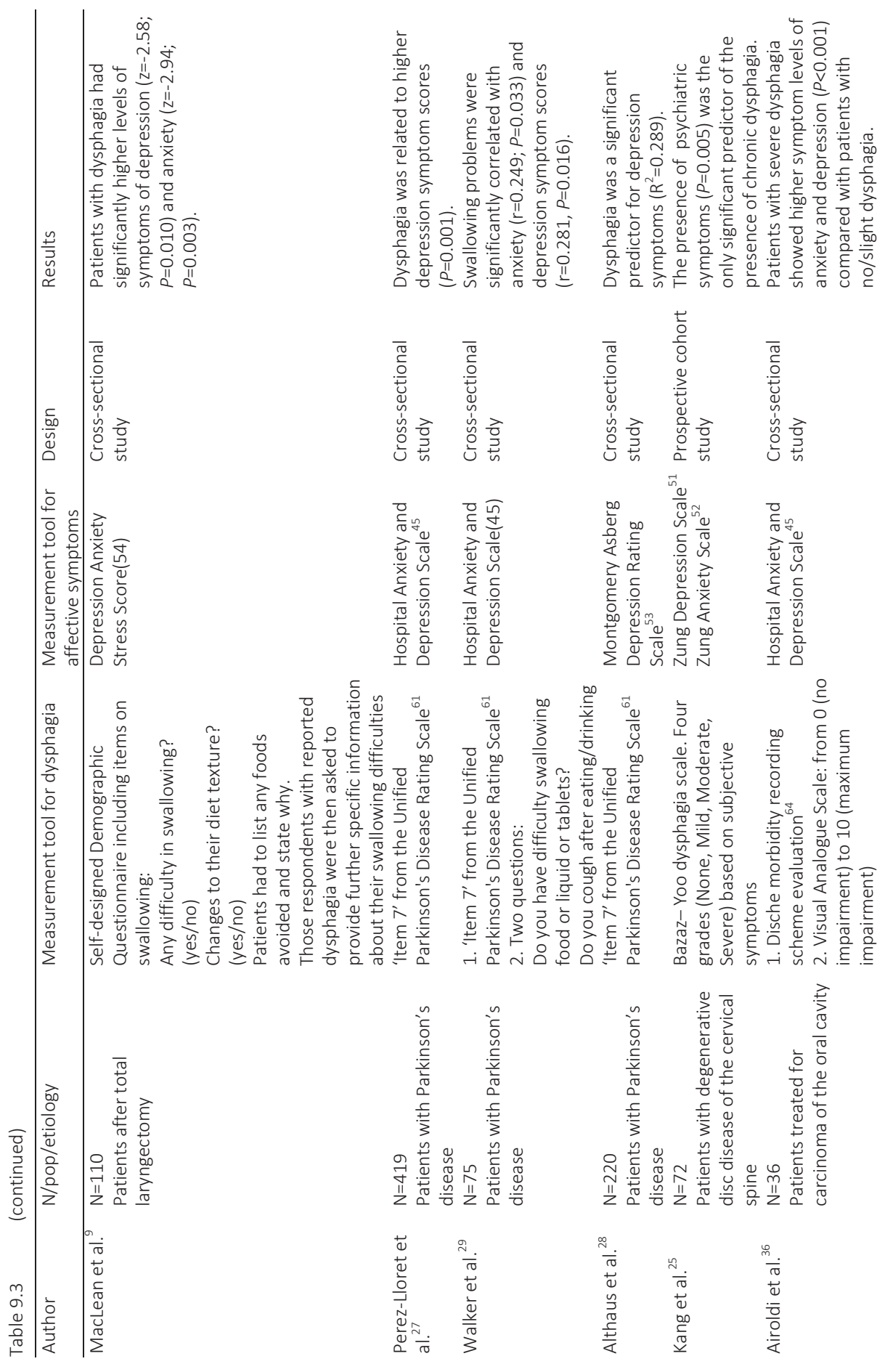




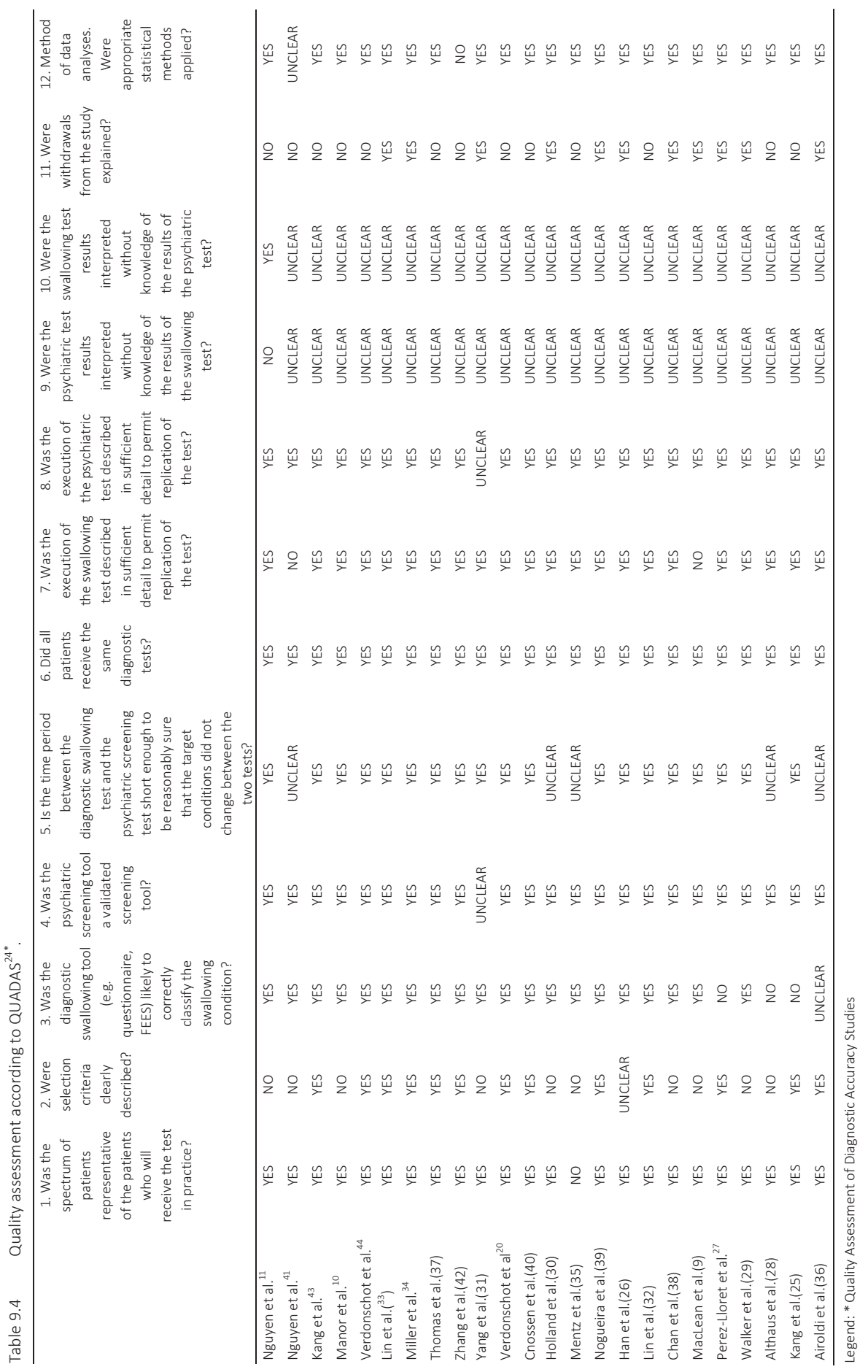




\section{Assessment tools}

Various assessment tools for swallowing function and affective symptoms were used. In order to assess swallowing function, most studies used self-reporting questionnaires or rating scales ${ }^{9,20,25-30,32,35,36,38}$. With regard to the assessment of affective symptoms, most studies used the Hospital Anxiety and Depression Scale (HADS) ${ }^{11,20,27,29,36,37,40,41,44}$. In 19 studies the swallowing assessment and the assessment of depression and/or anxiety symptoms were performed on the same day. In 5 studies time points of the assessments were unclear. See Table 9.3 for a complete overview of the assessment tools used for swallowing function and affective symptoms.

\section{Videofluoroscopic swallowing study and fiber-optic endoscopic evaluation of swallowing}

Nguyen et al. performed a study to evaluate OD in patients treated for head and neck cancer $^{11}$. One hundred and four patients treated for head and neck cancer (e.g., surgery, radiotherapy, chemoradiotherapy, or postoperative radiotherapy), with different disease sites and stages, were retrospectively analyzed. Patients with complaints of dysphagia $(n=73)$ underwent a videofluoroscopic swallowing study (VFSS) to assess its severity (mild, moderate, severe). The control group ( $n=31$ ) had no complaints. The HADS scores were significantly elevated in the dysphagic group. Scores on anxiety and depression symptoms were significantly higher in moderate and severe OD categories compared to mild OD. In addition, Nguyen et al. compared head and neck cancer patients retrospectively who had received chemoradiation $(\mathrm{N}=101)$ with patients who had received postoperative radiation ${ }^{41}$. They did not find any difference in symptoms of anxiety or depression between these 2 treatment groups. However, post-radiation patients who developed complications, of which OD was the most common, reported symptoms of anxiety and depression significantly more often. Kang et al. conducted a non-randomized controlled trial in 50 stroke patients with OD as confirmed by VFSS ${ }^{43}$. A control group of 25 patients received a conventional swallowing therapy (tactile-thermal stimulation, 5 days per week for 2 months). The experimental group of 25 patients received the same conventional therapy but also bedside training, which consisted of oral, pharyngeal, laryngeal, and respiratory exercises (1 hour per day for 2 months). Before and after therapy, all 50 patients underwent a VFSS and filled out the BDI. The experimental group showed a significantly better swallowing function and lower levels of depressive symptoms than the control group. In the study of Manor et al., 69 patients with Parkinson's disease underwent a clinical observation of swallowing by a speech and language pathologist who reported how the patient manipulated food in the mouth, the oral transit time, the presence of a swallowing reflex, and signs of aspiration (e.g., coughing, choking, change in vocal quality) ${ }^{10}$. If there were signs of reduced swallowing function, patients were referred for a fiber-optic endoscopic evaluation of swallowing (FEES) examination. Patients with swallowing disturbances showed significantly higher 
scores of anxiety and depressive symptoms compared with patients without swallowing disturbances. Verdonschot et al. performed a study in 107 patients with OD of various etiologies $^{44}$. Patients' performances on four FEES-variables were assessed by two independent observers and then compared with the results of the HADS questionnaire. Although clinically relevant symptoms of anxiety and depression were present in 46 (43\%) patients, the data revealed only few significant associations between anxiety symptoms and the FEES-variables. The more severe the dysphagia, the less important affective complaints were.

\section{Clinical evaluation of swallowing}

Several studies used a water-swallowing test as a measurement tool for OD. Lin et al. investigated swallowing function in institutionalized elderly ${ }^{33}$. Elderly with symptoms of depression showed a higher frequency of impaired swallowing compared to elderly without symptoms of depression. Miller et al. found an increased frequency of depressive symptoms related to a poorer outcome of the swallowing tests in Parkinson's patients $^{34}$. Thomas et al. and Zhang et al. found a significant positive association between impaired swallowing and depressive symptoms in patients with multiple sclerosis and tongue cancer, respectively ${ }^{37,42}$. Yang et al. performed a population-based study among 415 elderly $^{31}$. A clinical observation of swallowing was performed using water-swallowing tests and, if no dysfunction was noted, a meal observation was subsequently performed. Patients diagnosed with a major depressive disorder had dysphagia more often and major depression was an independent risk factor for dysphagia.

\section{Swallowing-related questionnaires}

Various questionnaires were used to assess swallowing and to record affective symptoms. Verdonschot et al. performed swallowing assessment in 96 patients with OD of various etiologies ${ }^{20}$. A high prevalence of symptoms of anxiety and depression in the total population was found. Cnossen et al. found that OD was significantly and positively associated with symptoms of anxiety and depression in head and neck cancer patients ${ }^{40}$. Holland et al. and Mentz et al. studied dysphagia in otherwise healthy elderly ${ }^{30,35}$. Both study populations consisted of individuals who represented the surviving members of the University of Manchester Longitudinal Study of Cognition in Normal Healthy Old $\mathrm{Age}^{58}$. They found significant associations between impaired swallowing scores and symptoms of depression. Han et al. used the Swallowing Disturbances Questionnaire and found a positive association between lower swallowing scores and symptoms of depression in Parkinson patients ${ }^{26,60}$. For a complete overview of all included articles, see Table 9.3. 


\section{Single swallowing questions}

The following studies used a single item or question to determine swallowing function. Perez-Lloret et al., Walker et al., and Althaus et al. studied Parkinson's disease and showed a significant positive association between OD and depressive symptoms ${ }^{27-29}$. The study of Walker et al. also showed an association of OD with symptoms of anxiety ${ }^{29}$. Kang et al. concluded that the presence of symptoms of anxiety and/or depression was a predictor for the presence of chronic dysphagia in patients who underwent surgery for degenerative disc disease of the cervical spine ${ }^{25}$. Airoldi et al. described a high prevalence of symptoms of anxiety and depression in oral cancer patients with severe OD after surgical flap reconstruction and postoperative radiotherapy ${ }^{36}$.

\section{Discussion}

This systematic review is the first to summarize and evaluate the evidence of an association between affective symptoms and oropharyngeal dysphagia. It consists of 24 articles that reported on swallowing function and affective complaints in different populations. Data pooling was prevented by heterogeneity of assessment tools, diversity of study populations, and poor methodological quality. Hence, although no metaanalytic conclusions can be drawn from the included articles, symptoms of anxiety and depression appear to be common in dysphagic patients. All studies concluded that symptoms of depression were significantly and positively associated with impaired swallowing function. Twelve studies investigated symptoms of anxiety as well, and 9 studies found a significant association with dysphagia too. Quality of the included studies varied. Thirteen of the included studies met all criteria for external validity ${ }^{20,25,27,32-}$ $34,36,37,39,40,42-44$, whereas none met all criteria for internal validity. Little is known about the relationship between the severity of OD and affective symptoms. Nguyen et al. concluded that anxiety and depressive symptoms scored higher in patients with moderate and severe OD compared to patients with mild $O D^{11}$, while the study of Verdonschot et al. concluded that affective symptoms were common in OD patients ${ }^{44}$, but patients with severe OD did not have a higher probability of affective complaints. Moreover, having affective symptoms might be a predictor of subjectively experienced dysphagia severity ${ }^{44}$. It remains unclear what the exact mechanism behind the association of OD with affective states is. However, at least in patients with medically unexplained otorhinolaryngological symptoms (MUORLS), including dysphagia, affective conditions are common ${ }^{12}$. This could be a reflection of a state of dysphagia amplification $^{44}$, which is comparable to the sensitization and alarm falsification process known from other functional somatic conditions ${ }^{65}$. Anxiety and depressive disorders might influence the perception of swallowing by influencing an 'alarm' set point to a dysfunctional state experienced as OD. From a neurobiological perspective, cerebral 
motor cortex areas may be related to the neural stress connectome in anxiety and depression ${ }^{66}$. Further research on this topic is deemed needed.

The results of the current review should be seen in the light of several limitations. The search strategy used MeSH terms and a limited number of free-text terms. Nonetheless, all of the included studies investigated symptoms of anxiety and/or depression. A broader search strategy could have generated different search results. Due to an assessable stratum, we decided to focus on specific symptomatology rather than on a wide range of sub-threshold psychological complaints. The search was conducted with an experienced university library information specialist, and together it was decided to not include "gray literature" in the search strategy. Moreover, a 12-item critical appraisal tool, as derived from the QUADAS ${ }^{24}$, was used for quality assessment. It is possible that a different tool would have led to a different assessment of the included studies. Overall, it was difficult to compare the results of the 24 studies for several reasons. First of all, despite the focus on affective complaints, the studies were considerably heterogeneous regarding patient populations and outcome parameters. The study populations consisted of stroke patients, head and neck oncology patients, the elderly, and others. Furthermore, the studies applied a wide range of assessment tools for affective symptoms or conditions and the swallowing function. Second, most articles had methodological limitations (e.g., no clear description of the selection criteria; little or incomplete information about diagnostic tools and procedures; no information about test result interpretations; and no explanation of withdrawals) (Table 4), which made it impossible to carry out data pooling and meta-analysis. Moreover, most studies used only 1 tool to assess swallowing, while a multidimensional approach is recommended for the diagnosis of OD and the assessment of OD severity. A broader protocol should include a structured interview, a clinical observation of oral intake by a speech and language pathologist, and a FEES and/or VFSS ${ }^{13}$. In addition, almost all studies used questionnaires that can only be applied as screening tools for symptoms of anxiety and depression. These tests are not suitable to come to a diagnosis of affective disorders. Although self-assessment questionnaires give an indication of patients' depressive or anxiety state, a consultation by an experienced psychiatrist or psychologist should be included in order to get a valid diagnosis ${ }^{12}$. Given that the use of psychotropic drugs may have led to an underestimation of affective states, reporting about psycho-pharmacy is essential in cohort studies as well. However, several articles did not mention the use of any psychotropic medication. In 19 articles it remained unclear whether drugs were used or not ${ }^{9-11,25,29-32,34-43}$. In addition, affective symptoms could be related to the underlying disease state instead of OD. It is unclear how well the studies controlled or adjusted for underlying diseases.

Overall, the question remains unsolved whether there is a causal relationship between affective symptoms and swallowing problems in OD patients. Therefore, future studies investigating this topic should use instrumental measurement tools for OD (e.g., FEES, VFSS), and a psychiatrist as a gold standard instead of assessment by retrospective 
self-reporting questionnaires should do psychiatric assessment. It is important that the execution of the swallowing test is standardized in every included patient. Moreover, the time period between swallowing assessment and psychiatric assessment should be short in order to assure that the conditions of OD and the affective state do not change between two test periods. Nevertheless, it is clear that affective symptoms are common in patients with OD. Screening for affective symptoms could be helpful for caregivers who are not familiar with psychiatric symptomatology in order to estimate influences on possible treatment refractoriness. To date, few treatment-effect studies have been conducted in OD patients with psychiatric comorbidity. Moreover, psychiatric symptoms or diagnoses other than anxiety disorders and depression might be prevalent in OD patients too. However, very few studies have been published on this topic. At least in complex patients with affective comorbidity, the management of OD requires a multidimensional approach to enable patients to adhere to swallowing rehabilitation. For that reason, psychological or psychiatric expertise should be integrated in future multidimensional OD approaches, which might contribute to the treatment satisfaction in OD.

\section{Conclusion}

The current literature revealed that affective symptoms in patients with OD are common. Screening for affective symptoms in patients with OD is clinically relevant and may help caregivers who are not aware of psychiatric comorbidity to detect cases. Although the likelihood of psychiatric comorbidity in OD patients seems obvious, therapy-effect studies to document effect of integrated care are scarce. As the present review of the literature demonstrates, there is a need for well-designed prospective research to investigate the possible benefit of integrated medical psychiatric care in patients with OD and affective comorbidity. 


\section{References}

1. Logemann, J. A. Evaluation and Treatment of Swallowing Disorders. 2nd Edition. Austin, Texas (1997).

2. Cook, I.J. \& Kahrilas, P. J. AGA technical review on management of oropharyngeal dysphagia. Gastroenterology 116, 455-478 (1999).

3. Cichero, J. A. Dysphagia. Foundation, Theory and Practice. Chichester, Manchester: In. John Wiley \& Sons, Ltd. 237-248 (2006).

4. Rofes, L. et al. Diagnosis and management of oropharyngeal dysphagia and its nutritional and respiratory complications in the elderly. Gastroenterol. Res. Pract. pii: 818979 (2011).

5. Takizawa, C. et al. A Systematic Review of the Prevalence of Oropharyngeal Dysphagia in Stroke, Parkinson's Disease, Alzheimer's Disease, Head Injury, and Pneumonia. Dysphagia 31, 434-441. (2016).

6. Heijnen, B. J. et al. Neuromuscular electrical stimulation versus traditional therapy in patients with Parkinson's disease and oropharyngeal dysphagia: Effects on quality of life. Dysphagia 27, 336-345 (2012).

7. Speyer, R. et al. Quality of life in oncological patients with oropharyngeal dysphagia: validity and reliability of the Dutch version of the MD Anderson Dysphagia Inventory and the Deglutition Handicap Index. Dysphagia 26, 407-414 (2011).

8. Ekberg, O. et al. Social and psychological burden of dysphagia: Its impact on diagnosis and treatment. Dysphagia 17, 139-146 (2002).

9. Mac Lean, J. et al. Dysphagia following a total laryngectomy: The effect on quality of life, functioning, and psychological well-being. Dysphagia 24, 314-321 (2009).

10. Manor, Y. et al. Anxiety, depression and swallowing disorders in patients with Parkinson's disease. Parkinsonism Relat. Disord. 15, 453-456 (2009).

11. Nguyen, N. P. et al. Impact of dysphagia on quality of life after treatment of head-and-neck cancer. Int. J. Radiat. Oncol. Biol. Phys. 61, 772-778 (2005).

12. Baijens, L. W. et al. Medically unexplained otorhinolaryngological symptoms: Towards integrated psychiatric care. Laryngoscope 125, 1583-1587 (2015).

13. Baijens LW. Multidisciplinaire polikliniek voor dysfagie. Dutch J. Ear Nose Throat 18, 5-6 (2012).

14. Janssen, D. J. et al. Symptoms of anxiety and depression in COPD patients entering pulmonary rehabilitation. Chron. Respir. Dis. 7, 147-157 (2010).

15. Thijssen, A. Y. et al. Dysfunctional cognitions, anxiety and depression in irritable bowel syndrome. J. Clin. Gastroenterol. 44, e236-241 (2010).

16. van Steenbergen-Weijenburg, K. M. et al. Collaborative care for co-morbid major depressive disorder in chronically ill outpatients in a general hospital. Tijdschr. Psychiatr. 57, 248-257 (2015).

17. Vrijens, D. et al. Affective symptoms and the overactive bladder: A systematic review. J. Psychosom. Res. 78, 95-108 (2015).

18. Schottke, H \& Giabbiconi, C. M. Post-stroke depression and post-stroke anxiety: Prevalence and predictors Int. Psychogeriatr. 27, 1805-1812 (2015).

19. Vrijens, D. M. et al. Associations of psychometric affective parameters with urodynamic investigation for urinary frequency. Low. Urin. Tract Symptoms doi: 10.1111/luts.12130 (2016).

20. Verdonschot, R. J. et al. Symptoms of anxiety and depression assessed with the Hospital Anxiety and Depression Scale in patients with oropharyngeal dysphagia. J. Psychosom. Res. 75, 451-455 (2013).

21. Higgins, J. P. \& Green, S. (Eds.) Cochrane Handbook for Systematic Reviews of Interventions: Version 5.1.0. London, United Kingdom: The Cochrane Collaboration, (2011). http://handbook.cochrane.org/. (Accessed March 30, 2016).

22. Leeflang, M. M. et al. Cochrane diagnostic test accuracy reviews. Syst. Rev. 2, 82 (2013).

23. Cochrane Screening and Diagnostic Tests Methods Group. Diagnostic Test Accuracy Handbook. Available at: http://methods.cochrane.org/sdt/handbook-dta-reviews. (Accessed May 23, 2016).

24. Whiting, P. et al. The development of QUADAS: A tool for the quality assessment of studies of diagnostic accuracy included in systematic reviews. BMC Med. Res. Methodol. 3, 25 (2003).

25. Kang, S. S. et al. The association between psychiatric factors and the development of chronic dysphagia after anterior cervical spine surgery. Eur. Spine J. 23, 1694-1698 (2014). 
26. Han, M. et al. Relationship between dysphagia and depressive states in patients with Parkinson's disease. Parkinsonism Relat. Disord. 17, 437-439 (2011).

27. Perez-Lloret, S. et al. Oro-buccal symptoms (dysphagia, dysarthria, and sialorrhea) in patients with Parkinson's disease: Preliminary analysis from the French COPARK cohort. Eur. J. Neurol. 19, 28-37 (2012).

28. Althaus, A. et al. Frequency and treatment of depressive symptoms in a Parkinson's disease registry. Parkinsonism Relat. Disord. 14, 626-632 (2008).

29. Walker, R. W. et al. Self-reported dysphagia and its correlates within a prevalent population of people with Parkinson's disease. Dysphagia 26,92-96 (2011).

30. Holland, G. et al. Prevalence and symptom profiling of oropharyngeal dysphagia in a community dwelling of an elderly population: A self-reporting questionnaire survey. Dis. Esophagus 24, 476-480 (2011).

31. Yang, E. J. et al. Oropharyngeal dysphagia in a community-based elderly cohort: The Korean longitudinal study on health and aging. J. Korean Med. Sci. 28, 1534-1539 (2013).

32. Lin, B. M. et al. The relationship between depressive symptoms, quality of life, and swallowing function in head and neck cancer patients 1 year after definitive therapy. Laryngoscope 122, 1518-1525 (2012).

33. Lin, L. C. et al. Depressive symptoms in long-term care residents in Taiwan. J. Adv. Nurs. 51, $30-37$ (2005).

34. Miller, N. et al. Swallowing problems in Parkinson disease: frequency and clinical correlates. J. Neurol. Neurosurg Psychiatry 80, 1047-1049 (2009).

35. Mentz, H. et al. Homozygosity in the ApoE 4 polymorphism is associated with dysphagic symptoms in older adults. Dis. Esophagus 28, 97-103 (2015).

36. Airoldi, M.et al. Functional and psychological evaluation after flap reconstruction plus radiotherapy in oral cancer. Head Neck 33, 458-468 (2011).

37. Thomas, F. J. \& Wiles, C. M. Dysphagia and nutritional status in multiple sclerosis. J. Neurol. 246, 677-682 (1999).

38. Chan, J. Y. et al. The relationship between depressive symptoms and initial quality of life and function in head and neck cancer. Laryngoscope 121, 1212-1218 (2011).

39. Nogueira, D. \& Reis, E. Swallowing disorders in nursing home residents: How can the problem be explained? Clin. Interventions Aging. 8, 221-227 (2013).

40. Cnossen, I. C. et al. Computerized monitoring of patient-reported speech and swallowing problems in head and neck cancer patients in clinical practice. Support. Care Cancer 20, 2925-2931 (2012).

41. Nguyen, N. P. et al. Quality of life following chemoradiation and postoperative radiation for locally advanced head and neck cancer. ORL 69, 271-276 (2007).

42. Zhang, L. et al. Effect of swallowing training on dysphagia and depression in postoperative tongue cancer patients. Eur. J. Oncol. Nurs. 18, 626-629 (2014).

43. Kang, J. H. et al. The effect of bedside exercise program on stroke patients with dysphagia. Ann. Rehabil. Med. 36, 512-520 (2012).

44. Verdonschot, R. J. et al. The relationship between fiberoptic endoscopic evaluation of swallowing outcome and symptoms of anxiety and depression in dysphagic patients. Laryngoscope 126, E199-207 (2016).

45. Zigmond, A. S. \& Snaith, R. P. The hospital anxiety and depression scale. Acta Psychiatr. Scand. 67, 361370 (1983).

46. Wancata, J. et al. The criterion validity of the Geriatric Depression Scale: A systematic review. Acta Psychiatr Scand. 114,398-410 (2006).

47. Beck, A. T. et al. Comparison of Beck Depression Inventories -IA and -II in psychiatric outpatients. J. Pers. Assess. 67, 588-597 (1996).

48. Beck, A. T. et al. An inventory for measuring depression. Arch. Gen. Psychiatry 4, 561-571 (1961).

49. Steer, R. A. et al. Use of the Beck Depression Inventory for Primary Care to screen for major depression disorders. Gen. Hosp. Psychiatry 21,106-111 (1999).

50. Richter, P. et al. On the validity of the Beck Depression Inventory: A review. Psychopathology 31,160-168 (1998).

51. Zung, W. W. A Self-rating depression scale. Arch. Gen. Psychiatry 12, 63-70 (1965).

52. Zung, W. W. A rating instrument for anxiety disorders. Psychosomatics 12, 371-379 (1971).

53. Asberg, M. et al. A comprehensive psychopathological rating scale. Acta Psychiatr. Scand. Suppl. 271,S 527 (1978). 
54. Lovibond, P. F. \& Lovibond, S. H. The structure of negative emotional states: Comparison of the Depression Anxiety Stress Scales (DASS) with the Beck Depression and Anxiety Inventories. Behav. Res. Ther. 33, 335-343 (1995).

55. Spielberger, C. Manual for the State-Trait Anxiety Inventory (STAI). Palo Alto (CA): Consulting Psychologists Press; (1983).

56. Diagnostic and Statistical Manual for Mental Disorders, fourth edition (DSM-IV). Washington DC: American Psychiatric Association (2000).

57. Chen, A. Y. et al. The development and validation of a dysphagia-specific quality-of-life questionnaire for patients with head and neck cancer: The M. D. Anderson dysphagia inventory. Arch. Otolaryngol. Head Neck Surg. 127, 870-876 (2001).

58. Rabbitt, P. et al. The University of Manchester longitudinal study of cognition in normal healthy old age, 1983 through 2003. Aging Neuropsychol. Cogn. 11, 245-279 (2004).

59. Wallace, K. L. et al. Development and validation of a self-report symptom inventory to assess the severity of oropharyngeal dysphagia. Gastroenterology 118, 678-687 (2000).

60. Manor, Y. et al. Validation of a swallowing disturbance questionnaire for detecting dysphagia in patients with Parkinson's disease. Mov. Disord. 22, 1917-1921 (2007).

61. Fahn, S. et al. Unified Parkinson's disease rating scale II. In: Fahn S, Marsden CD, Calne DB, Goldstein M, editors. Recent Developments in Parkinson's Disease. 153-163 (1987).

62. Bjordal, K. et al. Quality of life in head and neck cancer patients: Validation of the European Organization for Research and Treatment of Cancer Quality of Life Questionnaire-H\&N35. J. Clin. Oncol. 17, 1008-1019 (1999).

63. Logemann, J. A. et al. Dysphagia care among seniors in Los Angeles County: Planning and pilot study. Monterey, California (2008).

64. Cooper, J. S. et al. Late effects of radiation therapy in the head and neck region. Int. J. Radiat. Oncol. Biol. Phys. 31, 1141-1164 (1995).

65. Leue, C. et al. Functional urological disorders: a sensitized defense response in the bladder-gut-brain axis. Nat. Rev. Urol. 14, 153-163 (2017).

66. Dum, R. P. et al. Motor, cognitive, and affective areas of the cerebral cortex influence the adrenal medulla. Proc. Natl. Acad. Sci. U S A. 113, $9922-9227$ (2016). 


\section{Patientengeschichte}

Eine 82-jährige Patientin wird elektiv am offenen Herzen operiert. Die stenosierte Aortenklappe wird durch eine Schweineklappe ersetzt. Im präoperativen Verlauf besteht ein Risikoprofil, das durch einen Typ-2-Diabetes und eine Polymyalgia rheumatica mitbestimmt wird. Eine kognitive Beeinträchtigung ist nicht gegeben. Neben Antihypertensiva nimmt die Pat.in Metformin und niedrig dosiertes Kortison ein. Bis vor Kurzem wurde noch Methotrexat verabreicht. Unmittelbar bei Erwachen aus der Narkose ergibt sich ein hyperaktives Delir mit Agitation und visuellen Halluzinationen. Das Delir wird mit Haloperidol und Lorazepam symptomatisch behandelt. Vor allem die ängstliche Agitation macht den Pflegekräften zu schaffen. Der 24-Stunden-Einsatz der Familie am Bett beruhigt die Pat.in und entlastet den Pflegeaufwand. Nach 2 Wochen stellt sich in der Rehabilitation ein Dressler-Syndrom heraus, das hoch dosiert mit Kortison und Colchicin behandelt wird. Während bei der Pat.in im Nachhinein eine Amnesie über den Delirverlauf besteht, erlebt die Tochter noch nach Wochen Angstsymptome mit bedrohlichen Träumen und vielfältigen somatischen Äquivalenten. Unter einer gesprächstherapeutischen Aufarbeitung verblassen die letztgenannten Symptome schlussendlich. 


\section{Einleitung}

Das Delir ist ein komplexes neuropsychiatrisches Syndrom, das in allen medizinischen Versorgungs-situationen anzutreffen ist. In der Regel wird es multifaktoriell ausgelöst. Vor allem ältere Patienten sind betroffen (Inouye et al. 2014). Es ist durch eine Beeinträchtigung des Bewusstseins, des formalen und inhaltlichen Denkens, der Sprache und der Wahrnehmung gekennzeichnet. Darüber hinaus sind psychomotorische und affektive Symptome zu beobachten.

Der Verlauf entwickelt sich innerhalb weniger Stunden und fluktuiert während des Tages (WHO 1992 [ICD-10], APA 2013 [DSM-5]). Eine Verschlechterung ergibt sich besonders nachts. Nicht selten kündigen Prodromalphasen von bis zu 3 Tagen mit Schlafstörungen, Angstsymptomen und reduziertem Konzentrationsvermögen ein Delir an. Diese lassen sich als subsyndromales Delir beschreiben, wenn sie mild verlaufen (Trzepacz et al. 2012). Im Verlauf variiert die Bewusstseinslage von normaler Wachsamkeit bis hin zu Stupor und Koma. Eine deutliche Unterscheidung zwischen hypoaktivem Delir und Stupor oder Katatonie kann schwierig sein und verpflichtet zur Differenzialdiagnose. Ebenso können sich agitierte Verläufe mit der stillen Form abwechseln.

Anders als bei den meisten psychiatrischen Erkrankungen fluktuieren die Symptome innerhalb von 24 Stunden (WHO 1992; APA 2013). Das charakteristische Zu- und Abnehmen der Beschwerden kann die Diagnose erschweren. Dann kommt den Pflegekräften eine Signalfunktion zu, da sie Schwankungen am ehesten wahrnehmen (McCarthy 2003). Der Verlauf lässt sich bei Kindern, Erwachsenen und älteren Patienten gleichermaßen beschreiben (Turkel et al. 2006). Lediglich bei jungen Kindern und kommunikationsunfähigen Patienten ist die Diagnose ausschließlich von der Verhaltensobservation abhängig. Dabei kommt der Fremdanamnese besondere Bedeutung zu (Inouye et al. 2014). Vor allem bei älteren Patienten ist eine komorbide kognitive Erkrankung möglich, wobei Symptome des Delirs in der Regel eine Demenz überschatten (Trzepacz et al. 2002).

Das Delir verläuft meist reversibel. Insbesondere bei terminal kranken Patienten kann sich jedoch ein progressiver Verlauf ergeben. Auf der anderen Seite zeigt sich aber auch bei Krebs-patienten, dass sich ein Delir unter adäquater Behandlung beseitigen lässt (Ljubisavljevic und Kelly 2003; Lawlor und Bush 2015). Die Prognose ist abhängig von der Grunderkrankung und verschlechtert sich bei vielfältiger Symptomlage (Cole et al. 2003).

Ein Delir bleibt jedoch häufig unerkannt (Collins et al. 2010). Insbesondere die hypoaktive Form wird übersehen, und die Anwesenheit von Schmerz oder eine psychiatrische Erkrankung tragen dazu bei (Kishi et al. 2007). Besonders bei Übernahme der Patienten von chirurgischen oder Intensivstationen bleibt eine Diagnosestellung oft aus (Armstrong et al. 1997). Außerdem werden ältere Patienten wegen des Kostendrucks häufig aus dem Krankenhaus in Pflegeheime entlassen, bevor das Delir 
effektiv behandelt ist. Ein Delir kann jedoch auch zur Institutionalisierung prädisponieren (Kiely et al. 2003; Krogseth et al. 2014). Die Erkennung und Behandlung des Delirs ist eine Herausforderung, weil es mit einer schlechteren Prognose (einschließlich verlängertem Krankenhausaufenthalt und erhöhter Mortalität) einhergeht (Kakuma et al. 2003; Ely et al. 2004; Thomasson et al. 2005). Das Aufspüren der zugrunde liegenden somatischen Ursache ist von zentraler Bedeutung bei der kausalen Behandlung.

\section{Epidemiologie}

Das Delir tritt in jedem Lebensalter auf, auch wenn vorzugsweise ältere Patienten betroffen sind. Das häufigere Vorkommen von atherosklerotischen Veränderungen, Tumoren und Demenz beeinflusst die Vulnerabilität im Alter. Die höchste Inzidenz des Delirs bei älteren Patienten findet man auf Intensivstationen, im postoperativen Verlauf und in der Palliativversorgung (Inouye et al. 2014). Patienten mit Demenz haben auf Intensivstationen ein um 40\% erhöhtes Delirrisiko (McNicoll et al. 2003). Zudem weisen gebrechliche Patienten in Pflegeheimen noch 1 Monat nach Krankenhausaufnahme eine Delirinzidenz von mehr als 50\% auf (Kelly et al. 2001). Im Allgemeinen beeinflussen zugrunde liegende diagnostische Kriterien die Inzidenz und Prävalenz. Die ICD-10 legt striktere Einschlusskriterien zugrunde als das DSM-IV. Auch kann die Anwendung weiterentwickelter Kriterien zu unterschiedlichen Resultaten führen (Laurila et al. 2004). Da Studien unterschiedliche Definitionen zu Inzidenz und Prävalenz anwenden, empfiehlt es sich, der NICE-Leitlinie zu folgen und von „Häufigkeiten“ zu sprechen (NCGC 2010).

Studien zum Vorkommen des Delirs in der Allgemeinbevölkerung sind selten und zeigen eine Prävalenz von <1\% (Andrew et al. 2006). Die meisten Untersuchungen beschreiben Krankenhaus-populationen. Hierbei schwankt die Häufigkeit zwischen 15 und 50\%, bei älteren Patienten beträgt sie 30-65\% (Ryan et al. 2013; de Castro et al. 2014). Onkologische Patienten (70\%, bis zu $85 \%$ in der Palliativphase) und mechanisch beatmete Patienten (bis 80\%) sind häufiger betroffen (Lawlor et al. 2000; Pandharipande et al. 2008). Studien zur Häufigkeit des Delirs in der institutionellen Altenpflege schwanken zwischen 10 und 70\% (Arinzon et al. 2011; Boorsma et al. 2012). In den letzten Jahren hat sich der Fokus epidemiologischer Studien auf das Kindesalter erweitert (Kelly und Frosch 2012; Schieveld und Janssen 2014). Dies gilt besonders für die pädiatrische Intensivstation (Daoud et al. 2014). Vieles deutet darauf hin, dass ältere und jüngere Patienten bei schwersten Erkrankungen dem höchsten Delirrisiko ausgesetzt sind (Hatherill und Flisher 2010; Inouye et al. 2014). 


\section{Risikofaktoren}

Bei der Krankenhausaufnahme kommen prädisponierende Vulnerabilität und präzipitierende Faktoren zusammen. Je geringer die Vulnerabilität, desto einflussreicher müssen „Präzipitoren“ sein, um ein Delir hervorzurufen und umgekehrt (van Munster et al. 2014). Verschiedene patienten-, aber auch umgebungsabhängige Aspekte sind zu nennen. Inouye und Charpentier (1996) entwickelten ein Vorhersagemodell, das vier prädisponierende Faktoren (kognitive Beeinträchtigung, ernsthafte Erkrankung, Beeinträchtigung des Sehens, Dehydratation) und fünf präzipitierende Faktoren (Unterernährung, $\geq 3$ Pharmaka, Benutzung von Hilfsmitteln, Harnwegskatheter, iatrogene Ursachen) des Delirs definiert. Sie sagen eine bis zu 17-fache Variation des relativen Delirrisikos vorher.

Der am besten beeinflussbare Faktor ist das Absetzen eines auslösenden Medikaments, denn in ca. 30\% der Fälle ist ein Delir medikamentös induziert. Benzodiazepine, Opiate und Medikamente mit anticholinerger Wirkung sind eng mit dem Delir assoziiert (Brown 2000). Auch wenn Opiate ein Delir hervorrufen können, gilt, dass ältere Patienten eine erhöhte Delirwahrscheinlichkeit aufweisen, wenn auf die postoperative Schmerztherapie verzichtet wird (Morrison et al. 2003). Auf der Grundlage von Studien zu Krankenhauspopulationen unterscheidet die NICE-Leitlinie ca. 20 Risikofaktoren (NCGC 2010) (Tabelle 10.1).

Tabelle 10.1 Risikofaktoren zur Inzidenz des Delirs gemäß der NICE-Leitlinie (NCGC 2010).

\begin{tabular}{lc}
\hline Risikofaktoren & Odds-Ratio $(95 \%-\mathrm{Cl})$ \\
\hline Mit ausreichender Sicherheit* & \\
Fraktur bei Einweisung & $6,57(2,23-19,33)$ \\
Kognitive Beeinträchtigung & $6,30(2,89-13,74)$ \\
Alter > 80 Jahre & $5,22(2,61-10,44)$ \\
Schwere der Erkrankung (APACHE) & $3,49(1,48-8,23)$ \\
Alter >65 Jahre & $3,03(1,19-7,71)$ \\
Infektionen & $2,96(1,42-6,15)$ \\
Visusbeeinträchtigung & $1,70(1,01-2,85)$ \\
Mit geringer Sicherheit* & \\
Komorbidität (>3 Erkrankungen) & $15,94(4,60-55,27)$ \\
Gefäßchirurgie & $2,50(1,72-4,24)$ \\
Mit Unsicherheit* & \\
Polypharmazie (>3 Medikamente) & $33,60(1,90-591,60)$ \\
Harnwegskatheter & $2,70(1,44-5,05)$ \\
Depression & $2,43(0,93-6,35)$ \\
Elektrolytstörungen & $2,40(1,09-5,27)$ \\
Dehydratation & $2,02(0,72-5,64)$ \\
Polypharmazie (>7 Medikamente) & $1,90(1,10-3,24)$ \\
Geschlecht (Mann) & $1,36(0,64-2,89)$ \\
\hline
\end{tabular}

* Die Einteilung mit „ausreichender/geringer Sicherheit oder Unsicherheit“ basiert auf der Stärke der Assoziation sowie auf der Qualität der herangezogenen wissenschaftlichen Studien.

In der neueren Literatur werden Lebensalter, kognitive Probleme, Immobilisation, Schwere der somatischen Erkrankung, mechanische Beatmung, akute 
In der neueren Literatur werden Lebensalter, kognitive Probleme, Immobilisation, Schwere der somatischen Erkrankung, mechanische Beatmung, akute Krankenhauseinweisung und Krankenhaus-verweildauer als Risikofaktoren beschrieben. Zudem bestätigt sich, dass risikoreiche Medikamente, Unterernährung, Harnwegskatheter und eine Krankengeschichte mit Nikotin- und übermäßigem Alkoholgebrauch eine Rolle spielen (Brouquet et al. 2010; Ahmed et al. 2014; Huai und Ye 2014; Zaal et al. 2015). Zudem wird deutlich, dass das Vorliegen von präoperativen Schmerzen und depressiven Symptomen die postoperative Delirhäufigkeit erhöht (Kosar et al. 2014).

\section{Ätiologie}

Für das Delir ist eine ganze Bandbreite ätiologischer Faktoren bekannt (Tabelle 10.2). Eine kausale Beziehung ergibt sich, wenn einer dieser Faktoren nachweislich in zeitlichem Zusammenhang mit dem Delir steht. Dennoch gibt es Verläufe, in denen die Ätiologie unklar bleibt. Ein monokausales Delir liegt in weniger als der Hälfte der Fälle vor. Mehrere ursächliche Faktoren spielen vor allem bei älteren Patienten eine Rolle (Camus et al. 2000; Inouye et al. 2014).

Tabelle 10.2 Ätiologische Faktoren, die zur Entstehung eines Delirs beitragen: das Akronym „End Acute Brain Failure"*.

\begin{tabular}{lll}
\hline Englisches Akronym & Deutsche Erläuterung \\
\hline E & (Electrolyte imbalance) & Elektrolytstörungen und Dehydratation \\
$\mathrm{N}$ & (Neurological disorder) & Neurologische Erkrankung, Schädel-Hirn-Trauma \\
$\mathrm{D}$ & (Deficiencies) & Mangelernährung \\
$\mathrm{A}$ & (Age and gender) & Alter und Geschlecht \\
$\mathrm{C}$ & (Cognition) & Kognition \\
$\mathrm{U}$ & (U-Tox) & Drogenintoxikation oder -entzug \\
$\mathrm{T}$ & (Trauma) & Trauma \\
$\mathrm{E}$ & (Endocrine disturbance) & Endokrinopathie \\
$\mathrm{B}$ & (Behaviour and psychiatric disorders) & Psychische und Verhaltensstörungen \\
$\mathrm{R}$ & (Recipe and other toxins) & Medikamente und Toxine \\
$\mathrm{A}$ & (Anaemia and anoxia) & Anämie, Anoxie \\
$\mathrm{I}$ & (Infections) & Infektionen \\
$\mathrm{N}$ & (Noxious stimulus) & Schmerz \\
$\mathrm{F}$ & (Failure) & Organversagen \\
$\mathrm{A}$ & (APACHE score) & Krankheitsschweregrad (APACHE-Score) \\
$\mathrm{I}$ & (Intracranial process) & Intrakranielle Prozesse \\
$\mathrm{L}$ & (Light) & Licht (zirkadianer Rhythmus) \\
$\mathrm{U}$ & (Uraemia and metabolic disorders) & Urämie und Stoffwechselstörungen \\
$\mathrm{R}$ & (Restraints) & Beschränkungen/Hilfsmittel (Brille, Hörgerät etc.) \\
$\mathrm{E}$ & (Emergence delirium) & Durchgangssyndrom \\
\hline
\end{tabular}

*Maldonado JR (2015), Vortrag anlässlich der Jahreskonferenz der Europäischen Vereinigung für Psychosomatik (EAPM) 2015 (mit Zustimmung des Autors). 


\section{Pathogenese}

Ätiopathogenetisch sind diverse Systeme betroffen, wenn es zu einer neuronalen Netzwerkstörung auf der gemeinsamen Endstrecke (common final pathway) kommt (Maldonado 2013; Inouye et al. 2014). Sowohl kortikale als auch subkortikale Hirnregionen sind beteiligt. Die cholinerge Aktivität ist beeinträchtigt, wobei einem Delir anticholinerge Pharmaka zugrunde liegen können (Campbell et al. 2009). Bei der Alzheimer-Erkrankung, der vaskulären Demenz und der Lewy-Body-Demenz ist die hohe Assoziation zum Delir ebenfalls durch die reduzierte cholinerge Kompetenz, verbunden mit einem Verlust cholinerger Neuronen in basalen Ganglien, erklärbar (Gore et al. 2015). Auch apoplektische und traumatische Hirnschäden gehen mit einer reduzierten cholinergen Kapazität einher und prädisponieren zur Empfindlichkeit für antimuskarinerge Medikamente oder eine nikotinerge Behandlung (Chaisuksunt et al. 2003; Kelslo und Oestreich 2012).

Neben dem cholinergen Defizit spielt eine erhöhte dopaminerge Aktivität eine Rolle. Parkinsonmittel und Intoxikationen mit Stimulanzien oder Opiaten sind involviert. Die Wirksamkeit antidopaminerger Antipsychotika unterstreicht die pathogenetische Rolle des Dopamins (Lauretani et al. 2010). Darüber hinaus ist Serotonin mit dem Delir assoziiert. Mit Blick aufs GABAerge System verursachen sowohl Intoxikationen als auch Entzugssituationen ein Delir. Beim Alkoholentzugsdelir ist ebenfalls eine Dysbalance hemmender und aktivierender Signalwege entscheidend (Maldonado 2013).

Neben der Neurotransmitterhypothese bestehen andere Theorien, z.B. zum zellulären Stoffwechsel oder zum oxidativen Stress, die eine deutliche Interferenz mit Transmittersystemen aufweisen (Caplan et al. 2010; Schoen et al. 2011; Maldonado 2013). Entzündungskaskaden interferieren z.B. mit Neurotransmittern. Untersuchungen von Blutproben oder zerebrospinaler Flüssigkeit bestätigen den Einfluss von Entzündungsmediatoren bei der Pathogenese (MacLullich et al. 2013). Dabei lässt sich sowohl eine periphere als auch zentrale Inflammation beschreiben (Hughes et al. 2012; Cape et al. 2014). Ein Teil dieser Kaskaden lässt sich jedoch bei älteren Patienten nach Femurfraktur nicht belegen (de Jonghe et al. 2012). Gegenwärtig ist der immunologische Mechanismus, der das Delir umfassend erklären könnte, noch offen (Cerejeira et al. 2014).

\section{Klinische Symptome}

Das Delir zeigt Kernsymptome, die Ausdruck einer gemeinsamen Endstrecke verschiedener Störungen sind (Inouye et al. 2014). Dazu gehören: Aufmerksamkeitsdefizit, Desorientierung, Gedächtnisstörungen, Störungen der exekutiven Funktionen, Sprachstörungen, ein gestörter zirkadianer Rhythmus und psychomotorische Auffälligkeiten (WHO 1992; APA 2013). 
Diagnoseweisende Eigenschaften wie das Ausmaß der Erregung und die Bewusstseinstrübung gehören „noch“ nicht zum Kern (Franco et al. 2013). Die gemeinsame Empfehlung der European Delirium Association und der American Delirium Society (2014) das Delirkonzept über den Aspekt der kognitiven Störung hinaus zu erweitern, könnte die beträchtlichen Unterschiede zwischen DSM-IV und -V-Kriterien überwinden helfen (Meagher et al. 2014a). Zu den Symptomen zählen des Weiteren Wahrnehmungsstörungen, inhaltliche Denkstörungen und affektive Symptome. Während Aufmerksamkeitsstörungen bei allen Patienten anzutreffen sind, wird desorganisiertes Denken in beinahe allen Fällen beschrieben. Gedächtnisstörungen treten häufig auf und betreffen das Kurz- und Langzeitgedächtnis. Über den Verlauf besteht oft eine Amnesie (Breitbart et al. 2002). Wahrnehmungsstörungen können sich in visuellen, taktilen, olfaktorischen und gustatorischen Halluzinationen äußern. Rasch wechselnde Affektlabilität beinhaltet u. a. Angst und Dysphorie. Inhaltliche Denkstörungen treten in $25 \%$ der Fälle auf und äußern sich meist als wenig differenzierter Verfolgungswahn (Webster und Holroyd 2000).

Auch wenn im überwiegenden Teil der Fälle eine Amnesie dafür sorgt, dass der postdelirante Verlauf affektiv unkompliziert bleibt, entwickelt sich bei einem Teil der Patienten und deren Angehörigen nach dem Delir eine posttraumatische Belastungsstörung (PTBS). Vor allem bedrohliche Halluzinationen und der Verlust der persönlichen Integrität äußern sich nachhaltig als angstbesetzte Erinnerungen mit Schlafstörungen und körperlichen Angstäquivalenten (Long et al. 2014; Colville und Pierce 2012).

Eine Einteilung des Delirs erfolgt anhand psychomotorischer Eigenschaften. Dabei werden drei Subtypen unterschieden: das hyperaktive Delir, eine hypoaktive und eine Mischform (Stagno et al. 2004; Peterson et al. 2006). Während den hyperaktiven Verlauf ein erhöhtes Aktivitätsniveau mit Unruhe kennzeichnet, ist das Aktivitätsniveau bei der hypoaktiven Form bis hin zur Apathie erniedrigt (Meagher et al. 2008). Differenzialdiagnostisch ist an Stupor oder Katatonie zu denken. Häufigkeit und Verlauf der Subtypen sind populationsabhängig. Bei älteren Patienten mit Femurfraktur ist das hyper- und hypoaktive Delir anteilsgleich ausgeprägt, während die Mischform nur bei einer Minderheit der Patienten auftritt (Slor et al. 2013). In der Palliativversorgung dominiert dagegen das hypoaktive Delirium vor der Mischform. Bis auf den motorischen Anteil verlaufen die verschiedenen Subtypen phänomenologisch gleich (Meagher et al. 2012). Trotzdem werden auf Intensivstationen hyperaktive Verläufe eher erkannt und behandelt. Vor allem das stumme Delir wird übersehen und hat die schlechteste Langzeitprognose (Peterson et al. 2003; Meagher et al. 2011; (Tabelle 10.3). 
Tabelle 10.3 Symptome des Delirs, abhängig vom psychomotorischen Subtyp.

\begin{tabular}{lll}
\hline & Hyperaktives Delir & Hypoaktives Delir \\
Motorische Symptome & Aktivitätsniveau $\uparrow$ & Aktivitätsniveau $\downarrow$ \\
& Kontrollverlust & Apathie \\
Unruhe & \\
Nichtmotorische Symptome & Hachsamkeit $\uparrow$ & Hypoaktives Delir \\
& Fehlende Kooperation & Aufmerksamkeit $\downarrow$ \\
Halluzinationen & Somnolenz \\
Kognitive Symptome & Hggression & Halluzinationen \\
\hline
\end{tabular}

\section{Verlauf}

Das Delir wird von der Demenz traditionell durch den reversiblen Verlauf unterschieden. Nicht reversibel verlaufen mitunter Delirien bei terminalen Erkrankungen, traumatischer oder vaskulärer Hirnschädigung und präexistenter Demenz. Auch bei nichtdementen Patienten mit geringer kognitiver Reserve ist das Delir oft mehr als ein vorübergehendes Phänomen. Ein schlechteres kognitives Funktionieren nach dem Delir tritt bei schwerem Verlauf auf (Inouye et al. 2014). Darüber hinaus bestehen 30\% der Delirien älterer Patienten noch 6 Monate nach der Krankenhausentlassung fort (Levkoff et al. 1994). Teilweise mag dies auf eine fehlende Diagnose und Behandlung zurückzuführen sein, denn die symptomatische Therapie wird nicht durch die kognitive Beeinträchtigung bestimmt (Meagher et al. 1996). Generell bestehen aber nach $1 \mathrm{Jahr}$ sowohl bei dementen als auch bei nichtdementen Patienten Gedächtnisstörungen fort.

Das Delir ist also ein Risikofaktor für eine demenzielle Erkrankung (McCusker et al. 2003; Jackson et al. 2004). Relevant ist hierbei eine eingeschränkte kognitive Reserve, die Hinweis auf eine vorbestehende Grunderkrankung sein kann (Camus et al. 2000). Einiges weist darauf hin, dass chronisch entzündliche Prozesse neurodegenerative Auswirkungen haben (Popp 2013). Insbesondere eine längere Dauer des Delirs geht auch bei jüngeren schwerstkranken Patienten mit kognitiven Defiziten einher (Pandharipande et al. 2013).

Der wichtigste Verlaufsaspekt betrifft die erhöhte Mortalität. Die niedrigste Rate ergibt sich beim hyperaktiven Delir (10\%) (Olofsson et al. 1996). Metaanalysen zeigen, dass Intensivpatienten mit Delir eine signifikant höhere Mortalität bei zudem längerer Beatmung und längerem Krankenhaus-aufenthalt aufweisen (Salluh et al. 2015). Insbesondere eine längere Dauer des Delirs ist auf der Intensivstation mit erhöhter Mortalität assoziiert (Pisani et al. 2009). Auch im Langzeitverlauf ergibt sich eine erhöhte Mortalität, was nicht nur für delirante beatmete, sondern auch für delirante psychiatrische Patienten gilt (Brown 2014; Lundberg et al. 2014). Eine erhöhte Mortalität nach Krankenhausaufenthalt, ein erhöhtes Demenzrisiko und ein Risiko zur weiteren Institutionalisierung besteht beim Delir älterer Patienten unabhängig von den üblichen Störgrößen (Witlox et al. 2010). 
Inwieweit eine adäquate Erkennung und Behandlung des Delirs die Mortalität günstig beeinflussen könnte, lässt sich anhand einer prospektiven Studie zum Delir in der Notaufnahme vermuten. Patienten, deren Delir nicht erkannt wurde, wiesen im Halbjahresverlauf die höchste Mortalität auf (Kakuma et al. 2003). Umso mehr erschreckt die Tatsache, dass ein Delir in bis zu zwei Dritteln der Fälle nicht erkannt wird, und dies gilt über alle Fachdisziplinen (einschließl. Psychiatrie) hinweg (Johnson et al. 1992). Neuere Ergebnisse zur Behandlung in der Intensivmedizin zeigen jedoch, dass sich die Kurzzeitmortalität durch delirspezifische Interventionen nicht reduzieren lässt (AlQadheeb et al. 2014). Prospektiv untersucht scheint ein Delir den Aufenthalt auf der Intensivstation zu verlängern, die Kurzzeitmortalität aber nicht zu beeinflussen (Klein Klouwenberg et al. 2014).

\section{Diagnosestellung}

Diagnostische Kriterien sind unverzichtbar bei der Diagnosestellung und werden deshalb ständig weiterentwickelt. Die diagnostischen Delirkriterien der WHO sind im Wesentlichen mit denen der American Psychiatric Association (APA) vergleichbar (Treloar und McDonald 1997). Trotz Weiter-entwicklung variiert jedoch die Konkordanz zwischen DSM-IV und neuen DSM-V Kriterien abhängig von deren Interpretation erheblich (Meagher et al. 2014). Kognitive Tests wie die bewährte Mini Mental State Examination (MMSE) helfen bei der Erfassung kognitiver Störungen, können aber nicht zwischen Delir und Demenz unterscheiden (Folstein et al. 1975). Zur Bewertung der Symptome sind deshalb andere Methoden erforderlich. Zum Screening, zur Diagnosestellung und zur Erfassung der Schwere des Delirs sind verschiedene Instrumente in Gebrauch. Die Confusion Assessment Method (CAM) ist das am weitesten verbreitete Screening-Instrument für das Delir im Krankenhaus (Inouye et al. 1990). Die CAM basiert auf dem DSM-III-R und existiert in mehreren Varianten. Der weit verbreitete Algorithmus mit vier Kardinalsymptomen ermöglicht nicht psychiatrisch geschulten Spezialisten und Pflegekräften die Delirerkennung (Rolfson et al. 1999). Eine Erweiterung bildet die CAM-ICU für den Gebrauch durch Pflegekräfte bei Intensivpatienten; es liegt eine deutsche Übersetzung vor (Ely et al. 2001; Guenther et al. 2010). Das am häufigsten durch Pflegekräfte eingesetzte Instrument ist die Neelon Champagne Confusion Scale (NEECHAM) (Neelon et al. 1996). Die Qualität wissenschaftlicher Unter-suchungen zu Screening-Instrumenten wird für CAM und NEECHAM als am besten beurteilt (NCGC 2010). Die Delirium Rating Scale (DRS) oder DRS-R-98 in der revidierten Variante gilt diagnostisch gesehen als am besten untersucht. Mit einer Sensitivität und Spezifität von 90-100\% eignet sie sich gut zur Abgrenzung des Delirs von anderen psychischen Erkrankungen (NCGC 2010). Eine Beurteilung des Schweregrades ist ebenfalls möglich (Wong et al. 2010). Die DSR-R-98 ermöglicht zudem die Unterscheidung zwischen motorischen Subtypen (de Rooij et al. 2006). Als kürzeres Instrument lässt sich dafür 
auch die Delirium Motor Subtype Scale (DMSS) einsetzen (Slor et al. 2014). Im Rahmen der Testdiagnostik liegt der wissenschaftliche Fokus heutzutage auf Früherkennung und subsyndromalem Delir (Meagher et al. 2014b).

Die apparative Delirdiagnostik ist wenig sensitiv und wenig spezifisch. Im EEG ruft ein Delir einen diffus verlangsamten Hintergrundrhythmus hervor, der mit dem Schweregrad korreliert (Jacobson und Jerrier 2000). Es dient vor allem zur Unterscheidung zwischen atypischen komplex partiellen Insulten und einem nicht eindeutigen Status epilepticus. Das MRT empfiehlt sich bei traumatischer Hirnschädigung, Infarkt, Hämorrhagie und metabolischen oder infektiösen Auffälligkeiten (Lai et al. 2012; Krishnan et al. 2014).

Im Zweifel gilt: Bis zum Beweis des Gegenteils ist von einem Delir auszugehen, und es sollte kausal, wenn nötig auch symptomatisch behandelt werden.

\section{Delirmanagement}

\section{Präventionsstrategien}

Am günstigsten verläuft ein Delir, das sich vermeiden lässt. Schon die präoperative Patienten-aufklärung reduziert die Zahl deliranter Symptome (Williams et al. 1985). Ein Standardprotokoll mit nichtmedikamentösen Interventionen zur Prävention von Sehund Gehörbeeinträchtigungen, Schlafstörungen, kognitiven Defiziten und Immobilisation sowie zur Flüssigkeitsaufnahme und Ernährung kann die Anzahl und Dauer der Episoden senken. Der Schweregrad bleibt unbeeinflusst. Je konsequenter das Protokoll angewendet wird (staff adherence), umso günstiger ist der Verlauf mit einer um bis zu 90\% reduzierten Delirrate (Inouye et al. 2003). Besonders bei älteren Patienten hat sich die Mehrkomponentenstrategie bewährt und ist kosteneffektiv (Bo et al. 2009; Vidan et al. 2009; Inouye et al. 2014). Die Übersichtsliteratur zur nichtmedikamentösen Prävention kommt i. Allg. zu dem Schluss, dass die Anzahl positiver Befunde noch spärlich ist (Clegg et al. 2014). Optimistischer ist die Über-sichtsliteratur zur Mehrkomponentenprävention in der Intensivmedizin. Hiermit lassen sich Inzidenz und Dauer sowie die Länge des Krankenhausaufenthalts reduzieren (Collinsworth et al. 2014; Trogrlic et al. 2015). Beindruckend ist vor allem der Erfolg der frühen Physiotherapie beatmeter Patienten (Schweikert et al. 2009). Medikamentöse Präventionsstrategien werden in der NICE-Leitlinie vorsichtig positiv beurteilt, mit geringer wissenschaftlicher Beweiskraft (NCGC 2010).

Neuere Übersichtsartikel zur prophylaktischen Pharmakotherapie betreffen die Intensivmedizin, z.T. im postoperativen Verlauf. Hirota und Kishi (2013) identifizierten für ihre Metaanalyse sechs Artikel, wobei vor allem atypische Antipsychotika die Inzidenz zu senken scheinen. In die Metaanalyse von Zhang et al. (2013) zum postoperativen Delir wurden 38 Artikel zur pharmako-logischen und nichtmedikamentösen Prävention eingeschlossen; danach sind Dexmedetomidin, Antipsychotika und die 
Mehrkomponentenintervention effektiv. Eine systematische Übersicht von zehn Studien zur medikamentösen Prävention bei kardiothorakalem Eingriff zeigt, dass neben Risperidon auch verschiedene andere Pharmaka das Delirrisiko senken können:

Dexamethason, Rivastigmin, Ketamin, Propofol, Clonidin und Dexmedetomidin. Einschränkend muss erwähnt werden, dass ein Großteil der Daten einer einzigen randomisierten Multicenterstudie entstammt ( $\mathrm{Mu}$ et al. 2015). In einem weiteren Übersichtsartikel mit 15 Präventionsstudien zu Intensivpatienten senkte die präventive Anwendung von Antipsychotika nach chirurgischen Eingriffen die Prävalenz des Delirs auf der Intensivstation (Serafim et al. 2015).

\section{Therapie}

Wegen der komplexen klinischen Situation deliranter Patienten bedarf es einer integralen multidisziplinären Zusammenarbeit (Blot et al. 2014; Flikweert et al. 2014). Konnten sich die Empfehlungen in Leitlinien bis vor Kurzem nur auf schwache wissenschaftliche Evidenz stützen, so ist inzwischen die Anzahl der Studien so weit angestiegen, dass sich Leitlinien und Behandlungs-programme zur multidisziplinären Versorgung gegenseitig befruchten (Yue et al. 2014). In den meisten Leitlinien und in der neueren Übersichtsliteratur wird eine „proaktive“ Haltung empfohlen (O'Hanlon et al. 2014). Bei einer Delirprävalenz von 10-80\% stellt sich die Frage, wie die medizinische Versorgung im Routinefall aussehen muss.

Im Gegensatz zur NICE-Leitlinie nimmt die multidisziplinäre niederländische Leitlinie dazu deutlich Stellung. Jedes Krankenhaus sollte über ein lokales multidisziplinäres Protokoll verfügen, das für alle Abteilungen (inkl. Notaufnahme und Intensivstation) Gültigkeit hat. Die Behandlung des Delirs gehört zur Grundversorgung im Krankenhaus und sollte rund um die Uhr gewährleistet sein. Es ist von einer multidisziplinären Teamleistung auszugehen, die täglich durch den behandelnden Arzt koordiniert wird. Grundsätzlich wird der Patient auf der Station behandelt, auf der sich das Delir entwickelt hat (NVKG 2013). Voraussetzung ist, dass dort die somatische, psychische, funktionelle und soziale Wiederherstellung gewährleistet werden kann (Young et al. 2010).

Kern der Behandlung ist die Therapie der dem Delir zugrunde liegenden Ursache. Man sollte umgehend mit der Diagnostik und Behandlung des Auslösers beginnen. Darüber hinaus greifen adjuvante Maßnahmen: symptomatische Pharmakotherapie, psychosoziale Unterstützung (Familie), Anpassung der Krankenbettumgebung sowie Behandlung präzipitierender Faktoren (Umstellung der Medikation, Hilfestellung bei Sehund Hörbeeinträchtigungen, schlafunterstützende Maßnahmen, Schmerztherapie, Regulierung des Flüssigkeitshaushalts, Behebung von Mangelernährung) (NCGC 2010). Feedbackhilfen im Rahmen der Pflege (Uhr, Kalender, Familienfoto, Licht) sind wichtig für die Orientierung und den zirkadianen Rhythmus. Das Einbeziehen von Angehörigen 
trägt zur Stressreduktion bei. Versorgende sollten deeskalieren und eine bevormundende und kritische Haltung vermeiden (NCGC 2010). Ein strukturierender, dabei jedoch verständnisvoller und unterstützender Umgang wirkt sich möglicherweise auch im Hinblick auf die Entwicklung einer PTBS präventiv aus (Long et al. 2014).

Das niederländische multidisziplinäre Protokoll empfiehlt die Anwendung der Pharmako-therapie-anders als die NICE-Leitlinie-auch bei der nicht hyperaktiven Form des Delirs. Die Anwendung von Antipsychotika wird in der niederländischen Leitlinie nur eingeschränkt, wenn das Delir nicht mit Angst, Verhaltensstörungen oder psychotischen Symptomen einhergeht (NVKG 2013). Die NICE-Empfehlung, ein hypoaktives Delir nicht medikamentös zu behandeln, hat Diskussionen ausgelöst. Folgende Argumente sprechen für dieses Vorgehen:

- Der Einsatz von Antipsychotika beruht auf geringer wissenschaftlicher Evidenz.

- Antipsychotika sind bislang nicht für die Indikation Delir zugelassen.

- Nebenwirkungen, vor allem eine erhöhte Schlaganfallinzidenz während einer längeren Behandlung (NCGC 2010; Inouye et al. 2014).

Aber auch der Nutzen des weniger restriktiven Ansatzes lässt sich belegen. Antipsychotika sollten beim hypoaktiven Delir mit Angst- oder psychotischer Symptomatik eingesetzt werden, weil:

- die wissenschaftliche Evidenz (wenn auch bisher geringerer Qualität) konsistent in Richtung Wirksamkeit weist;

- kleinere Studien, die in der NICE-Leitlinie keine Berücksichtigung fanden, in dieselbe Richtung weisen;

- sich ein erhöhtes Apoplexrisiko nur bei längerer Einnahme ergibt;

- Patienten mit hypoaktivem Delir die schlechteste Prognose haben (Maher et al. 2011; Mittal et al. 2011; Leentjens et al. 2014).

Neuere Literatur weist darauf hin, dass klassische und atypische Antipsychotika gleich wirksam sind, und zwar unabhängig vom Subtyp, auch wenn höheres Lebensalter die Wirksamkeit zu mindern scheint (Meagher et al. 2013; Yoon et al. 2013). Dies geht bei den klassischen Antipsychotika beim älteren Patienten nicht mit einer erhöhten Mortalität einher (Hulshof et al. 2015). Kritisch ist anzumerken, dass Patienten mit Demenz in pharmazeutischen Studien zur Prophylaxe und Therapie des Delirs unterrepräsentiert bleiben, was die Übertragbarkeit der Ergebnisse erschwert (de Jonghe et al. 2014).

Die symptomatische Pharmakotherapie hat ihren praktischen Wert unter Beweis gestellt. Die am häufigsten verwendete Substanz ist Haloperidol (NCGC 2010; NVKG 2013). Die Verabreichung erfolgt in der Regel oral oder intramuskulär. Bei vorhandenem intravenösen Zugang kommt ggf. auch ein niedriger dosierter i.v. Bolus infrage. Für die palliative Situation in Pflegeheimen besteht auch eine subkutane Anwendungsmöglichkeit (Tanguy-Goarin und Cogulet 2010). Bei Erregungszuständen ist auch der zusätzliche Einsatz von Benzodiazepinen zu erwägen (AWMF 2010; NVKG 2013). In der neueren Literatur zum nicht entzugsbedingten Delir werden auch andere Medikamente als 
effektiv bewertet: atypische Antipsychotika, Gabapentin, Melatonin, Dexmedetomidin. Alles in allem ist der Präventionseffekt jedoch höher zu bewerten als der Therapieeffekt (Friedman et al. 2014). Zum vielversprechenden Einsatz von Alpha-2-Agonisten laufen zur Zeit Studien (Neerland et al. 2015).

Eine Alternative zum Haloperidol ist bei Patienten wichtig, denen man kein klassisches Antipsychotikum verabreichen sollte, z. B. Patienten mit Morbus Parkinson. Die Anwendungssicherheit wird allgemein durch Nebenwirkungen bestimmt (Grover et al. 2011). Neben Tremor, Rigidität und Konstipation bestehen potenziell letale Nebenwirkungen wie das maligne neuroleptische Syndrom (MNS) oder eine QT-ZeitVerlängerung im EKG mit möglichen polymorphen ventrikulären Tachykardien (MeyerMassetti et al. 2011). Die niederländische Leitlinie empfiehlt, vor und nach Verabreichung einer Haloperidol-Dosis von $>2 \mathrm{mg}$ i.v. ein EKG anzufertigen (NVKG 2013). Für demente Patienten besteht bei atypischen Antipsychotika neben einem erhöhten Risiko von Harnwegsinfekten und extrapyramidalen Symptomen auch ein leicht erhöhtes Mortalitätsrisiko (Schneider et al. 2005, 2006).

Die Pharmakotherapie des Delirium tremens dient der Sedierung; sie muss die Krampfschwelle erhöhen, autonome Überaktivität mindern, antipsychotisch wirksam sein und hat während des Krankenhausaufenthaltes stattzufinden (Einweisungsindikation) (Deutsche Gesellschaft für Neurologie 2012). Während die Clinical Institute Withdrawal Assessment Scale (CIWA-Skala) ein etabliertes Instrument zur Feststellung des Entzugsschweregrades ist, fehlen prädiktive Instrumente in der Intensivmedizin (Sullivan et al. 1989; Maldonado et al. 2014). Beim Entzugsdelir muss vor der Glukose-Kalium-Infusion oder Nahrungsaufnahme Thiamin (50-100mg i.v.) zur Vermeidung einer Wernicke-Korsakow-Enzephalopathie parenteral gegeben werden. Eine bilanzierte Flüssigkeitszufuhr mit Elektrolytausgleich muss wegen der Gefahr einer pontinen Myelinolyse langsam erfolgen. Bei Multimorbidität (Alkoholabhängigkeit plus somatische Erkrankung) empfiehlt sich, Lorazepam mit Haloperidol zu kombinieren (Amato et al. 2010; Deutsche Gesellschaft für Neurologie 2012).

\section{Zukünftige Entwicklung}

Die integrale multidisziplinäre Versorgung (zeitgleiche und koordinierte Zusammenarbeit verschiedener Disziplinen) gehört bei Geriatern, Psychiatern, Psychosomatikern, Neurologen oder Intensivmedizinern zum konsiliarischen Deliralltag (Barr und Pandharipande 2013). Defizite zur integralen pädiatrischen Delirversorgung und zur multidisziplinären Palliativmedizin werden gerade angegangen (Bush et al. 2014; Turkel und Hanft 2014).

Ein kritischer Punkt im Delirverlauf ist ähnlich wie bei anderen schweren, komplexen oder chronischen Erkrankungen der Moment des Versorgungsübergangs von der Intensivstation auf die Normalstation, vom Krankenhaus zum Hausarzt, Pflegeheim 
oder Hospiz (Coleman und Berenson 2004). Will die Delirbehandlung (kosten-) effektiv sein, muss sie diese Versorgungsübergänge einbeziehen; dies gilt auch für die Unterstützung von Familienmitgliedern (Coleman et al. 2015). Zukünftige Modelle erfordern multidisziplinäre Programme, welche die Versorgungsübergänge mit berücksichtigen (integrated transitional delirium care). Zu diesem Programm gehört angesichts der Risikofaktoren, der differenzialdiagnostischen Aspekte und einer etwaigen neuropsychiatrischen Anschlussbehandlung selbstverständlich ein Konsiliarpsychiater oder Psychosomatiker. 


\section{Literaturauswah|}

Ahmed S, Leurent B, Sampson EL (2014). Risk factors for incident delirium among older people in acute hospital medical units: A systematic review and meta-analysis. Age Ageing 43: 326-333.

Al-Qadheeb NS, Balk EM, Fraser GL, et al. (2014). Randomized ICU trials do not demonstrate an association between interventions that reduce delirium duration and short-term mortality: A systematic review and meta-analysis. Crit. Care Med. 42(6): 1442-1454.

Amato L, Minozzi S, Vecchi S, et al. (2010). Benzodiazepines for alcohol withdrawal. Cochrane Database Syst Rev 3: CD005063.

APA - American Psychiatric Association (2013). Diagnostic and Statistical Manual of Mental Disorders, $5^{\text {th }}$ ed. Washington, DC: American Psychiatric Association Publishing.

Andrew MK, Freter SH, Rochwood K (2006). Prevalence and outcomes of delirium in community and non-acute care settings in people without dementia: A report from the Canadian study of health and aging. BMC Med. 4: 15.

Arinzon Z, Peisakh A, Schrire S, Berner YN (2011). Delirium in long-term care setting: Indicator to severe morbidity. Arch. Gerontol. Geriatr. 52: 270-275.

Armstrong SC, Cozza KL, Watanabe KS (1997). The misdiagnosis of delirium. Psychosomatics 38: 433-439.

AWMF-Arbeitsgemeinschaft der Wissenschaftlichen Medizinischen Fachgesellschaften (2010). Leitlinie: Analgesie, Sedierun und Delirmanagement in der Intensivmedizin. (letzter Zugriff: 12.4.2016).

www.dggeriatrie.de/images/stories/pdf/analgesie_sedierung_und_delirmanagement_in_der_intensivmedizin. pdf

Barr J, Pandharipande PP (2013). The pain, agitation, and delirium care bundle: Synergistic benefits of implementing the 2013 Pain, Agitation, and Delirium Guidelines in an integrated and interdisciplinary fashion. Crit. Care. Med. 41(9Suppl1): S99-115.

Blot S, Afonso E, Labeau S (2014). Insights and advances in multidisciplinary critical care: A review of recent research. Am. J. Crit. Care 23: 70-80.

Bo M, Martini B, Ruatta C, et al. (2009). Geriatric ward hospitalization reduced incidence delirium among older medical inpatients. Am. J. Geriatr. Psychiatry 17: 760-768.

Boorsma M, Joling KJ, Frijters DH, et al. (2012). The prevalence, incidence and risk factors for delirium in Dutch nursing homes and residential care homes. Int. J. Geriatr. Psychiatry 27: 709-715.

Breitbart W, Gibson C, Tremblay A (2002). The delirium experience: delirium recall and delirium-related distress in hospitalized patients with cancer, their spouses/caregivers, and their nurses. Psychosomatics 43: 183-194.

Brouquet A, Cudennec T, Benoist S, et al. (2010). Impaired mobility, ASA status, and administration of tramadol are risk factors for postoperative delirium in patients aged 75 years or more after major abdominal surgery. Ann. Surg. 251: 759-765.

Brown CH (2014). Delirium in the cardiac surgical ICU. Curr. Opin. Anaesthesiol. 27: 117-122.

Brown TM (2000). Drug induced delirium. Semin. Clin. Neuropsychiatry 5: 113-125.

Bush SH, Bruera E, Lawlor PG, et al. (2014). Clinical practice guidelines for delirium management: Potential application in palliative care. J. Pain. Symptom Manage. 48: 249-258.

Campbell N, Boustani M, Limbil T, et al. (2009). The cognitive impact of anticholinergics: A clinical review. Clin. Interven. Ageing 4: 225-233.

Camus V, Gonthier R, Dubos G, et al. (2000). Etiologic and outcome profiles in hypoactive and hyperactive subtypes of delirium. J. Geriatr. Psychiatry Neurol. 13: 38-42.

Cape E, Hall RJ, van Munster BC, et al. (2014). Cerebrospinal fluid markers of neuroinflammation in delirium: a role for interleukin-1 $\beta$ in delirium after hip fracture. J. Psychosom. Res. 77: 219-225.

Caplan GA, Kvelde T, Lai C, et al. (2010). Cerebrospinal fluid in long-lasting delirium compared with Alzheimer's dementia. J. Gerontol. A. Biol. Sci. Med. Sci. 65: 1130-1136.

Cerejeira J, Lagarto L. Mukaetova-Ladinska EB (2014). The immunology of delirium. Neuroimmunomodulation 21: $72-78$.

Chaisuksunt V, Campbell G, Zhang Y, et al. (2003). Expression of regeneration-related molecules in injured and regenerating striatal and nigral neurons. J. Neurocytol. 32: 161-183. 
Clegg A, Siddiqi N, Heaven A, et al. (2014). Interventions for preventing delirium in older people in institutional long-term care. Cochrane Database Syst. Rev. 31: CD009537.

Cole M, McCusker J, Dendukuri N, et al. (2003). The prognostic significance of subsyndromal delirium in elderly medical inpatients. J. Am. Geriatr. Soc. 51: 754-760.

Coleman EA, Berenson RA (2004). Lost in transition: challenges and opportunities for improving the quality of transitional care. Ann. Intern. Med. 141: 533-536.

Coleman EA, Roman SP, Hall KA, Min SJ (2015). Enhancing the care transitions intervention protocol to better address the needs of family caregivers. J. Health Qual. 37(1): 2-11.

Collins N, Blanchard MR, Tookman A, Sampson EL (2010). Detection of delirium in the acute hospital. Age Ageing 39: 131-135.

Collinsworth AW, Priest EL, Campbell CR, et al. (2014). A review of multifaceted care approaches for the prevention and mitigation of delirium in intensive care units. J. Intensive Care Med. 31(2): 127-141.

Colville G, Pierce C (2012). Patterns of post-traumatic stress symptoms in families after paediatric intensive care. Intensive Care Med. 38: 1523-1531.

Daoud A, Duff JP, Joffe AR (2014). Diagnostic accuracy of delirium diagnosis in pediatric intensive care: A systematic review. Crit. Care 18: 489.

De Castro SM, Ünlü C, Tuynman JB, et al. (2014). Incidence and risk factors of delirium in the elderly general surgical patient. Am J Surg 208: 26-32.

De Jonghe A, van Munster BC, Fekkes D, et al. (2012). The tryptophan depletion theory in delirium: not confirmed in elderly hip fracture patients. Psychosomatics 53: 236-243.

De Jonghe A, van de Glind EM, van Munster BC, de Rooij SE (2014). Underrepresentation of patients with preexisting cognitive impairment in pharmaceutical trials on prophylactic or therapeutic treatments for delirium: A systematic review. J. Psychosom. Res. 76: 193-199.

De Rooij SE, van Munster BC, Korevaar JC, et al. (2006). Delirium subtype identification and the validation of the delirium rating scale-revised-98 (Dutch version) in hospitalized elderly patients. Int. J. Geriatr. Psychiatry 21: 876-882.

Deutsche Gesellschaft für Neurologie (2012). Leitlinien für Diagnostik und Therapie in der NeurologieAlkoholdelir und Verwirrtheitszustände.www.dgn.org/leitlinien/11-leitlinien-der-dgn/3056-|I-85-IIalkoholdelir-und-verwirrtheitszustaende (letzter Zugriff: 12.4.2016).

Ely EW, Inouye SK, Bernard GR, et al. (2001). Delirium in mechanically ventilated patients: validity and reliability of the Confusion Assessment Method for the Intensive Care Unit (CAM-ICU). JAMA 286: 2703-2710.

Ely EW, Shintani A, Truman B, et al. (2004). Delirium as a predictor of mortality in mechanically ventilated patients in the intensive care unit. JAMA 51: 1753-1762.

European Delirium Association, American Delirium Society (2014). The DSM-V criteria, level of arousal and delirium diagnosis. BMC Med. 12: 141.

Flikweert ER, Izaks GJ, Knobben BA, et al. (2014). The development of a comprehensive multidisciplinary care pathway for patients with hip fracture: Design and results of a clinical trial. BMC Musculoskelet Disord 15: 188.

Folstein MF, Folstein SE, McHugh PR (1975). Mini-Mental State: A practical method for grading the cognitive state of patients for the clinician. J. Psychiatr. Res. 12: 189-198.

Franco JG, Trzepacz PT, Meagher DJ, et al. (2013). Three core domains of delirium validated using exploratory and confirmatory factor analyses. Psychosomatics 54: 227-238.

Friedman JI, Soleimani L, McGonigle DP, et al. (2014). Pharmacological treatments of non-substancewithdrawal delirium: A systematic review of prospective trials. Am. J. Psychiatry 171: 151-159.

Gore RL, Vardy ER, O'Brien JT (2015). Delirium and dementia with Lewy bodies: Distinct diagnoses or part of the same spectrum? J. Neurol. Neurosurg. Psychiatry 86: 50-59.

Grover S, Mattoo SK, Gupta N (2011). Usefulness of atypical antipsychotics and choline esterase inhibitors in delirium: A review. Pharmacopsychiatry 44: 43-54.

Guenther U, Popp J, Koecher L, et al. (2010). Validity and reliability of the CAM-ICU flow sheet to diagnose delirium in surgical ICU patients. J. Crit. Care 25: 144-151.

Hatherill S, Flisher AJ (2010). Delirium in children and adolescents: A systematic review of the literature. J. Psychosom. Res. 68: 337-344.

Huai J, Ye X (2014). A meta-analysis of critically ill patients reveals several potential risk factors for delirium. Gen. Hosp. Psychiatry 36: 488-496. 
Hughes CG, Patel MB, Pandharipande PP (2012). Pathophysiology of acute brain dysfunction: What's the cause of all this confusion? Curr. Opin. Crit. Care 18: 518-526.

Hulshof TA, Zuidema SU, Ostelo RW, Luijendijk HJ (2015). The mortality risk of conventional antipsychotics in elderly patients: A systematic review and meta-analysis of randomized placebo-controlled trials. J. Am. Med. Dir. Assoc. 16(10): 817-824.

Hirota T, Kishi T (2013). Prophylactic antipsychotic use for postoperative delirium: A systematic review and meta-analysis. J Clin Psychiatry 74: e1136-1144.

Inouye SK, van Dyke CH, Alessi CA, et al. (1990). Clarifying confusion: The Confusion Assessment Method. Ann. Intern. Med. 113: 941-948.

Inouye SK, Charpentier PA (1996). Precipitating factors for delirium in hospitalized elderly patients: predictive model and interrelationships with baseline vulnerability. JAMA 275: 852-857.

Inouye SK, Bogardus ST, Williams CS, et al. (2003). The role of adherence on the effectiveness of nonpharmacological interventions: Evidence from the Delirium Prevention Trial. Arch. Intern. Med. 163: 958-964.

Inouye SK, Westendorp RG, Saczynski JS (2014). Delirium in elderly people. Lancet 383(9920): 911-922.

Jackson JC, Gordon SM, Hart RP, et al. (2004). The association between delirium and cognitive decline: A review of the empirical literature. Neuropsychol Rev. 14: 87-98.

Jacobson SA, Jerrier S (2000). EEG in delirium. Semin. Clin. Neuropsychiatry 5: 86-93.

Johnson JC, Kerse NM, Gottlieb G, et al. (1992). Prospective versus retrospective methods of identifying patients with delirium. J. Am. Geriatr. Soc. 40: 316-319.

Kakuma R, du Fort GG, Arsenault L, et al. (2003). Delirium in older emergency department patients discharged home: Effect on survival. J. Am. Geriatr. Soc. 51: 443-450.

Kelly KG, Zisselman M, Cutillo-Schmitter T, et al. (2001). Severity and course of delirium in medically hospitalized nursing facility residents. Am. J. Geriatr. Psychiatry 9: 72-77.

Kelly P, Frosch E (2012). Recognition of delirium on pediatric hospital services. Psychosomatics 53: 446-451.

Kelslo ML, Oestreich JH (2012). Traumatic brain injury: Central and peripheral role of $\alpha 7$ nicotinic acetylcholine receptors. Curr. Drug. Targets 13: 631-636.

Kiely DK, Bergmann MA, Murphy KM, et al. (2003). Delirium among newly admitted post-acute facility patients: Prevalence, symptoms and severity. J. Gerontol. A. Biol. Sci. Med. Sci. 58: M441-445.

Kishi Y, Kato M, Okuyama T, et al. (2007). Delirium: Patient characteristics that predict a missed diagnosis at psychiatric consultation. Gen. Hosp. Psychiatry 29: 442-445.

Klein Klouwenberg PM, Zaal IJ, Spitoni C, et al. (2014). The attributable mortality of delirium in critically ill patients: Prospective cohort study. BMJ 349: g6652.

Kosar CM, Tabloski PA, Travison TG, et al. (2014). Effect of preoperative pain and depressive symptoms on the development of postoperative delirium. Lancet Psychiatry 1: 431-436.

Krishnan V, Leung LY, Caplan LR (2014). A neurologist's approach to delirium: Diagnosis and management of toxic metabolic encephalopathies. Eur. J. Intern. Med. 25: 112-116.

Krogseth M, Wyller TB, Engedal K, Juliebo V (2014). Delirium is a risk factor for institutionalization and functional decline in older hip fracture patients. J. Psychosom. Res. 76: 68-74.

Lai MM, Wong Tin Niam DM (2012). Intracranial cause of delirium: Computed tomography yield and predictive factors. Intern. Med. J. 42: 422-427.

Lauretani F, Ceda GP, Maggio M, et al. (2010). Capturing side-effect of medication to identify persons at risk of delirium. Aging. Clin. Exp. Res. 22: 456-448.

Laurila JV, Pitkala KH, Strandberg TE, Tilvis RS (2004). Impact of different diagnostic criteria on prognosis of delirium: A prospective study. Dement. Geriatr. Cogn. Disord. 18: 240-244.

Lawlor PG, Bush SH (2015). Delirium in patients with cancer: Assessment, impact, mechanisms and management. Nat. Rev. Clin. Oncol. 12: 77-92.

Lawlor PG, Gagnon B, Mancini IL, et al. (2000). Occurrence causes and outcomes of delirium in advanced cancer. Arch. Intern. Med. 160: 786-794.

Leentjens AF, Molag ML, van Munster BC, et al. (2014). Changing perspectives on delirium care: the new Dutch guideline on delirium. J. Psychosom. Res. 77: 240-241.

Levkoff SE, Liptzin B, Evans D, et al. (1994). Progression and resolution of delirium in elderly patients hospitalized for acute care. Am. J. Geriatr. Psychiatry 2: 230-238. 
Ljubisavljevic V, Kelly B (2003). Risk factors for development of delirium among oncology patients. Gen. Hosp. Psychiatry 25: 345-352.

Long AC, Kross EK, Davydow DS, Curtis JR (2014). Posttraumatic stress disorder among survivors of critical illness: Creation of a conceptual model addressing identification, prevention, and management. Intensive Care Med. 40: 820-829.

Lundberg AS, Gustafsson LN, Meagher D, Munk-Jorgensen P (2014). Delirium during psychiatric admission increases mortality in psychiatric patients during and after hospitalization. A nationwide study from 1995 through 2012. J. Psychosom. Res. 77: 226-231.

MacLullich AM, Anand A, Davis DH, et al. (2013). New horizons in the pathogenesis, assessment, and management of delirium. Age Ageing 42: 667-674.

Maher AR, Maglione M, Bagley S, et al. (2011). Efficacy and comparative effectiveness of atypical antipsychotic medications for off-label uses in adults: A systematic review and meta-analysis. JAMA 306: 1359-1369.

Maldonado JR (2013). Neuropathogenesis of delirium: review of current etiologic theories and common pathways. Am. J. Geriatr. Psychiatry 21: 1190-1222.

Maldonado JR, Sher Y, Ashouri JF, et al. (2014). The „Prediction of Alcohol Withdrawal Severity Scale“ (PAWSS): Systematic literature review and pilot study of a new scale for the prediction of complicated alcohol withdrawal syndrome. Alcohol 48: 375-390.

McCarthy MC (2003). Detecting acute confusion in older adults: Comparing clinical reasoning of nurses working in acute, long-term, and community health care environments. Res. Nurs. Health 26: 203-212.

McCusker J, Cole MG, Dendukuri N, et al. (2003). The course of delirium in older medical inpatients: A prospective study. J. Gen. Intern. Med. 18: 696-704.

McNicoll L, Pisani MA, Zhang Y, et al. (2003). Delirium in the intensive care unit: occurrence and clinical course in older patients. J. Am. Geriatr. Soc. 51: 591-598.

Meagher DJ, O'Hanlon D, O'Mahony E, et al. (1996). Use of environmental strategies and psychotropic medication in the management of delirium. Br. J. Psychiatry 168: 512-515.

Meagher DJ, Moran M, Raju B, et al. (2008). A new data-based motor subtype schema for delirium. J. Neuropsychiatry Clin. Neurosci. 20: 185-193.

Meagher DJ, Leonard M, Donnelly S, et al. (2011). A longitudinal study of motor subtypes in delirium: relationship with other phenomenology, aetiology, medication exposure and prognosis. J. Psychosom. Res. 71: 395-403.

Meagher DJ, Leonard M, Donnelly S, et al. (2012). A longitudinal study of motor subtypes in delirium: Frequency and stability during episodes. J. Psychosom. Res. 72: 236-241.

Meagher DJ, McLoughlin, Leonard M, et al. (2013). What do we really know about the treatment of delirium with antipsychotics? Ten key issues for delirium pharmacotherapy. Am. J. Geriatr. Psychiatry 21: 12231238.

Meagher DJ, Morandi A, Inouye SK, et al. (2014a). Concordance between DSM-IV and DSM-V criteria for delirium diagnosis in a pooled database of 768 prospectively evaluated patients using the delirium rating scale-revised-98. BMC Med. 12: 164.

Meagher DJ, O'Regan, Ryan D, et al. (2014b). Frequency of delirium and subsyndromal delirium in an adult acute hospital population. Br. J. Psychiatry 205: 478-485.

Meyer-Massetti C, Vaerini S, Rätz Bravo AE, et al. (2011). Comparative safety of antipsychotics in the WHO pharmacovigilance database: The haloperidol case. Int. J. Clin. Pharm. 33: 806-814.

Mittal V, Kurup L, Williamson D, et al. (2011). Risk of cerebrovascular adverse events and death in elderly patients with dementia when treated with antipsychotic medication: A literature review of evidence. Am. J. Alzheimers Dis. Other Demen. 26: 10-28.

Morrison RS, Magaziner J, Gilbert M, et al. (2003). Relationship between pain and opioid analgesics on the development of delirium following hip fracture. J. Gerontol. A. Biol. Sci. Med. Sci. 58: 76-81.

Mu JL, Lee A, Joynt GM (2015). Pharmacological agents for the prevention and treatment of delirium in patients undergoing cardiac surgery: Systematic review. Crit. Care Med. 43: 194-204.

NCGC - National Clinical Guideline Centre (2010). Delirium: Prevention, diagnosis, and management. (letzter Zugriff: 12.4.2016). www.nice.org.uk/guidance/cg103

NVKG - Nederlandse Vereniging voor Klinische Geriatrie (2013). Richtlijn Delir Volwassenen. (letzter Zugriff: 12.4.2016). www.nvkg.nl/uploads/bB/zw/bBzwOYCHaGtVRSwyW-epTw/Richtlijn-Delier-Volwassenenvoor-autorisatie.pdf 
Neelon VJ, Champagne MT, Carlson JR, et al. (1996). The NEECHAM Scale: Construction, validation, and clinical testing. Nurs. Res. 45: 324-330.

Neerland BE, Roksund Hov K, Bruun Wyller V, et al. (2015). The protocol of the Oslo study of clonidine in elderly patients with delirium; LUCID: A randomised placebo-controlled trial. BMC Geriatr. 15: 7.

O'Hanlon S, O'Regan N, MacLullich AM, et al. (2014). Improving delirium care through early intervention: From bench to bedside to boardroom. J. Neurol. Neurosurg. Psychiatry 85: 207-213.

Olofsson SM, Wetzner MA, Valentine AD, et al. (1996). A retrospective study of the psychiatric management and outcome of delirium in the cancer patient. Support. Care Cancer 4: 351-357.

Pandharipande P, Cotton BA, Shintani A, et al. (2008). Prevalence and risk factors for the development of delirium in surgical and trauma intensive care unit patients. J Trauma 65: 34-41.

Pandharipande PP, Girard TD, Jackson JC, et al. (2013). Long-term cognitive impairment after critical illness. N. Engl. J. Med. 369: 1306-1316.

Peterson JF, Truman B, Shintani A, et al. (2003). The prevalence of delirium subtypes in medical ICU patients. J. Am. Geriatr. Soc. 51: S174.

Peterson JF, Pun BT, Dittus RS, et al. (2006). Delirium and its motoric subtypes: A study of 614 critically ill patients. J. Am. Geriatr. Soc. 54: 479-484.

Pisani MA, Kong SY, Kasl SV, et al. (2009). Days of delirium are associated with 1-year mortality in an older intensive care unit population. Am. J. Respir. Crit. Care Med. 180: 1092-1097.

Pop J (2013). Delirium and cognitive decline: More than a coincidence. Curr. Opin. Neurol. 26: 634-639.

Robinson MJ (2002). Probable Lewis body dementia presenting as delirium. Psychosomatics 43: 84-86.

Rolfson DB, McElhaney JE, Jhangri GS, et al. (1999). Validity of the confusion assessment method in detecting post-operative delirium in the elderly. Int. Psychogeriatr. 11: 431-438.

Ryan DJ, O'Regan NA, Caoimh RO, et al. (2013). Delirium in an adult acute hospital population: predictors, prevalence and detection. BMJ Open 3 pii: e001772.

Salluh JI, Wang H, Schneider EB, et al. (2015). Outcome of delirium in critically ill patients: systematic review and meta-analysis. BMJ 350: h2538.

Schieveld JN, Janssen NJ (2014). Delirium in the pediatric patient: on the growing awareness of its clinical interdisciplinary importance. JAMA Pediatr. 168: 595-596.

Schneider LS, Dragerman KS, Insel P (2005). Risk of death with atypical antipsychotic drug treatment for dementia: Meta-analysis of randomized placebo-controlled trials. JAMA 15: 1934-1943.

Schneider LS, Dragerman KS, Insel P (2006). Efficacy and adverse effects of atypical antipsychotics for dementia: Meta-analysis of randomized placebo-controlled trials. Am. J. Geriatr. Psychiatry 14: 191-210.

Schoen J, Meyerrose J, Paarmann H, et al. (2011). Preoperative regional cerebral oxygen saturation is a predictor of postoperative delirium in on-pump cardiac surgery patients: A prospective observational trial. Crit Care 15: R218.

Schweikert WD, Pohlman MC, Pohlman AS, et al. (2009). Early physical and occupational therapy in mechanically ventilated, critically ill patients: A randomised controlled trial. Lancet 373: 1874-1882.

Serafim RB, Bozza FA, Soares M, et al. (2015). Pharmacological prevention and treatment of delirium in intensive care patients: A systematic review. J. Crit. Care 30(4): 799-807.

Slor CJ, Adamis D, Jansen RW, et al. (2013). Delirium motor subtypes in elderly hip fracture patients: Risk factors, outcomes and longitudinal stability. J. Psychosom. Res. 74: 444-449.

Slor CJ, Adamis D, Jansen RW, et al. (2014). Validation and psychometric properties of the Delirium Motor Subtype Scale in elderly hip fracture patients (Dutch version). Arch. Gerontol. Geriatr. 58: 140-144.

Stagno D, Gibson C, Breitbart W (2004). The delirium subtypes: a study of prevalence, phenomenology, pathophysiology, and treatment response. Palliat. Support. Care 2: 171-179.

Sullivan JT, Sykora K, Schneidermann J, et al. (1989). Assessment of alcohol withdrawal: The revised clinical institute withdrawal assessment for alcohol scale (CIWA-Ar). Br. J. Addict. 84: 1353-1357.

Tanguy-Goarin C, Cogulet V (2010). Drugs administration by subcutaneous injection within palliative care. Therapie 65: 525-531.

Thomasson JW, Shintani A, Peterson JF, et al. (2005). Intensive care unit delirium is an independent predictor of longer hospital stay: A prospective analysis of 261 non-ventilated patients. Crit. Care 9: R375-381.

Treloar AJ, Mcdonald AJ (1997). Outcome of delirium, part I: Outcome of delirium diagnosed by DSM III-R, ICD10 and CAMDEX and derivation of the Reversible Cognitive Dysfunction Scale among acute geriatric patients. Int. J. Geriatr. Psychiatry 12: 609-613. 
Trogrlic Z, van der Jagt M, Bakker J, et al. (2015). A systematic review of implementation strategies for assessment, prevention, and management of ICU delirium and their effect on clinical outcomes. Crit. Care 19: 157.

Trzepacz PT, Mittal D, Torres R, et al. (2002). Delirium vs. Dementia symptoms: Delirium Rating Scale-Revised98 (DRS-R-98) and Cognitive Test for Delirium (CTD) item comparisons. Psychosomatics 43: 156-157.

Trzepacz PT, Franco JG, Meagher DJ, et al. (2012). Phenotype of subsyndromal delirium using pooled multicultural Delirium Rating Scale-Revised-98 data. J. Psychosom. Res. 73: 10-17.

Turkel SB, Hanft A (2014). The pharmacological management of delirium in children and adolescents. Pediatr. Drugs 16: 267-274.

Turkel SB, Trzepacz PT, Tavare CJ (2006). Comparison symptoms of delirium in adults and children. Psychosomatics 47: 320-324.

Van Munster BC, de Rooij SE (2014). Delirium: A synthesis of current knowledge. Clin. Med. 14: 192-195.

Vidan MT, Sanchez E, Alonso M, et al. (2009). An intervention integrated into daily clinical practice reduces the incidence of delirium during hospitalization in elderly patients. J. Am. Geriatr. Soc. 57: 2029-2036.

Webster R, Holroyd S (2000). Prevalence of psychotic symptoms in delirium. Psychosomatics 41: 519-522.

WHO - World Health Organization (1992). International Statistical Classification of Diseases and Related Health Problems. $10^{\text {th }}$ Revision. Geneva: WHO.

Williams MA, Campbell EB, Raynor WJ, et al. (1985). Reducing acute confusional states in elderly patients with hip fractures. Res. Nurs. Health 8: 329-337.

Witlox J, Eurlings LS, de Jonghe JF, et al. (2010). Delirium in elderly patients and the risk of postdischarge mortality, institutionalization, and dementia: A meta-analysis. JAMA 304: 443-451.

Wong CL, Holroyd-Leduc J, Simel DL, Straus SE (2010). Does this patient have a delirium?: Value of bedside instruments. JAMA 304: 779-786.

Yoon HJ, Park KM, Choi WJ, et al. (2013). Efficacy and safety of haloperidol versus atypical antipsychotic medications in the treatment of delirium. BMC Psychiatry 13: 240.

Young J, Murthy L, Westby M, et al. (2010). Diagnosis, prevention and management of delirium: A summary of NICE guidance. BMJ 341: c3704.

Yue J, Tabloski P, Dowal SL, et al. (2014). The National Institute for Health and Clinical Excellence (NICE) to Hospital Elder Life Program (HELP): Operationalizing Nice guidelines to improve clinical practice. J. Am. Geriatr. Soc. 62: 754-761.

Zaal IJ, Devlin JW, Peelen LM, et al. (2015). A systematic review of risk factors for delirium in the ICU. Crit. Care Med. 43: 40-47.

Zhang H, Lu Y, Liu M, et al. (2013). Strategies for prevention of postoperative delirium: A systematic review and meta-analysis of randomized trials. Crit. Care 17: R47. 



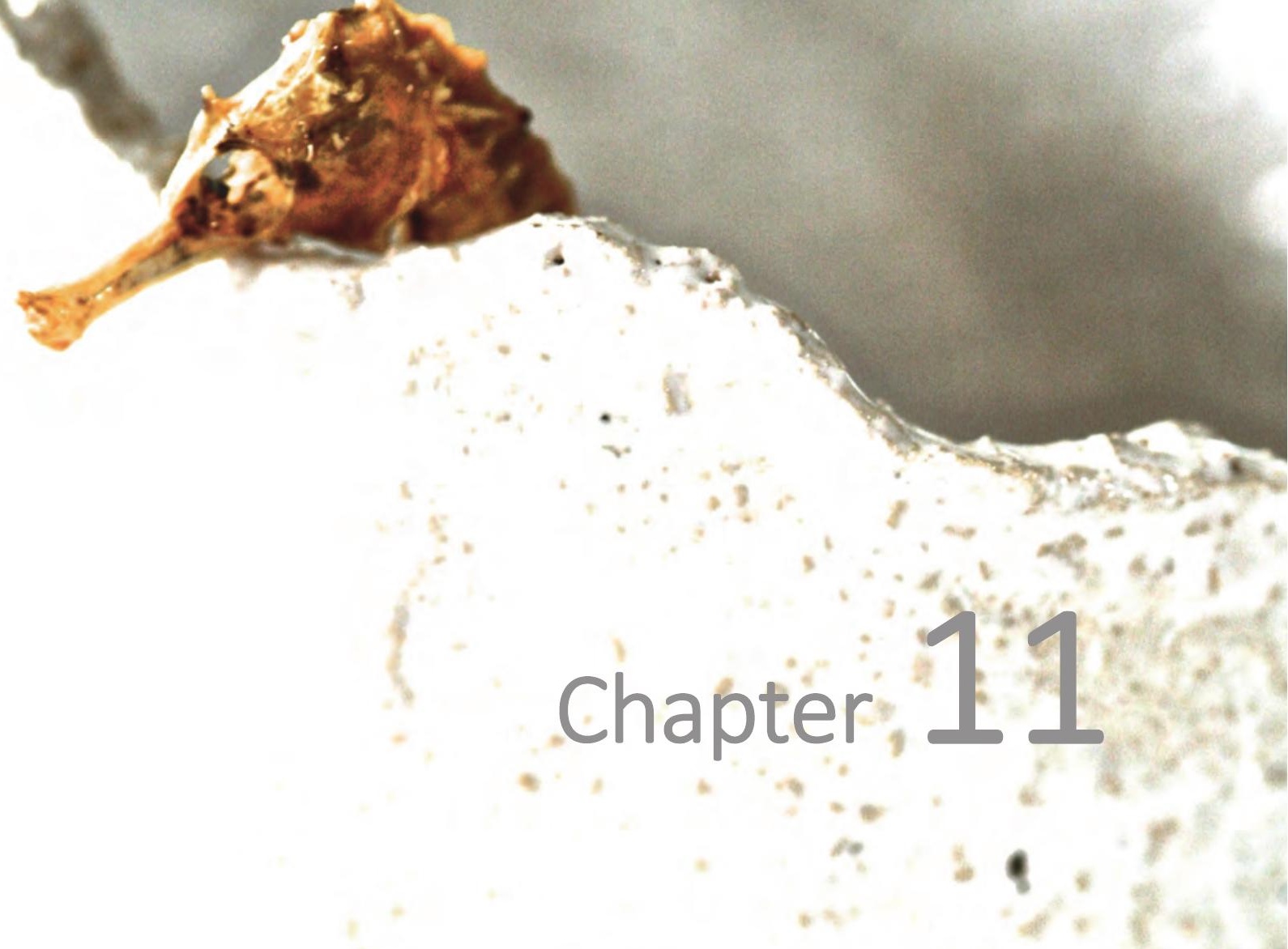

Chronic pain and psychiatry - an âppeal for integrated care beyond the pain depression diade [Chronische pijn en psychiatrie - een,betoog voor integrale zorg voorbij de pijn-depressie diade]

Leue C

Handboek Psychiatrie in het Ziekenhuis. 1e Editie. Hoofdstuk 19 (2018). In press. 


\section{Samenvatting}

Psychiatrie en somatiek ontmoeten elkaar vaak niet als het gaat om chronische pijnpatiënten. Dit is allesbehalve terecht, aangezien er ruim overlap bestaat in symptomatologie en etiologie van pijn- en affectieve stoornissen. In dit hoofdstuk worden gemeenschappelijke neuronale, inflammatoire en gedragsmatige aspecten van beide condities belicht en wordt uiteengezet waarom integrale zorg teleurstellende behandelresultaten zou kunnen voorkomen. In deze is het aan de psychiater om de kennis over antidepressiva en psychotherapie met zijn multidisciplinaire collega's te delen. Hierin biedt geïntegreerde psychiatrische en somatische zorg op zowel de medisch-psychiatrische unit (MPU) als ook binnen de consultatieve- en liaison psychiatrische dienst in een ziekenhuis kansen op verbetering van complexe zorg. Verder wordt aangestipt hoe multidisciplinair wetenschappelijk onderzoek remedie kan bieden tegen onderdiagnostiek, onderbehandeling en beperkte uitkomstmeting. 


\section{Inleiding}

Pijn ontstaat als gevolg van een verwonding, ontsteking of andere pathologie die van invloed is op somatosensorische paden van het perifere en centrale zenuwstelsel. Een perifere pijnstimulus gaat via primair sensorische neuronen langs de dorsale horens en de opstijgende pijnbanen van het ruggenmerg naar diverse hersenstructuren. In het ruggenmerg wordt als tegenregulatie via dalende banen vanuit de hersenstam pijn tot een homeostatisch evenwicht onderdrukt. Dit evenwicht weerspiegelt de staat van bedreiging uitgaande van het verwondend gevaar. Neurotransmitters die hierbij een rol spelen zijn serotonine en noradrenaline. Dysfunctionele processen binnen dit homeostatisch systeem kunnen een falende pijnonderdrukking tot gevolg hebben ${ }^{1}$. Pijn kent sensorische, gedragsmatige, cognitieve maar ook emotionele of affectieve aspecten. De affectieve component omvat niet alleen depressieve klachten maar ook angstgerelateerde symptomen. Vooral chronische pijn en depressie komen in hoge mate samen voor. Conceptueel gezien verdient deze verwevenheid een andere dan een alleen op symptomen gebaseerde diagnostische beschrijving of classificatie. Men kan ook spreken van een sensorische, gedragsmatige, cognitieve en emotie regulatieve aspecten omvattende alarmfalsificatie, een hypersensitiviteitssyndroom. Dit geldt met name voor functionele somatische aandoeningen [2]. De invloed van stress vormt hierbij niet alleen het ontstaan van affectieve stoornissen en functionele klachten maar ook de overgang van acute naar chronische pijn ${ }^{2-4}$.

$\mathrm{Na}$ een operatieve ingreep gaat acute pijn gepaard met affectieve klachten als onderdeel van ziektegedrag. In de periode aansluitend op een ingreep worden in $20-50 \%$ van de patiënten depressieve symptomen gerapporteerd, ook in een populatie die vooraf nauwelijks depressief was (0-10\% van de geopereerde populatie) ${ }^{5}$. De mate van pijnintensiteit hangt af van de mate van depressieve klachten. Een comorbide depressie is hierbij verder een voorspeller voor de duur van de ziekenhuisopname en de functionele uitkomst na een operatie. Het is van belang dat met name chronische pijn en een depressieve stoornis samen worden waargenomen. Bij patiënten met een depressie ligt de prevalentie van chronische pijn tussen 50 en $60 \%{ }^{5}$. In longitudinaal perspectief vormt de depressie een risicofactor voor het ontstaan van chronische pijn. Andersom vormt de ernst en de chroniciteit van pijn een risicofactor voor het ontstaan van een depressieve stoornis ${ }^{5}$. Hierbij bestaat een dosis-response relatie tussen pijn en depressie: hoe ernstiger en chronischer de pijn is en hoe groter het aantal van pijnlocaties, hoe ernstiger de depressieve stoornis wordt. Andersom ligt de prevalentie van een depressieve stoornis bij chronische pijn patiënten daarom ook tussen 20 en $80 \%$, afhankelijk van de zorgsetting. Bovendien beïnvloedt de ernst van pijn de uitkomst van een antidepressieve behandeling nadelig, en omgekeerd beperkt een depressie ook de prognose van de pijnbehandeling ${ }^{6,7}$. Vanwege het samen voorkomen van affectieve stoornissen en pijn ligt het voor de hand dat men moet uitgaan van een gedeelde origine. Dit wordt ook onderstreept door het feit dat dezelfde behandelingen in beide 
condities worden gebruikt. Cognitieve gedragstherapie biedt een aangrijpingspunt voor emotieregulatie en de acceptatie van een gestoorde signaaltransductie. Tevens worden antidepressiva uit diverse groepen in beide condities gebruikt om de pijngevoeligheid en de stemmingslast te verminderen ${ }^{1,5}$.

De vraag is dus gerechtvaardigd of pijn en depressie comorbide condities zijn of, ten minste in complexe patiënten, toebehoren tot een en dezelfde aandoening. Viscerale en perifere pijnen bij patiënten met functionele somatische aandoeningen, bijvoorbeeld prikkelbare darm (PDS), overactieve blaas (OAB) en fibromyalgie (FM), tonen een onderlinge sterkte associatie en gaan vaak gepaard met affectieve klachten. Angst, stemming, pijn en functionele somatische klachten voorspellen elkaars optreden afhankelijk van klachtenintensiteit en -complexiteit ${ }^{2}$. De bestaande diagnostische classificatie systemen (DSM V, Rome IV, etc.) zijn op symptomen gebaseerde constructies, voortkomend uit een geïsoleerde specialisme-specifieke consensus. Deze weerspiegelt de klinische en pathofysiologische werkelijkheid maar gedeeltelijk ${ }^{8,9}$. Dit hoofdstuk kiest een andere invalshoek. Aan de hand van diverse neurobiologische aspecten worden overeenkomsten tussen pijn en depressie beschreven. Een beschouwende integratie zal tenslotte conceptueel wegen hoe wij bestaande tekorten in de reductionistische symptoom gebaseerde diagnostiek voorbij kunnen streven om tot patiëntgerichte zorg op maat bij te dragen ${ }^{2}$.

\section{Neuro-imaging van pijn en depressie netwerken}

Acute experimentele pijn activeert vooral de volgende corticale en mesolimbische hersenarealen: de primaire somatosensorische cortex (pariëtaal, rond de gyrus postcentralis) en de secundaire somatosensorische cortex (pariëtaal, vanuit de sulcus lateralis naar de centraler gelegen insula cortex), de insula cortex (IC), de anterieure gyrus cingulus (ACC) en de prefrontale cortex (PFC), tevens de thalamus, de nucleus accumbens (NAC) en de amygdala. Het gaat om gebieden die in beloning, bedreiging en emotieregulatie geïnvolveerd zijn (zie Figuur 11.1 en 11.2).

De ACC, PFC, IC en de amygdala zijn vooral bij affectieve pijncomponenten betrokken ${ }^{5}$. Met name de transitie van acute naar chronische pijn gaat gepaard met een verplaatsing in activiteit van sensorische naar affectieve circuits. Bij chronische pijn gaat een reductie van grijze substantie in corticale arealen van de insula, de ACC en de PFC gepaard met negatieve emotionele en cognitieve pijnaspecten ${ }^{10}$. Opvallend is dat met name de bij chronische pijn betrokken hersenarealen dezelfde regio's weerspiegelen die ook bij depressieve patiënten verlies van grijze substantie laten zien $^{10,11}$. 
Figure 11.1 Legende: De primaire somatosensorische cortex.

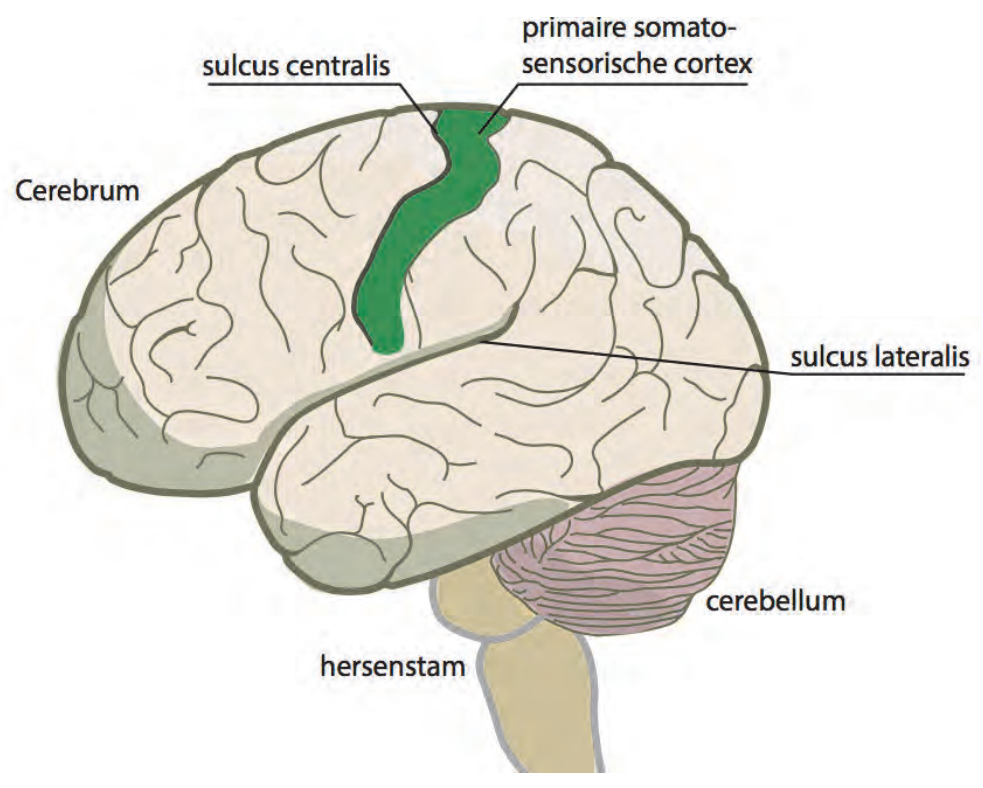

In de nieuwere literatuur worden functionele MRI-resultaten beschreven die zich met name focussen op netwerken die de staat van rustactiviteit weerspiegelen. Het zogenaamde 'default mode netwerk' (DMN) is bij chronische pijn patiënten veranderd. Het DMN bestaat uit hersengebieden die bij rust actief zijn en inactief worden onder een gerichte taakuitvoering. Het DMN is derhalve betrokken bij de staat van opwinding en oplettendheid. Er bestaat bij chronische pijn patiënten vergeleken met gezonde controlepersonen een grotere activiteitenrepresentatie in het gebied van de posterieure gyrus cingulus (PCC) van het DMN en een geringere activiteitenrepresentatie in de PFC. Ook is de connectiviteit van de PFC met de PCC bij chronische pijn patiënten gereduceerd. Vergelijkbare veranderingen van het DMN worden gezien bij depressieve patiënten. Al met al lijken met name connecties tussen de PFC en de ACC geïnvolveerd in comorbide chronische pijn en depressiviteit ${ }^{5,12}$.

Vertrekkend vanuit het concept dat emotioneel lijden betrokken is bij pijn en depressie werd in neuro-imaging studies onderzocht of de ervaring van sociale uitstoting neuronale pijn en depressie paden beïnvloedt. Bij gezonde personen zijn hersenarealen die bij sociale rejectie betrokken zijn dezelfde gebieden als de arealen die geactiveerd raken onder fysieke experimentele pijn. Dit betreft somatosensorische maar ook affectieve arealen. Experimentele pijn en intense sociale rejectie activeren dezelfde gebieden (IC, thalamus, het peri-aquaeductale grijs (PAG)), subtiel verschil binnen deze gebieden is als reactie op fysieke en emotionele prikkeling echter wel aan te tonen ${ }^{5,13,14}$. Er bestaat daarom ook kritiek op een geïsoleerde theorie van gedeelde fysieke-sociale 
pijn paden. Neuronale responsen op fysieke en sociale pijn zouden ook processen kunnen weerspiegelen die met 'salience' te maken hebben in plaats van bedreiging. 'Salience' laat zich in de neurowetenschappen definiëren als een staat van onderscheiding waarmee fenomenen oplettendheid induceren, in het pijngeval mogelijk meetbaar als neuronale activatie ${ }^{15}$. Hoe dan ook, de stelling dat affectieve processen neurale gebieden beïnvloeden die bij pijn betrokken zijn blijft overeind. De interactie van chronische pijn met depressie is hierbij nog complexer dan het samenspel van acute pijn met affectieve aspecten van ziektegedrag.

Figure 11.2 Legende: VTA = ventraal tegmentale areaal.

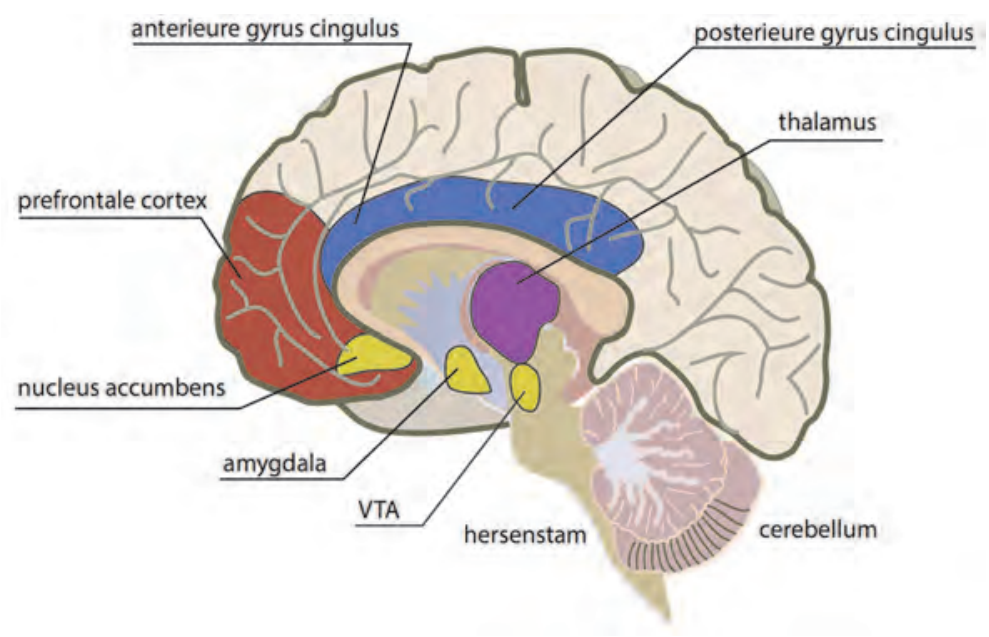

\section{Plasticiteit van neurocircuits bij comorbide pijn en depressie}

Moderne neurowetenschappelijke systeem-level methoden hebben hersengebieden geïdentificeerd die bij pijn en depressie betrokken zijn. Een groter systeem, naast de boven beschreven mesolimbische en corticale gebieden, laat zich beschrijven onder inclusie van het afdalende pijn modulerende systeem, met het PAG en de rostrale ventromediale medulla (RVM) alsmede lagere gebieden van het 'reward' systeem (het ventraal tegmentale areaal (VTA)) (zie Figuur 11.2). Pijn involveert als een multidimensionele ervaring bovengenoemde gebieden van het zenuwstelsel. De betrokken componenten worden hierbij dynamisch geactiveerd, afhankelijk van de pijnstimulus, de context, cognities, emoties en gedrag ${ }^{5,10}$. Preklinische studies beschrijven veranderingen van betrokken neurocircuits. De ACC is betrokken bij cognitieve, emotionele en autonome aspecten van pijn en depressie. Hypermetabolisme, volumereductie en elektrische hyperoscillaties werden waargenomen bij depressieve en neuro-pathische 
pijn patiënten. En neuro-pathisch pijnmodel laat zien dat volumevermindering van de AAC tezamen met angstig depressieve klachten pas met vertraging optreedt. Dit suggereert dat structurele veranderingen van de ACC emotionele veranderingen als reactie op bestaande pijn representeren ${ }^{5,16}$.

De hippocampus en amygdala zijn twee verdere structuren die een rol spelen bij pijn geïnduceerde depressieve klachten. Recente dierexperimentele onderzoeken laten zien dat cognitieve en affectieve processen vertragen in een chronische staat van neuropathische pijn. Deze veranderingen gaan gepaard met verlies van het hippocampale volume, hetgeen correleert met angstig depressieve klachten. In tegenstelling tot het hippocampale volumeverlies wordt er neuronale proliferatie en volumetoename binnen kerngebieden van de amygdala gezien. De amygdala spelen een centrale rol in stressperceptie en emotieregulatie en zijn derhalve ook betrokken bij affectieve stoornissen. Veelvuldige studies hebben aangetoond dat de amygdala geïnvolveerd zijn in angstig depressief en pijngedrag bij neuropathische en inflammatoire pijn ${ }^{5,17,18}$. Inflammatoire pijn modellen laten verder zien dat serotonine levels in de hippocampus dalen, gepaard gaande met depressief gedrag. Al met al lijkt matige inflammatie de hefboom te zijn tussen pijn en angstig depressieve klachten, werkzaam als een neuro-immuun netwerk dat diverse orgaansystemen overspant ${ }^{4,5,19}$. Uitgangspunt van deze aanname is de hypothese dat vroege stresservaringen de wisselwerking tussen inflammatoire activiteiten en het bovengenoemde corticoamygdala bedreigingscircuit alsmede het cortico-basale ganglia beloningscircuit versterken. Dat bedreiging op vroege leeftijd niet alleen inflammatoire cascaden maar ook de hersenen gevoelig maakt blijkt uit een overweldigend aantal aan diverse humane en dierexperimentele studies. Betrokken zijn hierbij natuurlijk banen die bij bedreigingsdetectie (dus ook het somatosensorische systeem) en emotieregulatie betrokken $\mathrm{zijn}^{2,4,20}$. Gevoeligheid voor bedreiging veroorzaakt door eerdere stresservaringen betreft het hele lijf en laat zich het best beschrijven als een hypersensitiviteits- of alarmfalsificatie syndroom. Gevoeligheid ('vulnerability') hangt af van genetische, contextuele en psychologische factoren, waaronder een tendens tot negatieve affectiviteit ${ }^{2}$. Echter, stress gerelateerde veranderingen van de neuromatrix zijn dynamisch en zelfs reversibel ${ }^{21}$. De klinische expressie van pijn en geassocieerde affectieve lasten wordt in deze ook bepaald door weerbaarheid ('resilience'). Weerbaarheid wordt psychologisch mede beïnvloed door positieve affectiviteit, hetgeen de functionaliteit van het cortico-basale ganglia-beloningscircuit impliceert ${ }^{2}$. De PFCVTA-NAc-as onderhoudt het evenwicht van normale hedonisch motivationele functies en speelt in dysfunctionele staat een rol bij de pathogenese van depressie en chronische $\operatorname{pijn}^{22}$. 


\section{Inflammatie als hefboom op de 'Brain-Body-Axis' bij comorbide pijn en depressie}

Inflammatie en excitotoxiciteit spelen als langdurende en uitputtende processen gerelateerd aan serotonerge en glutamaterge neurotransmissie een belangrijke rol in de pathofysiologie van pijn en depressie. Naast functionele somatische aandoeningen, speelt een defensieve, inflammatoire reactie van het hele lijf ook een rol bij chronisch inflammatoire somatische aandoeningen. Bij kanker of reumatoïde artritis bijvoorbeeld kan het aanvankelijk acute ziektegedrag (van verhoogde pijnsensitiviteit, tot aan moeheid, motivatieverlies, gebrek aan eetlust of seksuele belangstelling) gedurende een langdurend beloop in chronische pijn en een depressieve staat veranderen, uitgelokt door minimale maar chronische inflammatie, die niet meer overeenkomt met de ontsteking van de initiële acute ziekteschub. Processen die op de neuronale omgeving van invloed zijn betreffen effecten van pro-inflammatoire cytokines, de activatie van de tryptofaan cataboliserende indolamine-dioxygenase (IDO) en een verschuiving van de glutamaterge en GABAerge neurotransmissie van inhibitie naar excitatie ${ }^{4,19,23}$.

Dat perifere inflammatie pijn en depressie uitlokt is een goed gedocumenteerd gegeven. De vraag is, hoe deze informatietransfer van de periferie naar het centraal zenuwstelsel plaatsvindt. Grof samengevat prikkelt weefselschade door fysieke, chemische of infectieuze aantasting moleculaire paden van de ontstekingsinitiatie die worden onderscheiden in pathogeen geassocieerde moleculaire paden (PAMPs) en 'damage' geassocieerde moleculaire paden (DAMPs). PAMPs signaleren de intrusie van kiemen. Een voorbeeld zijn de lipopolysaccharides (LPS) als membraanonderdelen van bacteriën, die de cytokineproductie en -uitstorting induceren. DAMPs zijn lichaamseigen moleculen die weefselschade signaleren, bijvoorbeeld ATP of interleukine-1 $\alpha$ (IL-1 $\alpha)$. PAMPs en DAMPs activeren het immuunsysteem door middel van binding aan 'Toll-like receptors' (TLRS) die aan het begin staan van verdere processen in de signaal transductiecascade met second messengers, transcriptiefactoren en het uiteindelijke aanwakkeren van de cytokineproductie als gevolg. Cytokines spelen een centrale rol in de emotieregulatie en bij cognitieve en gedragsmatige veranderingen gedurende ziekteprocessen. Zowel pro-inflammatoire cytokines als ook de LPS geïnduceerde immuun-activatie zijn in staat om een angstige stemmingsdaling en pijngedrag bij fysiek gezonde mensen op te wekken ${ }^{4,19,24}$. Inflammatie gerelateerde klinische symptomen gaan hierbij gepaard met een verhoogde activiteit in depressie en pijn gerelateerde hersengebieden. De activiteit in de ACC wordt verhoogd en zijn connectiviteit met de amygdala, de PFC en de NAc wordt verlaagd ${ }^{25}$. Aangezien de meeste inflammatoire schade in de periferie plaatsvindt, wordt een complex signaalsysteem voor de immuunhersenoverdracht benodigd om ziekte gerelateerde lijfelijke responsen, waaronder pijngedrag en affectieve reacties te bewerkstelligen. De productie van pro-inflammatoire cytokines door macrofagen hoort erbij, bijvoorbeeld tumor necrose factor- $\alpha$ (TNF- $\alpha$ ) of $\mathrm{IL}-1 \beta$. De concentratie van circulerende cytokines is echter buiten acute ziektefasen 
(infectie, reumaschub, etc.) vrij klein en deze grote moleculen passeren de bloed-hersen barrière grotendeels niet ${ }^{19,24}$. Er moet dus een vertalend mechanisme bestaan dat inflammatoire perifere prikkels naar pijngedrag kan omzetten. Aangezien cytokines vooral lokaal ageren ontwikkelen zij hun centrale zenuwstelseleffecten vooral via neuronale afferente zenuwbanen. Cytokine-signalen die bijvoorbeeld van peritoneale oorsprong zijn informeren de hersenen via vagale afferente banen. Geactiveerde IL-1 $\beta$ receptoren van hepatische sensorische neuronen van de nervus vagus stimuleren sensorische neuronen die projecteren naar kerngebieden van de tractus solitarius $(\mathrm{NTS})^{26}$. Een aanvullend mechanisme is het proces van de zogenaamde volume transmissie van cerebrale cytokines. In antwoord op perifere inflammatoire signalen produceren centrale microgliacellen cytokines die diffuus het ontstekingssignaal over de extracellulaire ruimte van de hersenen verspreiden. Uit extracellulaire gebieden wordt dan vrij snel het parenchym van andere hersengebieden binnengedrongen ${ }^{27}$.

Dierexperimenteel onderzoek middels LPS gerelateerde inflammatie heeft de overgang van het acute ziektegedrag (inclusieve gereduceerde lijfelijke activiteit, gewichtsverlies en verhoogde pijngevoeligheid) naar depressie of chronische pijn kunnen beschrijven ${ }^{4,19}$. Dit geldt ook voor neuropathische pijn die door perifere weefselen zenuwbeschadiging tot stand komt en tot cross-sensitisaties over orgaansystemen heen kan leiden. TLRs zijn hierbij de sleutel-receptoren van een verhoogde pijngevoeligheid. De DAMPs activatie van TLRs leidt tot activatie van 'nuclear factor kappa B' (NF-kB), een proteïnecomplex die de DNA transcriptie en hiermee verdere productie en vrij zetting van cytokines en chemokines reguleert ${ }^{28}$. Met betrekking tot pijn sensitisatie en chronificatie toont humaan onderzoek onder gebruik van bloed en het cerebrospinale vloeistof aan dat de balans van pro- en anti-inflammatoire cytokines hiervoor belangrijk is. Zo correleert IL-1 $\beta$ positief met pijnintensiteit in polyneuropatische patiënten, terwijl IL-10 de ernst van pijn afzwakt ${ }^{29}$.

\section{Neuronale en inflammatoire basis voor het gebruik van antidepressiva bij chronische pijn}

De huidige kennis over het gebruik van antidepressiva bij patiënten met chronische pijn moet worden gekaderd binnen de geschiedenis van de ontwikkeling van antidepressieve middelen. Tricyclische antidepressiva (TCAs) vormen historisch gezien de hoofdgroep van ingezette antidepressieve psychofarmaca bij verschillende pijn condities. Al met al is er minder onderzoek gedaan met later ontwikkelde antidepressiva, bijvoorbeeld selectieve serotonine heropname remmers (SSRIs) of serotonine en noradrenaline heropname remmers (SNRIS) ${ }^{1}$. Vanwege de gedeelde etiologie en de consistente evidentie omtrent het gezamenlijk voorkomen van pijn en depressie worden antidepressiva vaak bij chronische pijn voorgeschreven. Antidepressiva worden bijvoorbeeld voorgeschreven bij pijn als gevolg van reumatoïde artritis, diabetische polyneuropathie en functionele somatische aandoeningen (fibromyalgie, prikkelbare darm, lage rugpijn, etc. $)^{1,2}$. Al met al bestaat er inmiddels robuuste evidentie dat het 
gebruik van antidepressiva pro-inflammatoire cytokineconcentraties in het serum verlaagd $^{30,31}$. De analgetische potentie resulteert verder uit de inhibitie van de centrale monoamine-heropname, met als consequentie een verhoogde activiteit van de afdalende antinociceptieve paden ${ }^{1,31}$.

Het analgetisch effect staat hierbij deels los van het antidepressieve effect. De dosering die noodzakelijk is om niet complexe pijn te bestrijden is lager dan bij een antidepressieve behandeling en analgesie treedt eerder op dan het antidepressieve effect. TCAs zoals amitriptyline, nortriptyline en desipramine gelden historisch gezien als analgetica, welke aan een lage dosis onafhankelijk van het antidepressieve effect werkzaam zijn ${ }^{1,31}$. Hiervoor worden verschillende mechanismen verantwoordelijk gemaakt. De interactie van amitriptyline met opioïdreceptoren waarmee de cerebrale dichtheid van opioïdreceptoren gemodificeerd wordt is een mechanisme. Een ander betreft de blokkade van excitatoire paden via de $\mathrm{N}$-methyl-D-aspartaat (NMDA) receptor. Verder wordt de functie van GABA-receptoren versterkt. Ernaast bestaat een inhibitie van histaminerge receptoren. Er bestaat tevens robuuste evidentie dat neuroinflammatoire mechanismen worden beïnvloed. Activatie van spinale gliacellen met als gevolg productie van pro-inflammatoire cytokines (TNF- $\alpha$, IL-6 en IL-1B) wordt door serotonerge en adrenerge blokkade voorkomen ${ }^{31,32}$. Ondanks de lange traditie van TCAgebruik ter analgesie bestaat er nog steeds teleurstelling over de effecten in chronische pijncondities, bijvoorbeeld bij neuropathishe $\mathrm{pijn}^{33,34}$. De vraag is gerechtvaardigd, of dit te maken kan hebben met het gebruik van lage doseringen bij een analgesie indicatie, terwijl in complexe populaties eigenlijk antidepressief zou moeten worden behandeld ${ }^{2}$.

SSRIs en SNRIs zijn minstens even effectief in de behandeling van chronische pijn condities. Met name een reductie van pro-inflammatoire cytokines wordt door hun bereikt. Vooral de behandeling van diabetische polyneuropathische pijn door SNRIs en SSRIs wordt in de recentere literatuur beschreven ${ }^{1,35,36}$. De RVM is hierbij door vrij zetting van serotonine (5-HT) een van de sleutelspelers en geïnvolveerd in de modulatie van afdalende pijnpaden. Deze betrokkenheid is bi-directioneel, inhiberend en faciliterend. Tijdens een vroege ziektefase vindt het faciliteren van pijn plaats. Dit geschiedt langs drie mechanismen: 1) stijging van de 5-HT expressie ter hoogte van de RVM; 2 ) in het vervolg stijging van serotoninelevels in het ruggenmerg samenlopend met up-regulatie van lokale pijn faciliterende 5HT3-receptoren; 3) tenslotte versterking van de spontane activiteit van faciliterende RVM neuronen met up-regulatie van 'transient receptor potential vanilloid type $1^{\prime}$ (TRPV1) receptor, een membraanreceptor die ook bij nociceptie betrokken is. Met de progressie van de diabetes stijgen de veranderingen in de RVM verder dramatisch. Oxidatieve stress en neuronale celdood leiden tot microglia gemedieerde inflammatie. Vergelijkbaar met de thalamus is de RVM een pijngenerator of een plaats van pijn amplificatie. Een vroege interventie in het geval van de diabetische polyneuropathie lijkt veelbelovend indien de uitbreiding van spinale 5-HT3-recptoren en de pro-inflammatoire microgliale RVM-activiteit voorkomen kan worden ${ }^{37}$. Hierbij hebben ook SSRIs en SNRIs een plek; bijvoorbeeld fluoxetine, paroxetine en citalopram, 
of duloxetine en venlafaxine ${ }^{31,35,36}$. Het analgetisch effect van SSRIs berust niet op initiële effecten van de serotonine-receptorblokkade aangezien SSRIs bijdragen tot een vroege verhoging van 5 -HT-levels in het ruggenmerg. Recenter onderzoek doet vermoeden dat het analgetisch SSRI-effect veroorzaakt wordt door langere termijn gevolgen waaronder protectie tegen excitatie en microglia gemedieerde inflammatie. Dit neuroprotectieve effect ligt mogelijk ten grondslag aan de werking van SSRIs en SNRIs, bij complexe depressieve pijnpatiënten ${ }^{31,37-40}$.

\section{Psychotherapie bij chronische pijn}

Zoals hierboven aangeduid gaat chronische pijn gepaard met progressieve veranderingen van mesolimbische en corticale belonings- en bedreigingspaden. Dit uit zich in pijnsensitisatie en cross-sensitisaties, uiteindelijk leidend tot stemmings- en angststoornissen, verslaving en complexe functionele somatische aandoeningen ${ }^{2,41}$. Psychotherapeutische behandeling focust zich op de modificatie van pijngedrag (vermijding en inactiviteit), pijncognities (catastroferen en anticiperen) en emotieregulatie (angst, frustratie, dysforie en depressie) ${ }^{1,41}$. Cognitieve gedragstherapie (CGT) grijpt bijvoorbeeld bij lage rugpijn aan op negatieve assumpties en vermijdende copingstrategieën. Analoog met de verstoorde emotieregulatie bij angst- en stemmingsstoornissen worden misattributies geherstructureerd en inactiviteit met vermijdingsgedrag in het kader van een graduele exposure met responspreventie opgeheven. Het opheffen van cognitieve misinterpretaties en negatieve automatische gedachten staat veelal aan de basis van pijnrehabilitatie programma's. Hierbij ligt de focus op catastroferen als cognitieve attributiestijl die gekarakteriseerd wordt door een bias richting negatieve affecten, alsmede het uitvergroten van en rumineren over negatieve pijnassumpties. Catastroferen is niet alleen geassocieerd met een breed scala aan pijncondities maar ook een vulnerabiliteitsfactor die pijnsensitisatie en de ontwikkeling van depressieve klachten vooraf gaat ${ }^{2,41}$. Recent onderzoek laat zien dat CGT niet alleen bijdraagt tot reductie van catastroferen bij chronische pijn maar ook tot neurobiologisch meetbare volumetoename van de dorsolaterale prefrontale cortex ${ }^{42}$. Verder bestaat er klinische evidentie dat psychotherapie, waaronder CGT, een effectieve behandeling van zowel chronische pijn- als depressieve staten biedt ${ }^{43,44}$. Het blijft onduidelijk welke CGT-component voor welke individuele pijnpatiënt werkt aangezien CGT niet voor iedere chronische pijnconditie effectief blijkt te zijn $^{45}$. Bovendien lijken psychotherapeutische interventies geen effect te hebben op objectieve uitkomstmaten zoals werkverzuim of medicatiereductie ${ }^{46}$. Er zijn derhalve psychotherapeutische ontwikkelingen op komst die pogen om huidige uitkomsten te overtreffen. Op acceptatie en achtzaamheid gebaseerde moderne CGT-vormen lijken ook effectief te zijn, hoewel deze tot nu toe niet superieur zijn gebleken ten opzichte van conventionele $\mathrm{CGT}^{47}$. 


\section{Neuromodulatie en Elektro-Convulsieve-Therapie bij complexe pijnpatiënten}

Gevoed vanuit de anesthesiologie en chirurgische vakgebieden (neurochirurgie, urologie, etc.) neemt het onderzoek en de toepassing van invasieve en non-invasieve stimulatie technieken bij pijnpatiënten vaart op. Bij complex regionale pijnsyndromen bereikt 'spinal cord' stimulatie met betrekking tot pijnvermindering 'high-level' evidentie. Dit geldt echter niet voor functie gerelateerde en psychologisch/ psychiatrische uitkomstmaten $^{48}$. Non-invasieve neuromodulatie, bijvoorbeeld noninvasieve nervus vagus stimulatie (nVNS) of nervus supra-orbitalis stimulatie (nSONS) evenals transcraniële magnetische neurostimulatie (TMS), is op het niveau van 'openlabel case series' onderzocht. Gesuggereerd wordt dat nVNS bij de acute behandeling en de preventie van clusterhoofdpijn effect toont. Voor TMS moet de bewijslast bij chronische hoofdpijnen als matig worden ingeschat ${ }^{49}$. Diepe hersenstimulatie kent een dierexperimentele traditie bij behandelpogingen van chronische pijn en richt zich op diverse structuren, o.a. de ACC, de NAc, de thalamus en het PAG. Bij de mens overschrijdt de bewijslast het niveau van 'case series' echter nog niet ${ }^{50}$. Hetzelfde geldt voor elektro-convulsie-therapie (ECT); alleen enkele casusbeschrijvingen en retrospectief dossier-onderzoek doet naast dierexperimenteel onderzoek vermoeden dat ECT effectief kan zijn in gevallen van chronische pijn $^{51-53}$.

\section{Integratie van zorg en onderzoek als remedie tegen behandelresistentie bij complexe pijnsyndromen}

De vraag luidt dus niet alleen, wat weten we tot nu toe over chronische pijncondities en het management ervan; maar ook, wat doen we ermee; en, moeten wij niet onze assessment- en behandelpogingen heroverwegen om ten minste bij complexe pijncondities met affectieve comorbiditeit tot patiëntgerichtere zorgmodellen te komen? Mogelijk biedt een multimodale behandeling die ook de ziekenhuispsychiatrische setting omvat betere uitkomst ${ }^{54,55}$.

Pijn, angst en depressie zijn klinisch en pathofysiologisch met elkaar verbonden. Echter, de complexiteit en ernst van functionele pijnsyndromen met affectieve comorbiditeit wordt vaak niet herkend. Komen pijn en depressie nog in een laag percentage samen voor bij de algemene populatie, stijgt deze naar $30 \%$ in de eerstelijnszorg tot $80 \%$ binnen geïntegreerde somatisch psychiatrische poliklinieken in

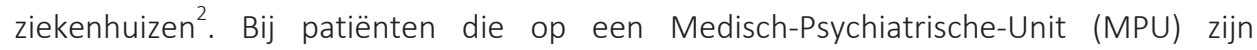
opgenomen is er zelfs in $90 \%$ sprake van psychiatrische (meestal affectieve) en somatische comorbiditeit, met pijn als een van de hoofdklachten. Nota bene: als dezelfde patiëntenpopulatie herhaaldelijk op somatische afdelingen werd opgenomen blijft de detectie van het complexe pijnsyndroom inclusieve psychiatrische comorbiditeit, 
ondanks aanwezigheid van psychiatrische consultatie-liaison diensten, laag en slechts tot $5 \%$ beperkt $^{56}$. Onderdetectie van complexe multimorbiditeits-fenotypen is een van de oorzaken voor teleurstellende behandelresultaten of behandelresistentie met toename van het oorspronkelijke klachtenprofiel of zelfs het ontstaan van iatrogene schade ${ }^{2}$. Derhalve blijft het therapeutisch resultaat suboptimaal als er geen integrale multidisciplinaire zorg wordt geboden. Zowel dokters als patiënten hebben inmiddels oog voor 'unmet clinical needs' bij functionele somatische syndromen met viscerale en ook perifere pijnklachten. Een integrale multidisciplinaire benadering is daarom de aanbevolen standard. In het bijzonder geldt dit voor patiënten met complexe syndromen, inclusief affectieve problematiek en aan comorbiditeit gerelateerde behandelresistentie $e^{2,57}$. Afhankelijk van de zorgzwaarte dient een integrale multimodale behandeling beschikbaar te zijn op verschillende zorglevels; bij de huisarts, maar ook in het ziekenhuis: van de spoedeisende hulp tot in de kliniek (met consultatie-liaison activiteiten en MPU). Een multidisciplinair pijnteam bestaat idealiter uit alle disciplines die bij de pijnzorg betrokken zijn: anesthesiologen, orthopeden, neurochirurgen, internisten, neurologen, revalidatieartsen, ziekenhuis-psychiaters en medisch psychologen. Doel van een mulitmodale benadering is integrale diagnostiek en behandeling. De kunst van samenwerking ligt hierbij in de multidisciplinaire afstemming, ook over bestaande zorgechelons heen.

Een van de 'unmet clinical needs' van de chronische pijnpatiënt ligt op het gebied van communicatie tussen dokters maar ook tussen dokters en patiënten. Deels heeft dit te maken met verouderde diagnostische concepten. Gedurende decennia is de conceptualisatie van functionele syndromen reductionistisch symptoom gebaseerd gebleven. Dit geldt voor somatische specialismen als de psychiatrie (DSM 5 en ROME IV criteria bijvoorbeeld $)^{2,8,9}$. Een dergelijke methodiek is goed voor intra-specialistische aspecten van complexe syndromen, doet echter de complexiteit en daarmee de klinische realiteit van functionele pijnsyndromen tekort en vernevelt de blik op comorbiditeit. Een wezenlijke stap tot verbetering van ons begrip van ziektecomplexiteit zou kunnen worden gezet door het gebruik van moderne diagnostische methoden die klachten in het dagelijks leven meten, het zogenaamde 'momentary assessment' (MA). Middels MA is het mogelijk om het beloop van klachten en hun verhouding tot elkaar in de dagelijkse context te documenteren. Zo kan bijvoorbeeld de volgtijdelijkheid van pijn en depressieve klachten worden vastgelegd en hoe deze zich verhoudt tot contextuele verandering of stressoren. Tot op heden zijn retrospectieve vragenlijsten in gebruik met impliciete veelvuldige bias ('recall bias' en contextuele aspecten die het meetresultaat kunnen beïnvloeden). Bovendien is de variabiliteit van gebruikte instrumenten enorm en maakt een vergelijking tussen studies in complexe syndromale pijnpatiënten moeilijk ${ }^{2}$. Deze beperkingen zouden kunnen worden overwonnen door herhaaldelijke en toevallige momentane metingen in het dagelijks leven door middel van de 'experience sampling method' (ESM), welke succesvol in een pilotstudie bij functionele gastro-intestinale aandoeningen werd gebruikt. Een van de belangrijkste uitkomsten is hierbij dat 
patiënten tijdens het gebruik van retrospectieve lijsten pijnscheuten overschatten en lijken te generaliseren ${ }^{58}$. De vraag is dus gerechtvaardigd, welke door symptoom gebaseerde diagnostiek niet in acht genomen patiënten karakteristieken bijdragen tot klachtenbeleving. Het opmerken van bijvoorbeeld psychosociale risicofactoren zou zorgindicering moeten beïnvloeden en iatrogene schade kunnen voorkomen ${ }^{59}$. De plek voor een dergelijke multidisciplinaire zorgintegratie is naast de eerste lijn ook het ziekenhuis, in het bijzonder bij complexe patiënten met frequent zorggebruik. Veelbelovende integrale 'stepped care' en 'collaborative care' modellen kunnen bij complexe pijnpatiënten met impact op pijn, depressie en kosten starten vanuit de ziekenhuis setting $^{60,61}$. 


\section{References}

1. Stannard CF, Kalso E, Ballantyne J. Evidence-based chronic pain management. BMJ Books. (2010) WileyBlackwell Publishing, Oxford, USA. ISBN: 9781405152914.

2. Leue C, Kruimel J, Vrijens D, et al. Functional urological disorders: A sensitized defence response in the bladder-gut-brain axis. Nature Reviews Urology. 2016; doi: 10.1038/nrurol.2016.227.

3. Li X, Hu L. The role of stress regulation on neural plasticity in pain chronification. Neural Plasticity. 2016; doi: 10.1155/2016/6402942.

4. Nusslock R, Miller GE. Early-life adversity and physical and emotional health across the lifespan: A neuroimmune network hypothesis. Biological psychiatry. 2016;80:23-32.

5. Doan L, Manders T, Wang J. Neuroplasticity underlying the comorbidity of pain and depression. Neural Plasticity. 2015; doi: 10.1155/2015/504691.

6. Gerrits MM, Vogelzangs N, van Oppen P, et al. Impact of pain on the course of depressive and anxiety disorders. Pain. 2012;153:429-36.

7. Bair MJ, Robinson RL, Katon W, Kroenke K. Depression and pain comorbidity: A literature review. Archives of Internal Medicine. 2003;163:2433-45.

8. American Psychiatric Association. Diagnostic and Statistical Manual of Mental Disorders. 2013; $5^{\text {th }}$ edition, American Psychiatric Publishing.

9. Sood R, Ford AC. Diagnosis: Rome IV criteria for FGIDs - an improvement or more of the same? Nature Reviews Gastroenterology and Hepatology. 2016;13:501-502.

10. Bushnell MC, Ceko M, Low LA. Cognitive and emotional control of pain and its disruption in chronic pain. Nature Reviews Neuroscience. 2013;14:502-11.

11. Russo SJ, Nestler EJ. The brain reward circuitry in mood disorders. Nature Reviews Neuroscience. 2013;14:609-25.

12. Baliki MN, Mansour AR, Baria AT, et al. Functional reorganization of the default mode network across chronic pain conditions. PLOS ONE. 2014;9:e106133.

13. Eisenberger NI. The pain of social disconnection: Examining the shared neural underpinnings of physical and social pain. Nature Reviews Neuroscience. 2012;13:421-34.

14. Ushinsky A, Reinhardt LE, Simmons AN, Strigo IA. Further evidence of emotional allodynia in unmediated young adults with major depressive disorder. PLOS ONE. 2013;8:e80507.

15. Eisenberger NI. Social pain and the brain: Controversies, questions, and where to go from here. Annual Review of Psychology. 2015;66;601-29.

16. Denk F, McMahon SB, Tracey I. Pain vulnerability: A neurobiological perspective. Nature Neuroscience. 2014;17:192-200.

17. Mutso AA, Radzicki D, Baliki MN, et al. Abnormalities in hippocampal functioning with persistent pain. Journal of Neuroscience. 2012;32:5747-56.

18. Boyle LM. A neuroplasticity hypothesis of chronic stress in the basolateral amygdala. Yale Journal of Biology and Medicine. 2013;86:117-25.

19. Walker AK, Kavelaars A, Heijnen CJ, Dantzer R. Neuroinflammation and comorbidity of pain and depression. Pharmacological Reviews. 2014;66:80-101.

20. Teicher $\mathrm{MH}$, Samson JA, Anderson CM, Ohashi K. The effects of childhood maltreatment on brain structure, function and connectivity. Nature Reviews Neuroscience. 2016;17:652-66.

21. Sousa N. The dynamics of the stress neuromatrix. Molecular Psychiatry. 2016;21:302-12.

22. Nestler EJ, Carlezon WA. The mesolimbic dopamine reward circuit in depression. Biological Psychiatry. 2006;59:1151-59.

23. Sanacora G, Treccani G, Popoli M. Towards a glutamate hypothesis of depression: An emerging frontier of neuropsychopharmacology for mood disorders. Neuropharmacology. 2012;62:63-77.

24. Piccinini AM, Midwood KS. DAMPening inflammation by modulating TLR signaling. Mediators of Inflammation. 2010; doi: 10.1155/2010/672395.

25. Harrison NA, Brydon L, Walker C, et al. Inflammation causes mood changes through alterations in subgenual cingulate activity and mesolimbic connectivity. Biological Psychiatry. 2009;66:407-14.

26. Goehler LE, Gaykema RP, Nguyen KT, et al. Interleukin-1beta in immune cells of the abdominal vagus nerve: A link between the immune and nervous system? Journal of Neuroscience. 1999;19:2799-2806. 
27. Konsman JP, Tridon V, Dantzer R. Diffusion and action of intra-cerebroventricolarly injected interleukin-1 in the CNS. Neuroscience. 2000;101:957-67.

28. Guo LH, Schluesener HJ. The innate immunity of the central nervous system in chronic pain: The role of Toll-like receptors. Cellular and Molecular Life Sciences. 2007;64:1128-36.

29. Backonja MM, Coe CL, Muller DA, Schell K. Altered cytokine levels in the blood and cerebrospinal fluid of chronic pain patients. Journal of Neuroimmunology. 2008;195:157-63.

30. Hannestad J, Della Gioia N, Bloch M. The effect of antidepressant medication treatment on serum levels of inflammatory cytokines: A meta-analysis. Neuropsychopharmacology. 2011;36:2452-9.

31. Mika J, Zychowska M, Makuch W, et al. Neuronal and immunological basis of action of antidepressants in chronic pain - clinical and experimental studies. Pharmacological Reports. 2013;65:1611-21.

32. Mika J, Zychowska M, Popiolek-Barczyk K, et al. Importance of glial activation in neuropathic pain. European Journal of Pharmacology. 2013;716:106-19.

33. Derry S, Wiffen PJ, Aldington D, Moore RA. Nortriptyline for neuropathic pain in adults. Cochrane Database of Systematic Reviews. 2015;doi: 10.1002/14651858.CD011209.

34. Moore RA, Derry S, Aldington D, et al. Amitriptyline for neuropathic pain in adults. Cochrane Database of Systematic Reviews. 2015;doi: 10.1002/14651858.CD008242.

35. Aiyer R, Barkin RL, Bhatia A. Treatment of neuropathic pain with venlafaxine: A systematic review. Pain Medicine. 2016; pii: pnw261.

36. Patetsos E, Horjales-Araujo E. Treating chronic pain with SSRIs: What do we know? Pain Research and Management. 2016; doi: 10.1155/2016/2020915.

37. Silva M, Costa-Pereira JT, Martins D, Tavares I. Pain modulation from the brain during diabetic neuropathy: Uncovering the role of the rostroventromedial medulla. Neurobiology of Disease. 2016;96:343-56.

38. Zhang $\mathrm{F}$, Zhou H, Wilson BC, et al. Fluoxetine protects neurons against microglial activation-mediated neurotoxicity. Parkinsonism Related Disorders. 2012;18:S213-17.

39. Jaracz J, Gattner K, Jaracz K, Górna K. Unexplained painful physical symptoms in patients with major depressive disorder: prevalence, pathophysiology and management. CNS Drugs. 2016;30:293-304.

40. Gebhardt S, Heinzel-Gutenbrunner M, König U. Pain relief in depressive disorders: a meta-analysis of the effects of antidepressants. Journal of Clinical Psychopharmacology. 2016;36:658-68.

41. Simons L, Elman I, Borsook D. Psychological processing in chronic pain: A neural systems approach. Neuroscience and Biobehavioral Reviews. 2014;39:61-78.

42. Seminowicz DA, Shpaner M, Keaser ML, et al. Cognitive-behavioral therapy increases prefrontal cortex gray matter in patients with chronic pain. The Journal of Pain. 2013;14:1573-84.

43. Williams AC, Eccleston C, Morley S. Psychological therapies for the management of chronic pain (excluding headache) in adults. Cochrane Database of Systematic Reviews. 2012; doi: 10.1002/14651858.CD007407.

44. Cuijpers $P$, Berking $M$, Andersson $G$, et al. A meta-analysis of cognitive-behavioural therapy for adult depression, alone and in comparison with other treatments. Canadian Journal of Psychiatry. 2013;58:376-85.

45. Eccleston C, Hearn L, Williams AC. Psychological therapies for the management of chronic neuropathic pain in adults. Cochrane Database of Systematic Reviews. 2015;doi: 10.1002/14651858.CD011259.

46. Pike A, Hearn L, Williams AC. Effectiveness of psychological interventions for chronic pain on health care use and work absence: A systematic review and meta-analysis. Pain. 2016;157:777-85.

47. Veehof MM, Tompetter HR, Bohlmeijer ET, Schreurs KM. Accetptance- and mindfulness-based interventions for the treatment of chronic pain: a meta-analytic review. Cognitive Behavioral Therapy. 2016;45:5-31

48. Visnjevac O, Costandi S, Patel BA, et al. A comprehensive outcome-specific review of the use of spinal cord stimulation for complex regional pain syndrome. Pain Practice. 2016; doi: 10.1111/papr.12513.

49. Miller S, Matharu M. Non-invasive neuromodulation in primary headaches. Current Pain Headache Reports. 2017; doi: 10.1007/s11916-017-0608-x.

50. Hollingworth $M$, Sims-Williams HP, Pickering AE, et al. Single electrode deep brain stimulation with dual targeting at the dual frequency for the treatment of chronic pain: A case series and review of the literature. Brain Sciences. 2017;7. pii: E9. 
51. Kimura Y, Ishikawa M, Hori Y, et al. Effect of electroconvulsive stimulation on messenger RNA expression in the prefrontal cortex in a rat pain model. Biomedical Reports. 2015;3:802-6.

52. Leong K, Tham JC, Scamvougeras A, Vila-Rodriguez F. Electroconvulsive therapy treatment in patients with somatic symptom and related disorders. Neuropsychiatric Disease and Treatment. 2015;11:256572.

53. Borisovskaya A, Augsburger JA. Somatic symptom disorder treated with electroconvulsive therapy. Pain Management. 2016; doi: 10.2217/pmt-2016-0048.

54. Bollard ER. The management of chronic pain: What do we know, what do we do, and how should we redesign our comprehensive assessment and treatment in order to provide for more patient-centered care? Medical Clinics of North America. 2016; doi: 10.1016/j. mcna.2015.10.002.

55. Dale R, Stacey B. Multimodal treatment of chronic pain. Medical Clinics of North America. 2016; doi: 10.1016/j. mcna.2015.08.012.

56. Leue C, Driessen G, Strik JJ, et al. Managing complex patients on a Medical Psychiatric Unit: an observational study of university hospital costs associated with medical service use, length of stay, and psychiatric intervention. Journal of Psychosomatic Research. 2010;98:295-302.

57. Sharpe M, Burton C, Sawhney A, et al. Is co-morbid depression adequately treated in patients repeatedly referred to specialist medical services with symptoms of a medical condition? Journal of Psychosomatic Research. 2012;72:419-21.

58. Mujagic Z, Leue C, Vork L, et al. The Experience Sampling Method - a new digital tool for momentary symptom assessment in IBS: an exploratory study. Neurogastroenterology and Motility. 2015;27:12951302.

59. Leue C, Buijs S, Strik J, et al. Observational evidence that urbanisation and neighbourhood deprivation are associated with escalation in chronic pharmacological pain treatment: A longitudinal populationbased study in the Netherlands. BMJ Open. 2012;doi: 10.1136/ bmjopen-2011-000731.

60. Kroenke K, Bair MJ, Damush TM, et al. Optimized antidepressant therapy and pain self-management in primary care patients with depression and musculoskeletal pain: A randomized controlled trial. JAMA. 2009;301:2099-110.

61. Thielke S, Corson K, Dobscha SK. Collaborative care for pain results in both symptom improvement and sustained reduction of pain and depression. General Hospital Psychiatry. 2015;37:139-43. 



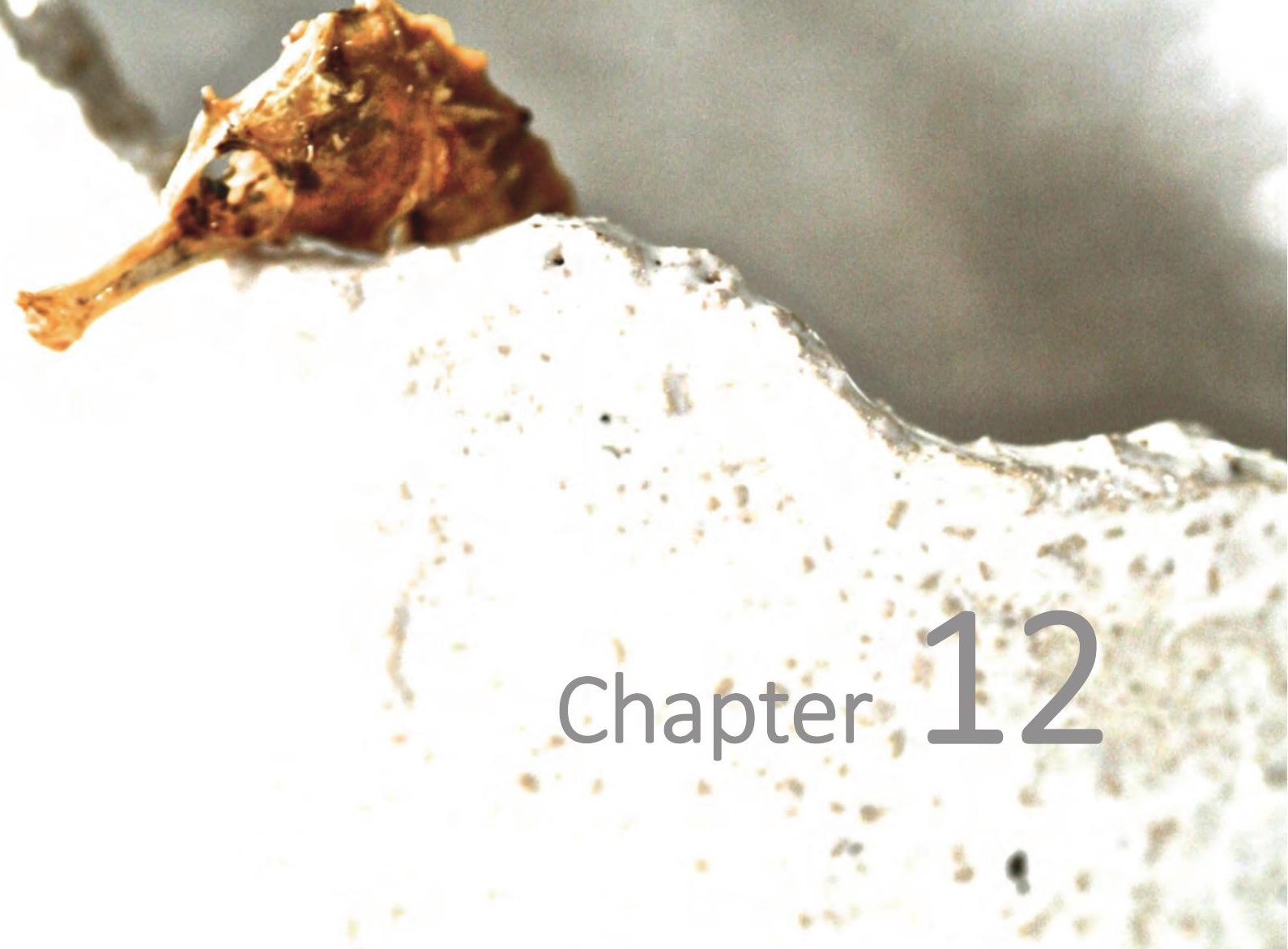

Affective symptoms and the overactive bladder:

A systematic review

Vrijens $D^{a}$, Drossaerts J ${ }^{a}$, van Koeveringe G, Van Kerrebroeck Ph, van Os J, Leue C ${ }^{a}$ These authors contributed equally to the present article J. Psychosom. Res. 78, 95-108 (2015) 


\section{Abstract}

\section{Background}

Overactive bladder syndrome (OAB) is characterised by urgency symptoms, with or without urgency incontinence, usually with frequency and nocturia. Although literature suggest an association between $\mathrm{OAB}$, depression and anxiety, no systematic review has been presented.

\section{Objective}

Systematically review the literature on the association of affective conditions with OAB.

\section{Methods}

Systematic review according to the PRISMA guidelines. This review is registered in the PROSPERO prospective register (CRD4201400664).

\section{Results}

Forty-three articles were included, describing more than 80000 subjects. Depression and $O A B$ were positively associated in 26 studies, anxiety and $O A B$ in 6 studies. Longitudinal studies reported: a) $O A B$ subjects who developed depression/anxiety or b) depressed/anxious subjects developing $O A B$, or c) both. The quality of evidence in studies reporting an association between the co-occurrence of $O A B$ and depression, was rated level 3 conform the GRADE framework. Evidence reporting on the co-occurrence of anxiety and $O A B$ was rated GRADE level 2. Longitudinal associations between new onset of $O A B$ in depressive subjects was GRADE level 2. Evidence reporting association of OAB with anxiety in longitudinal studies was of GRADE level 1.

\section{Conclusion}

To our knowledge, this systematic review is the first to give an comprehensive qualitative overview on the association between $O A B$ and affective symptoms. Many evaluated studies failed to note longitudinal changes and lacked evidence of causality. Still, results revealed an association between $O A B$ and affective symptoms and there is evidence for new onset of $O A B$ in depressive subjects, but further research is necessary to examine the strength of the effect. 


\section{Introduction}

Depression and anxiety are classified as affective disorders according to the DSM-IV psychiatric diagnostic criteria ${ }^{1}$. According to a survey of the World Health Organisation, anxiety is globally the most common psychiatric disorder ${ }^{2}$. The lifetime prevalence of anxiety disorders in the US adult population is around $18 \%{ }^{2}$. Depression has a high lifetime prevalence as well, ranging from 2 to $15 \%^{3}$.

Overactive bladder $(\mathrm{OAB})$ is defined by the International Continence Society (ICS) as a symptom complex of urgency, usually accompanied by frequency (voiding 8 or more times in a 24 hour period) and nocturia (awakening at night to void), with (OAB wet) or without ( $O A B$ dry) urinary urgency incontinence (UUI) $)^{4}$. Approximately $16-17 \%$ of the adult population is affected by OAB: $43 \%$ of adult women experience OAB symptoms 'sometimes' and $33 \%$ 'often',5, The health care costs of $O A B$ are high and around 12 billion USD per year".

Although high prevalence rate would suspect the opposite, OAB is often underdiagnosed and subsequently undertreated, mainly because of patients' reluctance to seek medical help ${ }^{8}$. Only $27 \%$ of people with $O A B$ were receiving treatment in a large population based survey ${ }^{6}$. One possible reason for not seeking help in OAB may be caused by stigma perception ${ }^{8}$. Another is that people may assume that $\mathrm{UI}$ is part of the normal aging process $^{9}$. In addition, an inverse relationship between depressive symptoms and healthcare seeking in patients with OAB is reported ${ }^{10}$.

A relationship between common affective disorders (e.g., depression and anxiety disorders) and lower urinary tract symptoms (LUTS) has been described as early as $1964^{11}$. The importance of mental disorders in the aetiology of urgency incontinence was again emphasized more recently ${ }^{12,13}$. In 2011, the International Consultation on Incontinence-Research Society (ICI-RS) organized a think tank on psychological factors and LUTS $^{14}$. It was noted that not only OAB but also other LUTS may be associated with affective conditions, concluding that the possibility of causation and or maintenance of lower urinary tract symptoms through psychological causes needs further research' ${ }^{14}$. At present, there has been renewed interest in the matter, reflected by a quadrupling in publications found through PubMed/EMBASE search on the topic since 2000 (searched for anxiety, depression and lower urinary tract symptoms before and after 2000).

Despite this growing interest, the exact nature of the association between bladder symptoms and mental conditions, specifically affective disorders, remains unknown. A systematic review using strict definitions may inform the field regarding the strength of the associations between affective disorders and $O A B$ and may possibly enhance insight in underlying pathophysiological mechanisms and treatment recommendations at the "bladder-brain-axis".

The aim of the current study was to (i) review articles providing evidence on the association of affective conditions with $\mathrm{OAB}$; (ii) to examine the direction and strength of the association; (iii) to gauge the possible influence of method and design factors in 
terms of occasioning bias or confounding; (iv) to formulate considerations for future research.

\section{Methods}

This systematic review was registered in PROSPERO under number CRD42014006641 ${ }^{15}$.

\section{Inclusion and exclusion criteria}

A literature search for full length original articles meeting the following inclusion criteria was carried out: a) adults; b) patients with overactive bladder, urinary urgency incontinence (and/or frequency or nocturia); not initiated by surgery, using a questionnaire, voiding diaries or urodynamic evaluation to state symptoms; c) assessment of depressive symptoms and/or anxiety using a validated multi-item scale or a structured diagnostic interview; d) reporting an association between urinary symptoms and depression or anxiety; e) published in English in a peer-reviewed journal. Reviews, expert opinions, abstracts and comments were excluded.

\section{Literature search}

PRISMA guidelines were used concerning preferred reporting items for systematic reviews ${ }^{16}$. The following databases were searched: Medline (PubMed), Embase, Web of Knowledge, Cochrane and PsychInfo. There was no age restriction on the searched articles, and the last search was dated December $31^{\text {st }}$ 2013. The detailed query is listed in the appendix.

These database searches were supplemented by hand searching the reference lists of eligible articles. Two authors (DV and CL) independently examined titles, abstracts and full-text articles. Name of authors, institution and journal of publication were not blinded. One author was a content expert on overactive bladder (DV), the other an expert in the field of affective disorders in somatic conditions (CL). Consensus was obtained in $85 \%$ of the manuscripts; the remaining $15 \%$ of disagreements were handled by discussion. 1249 abstracts were identified in the database searches, and after removal of duplicates, 925 remained for screening on relevance. In the reference lists an additional 54 abstract were found. Eventually, 117 full text articles were assessed for eligibility, 74 articles were excluded resulting in a final set of 43 articles (out of 37 studies), which were included in this review. Errata were examined if articles were retracted since publication; there were none. Attempts were made to obtain unpublished results to diminish effects of reporting bias. The flow-chart of the selection process is depicted in Figure 12.1. 


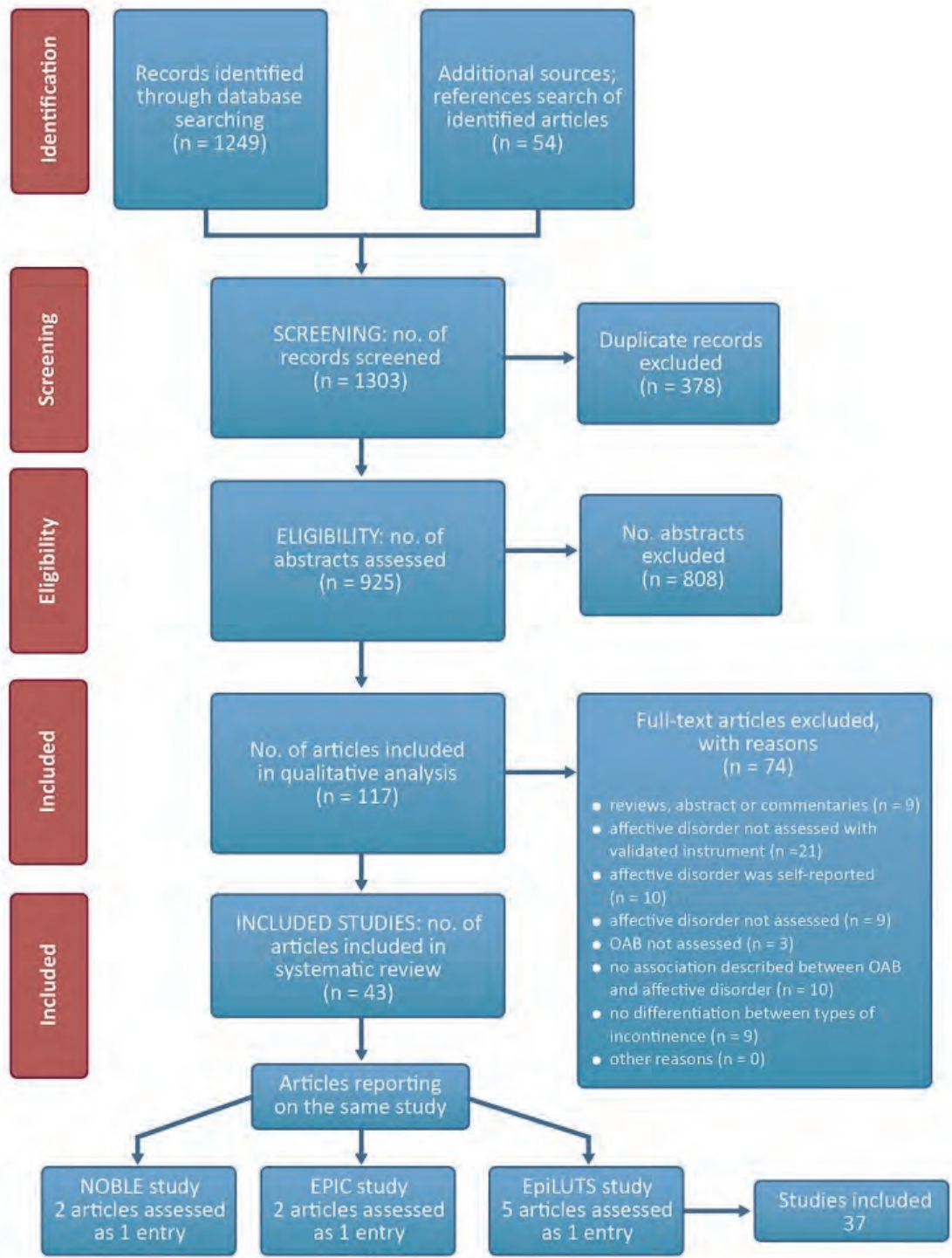

Figure 12.1 Flow-chart of selection process, according to the PRISMA guidelines, 200916.

\section{Data extraction and quality assessment}

Two authors performed data extraction and quality assessment independently (DV, urologist and JD, PhD student), using a standard data extraction form. The data extraction form was pilot tested and modified accordingly before further use. Consensus was obtained in $90 \%$ of the articles; the remaining $10 \%$ of disagreements were solved by 
discussion. Multiple reports on the same study were collated as one entry of interest. Level of evidence of each article was stated according to the University of Oxfords' Centre for Evidence-based Medicine (CEBM) documents ${ }^{17}$. Assessment of risk of bias was performed by using the Cochrane Collaboration's tool for assessing risk of bias ${ }^{18}$. Six domains of bias were taken into account: selection bias, performance bias, detection bias, attrition bias, reporting bias and other bias. The assessment was finished by applying the 'Grading of Recommendations, Assessment, Development, and Evaluation' (GRADE) framework to rate overall quality of evidence for each reported outcome measurement ${ }^{19}$.

\section{Results}

Forty-three articles regarding 37 studies were included in the review. Thirty-two articles were about depression, 9 about anxiety (and depression) and two about anxiety only. Diagnosing OAB was carried out according to the ICS definition of OAB in 33 articles. In 10 articles, the ICS definition had not been used. Three of these articles were published before establishment of the ICS definition in $2002^{20-22}$. Retrospectively, these 10 articles adhered to the ICS definition and were included. Twelve studies used additional instruments, such as voiding diaries or urodynamic studies. A validated measurement of depressive and/or anxiety symptoms was used in all studies (Table A.1). Most articles described cross-sectional $(n=29)$ study designs. The remainder were cohort studies $(n=10)$ and randomised controlled trials $(n=4)$ (Table 12.1). There was a wide range in mean age (between 29 and 89 years old) and the number of people included per study (35 to 30000). Overall, a little more than 80000 subjects were included. Most included subjects were women, but for approximately 30000 men, gender-specific analysis was reported. The eligibility of performing a meta-analysis was examined. Due to the heterogeneity of the included studies and the small number of longitudinal studies, it was not feasible to perform a meaningful meta-analysis. Hence, a qualitative synthesis was undertaken.

Overall in most articles all risk of bias domains were assessed as low risk or unclear risk. Also some domains were not applicable for several articles (inherent to study design) (Table 12.2). "Other bias" was around $47 \%$ across articles, such as ascertainment bias $^{23,24}$ and sick quitter bias ${ }^{25-27}$.

A positive association between depression and $O A B$ was found in the majority of studies $(n=26)$ (Table 12.3). There was no association of $O A B$ with depression in 9 studies (Table 12.3). Two studies reported about anxiety and OAB only (not depression); both revealed a positive association. In the longitudinal studies, associations in different directions were present: $O A B$ subjects developing depression/anxiety and/or depressed/anxious people developing OAB, or both (all compared with control subjects). 


\section{Depressive symptoms and OAB: cross-sectional studies}

Of the 29 cross-sectional studies, nine studies did not show an association of OAB with affective conditions ${ }^{20,22-24,28-32}$. The others revealed positive associations. No studies reported a negative association. Nine articles reported results from the same studies, respectively the NOBLE, EPIC and EpiLUTS studies (Table 12.1). The National Overactive Bladder Evaluation (NOBLE) study was conducted to establish estimates of the prevalence of $O A B$ and of the individual burden of illness; the sample was 5204 US adults. Women suffering from OAB, with or without UUI, had significantly worse scores on the Centre of Epidemiologic Studies Depression Scale (CES-D), than OAB-negative controls $^{33}$. Another report did not find an association between OAB and depression ${ }^{34}$. The Epidemiology Urinary Incontinence and Comorbidities (EPIC) study was a crosssectional survey in five countries representing 19165 subjects. The two EPIC articles reported significantly more cases with depression in OAB, with or without UUI, compared to controls ${ }^{35,36}$. The Epidemiology of Lower Urinary Tract Symptoms (EpiLUTS) study was a cross-sectional survey conducted in the USA, UK and Sweden, representing 30000 subjects $^{10,26,37-39}$. Subjects with OAB reported the highest rate of anxiety (31\%) and depressive symptoms $(27 \%)^{37}$.

The presence of major depression led to a significant increase in a patient's urinary incontinence symptom reporting ${ }^{40}$. A Japanese study of 833 elderly revealed a significant association between $\mathrm{OAB}$, and depressive symptoms based on the Geriatric Depression Scale (GDS-30 $)^{25}$. Multivariate analysis showed that the risk of having OAB was significantly higher in subjects with depressive symptoms. In contrast, in incontinent obese women there was no association between depressive symptoms with overall incontinent episodes ${ }^{41}$.

However, there was an association of depressive complaints with the number of stress incontinence episodes ${ }^{41}$. Another cross-sectional study revealed the strongest association between incontinence and depression for mixed urinary incontinence (MUI) and $\mathrm{UUI}^{42}$. Ito et al. compared people with depression diagnosed at the psychiatric clinic, with age-matched individuals without depression. There was significantly more urinary urgency and UUI in the medicated depressed women compared to controls ${ }^{43}$. A large Swedish twin study representing 14098 women aged 20-46 showed that major depression assessed by Composite International Diagnostic Interview-Short Form (CIDISF) and depressive symptoms by CES-D were positively associated with all subtypes of $\mathrm{UI}^{44}$. In contrast, Chiara et al. did not find any difference in subjects with OAB concerning depressive mood or anxiety ${ }^{20}$, but found anger, irritability and general hypochondria being more prevalent in subjects with $O A B$ than in subjects with stress urinary incontinence (SUI) (Table 12.1). 


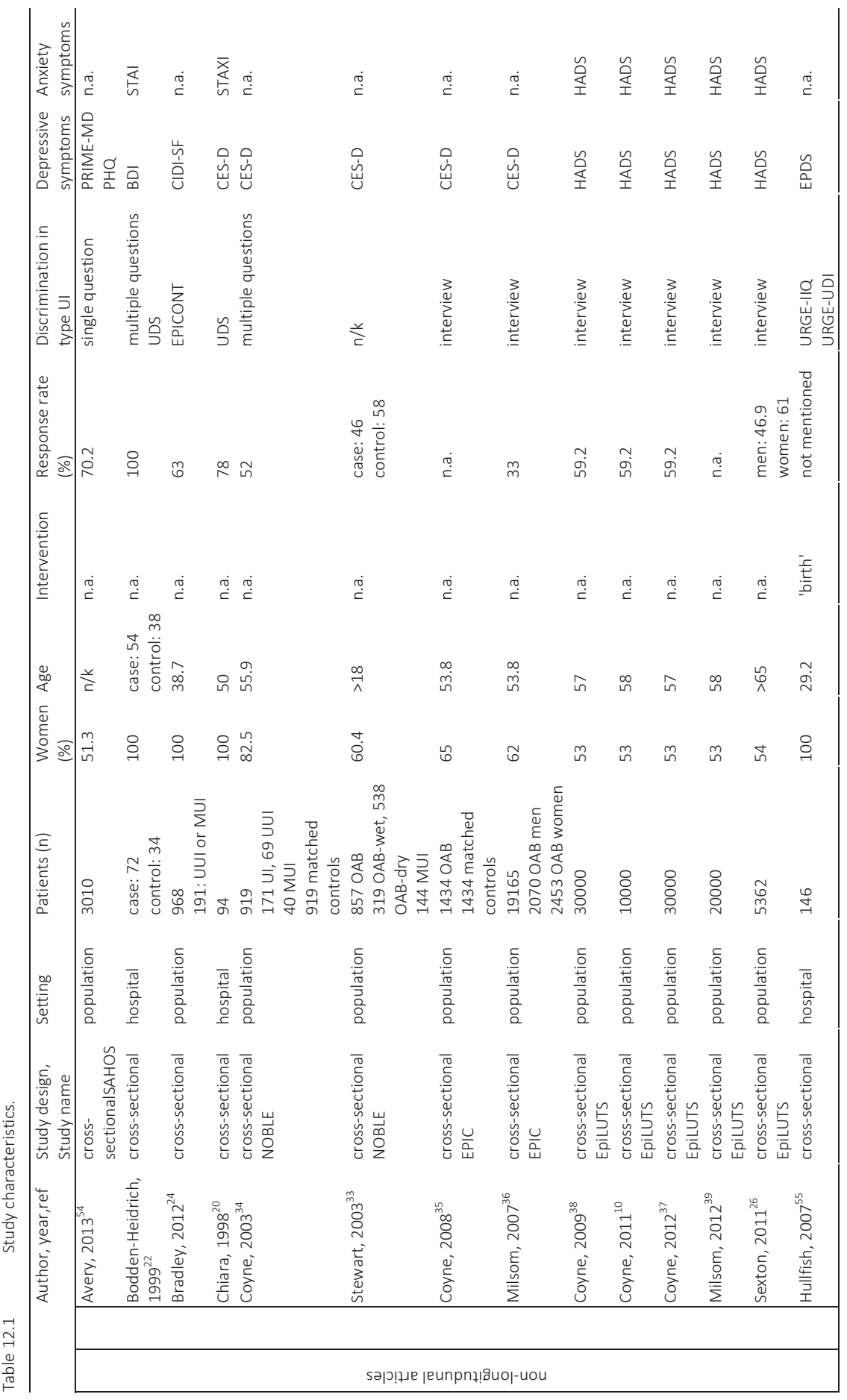




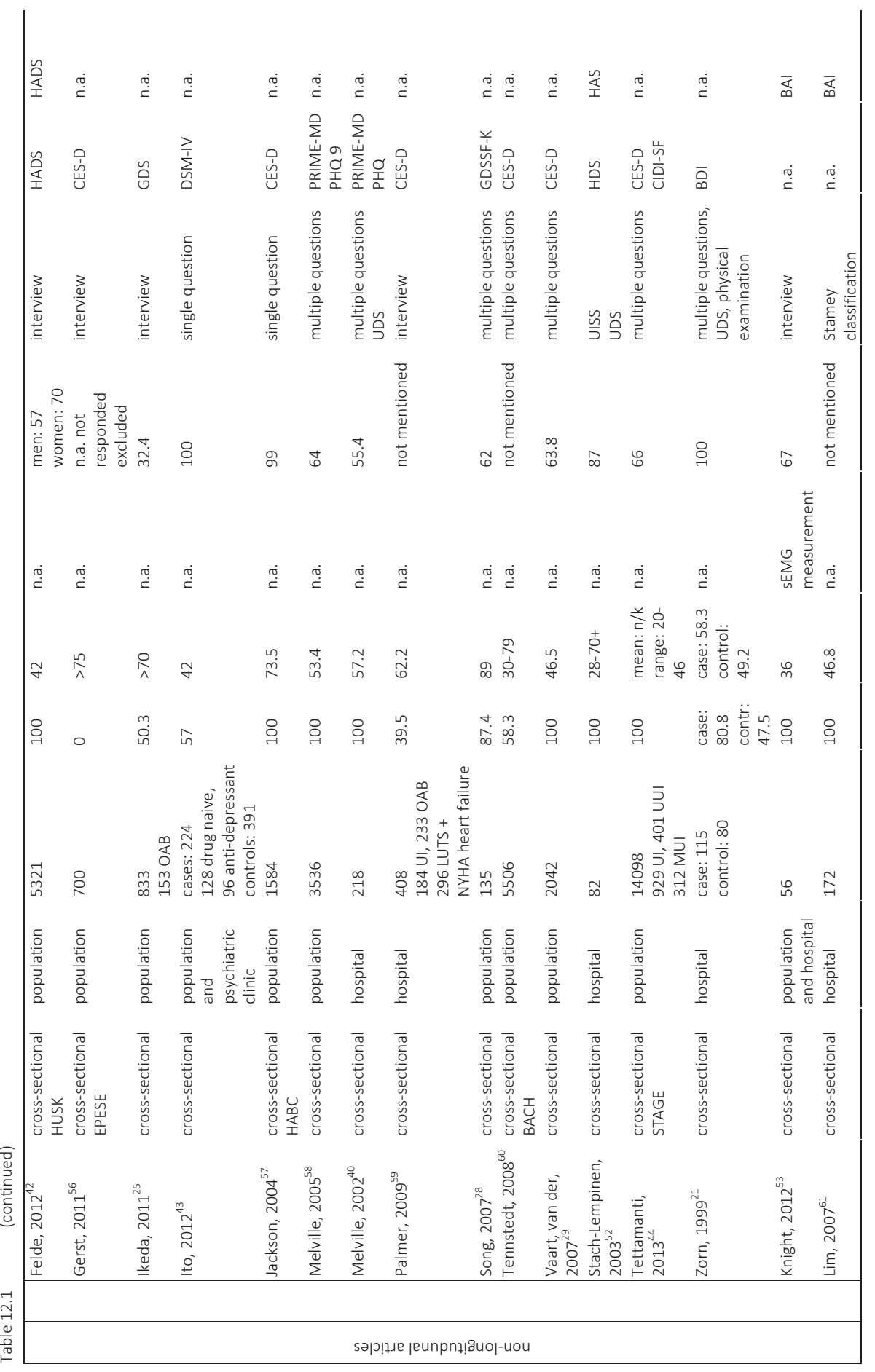




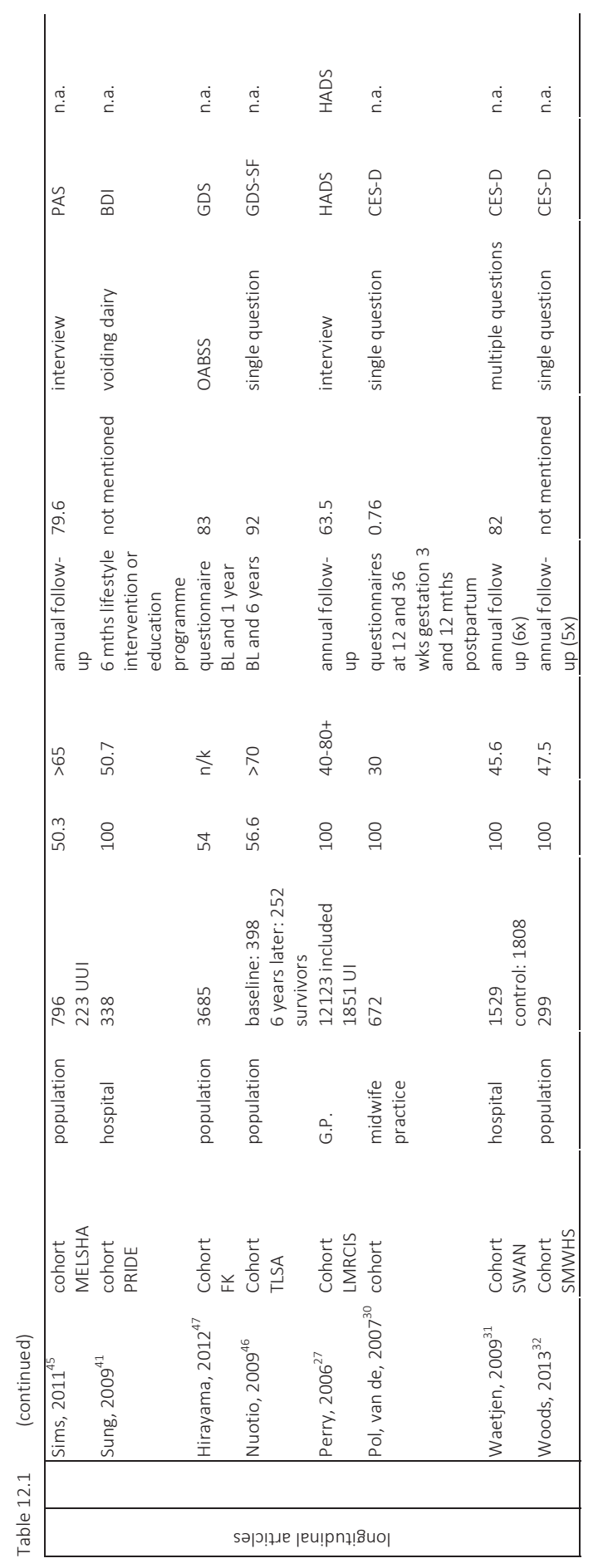




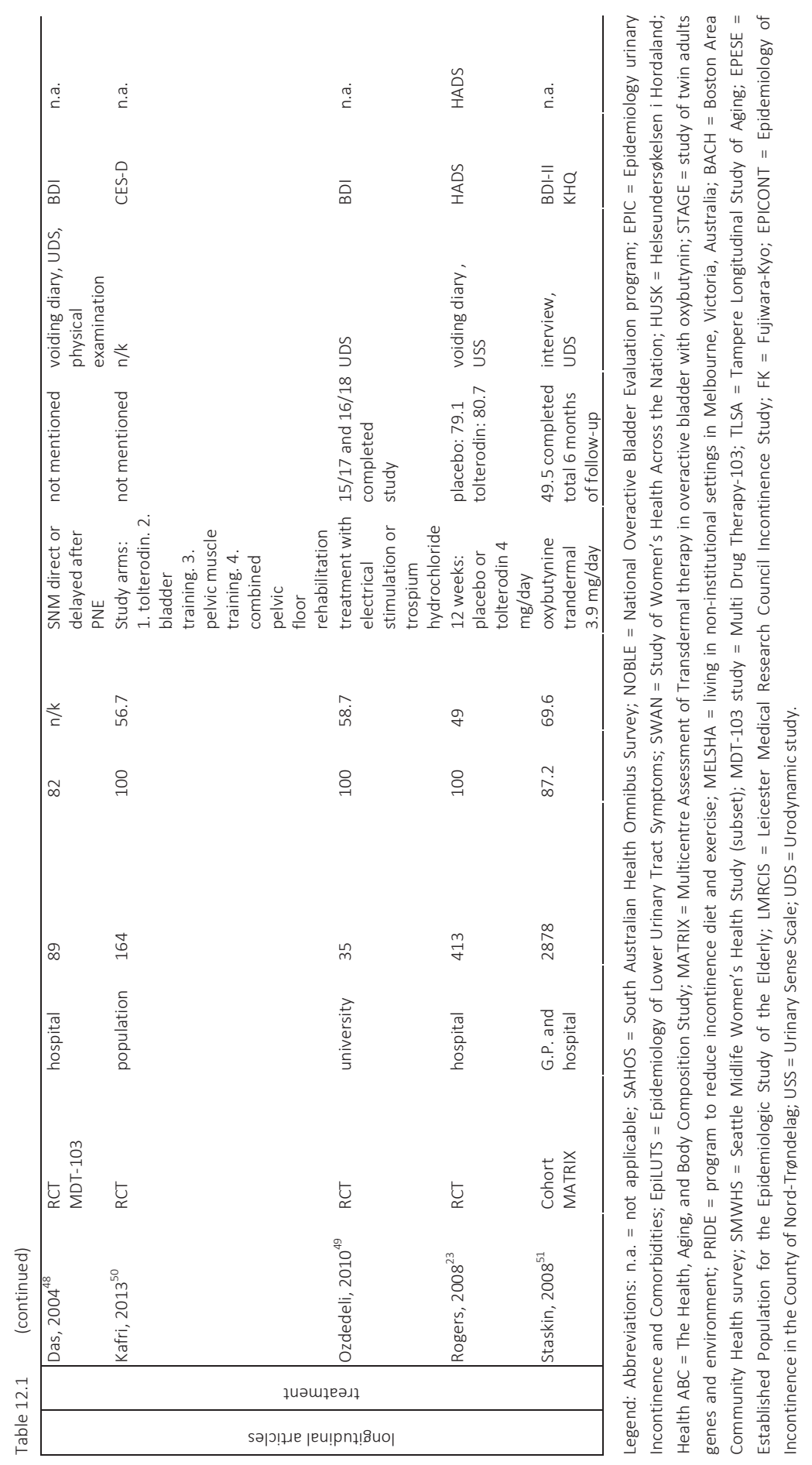




\section{Depressive symptoms and OAB: longitudinal studies}

Thirteen longitudinal studies were included, of which 9 were cohort studies and 4 were randomised controlled trials. Overall, almost 40000 subjects were analysed in these studies. Regarding affective complaints, the data of two longitudinal studies were only cross-sectional analysed and focuses upon the presence of depressive symptoms and $\mathrm{OAB}$ at baseline ${ }^{41,45}$.

Most longitudinal studies $(n=9)$ revealed a positive association of OAB with depressive symptoms, whereas the remaining four did not find any association ${ }^{23,30-32}$. Several studies $(n=4)$ showed a positive association of OAB onset in persons with depressive symptoms ${ }^{27,30,46,47}$. The studies of Perry et al. and van de Pol et al. revealed a positive association of onset of depressive symptoms in persons with $O A B^{27,30}$. One study revealed a positive association of onset of anxiety symptoms in persons with $O A B$ and reported a positive association between the onset of $O A B$ in anxious patients ${ }^{27}$. $A$ population based longitudinal study with six years of follow up revealed that depressive mood was a sign predictor of incident urgency related $\mathrm{UI}^{46}$. A large prospective study of people older than 65 years demonstrated that depression measured by GDS was an independent risk factor for new onset $O A B$ one year later ${ }^{47}$. The aim of another important prospective longitudinal study was to investigate the association between anxiety and depression and urgency incontinence in women ${ }^{27}$. Incident cases of anxiety and depression were predicted by the presence of UUI at baseline. Incident cases of UUI were predicted by anxiety, but not by depression, at baseline. Anxiety, UUI and frequency appeared to interact and exacerbate each other ${ }^{27}$. At 36 weeks gestation OAB was significantly associated with depression in multivariate analysis, but there was no association one year after childbirth ${ }^{30}$. Similarly, no association was found between depression and urinary incontinence in women followed six years through menopausal transition $^{31}$.

\section{Randomised controlled trials}

Among the longitudinal studies there were four randomised controlled trials which showed that successful treatment of $O A B$ led to less affective symptoms regardless of the investigated intervention ${ }^{23,48-50}$. Furthermore, one study showed only significantly less anxiety ${ }^{23}$. A cohort study without a control arm in solely men revealed that mean Beck Depression Inventory (BDI) and bladder symptoms improved significantly after 6 months of treatment with oxybutynin. The proportion of men with BDI scores indicating depression (>12) decreased from 23.9 to $17.9 \%{ }^{51}$. The randomised clinical trials consisted of comparing interventions ${ }^{49,50}$, interventions with placebo ${ }^{23}$ or timing of intervention ${ }^{48}$. OAB symptoms improved significantly more in the medication group after 12 weeks of treatment in a double blind placebo controlled trial of tolterodin in sexually active women. Baseline HADS scores were indicative for anxiety, but not for depression. There was a significant decrease of the mean HADS-A score, from aberrant to normal, 
between the two groups ${ }^{23}$. Ozededeli et al. compared effects of trospium hydrochloride and electrical stimulation during a 6 week follow up in two groups of women with OAB on urodynamic parameters, bladder diaries and BDI. There was significant improvement on all parameters in both groups ${ }^{49}$. Similar results were found by a secondary analysis of an assessor- blinded randomised controlled trial comparing three forms of physiotherapy with medication for self-reported UUI ${ }^{50}$. Although clinically diagnosed depression was an exclusion criterion, 36 (22\%) women had a CES-D score $>16$ at baseline. These women had a significant decrease in CES-D scores 3 and 12 months after therapy, which was accompanied by less $O A B$ related symptoms.

At the baseline of a study population with $O A B, 73 \%$ of the subjects had some level of depression. After randomisation between early and late implantation of a sacral neuromodulator only in the early implant group the BDI scores declined significantly ${ }^{48}$ (Table 12.1).

\section{Anxiety and OAB studies}

Thirteen articles concerning nine studies reported about anxiety. Six studies revealed a positive association (Table 12.2). Three studies did not show any association ${ }^{20,22,52}$. The two case-control studies yielded contradictory conclusions ${ }^{22,53}$. Most cross-sectional studies showed a positive association between urological symptoms and anxiety. In a cross-sectional population-based study amongst 5321 young women UI was associated with both anxiety and depression. The strongest associations were found for MUI and $\mathrm{UUI}^{42}$.

American, English and Swedish EpiLUTS data, including 30000 men and women, showed that OAB symptoms were significantly associated with positive HADS anxiety sub-scores in men and women ${ }^{38}$. In the US, EpiLUTS' respondents who experienced bothersome $O A B$ symptoms, were more likely to report anxiety than those with $O A B$ without bothersome symptoms ${ }^{39}$. Data from the UK and Sweden yielded similar results. Increasing levels of anxiety were strongly predictive of $O A B$ bother in both men and women $^{10}$. From a longitudinal perspective, a positive association between anxiety and urge incontinence was reported in a prospective postal survey of women with follow up one year later. Anxiety measured by HADS was present in $56.6 \%$ of those with UUI. Anxiety was not only associated with UI, but also with a number of other urinary symptoms. The presence of $\mathrm{UI}$ and/or frequency (but not SI or urgency) predicted incident cases of anxiety one year later. Vice versa, the presence of anxiety at baseline predicted incident cases of UI one year later. Anxiety, UUI and frequent micturition, appeared to interact by exacerbating each other ${ }^{10}$ (Table 12.2). 


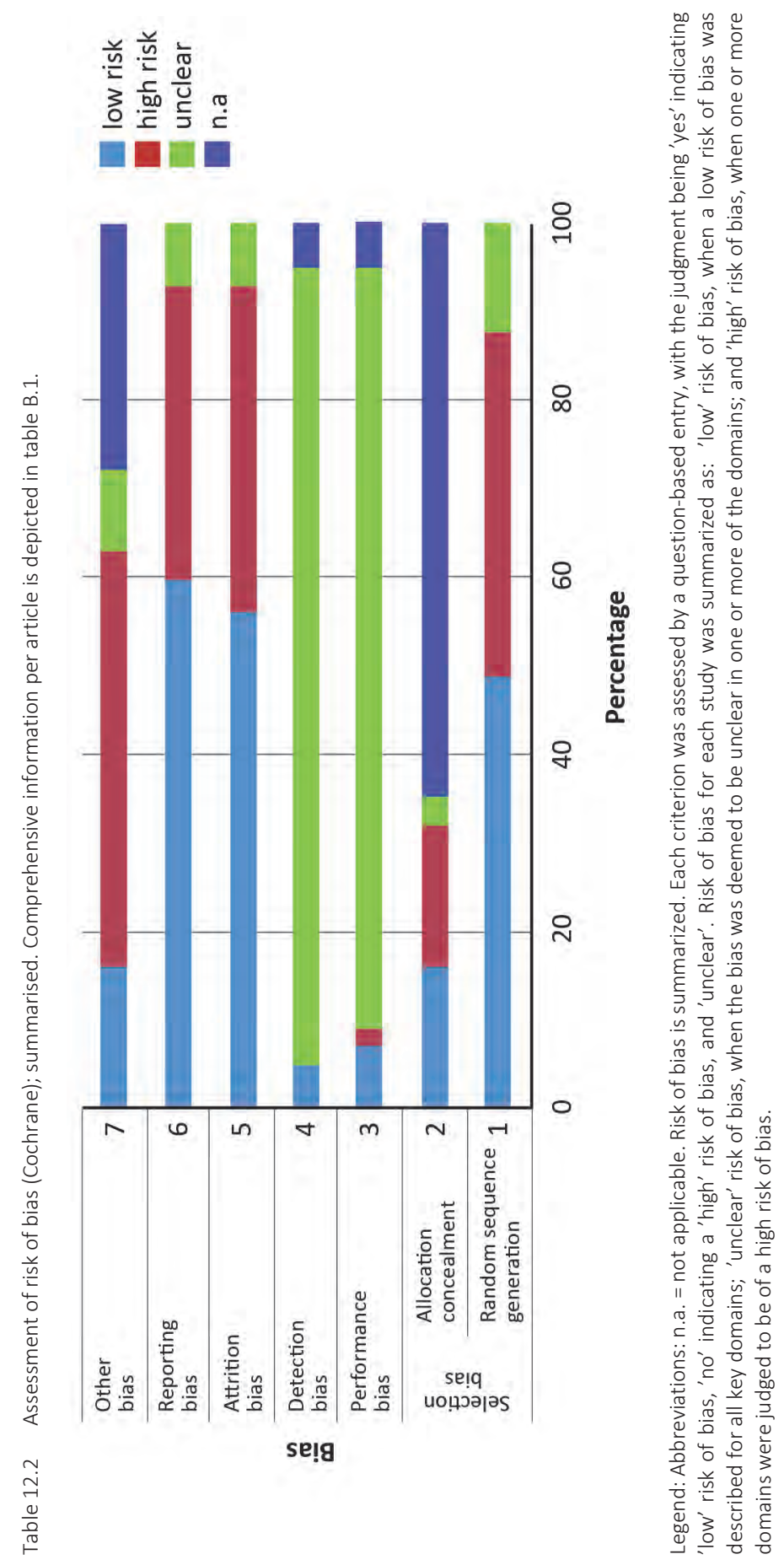




\section{Depressive symptoms and/or anxiety and OAB in men}

Twenty-two articles included men, Table 12.1. Of these, ten studies reported specifically about men (NOBLE, EPIC, EpiLUTS), see Table 12.3. Eight studies concluded that there is an association of $O A B$ with depressive or anxiety symptoms. The remaining two studies including men and women reported an association of $O A B$ with anxiety and depression for women, but not for men ${ }^{45,48}$.

Five articles from the EpiLUTS study included $47 \%$ men. Men with storage symptoms reported lower rates of depression (15.4\% vs. $26.6 \%$ ) and anxiety (12\% vs $14.9 \%)$ than women ${ }^{38}$. Men and women with bothersome OAB symptoms reported the highest rate of anxiety and depressive complaints ${ }^{10,39}$. In the UK, this was higher in men than in women and increased anxiety was predictive of $O A B$ bother ${ }^{10}$. Depressive symptoms predicted treatment seeking in men but not in women ${ }^{10,39}$. In an older subset of EpiLUTS, anxiety $(16.4 \%$ vs $3.3 \%, P<0.001))$ and depressive symptoms $(17.7 \%$ vs $4.0 \%$, $\mathrm{p}<0.001$ ) were more frequent in men with $O A B$ versus men without $O A B$. Furthermore, the rate of urgency $(25.9 \%)$ was higher than in aged-matched controls $(9 \%, p \leq 0.01)$ in depressed men without anti-depressants, recruited from the psychiatric clinic ${ }^{43}$. Hirayama et al. reported results of a questionnaire based follow up in 1410 men $^{47}$. Depression and the number of men was significantly more frequent in the new onset $O A B$ group. Both parameters were independent risk factors for $O A B$ in multivariate analyses $^{62}$. Moreover, men with OAB-wet had significantly poorer scores on the CES-D scale compared to men who had $O A B$ without incontinence ${ }^{33}$.

\section{Quality assessment of the included literature}

Level of evidence was stated per article as defined in publications of the University of Oxfords' Centre for Evidence-based Medicine (CEBM) ${ }^{17}$ (Table B.1 and summarised in Table 12.3). Risk of bias assessment was performed according to the Cochrane Collaboration guidelines (Table 3). The GRADE approach was applied to rate the quality of evidence for each outcome measure ${ }^{19}$ (Appendix D). An outcome-level assessment of risk of bias was maintained, after assessing the risk of bias for the studies as a whole, as the quality of evidence may differ across outcomes within one study.

The GRADE level of the outcome 'co-occurrence OAB and depressive symptoms' was upgraded $(+1)$, because of the very large group of subjects. In male subjects this cooccurrence was downgraded (-1), because of evident selection bias (dismissing existing prostate (obstruction) problems). Hirayama et al. did not report about new onset of depressive outcomes, additional inquiry revealed that only 61 (7\%) of 834 OAB patients developed depression, compared to $5 \%$ developing depressive symptoms in the control group $^{47}$. 


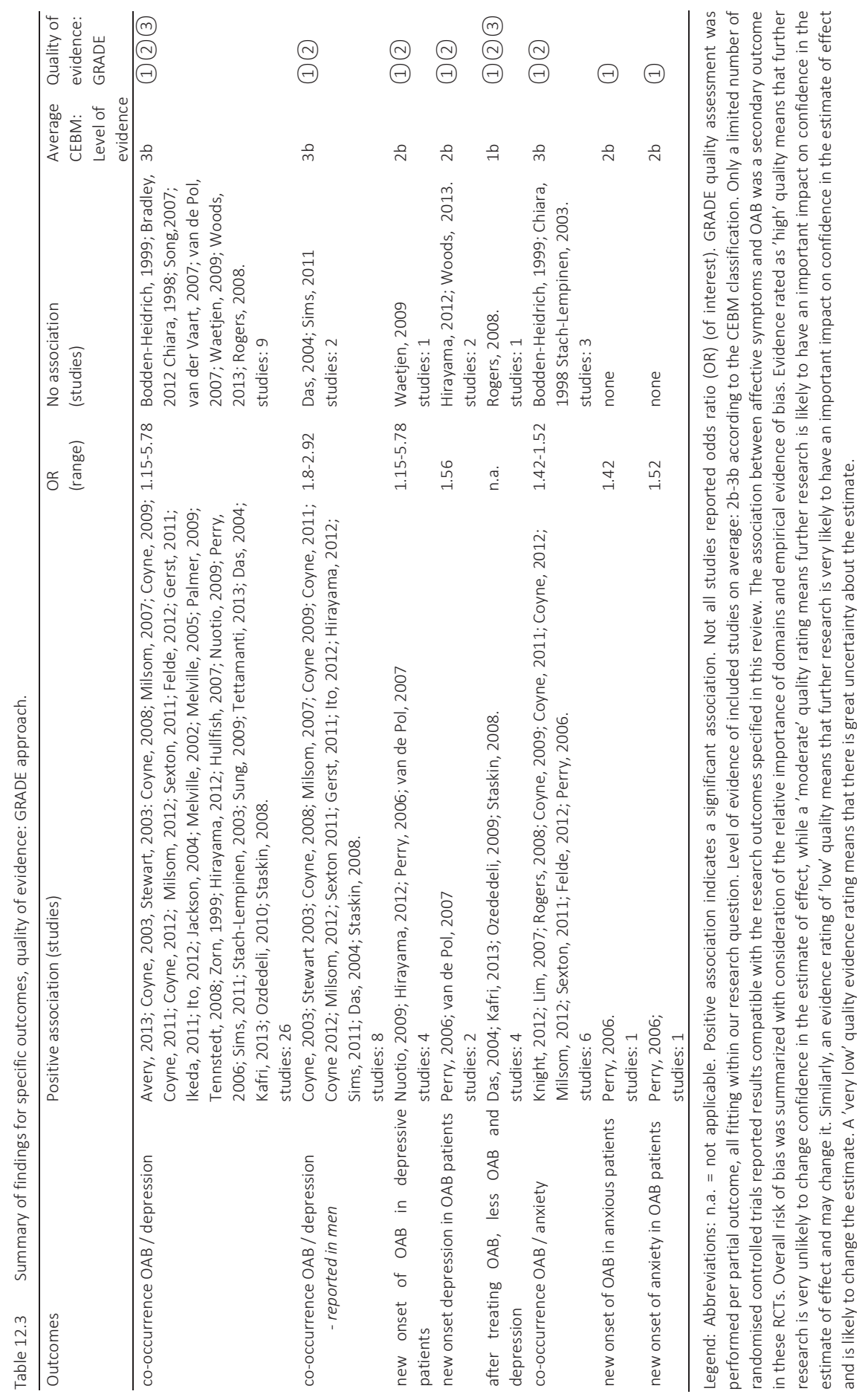


Adhering to the GRADE approach, quality of evidence concerning co-occurrence of OAB and depression was rated GRADE level 3. Evidence reporting an association the cooccurrence of anxiety and $O A B$ was GRADE level 2. Longitudinal associations between new onset of $O A B$ in depressive subjects was GRADE level 2. Evidence reporting OAB in longitudinal association with anxiety was GRADE level 1.

\section{Discussion}

To our knowledge, this systematic review is the first to give an extensive qualitative overview of the current literature on the association between $O A B$ and affective symptoms. The aim of the current study was to (i) review articles providing evidence on the association of affective conditions with $O A B$; (ii) to examine the direction and strength of the association; (iii) to gauge the possible influence of method and design factors in terms of occasioning bias or confounding; and (iv) to formulate considerations for future research.

Forty-three articles reporting 37 studies were included, comprising more than 80000 subjects in total. Of these articles 32 concern depression, 2 are solely about anxiety and 9 concern both. Regarding depression and overactive bladder there is a positive association in 26 studies. Regarding anxiety six studies reveal a positive association. In longitudinal studies, different directional associations were reported: a) $O A B$ subjects who develop depression/anxiety or b) depressed/anxious subjects who develop $O A B$, or $c$ ) both. The quality of the evidence in studies reporting an association between the co-occurrence of $O A B$ and depression was rated level 3 conform the GRADE framework. Evidence reporting an association on the co-occurrence of anxiety and $O A B$ was GRADE level 2. Longitudinal associations between new onset of $O A B$ in depressive subjects was GRADE level 2. Evidence reporting an association of $O A B$ in longitudinal association with anxiety was GRADE level 1.

No RCT was found with exact outcome measures as stated in this systematic review. Nevertheless, 4 RCTs were included, because they reported outcome measures of interest. These trials reveal a positive association between $O A B$ and affective symptoms because successful treatment of $O A B$ resulted in a significant decrease of affective complaints.

The main findings of this systematic review are that there is abundant and quality evidence of a positive association between co-occurrence of $O A B$ and depression and to a lesser extent the same accounts for an association between co-occurrence of OAB and anxiety. However, many evaluated studies failed to note longitudinal changes and lacked evidence of causality. The association between $O A B$ and depressive symptoms might be bidirectional in nature. Only strong evidence is found concerning the new onset of OAB in depressive patients (GRADE level 2). Evidence of the uni- or bidirectional association between $O A B$ and anxiety was too poor to make meaningful statements (GRADE level 1). 
Furthermore it is hard to identify the role of urgency incontinence in $O A B$, because only a few articles reported about separate results for OAB dry and wet. There was increased rate of depression independent of continence status ${ }^{33,36}$ and higher CES-D scores in $O A B$ wet were reported ${ }^{33,35}$. On the other hand, Ikeda et al. found an association between OAB-dry (without incontinence) and depression ${ }^{25}$.

\section{Possible explanations for association}

One possible explanation for the association of affective disorders with $O A B$ may be that $\mathrm{UI}$ can lead to social and functional impairment resulting in the accumulation of stress. This, in turn, may lead to depression ${ }^{58}$. The association between affective disorders and $O A B$ may further be explained by the fact that both syndromes share common biological pathways. For instance, serotonin and norepinephrine, as well as the interactions between the two neurotransmitter systems are thought to play a role in the aetiology of depression ${ }^{47}$. Serotonin depletion is thought to contribute to depression and selective serotonin reuptake inhibitors (SSRIs) are effective in depression treatment ${ }^{63}$. Moreover, in anxiety disorders, it is likely that the serotonergic system plays a role in regulation of anxiety both during development and in adulthood ${ }^{64}$. Additionally, serotonin plays a role in bladder function. Several animal studies revealed that lowering serotonin levels in the central nervous system (CNS) was accompanied by urinary frequency and detrusor overactivity $^{65}$. Most experimental studies in rats and cats indicated that activation of the central serotonergic system depresses reflex bladder contractions and increases the bladder volume threshold for micturition induction ${ }^{66}$. Affective disorders may thus be linked to $O A B$, due to altered serotonin function. Moreover, a rat model of depression caused by lowering of serotonin in the CNS, produced $O A B$ manifestation (urinary frequency due to non-neurogenic detrusor overactivity and lowered bladder capacity) in female rats. Administration of a selective serotonin reuptake inhibitor (SSRI) reversed urinary symptoms ${ }^{67}$. In humans, duloxetine, a serotonin-noradrenaline reuptake inhibitor (SNRI) improved frequency and urgency in female OAB patients significantly ${ }^{68}$.

Another possible explanation may be a shared dysregulation of the hypothalamicpituitary-adrenal (HPA) axis resulting in increased corticotroponin-releasing factor (CRF), increased adrenocorticothropic hormone (ACTH) and increased cortisol; the HPA-axis is thought to play a role in depression and anxiety disorders ${ }^{63,69,70}$ and there is mounting evidence that CRF is involved in bladder function as well. In rats, repeated psychological distress resulted in increased urinary frequency and lower voiding volume ${ }^{71}$. CRF stimulated bladder function (lowered micturition threshold accompanied by lower urine volume) in a rodent model of detrusor overactivity and anxiety, and administration of a CRF antagonist reduced detrusor overactivity ${ }^{72}$. Additionally, several investigations from functional brain magnetic resonance imaging (MRI) during urodynamic studies revealed that increased brain activation in the anterior cingulate cortex (ACC) was associated with increased urgency at large bladder volumes, especially in young females with UI and detrusor overactivity ${ }^{73-75}$. Accordingly, in young people with depressive disorder 
increased activity levels were found in the ACC. The ACC is part of a neural stressnetwork, which is involved in (social) pain aspects ${ }^{76,77}$. Single photon emission computed tomography (SPECT) of older patients with UI showed hypo-perfusion in the regions of the prefrontal cortex ${ }^{78}$. Similarly, hypo-perfusion in the ACC and the prefrontal cortex was also seen in patients with major depressive disorder ${ }^{79}$. These findings suggest that prefrontal brain regions and parts of the default mode network may mediate both affective disorders and functional somatic complaints, like OAB.

\section{Strengths and limitations of the review}

The validity of the conclusion of our systematic review is influenced by the quality of the included studies. A large part of the initially retrieved articles did not employ validated screening instruments for the assessment of affective symptoms. The exclusion of these articles minimized the amount of bias. The most important biases are present in the definition and selection of the study population and the data collection. The quality of evidence may differ across outcomes within one study. An outcome-level assessment of risk of bias was maintained, after assessing the risk of bias for the studies as a whole. In the longitudinal studies, associations in different directions were present: OAB patients developing depression/anxiety and/or depressed/anxious people developing $O A B$, or both, all compared with control subjects. Furthermore, it is difficult to assess the prevalence of affective symptoms across different groups, as most of the studies reported differences in mean scores and odds ratios rather than percentages. Moreover, there was substantial heterogeneity with regard to diagnostic assessment. Only a few studies controlled for confounding variables and performed multivariate analysis ${ }^{42}$. Risk of selection bias was kept to a minimum because two reviewers independently selected the articles to be in- or excluded. Only studies with adult subjects were included, this could have introduced some selection, affecting generalizability across all age groups. However, the overall conclusion stands in different age groups, strengthening our conclusions. Two authors, to avoid information bias, independently conducted quality assessment and risk of bias assessment of the 43 included articles. Language restriction may have led to biased results. Although articles not published in English are more common to exclude, Egger et al. demonstrated that there was a trend to publish in English if results achieved statistical significance ${ }^{80}$. On the other hand, the same authors concluded that language bias had no real effect on summary effect estimates most of the time. Thus, language restriction may possibly induce publication bias.

The most recent definition of OAB was launched in 2002. Three studies were published before $2002^{20-22}$. The definition of OAB may be less precise before that date. However, the current ICS definition is still not very specific, leaving the diagnosis of OAB heterogeneous. For instance, in research practice, different cut-off values concerning frequency were used. Thus, patients with OAB according to the ICS definition remain to be a heterogeneous group. Consecutively, Lee et al. suggested that there is disagreement among physicians in the way $O A B$ is defined ${ }^{81}$. A more defined 
specification of the ICS definition is needed, since diagnosing multi-conditional disorders may be affected by underdetection ${ }^{82}$. Furthermore, some of the excluded studies focussed on quality of life (QoL) only. This is a broad category, in which affective aspects are hard to specify. These studies showed that subjects with OAB had significantly lower scores on QoL scales (mostly used: Short Form Health Survey (SF-36)) ${ }^{83-85}$. Some longitudinal research showed that patients with low QoL scores, tended to develop OAB significantly more often ${ }^{84,85}$. In order to strengthen the conclusions found in $O A B$ literature in the area of psychological wellbeing, psychological dimensions need to be specified more precisely, particularly with regard to affective conditions. Studies in men did not correct (sufficiently) for obstruction induced $O A B$ in (elderly) men e.g. ${ }^{51,62}$, thus making the results not automatically generalizable for men.

\section{Future research and implications}

Currently there are no on-going studies listed in the clinical trial database "ClinicalTrials.gov" to further explore the association of affective disorders with OAB. Hence, there is need for well-designed studies in order to clarify the exact nature of this association. Relevant pathophysiological mechanisms need to be integrated in these studies. Furthermore, a standardised, but also more personalized diagnostic and treatment-evaluation tool is required. The Experience Sampling Method (ESM), applied using an m-health device, may fulfil this need. By exploring subjective reactions in daily life to experiences as they occur in the natural environment a better understanding of the relation between perceived stress and bladder function would be possible. ESM research in the area of affective conditions is promising ${ }^{86}$.

Considering the shortcomings of care management in patients with $\mathrm{OAB}^{6}$ and given the strong association with affective symptoms, which are associated with treatment non-adherence ${ }^{87}$, an integrated approach involving urology, (uro-)gynaecology and psychiatry is recommended.

\section{Conclusions}

The results of this systematic review reveal a positive association between the cooccurrence of $O A B$ and affective symptoms. Furthermore, there is evidence for new onset of $O A B$ in depressive subjects, but further research is necessary to examine the strength of the effect. However, many evaluated studies relied on a single survey instrument, failed to note longitudinal changes and lacked information allowing assessment of epidemiological criteria for causality. Affective dysregulation may occasionally worsen $\mathrm{OAB}$ symptoms and poorer treatment outcome. Results also indicate that mental health professionals would do well to take into account urological symptoms in patients with affective complaints. The precise relationship of OAB with 
affective conditions, is difficult to establish, but may involve a mechanism in the "bladder-brain-axis". Further research is needed in order to determine the direction of the association and the underlying pathophysiological pathways. 


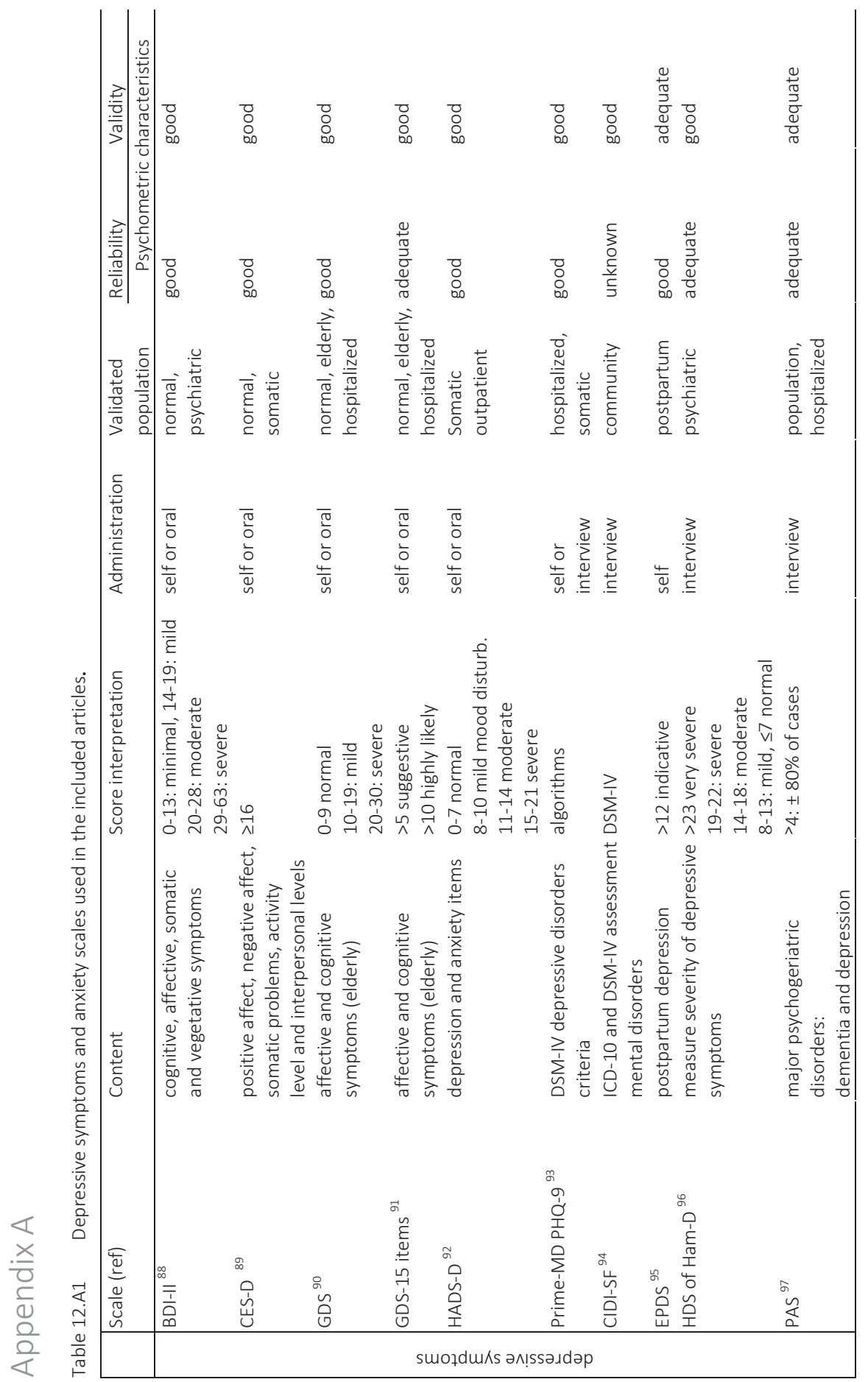




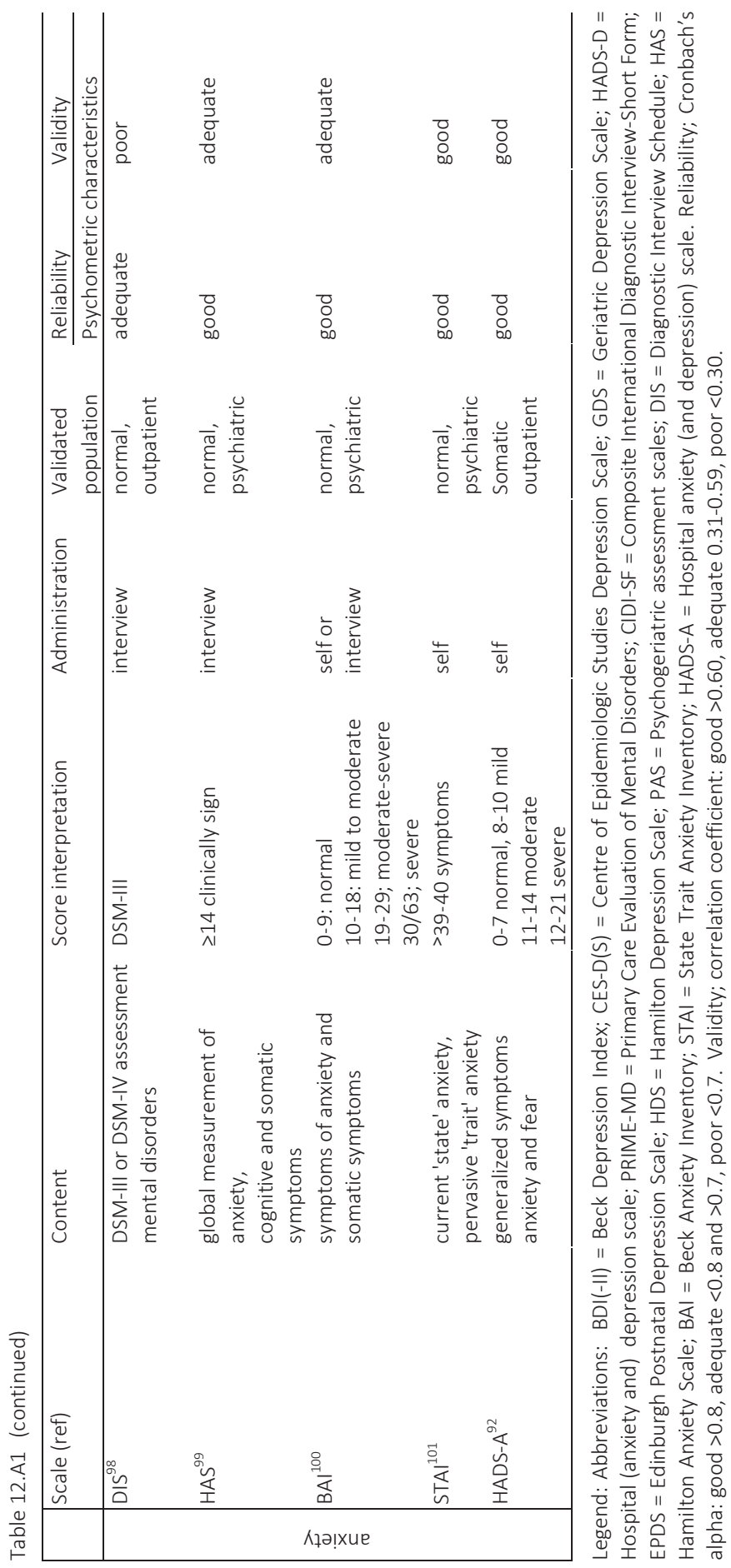




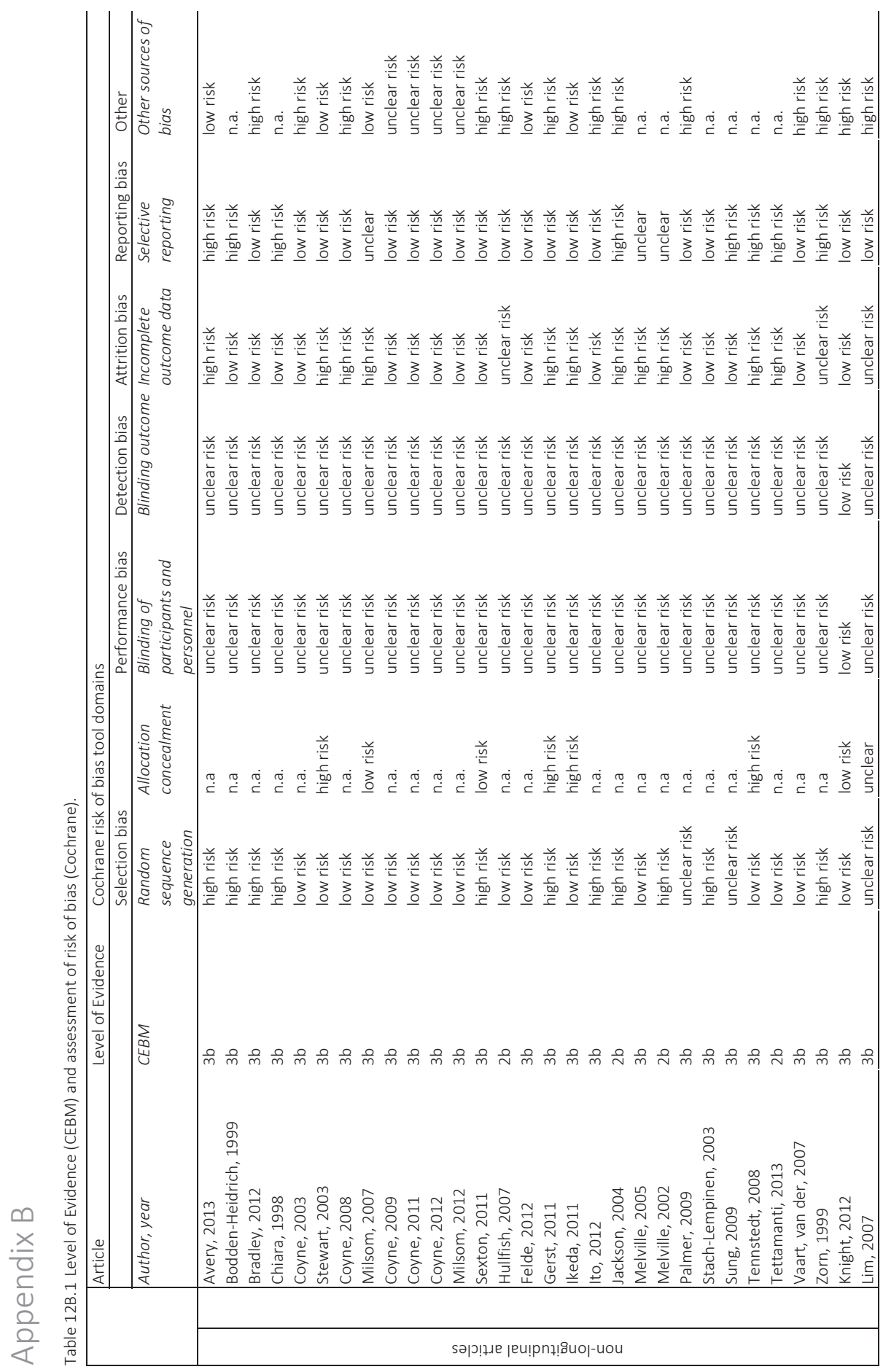




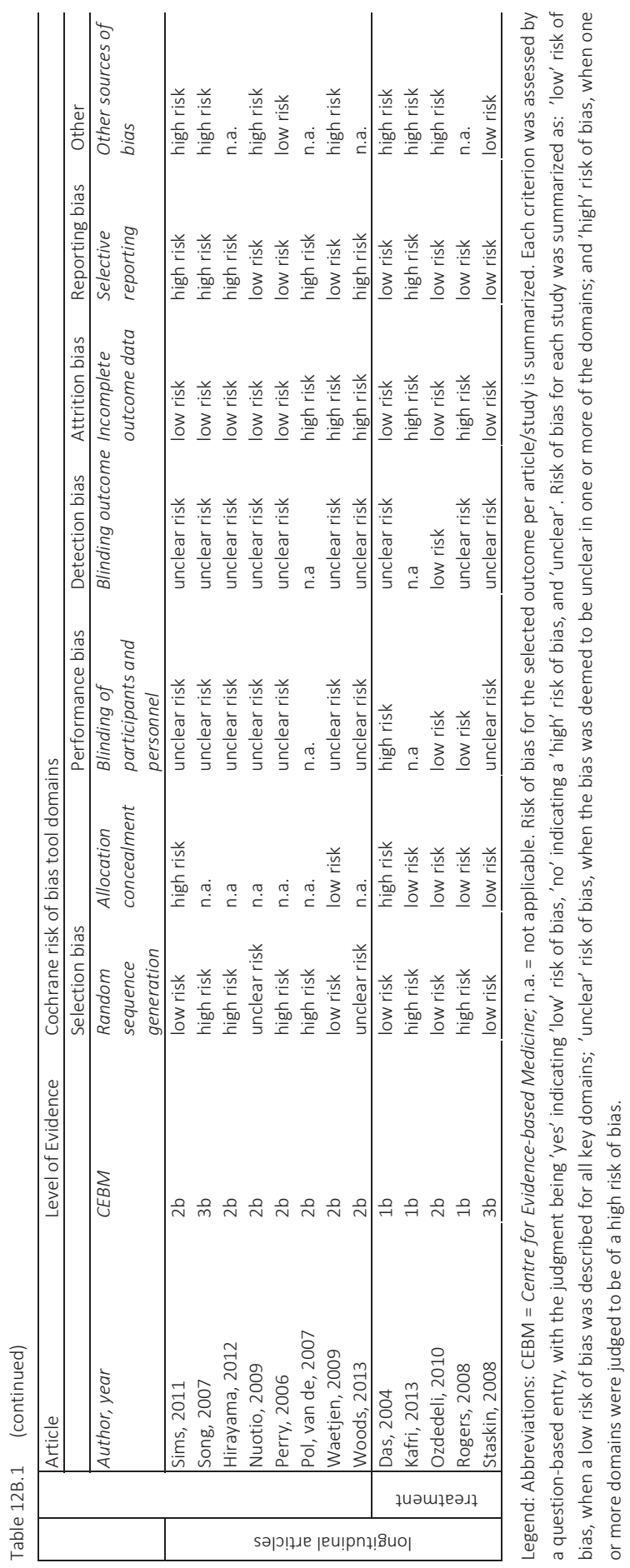




\section{Appendix C.}

\section{C.1 Literature search}

Detrusor overactivity, bladder overactivity, urinary bladder overactive, overactive bladder (syndrome), overactive detrusor function, overactive detrusor, urinary incontinence urge, urinary urge incontinence, $O A B$, urge incontinence, urgency/frequency (syndrome), urinary urgency/frequency (syndrome), urinary urgency incontinence was combined with depression, depressive disorder, depressive traits, depressive symptoms, emotional depression, anxiety, anxiety disorder, anxiety symptoms, anxiety traits, generalized anxiety disorder, panic disorder, Hospital anxiety and depression scale, Hamilton anxiety scale, Beck depression scale, Center for epidemiological studies depression scale, affective disorder, affective symptoms, mental health.

\section{Appendix D.}

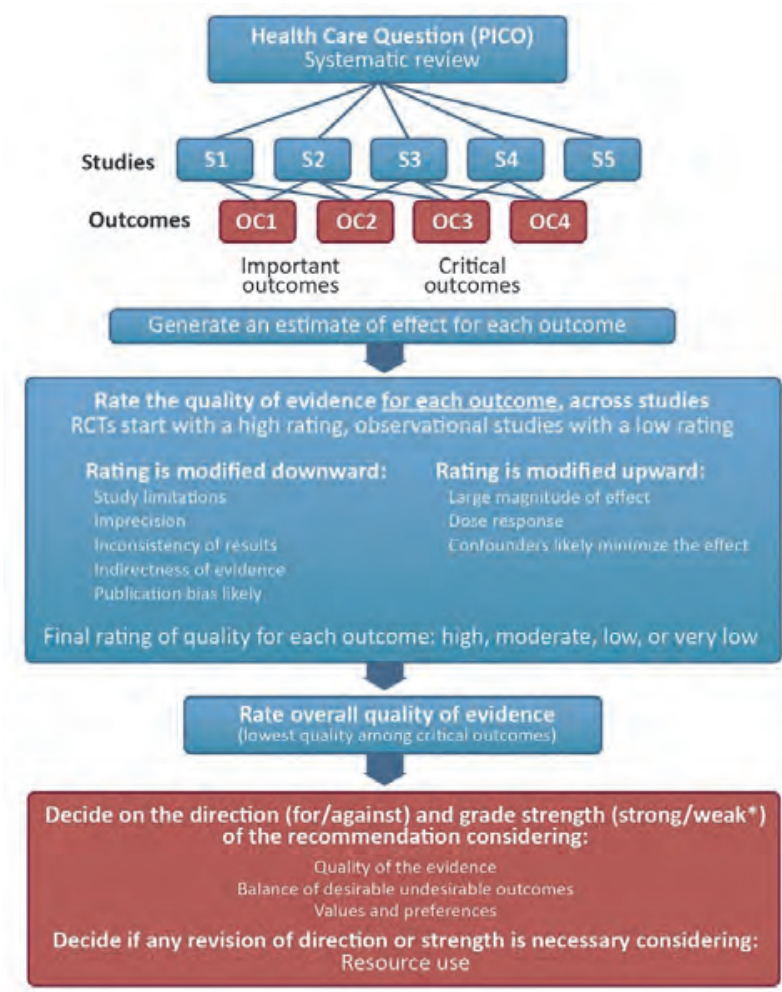

Figure 12D.1 Schematic view of GRADE approach. Abbreviation: RCT, randomized controlled trials. Reprinted with permission from G. Guyatt et al. 2011 ${ }^{(102)}$. * Also labeled "conditional" or "discretionary". 


\section{References}

1. Diagnostic and Statistical Manual of Mental Disorders. DSM-IV. 4th Edition ed (2000).

2. Wang, P. S. et al. Twelve-month use of mental health services in the United States: Results from the National Comorbidity Survey Replication. Arch. Gen. Psychiatry 62, 629-640 (2005).

3. Ustun, T. B. et al. Global burden of depressive disorders in the year 2000. Br J Psychiatry 184, 386-392 (2004).

4. Abrams, P. et al. The standardisation of terminology of lower urinary tract function: Report from the standardisation sub-committee of the International Continence Society. Neurourol. Urodyn. 21, 167-178 (2002).

5. Coyne, K. S. et al. National community prevalence of overactive bladder in the United States stratified by sex and age. Urology 77,1081-1087 (2011).

6. Milsom, I. et al. How widespread are the symptoms of an overactive bladder and how are they managed? A population-based prevalence study. BJU Int. 87, 760-766 (2001).

7. $\mathrm{Hu}, \mathrm{T} . \mathrm{W}$. et al. Estimated economic costs of overactive bladder in the United States. Urology 61, 11231128 (2003).

8. Tubaro, A. Defining overactive bladder: Epidemiology and burden of disease. Urology 64 (SUPPL. 1), 2-6 (2004).

9. Coyne, K. S. et al. Assessing patients' descriptions of lower urinary tract symptoms (LUTS) and perspectives on treatment outcomes: Results of qualitative research. Int. J. Clin. Pract. 64, 1260-1278 (2010).

10. Coyne, K. S. et al. The impact of overactive bladder on mental health, work productivity and healthrelated quality of life in the UK and Sweden: Results from EpiLUTS. BJU Int. 108, 1459-1471 (2011).

11. Engel, W. J. Uropsychiatry. Journal - Michigan State Medical Society. 63, 273-277 (1964).

12. Frewen, W. K. Urgency incontinence: Review of 100 cases. J. Obstet. Gynaecol. Br. Commonw. 79, 77-79 (1972).

13. Macaulay, A. J. et al. Micturition and the mind: Psychological factors in the aetiology and treatment of urinary symptoms in women. Br. Med. J. 294, 540-543 (1987).

14. Cortes E et al. The psychology of LUTS: ICI-RS 2011. Neurourol. Urodyn. 31, 340-343 (2012).

15. Uo York, dissemination Cfra. The overactive bladder and affective symptoms 2013. Available from: http://www.crd.york.ac.uk/PROSPERO/.

16. Moher, D. et al. Preferred reporting items for systematic reviews and meta-analyses: The PRISMA statement. J. Clin. Epidemiol. 62, 1006-1012 (2009).

17. Centre for Evidence Based Medicine UoO, US. Levels of Evidence. Medical Sciences Division; March 2009.

18. Higgins, J. P. Cochrane Handbook for Systematic Reviews of Interventions; 2011.

19. Guyatt, G. H. et al. GRADE guidelines: A new series of articles in the Journal of Clinical Epidemiology. J. Clin. Epidemiol. 64, 380-382 (2011).

20. Chiara, G. et al. Psychological investigation in female patients suffering from urinary incontinence. Int. Urogynecol. J. Pelvic Floor Dysfunct. 9, 73-77 (1998).

21. Zorn, B. H. et al. Urinary incontinence and depression. J. Urol. 162, 82-84 (1999).

22. Bodden-Heidrich, R. et al. Psychosomatic aspects of urinary incontinence. Arch. Gynecol. Obstet. 262, 151-158 (1999).

23. Rogers, R. et al. Efficacy of tolterodine on overactive bladder symptoms and sexual and emotional quality of life in sexually active women. Int. Urogynecol. J. Pelvic Floor Dysfunct. 19, 1551-1557 (2008).

24. Bradley, C. S. et al. Urinary incontinence, depression and posttraumatic stress disorder in women veterans. Am. J. Obstet. Gynecol. 206, 502.e1-8 (2012).

25. Ikeda, Y. et al. Risk factors for overactive bladder in the elderly population: A community-based study with face-to-face interview. Int. J. Urol. 18, 212-218 (2011).

26. Sexton, C. C. et al. Prevalence and effect on health-related quality of life of overactive bladder in older americans: Results from the epidemiology of lower urinary tract symptoms study. J. Am. Geriatr. Soc. 59, 1465-1470 (2011). 
27. Perry, S. et al. An investigation of the relationship between anxiety and depression and urge incontinence in women: Development of a psychological model. Br J Health Psychol. 11, 463-482 (2006).

28. Song, H. J. \& Bae, J. M. Prevalence of urinary incontinence and lower urinary tract symptoms for community-dwelling elderly 85 years of age and older. J. Wound Ostomy Continence Nurs. 34, 535-541 (2007).

29. van der Vaart, C. H. et al. Association between urogenital symptoms and depression in communitydwelling women aged 20 to 70 years. Urology 69, 691-696 (2007).

30. van de Pol, G. et al. Is there an association between depressive and urinary symptoms during and after pregnancy? Int. Urogynecol. J. Pelvic Floor Dysfunct. 18, 1409-1415 (2007).

31. Waetjen, L.E. et al. Association between menopausal transition stages and developing urinary incontinence. Obstet. Gynecol. 114, 989-998 (2009).

32. Woods, N. F. \& Mitchell, E. S. Consequences of incontinence for women during the menopausal transition and early postmenopause: Observations from the Seattle Midlife Women's Health Study. Menopause 20, 916-021 (2013).

33. Stewart, W. F. et al. Prevalence and burden of overactive bladder in the United States. World J Urol. 20, 327-336 (2003).

34. Coyne, K. S. et al. The impact on health-related quality of life of stress, urge and mixed urinary incontinence. BJU Int. 92, 731-735 (2003).

35. Coyne, K. S. et al. The impact of overactive bladder, incontinence and other lower urinary tract symptoms on quality of life, work productivity, sexuality and emotional well-being in men and women: Results from the EPIC study. BJU Int. 101, 1388-1395 (2008).

36. Milsom, I. \& Irwin, D. E. A cross-sectional, population-based, multinational study of the prevalence of overactive bladder and Lower Urinary Tract Symptoms: Results from the EPIC study. Eur. Urol. (Suppl) 6, 4-9 (2007).

37. Coyne, K. S. et al. Urinary incontinence and its relationship to mental health and health-related quality of life in men and women in Sweden, the United Kingdom, and the United States. Euo. Urol. 61, 88-95 (2012).

38. Coyne, K. S. et al. The burden of lower urinary tract symptoms: Evaluating the effect of LUTS on healthrelated quality of life, anxiety and depression: EpiLUTS. BJU Int. 103 (Suppl 3) 4-11 (2009).

39. Milsom, I et al. Effect of bothersome overactive bladder symptoms on health-related quality of life, anxiety, depression, and treatment seeking in the United States: Results from EpiLUTS. Urology 80, 9096 (2012).

40. Melville, J. L. et al. Prevalence of comorbid psychiatric illness and its impact on symptom perception, quality of life, and functional status in women with urinary incontinence. Am. J. Obstet. Gynecol. 187, 8087 (2002).

41. Sung, V. W. et al. Association between urinary incontinence and depressive symptoms in overweight and obese women. Am. J. Obstet. Gynecol. 200, 557.e1-5 (2009).

42. Felde, G. et al. Anxiety and depression associated with incontinence in middle-aged women: A large Norwegian cross-sectional study. Int. Urogynecol. J. Pelvic Floor Dysfunct. 23, 299-306 (2012).

43. Ito, T et al. Is major depression a risk for bladder, bowel, and sexual dysfunction? Low. Urin. Tract Symptoms 4, 87-95 (2012).

44. Tettamanti, G. et al. Depression, neuroticism, and urinary incontinence in premenopausal women: A Nationwide Twin Study. Twi.n Res. Hum. Genet. 16, 977-984 (2013).

45. Sims, J. et al. Urinary incontinence in a community sample of older adults: prevalence and impact on quality of life. Disabil. Rehailb. 33, 1389-1398 (2011).

46. Nuotio, M. et al. Six-year follow-up and predictors of urgency-associated urinary incontinence and bowel symptoms among the oldest old: a population-based study. Arch. Gerontol. Geriatr. 49, e85-90 (2009).

47. Hirayama, A. et al. Risk factors for new-onset overactive bladder in older subjects: results of the Fujiwara-kyo study. Urology 80, 71-76 (2012).

48. Das, A. K. et al. Improvement in depression and health-related quality of life after sacral nerve stimulation therapy for treatment of voiding dysfunction. Urology 64, 62-8 (2004).

49. Ozdedeli, S. et al. Comparison of intravaginal electrical stimulation and trospium hydrochloride in women with overactive bladder syndrome: a randomized controlled study. Clin. Rehabil. 24, 342-351 (2010). 
50. Kafri, R. et al. Depressive symptoms and treatment of women with urgency urinary incontinence. Int. Urogynecol. J. 24, 1953-1959 (2013).

51. Staskin, D. R. et al. Effects of oxybutynin transdermal system on health-related quality of life and safety in men with overactive bladder and prostate conditions. Int. J. Clin. Pract. 62, 27-38 (2008).

52. Stach-Lempinen, B. et al. Severe depression determines quality of life in urinary incontinent women. Neurourol. Urodyn. 22, 563-568 (2003).

53. Knight, S. et al. Comparisons of pelvic floor muscle performance, anxiety, quality of life and life stress in women with dry overactive bladder compared with asymptomatic women. BJU Int. 109, 1685-1689 (2012).

54. Avery, J. C. et al. Identifying the quality of life effects of urinary incontinence with depression in an Australian population. BMC Urol. 13, 11 (2013).

55. Hullfish, K. L. et al. Postpartum depression, urge urinary incontinence, and overactive bladder syndrome: Is there an association? Int. Urogynecol. J. Pelvic Floor Dysfunct. 18, 1121-1126 (2007).

56. Gerst, K et al. Self-reported urge urinary incontinence (UUI) among older Mexican-American men: Risk factors and psycho-social consequences. J. Immigr. Minor. Health 13, 1110-1115 (2011).

57. Jackson, R. A. et al. Urinary incontinence in elderly women: Findings from the health, aging, and body composition study. Obstet. Gynecol. 104, 301-7 (2004).

58. Melville, J.L. et al. Incontinence severity and major depression in incontinent women. Obstet Gynecol. 106, 585-592 (2005).

59. Palmer, M. H. et al. Urinary incontinence and overactive bladder in patients with heart failure. J Urol. 182, 196-202 (2009).

60. Tennstedt, S. L. et al. Prevalence of and risk factors for urine leakage in a racially and ethnically diverse population of adults: The Boston Area Community Health (BACH) Survey. Am J Epidemiol. 167, 390-399 (2008).

61. Lim, J. R. et al. Comparison of anxiety between patients with mixed incontinence and those with stress urinary incontinence. Scand. J. Urol. Nephrol. 41, 403-406 (2007).

62. Hirayama, A. et al. Risk factors for new onset of overactive bladder in older subjects: Results of the Fujiwara-kyo study. Neurourol. Urodyn.. 31, 765-766 (2012).

63. Nemeroff CB. The neurobiology of depression. Scientific American 278, 42-49 (1998).

64. Gordon, J. A. \& Hen, R. The serotonergic system and anxiety. Neuromolecular Med. 5, 27-40 (2004).

65. Steers, W. Potential targets in the treatment of urinary incontinence. Rev. Urol. 3, S19-26 (2001).

66. de Groat, W. C. Influence of central serotonergic mechanisms on lower urinary tract function. Urology 59, S1 30-36 (2002).

67. Lee, K. S. et al. Alterations in voiding frequency and cystometry in the clomipramine induced model of endogenous depression and reversal with fluoxetine. J. Urology 170, 2067-2071 (2003).

68. Steers, W. D. et al. Duloxetine compared with placebo for treating women with symptoms of overactive bladder. BJU Int. 100, 337-345 (2007).

69. Arborelius, L. et al. The role of corticotropin-releasing factor in depression and anxiety disorders. J. Endocrinol. 160, 1-12 (1999).

70. Nemeroff, C. B. Recent advances in the neurobiology of depression. Psychopharmacol. Bull. 36, S2 6-23 (2002).

71. Smith, A. L. et al. The effects of acute and chronic psychological stress on bladder function in a rodent model. Urology 78, 967e1-e7 (2011).

72. Klausner, A. P. et al. The role of corticotropin releasing factor and its antagonist, astressin, on micturition in the rat. Auton. Neurosci. 123, 26-35 (2005).

73. Griffiths, D. Imaging bladder sensations. Neurourol. Urodyn. 26, S899-903 (2007).

74. Griffiths, D. \& Tadic, S. D. Bladder control, urgency, and urge incontinence: Evidence from functional brain imaging. Neurourol. Urodyn. 27, 466-474 (2008).

75. Komesu, Y. M. et al. Functional MRI of the Brain in Women with Overactive Bladder: Brain Activation During Urinary Urgency. Female Pelvic Med. Reconstr. Surg. 17, 50-54 (2011).

76. Lederbogen, F. et al. City living and urban upbringing affect neural social stress processing in humans. Nature 474, 498-501 (2011).

77. Eisenberger, N. I. The neural bases of social pain: evidence for shared representations with physical pain. Psychosom. Med. 74,126-135 (2012). 
78. Griffiths, D. Clinical studies of cerebral and urinary tract function in elderly people with urinary incontinence. Behav. Brain. Res. 92,151-155 (1998).

79. Awata, S. et al. Regional cerebral blood flow abnormalities in late-life depression: Relation to refractoriness and chronification. Psychiatry Clin. Neurosci. 52, 97-105 (1998).

80. Egger, M. et al. Language bias in randomised controlled trials published in English and German. Lancet 350, 326-329 (1997).

81. Lee, H. E. et al. Short-term effects of a systematized bladder training program for idiopathic overactive bladder: A prospective study. Int. Neurourol. J. 17, 11-17 (2013).

82. Leue, C.et al. Managing complex patients on a medical psychiatric unit: An observational study of university hospital costs associated with medical service use, length of stay, and psychiatric intervention. J. Psychosom. Res. 68, 295-302 (2010).

83. Araki, I. et al. Prevalence and impact on generic quality of life of urinary incontinence in Japanese working women: assessment by ICl questionnaire and SF-36 Health Survey. Urology 66, 88-93 (2005).

84. Tadic, S. D. et al. Effect of biofeedback on psychological burden and symptoms in older women with urge urinary incontinence. J. Am. Geriatr. Soc. 55, 2010-2015 (2007).

85. Cheung, R. Y. et al. Quality of life in women with urinary incontinence is impaired and comparable to women with chronic diseases. Hong Kong Med. J. 18, 214-220 (2012).

86. Kramer, I. et al. A therapeutic application of the experience sampling method in the treatment of depression: A randomized controlled trial. World Psychiatry 13, 68-77 (2014).

87. Kondryn, H. J. et al. Treatment non-adherence in teenage and young adult patients with cancer. Lancet Oncology 12, 100-108 (2011).

88. Beck, A. T. et al. Comparison of Beck Depression Inventories -IA and -II in psychiatric outpatients. J. Pers. Assess. 67, 588-597 (1996).

89. Boyd, J. H. et al. Screening for depression in a community sample. Understanding the discrepancies between depression symptom and diagnostic scales. Arch. Gen. Psychiatry 39, 1195-1200 (1982).

90. Yesavage, J. A. et al. Development and validation of a geriatric depression screening scale: A preliminary report. J. Psychiatr. Res. 7, 37-49 (1982).

91. Friedman, B. et al. Psychometric properties of the 15-item geriatric depression scale in functionally impaired, cognitively intact, community-dwelling elderly primary care patients. J. Am. Geriatr. Soc. 53, 1570-1576 (2005).

92. Zigmond, A. S. \& Snaith, R. P. The hospital anxiety and depression scale. Acta Psychiatr. Scand. 67, 361370 (1983).

93. Spitzer, R. L. et al. Validation and utility of a self-report version of PRIME-MD: The PHQ primary care study. Primary Care Evaluation of Mental Disorders. Patient Health Questionnaire. JAMA 282, 1737-44 (1999).

94. Haro, J. M. et al. Concordance of the Composite International Diagnostic Interview Version 3.0 (CIDI 3.0) with standardized clinical assessments in the WHO World Mental Health surveys. Int. J. Methods Psychiatr. Res. 15, 167-180 (2006).

95. Cox ,J. L. et al. Detection of postnatal depression. Development of the 10-item Edinburgh Postnatal Depression Scale. Br. J. Psychiatry 150, 782-786 (1987).

96. Hamilton, M. A rating scale for depression. J. Neurol. Neurosurg. Psychiatry 23, 56-62 (1960).

97. Jorm, A. F. et al. The psychogeriatric assessment scales (PAS): Further data on psychometric properties and validity from a longitudinal study of the elderly. Int. J. Geriatr. Psychiatry 12, 93-100 (1997).

98. Robins, L. N. et al. National Institute of Mental Health Diagnostic Interview Schedule. Its history, characteristics, and validity. Arch. Gen. Psychiatry 38, 381-389 (1981).

99. Hamilton M. The assessment of anxiety states by rating. Br. J. Med. Psychol. 32, 50-55 (1959).

100. Beck, A. T. et al. An inventory for measuring clinical anxiety: psychometric properties. J. Consult. Clin. Psychol. 56, 893-897 (1988).

101. Kvaal, K. et al. The Spielberger State-Trait Anxiety Inventory (STAI): The state scale in detecting mental disorders in geriatric patients. Int. J. Geriatr. Psychiatry 20, 629-634 (2005).

102. Guyatt, G. et al. GRADE guidelines: 1. Introduction-GRADE evidence profiles and summary of findings tables. J. Clin Epidemiol. 64, 383-934 (2011). 


\section{Abstract}

Functional urological and gastrointestinal disorders are interrelated and characterized by a chronic course and considerable treatment resistance. Urological disorders associated with a sizeable functional effect include overactive bladder (OAB), interstitial cystitis/bladder pain syndrome (IC/BPS), and chronic prostatitis/chronic pelvic pain syndrome (CP/CPPS). Poor treatment outcomes might be attributable to untreated underlying psychological and psychiatric disorders, as the co-occurrence of functional urological and gastrointestinal disorders with mood and anxiety disorders is common. The hypothetical bladder-gut-brain axis (BGBA) is a useful framework under which this interaction can be studied, suggesting that functional disorders represent a sensitized response to earlier threats such as childhood adversity or previous traumatic events, resulting in perceived emotional and bodily distress - the symptoms of functional disorders. Psychological and physical stress pathways might contribute to such alarm falsification, and neuroticism could be a risk factor for the co-occurrence of functional disorders and affective conditions. Additionally, physical threat - either from external sources or internal sources such as infection - might contribute to alarm falsification by influencing body-brain crosstalk on homeostasis and, therefore, affecting mood, cognition, and behaviour. Multidisciplinary research and an integrated care approach is, therefore, required to further elucidate and remediate functional urological and gastrointestinal polymorphic phenotypes. 


\section{Introduction}

Functional disorders of the urogenital tract such as overactive bladder (OAB), interstitial cystitis/bladder pain syndrome (IC/BPS) and chronic prostatitis/chronic pelvic pain syndrome (CP/CPPS) comprise a group of disorders for which the structural aspect remains to be elucidated. These disorders overlap and often co-occur with functional disorders of the gastrointestinal tract, such as irritable bowel syndrome (IBS) or functional dyspepsia, suggesting a common origin ${ }^{1-14}$. Functional urological and gastrointestinal disorders are highly prevalent and can, therefore, exert considerable strain on daily functioning and quality of life, representing a substantial socioeconomic burden owing to reduced work productivity, sick leave, and need for health care ${ }^{15-22}$. In addition, functional disorders often run a chronic course and display considerable treatment resistance ${ }^{23-25}$. For example, approximately $40 \%$ of patients with $O A B$ either do not achieve acceptable therapeutic benefit or remain refractory to treatment ${ }^{24}$. Furthermore, the treatment response is heterogeneous and what constitutes treatment success or failure is not well defined ${ }^{26}$. The symptoms of functional urological and intestinal disorders overlap, and include visceral pain and pelvic floor dysfunction, suggesting that these conditions could represent a spectrum or continuum across organ systems, sharing an underlying central hypersensitivity expressed as bodily distress ${ }^{9,12-}$ ${ }^{14,27}$. For example, in patients with IC/BPS the number of antecedent functional disorders is one of the strongest risk factors for the development of others, both within and across organ systems, adding to the suggestion of a transorgan pleiotropic phenotype ${ }^{28}$. Also, in IC/BPS, the number of functional disorders present is predictive of the number of different surgical interventions that a patient will undergo, which might be linked with treatment refractoriness or iatrogenic damage afterwards ${ }^{29}$, suggesting that several functional complaints might be present at the time of diagnosis, but that they are not considered together for the indication of surgical interventions ${ }^{30}$. The failure to codiagnose comorbid syndromes might be partly attributable to difficulties in phenotyping functional urological disorders, given that they remain poorly understood and treatment strategies often do not yield satisfactory results ${ }^{25}$. The concept of urological functional disorders has been directed away from the historical organ-centered disease formulation towards a general consensus that defines such disorders as a series of symptom-based syndromes ${ }^{31-41}$. However, improved stratification with regard to patient characteristics and a comprehensive model that unifies aetiological pathways and associated treatment strategies are needed ${ }^{25,36,40,42,43}$.

The hypothetical bladder-gut-brain-axis (BGBA) is a useful framework under which the pathophysiology of related functional urological and gastrointestinal disorders can be studied. The pathophysiology of urological and related functional disorders is complex and remains elusive, but several mechanisms linked to the hypothesized BGBA, might have a role in symptom formation. These include enhanced bladder permeability and sensitivity, alterations in bladder motility, altered visceral function and cross- 
sensitization between the gastrointestinal tract and the bladder, neuroendocrine balance, autonomic nervous system balance, low-grade inflammation, genetic and/or epigenetic susceptibility, infectious aetiology and composition of the microbiota, as well as psychological stress and psychiatric disorder comorbidity ${ }^{27,44-63}$. Evidence suggests that psychosocial factors, life stress, coping styles, and level of psychosocial support all have roles in the pathophysiology of functional urological disorders, affecting symptom severity, illness behaviour and outcomes ${ }^{63-71}$. Anxiety, depression, stressful experiences, and recurrent negative social interactions as well as comorbid nonurological syndromes are associated with health perception and help-seeking behaviour in functional disorders ${ }^{60,62,70-73}$. Patients with functional disorders in combination with comorbid psychiatric disorders are more often referred to somatic specialists than those without, and comorbid anxiety and depression are both consistently associated with increased severity of symptoms and a greater illness burden ${ }^{70,74,75}$. Coexistent mood and anxiety disorders are associated with neuroticism, also known as mood bias towards negative emotions or trait negative affectivity ${ }^{76-79}$, which also underlies somatic ill health ${ }^{61,62,69,80,81}$. Given that functional urological disorders and related functional gastrointestinal syndromes such as IBS are also associated with neuroticism, trait negative affectivity might be a nonspecific risk factor for multiple morbidities ${ }^{69,71,82-87}$. Under-recognition of neuroticism-associated conditions could be causally related to unsatisfactory treatment outcomes, and research suggests that neuroticism is associated with significantly worse treatment responses and higher levels of depression in patients with $\mathrm{CP} / \mathrm{CPPS}^{82}$. Furthermore, the economic cost of neuroticism is sizeable, exceeding that of other common psychiatric disorders, and suggesting that a focus on broadly defined psychosomatic comorbidity rather than affective or somatic disorders in isolation might be economically advantageous ${ }^{88,89}$. Neuroticism is not only crosssectionally associated with somatic and mental ill-health - it also predicts subsequent onset of mental disorders as well as a spectrum of somatic conditions ${ }^{90-93}$. In general hospitals, psychiatric and somatic comorbidities affect the prognosis of somatic conditions and the level of health-care use ${ }^{94,95}$. However, the underdetection of psychiatric comorbidities is not rare, particularly in patients with complex symptoms and medically unexplained functional complaints ${ }^{96}$.

Three mechanisms along the BGBA can be hypothesized to underlie symptom formation. The antecedent hypothesis states that depression and anxiety cause the onset of somatic symptoms ${ }^{97,98}$; the consequence hypothesis states that somatic symptoms predict the onset of depression and anxiety disorders ${ }^{97.99}$; the common pathway hypothesis states that different factors (psychological, environmental and biological) cause the onset of both somatic symptoms and depression as well as anxiety disorders $^{100}$. Given that both functional disorders and affective disorders are stressrelated, sensitization to threat might mediate false-alarm signals at the BGBA as a defence system. Thus, a common pathway of dysregulation in the BGBA has been proposed, which involves sensitization or alarm falsification, giving rise to both 
emotional distress (psychiatric disorders) and bodily distress (functional disorders) ${ }^{13,46,53,101-104}$. Sensitization refers to a state of hypersensitivity to visceral stimuli that manifests as increased sensitivity to normally bothersome stimuli (for example, hyperalgesia in response to mildly painful stimuli) or symptom formation in response to normally nonbothersome stimuli (for example, allodynia in response to nonpainful stimuli) $^{105}$. Alarm falsification refers to the process of normally neutral (mental) or nonnoxious (physical) stimuli provoking a range of emotional reactions (anxiety, fatigue, depressed mood), cognitive responses (hypervigilance, catastrophizing) and bodily distress (urgency, frequency, pain), respectively.

In this Review, we will consider the associations between functional urological (and related gastrointestinal) disorders and affective disorders and neuroticism, in order to investigate the hypothesis that functional disorders are associated with psychiatric comorbidity. In order to better understand the biological and psychological factors underlying this association, this Review describes the evidence for shared stress pathways that might contribute to sensitization and/or alarm falsification along the BGBA, resulting in symptomatic functional disorders. We propose methods to further investigate and remediate the process of alarm falsification in patients with complex, treatment-refractory functional urological disorders, raising the possibility of resetting the BGBA defence system.

\section{Epidemiology}

Epidemiological studies of the prevalence of functional urological disorders have been hampered by a lack of agreement with regard to diagnostic definitions, and, as a consequence, inadequate case detection ${ }^{106}$. In OAB, for example, prevalence estimates vary considerably between studies, ranging from $\sim 4$ to $40 \%{ }^{19}$. Despite the introduction of standardized diagnostic definitions, such as the ICS criteria $^{33,106}$, general population surveys still show considerable variation, with rates ranging from 6 to $32 \%$ in different countries, the majority showing an increased prevalence of symptoms among women (at least in individuals $<60$ years of age), although some studies showed an increased prevalence of symptoms in men, or no sex-related differences ${ }^{20,107-113}$. Epidemiological assessments of the prevalence of other lower urinary tract symptoms (LUTS)-related disorders might also benefit from standardization ${ }^{114-116}$. However, in ICS/BPS, for example, proposed criteria were found to be too restrictive, and attempts to address this problem did not produce a single definition of IC/BPS with sufficiently high levels of sensitivity and specificity ${ }^{117,118}$. A case definition based on meeting two different sets of criteria has been suggested as the best approach to reliably estimate the prevalence of IC/BPS ${ }^{118}$. Using the single definition, with high sensitivity, $6.5 \%$ of adult US women were found to meet IC/BPS symptom criteria, which translates to 5.5 million women in the USA alone ${ }^{119}$. 
Overlap between definitions of functional urological disorders is considerable $1,2,3,120$. Moreover, evidence is also accumulating that overlap is not limited to the bladder. In one study up to $50 \%$ of patients with IC/BPS had a comorbid condition, mostly IBS ${ }^{121}$. Another study showed that $40 \%$ of patients in a CPPS cohort had nonurological-associated syndromes, again particularly $\mathrm{IBS}^{71}$. In addition, approximately $60 \%$ of women with IC/BPS reported at least one nonbladder condition - such as depression, panic attacks or IBS - and $80 \%$ of female patients with IC/BPS reported pain beyond the pelvis ${ }^{122}$. Overall, the literature suggests the existence of considerable overlap between IBS and functional urological disorders, with comorbidity estimates of up to $80 \%{ }^{11}$.

Functional disorders - both urological and gastrointestinal - as well as mental disorders are highly prevalent ${ }^{112,115,123,124}$. Furthermore, data from epidemiological studies indicate that depression and anxiety disorders, and functional urological and related gastrointestinal disorders are both common comorbidities, and both have adverse effects on patient outcomes ${ }^{61,62,70-72,75,104,125,126}$. The common association between functional urological and gastrointestinal disorders affects the severity of physical and mental symptoms experienced in a dose-related fashion ${ }^{4-10,71,121,127}$. A substantial proportion of functional disorders overlap with other functional complaints and/or comorbidities with psychiatric disorders $9,63,70,81,128-131$, and the prevalence of anxiety and depression increases in a stepwise manner with the number of functional disorders and the frequency or severity of functional somatic symptoms ${ }^{68,73,103,104,132,133}$. Thus, the more severe the somatic symptoms are, the more prevalent the affective complaints become. Pain and depression are particularly closely associated ${ }^{65,66,68-}$ 72,97,100,103.

Affective complaints might, therefore, complicate existing functional disorders. By contrast, the onset of anxiety and depressive symptoms might precede that of urological functional disorders ${ }^{62,122,131}$. Similarly, depression and anxiety both dose dependently increase the risk of developing urinary incontinence, and the number of neuroticismassociated conditions could be a marker of a complex psychosomatic multimorbidity phenotype $e^{7,65,69,81,104,121,127}$.

\section{Shared aetiological pathways}

The existence of associations between functional urological and gastrointestinal disorders and affective disorders and negative affectivity traits raises the question of how these conditions might have a shared aetiology. Affective disorders are stressrelated conditions ${ }^{134-136}$, and the overlap between functional urological disorders and indicators of psychosocial stress is also considerable. For example, perceived stress and severity of urinary incontinence in $O A B$ are positively correlated ${ }^{104}$, and the rate of stress-related affective symptoms is high in patients with functional urological and related gastrointestinal disorders ${ }^{63,81,128-130}$. An interrelationship between CP/CPPS and psychological distress has also been described ${ }^{73}$. In IC/BPS, CPPS, and OAB a positive 
association exists between psychological distress and the severity of urgency and pain, becoming progressively stronger in patients with more symptoms ${ }^{68,73,104,132}$.

\section{Physical and psychological consequences of threat}

Stress was first defined more than 75 years ago as an acute threat to the homeostasis of an organism ${ }^{137}$. Stressors can be present in the form of physical threat, for example accidents or infections, or as a psychological stressor, such as social defeat or learned anticipation to threat. Exposure to stressors causes a complex sequence of physical, emotional, and behavioural reactions in order to cope with the perceived threat, and can, if severe, exceed the threshold of pathology. Cognitive-behavioural as well as emotional responses include enhanced awareness and altered pain sensitivity ${ }^{138,139}$. In parallel, physical reactions emerge, including increased heart rate and respiratory rate as well as altered metabolism and digestion. Under chronic stressful circumstances, normal physiological processes such as feeding, growth, reproduction, and immunity are inhibited $^{139,140}$. Thus, the stress-response sequence involves the autonomic nervous system, the endocrine system, and the immune system, and serves to regulate adaptations to threat ${ }^{138-141}$.

In response to chronic and uncontrollable stressors, maladaptive dysregulation can occur, giving rise to affective and functional symptoms, including visceral hypersensitivity and visceral pain ${ }^{139,141-145}$. Stress-related changes in voiding and bowel habits can result from the brain and can affect both internal organ function and emotion regulation, resulting in comorbid anxiety or depressive symptoms ${ }^{145-147}$.

\section{Psychological and physical threat}

Lifetime psychological and external physical stressful or traumatic experiences are prevalent, affecting $20-35 \%$ of the people as measured by a population based survey in the US, in the form of physical, emotional, or sexual abuse, neglect, or other related adversities. Women tend to report a greater prevalence of adult emotional abuse and lifetime sexual abuse ${ }^{148}$. The effect of stress on the severity of functional disorder symptoms is well recognized by patients and clinicians. For example, OAB, IC/BPS, and IBS symptoms tend to wax and wane, becoming more severe with higher levels of stress, which are related to the levels of anxiety and depression ${ }^{60,68,104,149,150}$. Furthermore, lifetime adversity is more frequent in patients with OAB, IC/BPS, and IBS; evidence for the role of early-life adversity - such as sexual abuse measured by anonymous questionnaires in women and children or maltreatment allegations obtained from Child Protective Services - in the development of functional urological and related gastrointestinal disorders is particularly strong ${ }^{63,67,151-155}$. These findings are supported by animal models of early-life stress (such as early maternally separated rodents) and 
forced swim and foot-shock stressors ${ }^{156,157}$. Evidence suggests that lower urinary tract symptoms (LUTS) are associated with war-related trauma in adults, and veterans with post-traumatic stress disorder (PTSD) are more likely to have LUTS than those without PTSD $^{158}$. An interesting line of research suggests that adversity-associated low-grade inflammation, manifesting as a neuroimmune network across different organ systems, is involved in mental and somatic ill-health. This research is based on the hypothesis that adversity early in life amplifies crosstalk between low-grade inflammation and brain circuitries involved in threat, reward, and executive function (that is, corticoamygdala threat pathways and corticobasal ganglia reward circuitries) ${ }^{159}$. Given the central role of stress and adversity, contemporary stress and childhood adversity are clearly areas of interest during clinical assessment of functional disorders, affecting diagnostic procedures and patient care ${ }^{129}$. Clinical assessment in functional disorders affected by different but merging pathways should take into account all relevant information, and questions concerning medical history should incorporate questions on adversity, embedded into a respectful conversation and a medical explanation of contributing causes.

\section{Psychological factors}

Vulnerability and resilience: Psychological factors moderate the severity and persistence of symptoms, influence decisions to seek treatment, and predict treatment response $^{133,160}$ and, therefore, seem to have an important role in stress-related vulnerability to threat. For instance, different psychosocial factors are associated with $O A B$ and IBS, suggesting that related factors tend to cluster together, for example negative health beliefs and negative cognitive coping such as catastrophizing (a maladaptive cognitive magnification of threat accompanied by feeling helpless) and negative affectivity and affective disorders ${ }^{62,161}$. Personality traits and temperamental factors might also reflect elements of vulnerability to functional disorders. Sensitivity to stress is mediated by neuroticism, a personality trait associated with a bias towards negative emotions ${ }^{162}$. Patients with high scores on neuroticism questionnaires are more reactive to stressors. Neuroticism is one of the few personality traits that is consistently associated with functional urological and related gastrointestinal disorders $^{69,81,82,84,87,163,164}$, and along with a history of being abused, a history of negative life events, somatization, catastrophizing, and anxiety, affects the severity of functional disorders, being associated with increased disability ${ }^{81,165-168}$. In 2011, the International Consultation on Incontinence-Research Society (ICl-RS) organized a thinktank on psychological factors influencing LUTS, and concluded that OAB and other LUTS are associated with affective conditions, and that "... the possibility of causation and/or maintenance of LUTS through psychological causes requires further research. It is important to recognize that amongst ... patients suffering with LUTS a significant psychological component exists. Failure to address this may lead to incomplete assessment and consequently inadequate treatment" ${ }^{169}$. 
Moreover, the response to stress might also be moderated by resilience. Resilience is defined as the ability to successfully adapt and recover after the experience of adversity during the life course, and is a dynamic process that regains and maintains homeostasis under threat and conditions of stress ${ }^{170,171}$. Early adversity can moderate genetic programming of nervous system circuits, which mediate cognitive, emotional, and behavioural adaptation to threat later in life. The three-hit concept - hit 1: genetic predisposition; hit 2: early-life environment; hit 3: later-life environment - describes the interaction between vulnerability and resilience underlying stress-related disorders, proposing that, under certain circumstances, vulnerability increases when resilience to adversity is reduced ${ }^{172-174}$.

\section{Internal physical threat}

Infection: Physical threat can originate from outside or inside the body. The concept of postinfection functional gastrointestinal disorders is well established ${ }^{175}$, and infection might have a similar role in the development of functional urogenital disorders. Early cross-sectional data suggest a relationship between urinary tract infections (UTI) and all causes of incontinence, which is supported by more studies showing that a history of one or more UTIs in prior years is associated with new onset of urge urinary incontinence $^{176-178}$. Hence, ongoing colonization of the bladder could be suggested as a mechanism of persistent functional complaints. However, longitudinal evidence of the association between recurrent UTI and functional urological disorders is scarce. A low incidence of recurrent UTIs has been reported in patients with IC/BPS, indicating that mechanisms other than infection or colonization alone are likely to be involved ${ }^{179}$; however, many patients with IC/BPS report one or more UTIs shortly before the onset of the IC/BPS, which suggests that UTI might be a causative event. In the acute phase of infection, UTIs are associated with symptoms that resemble those of OAB and/or IC/BPS, perhaps even with concomitant urgency incontinence. However, urgency incontinence generally improves after remission of the infection. Thus, in cases of persistent or recurrent symptomatology, recurrent or persistent infection or sensitization and alarm falsification might be occurring. As some studies have shown that low-level bacteriuria is associated with a wide range of LUTS, low-count bacteriuria might be an underestimated causative factor in patients presenting with $O A B^{180-182}$. Low-level bacteriuria might, therefore, continue to represent an ongoing cause of functional symptoms, or it could be indicative of a physiological or dysbiotic colonization of the bladder (microbiome, dysbiosis) accompanied by functional complaints. As the definition of OAB excludes infections, and the bladder has historically been assumed to be physiologically sterile, assessment of bacteriuria in sensitized patients with frequency, urgency, and nocturia is not currently a standard procedure. However, evidence of the presence of uncultivated bacteria in the adult bladder suggests that more detailed assessment and treatment is required to identify and remedy infections ${ }^{183-187}$. The presence of asymptomatic bacteriuria further complicates matters, and only a subset of patients with refractory 
OAB have bacteriuria or bacterial cystitis ${ }^{188,189}$. Thus, diagnostic assessment might need to be enhanced in order to detect hidden infections that underlie bacteria-induced stress. The existence of a physiological bladder microbiota is also possible, which might contribute to the risk of sensitized functional disorders. Such possibilities point to other theoretical considerations as a consequence of cumulative threat, both host and pathogen-dependent, rather than recurrent UTI simply being a causative agent in functional urological disorders ${ }^{170,187-190}$. Although epidemiological evidence supports the observation that a previous UTI is a risk factor for IC/BPS, whether acute and chronic pelvic pain share common infection-related aetiological pathways is unclear ${ }^{191-193}$. However, as far as visceral pain pathways are concerned, further research on infectionrelated pathophysiology is merited.

Infection with uropathogenic Escherichia coli (UPEC) is the most important cause of acute UTI in adults, and UPEC accounts for $>80 \%$ of all community-acquired infections ${ }^{194}$. UPEC suppresses the local production of cytokines and chemokines by the urothelium and evades the host innate immune respons ${ }^{194-197}$. UPEC is also able to induce urothelial apoptosis, facilitating the purging of superficial cells, and, therefore, providing access to the underlying urothelial layers, which might introduce silent bacterial intracellular reservoirs ${ }^{193-196}$. Both the host immune response and microbial virulence contribute to the wide range of inflammatory responses and disease-specific pathology caused by $U_{P E C}{ }^{195}$. However, these responses are divergent in the post-acute state, leading to asymptomatic bacteriuria (ASB) at one end of the spectrum and, at the other, destructive infections or, consequently, chronic aseptic functional urological disorders ${ }^{196}$, ${ }^{198}$. In chronic functional urological disorders, pain is thought to result from repetitive super- threshold stimuli that trigger neuroimmunological changes that result in central sensitization along ascending pain-processing afferents ${ }^{199}$. In a rodent model of repetitive infection, such induction of central sensitization could be demonstrated ${ }^{200}$. Whereas UPEC induces transient pelvic pain, an ASB Escherichia coli isolate might cause no transient pain. Differential pain responses are not related to bladder colonization or inflammatory responses but instead to Escherichia coli lipopolysaccharides (LPS) ${ }^{193,200}$. LPS consist of different domains: the acetylated lipid A component, embedded in the outer membrane is known to drive inflammation ${ }^{200}$. The O-antigen lipopolysaccharide is another domain of LPS; in studies using a mouse model, only the O-antigen mutant, which lacked O-antigen gen clusters and was, therefore, defective for O-antigen biosynthesis, was able to induce pronounced and persistent pain states; whereas other serial infections induced only transient allodynia (hypersensitivity) in response to initial and subsequent infections ${ }^{193,200}$. This effect was not a result of persistent colonization. Remarkably, the mutant infection induced no allodynia in response to initial infection, but subsequent mutant infections triggered pronounced pain responses, exceeding those resulting from other non-mutant-infection-related acute pain responses in magnitude and persisting for weeks despite rapid clearance of bacteria from the bladder $^{193,200}$. Thus, it seems that an uropathogen-dependent sensitization process 
might have been induced, possibly leading to enduring alarm falsification in the absence of an initial inflammatory threat response ${ }^{200}$. Post UTI alarm falsification does not only include chronic pain but also voiding dysfunction and anxious/depressive behaviour, representing functional urological disorders ${ }^{193}$. These symptoms might be partly related to increased spontaneous firing of the spinal cord, as demonstrated in the rodent model, which is consistent with centralized pain states ${ }^{200}$.

Dysbiosis and microbiota: Little is known about the development of the human urinary microbiota and the way it alters and might influence functional urological disorders over the course of a lifetime, whereas the human gut microbiota are well studied with regard to functional intestinal disrorders ${ }^{201}$. The bladder was not included within the human microbiome project, owing to historical assumptions of the urine being sterile ${ }^{202}$. Nevertheless, emerging evidence suggests that the bladder contains bacteria under normal circumstances, indicating a physiological state of colonization with a microbiota that is different to that of the intestines ${ }^{59,203-206}$. Although many species of the urinary microorganisms carry virulence factors, they do not frequently cause infections, suggesting that host factors might have a role in both infectious and noninfectious urinary tract colonization ${ }^{207}$. Furthermore, a proportion of UTIs resolve without any intervention, suggesting the urinary microbiota require time to reconstitute after an acute infection ${ }^{208,209}$. Both host-dependent and microorganism-dependent factors could trigger chronic infectious states or postinfectious functional urological complaints. One of the mechanisms of such a trigger could be related to alterations in the urinary microbiota, as investigations of bacterial colonization of the urinary tract of patients with IC/CPPS and urgency incontinence reveal changes in the microbiota compared with that of individuals without such symptoms ${ }^{59,205,206}$. These changes pertain to the richness and composition of the IC/CPPS microbiota. More than $90 \%$ belongs to the genus Lactobacillus, which is a marked increase compared to $60 \%$ in urine of healthy women $^{206}$. Moreover, the balance between certain groups of microorganisms might be more important than the overall composition of the urinary microbiota ${ }^{59}$. For example, urine from patients suffering from IC/CPPS had a lower level of bacterial diversity compared with that of healthy women ${ }^{205}$. Loss of bacterial diversity is also associated with other inflammatory conditions such as obesity and inflammatory bowel disorders (IBD), and commensal bacterial strains have been shown to attenuate mucosal inflammation via inhibition of IL-6 and NF-KB signalling pathways ${ }^{210-212}$. These data suggest that changes in the composition of the urinary microbiota might be related to inflammation and, as affective disorders are associated with inflammatory pathways, affective and functional disorders might both be immunologically connected with dysbiosis, and consecutive tissue damage and subsequent changes associated with urological and related intestinal functional disorders might be part of a pelvic organ cross- sensitization effect ${ }^{48,49,159,213,214}$ (Figure 13.1). 


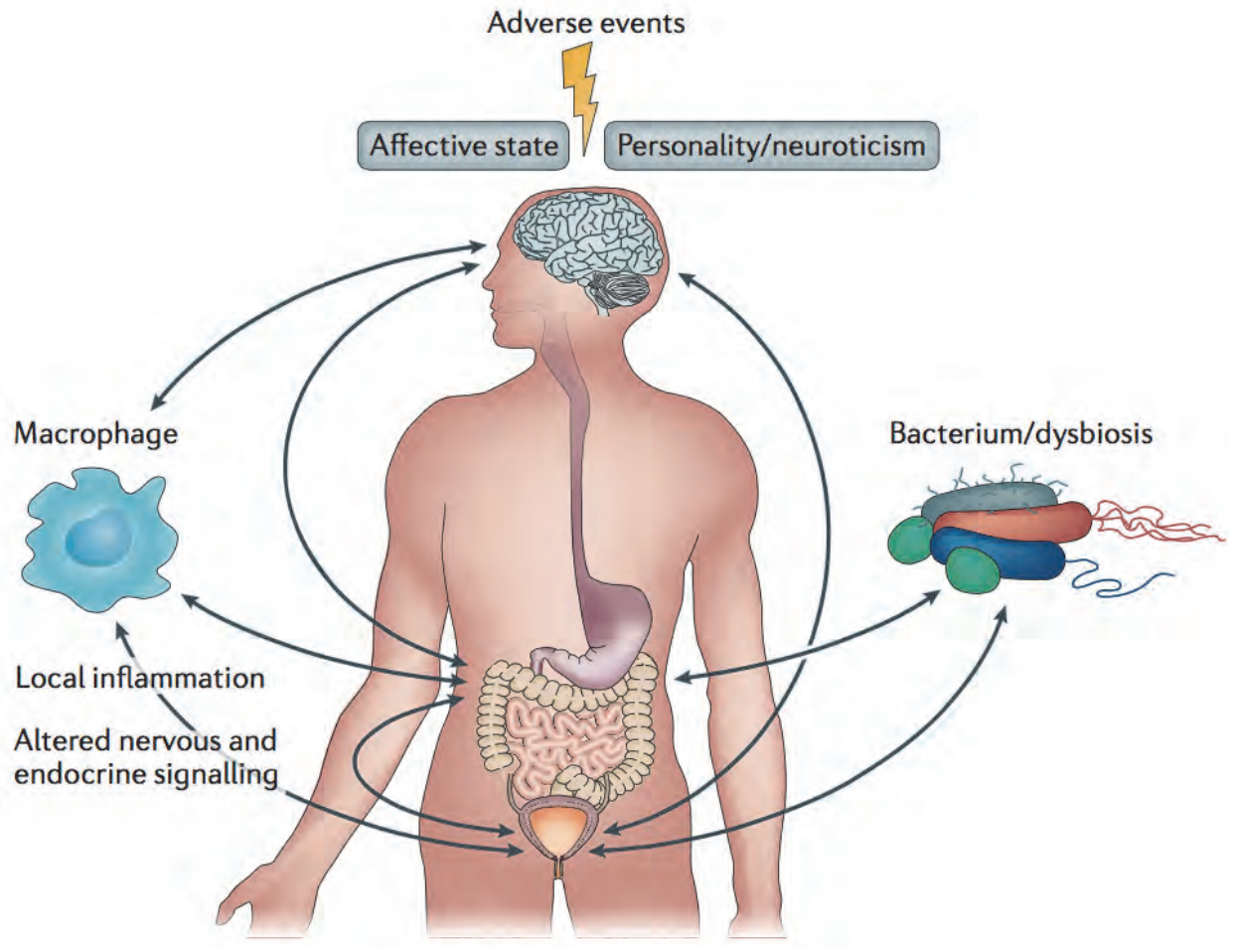

Figure 13.1 The threatening environment and the bladder-gut-brain axis (BGBA).

Legend: External psychological and/or physical adverse events can negatively affect the body via the BGBA, which can also be affected by internal threats such as infections or dysbiosis. Emotional, cognitive, and behavioural consequences include functional urological and gastrointestinal disorders, parts of which might be related to altered immunological, endocrine, and nervous system signalling.

Thus, stress and negative emotions - pain, anxiety, fear, decreased mood - and threat-related behaviour - fight, flight, or freeze, immune defence, and hypermobility of the bladder or the gut - could all be part of the same defence system that serves to reduce threat (Figure 13.2). In the case of functional disorders, this defence reaction can be best described as alarm falsification caused by a shared sensitization to threat, which can endure long after the occurrence of the original stressor.

\section{Integration of care}

Complexity of functional disorders including affective comorbidity increases in a stepwise manner with the number of functional disorders, from low rates at the general 
population level to $\sim 30 \%$ in primary care and up to $80 \%$ in an integrated hospital outpatient setting - a well-known comorbidity bias phenomenon, associated with the fact that any illness is more likely to have comorbidities at greater levels of severity ${ }^{215-217}$. In inpatients with complex conditions and treatment resistance that are medically unexplained, the level of psychiatric comorbidity increases up to $90 \%$ of admitted inpatients at integrated multidisciplinary units (Med-Psych-Units), whereas only $5 \%$ of these patients were detected at the level of nonintegrated monodisciplinary medical settings (internal medicine ward or surgical ward) ${ }^{96}$. Indeed, under-recognition of a complex psychosomatic multimorbidity phenotype consisting of urological, gastrointestinal and affective comorbidities might contribute to treatment resistance in functional urological and gastrointestinal disorders ${ }^{23-25}$. Thus, the therapeutic benefit might be suboptimal if nonintegrated diagnostic and treatment plans, without multidisciplinary support and understanding, are offered ${ }^{23-25,218}$. For example, multidisciplinary Pelvic Care Centres (PCC) have been introduced at some institutions in order to jointly diagnose and treat patients with urogenital and gastrointestinal symptoms. Such centres include specialists with urological, gynaecological, proctosurgical, gastrointestinal, sexological and psychiatric expertise, and see patients on an outpatient basis. Local PCC data suggest that $15-25 \%$ of PCC patients have multifactorial problems at initial triage, requiring more than one specialist for diagnosis and initial treatment advice 219,220 .

Both doctors and patients become more aware of unmet needs in the treatment of visceral pain and other functional complaints, thus an integrated multidisciplinary approach should be recommended as standard, particularly in patients with complex syndromes and comorbidity-related treatment resistance ${ }^{221,222}$. Furthermore, the need for improved communication among doctors and between doctors and patients is currently unmet. For decades, the conceptualization of medical syndromes has been symptom-based - functional urological disorders, functional gastrointestinal disorders, and affective disorders are all classified according to their symptomatology using the ICS consensus on OAB, ROME III criteria for IBS, and DSM-5 criteria for mood and anxiety disorders, respectively. Although such a system is useful for clinical practice within different specialities, it might obscure the natural comorbidity between functional syndromes across specialties that require an integrated approach in diagnosis and treatment, and combining speciality knowledge to treat the symptoms of functional syndromes with pleiotropic expression across different organ systems could improve satisfaction levels of both patients and doctors. Patients with medical conditions are often depressed and comorbid depression or anxiety frequently remains undetected, even in patients who are very frequently seen across different medical departments, negatively affecting quality of life and increasing the cost of care ${ }^{223}$. Although encouraging multidisciplinary initiatives to produce more sensitive (and specific) patient phenotyping have been launched, including the UPOINT system and the multidisciplinary MAPP research network studies ${ }^{81,224-226}$, assessment of functional syndromes with 
comorbid somatic and psychological expressions remains limited as they typically rely on retrospective questionnaires that do not inform on real-life intensity, variability, and reactivity, and are affected by recall bias and a host of other contextual and psychological factors. In addition, the multitude of different instruments that are available to measure, for example, chronic abdominal pain makes it difficult to compare outcomes across studies ${ }^{227}$. Thus, further development and standardization of assessments is needed to overcome the limitations of available methods and enable the field to move beyond use of symptom-based assessments and the various shortcomings of such approaches. These limitations could be overcome by randomly repeated momentary assessment during the day in a real-life environment, using the Experience Sampling Method (ESM), which has been successfully piloted in gastrointestinal functional disorders 228,229, although, to date, this method has not been used in functional urological disorders. The ESM combined with urgency and assessment of bladder fullness sensations could certainly be expected to deliver new insight in diagnostic aspects and treatment follow-up monitoring in patients with functional urological disorders.

Hence, a more flexible and insightful diagnostic approach might help to better map psychiatric and somatic comorbidities in functional disorders, while multidisciplinary meta-communication also enables clinicians to provide more integrated care, resulting in improved treatment satisfaction and reduced health-care $\operatorname{costs}^{230}$, although no consistent evidence exists showing that collaborative care improves physical quality of life ${ }^{231,232}$. In primary care, collaborative approaches have resulted in encouraging reductions in comorbid depression and anxiety ${ }^{233}$, but even in the Netherlands, where collaborative care have been offered for more than a decade, unmet needs concerning integrated psychosomatic care remain ${ }^{234}$. A transitional integrated care model with multidisciplinary staff input would be likely to help bridge the gap between hospital and primary care in complex urogenital and related gastrointestinal functional disorders ${ }^{217}$.

Thus, at least in polysyndromatic patients with persisting complaints after appropriate medical treatment, a collaborative approach integrating medical and psychosocial elements is necessary in order to deliver individually tailored solutions for any identifiable disease phenotype ${ }^{9,25,40,41}$. Reluctance in the field to consider the broader multidisciplinary treatment advice recommended in the NICE urinary incontinence guidelines might be partly related to low diagnostic sensitivity for comorbidity $^{235,236}$. However, continuation of care without taking serious life stressors into account is not recommended ${ }^{237,238}$. In integrated care, the whole becomes larger than its parts ${ }^{239,240}$, eventually leading to a multidisciplinary system view of defence in functional disorders. 


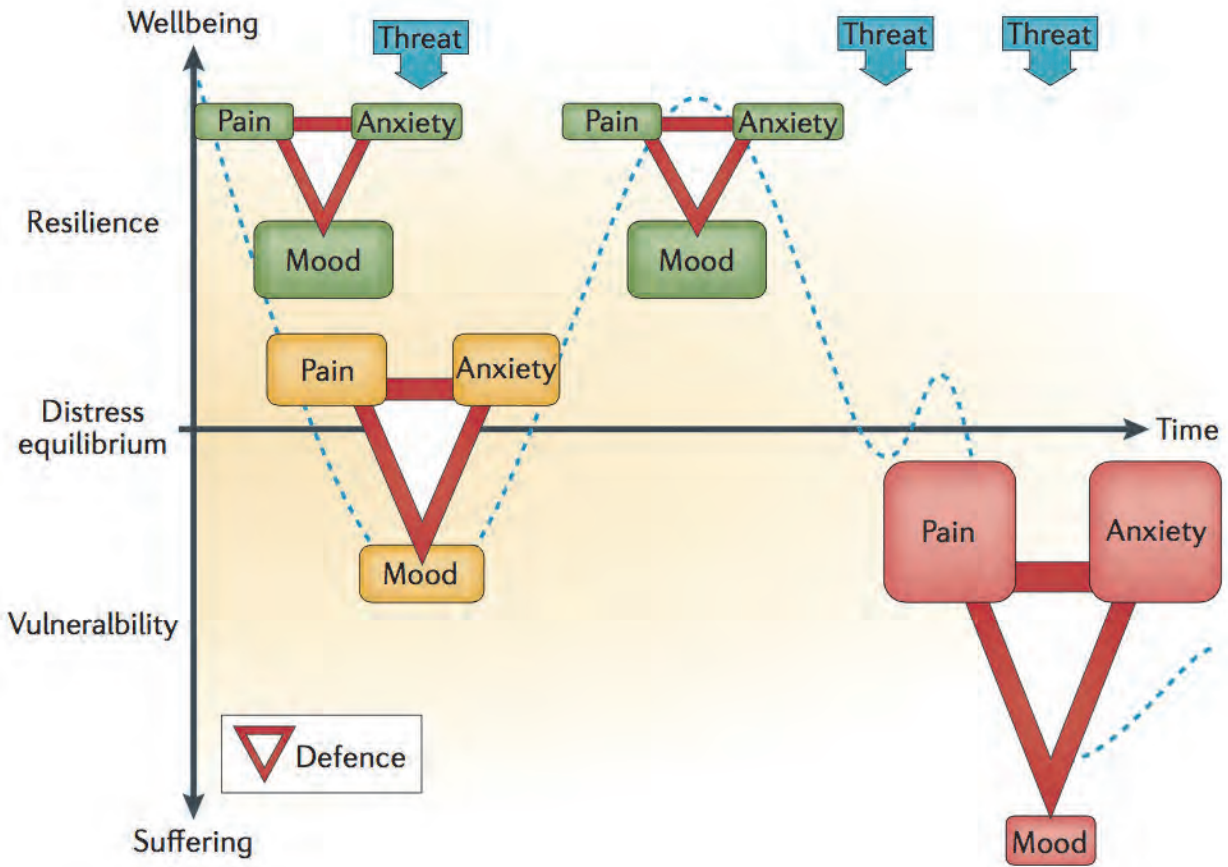

Figure 13.2 Timecourse of cumulative threat and the consecutive alarm falsification of defence.

Defensive behaviour (fight, flight or freeze; voiding; vomiting; diarrhoea; depicted as a red triangle) and corresponding emotional reactions (pain, anxiety, mood) to threat (trauma, childhood adversity, infections) during a timecourse of repetitive cumulative stress show that defensive behaviour and related pain and anxiety levels increase after threat, whereas wellbeing (mood) decreases (yellow). Stress-related behaviour and emotions recover after exposure to a single or less-intense stressor (green), but after cumulative stress, pain and/or anxiety increases and related defensive behaviours become bothersome and enduring functional complaints, reflecting a sensitized defence reaction and alarm falsification (red) $)^{173,174}$.

\section{Conclusions}

Undoubtedly, functional urological and functional gastrointestinal disorders are interrelated and associated with affective comorbidity - the union between these comorbidities might be neuroticism, or bias to negative affectivity. In this context, emotional, cognitive and behavioural reactions, including functional somatic complaints, might be best described as sensitized alarm falsification along a defence response to earlier threats. Physical and psychological stressors might contribute to such alarm falsification and depend on vulnerability and resilience factors, which are both patientrelated and environment-related. A useful framework under which this defence reaction 
can be studied is the bladder-gut-brain axis (BGBA). Future research and clinical management requires an integrated multidisciplinary approach to further elucidate aspects of a complex multimorbidity phenotype and underlying stress pathways, and to avoid treatment resistance. 


\section{References}

1. Chung, M. K. et al. The overlap of interstitial cystitis/painful bladder syndrome and overactive bladder. JSLS. 14, 83-90 (2010).

2. Lai, H. H. et al. The overlap and distinction of self-reported symptoms between interstitial cystitis/bladder pain syndrome and overactive bladder: A questionnaire based analysis. J. Urol. 192, 1679-1685 (2014).

3. Suskind, A. M. et al. The prevalence and overlap of interstitial cystitis/bladder pain syndrome and chronic prostatitis/chronic pelvic pain syndrome in men: Results of the RAND Interstitial Cystitis Epidemiology male study. J. Urol. 189, 141-145 (2013).

4. Matsumoto, S. et al. Relationship between overactive bladder and irritable bowel syndrome: A largescale internet survey in Japan using the overactive bladder symptom score and Rome III criteria. BJU Int. 111, 647-652 (2013).

5. Matsuzaki, J. et al. High frequency of overlap between functional dyspepsia and overactive bladder. Neurogastroenterol. Motil. 24, 821-827 (2012).

6. Chelimsky, G. et al. Co-morbidities of interstitial cystitis. Front. Neurosci. doi:10.3389/fnins.2012. 00114 (2012).

7. Fan, Y. $\mathrm{H}$. et al. Non-bladder conditions in female Taiwanese patients with interstitial cystitis/hypersensitive bladder syndrome. Int. J Urol. 21, 805-809 (2014).

8. Persson, R. et al. The relationship between irritable bowel syndrome, functional dyspepsia, chronic fatigue and overactive bladder syndrome: A controlled study 6 years after acute gastrointestinal infection. BMC Gastroenterol. 15, 66 (2015).

9. Lai, H. H. et al. Polysymptomatic, polysyndromic presentation of patients with urological chronic pelvic pain syndrome. J. Urol. 187, 2106-2112 (2012).

10. Fuoco, M. B. et al. Multiple sensitivity phenotype in interstitial cystitis/bladder pain syndrome. Can. Urol. Assoc. J. 8, 758-761 (2014).

11. Bullones Rodriguez, M. A. et al. Evidence for overlap between urological and nonurological unexplained clinical conditions. J. Urol. 189, S66-74 (2013).

12. Kanaan, R. A. et al. The association or otherwise of the functional somatic syndromes. Psychosom. Med. 69, 855-889 (2007).

13. Fink, P. \& Schröder, A. One single diagnosis, bodily distress syndrome, succeeded to capture 10 diagnostic categories of functional somatic syndromes and somatoform disorders. J. Psychosom. Res. 68, 415-426 (2010).

14. Wessely, S. \& White, P. D. There is only one functional syndrome. Br. J. Psychiatry 185, 95-96 (2004).

15. Mahadeva, S. \& Goh, K. L. Epidemiology of functional dyspepsia: a global perspective. World J. Gastroenterol. 12, 2661-2666 (2006).

16. Saito, Y. A. et al. The epidemiology of irritable bowel syndrome in North America: A systematic review. Am. J. Gastroenterol. 97, 1910-1915 (2002).

17. Agarwal, N. \& Spiegel, B. M. The effect of irritable bowel syndrome on health-related quality of life and health care expenditures. Gastroenterol. Clin. North Am. 40, 11-19 (2011).

18. Fortea, J. \& Prior, M. Irritable bowel syndrome with constipation: A European-focused systematic literature review of disease burden. J. Med. Econ. 16, 329-41 (2013).

19. Milsom, I. et al. The prevalence of overactive bladder. Am. J. Manag. Care 6, S565-573 (2000).

20. Tikkinen, K. A. et al. Is the prevalence of overactive bladder overestimated? A population-based study in Finland. PLOS ONE doi: 10.1371/journal.pone.0000195 (2007).

21. Coyne, K. S. et al. Economic burden of urgency urine incontinence in the United States: A systematic review. J. Manag. Care Pharm. 20, 130-140 (2014).

22. Reynolds, W. S. et al. The burden of overactive bladder on US public health. Curr. Bladder Dysfunct. Rep. 11, 8-13 (2016).

23. El-Serag, H. B. et al. Systematic review: Natural history of irritable bowel syndrome. Aliment. Pharmacol. Ther. 19, 861-870 (2004).

24. Giarensis, I. \& Cardozo L. Management of refractory overactive bladder. Minerva Ginecol. 65, 41-52 (2013). 
25. Mullins, C. et al. Novel research approaches for interstitial cystitis/bladder pain syndrome: Thinking beyond the bladder. Transl. Androl. Urol. 4, 524-533 (2015).

26. Goldman, H. B. et al. Defining response and non-response to treatment in patients with overactive bladder: A systematic review. Curr. Med. Res. Opin. 30, 509-526 (2014).

27. Kim, S. E. \& Chang, L. Overlap between functional Gl disorders and other functional syndromes: What are the underlying mechanisms? Neurogastroenterol. Motil. 24, 895-913 (2012).

28. Warren, J. W. et al. The number of existing functional somatic syndromes (FSSs) is an important risk factor for new, different FSSs. J. Psychosom. Res. 74, 12-17 (2013).

29. Warren, J. W. et al. Before the onset of interstitial/bladder pain syndrome, the presence of multiple nonbladder syndromes is strongly associated with a history of multiple surgeries. J. Psychosom. Res. 76, 7579 (2014).

30. Warren, J. W. et al. Functional somatic syndromes as risk factors for hysterectomy in early bladder pain syndrome/interstitial cystitis. J. Psychosom. Res. 77, 363-367 (2014).

31. Drossman, D. A. The functional gastrointestinal disorders and the Rome III process. Gastroenterology 130, 1377-1390 (2006).

32. Longstreth, G. F. et al. Functional bowel disorders. Gastroenterology 130, 1480-1491 (2006).

33. Abrams, P. et al. The standardisation of terminology of lower urinary tract function: Report from the standardisation subcommitee of the International Continence Society. Neurourol. Urodyn. 21, 167-178 (2002).

34. Fall, M. et al. EAU guidelines on chronic pelvic pain. Eur. Urol. 46, 681-689 (2004).

35. van de Merwe, J. P. et al. Diagnostic criteria, classification, and nomenclature for painful bladder syndrome/ interstitial cystitis: An ESSIC proposal. Eur. Urol. 53, 60-67 (2008).

36. van Ophoven, A. et al. From end-organ disease to a classable bladder pain syndrome: Paradigm shift in the understanding of urological pain syndromes exemplified by the condition currently called interstitial cystitis. Urologe A. 48, 152-162 (2009).

37. Fall, M. et al. EAU guidelines on chronic pelvic pain. Eur. Urol. 57, 35-48 (2010).

38. Engeler, D. S. et al. The 2013 EAU guidelines on chronic pelvic pain: is management of chronic pelvic pain a habbit, a philosophy, or a science? 10 years of development. Eur. Urol. 64, 431-439 (2013).

39. Wesselmann, U. A new paradigm in chronic bladder pain. J. Pain Palliat. Care Pharmacother. 28, 406-408 (2014).

40. Twiddy, H. et al. The development and delivery of a female chronic pelvic pain management programme: A specialised interdisciplinary approach. Br. J. Pain. 9, 233-240 (2015).

41. Hanno, P. M. et al. Diagnosis and treatment of interstitial cystitis/bladder pain syndrome: AUA guideline amendment. J. Urol. 193, 1545-1553 (2015).

42. Parsons, C. L. The role of a leaky epithelium and potassium in the generation of bladder symotoms in interstitial cystitis/overactive bladder, urethral syndrome, prostatitis and gynaecological chronic pelvic pain. BJU Int. 107, 370-375 (2011).

43. Homma, Y. Hypersensitive bladder: A solution to confused terminology and ignorance concerning interstitial cystitis. Int. J. Urol. 21, S41-47 (2014).

44. Hurst, R. E. et al. Increased bladder permeability in interstitial cystitis/painful bladder syndrome. Transl. Androl. Urol. 5, 563-571 (2015).

45. Camilleri, M. \& Gorman, H. Intestinal permeability and irritable bowel syndrome. Neurogastroenterol. Motil. 19, 545-552 (2007).

46. Das, R. et al. Dimensions of sensation assessed in urinary urgency: a systematic review. J. Urol. 190, 1165-1172 (2013).

47. Juszczak, K. et al. Hyperosmolarity alters micturition: a comparison of urinary bladder motor activity in hyperosmolar and cyclophosphamide-induced models of overactive bladder. Can. J. Physiol. Pharmacol. 88, 899-906 (2010).

48. Greenwood-van Meerveld, B. et al. Mechanisms of visceral organ crosstalk: Importance of alterations in permeability in rodent models. J. Urol. 194, 804-811 (2015).

49. Yoshikawa, S. et al. Pelvic organ cross-sensitization to enhance bladder and urethral pain behaviors in rats with experimental colitis. Neuroscience 284, 422-429 (2015).

50. Stanghellini, V. et al. Dyspeptic symptoms and gastric emptying in the irritable bowel syndrome. Am. J. Gastroenterol. 97, 2738-2743 (2002). 
51. Valentino, R. J. et al. The bladder-brain connection: putative role of corticotropin-releasing factor. Nat. Rev. Urol. 8, 19-28 (2011).

52. Martinez-Martinez, L. A. et al. Sympathetic nervous system dysfunction in fibromyalgia, chronic fatigue syndrome, irritable bowel syndrome, and interstitial cystitis: A review of case-control studies. J. Clin. Rheumatol. 20, 146-150 (2014).

53. Williams, D. P. Effects of chronic pelvic pain on heart rate variability in women. J. Urol. 194, 1289-1294 (2015).

54. Hughes, P. A. et al. Immune activation in irritable bowel syndrome: Can neuroimmune interactions explain symptoms? Am. J. Gastroenterol. 108, 1066-1074 (2013).

55. Cartwright, R. et al. Novel biomarkers for overactive bladder. Nat. Rev. Urol. 8, 139-145 (2011).

56. Qu, H. C. et al. Association between polymorphism of $\beta 3$-adrenoceptor gene and overactive bladder: A meta-analysis. Genet. Mol. Res. 14, 2495-2501 (2015).

57. Talati, A. et al. Panic disorder, social anxiety disorder, and a possible medical syndrome previously linked to chromosome 13. Biol. Psychiatry 63, 594-601 (2008).

58. Villani, A. C. et al. Genetic risk factors for post-infectious irritable bowel syndrome following a waterborne outbreak of gastroenteritis. Gastroenterology 138, 1502-1513 (2010).

59. Whiteside, S.A. et al. The microbiome of the urinary tract - a role beyond infection. Nat. Rev. Urol. 12, 81-90 (2015).

60. Pierce, A. N. \& Christianson, J. A. Stress and chronic pelvic pain. Prog. Mol. Biol. Transl. Sci. 131, 509-535 (2015).

61. Mykletun, A. et al. Prevalence of mood and anxiety disorder in self-reported irritable bowel syndrome (IBS): An epidemiological population based study of women. BMC Gastroenterol. 10, 88 (2010).

62. Vrijens, D. et al. Affective symptoms and the overactive bladder: A systematic review. J. Psychosom. Res. 78, 95-108 (2015).

63. Choung, R. S. et al. Psychosocial distress and somatic symptoms in community subjects with irritable bowel syndrome: A psychosocial component is the rule. Am. J. Gastroenterol. 104, 1772-1779 (2009).

64. Koloski, N.A. et al. Does psychological distress modulate functional gastrointestinal symptoms and health care seeking? Am. J. Gastroenterol. 98, 789-797 (2003).

65. Lackner, J. M. et al. Testing the sequential model of pain processing in irritable bowel syndrome: A structural equation modeling analysis. Eur. J. Pain 9, 207-18 (2005).

66. Koloski, N. A. et al. What level of IBS symptoms drives impairment in health-related quality of life in community subjects with irritable bowel syndrome? Are current IBS syndrome thresholds clinically meaningful? Qual. Life Res. 21, 829-836 (2012).

67. Link, C. L. et al. Is abuse causally related to urologic symptoms? Results from the Boston Area Community Health (BACH) survey. Eur. Urol. 52, 397-406 (2007).

68. Rothrock, N. E. et al. Stress and symptomatology in patients with interstitial cystitis: A life stress model. Urology 57, 422-427 (2001).

69. Hsiao, S. M. et al. Psychometric assessment of female overactive bladder syndrome and antimuscarinicsrelated effects. Maturitas 79, 428-434 (2014).

70. Lai, H. H. et al. Urological symptoms in a subset of patients with urological chronic pelvic pain syndrome and a polysymptomatic, polysyndromatic pattern of presentation. J. Urol. 191, 1802-1807 (2014).

71. Krieger, J. N. et al. Relationship between chronic nonurological associated somatic syndromes and symptom severity in urological chronic pelvic pain syndromes: Baseline evaluation of the MAPP study. J. Urol. 193, 1254-1262 (2015).

72. Lackner, J. M. et al. The impact of physical complaints, social environment, and physical functioning on IBS patients' health perception: looking beyond GI symptom severity. Am. J. Gastroenterol. 109, 224-233 (2014).

73. Riegel B. et al. Assessing psychological factors, social aspects and psychiatric co-morbidity associated with Chronic Prostatitis/Chronic Pelvic Pain Syndrome (CP/CPPS) in men: A systematic review. J. Psychosom. Res. 77, 333-350 (2014).

74. olde Hartman, T. C. et al. Chronic functional somatic symptoms: A single syndrome? Br. J. Gen. Pract. 54, 922-927 (2004).

75. Lackner, J. M. Type, rather than number, of mental physical comorbidities increases the severity of symptoms in patients with irritable bowel syndrome. Clin. Gastroenterol. Hepatol. 11, 1147-1157 (2013). 
76. Diagnostic and Statistical Manual of Mental Disorders. $5^{\text {th }}$ edition. Washington, DC: American Psychiatric Association (2013).

77. The ICD-10 Classification of Mental and Behavioural Disorders: Diagnostic Criteria for Research. World Health Organization, Geneva (1993).

78. Jylhä, P. \& Isometsä, E. The relationship of neuroticism and extraversion to symptoms of anxiety and depression in the general population. Depress. Anxiety 23, 281-289 (2006).

79. Klein, D. N. et al. Personality and depression: Explanatory models and review of the evidence. Annu. Rev. Clin. Psychol. 7, 269-295 (2011).

80. Neeleman, J. et al. The distribution of psychiatric and somatic ill health: Associations with personality and socioeconomic status. Psychosom. Med. 63, 239-247 (2001).

81. Lai, H. H. et al. Painful bladder filling and painful urgency are distinct characteristics in men and women with urological chronic pelvic pain syndromes: A MAPP research network study. J. Urol. 194, 1634-1641 (2015).

82. Koh, J. S. et al. The association of personality trait on treatment outcomes in patients with chronic prostatitis/chronic pelvic pain syndrome: An exploratory study. J. Psychosom. Res. 76, 127-133 (2014).

83. Koh, J. S. et al. The impact of depression and somatic symptoms on treatment outcomes in patients with chronic prostatitis/chronic pelvic pain syndrome: A preliminary study in a naturalistic treatment setting. Int. J. Clin. Pract. 68, 478-485 (2014).

84. Tayama, J. et al. Effects of personality traits on the manifestations of irritable bowel syndrome. Bio. Psycho. Soc. Med. 6, 20 (2012).

85. Goldstein, B. L. \& Klein D. N. A review of selected candidate endophenotypes for depression. Clin. Psychol. Rev. 34, 417-27 (2014).

86. Ormel, J. et al. Neuroticism and common mental disorders: Meaning and utility of a complex relationship. Clin. Psychol. Rev. 33, 686-697 (2013).

87. Muscatello, M. R. et al. Role of negative affects in pathophysiology and clinical expression of irritable bowel syndrome. World J. Gastroenterol. 20, 7570-7586 (2014).

88. Cuijpers, P. et al. Economic costs of neuroticism: a population-based study. Arch. Gen. Psychiatry 67, 1086-1093 (2010).

89. den Boeft, M. et al. The association between medically unexplained physical symptoms and health care use over two years and the influence of depressive and anxiety disorders and personality traits: A longitudinal study. BMC Health Serv. Res. doi: 10.1186/s12913-016-1332-7 (2016).

90. Hotopf, M. et al. Temporal relationships between physical symptoms and psychiatric disorder. Results from a national birth cohort. Br. J. Psychiatry 173, 255-261 (1998).

91. Neeleman, J. et al. Propensity to psychiatric and somatic ill-health: Evidence from a birth cohort. Psychol. Med. 32, 793-803 (2002).

92. Kroenke, K. \& Spitzer, R. L. Gender differences in the reporting of physical and somatoform symptoms. Psychosom. Med. 60, 150-155 (1998).

93. Goodwin, R. D. \& Gotlib, I. H. Gender differences in depression: The role of personality factors. Psychiatry Res. 126, 135-142 (2004).

94. Koenig, H. G. Depression in hospitalized older patients with congestive heart failure. Gen. Hosp. Psychiatry 20, 29-43 (1998).

95. Strik, J. J. et al. One-year cumulative incidence of depression following myocardial infarction and impact on cardiac outcome. J. Psychosom. Res. 56, 59-66 (2004).

96. Leue $\mathrm{C}$, et al. Managing complex patients on a Medical Psychiatric Unit: An observational study of university hospital costs associated with medical service use, length of stay and psychiatric intervention. J. Psychosom. Res. 68, 295-302 (2010).

97. Fishbain, D. A. et al. Chronic pain-associated depression: Antecedent or consequence of chronic pain? A review. Clin. J. Pain 13, 116-137 (1997).

98. Bair, M. J. et al. Anxiety but not Social Stressors Predict 12-Month Depression and Pain Severity. Clin. J. Pain 29, 95-101 (2013).

99. Gerrits, M. M. et al. Pain and the onset of depressive and anxiety disorders. Pain 155, 53-59 (2014).

100. Kroenke K, et al. Reciprocal Relationship between Pain and Depression: A 12-month longitudinal analysis in Primary Care. J Pain 12, 964-973 (2011). 
101. Henningsen, P. et al. Medically unexplained physical symptoms, anxiety, and depression: A meta-analytic review. Psychosom. Med. 65, 528-533 (2003).

102. Koloski, N. A. et al. The brain-gut pathway in functional gastrointestinal disorders is bidirectional: A $12-$ year prospective population-based study. Gut 61, 1284-1290 (2012).

103. Pinto-Sanchez, M. I. et al. Anxiety and depression increase in a stepwise manner in parallel with multiple FGIDs and symptom severity and frequency. Am. J. Gastroenterol. 110, 1038-1048 (2015).

104. Lai, H. et al. Correlation between psychological stress levels and the severity of overactive bladder symptoms. BMC Urology 15, 14 (2015).

105. Yunus, M. B. Fibromyalgia and overlapping disorders: The unifying concept of central sensitivity syndromes. Semin. Arthritis Rheum. 36, 339-356 (2007).

106. Rovner, E. S. \& Wein, A. J. Incidence and prevalence of overactive bladder. Curr. Urol. Rep. 3, 434-438 (2002).

107. Temml, C. et al. Prevalence of the overactive bladder syndrome by applying the International Continence Society definition. Eur. Urol. 48, 622-627 (2005).

108. Kogan, M. I. et al. Epidemiology and impact of urinary incontinence, overactive bladder, and other lower urinary tract symptoms: Results of the EPIC survey in Russia, Czech republic, and Turkey. Curr. Med. Res. Opin. 30, 2119-2130 (2014).

109. Moorthy, P. et al. Prevalence of overactive bladder in Asian men: An epidemiological survey. BJU Int. 93, 528-531 (2004).

110. Irwin, D. E. et al. Population-based survey of urinary incontinence, overactive bladder, and other lower urinary tract symptoms in five countries: Results of the EPIC study. Eur. Urol. 50, 1306-1314 (2006).

111. Coyne, K. S. et al. National community prevalence of overactive bladder in the United States stratified by sex and age. Urology 77, 1081-1087 (2011).

112. Coyne, K. S. et al. The prevalence of lower urinary tract symptoms (LUTS) and overactive bladder (OAB) by racial/ethnic group and age: Results from OAB-POLL. Neurourol. Urodyn. 32, 230-237 (2013).

113. Stewart, W. E. et al. Prevalence and burden of overactive bladder in the United States. World J. Urol. 20, 327-336 (2003).

114. Bedretdinova, D. et al. Prevalence of female urinary incontinence in the general population according to different definitions and study designs. Eur. Urol. 69, 256-264 (2016).

115. Milsom, I. et al. Global prevalence and economic burden of urgency urinary incontinence: A systematic review. Eur. Urol. 65, 79-95 (2014).

116. Serati, M. \& Ghezzi, F. The epidemiology of urinary incontinence: A case still open. Ann. Transl. Med. 4, 123 (2016).

117. Kusek, J. W. \& Nyberg, L. M. The epidemiology of interstitial cystitis: is it time to expand our definition? Urology. 57, S95-99 (2001).

118. Berry, S. H. et al. Development, validation and testing of an epidemiological case definition of interstitial cystitis/painful bladder syndrome. J. Urol. 183, 1848-1852 (2010).

119. Berry, S. H. et al. Prevalence of symptoms of bladder pain syndrome/interstitial cystitis among adult females in the United States. J. Urol. 186, 540-544 (2011).

120. Arora, H. C. \& Shoskes, D. A. The enigma of men with interstitial cystitis/bladder pain syndrome. Ann. Transl. Med. 4, 668-676 (2015).

121. Clemens, J. Q. et al. Temporal ordering of interstitial cystitis/bladder pain syndrome and non-bladder conditions. Urology. 80, 1227-1231 (2012).

122. Nickel, J. C. et al. Clinical and psychological parameters associated with pain pattern phenotypes in women with interstitial cystitis/bladder pain syndrome. J. Urol. 192, 138-144 (2015).

123. Lovell RM, Ford AC. Global prevalence of and risk factors for irritable bowel syndrome: A meta-analysis. Clin. Gastroenterol. Hepatol. 10, 712-721 (2012).

124. Kessler, R. C. et al. Lifetime prevalence and age-of-onset distributions of DSM-IV disorders in the national comorbidity survey replication. Arch. Gen. Psychiatry 62, 593-602 (2005).

125. Cole J. A. et al. Migraine, fibromyalgia and depression among people with IBS: A prevalence study. BMC Gastroenterology 6, 26 (2006).

126. Felde, G. et al. Anxiety and depression associated with urinary incontinence. A 10-year follow-up study from the Norwegian HUNT study (EPINCONT). Neurourol. Uroldyn. doi: 10.1002/nau.22921 (2015). 
127. Nickel, J. C. et al. Interstitial cystitis/painful bladder syndrome and associated medical conditions with an emphasis on irritable bowel syndrome, fibromyalgia and chronic fatigue syndrome. J. Urol. 184, 13581363 (2010).

128. Nickel, J. C. et al. Psychosocial phenotyping in women with interstitial cystitis/painful bladder syndrome: A case control study. J. Urol. 184, 167-172 (2010).

129. Naliboff, B. D. et al. Widespread psychological difficulties in men and women with urologic chronic pelvic pain syndromes: Case-control findings from the Multidisciplinary Approach to the Study of Chronic Pelvic Pain Research Network. Urology 85, 1319-1327 (2015).

130. Clemens, J. Q. et al. Mental health diagnoses in patients with interstitial cystitis/painful bladder syndrome and chronic prostatitis/chronic pelvic pain syndrome: A case/control study. J. Urol. 180, 13781382 (2008).

131. Chung, K. H. et al. Bladder pain syndrome/interstitial cystitis is associated with anxiety disorder. Neurourol. Uroldyn. 33, 101-105 (2014).

132. Lutgendorf, S. K. et al. Stress and symptomatology in patients with interstitial cystitis: A laboratory stress model. J. Urol. 164, 1265-1269 (2000).

133. Drossman, D. A. et al. Severity in irritable bowel syndrome: A Rome foundation working team report. Am J. Gastroenterol. 106, 1749-59 (2011).

134. Clauss, J. A. et al. The nature of individual differences in inhibited temperament and risk for psychiatric disease: A review and meta-analysis. Prog. Neurobiol. 127-128, 23-45 (2015).

135. Iniguez, S. D. et al. Social defeat stress induces a depression-like phenotype in adolescent male c57b1/6 mice. Stress 17, 247-255 (2014).

136. Mahar, I. et al. Stress, serotonin, and hippocampal neurogenesis in relation to depression and antidepressant effects. Neurosci. Biobehav. Rev. 38, 173-192 (2014).

137. Selye, H. A syndrome produced by diverse nocuous agents. 1936. J. Neuropsychiatry Clin. Neurosci. 10, 230-231 (1998).

138. McEwen, B. S. Physiology and neurobiology of stress and adaptation. Physiol. Rev. 87, 873-904 (2007).

139. Moloney, R. D. et al. Stress-induced visceral pain: Toward animal models of irritable bowel syndrome and associated comorbidities. Front. Psychiatry 6, 15 (2015).

140. Charmandari, E. et al. Endocrinology of the stress response. Annu. Rev. Physiol. 67, 259-284 (2005).

141. McEwen, B. S. The neurobiology of stress: Form serendipity to clinical relevance. Brain Res. 886, 172-89 (2000).

142. Larauche, M. et al. Stress and visceral pain: From animal models to clinical therapies. Exp. Neurol. 233,49- 67 (2012).

143. O'Malley, D. et al. Do interactions between stress and immune responses lead to symptom exacerbation in irritable bowel syndrome? Brain Behav. Immun. 25, 1333-1341 (2011).

144. McEwen, B. S. Brain on stress: How the social environment gets under the skin. Proc. Natl. Acad. Sci. USA. 109 (Suppl. 2), 17180-17185 (2012).

145. Zhou, Q. Q. \& Verne, G. N. New insights into visceral hypersensitivity - clinical implications in IBS. Nat. Rev. Gatsroenterol. Hepatol. 8, 349-355 (2011).

146. Cheung, W. W. et al. Prevalence of overactive bladder, its under-diagnosis, and risk factors in a male urologic veterans population. Int. J. Med. Sci. 7, 391-394 (2010).

147. Geuze E. et al. Altered pain processing in veterans with posttraumatic stress disorder. Arch. Gen. Psychiatry 64, 76-85 (2007).

148. Chiu, G. R. et al. Prevalence and overlap of childhood and adult physical, sexual, and emotional abuse: A descriptive analysis of results from the Boston Area Community Health (BACH) survey. Violence Vict. 28, 381-402 (2013).

149. Blanchard, E. B. et al. The role of stress in symptom exacerbation among IBS patients. J. Psychosom. Res. 64, 119-128 (2008).

150. Levy, R. L. et al. The relationship between daily life stress and gastrointestinal symptoms in women with irritable bowel syndrome. J. Behav. Med. 20, 177-193 (1997).

151. Jundt, K. et al. Physical and sexual abuse in patients with overactive bladder. Int. Urogynecol. J. Pelvic Floor Dysfunct. 18, 449-53 (2007).

152. Mayson, B. E. \& Teichman, J. M. The relationship between sexual abuse and interstitial cystitis/painful bladder syndrome. Curr. Urol. Rep. 10, $441-447$ (2009). 
153. Klooker, T. K. et al. Exposure to severe wartime conditions in early life is associated with an increased risk of irritable bowel syndrome: A population-based cohort study. Am. J. Gastroenterol. 104, 2250-2256 (2009).

154. Chitkara, D. K. et al. Early life risk factors that contribute to irritable bowel syndrome in adults: A systematic review. Am. J. Gastroenterol. 103, 765-774 (2008).

155. van Tilburg, M. A. et al. Unexplained gastrointestinal symptoms after abuse in a prospective study of children at risk for abuse and neglect. Ann. Fam. Med. 8, 134-140 (2010).

156. O'Mahony, S. M. et al. Maternal separation as a model of brain-gut axis dysfunction. Psychopharmacology 214, 71-88 (2011).

157. Merrill, L. et al. Repeated variate stress in male rats induces increasing voiding frequency, somatic sensitivity, and urinary bladder nerve growth factor expression. Am. J. Physiol. Regul. Integr. Comp. Physiol. 305, R147-156 (2013).

158. Breyer, B. N. et al. Lower urinary tract dysfunction in male Iraq and Afghanistan war veterans: Association with mental health disorders - a population-based cohort study. Urology 83, 312-319 (2014).

159. Nusslock, R. \& Miller, G. E. Early-life adversity and physical and emotional health across the lifespan: A neuroimmune network hypothesis. Biol. Psychiatry Pii: S0006-3223 (15) 00466-7 (2015).

160. Drossman, D. A. Do psychosocial factors define symptom severity and patient status in irritable bowel syndrome? Am. J. Med. 107, 41S-50 (1999).

161. Surdea-Blaga, T. et al. Psychosocial determinants of irritable bowel syndrome. World J. Gastroenterol. 18, 616-626 (2012).

162. Costa, P. T. \& McCrae, R. R. NEO PI-R professional manual. Odessa, FL. Psychological Assessment Resources, Inc. (1992)

163. Hazlett-Stevens, H. et al. Prevalence of irritable bowel syndrome among university students: The roles of worry, neuroticism, anxiety sensitivity and visceral anxiety. J. Psychosom. Res. 55, 501-505 (2003).

164. Farnam, A. et al. Personality factors and profiles in variants of irritable bowel syndrome. World J. Gastroenterol. 13, 6414-6418 (2007).

165. van Tilburg, M. A. et al. Which psychological factors exacerbate irritable bowel syndrome? Development of a comprehensive model. J. Psychosom. Res. 74, 486-492 (2013).

166. Sullivan, M. J. et al. Theoretical perspectives on the relation between catastrophizing and pain. Clin. J. Pain 17, 52-64 (2001).

167. Lackner, J. M. et al. Depression and abdominal pain in IBS patients: the mediating role of catastrophizing. Psychosom. Med. 66, 435-441 (2004).

168. Lackner, J. M. et al. Patient reported outcomes for irritable bowel syndrome are associated with patients' severity ratings of gastrointestinal symptoms and psychological factors. Clin. Gastroenterol. Hepatol. 9,957-964 (2011).

169. Cortes, E. et al. The psychology of LUTS: ICI-RS 2011. Neurourol. Urodyn. 31, 340-343 (2012).

170. Davydov, D.M. et al. Resilience and mental health. Clin. Psychol. Rev. 30, 479-495 (2010).

171. Lutha, S. S. \& Cicchetti, D. The construct of resilience: Implications for interventions and social policies. Dev. Psychopathol. 12, 857-885 (2000).

172. Daskalakis, N. P. et al. The three-hit concept of vulnerability and resilience: Toward understanding adaptation to early-life adversity outcome. Psychoneuroendocrinology 38, 1858-1873 (2013).

173. Daskalakis, N. P. et al. Testing the cumulative stress and mismatch hypotheses of psychopathology in a rat model. Physiol. Behav. 106, 707-721 (2012).

174. Sousa, N. The dynamics of the stress neuromatrix. Mol. Psychiatry. 21, 302-312 (2016).

175. Spiller, R. Postinfectious functional dyspepsia and postinfectious irritable bowel syndrome: Different symptoms but similar risk factors. Gastroenterology 138, 1660-1663 (2010).

176. Herzog, A. R. \& Fultz, N. H. Prevalence and incidence of urinary incontinence in community-dwelling populations. J. Am. Geriatr. Soc. 38, 273-281 (1990).

177. Brown J. S. et al. Prevalence of urinary incontinence and associated risk factors in postmenopausal women. Heart \& Estrogen/Progestin Replacement Study (HERS) Research Group. Obstet. Gynecol. 94, 66-70 (1999).

178. Moore, E. E. et al. Urinary incontinence and urinary tract infection: Temporal relationships in postmenopausal women. Obstet. Gynecol. 111, 317-323 (2008). 
179. Stanford, E. \& McMurphy, C. There is a low incidence of recurrent bacteriuria in painful bladder syndrome/ interstitial cystitis patients followed longitudinally. Int. Urogynecol. J. Pelvic Floor Dysfunct. 18, 551-554 (2007).

180. Walsh, C. A. \& Moore, K. H. Overactive bladder in women: does low-count bacteriuria matter? A review. Neurourol. Urodyn. 30, 32-37 (2011).

181. Walsh, C. A. et al. Prevalence of "low-count" bacteriuria in female urinary incontinence versus continent female controls: A cross-sectional study. Int. Urogynecol. J. 22, 1267-1272 (2011).

182. Sorrentino, F. et al. Associations between individual lower urinary tract symptoms and bacteriuria in random urine samples in women. Neurourol. Urodyn. 34, 429-433 (2015).

183. Kashriya, R. et al. The inadequacy of urinary dipstick and microscopy as surrogate markers of urinary tract infection in urological outpatients with lower urinary tract symptoms without acute frequency and dysuria. J. Urol. 183, 1843-1847 (2010).

184. Hessdoerfer, E. et al. Is a dipstick test sufficient to exclude urinary tract infection in women with overactive bladder? Int. Urogynecol. J. 22, 229-232 (2011).

185. Wolfe, A. J. et al. Evidence of uncultivated bacteria in the adult female bladder. J. Clin. Microbiol. 50, 1376-1383 (2012).

186. Kashriya, R. et al. Spectrum of bacterial colonization with urothelial cells from patients with chronic lower urinary tract symptoms. J. Clin. Microbiol. 51, 2054-2062 (2013).

187. Hannan, T. J. et al. Host-pathogen checkpoints and population bottlenecks in persistent and intracellular uropathogenic E. coli bladder infection. FEMS Microbiol. Rev. 36, 616-648 (2012).

188. Nicolle, L. E. Asymptomatic bacteriuria. Curr. Opin. Infect. Dis. 27, $90-96$ (2014).

189. Moore, K. H. \& Malykhina, A. P. What is the role of covert infection in detrusor overactivity, and other LUTD? ICI-RS 2013. Neurourol. Urodyn. 33, 606-610 (2014).

190. Mabbett, A. N. et al. Virulence properties of asymptomatic bacteriuria Escherichia coli. Int. J. Med. Microbiol. 299, 53-63 (2009).

191. Ragnarsdottir, B. et al. Genetics of innate immunity and UTI susceptibility. Nat. Rev. Urol. 8, 449-468 (2011).

192. Peters, K. M. et al. Childhood symptoms and events in women with interstitial cystitis/ painful bladder syndrome. Urology 73, 258-262 (2009).

193. Rosen, J. M. \& Klumpp, D. J. Mechanisms of pain from urinary tract infection. Int. J. Urol. 21 (Suppl. 1), 26-32 (2014).

194. McLellan, L. K. \& Hunstad, D. A. Urinary tract infection: Pathogenesis and outlook. Trends Mol. Med. 22, 946-957 (2016).

195. Lüthje, P. \& Brauner, A. Virulence factors of uropathogenic E. coli and their interaction with the host. Adv. Microb. Physiol. 65, 337-372 (2014).

196. Mulvey, M. A. Schilling JD, Martinez JJ, Hultgren SJ. Bad bugs and beleaguered bladders: Interplay between uropathogenic Escherichia coli and innate host defenses. Proc. Natl. Acad. Sci. USA 97, 88298835 (2000).

197. Billips, B. K. et al. Molecular basis of uropathogenic Escherichia coli evasion of the innate immune response in the bladder. Infect. Immun. 76, 3891-3900 (2008).

198. Ragnarsdottir, B. \& Svanborg, C. Susceptibility to acute pyelonephritis or asymptomatic bacteriuria: Hostpathogen interaction in urinary tract infections. Pediatr. Nephrol. 27, 2017-2029 (2012).

199. Woolf, C. J. \& Salter, M. W. Neuronal plasticity: Increasing the gain in pain. Science 288, 1765-1769 (2000).

200. Rudick, C. N. et al. O-antigen modulates infection-induced pain states. PLoS One 7, e1273 (2012).

201. Collins, S. M. A role for the gut microbiota in IBS. Nat. Rev. Gastroenterol. Hepatol. 11, 497-505 (2014).

202. Proctor L. M. The human microbiome project in 2011 and beyond. Cell Host Microbe 10, 287-291 (2011).

203. Siddiqui, H. et al. Assessing diversity of the female urine microbiota by high throughput sequencing of 16S rDNA amplicons. BMC Micobiol. 11, 244 (2011).

204. Lewis, D. A. et al. The human urinary microbiome; Bacterial DNA in voided urine of asymptomatic adults. Front. Cell. Infect. Microbiol. 3, 41 (2013).

205. Pearce, M. M. et al. The female urinary microbiome: A comparison of women with and without urgency urinary incontinence. MBio 5, e01283-14 (2014). 
206. Siddiqui, H. et al. Alterations of microbiota in urine from women with interstitial cystitis. BMC Microbiol. 12, 205 (2012).

207. Stapleton, A. E. Urinary tract infection pathogenesis: Host factors. Infect. Dis. Clin. North. Am. 28, 149159 (2014).

208. Ferry, S. A. et al. The natural course of lower uncomplicated urinary tract infection in women illustrated by a randomized placebo controlled study. Scand. J. Infect. Dis. 36, 296-301 (2004).

209. Reid, G. et al. Microbiota restauration: Natural and supplemented recovery of human microbial communities. Nat. Rev. Microbiol. 9, 27-38 (2011).

210. Ott, S. J. et al. Reduction in diversity of the colonic mucosa associated bacterial microflora in patients with active inflammatory bowel disease. Gut 53, 685-693 (2004).

211. Turnbaugh, P. J. A core gut microbiome in obese and lean twins. Nature 457, 480-484 (2009).

212. Cosseau, C. et al. The commensal Streptococcus salivarius K12 downregulates the innate immune responses of human epithelial cells and promotes host-microbe homeostasis. Infect. Immun. 76, 41634175 (2008).

213. Naseribafrouei, A. et al. Correlation between the human fecal microbiota and depression. Neurogastroenterol. Motil. 26, 1155-1162 (2014).

214. Kaplan, S. A. et al. Systematic review of the relationship between bladder and bowel function: Implications for patient management. Int. J. Clin. Pract. 67, 205-216 (2013).

215. Janssens, K. A. et al. Mood and anxiety disorders in chronic fatigue syndrome, fibromyalgia, and irritable bowel syndrome: Results from the Life Lines cohort study. Psychosom. Med. 77, 449-457 (2015).

216. Thijssen, A. Y. et al. Dysfunctional cognitions, anxiety and depression in irritable bowel syndrome. J. Clin. Gastroenterol. 44, e236-241 (2010).

217. Kruimel, J. et al. Integrated medical-psychiatric outpatient care in functional gastrointestinal disorders improves outcome: A pilot study. Eur. J. Gastroenterol. Hepatol. 27, 721-727 (2015).

218. Marshall, J. K. et al. Eight year prognosis of post infectious irritable bowel syndrome following waterborne bacterial dysentery. Gut 59, 605-611 (2010).

219. Berghmans, B. et al. Prevalence and triage of first-contact complaints on pelvic floor dysfunctions in female patients at a Pelvic Care Centre. Neurourol. Urodyn. 35, 503-508 (2016).

220. Berghmans, B. et al. Prevalence and triage of first-contact complaints on pelvic floor dysfunctions in male patients referred to a Pelvic Care Centre. Neurourol. Urodyn. 35, 487-491 (2016).

221. Filipetto, F. A. et al. The patient perspective on overactive bladder: A mixed-methods needs assessment. BMC Fam. Pract. doi: 10.1186/1471-2296-15-96 (2014).

222. Bosch, P. C. \& Bosch, D. C. Treating interstitial cystitis/bladder pain syndrome as a chronic disease. Rev. Urol. 16, 83-87 (2014).

223. Sharpe, M. et al. Is co-morbid depression adequately treated in patients repeatedly referred to specialist medical services with symptoms of a medical condition? J. Psychosom. Res. 72, 419-421 (2012).

224. Shoskes, D. A. et al. Phenotypically directed multimodal therapy for chronic prostatitis/chronic pelvic pain syndrome: A prospective study using UPOINT. Urology 75, 1249-1253 (2010).

225. Kartha, G. K. et al. Clinical phenotyping of urological pain patients. Curr. Opion. Urol. 23, 560-564 (2013).

226. Clemens, J. Q. et al. The MAPP research network: a novel study of urologic pelvic pain syndromes. BMC Urol. 14, 57 (2014).

227. Mujagic, Z. et al. Systematic review: instruments to assess abdominal pain in irritable bowel syndrome. Aliment. Pharmacol. Ther. 42, 1064-1081 (2015).

228. Mujagic, Z. et al. The Experience Sampling Method - a new digital tool for momentary symptom assessment in IBS: an exploratory study. Neurogastroenterol. Motil. 27, 1295-1302 (2015).

229. Myin-Germeys, I. Experience sampling research in psychopathology: Opening the black box of daily life. Psychol. Med. 39, 1533-1547 (2009).

230. Konnopka, A. et al. Economics of medically unexplained symptoms: A systematic review of the literature. Psychother. Psychosom. 81, 265-275 (2012).

231. Archer, J. et al. Collaborative care for depression and anxiety problems. Cochrane Database Syst. Rev. 10, CD006525 (2012).

232. Tully, P.J. \& Baumaeister, H. Collaborative care for comorbid depression and coronary heart disease: A systematic review and meta-analysis of randomized controlled trials. BMJ Open 5, pii: e009128 (2015). 
233. Huffman, J. C. et al. Essential articles on collaborative care models for the treatment of psychiatric disorders in medical settings: A publication by the academy of psychosomatic medicine research and evidence-based practice committee. Psychosomatics 55, 109-122 (2014).

234. Leue, C. et al. Observational evidence that urbanisation and neighbourhood deprivation are associated with escalation in chronic pharmacological pain treatment: A longitudinal population-based study in the Netherlands. BMJ Open 2, pii: e101136 (2012).

235. Balachandran, A. et al. Management of female urinary incontinence: A survey of urogynaecologists' view on the NICE guideline. J. Obstet Gynaecol. 9, 1-5 (2015).

236. Warren, J. W. \& Clauw, D. J. Functional somatic syndromes: Sensitivities and specificities of self-reports of physicians' diagnosis. Psychosom. Med. 74, 891-895 (2012).

237. Gawronski, K.A. et al. Potentially traumatic events and serious life stressors are prospectively associated with frequency of doctor visits and overnight hospital visits. J. Psychosom. Res. 77, 90-96 (2014).

238. Pierce, A. N. et al. Urinary bladder hypersensitivity and dysfunction in female mice following early life and adult stress. Brain Res. 1639, 58-73 (2016).

239. Mayer, E. A. et al. Towards a systems view of IBS. Nat. Rev. Gastroenterol. Hepatol. 12, 592-605 (2015).

240. Teicher, M. H. et al. The effects of childhood maltreatment on brain structure, function and connectivity. Nat. Rev. Neurosci. 17, 652-666 (2016). 



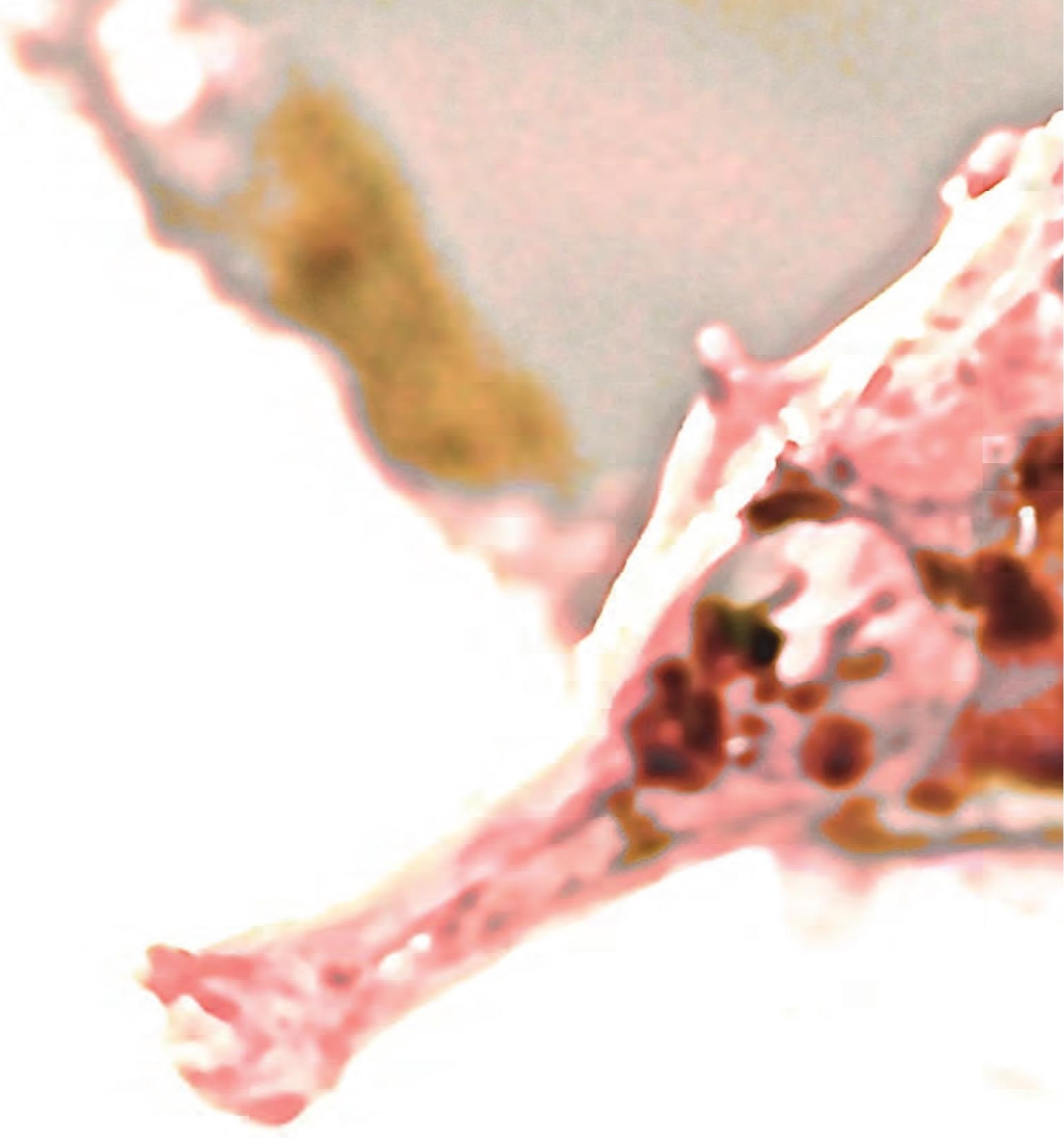




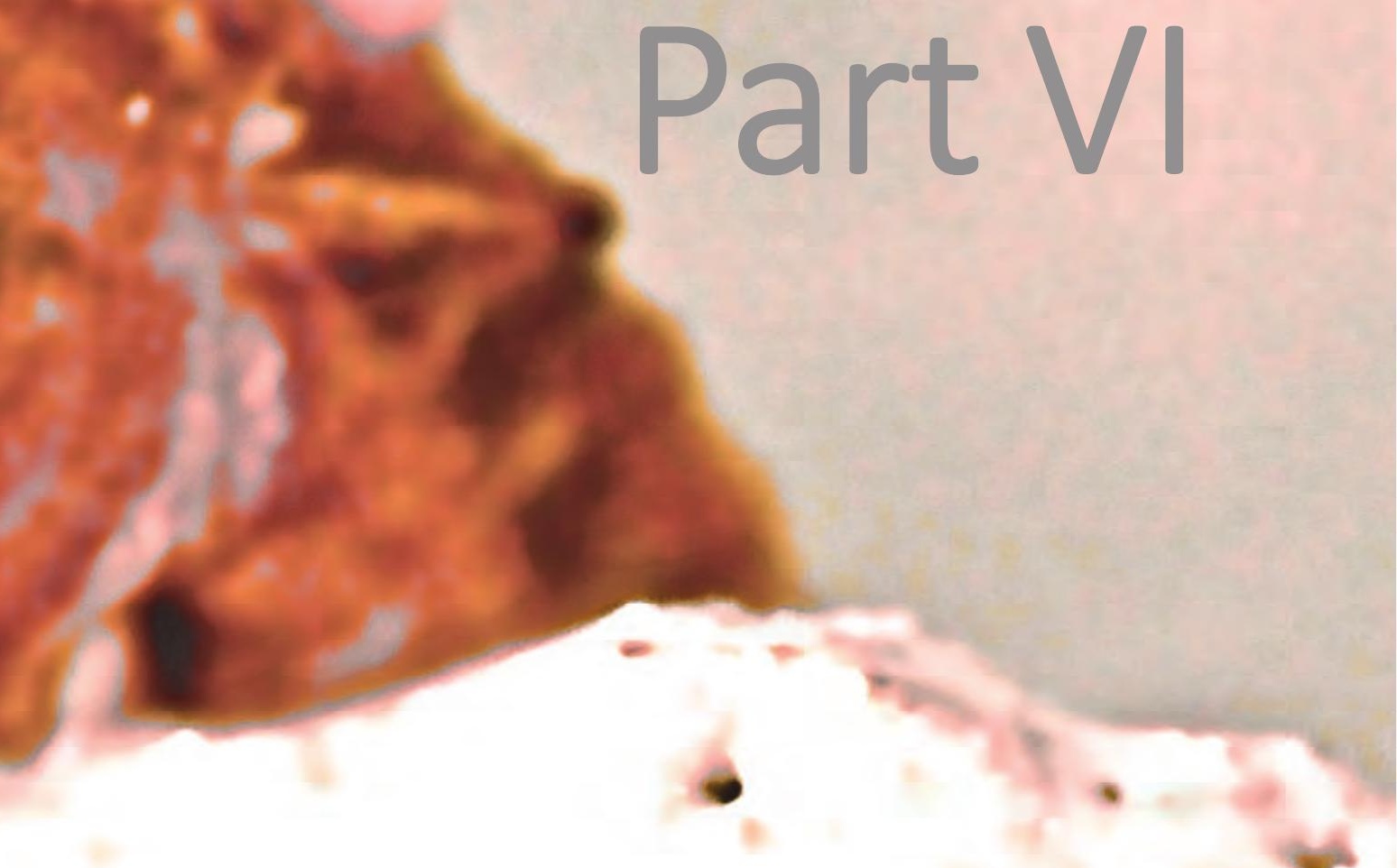

Integrated care fostering momentary assessment 



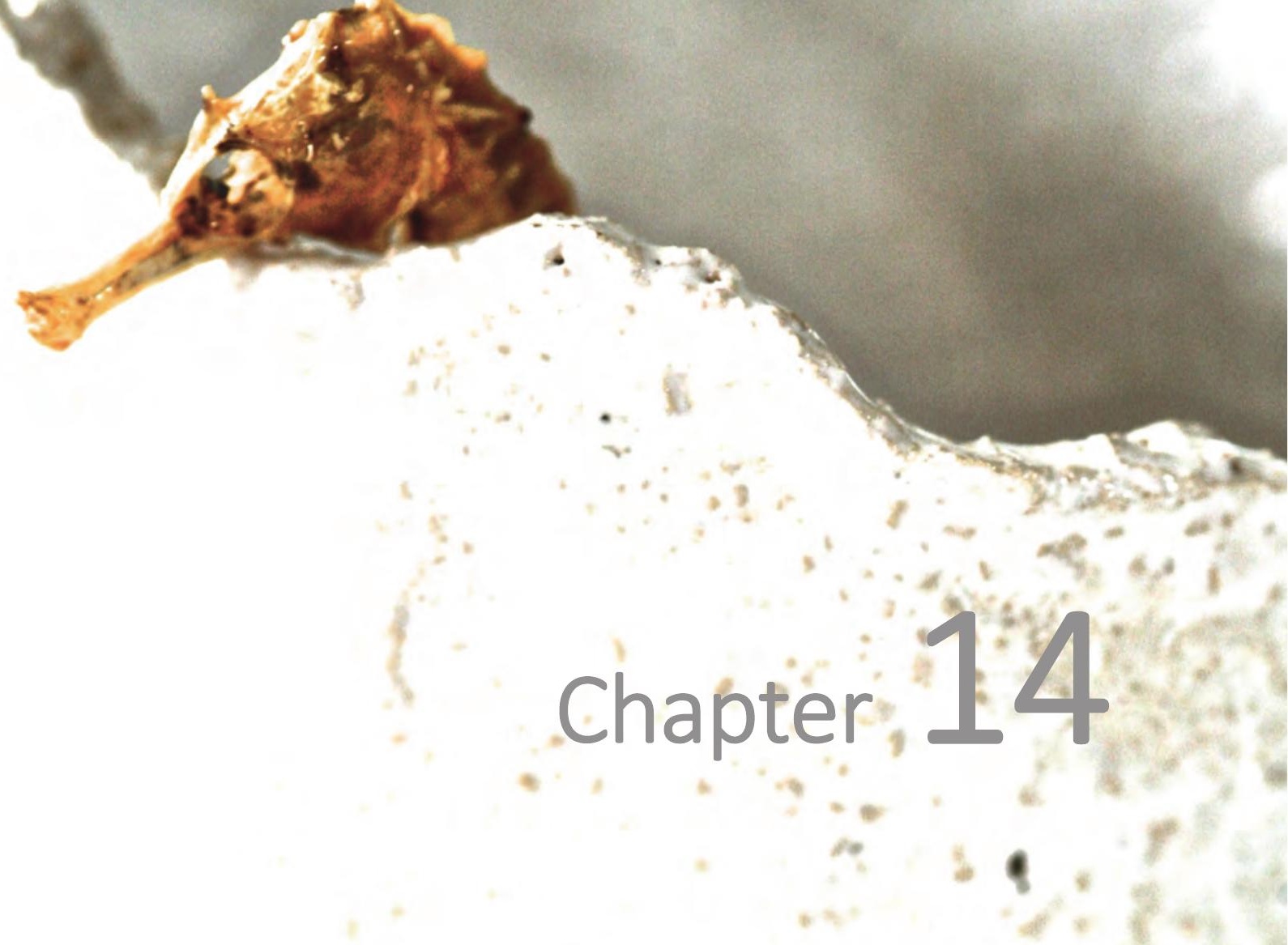

The experience sampling method - a new digital tool for momentary symptom assessment in IBS: An exploratory study

Mujagic Z, Leue $C^{a}$, Vork $L^{a}$, Lousberg R, Jonker D, Keszthelyi D, Hesselink M, van Schagen T, van Os J, Masclee A, Kruimel J ${ }^{a}$ These authors contributed equally to the present article Neurogastroenterol. Motil. 27, 1295-1302 (2015) 


\section{Abstract}

\section{Background}

Retrospective questionnaires are frequently used for symptom assessment in Irritable Bowel Syndrome (IBS) patients, but are influenced by recall bias and circumstantial and psychological factors. These limitations may be overcome by random, repeated, momentary assessment during the day, using electronic Experience Sampling Methodology (ESM). Therefore, we compared symptom assessment by ESM to retrospective paper questionnaires in IBS patients.

\section{Methods}

Twenty-six IBS patients (Rome III) were included, of which 16 were diagnosed with panic disorder (DSM-IV-TR). Patients scored symptoms using end-of-day diaries during 14 days and the gastrointestinal symptom rating scale (GSRS) once. ESM was used on seven consecutive days during the same time period.

\section{Key results}

End-of-day diary abdominal pain scores were 0.4 (SE 0.1, $P<0.001$ ) point higher (on a 1-to-5-point scale) compared to corresponding ESM mean-scores in IBS patients. The difference was even more pronounced for upper abdominal pain scores assessed by the GSRS (4.77 \pm 1.50$)$ compared to ESM mean-scores $(2.44 \pm 1.30, P<0.001)$, both on 1-to-7-point scale. For flatulence, comparable results were found. Nausea and belching scores showed small, but significant differences between end-of-day diary and ESM. All tested symptoms were scored higher on GSRS compared to ESM mean-scores $(P<0.01)$. Affective comorbidity did not influence differences in pain reporting between methods.

\section{Conclusions \& inferences}

IBS patients report higher scores for abdominal pain in retrospective questionnaires compared to ESM, with a tendency to report peak rather than average pain scores. ESM can provide more insight in symptom course and potential triggers, and may lead to a better understanding of IBS symptomatology. 


\section{Introduction}

A major hurdle for the development of new therapies in Irritable Bowel Syndrome (IBS) is the current lack of reliable instruments to measure symptoms over time and quantify therapy response. At present, retrospective self-reported paper questionnaires based on daily (i.e., by end-of-day diaries) or weekly symptom monitoring (i.e., by end-of-week questionnaires) are widely used as the standard method to assess symptoms in IBS patients $^{1-4}$. The Food and Drug Administration (FDA) recommends a 7-day mean of an 'end-of-day 11-point Numeric Rating Scale' as a primary endpoint for clinical trials assessing abdominal pain in IBS $^{5}$. In a recently published study, Lackner et al. demonstrated that (electronic) end-of-day diaries may more accurately quantify gastrointestinal (GI) symptoms in IBS patients compared to weekly symptom monitoring, in particular for average abdominal pain over a predefined period of time ${ }^{4}$. However, it should be taken into account that all retrospective questionnaires (e.g., end-of-day or end-of-week symptoms assessment) are based on autobiographical memory, thereby leading to recall bias, representing a reconstruction of specific moments, rather than a reliable reflection of symptoms over a time period ${ }^{6}$. Memory of pain can be influenced by contextual factors, as well as emotional and cognitive factors. This can affect perception and interpretation of symptoms, especially pain, and may result in statecongruent and ecological recall bias in retrospective questionnaires ${ }^{7-9}$. Affective comorbidity such as anxiety disorders, are prevalent in IBS patients and the presence of psychological comorbidity may contribute to a distorted perception and memory of $\mathrm{Gl}$ symptoms as well ${ }^{10,11}$. Furthermore, the inability to record temporal dynamics of symptoms renders retrospective questionnaires less suitable for measurement of symptoms fluctuating over time. Finally, it has been shown that paper diaries are subject to low compliance rates, as low as approximately $10 \%$, due to backfilling of questionnaires ${ }^{12}$.

The Experience Sampling Method (ESM) ${ }^{13}$, also referred to as Ecological Momentary Assessment $^{14}$, may overcome the significant limitations of retrospective questionnaires $^{15}$. This assessment method is characterized by the following criteria: data are collected repeatedly and randomly during the day, for several days, using a digital device, in the subject's natural environment, with a focus on the subject's in-themoment (i.e., momentary) physical and mental state and behavior. Digital momentary assessment reduces recall and ecological bias, may increase compliance rates and can take into account emotional, cognitive and contextual factors impacting IBS symptom reporting in general and abdominal pain in particular. One previous study by Weinland et al. used electronic momentary assessment in a group of 58 IBS patients to describe differences in symptom episodes between IBS subtypes. As secondary outcome they reported that abdominal pain scores were significantly higher in the traditional paper end-of-day diaries when compared to the electronic momentary assessment ${ }^{16}$. More research is needed to confirm their observation for abdominal pain, but also to compare 
ESM to current standards (i.e., daily and weekly retrospective symptom reporting) with regard to other Gl symptoms, and to investigate possible patient characteristics, such as anxiety, that may affect pain memory and therefore the differences between momentary and retrospective symptom assessment.

The aims of the present study were to explore possible advantages of electronic ESM over paper retrospective questionnaires for GI symptom measurement in IBS patients, by i) comparing abdominal pain and other Gl symptom scores assessed by a paper based end-of-day diary to corresponding scores assessed by ESM, by ii) comparing the GSRS, which includes a recall period of one week, to ESM, and by iii) investigating patient characteristics, that may affect memory of pain and may therefore contribute to a possible difference in scores between the momentary and retrospective symptom assessment.

We hypothesized that i) IBS patients will report higher levels of abdominal pain, and possibly also other GI symptoms, in end-of-day diaries and the GSRS compared to ESM average scores, and ii) that the presence of a comorbid panic disorder, more severe mental symptoms and negative cognitions will influence this difference in symptom reporting between the methods.

\section{Materials and methods}

The present study is part of a larger cohort study on the phenotypical and genotypical characterization of IBS (Maastricht IBS cohort) and of a 'Randomized Controlled Trial of Escitalopram versus Placebo for Patients with Irritable Bowel Syndrome and Panic Disorder', of which the baseline data (i.e., before start of any intervention) were used for the present analysis. Study protocols were approved by the Maastricht University Medical Center+ (MUMC+) Ethics Committee, were in compliance with the revised Declaration of Helsinki (64th WMA General Assembly, Fortaleza, Brazil, 2013) and were registered in the US National Library of Medicine (http://www.clinicaltrials.gov, NCT00775060 and NCT01551225, respectively).

\section{Study participants}

Subjects between 18 and 75 years of age with IBS, diagnosed by a gastroenterologist using the Rome III criteria $^{17}$, were recruited via the MUMC+ GastroenterologyHepatology outpatient clinic, which is a secondary and tertiary referral center for patients with functional GI disorders. For the diagnosis of IBS, GI endoscopy with biopsies, abdominal imaging by ultrasonography or CT scan, and/or blood, breath and fecal analysis were performed to exclude organic disease, when indicated by the physician. Patients with a history of abdominal surgery were excluded, with the exception of appendectomy, laparoscopic cholecystectomy, or hysterectomy. The 
presence of co-morbid panic disorder was diagnosed by an experienced psychiatrist based on the DSM-IV-TR criteria, by using the Mini-International-NeuropsychiatricInterview ${ }^{18}$. All subjects gave written informed consent prior to participation.

\section{Paper questionnaires and ESM}

Participants completed questionnaires on demographics, lifestyle factors and use of medication. Furthermore, they completed scales indexing psychopathology and negative cognitions. The Hospital Anxiety and Depression Scale (HADS), consisting of 14 items clustered into two subscales, i.e., anxiety and depression scale, with score of 0-21 per subscale, and a recall period of one week. Higher scores indicate higher symptom burden $^{19,20}$. The Cognitive Scale for Functional Bowel Disorders (CS-FBD), consisting of 31 items, scored on a 7-point Likert scale, and a total score of 217 , with a recall period of one month, was used to assess dysfunctional cognitions related to symptoms in FBD. A higher score indicates more dysfunctional cognitions ${ }^{21}$.

Gl symptoms were assessed over 14 consecutive days using an end-of-day diary. Complaints of abdominal discomfort, abdominal pain, nausea, bloating, belching, flatulence, diarrhea, constipation and overall symptom burden were scored on 5-point Likert scales ${ }^{2,3,22}$.

Electronic ESM ${ }^{13}$ was used to assess real-time Gl symptoms (e.g., abdominal pain, heartburn, nausea, belching, bloating, flatulence) in daily life, and momentary physical and mental experiences, activities and contextual aspects, during 7 consecutive days of the same time period. ESM was started at a random day during the first week of the 14 day end-of-day diary period. Subjects carried a digital device, the PsyMate (comparable to a mobile phone) $^{23}$. The device gives an auditory signal (a "beep") at ten random time points per day. At each beep, participants were instructed to complete 60 short questions on the device following every signal, using a 7-point Likert scale. The completion of the question set took 4-5 minutes on each occasion. The same set of questions was repeated in the same order at every beep. As subjects are usually not able to respond after every beep (for example because of driving a car), completion of $\geq 6$ of the 10 beep-questionnaires per day was considered as being compliant ${ }^{24}$.

In addition, participants were instructed to complete the Gastrointestinal Symptom Rating Scale (GSRS), consisting of 15 items, scored on a 7-point Likert scale, with a recall period of one week, during the same time period ${ }^{25}$.

Individual questions of both retrospective methods were compared to individual questions of ESM. Only GI symptoms assessed by ESM that corresponded to questions in the end-of-day diary and/or GSRS were included in the current analyses. The phrasing of the questions was similar, apart from the time frame explanation, and for the end-of day diary with regard to the used scale, i.e., a 1-to-5 point scale for the end-of-day diary compared a 1-to-7 point scale for the ESM and GSRS. 


\section{Data and statistical analysis}

4D software was used to program PsyMate devices and extract the data. Statistical analyses were performed using IBM SPSS Statistics version 22.0 (IBM Statistics for Windows, Armonk, New York).

In order to check if completing the ESM questionnaires at multiple time points each day influenced the scores of the end-of-day diary, we compared the end-of-day diary scores (i.e., 7-day mean of each symptom) of the 7 days during which participants also carried the PsyMate device to the 7 days during which subjects only completed the endof-day diary, using a paired sample $t$-test.

ESM data are based on a multi-level structure, including the variation between subjects and repeated measures within subjects. Associations between Gl symptom scores of the end-of-day diary and corresponding ESM scores were tested, using linear mixed models, based on a random intercept and an autoregressive (AR1) covariate structure. Currently, the end-of-day diary is the standard method and was therefore used as dependent variable in the analysis. For each participant, ESM day-mean scores of individual symptoms were used as predictor, to test whether average symptom scores of the day reflect the end-of-day-diary scores.

Scoring scales of the end-of-day diaries and ESM were harmonized by rescaling the ESM data from a 7-point to a 5-point scale, i.e., a score of 1 was 1, 2 or 3 was rescaled to 2, 4 to 3, 5 or 6 to 4 and 7 to 5 . In order to assess whether the rescaling affected the reliability of GI symptom related questions in ESM, a Cronbach's a was calculated for the original and rescaled data.

To quantify the difference of scores between the two methods, ESM data were subtracted from corresponding end-of-day diary scores, resulting in a delta score, which was statistically tested using the same multi-level analysis, adjusting for repeated measures. In like manner, the differences between end-of-day diary scores and ESM maximum scores of the day were tested.

In order to investigate if certain patient characteristics lead to a different retrospective perception of abdominal pain, possible predictors of a larger delta-score (i.e., the differences in abdominal pain reporting between ESM day-mean scores and the end-of-day diary scores) were tested: i.e. gender, age, presence of co-morbid panic disorder, psychological symptoms (HADS) and negative cognitions (CS-FBD). To further test the hypothesis that the presence of panic disorder in patients may influence $\mathrm{GI}$ symptom reporting, linear mixed models were used to investigate whether there was a difference in symptom score levels between IBS patients with and without a diagnosis of panic disorder, within ESM or end-of-day diary data.

Differences between GI symptom scores assessed by the GSRS and ESM could only be analyzed on subject level, by paired sample $t$-test, due to lack of intra-subject variation in the GSRS scores (only one measurement per subject). Subject-means (i.e., means over 7 days) of non-rescaled ESM data were calculated and compared to the corresponding GSRS scores (both ESM and GSRS use a 7-point scale). 


\section{Results}

Twenty-six IBS patients were included in the analysis. Female gender was predominant (69.2\%), mean age was $41.8 \pm 15.5$ years and mean BMI was $25.5 \pm 5.2 \mathrm{~kg} / \mathrm{m}^{2}$. IBS subtypes were defined based on bowel habits according to the ROME III criteria: 14 subjects were classified as diarrhea predominant (IBS-D), 4 as constipation predominant (IBS-C), 7 as mixed (IBS-M) and 1 as undefined subtype (IBS-U). Sixteen patients were diagnosed with co-morbid panic disorder. Mean scores for CS-FBD, measuring negative cognitions related to GI symptoms, and the HADS subscales for depression and anxiety were $112 \pm 44,4.2 \pm 3.7$ and $7.4 \pm 4.4$, respectively.

The scores of the 14-day paper end-of-day diary did not differ significantly between the 7 days on which also ESM was used compared to 7 days without ESM, indicating that carrying the PsyMate ${ }^{\mathrm{TM}}$ device did not influence the end-of-day diary scores.

All subjects reported some level of abdominal pain during the test period, and the rate of symptomatic days, on which abdominal pain was present, i.e., end-of-day diary score or at least once daily ESM score of $\geq 2$, was $84 \%$ and $86 \%$, respectively. The compliance rate was $76.8 \%$ for ESM, i.e., of the total number of days on which all study participants used ESM, on $76.8 \%$ of the days at least 6 beep-questionnaires had been completed. Not completed beep-questionnaires were considered as missing values in the analyses.

To compare end-of-day diary to ESM scores, the scales of the two methods were harmonized (i.e., ESM from a 7-point to a 5-point scale). A Cronbach's $\alpha$ was calculated for the original and rescaled ESM data; $\alpha=0.647$ versus 0.648 , respectively, demonstrating that the rescaling did not influence the data strongly.

\section{End-of-day diary compared to ESM scores}

There was a significant association between end-of-day diary scores and corresponding ESM day-mean scores for all GI symptoms analyzed (Table 14.1), demonstrating that both methods measure the same construct, with estimates (indicating the direction and strength of the association) ranging between 0.38 (SE 0.10) and 1.08 (SE 0.06), $P<0.001$.

In order to calculate possible differences in scores between both methods, the delta score was tested using the same multi-level analysis (Table 14.2). The estimate of the intercept represents the difference between the scores of the two methods. On a 1-to-5-point scale, abdominal pain and flatulence scores were 0.42 (SE 0.11) and 0.41 (SE 0.06) point higher, respectively $(P<0.001)$, for the end-of-day diary compared to the corresponding ESM scores. Small, though statistically significant differences were also observed for nausea (0.07 (SE 0.03), $P=0.032$ ), and belching (0.13 (SE 0.03), $P<0.001$ ), but the difference was not significant for bloating. 
Table 14.1 Association between end-of-day diary scores and corresponding ESM day-mean scores, using linear mixed models (random intercept and AR1 covariate structure) and corrected for repeated measures: the dependent variable is the end-of-day diary score and the predictor is the corresponding ESM item. Estimate indicates the direction and strength of the association.

\begin{tabular}{lcccc}
\hline Gl symptom & Estimate & Std. Error & $P$-value & $95 \% \mathrm{Cl}$ \\
\hline Abdominal pain & 0.38 & 0.10 & $<0.001$ & $0.19 ; 0.59$ \\
Nausea & 0.93 & 0.07 & $<0.001$ & $0.80 ; 1.07$ \\
Belching & 1.08 & 0.06 & $<0.001$ & $0.95 ; 1.20$ \\
Bloating & 0.75 & 0.08 & $<0.001$ & $0.59 ; 0.90$ \\
Flatulence & 0.49 & 0.08 & $<0.001$ & $0.33 ; 0.66$
\end{tabular}

Table 14.2 Difference between end-of-day diary scores and corresponding ESM scores, i.e. the delta score (as dependent), tested using linear mixed models (random intercept and AR1 covariate structure) and corrected for repeated measures. Estimate represents the difference between the scores of the two methods on a 1-to-5-point scale. A positive estimate indicates a higher score in the end-of-day diary when compared to ESM.

\begin{tabular}{lcccc}
\hline Gl symptom delta score & Estimate intercept & Std. Error & $P$-value & $95 \% \mathrm{Cl}$ \\
\hline Abdominal pain & 0.42 & 0.11 & $<0.001$ & $0.19 ; 0.65$ \\
Nausea & 0.07 & 0.03 & 0.032 & $0.01 ; 0.13$ \\
Belching & 0.13 & 0.03 & $<0.001$ & $0.06 ; 0.20$ \\
Bloating & 0.09 & 0.06 & 0.113 & $-0.02 ; 0.20$ \\
Flatulence & 0.41 & 0.06 & $<0.001$ & $0.30 ; 0.54$ \\
\hline
\end{tabular}

In contrast, ESM day-maximum scores did not differ when compared to paper endof-day diary scores for abdominal pain (Figure 14.1), but were significantly higher for bloating (Figure 14.2), nausea, belching and flatulence (see Appendix).

\section{Predictors of differences in abdominal pain reporting between ESM and end-of-day diary}

To identify possible predictors for the difference in abdominal pain reporting between the two methods, delta scores were used as dependent variable in the multilevel analysis. Only the interaction between the presence of panic disorder and male gender resulted in a statistically significant larger difference between end-of-day diary and the corresponding ESM pain score (estimate 1.88 (SE 0.87), $P=0.041$ ). When taking into account multiple testing, this result was no longer statistically significant. No significant effect could be demonstrated for age, presence of panic disorder in general, higher anxiety and depression scores (HADS), and negative cognitions (CS-FBD).

However, within each method (i.e., ESM and end-of-day diary), IBS patients with panic disorder reported higher abdominal pain scores compared to those without; mean difference between groups was $1.1 \pm 0.2$ point $(P<0.01)$ on a 1 -to-7-point scale in ESM, and $0.8 \pm 0.4$ point $(P<0.01)$ on a 1 -to-5-point scale in the end-of-day diary. This was also found for bloating (ESM: $1.6 \pm 0.2, P<0.01$, and end-of-day diary: $1.2 \pm 0.2, P<0.01$ ), while 
there were no significant differences for nausea, belching and flatulence between scores of patients with and without the diagnosis of panic disorder.

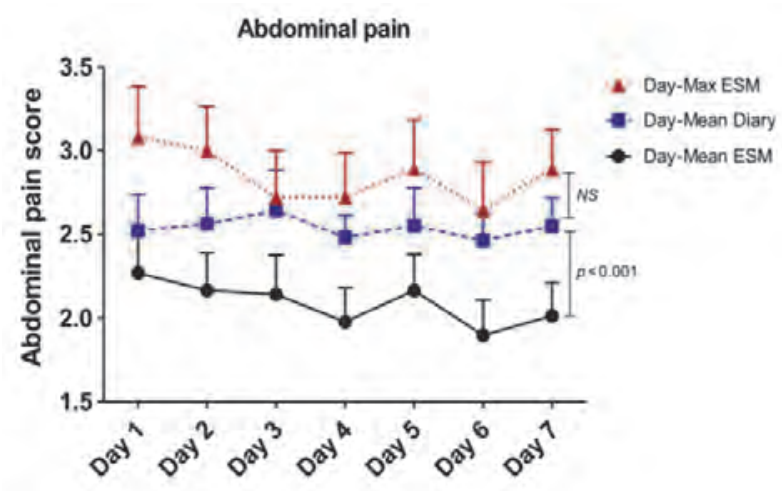

Figure 14.1 End-of-day diary scores compared to ESM day-mean and day-maximum sores, for abdominal pain (on a 1-to-5 point scale). Differences tested for both ESM day-mean and ESM day-max scores vs. end-of-diary scores, using linear mixed models.

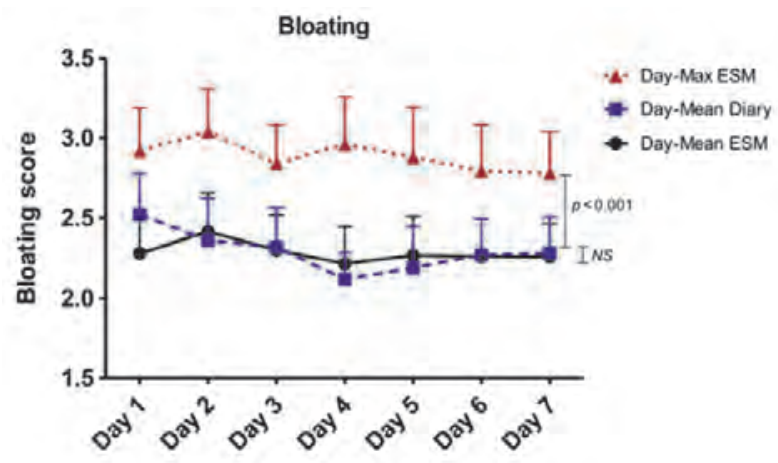

Figure 14.2 End-of-day diary scores compared to ESM day-mean and day-maximum scores, for bloating (on a 1-to-5 point scale). Differences tested for both ESM day-max scores vs. end-to-diary scores, using linear mixed models.

\section{GSRS compared to corresponding ESM scores}

Symptom scores of the GSRS, reflecting a one-week period, were also compared to corresponding ESM 7-day-mean scores per subject. For all symptoms (i.e., upper abdominal pain, nausea, heartburn, bloating and flatulence), GSRS scores were significantly higher compared to ESM scores $(P<0.01)$, with the most pronounced difference noted for pain and flatulence (Table 14.3). 
Table 14.3 GSRS scores compared to corresponding ESM symptom scores. Means calculated on subject level, SD indicates variation between subjects. Scores were not rescaled (original 1-to-7-point scale in GSRS and ESM). ${ }^{* *} P<0.01, * * * P<0.001$

\begin{tabular}{l|c|c}
\hline Scale & GSRS & ESM \\
\hline Gl symptom (mean \pm SD) & $\begin{array}{c}\text { IBS patients } \\
(\mathrm{N}=26)\end{array}$ & $(\mathrm{N}=26)$ \\
\hline 7 point scale: & & $2.44 \pm 1.30^{* * *}$ \\
Upper abdominal pain & $4.77 \pm 1.50$ & $1.11 \pm 0.28^{* * *}$ \\
Heartburn & $2.54 \pm 1.61$ & $1.45 \pm 0.88^{* *}$ \\
Nausea & $2.69 \pm 1.69$ & $2.94 \pm 1.67^{* *}$ \\
Bloating & $4.35 \pm 1.81$ & $2.13 \pm 1.38^{* * *}$ \\
\hline
\end{tabular}

\section{Discussion}

The current study demonstrates that retrospective symptom measurement by end-ofday diaries and an end-of-week questionnaire (GSRS), leads to higher scores when compared to electronic momentary symptom assessment (i.e., by ESM), in patients with IBS. This difference is most pronounced for abdominal pain and flatulence. Abdominal pain scores in the end-of-day dairies were similar to maximum pain reporting in ESM, indicating that subjects tend to report peak pain rather than average pain scores of the day in an end-of-day diary. This was not found for the other tested GI symptoms. With regard to GSRS versus ESM, in addition to abdominal pain and flatulence, also nausea, heartburn and bloating scores were higher in the retrospective method compared to momentary assessment. Furthermore, affective complaints in general did not lead to a larger difference in abdominal pain reporting between the end-of-day diary and ESM. However, within each method, IBS patients with panic disorder reported higher abdominal pain and bloating scores compared to those without.

The reporting of peak rather than average abdominal pain has been described previously by Weinland et al. for IBS patients ${ }^{16}$, and by Redelmeier et al. studying abdominal pain of other origin ${ }^{26}$, i.e., colonoscopy and lithotripsy associated pain. The observed patient preferences of peak pain reporting provide more understanding with regard to pain memory and perceived relevance in IBS patients. Interestingly, peak reporting was not found for nausea, bloating, belching and flatulence. The current findings may imply that pain is more prone to recall bias than other GI symptoms in IBS and may also be related to a considerable affective component in pain perception. However, it should be noted that some symptoms, especially nausea and belching, were less prevalent, and consequently the present study may lack power to identify potential differences between the methods for these symptoms. Accordingly, it might be interesting to assess a symptom as nausea in a population of functional dyspepsia 
patients in order to investigate whether a larger difference between ESM and end-ofday diaries can be observed.

When comparing ESM scores to retrospective end-of-week GSRS scores, even larger differences were found in comparison to ESM versus end-of-day diary scores, indicating that questionnaires with a longer recall period are associated with higher symptom scores in IBS patients. This is in line with previous research describing differences between end-of-day and end-of-week symptoms assessment in IBS ${ }^{4}$. When analyzing ESM data in more detail, GI symptoms in IBS patients were found to fluctuate considerably during the day and during the week. The 7-day mean of ESM data is an average of days with higher and lower symptom burden. Subjects are prone to base their scores on symptomatic days when filling in the GSRS, equivalent to the peak-pain tendency in the end-of-day diaries. This tendency may underlie the recall bias in retrospective methods. The differences between ESM and GSRS were tested parametrically on subject-level. However, non-parametric testing did not lead to different outcomes. The standard deviations of GSRS and ESM 7-day mean scores, indicating variation between subjects, in this analysis were broad, but differences between methods were highly significant.

Prevalent psychological factors in IBS, in particular anxiety, have been reported to affect pain processing, memory and interpretation ${ }^{11}$. To investigate how anxiety may influence the difference between the retrospective (based on memory) and momentary assessment of abdominal pain, we included IBS patients with and without a diagnosis of comorbid panic disorder in the current study. Although, the overall scores for abdominal pain were higher in patients with compared to the group without panic disorder, only the presence of panic disorder in male subjects was found to be a significant predictor of a higher pain score difference between ESM and end-of-day dairy. However, this predictor was no longer significant when corrected for multiple testing and should therefore be interpreted carefully. Furthermore, given the higher abdominal pain scores in IBS patients with panic disorder compared to those without, anxiety should be seen as a stable trait factor, which may be less dependent from momentarily fluctuating contextual influences in IBS. It has to be acknowledged that in the current study the IBS patients with panic disorder were overrepresented relative to the general IBS population. Therefore our data need to be replicated by future studies in large nonselected IBS cohorts.

In addition to higher overall scores and peak-symptom reporting in paper end-ofday diaries, this method is also compromised by high fake compliance, as demonstrates previously by Stone et al. ${ }^{12}$. Therefore, serious concern has risen with respect to the validity of retrospective paper end-of-day diaries. Fake compliance is excluded in ESM, since subjects can only respond within 10 minutes after the beep, and moreover, actual compliance can be monitored. We considered a subject to be compliant on a specific day if at least 6 out of 10 beep-questionnaires had been completed and found a good compliance rate for ESM, i.e., $76.8 \%$ for the total study population. 
Certain limitations of the present study should be mentioned. In the current study we have not assessed differences in reporting of consistency and frequency of defecation between the symptom assessment methods, while disordered defecation is a key symptom of IBS. Furthermore, the use of ESM may lead to higher patient burden compared to end-of-day paper diaries. This may lead to selection bias, because patient may decide not to participate beforehand. The number of questions asked was high and should be reduced in future investigations. The time needed to complete a beepquestionnaire should be less than 2 minutes. Questions used in ESM were selected based on available questionnaires, such as the end-of-day diary ${ }^{2,3,22}$ and the GSRS $^{25,27}$, and were previously not psychometrically tested for momentary symptom assessment. Therefore, future studies evaluating the potential of ESM for symptom assessment in IBS should study psychometric properties, regarding validity and reliability of the instrument, which were not subject to analysis in this preliminary study. It has to be acknowledged that for the comparison of ESM and end-of-day diaries, it was necessary to rescale the ESM data from a 7 to a 5-point scale, which could cause a distortion of the results. However, the Cronbach's $\alpha$ for ESM GI symptom scores did not change after rescaling, and moreover, comparable results were found by comparing ESM (nonrescaled data) with GSRS scores. Finally, the exploratory aim of the current study has resulted in a small sample size. However, this has not hampered the multilevel analyses, which are based on repeated measures.

Our results demonstrate certain advantages of ESM as a new digital symptom assessment tool in IBS patients, regardless of the presence of psychological complaints. Nevertheless, further research is needed to optimize the content and number of questions for the assessment of IBS symptoms and to validate the tool as well as the selected questions within the target population, before ESM can be implemented in medical science and clinical practice.

Because of the repeated measures, ESM offers the advantage to study symptom fluctuation during the day and to investigate possible triggers of pain or other symptoms in future investigations, including for example ecological and psychosocial factors as well as linkage to bowel habits. This insight may lead to improvement of therapies. Previously has been found that IBS symptom episodes on average last several hours ${ }^{16}$, which indicates that repeated measures over the day by ESM are suitable to capture the symptom variability in these patients. All patients in the current study had a debriefing interview with the researcher, and acknowledged that the number of repeated measures was sufficient to adequately measure the Gl symptom variation over the day. Furthermore, ESM could be used for more detailed evaluation of treatment response or for intervention purposes in behavioral therapies, in the context of personalized medicine, by giving IBS patients specific feedback with regard to triggers of symptoms identified by ESM. This has recently successfully been applied in a group of individuals with depressive disroder ${ }^{28}$. 


\section{Conclusion}

Irritable bowel syndrome patients report higher abdominal pain and flatulence scores in end-of-day diaries when compared to day-average scores of momentary symptom assessment by ESM, with a preference of peak-pain reporting at the end of the day. In retrospective symptom questionnaires that cover a 1-week period, the difference of symptom reporting when compared to electronic ESM is even higher for abdominal pain and the other GI symptoms. Affective comorbidity in IBS leads to higher abdominal pain and bloating scores compared to IBS patients without, but did not strongly influence differences in abdominal pain reporting between tested symptom assessment methods. 


\section{Appendix}

Table S14.1 End-of-day diary scores on 7 days with and 7 days without ESM, differences tested using paired samples t-test.

\begin{tabular}{l|ccc}
\hline \multicolumn{4}{c}{ End-of-day Gl symptom diary } \\
\hline Gl symptoms (mean \pm SD) & Days with ESM & Days without ESM & $P$-value \\
\hline Abdominal pain & $2.53 \pm 1.01$ & $2.43 \pm 1.03$ & NS \\
Discomfort & $2.54 \pm 1.01$ & $2.47 \pm 0.97$ & NS \\
Nausea & $1.31 \pm 0.76$ & $1.41 \pm 0.82$ & NS \\
Bloating & $2.35 \pm 1.20$ & $2.42 \pm 1.18$ & NS \\
Belching & $1.41 \pm 0.89$ & $1.44 \pm 0.88$ & NS \\
Flatulence & $2.16 \pm 0.99$ & $2.40 \pm 1.16$ & NS \\
Constipation & $1.58 \pm 1.02$ & $1.48 \pm 0.83$ & NS \\
Diarrhoea & $1.27 \pm 0.70$ & $1.36 \pm 0.82$ & NS \\
\hline
\end{tabular}

A

Flatulence

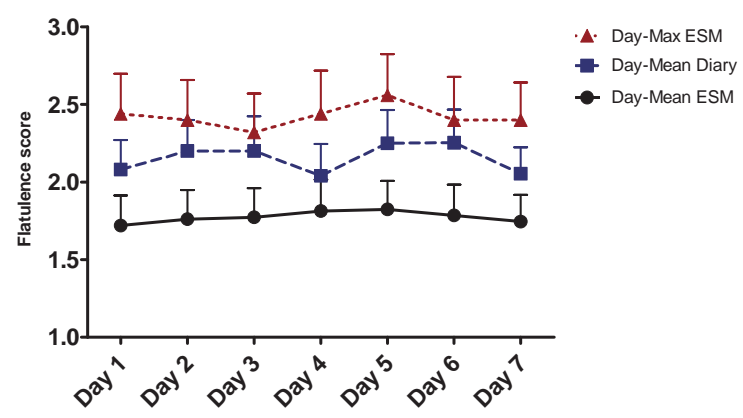

B

Nausea

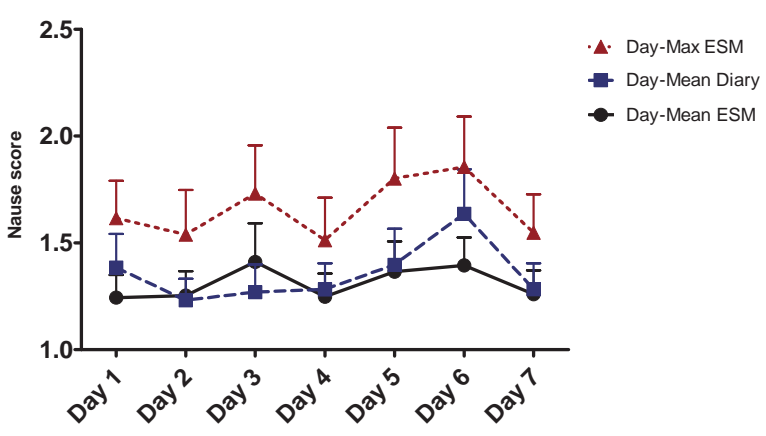


C

\section{Belching}

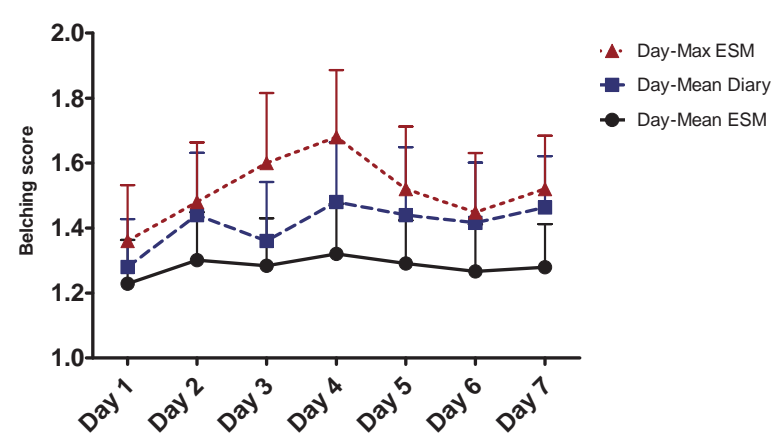

Figures S14 End-of-day diary scores compared to ESM day-mean and day-maximum scores, for flatulence (Figure A) nausea (Figure B) and belching (Figure C). Differences tested for both ESM day-mean and ESM day-max scores vs. end-of-diary scores, using linear mixed models. 


\section{References}

1. Spiegel, B. et al. Measuring irritable bowel syndrome patient-reported outcomes with an abdominal pain numeric rating scale. Aliment. Pharmacol. Ther. 30, 1159-1170 (2009).

2. Thijssen, A. Y. et al. Dysfunctional cognitions, anxiety and depression in irritable bowel syndrome. J. Clin. Gastroenterol. 44, e236-241 (2010).

3. Ludidi, S. et al. Markers for visceral hypersensitivity in patients with irritable bowel syndrome. Neurogastroenterol. Motil. 26, 1104-1111 (2014).

4. Lackner, J. M. et al. The accuracy of patient-reported measures for Gl symptoms: A comparison of real time and retrospective reports. Neurogastroenterol. Motil. 26, 1802-1811 (2014).

5. U.S. Department of Health and Human Services FaDA, Center for Drug Evaluation and Research (CDER). Guidance for Industry Irritable Bowel Syndrome - Clinical Evaluation of Drugs for Treatment (2012). http://wwwfdagov/downloads/Drugs/Guidances/UCM205269pdf.

6. Bradburn, N. M. et al. Answering autobiographical questions: the impact of memory and inference on surveys. Science 236,157-161 (1987).

7. Bower, G. H. Mood and memory. Am. Psychol. 36,129-148 (1981).

8. Babel P. et al. The effect of the type of pain on the accuracy of memory of pain and affect. Eur. J. Pain. 19, 358-368 (2014).

9. Bolger, N. et al. Diary methods: Capturing life as it is lived. Annu. Rev. Psychol. 54, 579-616 (2003).

10. Grzesiak M. et al. The lifetime prevalence of anxiety disorders among patients with irritable bowel syndrome. Adv. Clin. Ex. Med. 23, 987-992 (2014).

11. Gorczyca, R. et al. Psychological aspects of pain. Ann. Agric. Enviro Med. Spec no.1, 23-27 (2013).

12. Stone, A. A. et al. Patient compliance with paper and electronic diaries. Control. Clin. Trials 24, 182-199 (2003).

13. Csikszentmihalyi, M. \& Larson, R. Validity and reliability of the Experience-Sampling Method. J. Nerv. Ment. Dis. 175, 526-536 (1987).

14. Stone, A. \& Shiffman, S. Ecological Momentary Assessment (EMA) in behavioral medicine. Ann. Behav. Med. 16, 199-202 (1994).

15. Myin-Germeys, I. et al. Experience sampling research in psychopathology: Opening the black box of daily life. Psychol. Med. 39, 1533-1547 (2009).

16. Weinland, S. R. et al. Characterization of episodes of irritable bowel syndrome using ecological momentary assessment. Am. J. Gastroenterol. 106, 1813-1820 (2011).

17. Longstreth, G. F. et al. Functional bowel disorders. Gastroenterology 130, 1480-1491 (2006).

18. American Psychiatric Association. Diagnostic and statistical manual of mental disorders (DSM-IV-TR). 4th. ed. text rev. (2000).

19. Zigmond, A. S. \& Snaith, R. P. The hospital anxiety and depression scale. Acta Psychiatr Scand. 67, 361370 (1983).

20. Bjelland, I. et al. The validity of the Hospital Anxiety and Depression Scale. An updated literature review. J. Psychosom. Res. 52, 69-77 (2002).

21. Toner, B. B. et al. The development of a cognitive scale for functional bowel disorders. Psychosom. Med. 60, 492-497 (1998).

22. Mujagic, Z. et al. Small intestinal permeability is increased in diarrhoea predominant IBS, while alterations in gastroduodenal permeability in all IBS subtypes are largely attributable to confounders. Alimen. Pharmacol. Ther. 40, 288-297 (2014).

23. Myin-Germeys, I. et al. From environment to therapy in psychosis: A real-world momentary assessment approach. Schizophr. Bull. 37,244-247 (2011).

24. Courvoisier, D. S. et al. Compliance to a cell phone-based ecological momentary assessment study: The effect of time and personality characteristics. Psychol. Assess. 24, 713-720 (2012).

25. Revicki, D. A. et al. Reliability and validity of the Gastrointestinal Symptom Rating Scale in patients with gastroesophageal reflux disease. Qual. Life Res. 7, 75-83 (1998).

26. Redelmeier, D. A. \& Kahneman, D. Patients' memories of painful medical treatments: Real-time and retrospective evaluations of two minimally invasive procedures. Pain 66, 3-8 (1996). 
27. Svedlund, J. et al. GSRS--a clinical rating scale for gastrointestinal symptoms in patients with irritable bowel syndrome and peptic ulcer disease. Dig. Dis, Sci. 33, 129-134 (1988).

28. Kramer, I. et al. A therapeutic application of the experience sampling method in the treatment of depression: A randomized controlled trial. World Psychiatry 13, 68-77 (2014). 



\section{Abstract}

\section{Background}

Routine Outcome Monitoring (ROM) should provide a dynamic, within-treatment forward feedback loop to guide individual treatment decisions across diagnostic categories. It has been suggested that the Experience Sampling Method (ESM), capturing the film of daily life adaptive processes, offers a flexible, personalised and transdiagnostic feedback system for monitoring and adapting treatment strategies. This is the first study that uses an ESM application (the PsyMate ${ }^{\mathrm{TM}}$ ) as a routine mobile-ROM (mROM) tool in an ambulatory mental health setting.

\section{Objective}

To demonstrate adequate psychometric properties of the PsyMate ${ }^{T M}$ app assessing both symptom severity levels as well as daily life functioning.

\section{Method}

In a transdiagnostic sample of 64 outpatients, an mROM protocol (ESM for 6 days, at 10 semi-random moments a day) and a standard ROM instrument (HADS) were administered at baseline and at three-month follow-up. We measured positive affect (PA), negative affect (NA), quality of sleep, positive social interaction, activity-related stress, tiredness, and feeling unwell.

\section{Results}

Subjects completed $53 \%$ of the measurements at baseline $(N=64)$ and $48 \%$ at follow-up $(N=29)$. Factor analysis and subsequent reliability analysis of PA and NA confirmed the two constructs. Significant and meaningful correlations were found between PA, NA and HADS scores (ranging from $r=.4$ to $r=.7$ ). Multilevel analyses yielded significant change scores for all measures.

\section{Conclusion}

The ESM-based, transdiagnostic mROM tool can be used reliably in clinical settings: it shows adequate psychometric properties, as well as concurrent validity and sensitivity to change over time with respect to relevant ROM constructs. Person-tailored items can be added. In addition, mROM offers added value over standard symptom-based ROM, as it provides information on adaptive functioning in the daily environment of patients. 


\section{Introduction}

Routine Outcome Monitoring (ROM) in mental health care refers to the process of collecting clinical data, which can serve as feedback to guide informed treatment decisions ${ }^{1}$. ROM should be transdiagnostic, flexible and follow the flow of the clinical process. Furthermore, it must be adjusted to the needs and wishes of individual patients $^{2}$. Given these requirements, there is considerable debate as to what degree clinical ROM measures in mental health care can serve as input to benchmark outcomes across clinicians and institutions - a process referred to as Benchmark- $\mathrm{ROM}^{3}$. Benchmark-ROM requires rigid standardization, both in terms of instruments and data collection, to allow cross-patient and cross-centre comparisons. The implementation of rigid benchmark-ROM negatively influences clinical ROM, which requires a more flexible approach $^{4-6}$. Clinical ROM requires repeated assessments at specific time points over successive diagnostic and treatment phases. Although there is no agreed measure for ROM in mental health care, many ROM scales have been developed for use in clinical practice $^{7-9}$. In the literature, ROM is often referred to as patient-reported outcome measures (PROM), stressing the need for unbiased assessments of outcome collected by the patient rather than the treating clinician ${ }^{10,11}$. ROM instruments should be sensitive to change over time. Selected scales can be generic and, therefore, relevant across populations, or, in contrast, target specific patient groups. Moreover, ROM instruments should map several outcome domains, including psychopathology, daily life functioning, personal recovery, and quality of life ${ }^{3,12}$. Unfortunately, current ROM implementations mainly focus on psychopathology ${ }^{13}$, disregarding the other outcome domains. In addition, measures of psychopathology, expressed as total scores from traditional technical scales, do not reflect outcomes that are important to patients ${ }^{14,15}$. Given that ROM is part of clinical routine, instruments should be time efficient. Furthermore, ROM should be set up in such a way that it allows the patient to become the empowered coowner of the process of diagnosis and treatment. Therefore, ROM should be constructed as an informed forward feedback loop: the iterative process that includes clinical decision-making, outcome evaluation, treatment adjustment, and further outcome evaluation.

A wide variety of ROM instruments exists, often developed to assess psychopathology in specific patient populations (e.g., mood disorders and psychosis) using traditional rating scales that lack relevance to the daily life of patients. Comprehensive assessment batteries that incorporate all different outcome domains are cumbersome and time consuming. Inclusion of clinicians (or trained personnel) as assessors dramatically adds to the complexity and cost of data collection logistics and potentially introduces bias. In many cases, a trade-off has to be made between brevity and completeness ${ }^{7}$. Finally, ROM assessments often rely on retrospective assessments, which are vulnerable to recall bias and limit sensitive assessment of change ${ }^{16}$. Most individuals are poor assessors when asked retrospectively to assess emotional 
experiences $^{17}$. The most essential problem with traditional ROM assessments is that they only marginally inform adequate treatment selection as they do not inform on personal goals in real life settings and are not set up in such a way that a constructive forward feedback loop arises. For customized treatment selection, symptom variability and contextual variation is relevant. Mental health problems and related disabilities occur in the flow of daily life. Effective treatment should address the issue that patients are most vulnerable and consider the coping strategies available within their daily routines. Traditional rating scales, completed in a clinical setting, are often not representative of daily life functional adaption. A successful recovery process assumes functional adaptation and increasing resilience against mental illness. Resilience factors, such as experience of positive mood states and the strength of social connections, are increasingly being recognized and used in mental health treatment ${ }^{18}$.

Thus, ROM measurements should target contextual factors and positive mood states in addition to negative mood states and symptomatology, rather than being exclusively focused on negative symptomatic states. Experience sampling techniques take into account all these factors. The Experience Sampling Method (ESM), also referred to as Ecological Momentary Assessment (EMA), is an umbrella term for several ambulatory assessment strategies that randomly sample mental state in the context, following the flow of daily life. ESM is a structured diary technique, in which patients are asked to fill in short questionnaires in response to auditory cues (beep signals), at semirandom moments during the day, for a number of consecutive days. Patient assessments include momentary emotional experiences (e.g., positive and negative mood), symptoms, cognition, context information (e.g., activity, company and location), and an appraisal of the context. Because ESM is a repeated assessment strategy, additional constructs, such as stress-sensitivity or coping strategies, can be quantified ${ }^{18}$. ESM was proposed as a comprehensive, generic ROM instrument and has several advantages over typical ROM assessments ${ }^{3,18,19}$. First, ESM increases ecological validity, because reports are provided in patients' natural environment. Second, memory bias is minimized because patients report in the moment. Third, ESM is contextualized, allowing for a better understanding of the person-environment interactions that give rise to psychopathology. Being aware of environmental reactivity and pattern changes over time will aid the understanding of vulnerability, resilience and treatment progress ${ }^{18,20}$. Fourth, ESM is highly relevant to the individual and person-tailored when used as a clinical tool, thus creating transparency and facilitating patient engagement when discussing the data with the clinician. Last, ESM facilitates and enhances shared decision making processes, because patients are actively participating in the data collection, and data can be used to improve treatment efficiency. Study results show that ESM during treatment can enhance feelings of empowerment and the ability for self-management ${ }^{21}$. Across the psychopathology spectrum, ESM has been proven feasible, and can be successfully applied, in combination with feedback, as a treatment method for 
depression $^{22}$. Moreover, ESM has been found valuable for a range of uses, including ROM in clinical practice ${ }^{23}$.

Van Os and colleagues studied the use of ESM as a mobile ROM (mROM) tool. They assumed that ESM data was sensitive to change over time, using several clinically relevant parameters. Observational analyses were performed using data from the treatment arm of a randomized control trial in patients diagnosed with major depressive disorder ${ }^{24-26}$. The results confirmed the assumption, with the greatest sensitivity to change found for measures of positive adjustment, positive affect, and increases of positive affect due to natural rewards in daily life. These results show that resilience factors, such as positive affect, are informative for outcome assessment. Positive mood states are relevant for treatment outcome across mental disorders ${ }^{27,28}$ and can be seen as a generic trans-diagnostic indicator of underlying resilience that can be captured in ROM. Research showed that positive affect and negative affect are related but different dimensions, i.e., they are not the extremes of a single continuum. Positive affect is nonheritable and an indicator of resilience, whereas negative affect is highly heritable and an indicator of vulnerability ${ }^{29}$. As both dimensions are important in the clinical context, both should be captured in ROM. With the advent of modern mHealth applications for use on mobile devices, such as smartphones, ESM can now be carried out cheaply and routinely ${ }^{23}$. We refer to $\mathrm{mHealth}$ ESM for the purpose of ROM as mROM. There are multiple ESM mobile applications available (e.g., https://www.lifedatacorp.com/, http://experiencesampler.com/, https://pielsurvey.org/) Here, we present the first study that uses a mobile devise to implement mROM in an ambulatory hospital psychiatry setting, using an open trial design with PsyMate ${ }^{\mathrm{TM}}$, one of these ESM mobile applications (www.psymate.eu).

\section{Objective}

The aims of this study were twofold. First, to demonstrate transdiagnostically adequate psychometric properties of routine mROM with a PsyMate ${ }^{\mathrm{TM}}$ moderated mROM application, and second, to investigate sensitivity to change during treatment. Based on previous work, it was expected that (i) the psychometric properties of the PsyMate ${ }^{\mathrm{TM}}$ method would be adequate and suitable for therapeutic monitoring; and (ii) change over time could be demonstrated for a series of variables (e.g., mood, activity-related stress, and sleep quality) indexing both psychopathology and daily life functioning. In this regard, we expected to find a weak to moderate association between a traditional rating scale (the Hospital Anxiety and Depression Scale ${ }^{30}$ ) and the mROM mood measures, leaving sufficient room for the added value of mROM. 


\section{Methods}

\section{Sample}

Patients were recruited at the outpatient mental health service of the Maastricht University Medical Centre (MUMC). In total, 115 consecutively attending patients were asked to participate in routine mROM, of whom 75 provided their consent. All patients were 18 years or older and capacity for consent was established by the psychiatrist providing care to the patient. Exclusion criteria for inclusion in the analysis were not being able to read Dutch or not being able to handle a mobile device with the PsyMate ${ }^{\mathrm{TM}}$ app.

\section{Procedures}

\section{Analysis design}

mROM with the PsyMate ${ }^{\mathrm{TM}}$ app is applied routinely in MUMC. There were two measurement periods, one at baseline $(t=0)$, the second at follow-up approximately three months later $(t=1)$. Due to logistical reasons, the second visit did not always take place exactly 3 months later ( $M=111.59$ days later, $S D=27.3$, range 80-189 days). Each measurement consisted of six consecutive ESM sampling days and the administration of a traditional ROM questionnaire at the beginning of the ESM sampling days.

\section{Briefing}

During the baseline session, patients were helped while downloading the Psymate ${ }^{\mathrm{TM}}$ application on their smartphone via the App Store or Google play store. A specific code was required to enter the study protocol. However, a free demo app is available in different languages, for those wishing to implement the app in routine clinical practice. In case a patient could not use his own device, an iPod was provided for the duration of the $\mathrm{mROM}$ period. A clinician explained the PsyMate ${ }^{\mathrm{TM}}$ procedure during a briefing session and coached patients through the PsyMate ${ }^{\text {TM }}$ items. Patients were instructed to continue their normal routine during the Psymate ${ }^{\mathrm{TM}}$ data collection period. After the briefing, a sampling period of six days started, not including the briefing day on which the Psymate ${ }^{\mathrm{TM}}$ protocol was activated.

The standing medical ethical committee approved the anonymous use of routine clinical data, if patients provided informed consent. Thus, all patients provided informed consent to use routine clinical data for the purpose of scientific investigation. 


\section{mROM}

For the purpose of $\mathrm{mROM}$, the Psymate $^{\mathrm{TM}}$ application was programmed to emit 10 random beeps each day. Signals notify when a short questionnaire has to be completed (lasting approximately 1 minute). Beeps were semi-randomised in ten blocks of 90 minutes, between 7:30 AM and 10:30 PM. The questionnaire consisted of 13 mood items, 5 context items (what, where, and with whom the patient was spending time and whether they were enjoying it), 5 items about important events happening since the last beep, 1 item questioning specific somatic complaints, 5 items assessing patients' physical condition, and 1 item assessing levels of beep disturbance. In addition to the beep questionnaire, patients filled in some extra questions at the beginning and the end of each day. The morning questions were related to the quality of sleep of the night before, while the questions in the evening required the patient to give an estimate of their average mood and somatic complaints over the past day. Most items were presented on a 7-point Likert scale, ranging from 1 (not at all) to 7 (very).

In order to assess change over time, items were clustered to form measurable constructs. Previous work showed positive and negative mood items reliably form a Positive Affect (PA) and a Negative Affect (NA) cluster $^{31}$. In this analysis, PA consisted of the items: I feel cheerful, satisfied, relaxed and globally feeling well. The remaining nine mood items (I feel lonely, guilty, worried, down, threatened, insecure, irritated, frightened, and suspicious) formed the NA scale. The quality of sleep was assessed using the (ordinal-coded) items: (1) time needed to fall asleep, (2) number of times the person woke up during the night, (3) the time lying awake before getting up, (4) whether the person felt rested, and (5) how the person globally felt about their sleep quality last night.

Approximately three months after the initial visit, patients were asked to participate again in the second part of the PsyMate ${ }^{\mathrm{TM}}$ mROM procedure.

\section{Debriefing}

After each sampling period, a debriefing session was scheduled. Patients were asked whether the past week was representative for their daily life and whether the Psymate ${ }^{\mathrm{Tm}}$ interfered with their thoughts, feelings, activities, or social contacts. Furthermore, patients were asked whether unusual incidents occurred, and to what degree the use of Psymate $^{\mathrm{TM}}$ was bothersome.

\section{HADS}

The Hospital Anxiety and Depression Scale (HADS) ${ }^{30}$ is a reliable and validated rating scale $^{32,33}$ which is often used for mental health ROM purposes worldwide, particularly in somatic hospital settings ${ }^{34}$. The HADS consists of fourteen items (scaled from 0 to 3 ) assessing aspects of anxiety and depression experienced during the last week. The HADS was administered digitally (via the Psymate ${ }^{\mathrm{TM}}$ ) at the end of the briefing session. 


\section{Statistical analyses}

Analyses were performed to investigate the structure of the 13 mood items. First, a principal component analysis (PCA) was conducted on these items with orthogonal rotation (varimax), requiring two factors to be extracted. Second, a reliability analysis (i.e., Cronbach's alpha) was performed on each factor to determine the internal consistency of each scale.

The data collected with ESM have a multilevel structure - successive beeps (level 1) are nested within patients (level 2). Multilevel regression analyses took the variability of both levels into account. With respect to the analyses of change, there were 7 a priori dependent variables: NA, PA, quality of sleep, preferring to do something else (activityrelated stress), enjoying company (social stress), feeling unwell, and feeling tired. The following items were incorporated as covariates in the multilevel models: age, sex, a dichotomous variable indicating whether a patient participated in one or both measurement periods, and DSM-IV-TR diagnosis prior to participation (i.e., a dichotomous variable indicating whether or not a patient had been previously diagnosed with a mental disorder (e.g., depressive disorder, panic disorder, somatoform disorder, no diagnosis, etc.) $)^{35}$. Additionally, when examining the variable feeling unwell, a dichotomous variable describing the presence or absence of somatic complaints was taken into account and added as a covariate in the statistical model. The predictor variable of main interest was measurement period, indicating a possible change over time.

Analyses were carried out using SPSS Statistics version 23.0. Two-sided $P$-values $<.05$ were considered significant. All dependent variables were checked for approximately normal distribution before performing further analyses.

\section{Results}

\section{Sample}

In the period from February 2015 until May 2016, 75 patients at the MUMC outpatient mental health service fulfilled criteria for inclusion in the analysis. In case a patient completed less than ten beeps during a measurement period (either baseline or followup), the measurement period was excluded from further analysis. There were 11 patients who did not complete sufficient beeps during both measurement periods, leading to a final dataset of 64 patients. Thirty-six patients completed one measurement period, 28 patients completed both measurement periods. A logistic regression model examined whether these two groups differed with respect to age, sex and being diagnosed (yes or no). This was not the case for either the omnibus model ( $2 \mathrm{LL}=78.9$; $\mathrm{df}=3 ; P=.18$ ) or for the univariate tests (all $P$-values $>.075$ ). 
The average age of the $N=64$ analysable group was 48.7 years ( $S D=13.9$, range 18-73). There were 39 women and 25 men. All but 11 patients received a clinical diagnosis by the treating psychiatrist, based on DSM-IV-TR criteria. Diagnoses included depression $(N=25)$, panic disorder $(N=18)$, somatoform disorder $(N=4)$, posttraumatic stress disorder $(N=2)$, bipolar disorder $(N=1)$, anxiety disorder not otherwise specified $(N=1)$, dysthymia $(N=1)$ and psychotic disorder $(N=1)$.

\section{App statistics}

The complete protocol presented 120 beeps per patient ( 6 days $\times 10$ beeps $\times 2$ measurement periods). At baseline, the response percentage was $52.7 \%$ of 3780 presented beeps, comparable to the $48.2 \%$ of 1740 presented beeps at follow-up.

In October 2015, an update of the PsyMate ${ }^{\mathrm{TM}}$ app was introduced. Since then, the number of errors concerning the internet connection considerably reduced. The proportion of valid beeps with no missing items within the beep increased substantially after this update: from $71.1 \%$ to $86.2 \%$.

\section{Compliance}

There was no suggestion of relevant differences in response rates between the days of the week at either baseline or three-month follow-up. However, the data suggest that there is a difference in response rate over consecutive days of the ROM protocol (Figure 15.1). During the first day and last day of the protocol, patients tend to complete less beeps than on the remaining days. At both baseline and follow-up, a reduction in response rate over time was apparent. At follow-up, the reduction started two days earlier (day 3), compared to baseline. Regarding the hours of the day, there was a slight increase in response over time, as illustrated in Figure 15.2. The lowest response was in the early hours of the day (7:30 am - 10:30 am).

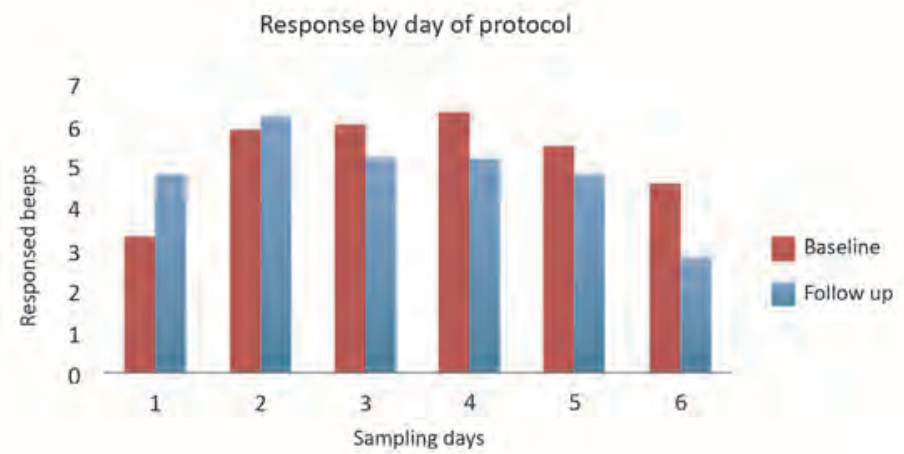

Figure 15.1 This figure shows the average number of beeps that were completed per day per subject, both for the baseline assessment and the three-month follow-up assessment, over the 6 day ESM period. 


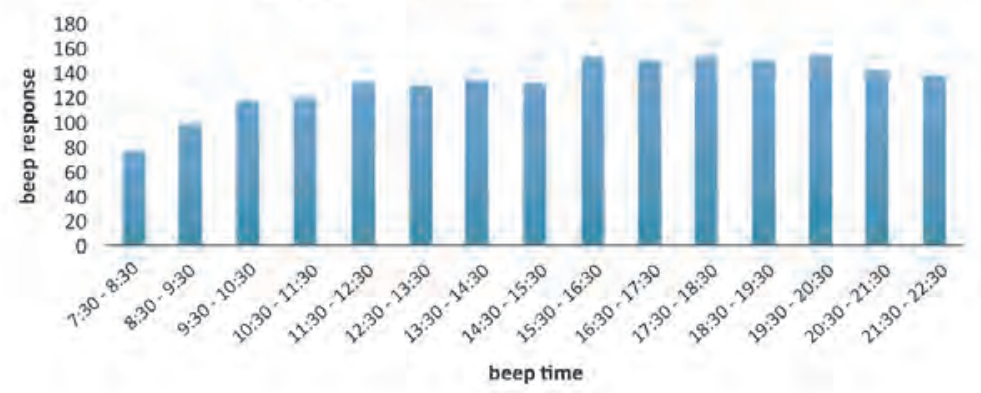

b)Follow up beep response within the day

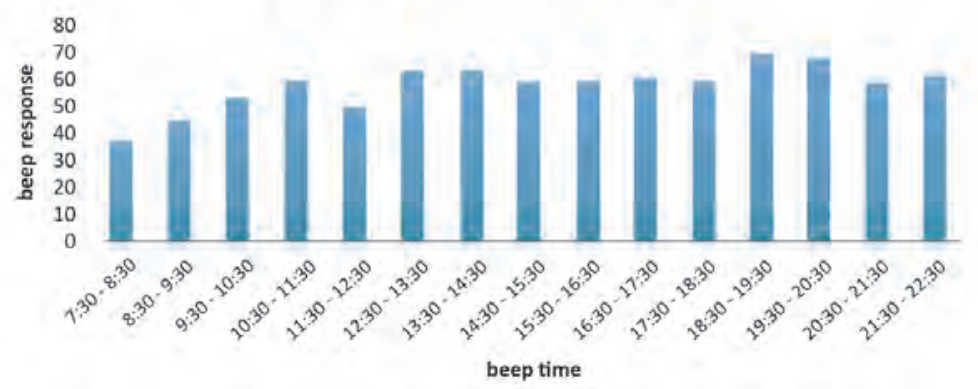

Figure 15.2 This figure shows the average number of responded beeps, per time window, within a day. a) Number of beep responses within the day, derived from baseline ESM data. b) Number of beep responses within the day, derived from the three-month follow-up ESM data.

\section{Factor structure of PA and NA}

The principal component analysis of the 13 PA and NA items resulted in two factors, each having a value larger than 1 (7.1 and 1.5 respectively), with a cumulative percentage of explained variance of $66.1 \%$. An item was assigned to a factor when the factor loading exceeded .45 with a difference larger than .1 for both factor loadings. Using these criteria, all items could be assigned unambiguously to either the PA or the NA factor. As a next step, a reliability analysis was performed. Cronbach's alphas for factor 1 and 2 (NA and PA) were .91 and .92 respectively.

Consequently, the PA subscale was constructed by computing the sum of the 4 positive affect items, the NA scale by computing the sum of the 9 negative affect items. Sub-scores containing missing items were computed by inserting the mean value of the available items. A maximum of two missing items for PA and 4 items for NA was allowed. PA and NA could not be computed for 41 beeps, leaving an analysable number of beeps (records) of 2832 . 


\section{Validation analyses}

A correlation matrix with the patient-level means of the PA and NA scores (beep-level data) and the patient-level means of HADS total, HADS anxiety, and HADS depression was computed (Table 15.1). The results confirmed the a priori hypothesized relationships between these five variables. Next, an analysis was performed to investigate whether a change in PA and NA over time correlated with a change on the HADS total score. Significant correlations between change scores (baseline versus 3 months) were found: $\triangle P A$ with $\triangle$ HADS $-0.634 p=0.002, \triangle N A$ with $\triangle$ HADS 0.603 $P=0.004$.

Table 15.1 Pearson correlates between positive affect scores, negative affect scores and hospital anxiety and depression scale scores.

\begin{tabular}{|c|c|c|c|c|c|}
\hline Variables & 1 & 2 & 3 & 4 & 5 \\
\hline 1. NA mean & - & & & & \\
\hline 2. PA mean & $-.71 * * *$ & - & & & \\
\hline 3. HADS anxiety & $.71 * * *$ & $-.51 * * *$ & - & & \\
\hline 4. HADS depression & $.56 * * *$ & $-.45 * * *$ & $.66^{* * *}$ & - & \\
\hline 5. HADS total & $.66^{* * *}$ & $-.51 * * *$ & $.89 * * *$ & $.92 * * *$ & - \\
\hline
\end{tabular}

Legend: Correlations between 1 and 2 were performed over $N=63$. All others were performed over $N=59$. NA = Negative affect, $\mathrm{PA}=$ Positive affect, $\mathrm{HADS}=$ Hamilton Anxiety and Depression Scale. NA and PA represent mean scores over all subjects for one measurement period. HADS anxiety, HADS depression, and HADS total represent subject scores for one measurement period. ${ }^{*} P<.05 .{ }^{* *} P<.01 .{ }^{* * *} P<.001$.

\section{Sensitivity to change}

Analyses were performed with multilevel regression to examine whether the positive change that was a priori expected could be demonstrated (Table 15.2). Each model used the same set of predictor variables (i.e., a dichotomous variable for the assessment moment, and age, sex, being diagnosed yes or no, and participation in both measurement periods as covariates). As reported in Table 15.2, the mROM Psymate ${ }^{\text {TM }}$ was able to detect significant changes over time in all the variables.

Table 15.2 Multilevel regression model estimates for the effects of measurement period on several variables.

\begin{tabular}{lccc}
\hline Dependent Variables & B (SE) & t-values & p-values \\
\hline Positive affect & $.65(.19)$ & 3.45 & .002 \\
Negative affect & $-.37(.14)$ & -2.61 & .015 \\
Quality of sleep & $1.89(.85)$ & 2.23 & .033 \\
Positive social interaction & $.44(.15)$ & 3.69 & .001 \\
Activity-related stress & $-.37(.18)$ & -2.10 & .046 \\
Feeling tired & $-.54(.18)$ & -2.94 & .007 \\
Feeling unwell & $-.67(.21)$ & -3.17 & .003 \\
\hline
\end{tabular}

Legend: The analyses are based on 2874 beeps nested within 64 persons. SE = standard error. 


\section{Discussion}

This study examined the applicability of a routine mobile-ROM tool within an ambulatory hospital mental health setting. The PsyMate ${ }^{\mathrm{TM}}$ application was used to implement experience sampling methodology (ESM). The first aim was to demonstrate adequate psychometric properties of $\mathrm{mROM}$, by investigating the reliability and validity of the mROM application in PsyMate ${ }^{\mathrm{TM}}$. The second aim was to investigate sensitivity to treatment change using PsyMate ${ }^{\mathrm{TM}}$ data.

Results demonstrated adequate psychometric properties of the PsyMate ${ }^{\mathrm{Tm}}$ app when used with the present mROM protocol. The reliability of the method was examined by exploring user characteristics of the PsyMate ${ }^{\mathrm{TM}}$. Compliance rates during both the baseline and follow-up periods (a period consisted of 60 possible beeps per person; 10 per day, for 6 days), were around 50 percent, in terms of beeps completed. Although ESM compliance may seem lower compared to previous ESM findings, which showed compliance rates around 80 percent $^{35}$, sufficient beeps were completed for reliable data analyses. There are a number of possible reasons for the reduced compliance. Even conscientious patients miss some beeps due to daily life demands. Furthermore, the compliance rates found in earlier studies were based on traditional sampling techniques, using booklets combined with wristwatches or Personal Digital Assistants. Therefore, a direct comparison to the PsyMate ${ }^{\mathrm{TM}}$ app is misleading. In addition, there were technical issues with the first release of the app, leading to data loss when the Wi-Fi communication was unstable. After a system update, these problems were reduced. Hence, the compliance with the PsyMate ${ }^{\mathrm{TM}}$ app is considered acceptable. Different strategies may be used to improve compliance. The briefing session is important. It should create transparency about the reasons to collect mROM data: to optimize treatment, participate in clinical research and/or to comply with administrative requirements. The briefing session is the start of building an alliance in which clinicians try to understand needs arising in the context of daily life. It helps to discuss ESM sampling situations that shed light on aspects of resilience and vulnerability (with the possibility to customize ESM-questionnaires if necessary). Elements of 'gamification' can be included to motivate patients to fill in the ESM sampling sheets. A crucial factor is the personalized feedback on daily life strengths and vulnerabilities. The PsyMate ${ }^{\mathrm{TM}}$ includes an automated web-based ESM feedback that clinicians and patients can consult. This option should be better advertised because it makes data collection more relevant to the individual patients. Looking at the response behaviour at week level, few differences were found across the days of the week, although the first and last day of the ESM protocol were characterised by slightly fewer responses. During the baseline assessment, the first day showed the lowest response rate, whereas in the follow-up period, the last day had the lowest response rate. A lack of familiarity with the ESM routine is arguably the reason for the low response rate on the first day, while the anticipation of completing the ESM period might account for the low numbers on the 
final day ${ }^{36}$. Considering data at day level, fewer beeps were completed in the morning hours. Again, this is to be expected, since patients were instructed to follow their own daily pattern and sometimes were still asleep at the early pre-programmed moments. When considering attrition rates, only 28 patients completed both measurement periods, or $37 \%$ of the 75 patients initially included. Some patients were excluded because there were not enough data and others did not complete the follow-up measurement. The loss of patients at follow-up could indicate that the method is too burdensome or not experienced as relevant. This seems unlikely, given that previous research has shown that the method was feasible in patients with a wide variety of mental disorders ${ }^{23}$ and current findings indicate feasibility within a single measurement period. Furthermore, a substantial proportion was already discharged from the hospital psychiatry outpatient service by the time of the three month follow-up, as can be expected within the dynamics of a university hospital outpatient-based mental health care setting. After three months, patients being seen in the context of diagnostic assessment would already have been referred back to their area of living. Other patients would have discontinued treatment in the high-attrition risk context of referral from a somatic department to a hospital psychiatry setting. Hence, to a degree, limited followup may reflect the natural flow of a general hospital mental health care setting. Future research on patients' illness attribution and care giver characteristics should further examine this issue. From a clinical perspective, $\mathrm{mROM}$ is most relevant at the beginning and during treatment; patients' motivation may naturally decrease as they start to leave the episode of mental distress behind ${ }^{37}$.

The constructs Positive Affect (PA) and Negative Affect (NA) were selected based on the existing ESM literature ${ }^{31}$. Factor analysis of these items confirmed the structure of the two concepts and the subsequent reliability analyses yielded excellent internal consistency coefficients. To assess the concurrent validity of mROM, comparisons where made between the traditional ROM questionnaire used in the hospital (HADS; assessing anxiety and depression) and the PsyMate ${ }^{\mathrm{TM}}$ moderated mROM protocol. Overall, significant and clinically relevant correlations were found between the PA and NA constructs on the one hand, and the HADS (total, anxiety, and depression) scores on the other. Although the measures show substantial overlap, the ESM based PsyMate ${ }^{\text {тM }}$ moderated $\mathrm{mROM}$ protocol offers a unique contribution to clinical routine outcome assessment given that recall bias and contextual biases are controlled for to a great extent. In addition, the ecological measures within the mROM protocol inform on daily life adaptive functioning. Seven measures (PA, NA, positive social interactions, quality of sleep, activity-related stress, tiredness, and feeling unwell), considered relevant in mental health care, were assessed and compared over baseline and follow-up. All measures changed significantly over time. Thus, the mROM measurements are sensitive to change over time. At follow-up, scores on NA, activity-related stress, tiredness and feeling unwell were reduced, whereas scores on PA, positive social interactions, and 
quality of sleep were increased. This overall sensitivity to change is in line with previous ESM research ${ }^{19,21}$.

The study sample was heterogeneous although mood and anxiety disorders were most prevalent in this general hospital setting. Despite a degree of heterogeneity, the same Psymate ${ }^{\mathrm{TM}}$-moderated $\mathrm{mROM}$ protocol was used transdiagnostically for all patients. The ESM questionnaires used in different mental health target populations typically share $80 \%$ of the items, in order to generically assess contextualized symptom variability and well-being in daily life ${ }^{20}$. The mROM tool shows initial usefulness as a transdiagnostic instrument. However, the small sample size and limited heterogeneity in our sample warrant further investigation across more diagnostic groups. Moreover, in contrast to traditional ROM tools, the data collection is not restricted to symptom intensity, but also includes assessment of functioning and quality of life. The ESM procedure allows for patient-reported outcome measures that index adaptive daily life functioning, avoiding potentially biased data collection methods based on clinical interviews conducted by the treating therapist. Self-reports of mental states can have their own biases; the ESM sampling procedure is designed to avoid these risks, which may be especially advantageous in general hospital patients with somatic complaints due to underlying mental conditions ${ }^{38,39}$.

The logistics of ESM data sampling, and thus the mROM data-collection, were simplified by using modern smartphones. Patients can use their own device to collect data in daily life. The Psymate ${ }^{\mathrm{TM}}$ methodology allows customization; beeps can be programmed, questionnaires adapted and person-tailored items can be added. Collected data is immediately sent to the database whenever a connection is available and remains continually available for feedback through a website with understandable graphs and figures (Figure 15.3). The improved logistics broaden the applicability of the ESM method from limited use in research centres to routine use in clinical care ${ }^{40}$.

ROM is often positioned as a management tool, which suggests that ROM-data can be used to benchmark different centres. ESM methodology, applied as an mROM tool, assures that ROM data has a far broader applicability. The high level of patient involvement increases its clinical relevance to users of services ${ }^{41}$. Moreover, clinical and patient relevance is further impacted by the possibility of accessing and viewing the data independent from services. Thus, in order to make ROM relevant to both the patient and the clinician, ESM may be rolled out routinely. 

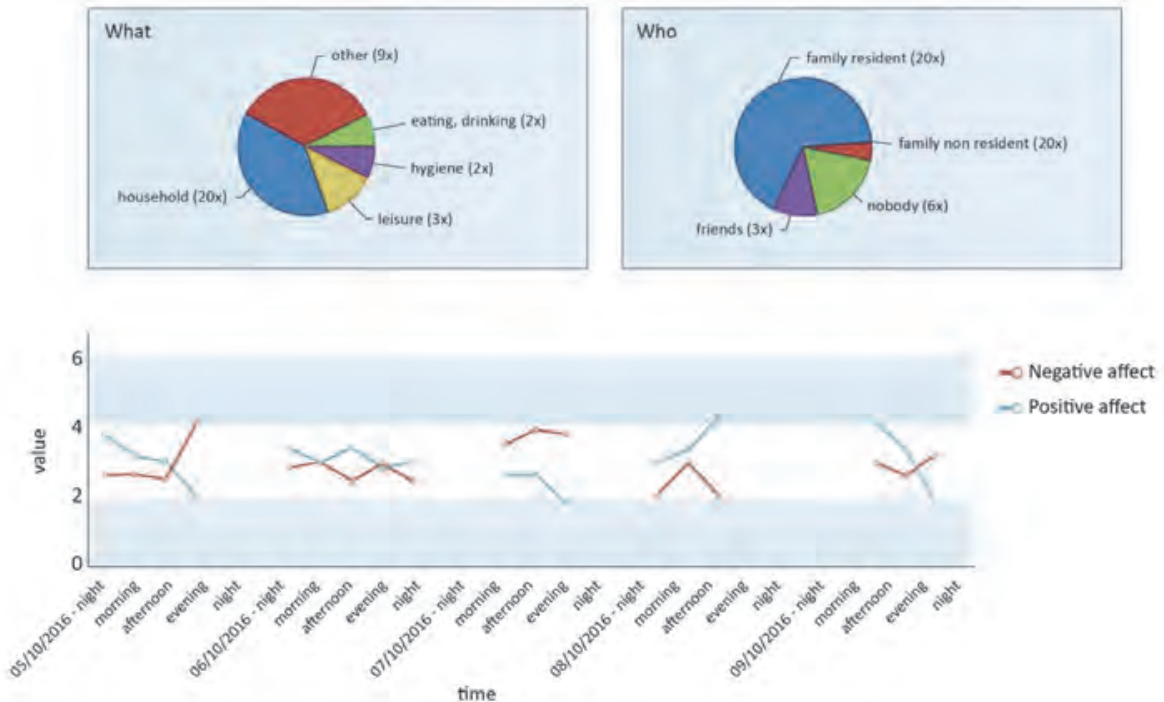

Figure 15.3 This figure is an example of the PsyMate ${ }^{\mathrm{TM}}$ ESM feedback, provided on a dedicated website with choice of 'contextual or functional analysis'. The data (here five consecutive days) are displayed over time (here clustered in day parts; morning, afternoon ...). Aggregated Likert scores of the scale items (here positive and negative affect is selected) reflect the mental state at the time points. What and Who pie charts display the time budgets for the sampling period. In an interactive feedback meeting with the patient, the clinician can select options of the pie chart to break down the subject's responses and assess whether mental states are contextualized (subject feels better in one situation, compared to the other) or zoom in on specific moments, that reflect vulnerability (crisis) or resilience (coping). 


\section{References}

1. Hafkenscheid, A. \& van Os, J. Naar een deugdelijke ROM. Maandblad Geestelijke Volksgezondheid 69, 2028 (2014).

2. Hafkenscheid, A. \& van Os, J. ROM van geïndividualiseerde behandeldoelen. Psychoparktijk 6, 29-32 (2014).

3. Delespaul, P. A. Routine outcome measurement in the Netherlands - a focus on benchmarking. Int. Rev. Psychiatry 27, 320-328 (2015).

4. Cofiel, L. et al. Detecting dissonance in clinical and research workflow for translational psychiatric registries. PloS One 8:e75167 (2013).

5. Hafkenscheid, A. \& van Os, J. Current routine outcome monitoring undermines valid quality measurement. Tijdschr. Psychiatr. 55, 179-181 (2013).

6. Hafkenscheid, A. \& van Os, J. ROM measurements in mental health care: Users need to be aware of the problems and pitfalls. Tijdschr. Psychiatr. 58, 388 (2016).

7. Roe, D. et al. Routine outcome monitoring: An international endeavour. Int. Rev. Psychiatry 27, 257-260 (2015).

8. Wing, J. et al. Health of the Nation Outcome Scales (HoNOS). Research and development. Br. J. Psychiatry 172, 11-18 (1998).

9. Evans, C. et al. CORE: clinical outcomes in routine evaluation. J. Ment. Health 9, 247-255 (2000).

10. Fitzpatrick, R. et al. Evaluating patient-based outcome measures for use in clinical trials. 2, 1-74 (1998).

11. Dawson J. et al. The routine use of patient reported outcome measures in healthcare settings. BMJ 340:c186 (2010).

12. Carlier, I. V. et al. Routine outcome monitoring and feedback on physical or mental health status: Evidence and theory. J. Eval. Clin. Pract. 18, 104-110 (2012).

13. Ferrero, A. et al. Psychopathological Functioning Levels (PFLs) and their possible relevance in psychiatric treatments: A qualitative research project. BMC Psychiatry 16:253 (2016).

14. Tylee, A. Major depressive disorder (MDD) from the patient's perspective: Overcoming barriers to appropriate care. Int. J. Psychiatry Clin. Pract. 5, 37-42 (2001).

15. Zimmerman, M. et al. A clinically useful depression outcome scale. Compr. Psychiatry 49, 131-140 (2008).

16. Solhan, M. B. et al. Clinical assessment of affective instability: Comparing EMA indices, questionnaire reports, and retrospective recall. Psychol. Assess. 21, 425-436 (2009).

17. Robinson, M. D. \& Clore, G. L. Belief and feeling: Evidence for an accessibility model of emotional selfreport. Psychol. Bull. 128, 934 (2002).

18. Van Os, J. et al. The Experience Sampling Method as an mHealth tool to support self-monitoring, selfinsight and personalised health care in clinical practice. Depress. Anxiety in press (2017).

19. van Os, J. et al. Testing an mHealth momentary assessment Routine Outcome Monitoring application: A focus on restoration of daily life positive mood states. PloS One 9:e115254 (2014).

20. Myin-Germeys, I. et al. Experience sampling research in psychopathology: Opening the black box of daily life. Psychol. Med. 39:1533 (2009).

21. Wichers, M. et al. Momentary assessment technology as a tool to help patients with depression help themselves. Acta Psychiatr. Scand. 124, 262-272 (2011).

22. Kramer, I. et al. A therapeutic application of the experience sampling method in the treatment of depression: A randomized controlled trial. World Psychiatry 13, 68-77 (2014).

23. van Os, J. et al. The Experience Sampling Method as an m-health tool to support self-monitoring, selfinsight and personalised health care in clinical practice. J. Depress. Anxiety 34, 481-493 (2017).

24. Barge-Schaapveld, D. Q. et al. Changes in daily life experience associated with clinical improvement in depression. J. Affect. Disord. 34, 139-514 (1995).

25. Barge-Schaapveld, D. Q. et al. Quality of life in depression: Daily life determinants and variability. Psychiatry Res. 88, 173-189 (1999).

26. Barge-Schaapveld, D. Q. \& Nicolson, N. A. Effects of antidepressant treatment on the quality of daily life: An Experience Sampling Study. J. Clin. Psychiatry 63, 477-485 (2002). 
27. Garland, E. L. et al. Upward spirals of positive emotions counter downward spirals of negativity: Insights from the broaden-and-build theory and affective neuroscience on the treatment of emotion dysfunctions and deficits in psychopathology. Clin. Psychol. Rev. 30, 849-864 (2010).

28. Fredrickson, B. L. \& Joiner, T. Positive emotions trigger upward spirals toward emotional well-being. Psychol. Sci. 13, 172-175 (2002).

29. Jacobs, N. et al. Deconstructing the familiality of variability in momentary negative and positive affect. Acta Psychiatr. Scand. 127, 318-327 (2013).

30. Zigmond, A. S. \& Snaith, R. P. The hospital anxiety and depression scale. Acta Psychiatr. Scand. 67, 361370 (1983).

31. Myin-Germeys, I. et al. Emotional reactivity to daily life stress in psychosis and affective disorder: An experience sampling study. Acta Psychiatr. Scand. 107, 124-131 (2003).

32. Spinhoven, P. et al. A validation study of the Hospital Anxiety and Depression Scale (HADS) in different groups of Dutch subjects. Psychol. Med. 27, 363-370 (1997).

33. Bjelland, I. et al. The validity of the Hospital Anxiety and Depression Scale: An updated literature review. J. Psychosom. Res. 52, 69-77 (2002).

34. Herrmann C. International experiences with the Hospital Anxiety and Depression Scale - a review of validation data and clinical results. J. Psychosom. Res. 42, 17-41 (1997).

35. Palmier-Claus, J. E. et al. Experience sampling research in individuals with mental illness: Reflections and guidance. Acta Psychiatr. Scand. 123,12-20 (2011).

36. Delespaul PA. Assessing schizophrenia in daily life: The experience sampling method: Maastricht university (1995).

37. de Beurs, E. et al. Bron voor kwaliteitsbeleid in de GGZ. Boom (2017).

38. Leue, C. et al. Functional urological disorders: A sensitized defence response in the bladder-gut-brain axis. Nat. Rev. Urol. 14, 153-163 (2017).

39. Mujagic, Z. et al. The Experience Sampling Method-a new digital tool for momentary symptom assessment in IBS: An exploratory study. J. Neurogastroenterol. Motil. 27, 1295-1302 (2015).

40. Vilardaga, R. et al. The promise of mobile technologies and single case designs for the study of individuals in their natural environment. J. Contextual. Behav. Sci. 3, 148-53 (2014).

41. Verhagen, S. J. et al. Use of the experience sampling method in the context of clinical trials. Evid. Based Ment. Health 19, 86-89 (2016). 



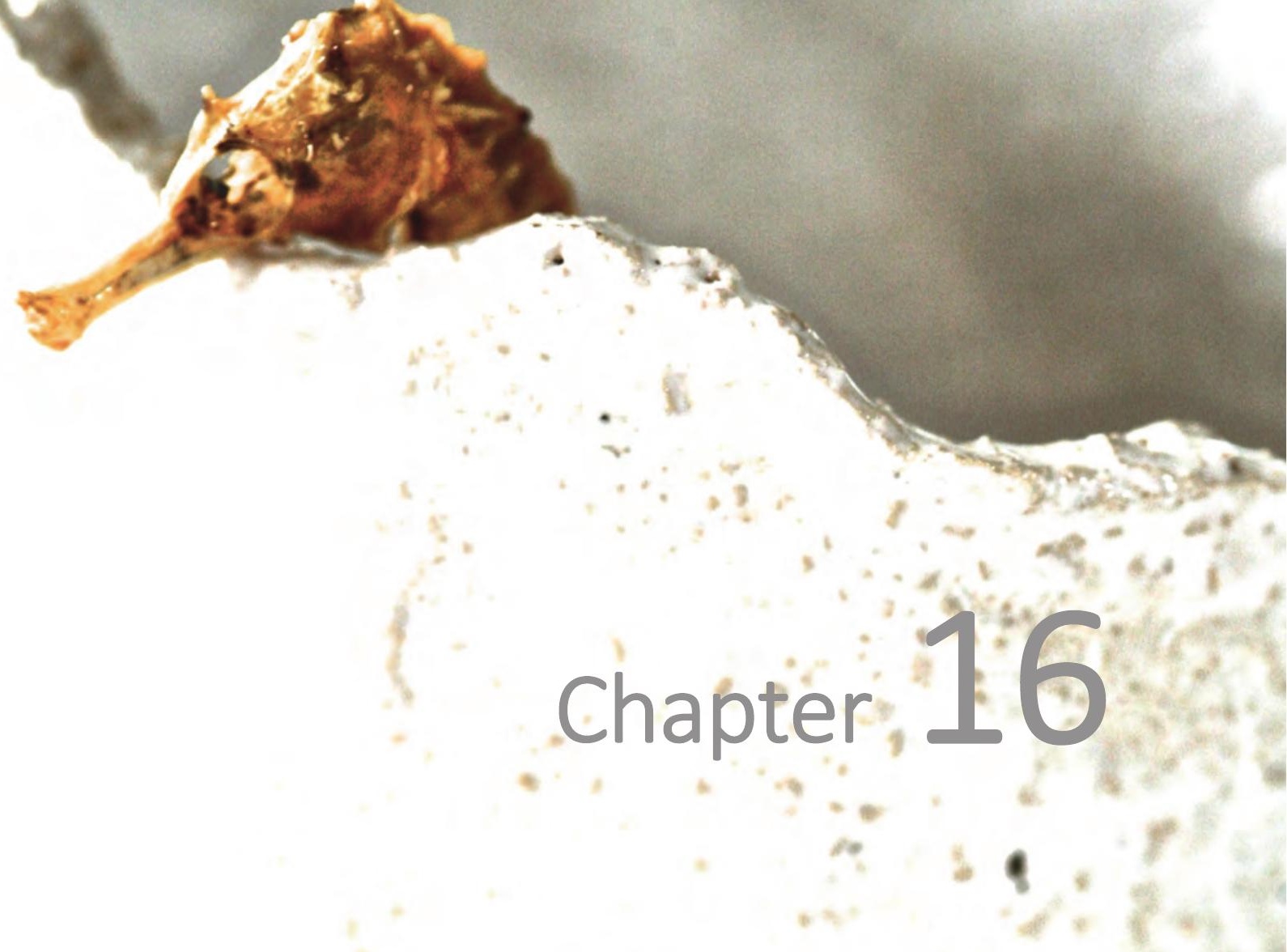

General discussion and valorisation 



\section{General discussion}

Although many clinical needs in multi-conditional patients are still unmet, the integration of physical and mental health care remains aspirational, and integrated medical and psychiatric services are often hard to find ${ }^{1}$. There is a false divide between mental health services (MHS) and somatic health care facilities across all settings ${ }^{1,2}$, a divide that is grounded in conceptual shortcomings due to that lack of integrated care. The aim of the studies underlying the present PhD thesis was to gain further insight into meaningful possibilities to integrate care in psychosomatic medicine. That research was conducted against the background of different levels of hospital-related and primary care-based health care and its associated medical specialties. Given that the MUMC's research on the brain and nervous system spans several medical specialties investigating functional somatic conditions, this thesis focuses mostly on integrated care regarding multi-morbidity aspects in functional disorders.

\section{The inpatient Med-Pych-Unit}

The first part of the thesis describes and investigates integrated psychosomatic care in the inpatient hospital setting. In chapter 2, the advantages and costs derived from admissions to a Med-Psych-Unit (MPU) are explored ${ }^{3}$. MPUs were established in the late 1980s to cater for the combination of somatic and psychiatric needs in inpatient hospital settings. Since then, however, only a few MPU descriptive studies have been published ${ }^{4-}$ ${ }^{11}$. One of these dealt indirectly with hospital-related costs by examining the length of hospital stay (LOS). The investigators found that the MPU was able to decrease LOS in patients with combined medical and psychiatric disorders, compared to admissions at other general hospital wards ${ }^{12}$. However, studies of cost-effectiveness in MPUs remain sparse, and the evidence regarding cost of hospital stay had to be replicated. This is a critical issue, given that LOS is a major contributor to overall costs, accounting for an estimated $80 \%$ of hospital costs ${ }^{13}$. Moreover, no controlled data have yet been presented with regard to medical costs before and after MPU admission other than LOS. While data from a naturalistic pre-post comparison could be useful, there are limitations related to possible confounding due to changing reimbursement or diagnostic and treatment circumstances over the period of observation. Despite these limitations, prepost comparison could represent an opportunity to inform the psychosomatic field in particular, provided that routine clinical data are available. In that framework, the current study is the first to report a controlled MPU pre-post comparison of university hospital-related costs (calculated in terms of LOS, medical service use and psychiatric interventions) with the costs of admission to medical wards. Hospital admission of the same multi-conditional patient population to the MPU on one occasion and to medical wards on another led to a different distribution across the categories of diagnosis. In the case of MPU admission, a psychiatric disorder was diagnosed in almost $90 \%$ of the patients. Interestingly, the most frequent diagnoses were affective disorders (e.g., 
unipolar depression and anxiety disorders) followed by somatoform disorders. However, if the patient was admitted to a medical ward, a psychiatric diagnosis was confirmed in only $6 \%$ of the cases, representing a remarkable difference in frequency of psychiatric diagnosis per setting. The reason for such a substantial divergence might be that patients admitted to medical wards were less psychiatrically and more physically ill compared to the period during which they had been admitted to the MPU. However, a more likely reason is that case detection was better in the MPU setting, as psychiatric disorders at the MPU were frequently chronic or repetitive in course ${ }^{14}$. Concurring with the literature, the divergence suggests that psychiatric disorders were probably underrecognized in the medical wards ${ }^{15-18}$. Concerning somatic complaints, there was almost no difference (functional complaints) or only a small one (somatic diagnoses) in case detection as a function of setting. This indicates that an integrated MPU approach guarantees adequate psychiatric and somatic case findings. Furthermore, the distribution of somatic diagnoses per affected organ system did not differ much between the two settings, suggesting a representative case detection of somatic illnesses at the MPU. Moreover, the findings suggest that patients admitted to the MPU were no less complex than during admission to the medical ward.

With regard to costs, data on the use of medical services revealed a significant difference in favour of the MPU. An explanation could be that the case mix at medical wards consisted predominantly of physical illness, which required more diagnostic tests and treatment procedures. This is not convincing, however, since the patient population did not differ much in terms of frequency or type of somatic diagnoses across the two settings. Additionally, there was no evidence that cost differences were moderated by somatic diagnosis. On the other hand, the costs associated with psychiatric interventions had increased compared to reference wards, reflecting the desired outcome of increased psychiatric treatment. The earlier evidence that MPUs may reduce LOS in complex patients has not been replicated ${ }^{12}$. LOS was much lower in medical wards, which explains why bed costs were significantly higher in the MPU setting. Apparently, the costs of extended hospitalization and psychiatric treatment outweigh the costbenefit derived from lower usage of medical services. At least, that conclusion would be reasonable for an MPU focusing on psychosomatic conditions (e.g., affective disorders with comorbid somatic diseases, somatoform disorders and functional somatic conditions). A very likely explanation for the extended LOS and in turn for the higher costs of treatment in an MPU may be that the setting where reattribution of physically experienced symptoms to stress-related experiences initially took place was inpatientrelated. The question arises whether psychiatric interventions offered at an MPU may be provided just as effectively in an outpatient setting, in view of the low recognition and detection of psychiatric comorbidity at medical wards. According to these data, it seems that for MPUs to provide cost-effective care, a shift from inpatient to day care and outpatient settings would be desirable. An exception may be made for MPUs targeting delirium in severe somatic conditions or self-harm in the context of severe mental 
illness. In such cases, LOS may be shortened when a multidisciplinary team provides integrated care clinically and treatment on an outpatient basis is initially not desirable ${ }^{19}$. Thus, a group of complex patients will continue to require MPU care, and for these patients multidisciplinary inpatient care must still be available. Notably, in cases of very severe complexity, no conclusions can be drawn from our investigation about the economic or clinical outcomes of interventions for more severe and multi-morbid patients. Medical patients who are also seriously ill psychiatrically with delirium or selfharm have been excluded from admission to the MPU despite the value that high acuity integrated care could bring ${ }^{3,20}$. This, however, does not justify maintaining a nonembedded unit without subsequent transitional integrated care solutions, since health care costs have generally been exploding. The exponential growth may be attributed largely, if you please, to the increasing severity, comorbidity and complexity of the conditions of patients targeted in health care delivery over the last decades ${ }^{1,2,21,22}$.

Over the last several decades, the changes in health care systems due to alterations in reimbursement policies and improvements in medical technology have profoundly affected the delivery of care. There has been a major shift away from inpatient to outpatient settings and LOS has become increasingly shorter ${ }^{23,24}$. The MPU data suggest that services at the interface of medical and psychiatric comorbidity are also under pressure to adjust to these trends. Concretely, they should focus more on proactively operating liaison psychiatric services with extended integrated day care and outpatient settings. In addition, transitional alliances with community mental health services (CMHS) and/or general practitioners (GPs) should be formed to create transitional collaborative services from inside the hospital back to primary care, hopefully resulting in cost reductions without compromising quality of care ${ }^{2,25,26}$. A hospital-based MedPsych-Centre (MPC) serving at different medical health care levels (i.e., inpatients, day care and outpatients) may more appropriately come to refer to a range of ambulatory solutions for a group of multi-conditional patients requiring integrated care, rather than a collection of hospital beds alone. Subsequently, a staff-guided transition of care initiated at the MPC from the hospital back to the GPs should be pursued. However, a complex group of patients will continue to require MPU care, and for these patients multidisciplinary inpatient care still must be available ${ }^{27-32}$.

Unfortunately, the scope of the current MPU study did not allow documentation of clinical outcomes ${ }^{11}$. Furthermore, its follow-up duration was only one year post admission, whereas other studies suggest a duration of at least two years to show total health care cost reduction ${ }^{30,33}$. Notably, inpatient treatment and follow-up of patients with somatization disorder revealed that prolonged hospitalization and psychotherapeutic treatment led to improved clinical and cost outcomes during a twoyear follow-up period ${ }^{34}$. Moreover, the current evidence does not support the use of 'frontline' primary caregivers for patients with functional somatic symptoms, as the benefit of treatment may be confined to more intensive interventions with regard to change of functional outcome ${ }^{35}$. As revealed by a recent systematic review, evidence 
supporting improved outcome of integrated models of care in medical inpatients is still sparse $^{22}$. Apparently, organizing health care at the medical-psychiatric interface is a clinical challenge. A main issue from the perspective of clinical practice, and a concern that hinders the implementation of new integrated care models, is that it takes a tremendous investment of time and sustained caregivers' effort to develop and establish integrated care changes in the long run ${ }^{36}$. In contrast to the US, the Netherlands did not stop development of MPU-based integrated care; starting with two MPUs in the late 1990s, more than 40 MPUs are currently operational ${ }^{37}$. Moreover, 'MPU field standards' have been developed to simplify and standardize the organization of MPUs and to guarantee their quality of care ${ }^{37}$. In view of these standardization efforts, we may expect more MPU-related research soon.

\section{The medical-psychiatric outpatient interface at the Med-Psych-Centre}

Our own MPU research indicates that a shift from inpatient to day care and outpatient settings is desirable in integrated psychosomatic care. In order to gain further insight into meaningful possibilities for integrated care in psychosomatic outpatient settings, a choice has been made to narrow the focus to certain medical specialties, which may overlap with regard to aetiology and pathophysiology. Functional somatic complaints of the upper as well as the lower body are frequently related to pain experiences, including anxiety and motility habits ${ }^{38,39}$. Therefore, as an aspect of defensive behaviour, threatrelated disturbances in emotion regulation (e.g., anxiety and mood conditions) and somatic functions with regard to functional syndromes were investigated in integrated outpatient settings of our university hospital's Med-Psych-Centre (1: ear, nose and throat (ENT) referrals with dysphagia; 2: uro-gynaecology referrals with overactive bladder or chronic pelvic pain; and 3: gastroenterology referrals with functional dyspepsia and irritable bowel). The findings from these three settings are described consecutively in the following paragraphs.

First, in order to establish an affective comorbidity pattern in medically unexplained physical symptoms (MUPS) referred to university hospital outpatient settings, we described the frequency of affective complaints associated with MUPS in ENT patients. At a tertiary ENT-psychiatry-outpatient clinic, dysphagia classified as a medically unexplained otorhinolaryngological symptom (MUORLS) associated with anxiety is common, although exact prevalence rates were unknown ${ }^{40,41}$. In general, medically unexplained symptoms frequently accompany psychiatric disorders ${ }^{20,42,43}$. Nonetheless, psychiatric co-morbidity is consistently under-recognized, which precludes effective treatment $^{17,20}$. Given that MUORLS are associated with high impairment, frequent care utilization, risk of iatrogenic damage, and frustration among both physicians and patients, it is critical to improve early detection ${ }^{17,20,44,45}$. The first step is to identify the underlying disorder, which might be psychiatric. The diagnostic process may be more lengthy than necessary due to the somatic focus of the physician or the patient, thereby 
delaying successfully targeted interventions ${ }^{46,47}$. For some otorhinolaryngological (ORL) subcategories, such as oropharyngeal dysphagia or benign paroxysmal positional vertigo, affective symptomatology has been reported ${ }^{41,48}$. However, the Dutch evidence-based guideline on various ORL complaints does not help the physician recognize and label MUORLS concerning comorbidity aspects because the guideline does not cover integrated care. Notably, neither consultation-liaison psychiatry nor psychosomatics is addressed by that recommendation ${ }^{49,50}$. For this reason, the current study investigated the presentation of outpatients with MUORLS in a tertiary ENT cohort. Patients with no clear medical explanation for their symptoms who had associated psychological distress and/or high health care utilization were enrolled in the study. The participants were referred by general practitioners, ORL specialists from other hospitals, or medical consultants from other specialties. To our knowledge, this study was the first to investigate MUORLS at an integrated ORL psychiatry outpatient setting of a university medical centre. Given the health care trajectories that are unsatisfactory to both patients and their physicians, MUORLS has become a topic of considerable interest ${ }^{41,51}$, and the task of adequately diagnosing non-specific conditions has become more challanging ${ }^{52}$. An important outcome of this study is the insight gained into the existence of this patient population and the detour they take to obtain appropriate treatment in daily clinical ORL practice. Quite often a diagnosis of MUORLS is made by exclusion. An integrated diagnosis of functional conditions with psychiatric comorbidity (e.g., affective or somatoform disorders) is frequently hampered by barriers (patient-related, doctorrelated, doctor-patient interactional, situational, operational, or conceptual) ${ }^{52}$. From a consultant's perspective, uncertainty about causation can make physicians uneasy, as they weigh the need to rule out serious illness and increasing chronicity against the cost, distress and even damage of extensive clinical testing. It may be the fear of missing a "serious diagnosis" that induces physicians to emphasize the physical aspect of the symptoms. Queries about psychological distress are subordinated to a physical examination or even forgotten during consultations. Amongst their medically unexplained physical symptoms, our patient cohort had a high prevalence of psychiatric comorbidity. The present study revealed that the majority of patients suffering from MUORLS were eventually diagnosed with a psychiatric disorder (78\%). A final psychiatric diagnosis was often delayed by requiring additional (instrumental) examinations to exclude a somatic disorder. Some of the patients visited the ORL outpatient clinic many times (up to 92 visits) or received care in many different disciplines for diverse medical complaints, thereby raising both the costs of health care and the risk of iatrogenic damage. Thirty-two per cent of the patients had received some ORL treatment without improvement of complaints before they had contact with our integrated approach. Furthermore, the importance of a psychiatric diagnosis in MUORLS patients with an established somatic ORL diagnosis should not be disregarded. The presence of a somatic ORL diagnosis has often delayed referral to the integrated ORL-psychiatry care setting. Among MUORLS patients without a somatic ORL diagnosis, 90\% were diagnosed with a 
psychiatric comorbidity. Given the under-detection of psychiatric morbidity along a nonintegrated trajectory ${ }^{20,51}$, evidence-based treatment of psychiatric comorbidity was implemented right from the start of our multidisciplinary outpatient approach. In patients with expected multi-morbidity, screening for psychiatric morbidity makes sense, and an integrated approach is deemed necessary, as it gets patients motivated for and referred to psychosomatic care.

Second, given the under-detection of psychiatric comorbidity in MUORLS patients, the question arises whether a comparable comorbidity pattern exists in hospital outpatient settings for lower body complaints, whether these are functional or structural. Pelvic floor dysfunctions (PFDs) such as voiding complaints, urinary and faecal incontinence (UI, FI), constipation, sexual problems and pelvic organ prolapse (POP) are prevalent and distressing health problems in both sexes. The incidence increases with age and PFDs are associated with a decrease in quality of life ${ }^{53}$. Since life expectancy is rising, the worldwide burden of PFDs is expected to increase significantly over time. The need for PFD-related health care is expected to rise by $50 \%$ over the next 30 years ${ }^{54}$. PFDs are complex and multi-factorial by nature; therefore a multidisciplinary approach is recommended ${ }^{55}$. Recently, we reported about the complexity of the PFDs represented at our Pelvic Care Centre (PCC), their multi-factorial character and their frequent interactions with each other ${ }^{56}$. One such complex PFD is overactive bladder (OAB), with a prevalence of around $17 \%^{57,58}$. It is often associated with affective disorders ${ }^{59}$ and may lead to significant social and functional impairment ${ }^{60}$. OAB and affective disorders may even share common biological pathways, and in that sense they are comparable to functional bowel disorders ${ }^{61}$. However, most physicians do not adequately identify affective conditions on the basis of self-reported symptoms ${ }^{15}$, a record similar to what has been reported for irritable bowel syndrome (IBS) ${ }^{62}$. Hence, a diagnostic insensitivity of medical consultants to psychiatric and somatic multi-morbidity exists independent from the setting. For example, physicians miss the diagnosis of somatoform disorders in around two-thirds of the cases, and psychiatric diagnoses remain undetected in as many as $95 \%$ of the most complex patients with somatic and psychiatric comorbidity ${ }^{20,52,63}$. Although multidisciplinary care is advocated for PFDs ${ }^{64}$, even now psychological or psychiatric assessment is not routinely incorporated in the assessment of PFDs. In order to elaborate on that diagnostic insensitivity, our aim was to describe, analyse and interpret the relation between PFDs and affective symptoms specified by Hospital Anxiety and Depression Scale (HADS) scores in patients referred to our PCC ${ }^{65}$. Concurring with the results of other multidisciplinary efforts, our cohort data demonstrate that functional complaints are strongly associated with emotion regulation in PFDs ${ }^{66}$. At our PCC, $30 \%$ of patients with PFDs had an abnormal anxiety score and $20 \%$ an abnormal depression score. Corrected for confounding factors, PFDs may account for $7.4 \%$ of the variance in anxiety scores and $12 \%$ of the variance in depression scores. These findings are in accordance with the literature concerning psychiatric and somatic comorbidity 
with regard to the urogenital tract in general and to PFDs in particular. For example, in cancer patients, abnormal HADS anxiety scores were found in $30 \%$ of the patients and abnormal depression scores were seen in approximately $25 \%{ }^{67}$. Moreover, the prevalence of affective disorders in patients with OAB is considerable. In the UK, HADS anxiety scores of 8 and higher were found in $30 \%$ and $47 \%$ of men with OAB without and with bothersome complaints, respectively. In women these figures were higher: $44 \%$ for $\mathrm{OAB}$ without bother and almost $60 \%$ with bothersome complaints ${ }^{68}$. Anxiety and depression scores, as measured by the HADS, were also high for women with urgency incontinence (UUI). The majority of women with UUI reported anxiety (56\%) and over a third reported depression ${ }^{69}$. A prevalence of $22 \%$ depressed patients among women seeking treatment for advanced pelvic organ prolapse (POP) has been reported in the United States, so depression is five times more prevalent in women with POP than in those without $i^{70}$. Additionally, within the group of women with prolapse, women experiencing symptoms of depression reported a lower quality of life and worse prolapse-related symptom scores compared to women without depressive symptoms, indicating that depression may amplify the symptom burden by bringing emotion regulation into the $\mathrm{mix}^{70}$. A qualitative study regarding the emotional burden experienced by women seeking treatment for prolapse revealed that women's psychological well-being is closely related to their pelvic floor symptoms ${ }^{71}$.

However, exact prevalence rates for depression and anxiety disorders in patients with overall PFDs have not been established. Our cohort includes many patients with multi-factorial causes of PFDs or interacting PFDs. Higher HADS depression and anxiety scores were found for patients with urinary incontinence, especially in cases of higher severity and complexity due to other health-related problems (e.g., headache or diabetes mellitus), suggesting a complexity-related somatic pathway in affective conditions. This implication is in line with the literature indicating that patients with lowcomplexity incontinence problems had fewer symptoms related to depression or anxiety, less distress, and a better clinical outcome at follow-up than high complexity patients $^{72}$. Thus, psychiatric and somatic multi-morbidity in PFDs would seem to consist of a bidirectional pathway where one spectrum of symptoms may induce or amplify the other. For all that, the field may be reluctant to consider a broader multidisciplinary approach as set forth in the guideline of the National Institute for Health and Care Excellence (NICE) on urinary incontinence. Part of the reluctance may derive from the low diagnostic sensitivity for comorbidity and complexity on mono-disciplinary trajectories $^{73}$. It may also be grounded in the medical specialists' experience in looking after patients with PFDs; since many will not have had sufficient training, they will not devote sufficient time and resources to implement complexity assessment or even integrated treatment. Nevertheless, psychometric questionnaires can detect mental comorbidity quite easily in somatic patients ${ }^{74}$. The HADS performs well in screening for depression or anxiety; it detects cases of anxiety disorders and depression in somatic, psychiatric, and primary care patients as well as in the general population ${ }^{75}$. Psychiatric 
case detection is important because psychiatric disorders affect the prognosis of somatic conditions and the level of health care consumption ${ }^{28}$. Depression can adversely affect self-care and may increase the risk of new medical complaints, complications or even mortality ${ }^{76}$. Similarly, anxiety can amplify symptoms of medical illness and may worsen clinical outcomes ${ }^{77}$. With regard to PFDs, in a longitudinal study, depression was at baseline associated with the persistence of urinary incontinence (UI) in women ${ }^{78}$, and anxiety and depression may intensify $\mathrm{UI}$ and decrease the benefit of treatment ${ }^{77,79}$. A large population-based survey revealed an inverse relationship between depressive symptoms and health care-seeking. Depressive symptoms may thus prevent patients from seeking treatment ${ }^{68}$. Indeed, our PCC team increasingly observes symptoms of depression and/or anxiety in patients with complicated and multi-factorial PFDs, a trend that is clearly supported by the literature. Since psychiatric disorders affect the prognosis of somatic conditions and the level of health care consumption in general, the present findings demonstrate the importance of an interdisciplinary approach for patients with PFDs to address the physical and emotional as well as the social and relational aspects of their symptoms. Thus, a multidisciplinary Pelvic Care Centre may be the best setting to cover an integration of care for patients with PFDs at the hospital level.

Third, the history of psychosomatic integrated care at the Maastricht University Medical Centre (MUMC) started in 1998 with the introduction of a level III/IV MPU M $^{3,8}$. Since then, the department of gastroenterology has always been involved in multidisciplinary care solutions. Because of that record, longitudinal data were available to investigate patient-related outcome measurement at an integrated gastroenterologypsychiatry outpatient clinic for functional gastrointestinal disorders (FGIDs), which is part of the MUMC's Med-Psych-Centre. The most common and well-known FGIDs are irritable bowel syndrome (IBS) and functional dyspepsia. These FGIDs are diagnosed using symptom-based criteria formulated by the Rome IV consensus ${ }^{39}$. FGIDs are highly relevant for health care because of their high prevalence, their impact on daily functioning and quality of life, their socio-economic burden due to reduced work productivity, illness-related sick leave and high health care utilization ${ }^{80-82}$. The pathophysiology of IBS is complex and remains elusive, but several mechanisms appear to play a role in symptom development, such as alterations in intestinal motility ${ }^{83}$, enhanced visceral sensitivity ${ }^{84}$, low grade inflammation, increased gut permeability ${ }^{85}$, and genetic susceptibility ${ }^{86}$. Furthermore, there is abundant evidence that psychosocial factors such as life stress, coping styles and level of psychosocial support play an important role by influencing symptom severity, illness behaviour and complexity, and eventually patient outcome ${ }^{87}$. Concerning psychiatric comorbidity, IBS patients are more likely to have coexistent mood and anxiety disorders ${ }^{88}$. In part, this may be related to the fact that the personality trait neuroticism is associated with psychiatric as well as somatic ill health ${ }^{89}$. The bi-directional interaction between somatic and psychological 
factors in the pathogenesis of gastrointestinal disorders is known as the 'gut-brain axis'; it is proposed that FGIDs result from a disturbed regulation along this axis ${ }^{87}$. However, in most complex patients with functional complaints, under-detection of psychiatric comorbidity still exists ${ }^{20}$. Reports and clinical experience have indicated that treatment of FGID patients with more severe symptoms by gastroenterologists has only been moderately successful. As a consequence, patients tend to consult many specialists in various hospitals, often undergoing multiple medical and alternative treatments, and sometimes even unnecessary surgical interventions. At our department, FGID patients with moderate to severe gastrointestinal symptoms who do not show sufficient improvement by standard medical management, including dietary and life style advice, and who are suspected of having psychiatric comorbidity are offered additional medicalpsychiatric consultation. The multidisciplinary outpatient joint simultaneous consultation provided by a gastroenterologist and a psychiatrist takes an integrated approach to management of both somatic and psychosocial factors in complex FGIDs. The aim of the current study was to evaluate the results of our integrated outpatient approach longitudinally. The focus was on patient-related direct outcome in terms of gastrointestinal and psychiatric symptoms and quality of life. The results of this prospective, open, non-controlled cohort study showed a beneficial effect of additional integrated treatment on gastrointestinal and psychiatric symptoms, as well as on quality of life, after six months. The beneficial effect was no longer present after one year with regard to the gastrointestinal symptoms but it persisted for the psychiatric symptoms and quality of life. The most reasonable explanation is that in daily practice, a gastroenterologist has already treated patients intensively before the MPC consultation. After the MPC consultation by a gastroenterologist and a psychiatrist, the patient is followed up, particularly in the area of mental health, with a focus on hitherto undetected mental symptoms. With respect to the gastrointestinal complaints, after the MPC consultation, patients were referred back to their general practitioner with advice on further management. The results suggest a choice between two options: a prolonged integrated approach at the MUMC by a gastroenterologist and a psychiatrist; or more collaboration with the general practitioner to maintain somatic symptom relief, since current evidence does not support the use of 'frontline' primary caregivers in patients with functional somatic symptoms ${ }^{34,35}$. The patient population included patients treated locally after their first MPC visit at the medical-psychiatric ward, at day-treatment facilities, and initially or eventually at our integrated outpatient clinic. It also included patients only receiving advice on treatment to be implemented by the referring consultant or general practitioner in their own region; this applied to those who lived outside the Maastricht catchment area (external group). More psychiatric symptoms and lower mean levels of mental and physical quality of life were observed at baseline in the local group compared with the external group, suggesting more psychiatric comorbidity among locally treated patients. The gastrointestinal symptoms were equal at baseline in both groups. Patients treated locally showed more improvement than patients treated 
externally after six months and/or one year of follow-up, except for physical quality of life. The greater improvement in psychiatric symptoms in the local Maastricht group could be the result of the more integrated expertise in our multidisciplinary approach. Alternatively, it could simply reflect the higher symptom severity at baseline in the local group.

A gastroenterologist and a psychiatrist deliver integrated care at the MPC outpatient clinic for FGIDs. The multidisciplinary joint consultation they offer is a tertiary centre approach for the referral of patients with complex FGIDs. Therefore, the results of this study cannot be extrapolated to a general hospital FGID population or to patients in primary care. Three-quarters of the patient population was female, between 40 and 50 years of age. Half of the patients had a history of abdominal surgery, and prior to receiving integrated care $10 \%$ of the patients had used opioids as pain medication. As expected, a high percentage of psychiatric comorbidity was observed, ranging from as much as $80 \%$ in the entire study population to $90 \%$ in the locally treated group. The results point towards a marked under-detection of psychiatric comorbidities among patients with complex FGIDs. Previously reported prevalence rates for psychiatric comorbidities were much lower in FGID patients, namely 40-60\%, whereas the rate of psychiatric comorbidities was less than $20 \%$ in healthy controls ${ }^{90}$. In short, frequently utilizing patients with severe and complex FGIDs being diagnosed and treated at the university hospital level show a very high incidence of psychiatric comorbidity.

Our investigation is a pre-post comparison, which is characterized by an observational naturalistic design. Given that this study is explorative in nature, its outcome will generate new hypotheses. In the future, these hypotheses will have to be evaluated by conducting investigations using a sophisticated prospective-controlled study design. However, because this is a pilot study, it does not require an initial control condition. In that sense, it is similar to explorative papers regarding neuromodulation under functional or chronic pain conditions or various functional somatic disorders ${ }^{91-93}$. There are several reasons why this study can inform the field further on unmet clinical needs in complex FGID patients with psychiatric comorbidities. First, almost no research had been conducted on integrated care (gastroenterology and psychiatry) in the complex population of FGIDs. Most research that has been carried out is characterized by a mono-disciplinary approach. For that reason, the comorbidity of psychiatric disorders often goes undetected ${ }^{20}$ and the treatment outcome is unsatisfactory due to undetected and thus unresolved heterogeneity issues in FGID populations ${ }^{94,95}$. Second, patients are referred to our integrated facility because of long-term suffering, unsolved diagnostic questions and treatment refractoriness. It is almost unethical to enrol such patients in a prospective case-control design if previously undetected psychiatric morbidity is present and evidence-based treatment for psychiatric disorders is available (e.g., for IBS and panic disorder). Third, the inclusion of a control group of FGID patients with less complex disease characteristics would have created an artificial situation as well. To estimate methodological influences on outcome, the external group 
representing advised treatment co-ordinated by the general practitioner or referring consultant is a 'kind of control condition'. Even though the two cohorts (local and external group) are not well matched, it is not likely that positive psychiatric and shortterm somatic outcomes of integrated care would simply mark a regression to the mean. It should be kept in mind that advised continuation of treatment co-ordinated externally by GPs did not influence outcome positively in FGID patients with psychiatric comorbidities. Indeed, the study population was heterogeneous concerning the 'control condition'. However, heterogeneity does not justify withholding a paper from being published in IBS research ${ }^{94,95}$. Furthermore, with regard to a prospective randomizedcontrolled trial (RCT), we know from experience that it is quite difficult to enrol patients in an RCT if standard therapy for comorbid psychiatric disorders is available ${ }^{96}$. We have just finalized a standardized RCT on selective serotonin reuptake inhibitors (SSRIs) for IBS and comorbid panic disorder, a project which has been prolonged a couple of times because of delays in inclusion. This experience is in accordance with RCT evidence in general, as almost $50 \%$ of surgical RCTs remain unpublished ${ }^{97}$. Thus, until high-quality research in integrated care for FGIDs gets published, papers with minor methodological quality are necessary. Moreover, this is justifiable since more than one-third of RCTs have prompted different conclusions at reanalysis ${ }^{98}$. It may be expected that RCTs directed by previous naturalistic clinical research might be less prone to error. However, given that this is a negative study of gastrointestinal symptom relief after one year of investigation, it is conceivable that the bio-psycho-social approach is somehow incomplete. The question is, what is missing here? Much has been done in terms of biological aspects, given that SSRIs were frequently prescribed in the majority of our patients in order to influence serotonergic transmission. However, frequent care utilizers with a long-term mono-disciplinary trajectory may profit more from prolonged integrated care (by a gastroenterologist and psychiatrist) including psychotherapy, especially if a somatoform disorder is present, in order to reattribute somatization of complaints to a more stress-dependent context ${ }^{35,99}$. This reattribution might even be more consistent if a case manager accompanies the patient in the transition back to primary care.

\section{Lack of integrated staff guidance in psychosomatic primary care}

So far, the best evidence of the benefit of psychosomatic integrated care has been obtained in primary care. Despite the high prevalence of mental illness - almost $25 \%$ of people suffer from a psychiatric disorder during their lifetime, and most of them receive treatment for mental disorders in primary care - in many patients it remains undetected and consequently untreated ${ }^{2}$. Part of the reason may be the somatic presentation of underlying disturbances in emotion regulation that are (not only) attributed to anxiety or mood disorders ${ }^{38}$. In order to enhance the detection rates, it is essential to offer guidance to primary caregivers concerning psychiatric comorbidity. Collaborative care (CC) in the form of co-ordinated case management, which is delivered by a medically 
supervised nurse in co-operation with the patients' general practitioner and a navigating psychiatrist, improves affective and chronic disease states in multiple conditions ${ }^{2,100,101}$. Getting case managers (CMs) into the frontline of treatment in addition to GPs may be advantageous to combined somatic and mental health care. ${ }^{2,100,102}$. However, there are also uncertainties about the effectiveness of primary care-based collaborative interventions targeting multi-morbidity ${ }^{103,104}$. Especially in patients with severe somatic conditions, CC did not contribute much to the reduction of major adverse somatic events $^{104,105}$. Moreover, as multi-conditional or frequently utilizing patients present themselves at in- and outpatient hospital settings, the question arises whether the results of CC may be as favourable in urban areas as they are in the rural parts of the US.

The answer touches upon the characteristics of city living, which might be associated with treatment outcome in multi-conditional patients. The well-known epidemiological association between an urban environment and mental ill-health is one of these characteristics. That link is supported by work showing that urban living is associated with increased amygdala activity ${ }^{106-109}$, which is a key region in the regulation of stress, affective conditions and pain ${ }^{110,111}$. Pain is the natural comorbid mental experience of somatic conditions ${ }^{112,113}$. In turn, pain is strongly influenced by comorbid mental disorders, particularly affective disorders ${ }^{114,115}$. In view of the evidence of an urban impact on risk for common mental disorders ${ }^{116}$, including a likelihood of psychiatric medication prescriptions ${ }^{117}$, we carried out a nationwide cohort study of primary and outpatient care-based analgesics prescriptions. We hypothesized that pain outcomes, as indexed through prescriptions, would be worse in urban environments and disadvantaged urban neighbourhoods. Indeed, escalation of chronic analgesic treatment was observed more often there, suggesting that pain outcomes are associated with individual characteristics that are more prevalent in urban and deprived areas or, alternatively, are subject to contextual influences, like area-level stress or social fragmentation, regardless of individual-level characteristics. One individual-level variable that may explain part of the association with urban life and deprivation is socioeconomic status ${ }^{118,119}$, but that variable was not available for inclusion in our model. Nevertheless, the fact that the association with urbanicity remained when deprivation was controlled for in the same model suggests that urban effects may not be reducible to individual-level socio-economic status. Furthermore, although neighbourhood deprivation and urbanicity are correlated, there is an additional association of deprivation with escalation of analgesics, indicating that parameters other than population density are involved too. However, the findings could also be attributed to reverse causation. That is, patients with worsening pain may move into more urbanized areas and deprived neighbourhoods as a consequence of ill-health and/or a need for hospital care. Regardless of the underlying mechanism, the results clearly echo the unconfounded higher rates of poor mental health in areas with higher levels of urbanization and greater neighbourhood deprivation ${ }^{118,120}$, and suggest that the outcome of mental disorder comorbidity associated with somatic disorders may show 
similar predictable variation. Functional pain syndromes and psychiatric disorders show high levels of interdependency, and psychiatric conditions enhance the severity of somatic symptoms $3,15,63,90,121,122$. Thus, part of the mechanism underlying the association between pharmacological pain escalation and urban environment may be that urbanization increases the risk for mental ill health. This hypothesis is supported by the observation in both the starter and the continuation groups of analgesic treatment that escalation of chronic analgesic treatment was associated not only with an urban environment and neighbourhood deprivation, but also with the prescription of various psychotropic medications. Overall, the positive association of escalating analgesic treatment with psychotropic medication was as strong or even stronger than the association with prescribed somatic co-medication. The exception was the observed deescalating effect in the continuation group of second-generation antipsychotics, which might possess powerful analgesic properties ${ }^{123,124}$. This observation is accordance with the literature, given the fact that psychiatric conditions can enhance symptom severity in somatic patients, which may have even more impact than the somatic condition itself $^{125}$.

Although the absolute difference between analgesic escalations in the more versus less urbanized environment was small, this difference may be relevant from a public health perspective in view of the high incidence of painful conditions in the general population. Furthermore, prevention of persistent pain states can reduce costs ${ }^{126}$. Given the well-known increase of health care costs in complex patients with frequent utilization of health care, with or without psychiatric comorbidity, even a small number of such patients can induce relevant changes in clinical costs ${ }^{127}$. Hence, a more effective treatment for persistent pain, including treatment of psychiatric comorbidity, may have a cost-saving effect. Targeting populations with painful conditions for early recognition and treatment of mental health problems may be cost-effective from a public health perspective. This also represents an area of considerable unmet clinical needs, since opioid escalation is an inflationary development in the treatment of painful conditions $^{128,129}$. Moreover, broadening the pain agenda to include a better understanding of associated mental health problems could minimize failed surgery outcomes in patients with undetected mental disorders. For instance, new surgical procedures were found to be more common in chronic back pain patients with posttraumatic stress disorder (PTSD) compared with those without PTSD ${ }^{130}$. Similarly, depression was demonstrated in $47.4 \%$ of patients with low back pain who had no surgery, in $50 \%$ of those with one surgical procedure and in $62.5 \%$ of those who had undergone surgery more than once ${ }^{131}$. Thus, influencing central pain sensitization by providing adequate antidepressant treatment in depressive conditions may help prevent surgical escalation.

Furthermore, odds ratios for escalation of analgesics in relation to the original level may represent ceiling effects in both the starter and the continuation groups. Patients already at level 5 have nowhere stronger to go; treatment of patients who were at level 
1 at baseline can escalate to stronger medication. Ceiling effects may reflect the pattern of prescribing analgesics in general practice. In that regard, it has been suggested that the WHO analgesic ladder is in need of updating ${ }^{132}$. For example, Vargas-Schaffer is broadening the ladder with a fourth surgical step. In the current study, however, we look in the opposite direction, namely at treatment aspects related to underestimated mental disorder comorbidity in persistent pain states. A speculative question remains to be considered: To what degree does the escalation of analgesic treatment and its association with psychotropic medication reflect therapeutic paradigms to remedy pain, treatment of psychiatric comorbidity or a cause of psychopathology? For instance, in the starter and the continuation groups of chronic analgesic treatment, escalation of analgesics was consistently and positively associated with the use of tricyclic antidepressants (TCAs). This prescription habit may reflect routine off-label paradigms in the pharmacological treatment of pain syndromes ${ }^{94,133,134}$. However, given the evidence regarding TCA's efficacy in pain conditions, negative rather than positive associations with escalation of analgesics should have been expected. More likely, since the association with TCAs was as strong as the association of sedatives with analgesic escalation, it may be a reflection of affective or addictive comorbidity in persistent pain such as in vulnerable cases of opiate-induced sensitization, tolerance and hyperalgesia ${ }^{135-142}$. Our data indicate that escalation of analgesics may represent an ongoing process even after months of treatment, a process not exclusive to the context of environmental deprivation. Since prescriptions of psychotropic and (attenuated) somatic medication show a similar pattern over time, escalation may to some degree be driven by patient factors such as opioid tolerance, opioid-induced hyperalgesia, or disease progression in psychiatric and somatic morbidity. The factors related to multi-morbidity in pain treatment at primary and outpatient levels therefore need integrated staff guidance to navigate patients along a more goal-orientated path through complexity, i.e., multi-morbidity treatment in chronic pain states.

\section{A hospital to primary care transition - the Med-Psych-Net}

Despite more than ten years of collaborative care in the Netherlands, there are still gaps to bridge from hospital to primary care, as evidenced by our own nationwide, predominantly primary care-based, analgesics prescription cohort ${ }^{143,144}$. Part of this lapse may be due to making hospital admissions increasingly brief, thereby limiting the amount of time that health care professionals can dedicate to psychosomatic consultation and intervention in complex patients with multi-morbidity ${ }^{145,146}$. Shortening the stay is likely to lower the rate of detection and treatment of psychiatric comorbidity in routine hospital settings ${ }^{20}$. Given the high rates of somatic and psychiatric multimorbidity ${ }^{147}$, which are predictors of a longer hospital stay and greater hospital $\operatorname{costs}^{20,147}$ as well as poorer outcomes and increased subsequent health care use ${ }^{148}$, alternative approaches to care should be considered. There is evidence that modern proactive inpatient integrated care is effective, despite the observed barriers to 
discharge ${ }^{149,150}$. However, although transitional communication between hospital consultants and primary caregivers is gaining ground ${ }^{151}$, the transition of care from inside the hospital back to the general practitioners deserves further improvement ${ }^{152,153}$. Furthermore, although hospital-initiated transitional care improves outcome in some somatic conditions $^{154}$, the quality of discharge practice remains deficient in the university hospital setting ${ }^{155}$. Transitional care may cut re-admissions in patients with complex chronic conditions ${ }^{156,157}$. However, research on cost developments in patients with psychosomatic multi-morbidity receiving transitional care is scarce. Moreover, the original formulation of care transitions intervention (CTI) specifically excluded patients with psychiatric comorbidity ${ }^{156}$. Previous work suggests that psychosomatic care in particular may gain in cost-effectiveness by shifting transitionally from inpatient to primary care solutions ${ }^{20}$. Primary care-based CC represents a useful supplement to clinical care in affective disorders ${ }^{33,100}$. However, there is a lack of evidence concerning re-admission rates and benefit to the physical quality of life ${ }^{103,104,158}$. Thus, the trend for increasingly brief hospital stays needs to be offset by a transitional care organization combining proactive liaison psychiatric services with extended integrated day care, outpatient and primary care settings $2,20,33,43,159-161$. In our current study, transitional care refers to bridging the gap between specialty hospital care, on the one hand, and primary mental health care at the level of a community mental health service and GPs, on the other. Care transition in our naturalistic study is organized by means of a medical psychiatric network. The Med-Psych-Net (MPN) is an umbrella term to denote a close collaboration to deliver integrated care, combining specialty medical and psychiatric hospital care and primary somatic and mental health care. The MPN of interest in the current investigation is not a physical centre. Rather, it is a virtual entity that is part of the Maastricht University Medical Centre working in combination with MUMC's associated GPs. The MPN consists of psychiatry and medical psychology consultation liaison services and a community mental health service in collaboration with hospitalbased medical consultants and primary care-based GPs. The combination provides a wide range of transitional inpatient (including MPU beds) as well as outpatient diagnostic and treatment options for a group of patients with somatic and psychiatric multi-morbidity. Besides medical care, the key elements are psychiatric diagnosis and management, psychotherapy and managed care, and delivering a graded transition from integrated care starting inside the hospital back to GPs or, if required, to the CMHS. At the level of hospital consultation-liaison-service (CLS) activities, psychosomatic care is embedded in the MUMC's Med-Psych-Centre, which bundles psychosomatic care inside the university hospital from inpatient (i.e., MPU and CLS) to outpatient care. In this regard, hospital psychologists and psychiatrists proactively carry out multidisciplinary psychiatric and psychological screening and case finding at medical wards or at outpatient clinics, for example at the multidisciplinary pain centre, the cancer centre, the centre for functional bowel disorders or the $\mathrm{PCC}^{43,162,163}$. A motivational procedure (towards accepting a transitional trajectory into primary mental health care) is 
administered prior or parallel to treatment at the medical ward and/or in the medical and psychiatric outpatient setting. This procedure is ultimately followed by a transition via CMHS professionals working initially inside the hospital and, at a later moment of patients' treatment, at the GPs' practices, or, if a more lengthy or specialized mental health care treatment trajectory is deemed necessary, at the nearby CMHS. This staffguided care transition from the hospital to primary care completes our approach to the Med-Psych-Net. The specific MPN intervention is that care managers from the CMHS see patients five to eight times at the MUMC's mental health care outpatient clinic and thus 'take over' treatment inside the hospital in order to accompany the patient on the way back to primary care. This clearly distinguishes the MPN approach from a standalone inhospital psychiatric CLS, which has an established link with GPs or the CMHS, or from somatically focused CTI (one inpatient contact by a professional, followed by primary care-based follow-up) or primary care-based CC (no initial inpatient face-to-face contact) ${ }^{103,156}$. The MPN physically combines professionals from both the medical hospital and the primary mental health care setting, providing a unique opportunity to gain patients' acceptance of any mental health issues underlying their somatic complaints. In addition, the MPN has the advantages of convenience and transitional continuity of care, which can be expected to have a positive impact on treatment adherence $^{160,164}$.

A pre-post MPN analysis was conducted as part of the present study. It was focused on cost reductions related to medical service use and length of stay (LOS) due to an increase of mental health care ( $\mathrm{MHC}$ ) interventions. In contrast to a previous publication regarding cost developments in an exclusively hospital-based $\mathrm{MPU}^{20}$, this pre-post analysis hypothesized an overall reduction in costs for the transitional collaborative MPN approach. This hypothesis was confirmed by the results of the present study. Almost every referred patient (95\%) was diagnosed with a psychiatric disorder, while the prevalence of somatic conditions (80\%) was lower (but still high). A portion of the latter was diagnosed as functional complaints $(37 \%)$, so the increase in costs for mental health care reflects a desirable and appropriate rise in $\mathrm{MHC}$ interventions for psychosomatic conditions. Even though MHC costs increased, overall costs decreased by $84.5 €$ per patient and month. This amounts to approximately $1000 €$ in cost-benefit per patient and year, which is associated with a reduction of LOS and medical service use. These developments did not require changes in the hospital's psychiatry or medical psychology fulltime equivalents (FTES), since CMHS caregivers started to join primary care in the Maastricht area during that period. Noteworthy with regard to $\mathrm{MHC}$ is that these CMHS caregivers were colocated in the MUMC and at GPs and not additionally employed. Compared with primary care-based collaborative care interventions in general, an amount of $1000 € / \$$ cost savings had already been realized one year after the transition of care ${ }^{165}$. This reduction was due to hospital-related cost-savings alone and did not include other costs of social relevance (e.g., illness-related sick leave, primary care consumption, etc.). Part of the reduction in costs for medical service use and LOS after 
referral to the MPN may be due to successfully delivered medical treatment of somatic conditions. This could occur if medical consultants had admitted patients to the hospital for urgent (costly) treatment of a medical condition, followed by referral to the MPN for emergent psychiatric conditions after initial medical interventions. It should be kept in mind that the proportion of psychiatric and functional diagnoses in the MPN population was high and somatic conditions were less prevalent. Hence, another explanation, in line with previous work, may therefore be more likely - that psychiatric disorders had gone unrecognized in the medical setting ${ }^{20}$. Integrated care by an MPN facilitates detection and treatment of psychiatric disorders, preventing physicians from misunderstanding psychiatric disorders as a somatic complication and consequently prescribing costly diagnostic and therapeutic procedures ${ }^{15,17,20}$. Due to data management changes in the hospital (replacement of paper-based records by electronic patient files), a well-matched comparison between the MPN and the randomly chosen control group was not possible. However, the development of medical costs did not differ between an MPN sub-cohort and the random MUMC medical cohort after the first medical consultation. A medical cost-benefit was apparent in the second year after integrated MPN care in comparison to the second year of medical care alone. The observed benefit indicates that MPN care may truly add value in terms of cost savings. It is not likely that the cost reduction for the MPN condition simply reflects a regression to the mean. In fact, somatic morbidity was prevalent in the MPN cohort too, and psychiatric comorbidity may also occur in chronic somatic diseases managed by non-psychiatrically integrated medical interventions only ${ }^{166-170}$. Moreover, there is no reason to assume that costs require three years to decrease, especially not in patients with chronic or repetitively recurring somatic and psychiatric comorbidites ${ }^{20,171-173}$. Thus, transitional integrated care delivered by a MedPsych-Net may fulfil unmet clinical needs of multi-conditional patients concerning treatment as indicated and may reduce the waiting lists of medical consultants through treatment transition back to GPs.

Traditional integrated care is focused on primary care or clinical care alone ${ }^{2,20,160}$, and transitional care in psychosomatic conditions is lacking. The MPN approach offers an organizational advantage - integrated psychiatric and psychological consultation and treatment in psychosomatic conditions independent of the setting. At the MUMC-based Med-Psych-Centre, multidisciplinary clinical, outpatient and primary care-based roundtable conferences provide psychiatrically or psychologically staff-guided casemanagement that includes consultation opportunities at different levels of care. As a result of integrated diagnosis and treatment, frequent utilizers of medical services may display better psychiatric/psychological treatment adherence, as evidenced by an increase in costs of transitional mental health care and a decrease in medical and overall costs. These savings were achieved by care delivery using pre-existing services. Neither the hospital CLS of psychiatry and medical psychology nor the CMHS had to hire extra staff. At both sides of the MPN (MUMC and CMHS/GPs), caregivers' FTEs remained the same, reflecting cost-effectiveness from both a societal and a health system perspective. 
Patients, if any comorbidity had been identified at all, would usually have been referred directly to the CMHS. Due to the activities of the MPN, case detection was better and the medical staff as well as GPs received guidance. Since patients adhered to their treatment and were, therefore, not lost in the transition, hospital discharge could be managed effectively without compromising FTEs. This consideration might be indirectly supported by records on negative hospital evaluations (e.g., re-admissions, adverse events) of our own MUMC concerning wards still running without an integrated transitional network approach, especially those without routinely integrated psychosomatic care in patients with a great chance of suffering from medical and psychiatric comorbidity ${ }^{174,175}$. As suggested by our investigation, transitional care by case managers can be provided effectively by caregivers receiving guidance from medical staff but operating in- and outside the MUMC if the CMs are supervised and trained by clinical navigators. From our clinical perspective, patient empowerment due to a "warm handover" in between settings as well as to medical skills guaranteed by integrated staffguidance are essential elements of care transition.

This investigation was not designed to be a case-control study, as the cost comparison with a randomly selected MUMC cohort is not well matched with regard to sex, age and diagnoses. The 'quasi experimental' comparison with a non-transitionally treated UMC cohort only allows for an estimation of general trends in cost development due to changes in reimbursement or to hospital-related changes in diagnostic and treatment procedures. Thus, the current MPN study is a non-controlled pre-post comparison of cost aspects. Nevertheless, as a naturalistic pilot study, its design served to generate the following hypothesis: Integrated transitional care in psychosomatic medicine may lead to relevant cost savings compared to care as usual. Verification of that hypothesis awaits a prospective randomized controlled trial (RCT). However, as in surgical RCTs, prospective comparative research better has to be preceded by naturalistic clinical research, such as this MPN study, since almost $50 \%$ of the surgical RCTs remain unpublished and more than one-third of overall clinical RCTs have prompted different conclusions upon re-analysis ${ }^{97,98}$. Thus, prospective comparative controlled research concerning (cost-) effectiveness of integrated transitional care has to follow a study like this one in order to test a clinically formulated hypothesis on its relevance. Overall, integrated psychosomatic care at our MUMC contributes to valuable clinical research concerning treatment of multi-conditional patients. This includes the Med-Psych-Centre with the clinical MPU setting and various multidisciplinary outpatient settings (e.g., PCC, centre for FGIDs, multidisciplinary pain centre, cancer centre). Most distinctively, the hospital-based MPC and the broader transitional Med-Psych-Net approach have overcome barriers and gone on to integrate monodisciplinarily-operating caregivers into an effectively collaborating network for complexity care. 


\section{Impact of integrated care on the understanding of psychosomatic aetiology}

Taking our MUMC's integrated care approaches as our point of departure, we observe that positive evaluations provide the conceptual background and framework for what may constitute an aetiologic nosology of multi-conditional complaints. Pain, dysphagia, urgency and other functional complaints are stress-related sensations. As such, these might be influenced by stress experiences and disturbed emotion regulation, such as anxiety or mood disorders. Indeed, oropharyngeal dysphagia (OD) is frequently associated with anxiety and depressive symptoms, leading to a bothersome symptom burden, but $O D$ is possibly amplified by affective mood states ${ }^{42}$. In order to objectively determine whether disturbed emotion regulation contributes to alarm sensitization, we carried out a 'biomarker' cohort study to investigate the relationship between the severity of OD, by means of fiberoptic endoscopic evaluation of swallowing (FEES), and clinically relevant depressive and anxiety symptoms using the Hospital Anxiety and Depression Scale. While our current study revealed high frequencies (43\%) of clinically relevant affective symptoms in patients with OD, it did not find a significant association between clinically relevant depressive symptoms and FEES outcome variables. This suggests that there may be no relationship between a depressive state and the experienced severity of OD. However, our study did find a significant association between clinically relevant anxiety symptoms and the FEES variable piecemeal deglutition, demonstrating a higher probability of anxiety symptoms in the lower ordinal categories of the piecemeal deglutition. Moreover, a significant association was found for clinically relevant symptoms of anxiety and the FEES variable postswallow vallecular pooling. Apparently, severe OD did not result in a higher probability of having affective complaints. These findings may indicate that less severe or medically unexplained and only subjectively experienced OD might be a manifestation of an anxious and somatically hyperaroused state of stress. Hyperarousal is characterized by somatic anxiety equivalents induced either by psychiatric disorders or by early stages of somatic diseases ${ }^{163,176}$. Another possible explanation may be that many of the patients in the study with more severe and chronic objectively measured OD had a long history of disease. They may have already adapted to their physical limitations, unlike patients with mild OD in an early stage or with acute onset of disease. Regarding Parkinson's patients, for example, the prevalence of depression peaks at the beginning of the disease and again in its latter stages; in between, the prevalence of affective complaints is lower ${ }^{177}$. Moreover, all head and neck oncological patients in this study were not in a palliative stage of care, meaning that they were cancer survivors. Head and neck oncological patients often show improvement in their psychological and psychiatric functioning during follow-up, while the severity of the disease or functional disability remains stable or even deteriorates. These patients may successfully adjust to living with their physical problems; as time passes, their fear of recurrence or death may be likely to decline ${ }^{178-180}$. 
This study also revealed a relationship between patients' subjective evaluation of their own swallowing as measured with a visual analogue scale (DSS), on the one hand, and the FEES variable postswallow pyriform sinus pooling, on the other. For the rest of the FEES variables, no significant association with the DSS was found. These results show no apparent relation between the measured severity of OD and patients' subjective evaluation of their own swallowing. Some patients with a nearly normal function considered their swallowing to be very impaired, while other patients with severe OD considered it normal, though acknowledging that they were not always aware of their diminished swallowing function. For example 'silent aspiration', a severe swallowing abnormality, can occur without subjectively suffering from it ${ }^{181,182}$. Moreover, several patient groups (e.g., neurodegeneration, stroke) tend to have sensory deficits that may contribute to inaccurate judgment ${ }^{183-189}$. Inaccuracy of perceptual judgment (underestimation of the problem) may manifest itself in fewer subjective complaints ${ }^{182}$. This could explain why the present study found very few significant associations between the DSS and the severity of OD as measured with FEES. Another explanation may be that higher anxiety levels influenced patients' experience, leading to suffering without severe OD. A previous study in the present patient population showed a significant association between DSS and anxiety ${ }^{41}$. That finding indicates that a patient's subjective evaluation of swallowing probably is a better indicator of affective symptoms than the FEES outcome. Our findings are in accordance with the literature on medically unexplained physical symptoms in general ${ }^{163,176}$. Recognizing that affective symptoms are common in OD patients ${ }^{11}$ and assuming that these problems are not solely dependent on the severity of OD may lead to new directions for future research. These insights also serve as grounds for a clinically relevant implementation of affective symptom screening in dysphagia management. Such screening could extend diagnostic skills throughout the field while preventing under-detection of relevant psychiatric comorbidities $^{20,41,74}$.

Little is known about the strength and direction of the association between OD and affective complaints, and investigations on pathophysiology, risk factors and treatment effect are scarce. We therefore carried out the first systematic review on affective symptoms in OD in which we summarized published studies on affective complaints in patients with OD and analysed the quality of the literature. The broader objective was to elucidate how affective comorbidities may impact symptom bother and treatment compliance, since attending to specific psychiatric complaints instead of general psychosocial aspects may more appropriately call for integrated or collaborative approaches. The review consists of 24 articles that reported on swallowing function and affective symptoms in different populations. Data pooling was prevented by the heterogeneity of assessment tools, diversity of study populations and poor methodological quality. Nevertheless, symptoms of anxiety and depression appear to be common in dysphagic patients. All of the studies concluded that symptoms of 
depression were significantly and positively associated with impaired swallowing function. Twelve investigated symptoms of anxiety as well, and 9 found a significant association with dysphagia too. The quality of the included studies varied. Thirteen met all criteria for external validity ${ }^{41,190-200}$, whereas none met all criteria for internal validity. Nor is much known about the relationship between the severity of OD and affective symptoms. Nguyen et al. concluded that anxiety and depressive symptoms scored higher in patients with moderate and severe OD compared to patients with mild $O D^{201}$, while the study of Verdonschot et al. concluded that affective symptoms were common in mildly disturbed OD patients ${ }^{200}$, but patients with severe OD did not have a higher probability of affective complaints. Moreover, having affective symptoms might be a predictor of subjectively experienced dysphagia severity ${ }^{200}$. It remains unclear what the exact mechanism behind the association of OD with affective states is. At least in patients with medically unexplained otorhinolaryngological symptoms, affective conditions may reflect a state of dysphagia amplification ${ }^{200}$, which is comparable to the sensitization and alarm falsification process known from other functional somatic conditions $^{38}$. Anxiety and depressive disorders might alter the perception of swallowing by setting off a false alarm and provoking one to experience OD. From a neurobiological perspective, cerebral motor cortex areas may be related to a neural stress connectome combining anxiety and depression states with sensory and motor function ${ }^{202}$. Further research on this topic is deemed necessary.

The results of the current review should be seen in the light of its limitations. The search strategy used MeSH terms and a limited number of free-text terms. A broader search strategy could have generated different search results. Moreover, we decided to focus on specific symptomatology rather than on a wide range of sub-threshold psychological complaints. A 12-item critical appraisal tool, as derived from the QUADAS, was used for quality assessment ${ }^{203}$. It is possible that a different tool would have led to a different assessment of the included studies. It was difficult to compare the results of the 24 studies for several reasons. First of all, despite the focus on affective complaints, the studies were still considerably heterogeneous regarding patient populations and outcome parameters. The study populations consisted of stroke patients, head and neck oncology patients, the elderly and others. Furthermore, studies applied a wide range of assessment tools for affective symptoms or conditions and the swallowing function. Second, most articles had methodological limitations (e.g., no clear description of selection criteria; little or incomplete information about diagnostic tools and procedures; no information about test result interpretations; no explanation of withdrawals), which made it impossible to carry out data pooling and meta-analysis. Most studies used only one tool to assess swallowing, while a multi-dimensional approach is recommended for the diagnosis of OD and the assessment of OD severity. A broader protocol should include a structured interview, both to assess OD and to establish a psychiatric diagnosis. In addition, a clinical observation of oral intake by a speech and language pathologist should be incorporated, as should a FEES or other objective measurement. Almost all 
studies used questionnaires that can only be applied as screening tools for symptoms of anxiety and depression. These tests are not suitable to come to a diagnosis of affective disorders. The question remains unanswered whether there is a causal relationship between affective symptoms and swallowing problems in OD patients. Therefore, future studies investigating this topic should use instrumental measurement tools for OD, as well as a psychiatrist as a gold standard plus repetitive momentary assessment, in order to assess time changes in symptoms due to contextual influences, instead of measurement by retrospective self-reporting questionnaires. Furthermore, it is important that the execution of the swallowing test is standardized in included patient populations. Moreover, the time period between swallowing assessment and psychiatric measurement should be short in order to assure that the conditions of OD and the affective state do not change between two test periods. Nevertheless, it is clear that affective symptoms are common in patients with OD. Screening for affective symptoms could also be helpful for caregivers who are not familiar with psychiatric symptomatology in order to estimate influences on possible treatment refractoriness ${ }^{74}$. To date, few treatment-effect studies have been conducted in OD patients with psychiatric comorbidity. Psychiatric symptoms or diagnoses other than anxiety disorders and depression might be prevalent in OD patients too; very few studies have been published on this topic, however. At least in complex patients with affective comorbidity, the management of OD therefore requires a multi-dimensional approach that would enable patients to adhere to swallowing rehabilitation, which might better contribute to treatment satisfaction in OD.

As in upper-body oropharyngeal dysphagia, lower urinary tract symptoms (LUTS) form a focus of interest concerning body-brain-axis pathophysiology and aetiologydriven treatment options. Although the high prevalence rates would suggest the opposite, $O A B$ is often under-diagnosed and subsequently undertreated, mainly because of patients' reluctance to seek medical help ${ }^{204}$. Only one-third of the patients with OAB receive treatment ${ }^{205}$. One reason for not seeking help may be its perceived stigma ${ }^{204}$. Another may be an inverse relationship between depressive symptoms and health care seeking in patients with $O A B^{68}$. A relationship between common affective disorders (e.g., depression and anxiety disorders) and LUTS was described as early as $1964^{206}$, and the importance of mental disorders in the aetiology of urgency incontinence was reemphasized more recently ${ }^{207}$. In 2011, the International Consultation on IncontinenceResearch Society (ICl-RS) set up a think tank on psychological factors and LUTS $^{208}$. It was noted that not only OAB but also other LUTS may be associated with affective conditions, concluding that the possibility of causation and or maintenance of lower urinary tract symptoms through psychological causes needs further research' ${ }^{208}$. Despite this growing interest, the exact nature of the association between bladder symptoms and mental conditions, specifically affective disorders, remains elusive. A systematic review using stricter definitions of psychopathology may inform the field further 
regarding the strength of the associations between affective disorders and $O A B$ and possibly provide insight in the underlying pathophysiological mechanisms and treatment recommendations at the "bladder-brain-axis". Hence, our systematic review is the first to give an extensive qualitative overview of the current literature on the association between $O A B$ and affective symptoms. Forty-three articles were included, covering more than 80,000 subjects. Of these articles, 32 concern depression, two are solely about anxiety and nine concern both. Regarding depression and overactive bladder, a positive association was observed in 26 studies. Regarding anxiety, six studies revealed a positive association. In longitudinal studies, different directional associations were reported: a) OAB subjects who develop depression/anxiety, or b) depressed/anxious subjects who develop $O A B$, or $c$ ) both. The quality of evidence in studies reporting an association between the co-occurrence of $O A B$ and depression was rated level 3 in the GRADE framework. Evidence of an association between the co-occurrence of anxiety and $O A B$ was rated GRADE level 2. Longitudinal associations between new onsets of $O A B$ in depressive subjects were GRADE level 2. Evidence of an association of $O A B$ in longitudinal association with anxiety was GRADE level 1. No RCT was found with exact outcome measures. Nevertheless, four RCTs were included because they reported outcome measures of interest. These trials revealed a positive association between $O A B$ and affective symptoms due to successful treatment of $O A B$, which resulted in a significant decrease of affective complaints. The main findings of this systematic review are that there is an abundance of high-quality evidence regarding a positive association between co-occurrence of $\mathrm{OAB}$ and depression and to a lesser extent regarding an association between co-occurrence of OAB and anxiety. However, many of the evaluated studies failed to note longitudinal changes and thus lacked evidence of causality. The association between $\mathrm{OAB}$ and depressive symptoms might, however, be bi-directional.

The quality of the studies included in our systematic review influences the validity of the conclusions we can draw from them. Many of the initially retrieved articles did not employ validated screening instruments to assess affective symptoms. Exclusion of these articles minimized the amount of bias. Risk of selection bias was kept to a minimum by having two reviewers independently select the articles to be in- or excluded. Two authors, to avoid information bias, independently conducted quality assessment and risk of bias assessment of the 43 included articles. Furthermore, it was difficult to assess the prevalence of affective symptoms across different groups, as most of the studies reported differences in the form of mean scores and odds ratios rather than percentages. Moreover, there was substantial heterogeneity with regard to diagnostic assessment. Very few studies controlled for confounding variables and performed multivariate analysis. Only studies with adult subjects were included, which could have introduced some selection bias, affecting generalizability across all age groups. In general, this systematic review revealed a positive association between the cooccurrence of $O A B$ and affective symptoms. Furthermore, there is evidence for new onset of $O A B$ in depressive subjects, but further research is necessary to examine the 
strength of the effect. One possible explanation for the association of affective disorders with OAB may be that UI can lead to social and functional impairment that results in the accumulation of social stress, which in turn may lead to depression ${ }^{60}$. Another explanation might be that both affective disorders and LUTS are mediated along common bladder-brain-axis pathways.

The association between affective disorders and OAB can be explained biologically by the fact that both syndromes share common pathways. For instance, serotonin and norepinephrine as well as the interactions between the two neurotransmitter systems are thought to be involved in the aetiology of depression ${ }^{209}$. Serotonin depletion is thought to contribute to depression and selective serotonin reuptake inhibitors are effective in depression treatment ${ }^{210}$. Moreover, in anxiety disorders, it is likely that the serotonergic system helps regulate anxiety both during development and in adulthood $^{211}$. Additionally, serotonin plays a role in bladder function. Several animal studies revealed that lowering serotonin levels in the central nervous system (CNS) was accompanied by urinary frequency and detrusor overactivity ${ }^{212}$. Most experimental studies in rats and cats indicated that activation of the central serotonergic system depresses reflex bladder contractions and increases the bladder volume threshold for micturition induction ${ }^{213}$. Affective disorders may thus be linked to $O A B$ due to altered serotonin function. Moreover, a rat model of depression caused by lowering the serotonin in the CNS led to OAB manifestations (urinary frequency due to nonneurogenic detrusor overactivity and lowered bladder capacity) in female rats. Administration of an SSRI reversed the urinary symptoms ${ }^{214}$. In humans, duloxetine, a serotonin-noradrenaline reuptake inhibitor (SNRI), improved the symptoms of frequency and urgency in female $O A B$ patients significantly ${ }^{215}$. Increasing the concentrations of both serotonin and norepinephrine in the peripheral nervous system leads to an enhanced stimulation of the pudendal nerve and, therefore, increases the resting tone and contraction of the urethral sphincter. That sequence suggests that an altered neurotransmission is accompanied by affective and lower urinary tract symptoms ${ }^{216}$. Another explanation is related to a shared deregulation of the hypothalamic-pituitaryadrenal (HPA) axis, which is involved in stress experiences and coping, resulting in increased corticotroponin-releasing factor (CRF), increased adrenocorticotropic hormone (ACTH) and increased cortisol. The HPA axis is thought to play a stressdependent role in depression and anxiety disorders and in bladder function as well ${ }^{217-219}$. In rats, repeated psychological distress resulted in increased urinary frequency and lower voiding volume ${ }^{219}$. CRF stimulated bladder function (a lower micturition threshold, accompanied by a lower urine volume) in a rodent model of detrusor overactivity and anxiety, and the administration of a CRF antagonist reduced detrusor overactivity ${ }^{220}$. A study with repeated social stress in rats revealed a urodynamic profile resembling detrusor overactivity, observed even days after exposure to threat, which indicates an enduring effect of alarm sensitization ${ }^{221}$. From a neuro-anatomical point of view, several investigations based on functional brain magnetic resonance imaging (MRI) during 
urodynamic studies revealed that increased brain activation in the anterior cingulate cortex (ACC) was associated with increased urgency at large bladder volumes, especially in young females with UI and detrusor overactivity ${ }^{222-224}$. Accordingly, in young people with depressive disorder, increased activity levels were found in the ACC as well. The ACC is part of a neural stress network, which is involved not only in emotion regulation but also in aspects of (social) pain ${ }^{109.225}$. Moreover, single photon emission computed tomography (SPECT) of older patients with UI showed hypo-perfusion in the regions of the prefrontal cortex ${ }^{226}$. Similarly, hypo-perfusion in the ACC and the prefrontal cortex was also seen in patients with major depressive disorder ${ }^{227}$. Acute experimental pain activates several cortical and mesolimbic brain regions: the primary and secondary somatosensory cortex, the insula cortex (IC), the anterior gyrus cingulus (ACC) and the prefrontal cortex (PFC), the thalamus, the nucleus accumbens (NAC) and the amygdala (see Figure 16.1). All of the above brain regions are involved in reward and threat experiences and in emotion regulation ${ }^{228-231}$. These findings suggest that the prefrontal brain and other parts of the default mode network may mediate both affective disorders and functional somatic complaints, as evidenced for the pain-depression diade too ${ }^{\text {[chapter }}$ 11, this thesis]. Unravelling how chronic bladder pain affects the (central) nervous system, neuro-imaging of the brain revealed that patients with CP/CPPS have increased grey matter in the primary somatosensory cortex, the insular cortex, the anterior cingulate cortex and the amygdala ${ }^{232}$. This finding is in accordance with the literature for pain and affective conditions, indicating that functional somatic syndromes and affective disorders are regulated by the same neuronal stress connectome. Thus, the question is, what is (dys-) functional in functional somatic disorders according to a conceptual framework that includes central sensitization and alarm falsification by means of symptom amplification due to affective conditions?

Figure 16.1 Brain areas relevant for pain and depressive states.

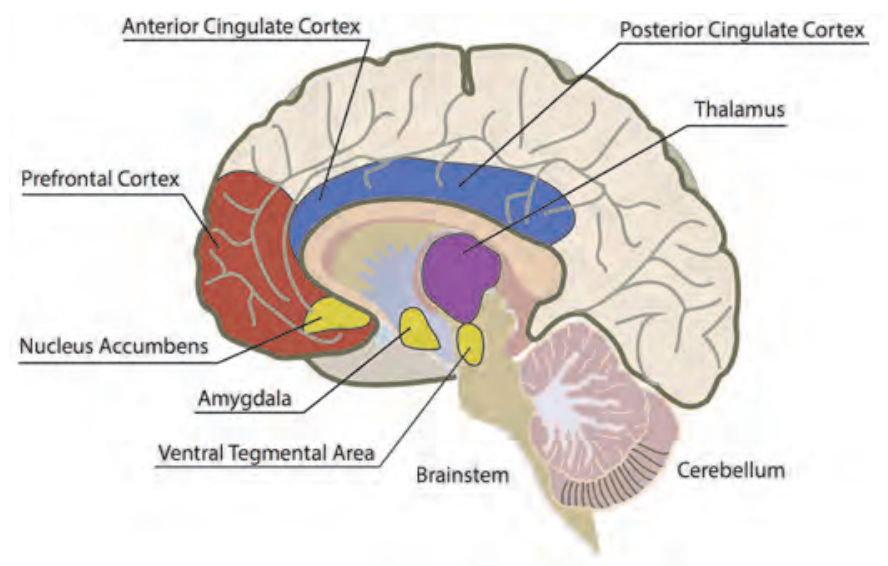




\section{Functional somatic disorders - a sensitized defence response including alarm falsification and hyperarousal}

Like other functional disorders, those of the urogenital or the gastrointestinal tract overlap and often co-occur with functional disorders of other tracts, suggesting a common origin ${ }^{233-246}$. The overlapping symptoms include visceral pain or pelvic floor dysfunction, suggesting that these conditions could represent a spectrum or continuum across organ systems, sharing an underlying central hypersensitivity expressed as bodily distress or hyperarousal ${ }^{241,244-247}$. In patients with IC/BPS, for example, having multiple antecedent functional disorders is one of the strongest risk factors for the development of other disorders, both within and across organ systems, adding to the suggestion of a transorgan pleiotropic phenotype ${ }^{248}$. Also, the number of functional disorders present in IC/BPS is predictive of the number of different surgical interventions that a patient will undergo. Recurrent surgery, in turn, might be linked with treatment refractoriness or iatrogenic damage afterwards ${ }^{249}$. While several functional complaints might be present at the time of diagnosis, they are not necessarily considered together for the indication of surgical interventions ${ }^{250}$. The failure to co-diagnose comorbid syndromes might be partly attributable to difficulties in phenotyping functional urological disorders, given that they remain poorly understood and, as a consequence of conceptual shortcomings, that treatment strategies often do not yield satisfactory results ${ }^{251}$. The concept of functional urological disorders, which is comparable to the concept of functional gastrointestinal disorders (FGIDs), has been reformulated. Previously couched in the historical paradigm of organ-centred disease, there is now a general consensus to define such disorders as a series of symptom-based syndromes ${ }^{251-262}$. However, there is a need for improved stratification of patient characteristics and for a comprehensive model that unifies aetiological pathways and associated treatment strategies $251,257,261,263,264$. The hypothetical bladder-gut-brain axis (BGBA) is a useful framework in which to study the pathophysiology of related functional urological and gastrointestinal disorders. Although the common pathophysiology is complex and remains elusive, several mechanisms linked to the hypothesized BGBA might be involved in symptom formation. These include enhanced bladder permeability and sensitivity, alterations in bladder motility, altered visceral function, cross-sensitization between the gastrointestinal tract and the bladder as well as with emotion regulation, neuroendocrine balance, autonomic nervous system balance, low-grade inflammation, genetic and/or epigenetic susceptibility, infectious aetiology and composition of the microbiota, as well as psychological stress and psychiatric disorder comorbidity $59,83,85,86,88,247,265-279$. Evidence suggests that psychosocial factors, life stress, coping styles, and level of psychosocial support all have a role in the pathophysiology of functional urological disorders, affecting symptom severity, illness behaviour and outcomes ${ }^{279-287}$. Anxiety, depression, stressful experiences, and recurrent negative social interactions as well as comorbid functional syndromes are associated with one's health perception and help-seeking behaviour in functional disorders ${ }^{59,278,286-288}$. Patients with functional disorders in combination with 
comorbid psychiatric disorders are more often referred to somatic specialists than those without combined symptoms, and comorbid anxiety and depression are both consistently associated with increased severity of symptoms and a greater illness burden $286,289,290$. Coexistent mood and anxiety disorders are associated with neuroticism, also known as mood bias towards negative emotions or trait-negative affectivity ${ }^{291-294}$, which also underlies somatic ill health ${ }^{59,88,89,285,295}$. Functional syndromes are also associated with neuroticism, so trait-negative affectivity might be a non-specific risk factor for multiple morbidities ${ }^{285,287,296-301}$. Under-recognition of neuroticism-associated conditions could be causally related to unsatisfactory treatment outcomes. Research suggests that neuroticism is associated with significantly worse treatment responses and higher levels of depression, for example in patients with CP/CPPS ${ }^{296}$. Furthermore, the economic cost of neuroticism is sizeable, exceeding that of other common psychiatric disorders, which suggests that a focus on broadly defined psychosomatic comorbidity rather than affective or somatic disorders in isolation might be economically advantageous ${ }^{172,302}$. Neuroticism is not only cross-sectionally associated with somatic and mental ill-health, but it also predicts subsequent onset of mental disorders as well as a spectrum of somatic conditions ${ }^{121,169,303,304}$. For all that, in general hospitals, psychiatric and somatic comorbidities affect the prognosis of somatic conditions and the level of health care use ${ }^{305,306}$. However, under-detection of psychiatric comorbidities is not rare, particularly in patients with complex multi-morbidity and medically unexplained functional complaints ${ }^{20}$. In this context, the real question concerning causal pathways comes to the fore: Does psychiatric morbidity amplify somatic symptom burden and malfunctioning or does psychiatry start in the body?

Three mechanisms along the BGBA can be hypothesized to underlie symptom formation. The antecedent hypothesis states that depression and anxiety cause the onset of somatic symptoms ${ }^{307,308}$; the consequence hypothesis states that somatic symptoms predict the onset of depression and anxiety disorders ${ }^{307,309}$; the common pathway hypothesis states that different factors (psychological, environmental and biological) cause the onset of both somatic symptoms and depression or anxiety disorders $^{310}$. Given that both functional and affective disorders are stress-related, sensitization to threat might mediate false alarm signals along the BGBA as a defence system. Thus, a common pathway of dysregulation or hyperarousal in the BGBA can be proposed, which involves sensitization and alarm falsification, giving rise to both emotional distress (psychiatric disorders) and bodily distress (functional disorders) ${ }^{176,245,266,272,311-313}$. Sensitization refers to a state of hypersensitivity to (visceral) stimuli that manifests as increased sensitivity to normally bothersome stimuli (for example, hyperalgesia in response to mildly painful stimuli) or symptom formation in response to normally non-bothersome stimuli (for example, allodynia in response to non-painful stimuli) ${ }^{314}$. Alarm falsification refers to the process of normally neutral (mental) or non-noxious (physical) stimuli provoking a range of emotional reactions (anxiety, fatigue, depressed mood), cognitive responses (hypervigilance, catastrophizing) 
and bodily distress or hyperarousal (urgency, frequency, pain), respectively. So, what are the stressors and stress-related factors that influence affective and functional symptom formation?

Psychological factors moderate the severity and persistence of symptoms, influence decisions to seek treatment, and predict treatment response ${ }^{315,316 .}$ Therefore, they seem to have an important role in stress-related vulnerability to threat. For example, different psychosocial factors are associated with $\mathrm{OAB}$ and IBS, suggesting that related factors tend to cluster together: negative health beliefs and negative cognitive coping such as catastrophizing (a maladaptive cognitive magnification of threat accompanied by feeling helpless) and negative affectivity or affective disorders ${ }^{59-61,317}$. Personality traits and temperamental factors might also reflect elements of vulnerability to functional disorders. As mentioned above, sensitivity to stress is mediated by neuroticism ${ }^{318}$. Patients with high scores on neuroticism questionnaires are more reactive to stressors. Moreover, neuroticism is one of the few personality traits that is consistently associated with functional urological and related gastrointestinal disorders $285,295,296,298,301,319,320$. Along with a history of being abused, a history of negative life events, as well as somatization, catastrophizing, anxiety and neuroticism affects the severity of functional disorders, being associated with increased disability ${ }^{295,321-324}$.

Furthermore, physical threat originating either outside or inside the body may influence hypersensitivity and hyperarousal. The concept of postinfection functional gastrointestinal disorders is well established ${ }^{325}$, and infection might have a similar role in the development of functional urogenital disorders. Moreover, it seems that a uropathogen-dependent sensitization process could be induced, possibly leading to enduring alarm falsification in the absence of an initial inflammatory threat response ${ }^{326}$. Whether sensitization leads to recurrent UTI, to alarm falsification in the absence of a recurrent infection or both remains elusive ${ }^{327}$. However, post-UTI alarm falsification includes not only chronic pain but also voiding dysfunction and anxious/depressive behaviour in the absence of recurrent infections, together presenting as complex functional disorders ${ }^{38,328}$. These symptoms might be partly related to increased spontaneous firing of the spinal cord, as demonstrated in a rodent model, which is consistent with centralized pain states ${ }^{326}$.

Thus, stress and negative emotions (e.g., pain, anxiety, fear, decreased mood) and threat-related behaviour (e.g., fight, flight, or freeze, immune defence, and hypermobility of the bladder or the gut) could all be part of the same defence reaction that serves to reduce threat. In the case of functional disorders, this reaction can be best described as alarm falsification caused by a shared sensitization to threat, which can endure as emotional and bodily distress (i.e., hyperarousal) long after the occurrence of the original stressor. Future research should focus on underlying mechanisms, for example, epigenetic changes due to earlier perceived stress, which persist after initial threat in order to be prepared for recurrent danger. 
Figure 16.2 Timecourse of cumulative threat and the consecutive alarm falsification of defence.

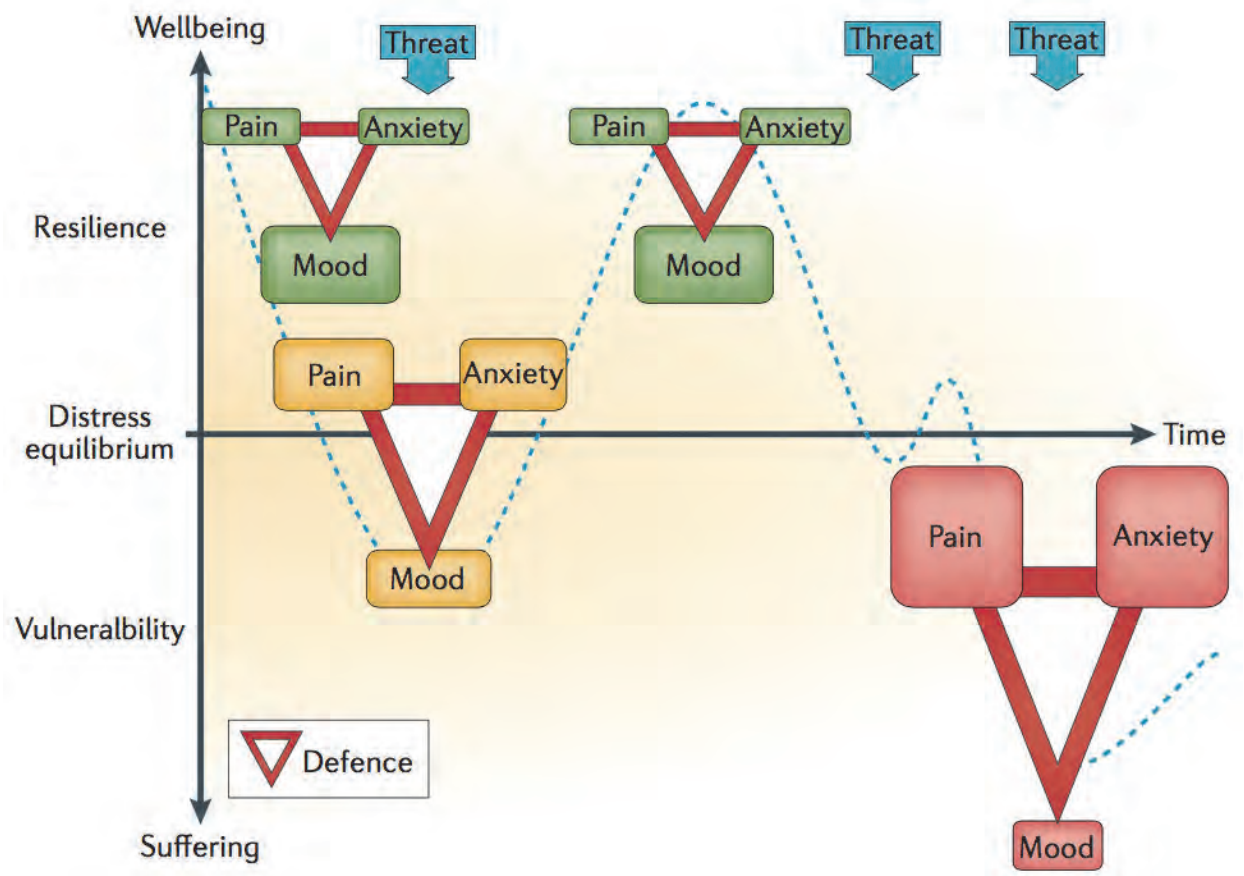

Legend: Defensive behaviour (flight or freeze; voiding; diarrhoea; depicted as a red triangle) and corresponding emotional reactions (pain, anxiety, mood) to threat (trauma, childhood adversity, infections) during a timecourse of repetitive cumulative stress show that defensive behaviour and related pain and anxiety levels increase after threat, whereas wellbeing (mood) decreases (yellow). Stress-related behaviour and emotions recover after exposure to a single or less-intense stressor (green), but after cumulative stress, pain and/or anxiety increases and related defensive behaviours become bothersome and enduring functional complaints, reflecting a sensitized defence reaction and alarm falsification (red).

\section{How to overcome desperation concerning treatment refractoriness in functional disorders?}

\section{Momentary assessment to identify unmet clinical needs and to guide transitional integrated care solutions}

Both the complexity and the severity of functional disorders, including affective comorbidity, increase in a stepwise manner with the number of functional disorders, from low rates at the general population level to approximately 30\% in primary care and up to $80 \%$ in an integrated hospital outpatient setting. This phenomenon, which is a well-known comorbidity bias, derives from the fact that any illness is more likely to have comorbidities at greater levels of severity ${ }^{43,329,330}$. For inpatients with medically 
unexplained complex conditions and treatment resistance, the level of psychiatric comorbidity increases up to $90 \%$ of inpatients admitted at integrated multidisciplinary units (Med-Psych-Units), whereas only $5 \%$ of these patients were detected at the level of non-integrated monodisciplinary medical settings (internal medicine ward or surgical ward $)^{20}$. Indeed, under-recognition of a complex psychosomatic multi-morbidity phenotype consisting of urological, gynaecological, gastrointestinal and affective comorbidities might contribute to treatment resistance in functional disorders ${ }^{251,331,332}$. Thus, the therapeutic benefit might be suboptimal if non-integrated diagnostic and treatment plans, i.e., without multidisciplinary support and understanding, are offered $^{20,251,333}$. At the MUMC, we deliver integrated care in different settings. We still offer MPU care for the most complex patients with multi-conditional complaints. However, instead of running MPU beds alone, we reduced our amount of hospital beds in order to intensify integrated care at other health care levels as well. For instance, at the level of outpatient hospital care, a multidisciplinary Pelvic Care Centre has been introduced in order to jointly diagnose and treat patients with urogenital and gastrointestinal symptoms. Such a centre includes specialists with urological, gynaecological, proctosurgical, gastrointestinal, sexological and psychiatric expertise. Local PCC data suggest that $15-25 \%$ of PCC patients have multifactorial problems at initial triage, requiring more than one specialist for diagnosis and initial treatment advice ${ }^{56,334}$. Further on, in order to bridge the gap between hospital and primary care, case managers (if indicated) accompany patients on their journey form the hospital back to GPs. To enhance the recognizability of integrated psychosomatic care across all settings and to support the collaboration between different care providers, we frame our integrated care efforts within our transitional Med-Psych-Network. Nowadays, both doctors and patients are becoming more aware of unmet needs in the treatment of functional complaints. Thus, an integrated transitional approach should be recommended as the standard, particularly in patients with complex syndromes and comorbidity-related treatment resistance ${ }^{335,336}$.

An integrated perspective may help to overcome shortcomings in monodisciplinary concepts of functional disorders. For decades, the conceptualization of medical syndromes has been symptom-based; functional urological disorders, functional gastrointestinal disorders and affective disorders are all classified according to their symptomatology using the ICS consensus on OAB, ROME IV criteria for IBS, and the DSM5 criteria for mood and anxiety disorders, respectively. Although such a system is useful for clinical practice within different specialities, it might obscure the natural comorbidity between functional syndromes across specialties that require an integrated approach to diagnosis and treatment. Instead, combining speciality knowledge to treat the symptoms of functional syndromes with pleiotropic expression across different organ systems could improve satisfaction levels among both patients and doctors. Today, assessment of functional syndromes with comorbid somatic and psychological or psychiatric expressions remains limited as it typically relies on retrospective questionnaires that do 
not inform on real-life intensity, variability or reactivity. Furthermore, these questionnaires are affected by recall bias and a host of other contextual and psychological factors. In addition, the multitude of instruments that are available to measure, for example chronic abdominal pain, makes it difficult to compare outcomes across studies $^{337}$. Moreover, making use of specialty-related questionnaires limits the perspectives and excludes comorbidity assessment. Thus, further development and standardization of assessment instruments is needed to overcome the limitations of available methods and enable the field to move beyond use of symptom-based assessments and the various shortcomings of such approaches. In order to gather the data, which may lead to a better concept of multi-morbidities, the limitations could be overcome by randomly repeated momentary assessment during the day in a real-life environment, using the Experience Sampling Method (ESM), which has already been piloted in gastrointestinal functional disorders ${ }^{338}$. Our current pilot study demonstrates that retrospective symptom measurement by end-of-day diaries or end-of-week questionnaires leads to higher scores when compared to electronic momentary symptom assessment (i.e., ESM) in patients with IBS. This difference is most pronounced for abdominal pain. Scores for abdominal pain recorded in the end-of-day dairies were similar to maximum pain reporting in ESM, indicating that subjects tend to report peak pain rather than average pain scores in an end-of-day diary. The current findings may imply that pain is more prone to recall bias than other GI symptoms in IBS and may also be related to a considerable affective component in its perception. Although the overall scores for abdominal pain were higher in patients with panic disorder compared to the IBS group without panic disorder, only the presence of panic disorder in male subjects was found to be a significant predictor of a higher pain score difference between ESM and the end-of-day dairy. Furthermore, given the higher abdominal pain scores in IBS patients with panic disorder compared to those without it, anxiety could be seen as a trait factor, which may make it less dependent on momentarily fluctuating contextual influences. Another explanation could be that in IBS patients with affective conditions, alarm falsification and symptom amplification take place, leading to more bodily distress (e.g., pain) and defensive behaviour (e.g., flatulence). Thus, ESM delivers different insights into symptom formation compared to retrospective diaries. Although momentary assessment has not been used in functional urological or ENT disorders, ESM combined with, for example, urgency and assessment of bladder fullness sensations could certainly be expected to deliver new insight into diagnostic aspects and treatment follow-up monitoring in patients with complex functional urological disorders too.

Regarding feasibility, in the hospital psychiatry outpatient setting ESM demonstrated adequate psychometric properties, and compliance with the ESM app is considered acceptable. Given that it was a complex and thus transdiagnostic hospital psychiatry outpatient cohort, the constructs of positive affect (PA) and negative affect (NA) were selected on the basis of existing ESM literature ${ }^{339}$. Factor analysis of these items confirmed the structure of the two concepts, and the subsequent reliability 
analyses yielded excellent internal consistency. In order to assess the concurrent validity of mobile Routine Outcome Measurement (mROM), comparisons were made between the traditional ROM questionnaire used in the hospital (HADS; assessing anxiety and depression) and the ESM-moderated mROM protocol. Overall, significant and clinically relevant correlations were found between the PA and NA constructs, on the one hand, and the HADS (total, anxiety, and depression) scores, on the other. Although the measures show substantial overlap, the ESM-moderated mROM protocol makes a unique contribution to clinical routine outcome assessment in the sense that recall bias and contextual biases are controlled for to a great extent. In addition, the ecological measures within the mROM protocol inform on daily life adaptive functioning. Seven measures (PA, NA, positive social interactions, quality of sleep, activity-related stress, tiredness, and feeling unwell) that are considered relevant in mental health care were assessed and compared over baseline and follow-up. All measures changed significantly over time due to integrated treatment. Thus, the mROM measurements are sensitive to change over time. At follow-up, scores on NA were reduced, whereas scores on PA were increased. This overall sensitivity to change is in line with previous ESM research and further suggests that hospital patients indicated for psychosomatic care improve by multidisciplinarily embedded hospital psychiatry outpatient interventions ${ }^{340,341}$. In conclusion, the ESM-based, transdiagnostic mROM tool can be used reliably in clinical settings and will be introduced in settings for functional disorders soon.

Thus, a more flexible and insightful diagnostic approach might help to better map psychiatric and somatic comorbidities in functional disorders. Furthermore, momentary assessment also enables clinicians to increase multidisciplinary meta-communication in order to provide integrated care, which may result in improved treatment satisfaction and reduced health care costs ${ }^{171}$. In this context, a transitional Med-Psych-Network may facilitate integrated care across all settings. In addition, ESM-based personal data concerning symptoms, symptoms impacting on other symptoms, the dynamics of multiconditional complaints, and patterns of contextual reactivity yield uniquely personalized data to support decision making that is shared between various specialty consultants and, last but not least, between caregivers and their patients ${ }^{342}$.

\section{In conclusion}

Functional somatic conditions, such as IBS, OAB or MUORLS, are frequently seen cooccurring with comorbid affective conditions in the general hospital setting. This cooccurrence is due to the complexity aspects of functional disorders including affective comorbidities. Comorbid affective conditions increase with the number of functional complaints (e.g., IBS, OAB, fibromyalgia, chronic fatigue syndrome), from low rates at the general population level to $\sim 30 \%$ in primary care and up to $80 \%$ in an integrated (i.e., gastroenterology and psychiatry) hospital outpatient setting. This well-known comorbidity bias regarding the co-occurrence of disorders is associated with the fact that any illness is more likely to have comorbidities at greater levels of severity and 
complexity ${ }^{43,329,330}$. The question is whether these observations are indicative of separate but comorbid disorders, are epiphenomena, or are part of a single but complex illness that is recognized and described in isolation from distinct clinical perspectives (e.g., gastroenterology, urology or psychiatry). Among inpatients with complex conditions that are medically unexplained who show treatment refractoriness, the level of psychiatric comorbidity runs as high as $90 \%$ of admitted inpatients at an integrated multi-disciplinary unit (i.e., Med-Psych-Unit), whereas only $5 \%$ of these patients have been recognized as psychiatrically comorbid at the level of nonintegrated monodisciplinary medical wards ${ }^{20}$. Indeed, under-recognition of a complex psychosomatic multi-morbidity phenotype consisting of, for example, urological, gastrointestinal and affective comorbidities may contribute to a muddled understanding of complex medical conditions and thereby to treatment resistance ${ }^{38,331,332}$. However, a mono-disciplinary and exclusively descriptive manner of identifying complex (functional) syndromes, such as those statistical manuals or consensus protocols based solely on the description and enumeration of symptoms (e.g., ROME IV or DSM V), may not be conducive to a valid diagnosis and, subsequently, to the successful treatment of syndrome complexity ${ }^{243,343}$. Instead, complex functional somatic conditions with comorbid affective disorders are best understood as a sensitized response to earlier threats (notably hyperarousal or alarm falsification) resulting in perceived emotional and bodily distress. Besides psychological stressors, also physical threat contributes to alarm falsification in bodybrain crosstalk and therefore affects mood, cognition and defensive behaviour - hence, evoking the symptoms of affective and functional disorders ${ }^{38}$. As mentioned before, attempts to distinguish purely physical from solely mental disorders have become obsolete. All somatic diseases are known to be affected by mental factors, as manifest in susceptibility, onset, clinical presentation and treatment adherence. Furthermore, mental illness is strongly associated with somatic pathways ${ }^{344-348}$. Current concepts of complex conditions have since moved toward convergence with systemic views, whereby disorders present themselves as a pleiotropic or multi-morbidity phenotype. A systemic approach is better at explaining what is (dys-) functional in complex conditions. The current concepts suggest a clinical need for integrated care in order to recognize clinical complexity and to understand threat-related pathways of mental ill-health which may amplify somatic symptom formation ${ }^{38,346-350}$. In other words, functional somatic symptoms are inseparable from emotion (dys-) regulation, since anxiety and pain are emotional and sensory aspects of the same complex fight-or-flight response.

Despite all that progress, situations still arise in clinical experience where comorbid mental and somatic illnesses or related factors are met with misdiagnosis, delayed treatment or even serious adverse events in general hospital settings. Factors leading to misattribution in this regard include i) patients' complex presentation, ii) consultants' lack of complexity concepts, iii) patients' challenging behaviour, iv) clinical pressure due to a crowded medical ward environment, and last but not least, $v$ ) the stigmatizing attitude of some staff members ${ }^{20,351}$. In that context, some recommendations are in 
order; these are intended to help optimize the diagnosis and treatment of multiconditional patients with medical and psychiatric comorbidity in the general hospital setting. In the first place, it is advisable to have a pro-active psychiatric $C L$ team available 24 hours a day, a capacity which presently does not fall under standard care in all general hospitals. This recommendation concurs with in-hospital findings that a more pro-active CLS involvement may prevent comorbid patients from going unrecognized ${ }^{352}$. With regard to the subsequent transition back to primary care at the end of hospital stay, there is evidence that GPs' compliance with CLS' recommendations may be enhanced by direct communication between the hospital and primary care providers ${ }^{353}$. Secondly, it is advisable to prepare detailed multi-disciplinary guidelines for the assessment and treatment of multi-conditional patients. Thirdly, the road to integrated multi-disciplinary management would be to hold regular meetings with staff members from all departments and across all settings involved. This avenue should be taken at the inpatient and outpatient hospital level as well as in primary care to improve clinical deliberations ${ }^{20,43,354}$. Fourthly, the importance of the therapeutic relationship should be emphasized $^{355}$. Moving case managers (CMs) into the frontline of treatment rather than offering mental health or somatic consultation by consultants or GPs alone may create advantages by empowering the therapeutic relation between care givers and patients. In primary care and in the hospital, CMs could then spend more time with the patient, which may evoke better engagement, compliance and treatment adherence, a change in performance which eventually might translate into better outcomes and favourable economic evaluations $2,100,102$, chapter7. However, there are uncertainties about the effectiveness of primary care-based collaborative case management targeting complexity or severity in multi-morbidity ${ }^{102-105}$. These uncertainties remain in 'hard-core' somatic conditions (e.g., cancer or cardiovascular disease) with comorbid psychiatric disorders (e.g., affective disorders or delirium) as well as in medically unexplained physical symptoms associated with affective or somatoform conditions ${ }^{35,103,356}$. Especially in patients with severe somatic conditions, CC did not significantly reduce the occurrence of major adverse somatic events ${ }^{104}$. Thus, as multi-conditional or frequently utilizing patients generally present themselves in hospital settings at some point in their patient career, there is a need to pursue integrated care, preferably by means of CC, in order to address disease severity and complexity in the general hospital setting too. Moreover, the current body of evidence does not support the use of 'frontline' primary care givers in cases of functional somatic symptoms. The benefit may be confined to more intensive interventions with regard to change of functional outcome. Such interventions should include staff-guidance in view of the well-known bottlenecks in primary care: i) the limited amount of time for consultation; ii) lack of skills; iii) the need for a degree of diagnostic openness; and iv) patients' resistance to psychosomatic attributions $^{35}$. There is a role for hospital-based integrated care approaches, since the momentum is building for a much wider range of collaboration than between primary care-based CC and hospital-based CL services alone. The thrust toward integration is fed 
by the growing recognition of comorbid somatic and psychiatric disorders, their frequency and overlap, as well as multi-morbidity-related poorer treatment outcome and the greater costs of care ${ }^{35,357,358}$. Obviously, management of multi-morbidity should include staff-guided care managers, for example, physician assistants, nurse practitioners, psychologists, and if appropriate (i.e., for non-medical concerns) social workers. Integrated care and related scientific research could recognize and identify patients' natural organ-related symptom attribution but might also be able to overcome treatment refractoriness related to complexity ${ }^{38}$. Furthermore, integrated care should respect the clinical and economic interests of various specialty care givers involved in psychosomatic medicine, which might paradoxically have a societal impact, namely of reducing cost -- which is counterintuitive because more specialist involvement tends to make care more expensive. Therefore, integrated care should best be construed as a virtual transitional network instead of as colocated medical services. In the light of the complexity and severity of medical conditions ${ }^{38}$, the introduction of a transitional medical-psychiatric-network (Med-Psych-Net) may contribute to the integration of psychosomatic care across different medical settings without violating the diverse and sometimes contradictory interests in the field. It is acknowledged that observational clinical data are not suitable for identifying causal inference in psychiatric comorbidity. Nonetheless, our integrated transitional Med-Psych-Net provided data that consistently recognized clinical complexity at various clinical levels, yielding insights which may help to understand and treat the pathways of mental ill-health that amplify somatic symptom formation - and vice versa ${ }^{20,38,43,359}$. Besides improving diagnostic skills, the advantage of integrated care has also been demonstrated with regard to patient-related outcomes and cost savings ${ }^{43, \text { chapter } 7 \& 15}$. Hence, a clinical network approach driven by aetiologybased medical concepts may help to establish integrated care, specifically by connecting specialty outpatient clinics in the hospital and bridging the gap between the hospital and primary care. This may be cost-effectively realized via medical staff-guided transitional CMs without compromising the quality of medical consultants' aetiology-based concept of care.

Based on the Med-Psych-Net as a transitional care platform, the introduction of experience sampling-based $\mathrm{m}$-health tools could contribute to a meaningful communication between multi-conditional patients and their care givers. ESM-based patient data -- notably concerning symptoms, symptoms impacting on other symptoms, and the dynamics of multi-conditional complaints, as well as patterns of contextual symptom reactivity -- may yield uniquely personalized profiles to support decision making on a platform that is shared between various specialty consultants and, crucially, between care givers and their patients ${ }^{32,360, \text { chapter15 }}$. The body of momentary assessment data may deliver less-biased insight in patients' stress-related experiences and elucidate how environmental threat may contribute to symptom formation. These insights may improve clinical reasoning with regard to psychotherapeutic possibilities, such as graded exposure and response prevention in situations of fear-related avoidance behaviour. On 
the other hand, the scientific understanding of multi-conditional pathways may profit from ESM-data too, especially if the course of complex symptoms in relation to treatment can be combined with repeatedly measured biomarkers or epigenetic changes. Eventually, ESM may serve as a reliable patient-related outcome measurement (PROM) tool, which may prepare the ground for reimbursement changes in the future, i.e., payment for performance instead of payment for service, that might help to overcome the current reimbursement dichotomy between soma and psyche chapter15,342,361.

\section{Valorisation}

This PhD thesis presents a series of studies and articles with diverse objectives: i) to investigate and describe the effect of psychosomatic integrated care on complex (functional) conditions at various clinical levels; ii) to develop an integrated transitional care model, i.e., the Med-Psych-Net, that enables care givers to accompany multiconditional patients from the hospital to primary care and vice versa, in order to overcome the disadvantages of mono-disciplinary trajectories; iii) to conceptualize a system-based etiologic nosology of complex (functional) syndromes; and iv) to introduce a modern m-health tool, the ESM, that may advance the development of tailored personalized medicine, clinical reasoning and the scientific understanding of pleiotropic multi-morbidity.

This valorisation section places the outcomes of this thesis in a broader societal context in order to address how this transitional Med-Psych-Net may be further implemented in daily clinical practice. Its societal importance will be considered from three perspectives: 1) the relevance of scientific findings for clinical practice; 2) the clinical target groups to whom the findings are relevant; and 3) the translation of the findings into Med-Psych-Net activities concerning innovativeness, feasibility, implementation and perspectives for future research.

\section{Relevance}

Functional somatic disorders as well as mental disorders are highly prevalent ${ }^{362-364}$. Data from epidemiological studies indicate that depression and anxiety disorders as well as, for instance, functional urological and related gastrointestinal disorders are common comorbidities having adverse effects on patients' outcomes ${ }^{59,88,286,287,290,313,365-367}$. Moreover, the common association between functional urological and gastrointestinal disorders affects the severity of experienced physical and mental symptoms in a dosedependent fashion ${ }^{236-242,287,368,369}$. Additionally, the prevalence of anxiety disorders and depression increases with the number of functional disorders and the frequency or severity of functional somatic symptoms ${ }^{284,288,312,313,315,370}$. Thus, the more severe the 
somatic symptoms are, the more prevalent the affective complaints become. Pain and depression are closely associated ${ }^{281,282,284-287,307,310,312}$. Affective complaints might therefore complicate or amplify existing functional complaints, and the onset of anxiety and depressive symptoms might precede that of, for example, urological functional disorders ${ }^{59,371,372}$. Similarly, depression and anxiety both dose-dependently increase the risk of developing urinary incontinence, and the number of neuroticism-associated conditions (i.e., functional as well as affective disorders) might be a marker of a complex psychosomatic multi-morbidity phenotype $239,281,285,295,313,373,374$. Thus, in cases of (functional) clinical complexity, psychiatric comorbidity has to be taken into account in order to avoid misdiagnosis and treatment resistance. However, in the medical hospital, where complex cases will present themselves at a certain point in a patient's career, clinical complexity, including psychiatric comorbidity, is frequently under-detected. For example, at the emergency department, in the outpatient medical hospital setting and in clinical general hospital wards, affective comorbidities frequently go undetected, which has an impact on care utilization later on ${ }^{17,20,43}$. This also applies to the most complex and severely ill patients in the intensive care unit (ICU), where psychiatric case detection concerning transitional care for delirium, which is traditionally considered organic and not functional, still deserves attention ${ }^{375}$.

Multi-morbidity and the characteristics of health care providers are predictors for complications of hospital interventions. Therefore, the length of hospital stay and the rate of unplanned hospital readmission are the main medical cost factors in complexity care that need to be kept in mind with regard to reducing the societal cost of care at the hospital level ${ }^{20,376-380}$. The need for this focus has become more and more obvious; the percentage of hospital stays for multi-conditional patients increased between 2003 and 2014 in the US from approximately $65 \%$ to $80 \%$, whereas hospital stays for adults without multi-conditional complaints decreased from roughly $35 \%$ to $20 \%$ during the same period $^{381}$. Moreover, given that hospital stays for adult patients with multiple conditions cost on average $20 \%$ more than stays for inpatients with a mono-conditional background, it would seem quite reasonable to pursue enhanced integrated hospital and transitional care from a hospital cost perspective ${ }^{381}$. Furthermore, integrated care has to incorporate psychosomatic complexity, as almost one-third of inpatient hospital stays in the US involved mental disorders in 2012, whereby affective disorders were the most common mental disorder diagnoses ${ }^{379}$. Comparable trends have been noted for unplanned readmissions in the US: readmission rates increased substantially between 2009 and 2013, and their average cost was higher than the average cost of index admissions $^{382}$. This is also true for readmissions involving mood disorders compared with initial hospital stays ${ }^{383}$. Thus, hospital readmissions within 30 days of discharge represent a negative clinical outcome. They might be due to a lack of integrated hospital care solutions and poor access to adequate primary or community-based aftercare. Clearly, transitional integrated approaches concerning multi-morbidity in complex psychosomatic syndromes face a challenge ${ }^{156,384,385}$. The level of comorbidity care and the 
quality of illness management across settings are very relevant to attempts to avoid every kind of hospital readmission ${ }^{386-388}$. This consideration is indirectly supported by our own hospital evaluations (MUMC) concerning wards which are still running without a transitional network approach, especially without routinely integrated psychosomatic care for patients with a high chance of medical and psychiatric comorbidities ${ }^{174,175}$.

\section{Target groups}

Besides complex multi-conditional patients, health care providers from in- and outside the general hospital and the patients' environment (i.e., family and social network) are important target groups for integrated transitional care in psychosomatic medicine. From our clinical experience, patient and social network empowerment due to a "warm handover" across settings by case managers as well as the availability of medical skills guaranteed by integrated medical staff-guidance are essential. Transitional care by case managers can be effectively realized by medical staff-guided care givers from outside the MUMC, as suggested by our investigation ${ }^{\text {chapter7 }}$, or instead by multi-disciplinarily guided care managers related to general hospital-based disease management programmes. Primary care-based collaborative care is very well suited to long-distance communication between care givers and patients in rural areas. But the Med-Psych-Net approach may be especially productive in urban areas, where complex patients accumulate at the hospital level and where GPs as well as mono-disciplinary general hospital-related treatment options might fail. Depending on the severity and complexity of patients' multi-conditional complaints, the Med-Psych-Net approach would start in the ICU setting, for example, in multi-disciplinary delirium care (i.e., proactive CLS involving integrated multi-disciplinary medical staff-guidance and transitional case management by nurse practitioners), or at the MPU and at medical wards for comorbidity care. Most importantly, all clinical settings require guided $\mathrm{CM}$ in order to accompany inpatients from care at a higher to a lower level of complexity, depending on the patients' state of clinical severity. In complex but less severe multi-conditional cases, integrated multidisciplinary care would start with the integrated outpatient setting (e.g., the pelvic care centre, the multi-disciplinary pain team) for comorbid (functional) somatic and psychiatric conditions, as evidenced by the findings reported in this

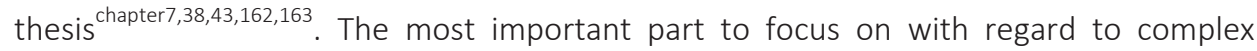
psychosomatic patients concerning LOS, unplanned hospital readmissions and frequent outpatient care utilization is the transition of inpatient or outpatient hospital care back to primary care ${ }^{\text {chapter7 }}$. For instance, in complex patients with a pleiotropic presentation of functional somatic complaints, the successful transition of care after intensive outpatient-based psychotherapy may profit from integrated staff-guided CM back to the patients' GPs ${ }^{35,38, \text { chapter7 }}$. Moving case managers into the frontline of care transition may improve the engagement, compliance and treatment adherence of patients, whose performance may eventually translate into better outcomes and more favourable economic evaluations ${ }^{2,100,102, \text { chapter7 }}$. 


\section{The Med-Psych-Net - innovativeness, feasibility, implementation and future perspectives}

To avoid unintended negative outcomes due to under-detection of psychosomatic complexity or treatment refractoriness in mono-disciplinary care trajectories, integration of care (i.e., bringing together diverse specialty skills and expertise concerning health care in cases of multi-morbidity with comorbid psychiatric and somatic disorders) is deemed necessary at the hospital level and between hospital and primary care. Different organizational aspects of integrated care should be taken into account. The presence of multi-disciplinary teams is indispensable. Furthermore, shared multi-disciplinary clinical protocols and a specialty-transcending information system (with electronically accessible patient files) have to be available. Colocated work would be the ideal arrangement for co-operating services; if not feasible, liaison services operating bi-directionally would be needed to approach the multi-conditional patient. In order to guarantee clinical expertise on a consultant level, navigators (medical staff) and 'steersmen' (care managers) should deliver guidance on the clinical course, either in face-to-face contact with patients and/or care givers or via digital information transferred to patients and their care managers. Furthermore, a multi-component strategy should include efforts to combat stigmatization concerning psychiatric comorbidity ${ }^{21}$.

Various clinical scenarios may illustrate how access to health care can influence patients' outcome negatively. Both low and high levels of access can lead to unplanned readmissions. Patients who are vulnerable from a psychosocial point of view and also show complex multi-morbidity may easily relapse, even if they had received high-quality inpatient care ${ }^{389}$. When these patients receive outpatient care after discharge, they might be less likely to seek timely appointments and more likely to enter the emergency room (ER $)^{389-391}$. With a low level of access to resources that enable self-care and outpatient follow-up, these patients are at a higher risk of readmission and stay in the hospital. Paradoxically, hospitals with expanded access to post-discharge health services also see a rise in unintended readmissions, since increased health care utilization may also occur in multi-conditional patients who lack the resources that enable self-care and are therefore at higher risk of relapse ${ }^{389,390}$. Thus, taking care of the patient and not just the disease is imperative in order to reduce costly hospital stays and unplanned readmissions ${ }^{20,392}$. Primary care-based CM through collaborative care leaves problems with physical well-being unsolved and it is not advisable to treat complex functional conditions in primary care. The solution seems to lie in a hospital-based integrated care model, which incorporates medical staff-guided case management, leading vulnerable complex patients back to primary care ${ }^{20,35,103,104}$. Therefore, we decided to create a psychosomatic network approach, which might be able to increase the patient flow through the hospital and could help bridge the gap between hospital and primary care in order to reduce length of stay or the rate of admissions ${ }^{43,162, \text { chapter } 7,393}$.

The Med-Psych-Net consists of the inpatients' MPU, a proactive CLS, different outpatient-based liaison activities (e.g., the multi-disciplinary pain team, the pelvic care 
centre) and most importantly the staff-guided CM-based transition of care to GPs. The engineering of care applications that would improve the affordability of hospital care has to incorporate mechanisms that enhance in- and outpatient flow through the medical hospital without proportionately adding staff $^{393}$. Our Med-Psych-Net approach succeeded in that sense. Cost savings have been booked without changing the hospital's fulltime-equivalents among the psychiatry or medical psychology staff, since community mental health service care mangers started to join hospital and primary care during that period. With regard to the Med-Psych-Net, it should be kept in mind that these CMHS care givers were only colocated in the MUMC and at GPS and were not additionally employed. For clarification, we engineered a care application that improved the affordability of hospital care via a guided CM mechanism, which enhanced the flow of outpatients through the medical hospital without proportionately adding staff. The MedPsych-Net is the first comprehensive transitional health care network to address psychosomatic multi-morbidity across various health care settings, starting by picking up patients face-to-face at the medical hospital, as community mental health services' case managers ease the transition from hospital outpatient settings to primary care. In other words, the journey of complex and vulnerable multi-conditional patients across the health care (dis-) continuum has been finalized by placing the role of the hospital within the community context ${ }^{394,395}$. Most programmes targeting care transition assume that hospital readmissions can be reduced by patient empowerment and improved relationships between the hospital and post-acute care settings ${ }^{394}$. The MPN adds another dimension which should not be underestimated: a clinical network approach based on etiologic medical concepts and implemented by medical staff-guidance. The approach might help establish integrated care by connecting specialty outpatient clinics in the hospital. It may also bridge the gap between the hospital and primary care via medical staff-guided transitional care managers without compromising the quality of consultants' etiologic conceptualization or treatment. Thus, in order to guarantee medical expertise across different settings, the MPN facilitates psychosomatic specialty skills in primary care and, vice versa, assures that the general practitioners have an overview of their patients' experiences in the medical hospital. This does not only happen via CMs as a link but also by multi-disciplinary direct or telephone/internetbased medical staff consultations independent from the setting. 
Figure 16.3 The Med-Psych-Net - a transitional network approach fostering personalized care in psychosomatic medicine.

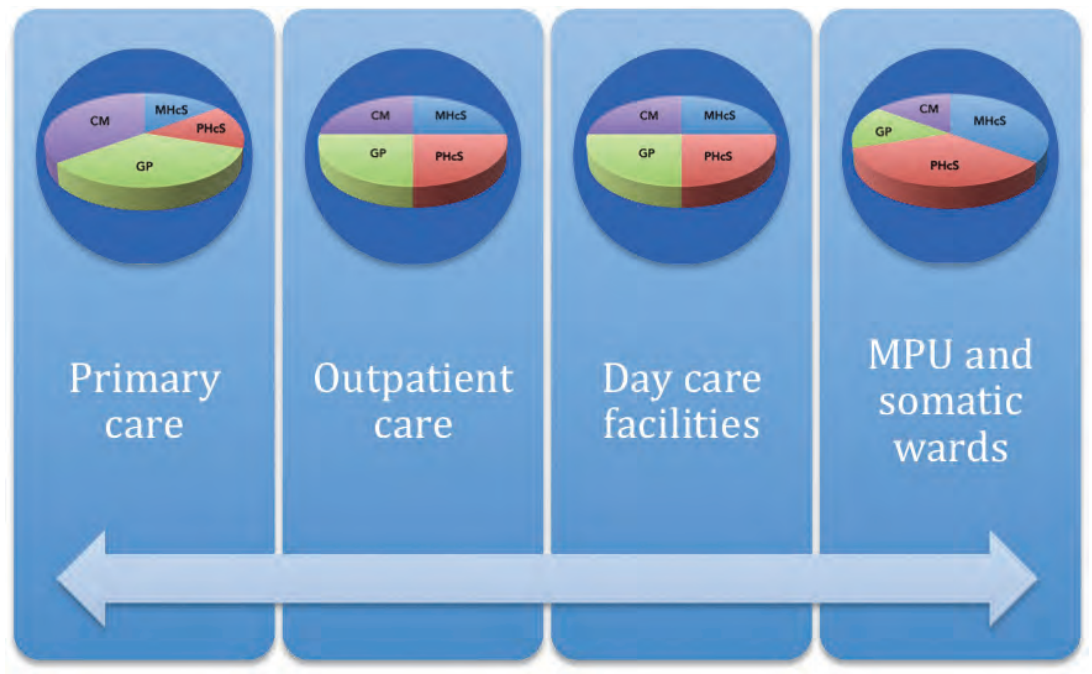

Legend: A Med-Psych-Net (MPN) is a virtual network of co-operating care givers who are treating patients with complex psychosomatic conditions, referring them from primary care to the hospital and vice versa. Care givers involved in this integrated approach provide treatment in different proportions depending on the severity and complexity of disease. Patients with the most severe conditions are admitted to the integrated hospital inpatient unit (Med-Psych-Unit (MPU)) or to somatic wards in co-operation with the mental health care consultation-liaison service (CLS). Patients with less impairment but whose medical and/or bio-psychosocial problems are still severe are followed on a day care or outpatient basis. The hospital-related integrated care, ranging from outpatient to inpatient interventions, is covered by the hospital-based Med-Psych-Centre (MPC). Transition of the patient back to primary care takes place at suitable moments when the roles of case manager (CM) and general practitioner (GP) can become more prominent. PHcS = Physical Health care Specialist; $\mathrm{MHcS}=$ Mental Health care Specialist.

In order to assess the feasibility of the MPN approach, it is necessary to chart the bottlenecks in psychosomatic complexity care related to primary as well as medical hospital care. As mentioned above, several bottlenecks in primary care are well known: i) the limited consultation time of general practitioners; ii) lack of GPs' specialty skills; iii) the need for a degree of diagnostic openness in psychosomatic patients; and iv) patients' stigma-related resistance to psychosomatic attributions ${ }^{35}$. But others are less well known and are discussed in this thesis. As clinical experience reveals, comorbid mental and somatic illnesses may lead to misdiagnosis, delayed treatment and serious adverse events in the general hospital setting. Several factors have led to misattribution in this regard: i) patients' complex presentation; ii) consultants' lack of concepts concerning complexity; iii) patients' challenging behaviour; iv) clinical pressure due to a crowded medical ward environment; v) patients' resistance to psychosomatic explanations; and 
last but not least, vi) the stigmatizing attitude of some staff members ${ }^{20,351}$. In that context, some recommendations may be made to optimize the diagnosis and treatment of multi-conditional patients in the general hospital. In the first place, a pro-active psychiatric CL team has to be available 24 hours a day. This recommendation concurs with in-hospital findings that a pro-active CLS involvement may prevent comorbid patients from going unrecognized ${ }^{352}$. Regarding the subsequent transition back to primary care at the end of hospital stays, GPs' compliance with CLS' recommendations may be enhanced by direct communication between the hospital and primary care providers $^{353}$. Secondly, detailed multi-disciplinary guidelines for the assessment and treatment of multi-conditional patients should be prepared. Thirdly, the hallmark of integrated multi-disciplinary management is to hold regular meetings with staff members from all departments and across all of the settings involved. This should be realized at the in- and outpatient hospital level as well as in primary care to improve clinical reasoning ${ }^{20,43,354}$. Fourthly, the importance of the therapeutic relationship should be emphasized by moving case managers into the frontline of treatment rather than offering mental health or somatic consultation by consultants or GPS alone $^{2,100,102,355, \text { chapter } 7}$. Last but not least, given the patients' resistance to accepting psychosomatic explanations across all care levels, multi-disciplinary staff-guidance of CMs might help to overcome stigmatization regarding psychosomatic conditions and may guarantee the level of medical expertise across different settings.

Future health care delivery and payment reforms may affect the care of complex multi-conditional patients more than programmes addressing clinical problems in monodisciplinary settings. Bundled payment for services providing care across settings (i.e., hospital stay and readmission reduction programmes targeting care transition to primary care) should align incentives for hospitals and primary care ${ }^{394,395}$. Given the exploding health care budget and the waiting lists for various hospital interventions in the Netherlands, the Med-Psych-Net approach may achieve multiple goals of care delivery; therefore, it deserves integrated reimbursement. The increasing patient flow through hospitals achieved by the transition of care may reduce frequent care utilization, the length of hospital stay and the amount of unplanned readmissions. Besides achieving favourable patient-related outcomes, the transitional MPN approach could reduce the cost of health care, which might have a societal impact without violating care givers' interests. For instance, the objection voiced by hospitals regarding a loss of income is unfounded. A decrease in the length of hospital stay and in readmissions is accompanied by an increase in first patient consultations at the medical hospital, as evidenced by existing waiting lists. Since health care policy and delivery is increasingly influenced by reimbursement changes to attenuate the health care-related financial burden, new models of care should be evaluated scientifically, taking special note of quality and safety, to minimize unintended outcomes. Thus, future research on care delivery concerning complex or multi-conditional (functional) disorders should take a perspective of complexity science as its point of departure ${ }^{380}$. Disease severity and complexity is 
strongly associated with the intensity and frequency of care activities during patients' index admission but also with unintended readmissions ${ }^{380,386}$. The lack of improvement in readmission rates over the past few years indicates that the relationships between risk factors, interventions and intended outcomes are more complex than hitherto acknowledged $^{380,382}$. In addition to current research efforts conducted with an eye to reducing hospital admissions or length of stay, a better scientific approach would entail studying intended outcomes of complex (functional) patients through the lens of complexity science ${ }^{380}$. Its focus is on the identification of participating 'agents', the unpredictability of agent actions, the interactions between multiple agents, and their effect on intended outcomes. In a complex system, it is deemed necessary to look beyond single causative factors and an expected linear response to interventions ${ }^{380}$. By this reasoning, the unintended outcome of unplanned readmissions or a long hospital stay is a result of a series of complex interactions among multiple agents. Hence the need to identify the factors in play: i) the complexity and severity of the underlying disease; ii) patients' openness to a psychosomatic diagnosis; iii) quality of care delivery (at all health care levels) including staffing; iv) co-ordination and continuity of care across settings; v) communication with care participants (between patients and care givers and mutually between care givers); and vi) assessment of the environment into which the patient is discharged ${ }^{380}$. The ability to initiate and sustain an integrated transitional care programme in psychosomatic medicine is increasingly important since the patients' psychosocial factors contribute significantly to the risk of unplanned readmission ${ }^{396}$. The transitional Med-Psych-Net may fulfil its expectations concerning its bridging function. However, the intended outcomes have to be further evaluated with complexity science models. Moreover, a tailored and therefore personalized care model should be constructed by incorporating modern m-health tools into transitional integrated care. Doing so may enable patients and care givers to investigate environmental influences on symptom formation and ways to prevent or overcome stressful threatening events. In this regard, the experience sampling methodology proves to be a valid tool for momentary assessment in complex psychosomatic

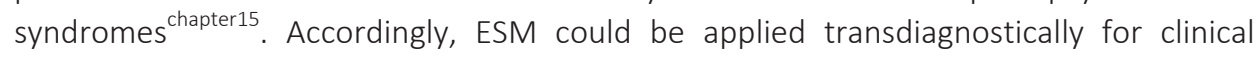
purposes to gain insights that can be used therapeutically, such as in psychotherapeutic trajectories concerning exposure and response prevention in situations of exaggerated harm avoidance. In addition, repeated measures over time using ESM could help to unravel complexity pathways by applying network analysis methodology. In psychiatry, network analysis is currently used to move away from a concept of symptom-based diagnostics in the direction of elementary syndromes. Apparently, 'bridge symptoms' seem to be responsible both for combining symptoms into syndromes and for combining various syndromes into a network structure ${ }^{397}$. In that sense, complexity science may not only improve the efficacy of network approaches with regard to care delivery but might also explain comorbidity patterns. Therefore, network models may predict the clinical course of multi-morbidity phenotypes and might thus help to designate state- and 
severity-related targets for complexity interventions. As in psychosis, affective symptoms may act as central network symptoms ${ }^{398}$, thereby moderating or amplifying complex (functional) somatic complaints and care utilization accordingly since stress-related disorders are associated with an increase in direct and indirect costs, depending on the complexity (i.e., comorbidity) and the severity of symptoms ${ }^{399,400}$. Regarding potential causation, cumulative serious life threat has been linked with both mental and physical complaints and is associated with an increased number of doctor visits and hospital admissions $^{401}$. However, the individual variation in the temporal relationship between stress and complex functional somatic conditions and the dynamics of the body's stress matrix warrant further investigation to elucidate the temporal complexity between stress and multi-conditional complaints over time ${ }^{402,403}$. Subjecting temporal complexity to time series analysis might contribute to the discovery of complexity pathways and, more importantly, to patient-tailored treatment ${ }^{402}$. A complex functional somatic syndrome shows very high frequencies of comorbid mental disorders at the specialized hospital outpatient level (i.e., affective conditions and somatoform disorders presenting with almost equal prevalence). In that light, the presence of multiple physical symptoms is longitudinally associated with the recurrence of affective conditions at the community, primary care or mental health care level. The question then arises: What comes first the chicken or the egg (i.e., complex (functional) somatic or affective complaints) ${ }^{42,404-}$ ${ }^{406}$ ? Following patients through various health care settings (e.g., via the Med-Psych-Net) by using the same patient-related mobile/e-health assessment tool across medical settings (e.g., ESM) might elucidate the sequence of complexity development and help answer the next question: What is (dys-) functional in complex functional somatic syndromes? From a systemic point of view, complex functional somatic conditions with comorbid affective disorders are probably best understood as a sensitized defence response to earlier threats resulting in perceived emotional and bodily distress (i.e., hyperarousal). Besides psychological stressors, physical threat contributes to an alarm falsification in the body-brain crosstalk, affecting mood, cognition and defensive behaviour -- the symptoms of affective and functional somatic disorders ${ }^{38}$. In order to further investigate the relation between contextual threat and symptom formation, a feasible and reliable momentary assessment tool for diagnostic and therapeutic purposes (i.e., ESM) can be implemented. ESM has been shown to be a reliable transdiagnostic mROM tool, which also can be used to facilitate shared decision making and monitoring of personalized treatment in psychosomatic medicine ${ }^{\text {chapter15. }}$.

With regard to integrated complexity treatment, MPUs embedded in a transitional Med-Psych-Net might help to reassess the consistent evidence for the negative association between concomitant physical symptoms and affective conditions in order to turn the course of affective conditions at the highest level of complexity. Moreover, the Med-Psych-Net may add value in the light of the fragile consensus between patients with comorbid conditions and health care professionals on the preventability of costly readmissions ${ }^{20,37,407-409}$. At moments of care transition, staff-guided case management 
supported by ESM can take over the care, eventually leading to primary care-based collaborative care ${ }^{\text {chapter } 7 \& 15}$. In the temporal relationship between stress and complex functional somatic conditions, the most care-relevant moment might be when complexity gets started. At that moment of initial complexity, the exclusive administration of web-based telemedicine or m-health management is a scenario that deserves further investigation, even with regard to multi-conditional complaints. The rationale is that patients with an initial but increasing somatic symptom burden rise instantly to the same level of health care utilization as patients with chronic physical symptoms ${ }^{410-412}$. Alternatively, immediate involvement of an integrated Med-Psych-Net approach should be considered if patients and primary care givers need medical staffguided CM to establish psychosomatic attributions. 


\section{References}

1. Sharpe, $M$ \& Naylor, C. Integration of mental and physical health care: From aspiration to practice. Lancet Psychiatry 3, 312-313 (2016).

2. Kroenke, K. \& Unützer, J. Closing the false divide: Sustainable approaches to integrating mental health services into primary care. J. Gen. Intern. Med. 32, 404-410 (2016).

3. Kathol, R. G. Cost outcomes on a medical psychiatry unit. J. Psychosom. Res. 68, 293-294 (2010).

4. Hall, R. \& Kathol, R. Developing a level III/IV medical psychiatric unit: Establishing a basis, design of the unit and pshysician responsibility. Psychosomatics 33, 368-375 (1992).

5. Campo, J. V. \& Raney, D. The pediatric medical-psychiatric unit in a psychiatric hospital. Psychosomatics 36, 438-444 (1995).

6. Nomura, S. et al. Evaluation of the first medical psychiatric unit in Japan. Psychiatry Clin. Neurosci. 50, 305-308 (1996).

7. Fennig, S. \& Fennig, S. Management of encopresis in early adolescence in a medical psychiatric unit. Gen. Hosp. Psychiatry 21, 360-367 (1999).

8. Kishi, Y. \& Kathol, R. Integrating medical and psychiatric treatment in an inpatient medical setting: The type IV program. Psychosomatics 40, 345-355 (1999).

9. van Waarde, J. et al. The medical psychiatric unit: Added value for patients, physicians and hospitals. Ned. Tijdschr. Geneeskd. 148, 209-211 (2004).

10. Hanna, S. J. et al. The coming of age of a joint elderly medicine-psychiatric ward: 18 years experience. Int. J. Clin. Pract. 62, 146-151 (2008).

11. Honig, A. et al. A medical-psychiatric unit in a general hospital: Effective combined somatic and psychiatric care? Ned Tijdschr. Geneeskd. 158, A6520 (2014).

12. Young, L. \& Harsch, H. H. Length of stay on a psychiatry medicine unit. Gen. Hosp. Psychiatry 11, 31-35 (1989).

13. Goldberg, R. \& Simundson, S. Managing Medicare reimbursement on medical psychiatry units. Gen. Hosp. Psychiatry. 13, 313-318 (1991).

14. Üstün, T. B. et al. Global burden of depressive disorder in the year 2000. Br. J. Psychiatry 184, 386-392 (2004).

15. Meyer, T. et al. Depression but not anxiety is a significant predictor of physicians' assessments of medical status in physically ill patients. Psychother. Psychosom. 69, 147-154 (2000).

16. Kroenke, K. et al. Depressive and anxiety disorders in patients with physical complaints: Clinical predictors and outcome. Am. J. Med. 103, 339-347 (1997).

17. Sharpe, M. et al. Is co-morbid depression adequately treated in patients repeatedly referred to specialist medical services with symptoms of a medical condition? J. Psychosom. Res. 72, 419-421 (2012).

18. Robinson, R. L. et al. Covariates of depression and high utilizers of healthcare: Impact on resource use and costs. J. Psychosom. Res. 85, 35-43 (2016).

19. Schieveld, J. N. et al. Pediatric illness severity measures predict delirium in a pediatric intensive care unit. Crit. Care Med. 36,1933-1936 (2008).

20. Leue C, et al. Managing complex patients on a Medical Psychiatric Unit: An observational study of university hospital costs associated with medical service use, length of stay and psychiatric intervention. J. Psychosom. Res. 68, 295-302 (2010).

21. Rodgers, M. et al. Integrated care to address the physical health needs of people with severe mental illness - a rapid review. Southampton (UK): NIHR Journals Library (2016).

22. Hussain, M. \& Seitz, D. Integrated models of care for medical inpatients with psychiatric disorders: A systematic review. Psychosomatics 55, 315-325 (2014).

23. Gemmill, E. H. \& McCulloch, P. Systematic review of minimally invasive resection for gastro-oesophageal cancer. Br. J. Surg. 94, 1461-1467 (2007).

24. Kane, R. L. et al. The association of registered nurse staffing levels and patient outcomes: Systematic review and meta-analysis. Med. Care. 45, 1195-1204 (2007).

25. Kinder, L. S. et al. Improving depression care in patients with diabetes and multiple complications. J. Gen. Intern. Med. 21, 1036-1041 (2006). 
26. Katon, W. J. et al. The Pathway Study: A randomized trial of collaborative care in patients with diabetes and depression. Arch. Gen. Psychiatry 61, 1042-1049 (2004).

27. Wilson, T. \& Holt, T. Complexity and clinical care. BMJ. 323, 685-688 (2001).

28. Stewart, M. Continuity, care and commitment: The course of patient-clinician relationships. Ann. Fam. Med. 2, 388-390 (2004).

29. de Jonge, P. et al. Care complexity in the general hospital: Results from a European study. Psychosomatics 42, 204-212 (2001).

30. Kathol, R. et al. Epidemiologic trends and costs of fragmentation. Med. Clin. N. Am. 90, 549-572 (2006).

31. Huyse, F. J. et al. Identifiers, or "red flags," of complexity and need for integrated care. Med. Clin. North Am. 90, 703-712 (2006).

32. Lobo, E. et al. Identification of components of health complexity on internal medicine units by means of the INTERMED method. Int. J. Clin. Pract. 69, 1377-1386 (2015).

33. Huffman, J. C. et al. Essential articles on collaborative care models for the treatment of psychiatric disorders in medical settings: A publication by the academy of psychosomatic medicine research and evidence-based practice committee. Psychosomatics 55, 109-122 (2014).

34. Hiller, W. et al. A controlled treatment study of somatoform disorders including analysis of healthcare utilization and cost-effectiveness. J. Psychosom. Res. 54, 369-380.

35. Rosendal, M. et al. Enhanced care by generalists for functional somatic symptoms and disorders in primary care. Cochrane Database Syst. Rev. 10, CD008142 (2013).

36. Janse, B. et al. Delivering integrated care for the frail elderly: The impact on professionlas' objective burden and job satisfaction. Int.J. Integr. Care 16:7 (2016).

37. van Schijndel, M. A. et al. Medical-psychiatric units in the Netherlands: An investigation into distribution and quality. Ned. Tijdschr. Geneeskd. 161, D890 (2017).

38. Leue, C. et al. Functional urological disorders: A sensitized defence response in the bladder-gut-brain axis. Nat.Rev. Urol. 14, 153-163 (2017).

39. Sood, R. \& Ford, A. C. Rome IV criteria for FGIDs - an improvement or more of the same? Nat. Rev. Gastroenterol.Hepatol.13, 501-502 (2016).

40. Van Dessel, N. et al. The PROSPECTS study: Design of a prospective cohort study on prognosis and perpetuating factors of medically unexplained physical symptoms (MUPS). J. Psychosom. Res. 76, 200206 (2014).

41. Verdonschot, R. J. et al. Symtoms of anxiety and depression assessed with the Hospital Anxiety and Depression Scale in patients with oropharyngeal dysphagia. J. Psychosom. Res. 75, 451-455 (2013).

42. Brünahl, C. et al. Mental disorders in patients with chronic pelvic pain syndrome (CPPS). J. Psychosom. Res. 98, 19-26 (2017).

43. Kruimel, J. et al. Integrated medical-psychiatric outpatient care in functional gastrointestinal disorders improves outcome: A pilot study. Eur. J. Gastroenterol. Hepatol. 27, 721-727 (2015).

44. Fink, P. et al. Somatization in primary care. Prevalence, health care utilization, and general practitioner recognition. Psychosomatics 40, 330-338 (1999).

45. Hahn, S. R. et al. The difficult doctor-patient relationship: Somatization, personality and psychopathology. J. Clin. Epidemiol. 47, 647-657 (1994).

46. Smith, B. J. et al. The identification in primary care of patients who have been repeatedly referred to hospital for medically unexplained symptoms: a pilot study. J. Psychosom. Res. 67, 207-211 (2009).

47. Smith, R. C. \& Dwamena, F. C. Classification and diagnosis of patients with medically unexplained symptoms. J. Gen. Intern. Med. 22, 685-691 (2007).

48. Ferrari, S. et al. Vertigo "in the pink": The impact of female gender on psychiatric-psychosomatic comorbidity in benign paroxysmal positional vertigo patients. Psychosomatics 55, 280-288 (2014).

49. http://www.cbo.nl

50. http://kims.orde.nl/het-kennisinstituut-voor-u/richtlijnen/

51. Murray, A. M. et al. Barriers to the diagnosis of somatoform disorders in primary care: Protocol for a systematic review of the current status. Syst. Rev. 2, 99 (2013).

52. Murray, A. M. et al. The challenge of diagnosing non-specific, functional, and somatoform disorders: A systematic review of barriers to diagnosis in primary care. J. Psychosom. Res. 80, 1-10 (2016). 
53. Milsom, I. et al. Epidemiology of urinary incontinence (IU) and other lower urinary tract symptoms (LUTS), pelvic orgen prolapse (POP) and anal incontinence. In: Abrams P, editor. Incontinence 5th ed, 15108 (2013).

54. Luber, K. M. et al. The demographics of pelvic floor disorders: Current observations and future projections. Am. J. Obstet. Gynecol. 184, 1496-1501 (2001).

55. Davis, K. J. et al. Pelvic floor dysfunction: A scoping study exploring current service provision in the UK, interprofessional collaboration and future management priorities. Int. J. Clin. Pract. 64, 1661-70 (2010).

56. Berghmans, B. et al. Prevalence and triage of first-contact complaints on pelvic floor dysfunctions in female patients at a Pelvic Care Centre. Neurourol. Urodyn. 35, 503-508 (2015).

57. Coyne, K. S. et al. National community prevalence of overactive bladder in the United States stratified by sex and age. Urology 77, 1081-1087 (2011).

58. Abrams, P. et al. The standardisation of terminology in lower urinary tract function: Report from the standardisation sub-committee of the International Continence Society. Urology 61, 37-49 (2003).

59. Vrijens, D. et al. Affective symptoms and the overactive bladder: A systematic review. J. Psychosom. Res. 78, 95-108 (2015).

60. Melville, J. L. et al. Incontinence severity and major depression in incontinent women. Obstet. Gynecol. 106, 585-592 (2005).

61. Leue, C. et al. Bidirectional associations between depression/anxiety and bowel disease in a population based cohort. J. Epidemiol. Community Health 59, 434 (2005).

62. Fukudo, S. et al. Evidence-based clinical practice guidelines for irritable bowel syndrome. J. Gastroenterol. 50, 11-30 (2015).

63. Fink, P. et al. The prevalence of somatoform disorders among internal medical inpatients. J. Psychosom. Res. 56, 413-418 (2004).

64. Chatoor, D. et al. Organising a clinical service for patients with pelvic floor disorders. Best Pract. Res. Clin. Gastroenterol. 23, 611-620 (2009).

65. Zigmond, A. S. \& Snaith, R. P. The hospital anxiety and depression scale. Acta Psychiatr Scand. 67, 361370 (1983).

66. Clemens, J. Q. et al. The MAPP research network: A novel study of urologic chronic pelvic pain syndromes. Bmc Urol. 14, doi: 10.1186/1471-2490-14-57 (2014).

67. Cardoso, G. et al. Depression and anxiety symptoms following cancer diagnosis: A cross-sectional study. Psychol. Health Med. 21, 562-570 (2016).

68. Coyne, K. S. et al. The impact of overactive bladder on mental health, work productivity and healthrelated quality of life in the UK and Sweden: Results from EpiLUTS. BJU Int. 108,1459-1471 (2011).

69. Perry, S. et al. An investigation of the relationship between anxiety and depression and urge incontinence in women: Development of a psychological model. Br. J. Health Psychol. 11, 463-482 (2006).

70. Ghetti, C. et al. Depressive symptoms in women seeking surgery for pelvic organ prolapse. Int. Urogynecol. J. 21, 855-860 (2010).

71. Ghetti, C. et al. The emotional burden of Pelvic Organ Prolapse in women seeking treatment: a qualitative study. Female Pelvic Med. Reconstr. Surg. 21, 332-338 (2015).

72. Di Gangi Herms, A. M. et al. Assessing health care needs and clinical outcome with urological case complexity: A study using INTERMED. Psychosomatics 44,196-203 (2003).

73. Balachandran, A. et al. Management of female urinary incontinence: a survey of urogynaecologists' view on the NICE guideline. J. Obstet. Gynaecol. 36,487-491 (2016).

74. Walker, J. et al. Screening medical patients for depression: Lessons from a national program in cancer clinics. Psychosomatics pii: S0033-3182(17)30007-5 (2017).

75. Bjelland, I. et al. The validity of the Hospital Anxiety and Depression Scale: An updated literature review. J. Psychosom. Res. 52, 69-77 (2002).

76. Penninx, B. W. et al. Depression and cardiac mortality: Results from a community-based longitudinal study. Arch. Gen. Psychiatry 58, 221-227 (2001).

77. Roy-Byrne, P. P. et al. Anxiety disorders and comorbid medical illness. Gen. Hosp. Psychiatry 30, 208-225 (2008).

78. Maserejian, N. N. et al. Treatment status and risk factors for incidence and persistence of urinary incontinence in women. Int. Urogynecol. J. 25, 775-782 (2014). 
79. Bogner, H. R. et al. Anxiety disorders and disability secondary to urinary incontinence among adults over age 50. Int. J. Psychiatry Med. 32, 141-154 (2002).

80. Talley, N. J. Functional gastrointestinal disorders as a public health problem. Neurogastroenterol. Motil. 20 (Suppl 1), 121-129 (2008).

81. Fortea, J. \& Prior, M. Irritable bowel syndrome with constipation: A European-focused systematic literature review of disease burden. J. Med. Econ. 16, 329-41 (2013).

82. Agarwal, N. \& Spiegel, B. M. The effect of irritable bowel syndrome on health-related quality of life and health care expenditures. Gastroenterol. Clin. North Am. 40, 11-19 (2011).

83. Stanghellini, V. et al. Dyspeptic symptoms and gastric emptying in the irritable bowel syndrome. Am. J. Gastroenterol. 97, 2738-2743 (2002).

84. Van der Veek, P. P. et al. Symptom severity but not psychopathology predicts visceral hypersensitivity in irritable bowel syndrome. Clin. Gastroenterol. Hepatol. 6, 321-328 (2008).

85. Camilleri, M. \& Gorman, H. Intestinal permeability and irritable bowel syndrome. Neurogastroenterol. Motil. 19, 545-552 (2007).

86. Villani, A. C. et al. Genetic risk factors for post-infectious irritable bowel syndrome following a waterborne outbreak of gastroenteritis. Gastroenterology 138, 1502-1513 (2010).

87. Drossman, D. A. et al. Psychosocial aspects of the functional gastrointestinal disorders. Gut 45, II25-30 (1999).

88. Mykletun, A. et al. Prevalence of mood and anxiety disorder in self-reported irritable bowel syndrome (IBS): An epidemiological population based study of women. BMC Gastroenterology 10, 88 (2010).

89. Neeleman, J. et al. The distribution of psychiatric and somatic ill health: Associations with personality and socioeconomic status. Psychosom. Med. 63, 239-247 (2001).

90. Levy, R. L. et al. Psychosocial aspects of the functional gastrointestinal disorders. Gastroenterology 130, 1447-1458 (2006).

91. Stidd, D. A. et al. Spinal cord stimulation with implanted epidural paddle lead relieves chronic axial low back pain. J. Pain. Res. 7, 465-470 (2014).

92. Lihua, P. et al. Spinal cord stimulation for cancer related pain in adults. Cochrane Database Syst. Rev. 2, CD009389 (2013).

93. Noblett, K. et al. Results of a prospectiev multicenter Study evaluating quality of life, safety, and efficacy of sacral neuromodulation at twelve months in subjects with symptoms of overactive bladder. Neurourol. Urodyn. 35, 246-251 (2016).

94. Ford, A. C. et al. Efficacy of antidepressants and psychological therapies in irritable bowle syndrome: Systematic review and meta-analysis. Gut 58, 367-378 (2009).

95. Ford, A. C. et al. Effect of antidepressants and psychological therapies, including hypnotherapy, in irritable bowel syndrome: Systematic review and met-analysis. Am. J. Gatsroenterol. 109, 1350-1365 (2014).

96. Clinical Trials.gov Protocol Registration System; Identifier:NCT01551225.

97. Chapman, S. J. et al. Discontinuation and non-publication of surgical randomised controlled trials: An observational study. BMJ 349, g6870 (2014).

98. Ebrahim, S. et al. Reanalyses of randomized clinical trial data. JAMA 312, 1024-1032 (2014).

99. Schweickhardt, A. et al. Short-term psychotherapeutic interventions for somatizing patients in the general hospital: A randomized controlled study. Psychother. Psychosom. 76, 339-346 (2007).

100. Katon, W. J. et al. Collaborative care for patients with depression and chronic illnesses. N. Engl. J. Med. 363, 2611-2620 (2010).

101. Katon, W. et al. Cost-effectiveness of a multicondition collaborative care intervention: A randomized controlled trial. Arch. Gen. Psychiatry 69, 506-514 (2012).

102. Reed, S. J. et al. Effectiveness and value of integrating behavioral health into primary care. JAMA Intern. Med. 176, 691-692 (2016).

103. Archer, J. et al. Collaborative care for depression and anxiety problems. Cochrane Database Syst. Rev. 10:CD006525 (2012).

104. Tully, P. J. \& Baumeister, H. Collaborative care for comorbid depression and coronary heart disease: A systematic review and meta-analysis of randomised controlled trials. BMJ Open 5, e009128 (2015).

105. Smith, S. M. et al. Interventions for improving outcomes in patients with multimorbidity in primary care and community settings. Cochrane Database Syst. Rev. 3, CD006560 (2016). 
106. Lofors, J. \& Sundquist, K. Low-linking social capital as a predictor of mental disorders: A cohort study of 4.5 million Swedes. Soc. Sci. Med. 64, 21-34 (2007).

107. Thomas, H. et al. Mental health and quality of residential environment. Br. J. Psychiatry 191, 500-505 (2007).

108. Ivory, V. C. et al. When does neighbourhood matter? Multilevel relationships between neighbourhood social fragmentation and mental health. Soc. Sci, Med. 72, 1993-2002 (2011).

109. Lederbogen, F. et al. City living and urban upbringing affect neural stress processing in humans. Nature 474, 498-501 (2011).

110. Hubbard, C. et al. Corticotropin-releasing factor receptor 1 antagonist alters regional activation and effective connectivity in an emotional-arousal circuit during expectation of abdominal pain. J. Neurosci. 31, 12491-12500 (2011).

111. Murrough, J. W. et al. Reduced amygdala serotonin transporter binding in posttraumatic stress disorder. Biol. Psychiatry 70, 1033-1038 (2011).

112. Smith, H. S. et al. Fibromyalgia: an afferent processing disorder leading to a complex pain generalized syndrome. Pain Physician 14, E217-245 (2011).

113. Price, D. D, et al. Widespread hyperalgesia in irritable bowel syndrome is dynamically maintained by tonic visceral impulse input and placebo/nocebo factors: Evidence from human psychophysics, animal models and neuroimaging. Neuroimage 47, 995-1001 (2009).

114. Wise, T. N. et al. Painful physical symptoms in depression: A clinical challenge. Pain Med. 8 (Suppl 2), $75-$ 82 (2007).

115. Goldenberg DL. Pain/Depression dyad: A key to a better understanding and treatment of functional somatic syndromes. Am. J. Med. 123, 675-682 (2010).

116. Peen, J. et al. The current status of urban-rural differences in psychiatric disorders. Acta Psychiatr. Scand. 121, 84-93 (2010).

117. Crump, C. et al. Neighborhood deprivation and psychiatric medication prescription: A Swedish national multilevel study. Ann. Epidemiol. 21, 231-237.

118. Drukker, M. \& van Os, J. Mediators of neighbourhood socioeconomic deprivation and quality of life. Soc. Psychiatry Psychiatr. Epidemiol. 38, 698-706 (2003).

119. Skapinakis, P. et al. Socio-economic position and common mental disorders: Longitudinal study in the general population in the UK. Br. J. Psychiatry 189, 109-117 (2006).

120. March, D. et al. Psychosis and place. Epidemiol. Rev. 30, 84-100 (2008).

121. Hotopf, M. et al. Temporal relationships between physical symptoms and psychiatric disorder: Results from a national birth cohort. Br. J. Psychiatry 173, 255-261 (1998).

122 Barsky, A. J. et al. Somatization increases medical utilization and costs independent of psychiatric and medical comorbidity. Arch. Gen. Psychiatry 62, 903-910 (2005).

123. Freedenfeld, R. N. et al. Decreased pain and improved quality of life in fibromyalgia patients treated with olanzapine, an atypical neuroleptic. Pain Pract. 6, 112-118 (2006).

124. Seidel, S. et al. Antipsychotics for acute and chronic pain in adults. Cochrane database Syst. Rev. 4, CD004844 (2008).

125. Kim, K. W. et al. Association between comorbid depression and osteoarthritis symptom severity in patients with knee osteoarthritis. J. Bone Joint. Surg. Am. 93, 556-563 (2011).

126. Maniadakis, N. \& Gray, A. The economic burden of back pain in the UK. Pain 84, 95-103 (2000).

127. de Jonge, P. et al. Case and Care Complexity in the Medically III. Med. Clin. North Am. 90, 679-692 (2006).

128. Seal, K. H. et al. Association of mental health disorders with prescription opioids and high-risk opioid use in US veterans of Iraq and Afghanistan. JAMA 307, 940-947 (2012).

129. Bohnert, A. S. et al. Association between opioid prescribing patterns and opioid overdose-related deaths. JAMA 305, 1315-1321 (2011).

130. Dersh, J. et al. Chronic pain and psychopathology: Research findings and theoretical considerations. Psychosom. Med. 64, 773-786 (2002).

131. Edit, V. et al. Psychosocial, educational, and somatic factors in chronic non-specific low back pain. Rheumatol. Int. 33, 587-592(2013).

132. Vargas-Schaffer G. Is the WHO analgesic ladder still valid? Twenty-four years of experience. Can. Fam. Physician 56, 514-517 (2010). 
133. Häuser, W. et al. Treatment of fibromyalgia syndrome with antidepressants: A meta-analysis. JAMA 301, 198-209 (2009).

134. Saarto, T. \& Wiffen, P. J. Antidepressants for neuropathic pain. Cochrane database Sys. Rev. 4, CD005454 (2007).

135. Krebs, E. E. et al. Treating the physical symptoms of depression with second-generation antidepressants: A systematic review and meta-analysis. Psychosomatics 49, 191-198 (2008).

136. Arnold, L. M. et al. Comparisons of the efficacy and safety of duloxetine for the treatment of fibromyalgia in patients with versus without major depressive disorder. Clin. J. Pain 25, 461-468 (2009).

137. Arnold, L. M. et al. Comorbidity of fibromyalgia and psychiatric disorders. J Clin. Psychiatry 67, 1219-1225 (2006).

138. Bekhit MH. Opioid-induced hyperalgesia and tolerance. Am. J. Ther. 17, 498-510 (2010).

139. Berton, O. et al. Essential role of BDNF in the mesolimbic dopamine pathway in social defeat stress. Science 311, 864-868 (2006).

140. Upadhyay, J. et al. Alterations in brain structure and functional connectivity in prescription opioiddependent patients. Brain 133, 2098-2114 (2010).

141. Angst, M. S. \& Clark, J. D. Opioid-induced hyperalgesia: A qualitative systematic review. Anesthesiology 104, 570-587 (2006).

142. Rayner, L. et al. Antidepressants for depression in physically ill people. Cochrane database Sys Rev 3, CD007503 (2010).

143. van der Feltz-Cornelis, C. M. Ten years of integrated care for mental disorders in the Netherlands. Int. J. Integr. Care SpecEd: e015 (2011).

144. Leue, C. et al. Observational evidence that urbanisation and neighbourhood deprivation are associated with escalation in chronic pharmacological pain treatment: A longitudinal population-based study in the Netherlands. BMJ Open 2: e101136 (2012).

145. Simon, G. E. \& Walker E. A. The primary care clinic. The American Psychiatric Publishing Textbook of Consultation-Liaison Psychiatry: Psychiatry in the Medically III, 2nd Edition. Edited by Wise, M. G. \& Rundell, J. R. Washington, DC, American Psychiatric Publishing, 917-925 (2002).

146. Hall, R. C. et al. Cost-effectiveness of the consultation-liaison service. The American Psychiatric Publishing Textbook of Consultation-Liaison Psychiatry: Psychiatry in the Medically III, 2nd Edition. Edited by Wise, M. G. \& Rundell, J. R. Washington, DC, American Psychiatric Publishing, 25-32 (2002).

147. Saravay, S. M. \& Lavin, M. Psychiatric comorbidity and length of stay in the general hospital. A critical review of outcome studies. Psychosomatics 35, 233-252 (1994).

148. Druss, B. G. \& Rosenheck, R. A. Patterns of health care costs associated with depression and substance abuse in a national sample. Psychiatr. Serv. 50, 214-218 (1999).

149. Stiefel, F. et al. Effects of a multifaceted psychiatric intervention targeted for complex medically ill: A randomized controlled trial. Psychother. Psychosom. 77, 247-256 (2008).

150. Desan, P. H. et al. Proactive psychiatric consultation services reduce length of stay for admission to an inpatient medical team. Psychosomatics 52,513-520 (2011).

151. Foy, R. et al. Meta-analysis: Effect of interactive communication between collaborating primary care physicians and specialists. Ann. Intern. Med. 152, 247-258 (2010).

152. Holland, D. E. et al. Problems and unmet needs of patients discharged "home to self-care". Prof. Case Manag. 16, 240-250 (2011).

153. Mesteig, M. et al. Unwanted incidents during transition of geriatric patients from hospital to home: A prospective observational study. BMC Health Serv. Res. 10: doi: 10.1186/1472-6963-10-1 (2010).

154. Prvu Bettger, J. et al. Transitional care after hospitalization for acute stroke or myocardial infarction: A systematic review. Ann. Intern. Med. 157, 407-416 (2012).

155. Horwitz, L. I. et al. Quality of discharge practices and patient understanding at an academic medical center. JAMA Intern. Med. 173, 1715-1722 (2013).

156. Coleman, E. A. et al. The care transitions intervention: Results of a randomized controlled trial. Arch. Intern. Med. 166, 1822-1828 (2006).

157. Jackson, C. T. et al. Transitional care cut hospital readmissions for North Carolina Medicaid patients with complex chronic conditions. Health Aff. 32, 1407-1415 (2013). 
158. Schaefert, R. et al. Specific collaborative group intervention for patients with medically unexplained symptoms in general practice: A cluster randomized controlled trial. Psychother. Psychosom. 82, 106-119 (2013).

159. Kathol, R. G. et al. Psychiatrists for medically complex patients: Bringing value at the physical health and mental health/substance-use disorder interface. Psychosomatics 50, 93-107 (2009).

160. Leentjens, A. F. et al. Can we increase adherence to treatment recommendations of the consultation psychiatrist working in a general hospital? A systematic review. J. Psychosom. Res. 68, 303-309 (2010).

161. Van der Feltz-Cornelis, C. M. et al. Effect of psychiatric consultation models in primary care. A systematic review and meta-analysis of randomized clinical trials. J. Psychosom. Res. 68, 521-533 (2010).

162. Vrijens, D. et al. Prevalence of anxiety and depressive symptoms and their association with pelvic floor dysfunctions: A cross sectional cohort study at a Pelvic Care Centre. Neurourol. Urodyn. doi: 10.1002/nau:23186 (2017).

163. Baijens, L. W. et al. Medically unexplained otorhinolaryngological symptoms: Towards integrated psychiatric care. Laryngoscope 125, 1583-1587 (2015).

164. DiMatteo, M. R. et al. Depression is a risk factor for non-compliance with medical treatment: Metaanalysis of the effects of anxiety and depression on patient adherence. Arch. Intern. Med. 160, 2101-07 (2000).

165. Unutzer, J. et al. Long-term cost effects of collaborative care for late-life depression. Am. J. Manag. Care. 14, 95-100 (2008).

166. Wells, K. B. et al. Psychiatric disorder in a sample of the general population with and without chronic medical conditions. Am. J. Psychiatry 145, 976-981 (1988).

167. Katon, W. J. Clinical and health services relationships between major depression, depressive symptoms, and general medical illness. Biol. Psychiatry 54, 216-226 (2003).

168. Katon, W. J. Epidemiology and treatment of depression in patients with chronic medical illness. Dialogues Clin. Neurosci. 13, 7-23 (2011).

169. Neeleman, J. et al. Propensity to psychiatric and somatic ill health: Evidence from a birth cohort. Psychol. Medicine 32, 793-803 (2002).

170. Härter, M. et al. Increased 12-month prevalence rates of mental disorders in patients with chronic somatic diseases. Psychother. Psychosom. 76, 354-360 (2007).

171. Konnopka, A. et al. Economics of medically unexplained symptoms: A systematic review of the literature. Psychother. Psychosom. 81, 265-275 (2012).

172. Cuijpers, P. et al. Economic costs of neuroticism: A population-based study. Arch. Gen. Psychiatry 67, 1086-1093 (2010).

173 Mitchell, A. J. Systematic review: Depression screening and management programmes with staff assistance in primary care increase response and remission rates, but programmes without staff assistance do not show benefits. Evid. Based Med. 15, 49-50 (2010).

174. Magdelijns, F. J. et al. Unplanned readmissions in younger and older adult patients: The role of healthcare-related adverse events. Eur. J. Med. Res. 21, 35 (2016).

175. Magdelijns, F. J. et al. Direct health care costs of hospital admissions duet o adverse events in The Netherlands.Eur. J. Public Health. 24, 1028-1033 (2014).

176. Henningsen, P. et al. Medically unexplained physical symptoms, anxiety and depression: A meta-analytic review. Psychosom. Med. 65, 528-533 (2003).

177. Rickards H. Depression in neurological disorders: Parkinson's disease, multiple sclerosis, and stroke. J. Neurol. Neurosurg. Psychiatry 76, i48-i52 (2005).

178. Hammerlid, E. \& Taft, C. Health-related quality of life in long-term head and neck cancer survivors: A comparison with general population norms. Br. J. Cancer 84, 149-56 (2001).

179. Ronis, D. L. et al. Changes in quality of life over 1 year in patients with head and neck cancer. Arch. Otolaryngol. Head Neck Surg. 134, 241-248 (2008).

180. De Graeff, A. et al. Long-term quality of life of patients with head and neck cancer. Laryngoscope 110, 98-106 (2000).

181. Rogus-Pulia, N. M. et al. Changes in swallowing physiology and patient perception of swallowing function following chemoradiation for head and neck cancer. Dysphagia 29, 223-233 (2014).

182. Troche, M. S. et al. Decreased cough sensitivity and aspiration in Parkinson's disease. Chest 146, 12941299 (2014). 
183. Mu, L. et al. Parkinson disease affects peripheral sensory nerves in the pharynx. J. Neuropathol. Exp. Neurol. 72, 614-623 (2013).

184. Onofri, SM. et al. Correlation between laryngeal sensitivity and penetration/aspiration after stroke. Dysphagia 29, 256-261 (2014).

185. Manikantan K, Khode S, Sayed SI et al. Dysphagia in head and neck cancer. Complications of treatment. Cancer Treat. Rev. 35, 724-732 (2009).

186. Jäghagen, A. L. et al. Pharyngeal swallowing dysfunction following treatment for oral and pharyngeal cancer association with diminished intraoral sensation and discrimination ability. Head Neck 10, 13441351 (2008).

187. Ku, P. K. et al. Laryngopharyngeal sensory deficits and impaired pharyngeal motor function predict aspiration in patients irradiated for nasopharyngeal carcinoma. Laryngoscope 120, 223-228 (2009).

188. Martens, K. A. \& Almeida, Q. J. Dissociating between sensory and perceptual deficits in PD: More than simply a motor deficit. Move. Disord. 27, 387-392 (2012).

189. Pilz, W. et al. Oropharyngeal dysphagia in myotonic dystrophy type 1: A systematic review. Dysphagia 29, 319-331 (2014).

190. Kang, S. S. et al. The association between psychiatric factors and the development of chronic dysphagia after anterior cervical spine surgery. Eur. Spine J. 23, 1694-1698 (2014).

191. Perez-Lloret, S. et al. Oro-buccal symptoms (dysphagia, dysarthria, and sialorrhea) in patients with Parkinson's disease: Preliminary analysis from the French COPARK cohort. Eur. J. Neurol. 19, 28-37 (2012).

192. Lin, B. M. et al. The relationship between depressive symptoms, quality of life, and swallowing function in head and neck cancer patients 1 year after definitive therapy. Laryngoscope 122, 1518-1525 (2012).

193. Lin, L. C. et al. Depressive symptoms in long-term care residents in Taiwan. J. Adv. Nurs. 51, $30-37$ (2005).

194. Miller, N. et al. Swallowing problems in Parkinson disease: frequency and clinical correlates. J. Neurol. Neurosurg.Psychiatry 80, 1047-1049 (2009).

195. Airoldi, M.et al. Functional and psychological evaluation after flap reconstruction plus radiotherapy in oral cancer. Head Neck 33, 458-468 (2011).

196. Thomas, F. J. \& Wiles, C. M. Dysphagia and nutritional status in multiple sclerosis. J. Neurol. 246, 677-682 (1999).

197. Nogueira, D. \& Reis, E. Swallowing disorders in nursing home residents: How can the problem be explained? Clin. Interventions Aging. 8, 221-227 (2013).

198. Zhang, L. et al. Effect of swallowing training on dysphagia and depression in postoperative tongue cancer patients. Eur. J. Oncol. Nurs. 18, 626-629 (2014).

199. Kang, J. H. et al. The effect of bedside exercise program on stroke patients with dysphagia. Ann. Rehabil. Med. 36, 512-520 (2012).

200. Verdonschot, R. J. et al. The relationship between fiberoptic endoscopic evaluation of swallowing outcome and symptoms of anxiety and depression in dysphagic patients. Laryngoscope 126, E199-207 (2016).

201. Nguyen, N. P. et al. Impact of dysphagia on quality of life after treatment of head-and-neck cancer. Int. J. Radiat. Oncol. Biol. Phys. 61, 772-778 (2005).

202. Dum, R. P. et al. Motor, cognitive, and affective areas of the cerebral cortex influence the adrenal medulla. Proc. Natl. Acad. Sci. U S A. 113, 9922-9227 (2016).

203. Whiting, P. et al. The development of QUADAS: A tool for the quality assessment of studies of diagnostic accuracy included in systematic reviews. BMC Med. Res. Methodol. 3, 25 (2003).

204. Tubaro, A. Defining overactive bladder: Epidemiology and burden of disease. Urology 64 (SUPPL. 1), 2-6 (2004).

205. Milsom, I. et al. How widespread are the symptoms of an overactive bladder and how are they managed? A population-based prevalence study. BJU Int. 87, 760-766 (2001).

206. Engel, W. J. Uropsychiatry. Journal - Michigan State Medical Society. 63, 273-277 (1964).

207. Macaulay, A. J. et al. Micturition and the mind: Psychological factors in the aetiology and treatment of urinary symptoms in women. Br. Med. J. 294, 540-543 (1987).

208. Cortes E et al. The psychology of LUTS: ICI-RS 2011. Neurourol. Urodyn. 31, 340-343 (2012).

209. Hirayama, A. et al. Risk factors for new-onset overactive bladder in older subjects: results of the Fujiwara-kyo study. Urology 80, 71-76 (2012). 
210. Nemeroff CB. The neurobiology of depression. Scientific American 278, $42-49$ (1998).

211. Gordon, J. A. \& Hen, R. The serotonergic system and anxiety. Neuromolecular Med. 5, 27-40 (2004).

212. Steers, W. Potential targets in the treatment of urinary incontinence. Rev. Urol. 3, S19-26 (2001).

213. de Groat, W. C. Influence of central serotonergic mechanisms on lower urinary tract function. Urology 59, S1 30-36 (2002).

214. Lee, K. S. et al. Alterations in voiding frequency and cystometry in the clomipramine induced model of endogenous depression and reversal with fluoxetine. J. Urology 170, 2067-2071 (2003).

215. Steers, W. D. et al. Duloxetine compared with placebo for treating women with symptoms of overactive bladder. BJU Int. 100, 337-345 (2007).

216. Jost, W. \& Marsalek, P. Duloxetine: mechanism of action at the lower urinary tract and Onuf's nucleus. Clin. Auton. Res. 14, 220-227 (2004).

217. Arborelius, L. et al. The role of corticotropin-releasing factor in depression and anxiety disorders. J. Endocrinol. 160, 1-12 (1999).

218. Nemeroff, C. B. Recent advances in the neurobiology of depression. Psychopharmacol. Bull. 36, S2 6-23 (2002).

219. Smith, A. L. et al. The effects of acute and chronic psychological stress on bladder function in a rodent model. Urology 78, 967e1-e7 (2011).

220. Klausner, A. P. et al. The role of corticotropin releasing factor and its antagonist, astressin, on micturition in the rat. Auton. Neurosci. 123, 26-35 (2005).

221. Wood, S. K. et al. Social stress-induced bladder dysfunction: Potential role of corticotropin-releasing factor. Am. J. Physiol. Regul. Integr. Comp. Physiol. 296, R1671-1678 (2009).

222. Griffiths, D. Imaging bladder sensations. Neurourol. Urodyn. 26, S899-903 (2007).

223. Griffiths, D. \& Tadic, S. D. Bladder control, urgency, and urge incontinence: Evidence from functional brain imaging. Neurourol. Urodyn. 27, 466-474 (2008).

224. Komesu, Y. M. et al. Functional MRI of the Brain in Women with Overactive Bladder: Brain Activation During Urinary Urgency. Female Pelvic Med. Reconstr. Surg. 17, 50-54 (2011).

225. Eisenberger, N. I. The neural bases of social pain: evidence for shared representations with physical pain. Psychosom. Med. 74,126-135 (2012).

226. Griffiths, D. Clinical studies of cerebral and urinary tract function in elderly people with urinary incontinence. Behav. Brain. Res. 92,151-155 (1998).

227. Awata, S. et al. Regional cerebral blood flow abnormalities in late-life depression: Relation to refractoriness and chronification. Psychiatry Clin. Neurosci. 52, 97-105 (1998).

228. Bushnell, M. C. et al. Cognitive and emotional control of pain and its disruption in chronic pain. Nat. Rev.Neurosci. 14, 502-511 (2013).

229. Russo, S. J. \& Nestler, E. J. The brain reward circuitry in mood disorders. Nat. Rev. Neurosci. 14, 609-625 (2013).

230. Baliki, M. N. et al. Functional reorganization of the default mode network across chronic pain conditions. PLoS One 9:e106133 (2014).

231. Eisenberger, N. I. The pain of social disconnection: Examining the shared neural underpinnings of physical and social pain. Nat. Rev. Neurosci. 13, 421-434 (2012).

232. Sadler, K. E. \& Kolber, B. J. Urine trouble: Alterations in brain function associated with bladder pain. J. Urology 196, 24-32 (2016).

233. Chung, M. K. et al. The overlap of interstitial cystitis/painful bladder syndrome and overactive bladder. JSLS. 14, 83-90 (2010).

234. Lai, H. H. et al. The overlap and distinction of self-reported symptoms between interstitial cystitis/bladder pain syndrome and overactive bladder: A questionnaire based analysis. J. Urol. 192, 1679-1685 (2014).

235. Suskind, A. M. et al. The prevalence and overlap of interstitial cystitis/bladder pain syndrome and chronic prostatitis/chronic pelvic pain syndrome in men: Results of the RAND Interstitial Cystitis Epidemiology male study. J. Urol. 189, 141-145 (2013).

236. Matsumoto, S. et al. Relationship between overactive bladder and irritable bowel syndrome: A largescale Internet survey in Japan using the overactive bladder symptom score and Rome III criteria. BJU Int. $111,647-652$ (2013). 
237. Matsuzaki, J. et al. High frequency of overlap between functional dyspepsia and overactive bladder. Neurogastroenterol. Motil. 24, 821-827 (2012).

238. Chelimsky, G. et al. Co-morbidities of interstitial cystitis. Front. Neurosci. doi:10.3389/fnins.2012. 00114 (2012).

239. Fan, Y. H. et al. Non-bladder conditions in female Taiwanese patients with interstitial cystitis/ hypersensitive bladder syndrome. Int. J Urol. 21, 805-809 (2014).

240. Persson, R. et al. The relationship between irritable bowel syndrome, functional dyspepsia, chronic fatigue and overactive bladder syndrome: A controlled study 6 years after acute gastrointestinal infection. BMC Gastroenterol. 15, 66 (2015).

241. Lai, H. H. et al. Polysymptomatic, polysyndromic presentation of patients with urological chronic pelvic pain syndrome. J. Urol. 187, 2106-2112 (2012).

242. Fuoco, M. B. et al. Multiple sensitivity phenotype in interstitial cystitis/bladder pain syndrome. Can. Urol. Assoc. J. 8, 758-761 (2014).

243. Bullones Rodriguez, M. A. et al. Evidence for overlap between urological and nonurological unexplained clinical conditions. J. Urol. 189, S66-74 (2013).

244. Kanaan, R. A. et al. The association or otherwise of the functional somatic syndromes. Psychosom. Med. 69, 855-889 (2007).

245. Fink, P. \& Schröder, A. One single diagnosis, bodily distress syndrome, succeeded to capture 10 diagnostic categories of functional somatic syndromes and somatoform disorders. J. Psychosom. Res. 68, 415-426 (2010).

246. Wessely, S. \& White, P. D. There is only one functional syndrome. Br. J. Psychiatry 185, 95-96 (2004).

247. Kim, S. E. \& Chang, L. Overlap between functional Gl disorders and other functional syndromes: What are the underlying mechanisms? Neurogastroenterol. Motil. 24, 895-913 (2012).

248. Warren, J. W. et al. The number of existing functional somatic syndromes (FSSs) is an important risk factor for new, different FSSs. J. Psychosom. Res. 74, 12-17 (2013).

249. Warren, J. W. et al. Before the onset of interstitial/bladder pain syndrome, the presence of multiple nonbladder syndromes is strongly associated with a history of multiple surgeries. J. Psychosom. Res. 76, 7579 (2014).

250. Warren, J. W. et al. Functional somatic syndromes as risk factors for hysterectomy in early bladder pain syndrome/interstitial cystitis. J. Psychosom. Res. 77, 363-367 (2014).

251. Mullins, C. et al. Novel research approaches for interstitial cystitis/bladder pain syndrome: Thinking beyond the bladder. Transl. Androl. Urol. 4, 524-533 (2015).

252. Drossman, D. A. The functional gastrointestinal disorders and the Rome III process. Gastroenterology 130, 1377-1390 (2006).

253. Longstreth, G. F. et al. Functional bowel disorders. Gastroenterology 130, 1480-1491 (2006).

254. Abrams, P. et al. The standardisation of terminology of lower urinary tract function: Report from the standardisation subcommitee of the International Continence Society. Neurourol. Urodyn. 21, 167-178 (2002).

255. Fall, M. et al. EAU guidelines on chronic pelvic pain. Eur. Urol. 46, 681-689 (2004).

256. van de Merwe, J. P. et al. Diagnostic criteria, classification, and nomenclature for painful bladder syndrome/ interstitial cystitis: An ESSIC proposal. Eur. Urol. 53, 60-67 (2008).

257. van Ophoven, A. et al. From end-organ disease to a classable bladder pain syndrome: Paradigm shift in the understanding of urological pain syndromes exemplified by the condition currently called interstitial cystitis. Urologe A. 48, 152-162 (2009).

258. Fall, M. et al. EAU guidelines on chronic pelvic pain. Eur. Urol. 57, 35-48 (2010).

259. Engeler, D. S. et al. The 2013 EAU guidelines on chronic pelvic pain: is management of chronic pelvic pain a habbit, a philosophy, or a science? 10 years of development. Eur. Urol. 64, 431-439 (2013).

260. Wesselmann, U. A new paradigm in chronic bladder pain. J. Pain Palliat. Care Pharmacother. 28, 406-408 (2014).

261. Twiddy, H. et al. The development and delivery of a female chronic pelvic pain management programme: A specialised interdisciplinary approach. Br. J. Pain. 9, 233-240 (2015).

262. Hanno, P. M. et al. Diagnosis and treatment of interstitial cystitis/bladder pain syndrome: AUA guideline amendment. J. Urol. 193, 1545-1553 (2015). 
263. Parsons, C. L. The role of a leaky epithelium and potassium in the generation of bladder symotoms in interstitial cystitis/overactive bladder, urethral syndrome, prostatitis and gynaecological chronic pelvic pain. BJU Int. 107, 370-375 (2011).

264. Homma, Y. Hypersensitive bladder: A solution to confused terminology and ignorance concerning interstitial cystitis. Int. J. Urol. 21, S41-47 (2014).

265. Hurst, R. E. et al. Increased bladder permeability in interstitial cystitis/painful bladder syndrome. Transl. Androl. Urol. 5, 563-571 (2015).

266. Das, R. et al. Dimensions of sensation assessed in urinary urgency: a systematic review. J. Urol. 190, 1165-1172 (2013).

267. Juszczak, K. et al. Hyperosmolarity alters micturition: a comparison of urinary bladder motor activity in hyperosmolar and cyclophosphamide-induced models of overactive bladder. Can. J. Physiol. Pharmacol. 88, 899-906 (2010).

268. Greenwood-van Meerveld, B. et al. Mechanisms of visceral organ crosstalk: Importance of alterations in permeability in rodent models. J. Urol. 194, 804-811 (2015).

269. Yoshikawa, S. et al. Pelvic organ cross-sensitization to enhance bladder and urethral pain behaviors in rats with experimental colitis. Neuroscience 284, 422-429 (2015).

270. Valentino, R. J. et al. The bladder-brain connection: putative role of corticotropin- releasing factor. Nat. Rev. Urol. 8, 19-28 (2011).

271. Martinez-Martinez, L. A. et al. Sympathetic nervous system dysfunction in fibromyalgia, chronic fatigue syndrome, irritable bowel syndrome, and interstitial cystitis: A review of case-control studies. J. Clin. Rheumatol. 20, 146-150 (2014).

272. Williams, D. P. Effects of chronic pelvic pain on heart rate variability in women. J. Urol. 194, 1289-1294 (2015).

273. Hughes, P. A. et al. Immune activation in irritable bowel syndrome: Can neuroimmune interactions explain6symptoms? Am. J. Gastroenterol. 108, 1066-1074 (2013).

274. Cartwright, R. et al. Novel biomarkers for overactive bladder. Nat. Rev. Urol. 8, 139-145 (2011).

275. Qu, H. C. et al. Association between polymorphism of $\beta 3$-adrenoceptor gene and overactive bladder: A meta-analysis. Genet. Mol. Res. 14, 2495-2501 (2015).

276. Talati, A. et al. Panic disorder, social anxiety disorder, and a possible medical syndrome previously linked to chromosome 13. Biol. Psychiatry 63, 594-601 (2008).

277. Whiteside, S.A. et al. The microbiome of the urinary tract - a role beyond infection. Nat. Rev. Urol. 12, 81-90 (2015).

278. Pierce, A. N. \& Christianson, J. A. Stress and chronic pelvic pain. Prog. Mol. Biol. Transl. Sci. 131, 509-535 (2015).

279. Choung, R. S. et al. Psychosocial distress and somatic symptoms in community subjects with irritable bowel syndrome: A psychosocial component is the rule. Am. J. Gastroenterol. 104, 1772-1779 (2009).

280. Koloski, N.A. et al. Does psychological distress modulate functional gastrointestinal symptoms and health care seeking? Am. J. Gastroenterol. 98, 789-797 (2003).

281. Lackner, J. M. et al. Testing the sequential model of pain processing in irritable bowel syndrome: A structural equation modeling analysis. Eur. J. Pain 9, 207-18 (2005).

282. Koloski, N. A. et al. What level of IBS symptoms drives impairment in health-related quality of life in community subjects with irritable bowel syndrome? Are current IBS syndrome thresholds clinically meaningful? Qual. Life Res. 21, 829-836 (2012).

283. Link, C. L. et al. Is abuse causally related to urologic symptoms? Results from the Boston Area Community Health (BACH) survey. Eur. Urol. 52, 397-406 (2007).

284. Rothrock, N. E. et al. Stress and symptomatology in patients with interstitial cystitis: A life stress model. Urology 57, 422-427 (2001).

285. Hsiao, S. M. et al. Psychometric assessment of female overactive bladder syndrome and antimuscarinicsrelated effects. Maturitas 79, 428-434 (2014).

286. Lai, H. H. et al. Urological symptoms in a subset of patients with urological chronic pelvic pain syndrome and a polysymptomatic, polysyndromatic pattern of presentation. J. Urol. 191, 1802-1807 (2014).

287. Krieger, J. N. et al. Relationship between chronic nonurological associated somatic syndromes and symptom severity in urological chronic pelvic pain syndromes: Baseline evaluation of the MAPP study. J. Urol. 193, 1254-1262 (2015). 
288. Riegel B. et al. Assessing psychological factors, social aspects and psychiatric co-morbidity associated with Chronic Prostatitis/Chronic Pelvic Pain Syndrome (CP/CPPS) in men: A systematic review. J. Psychosom. Res. 77, 333-350 (2014).

289. olde Hartman, T. C. et al. Chronic functional somatic symptoms: A single syndrome? Br. J. Gen. Pract. 54, 922-927 (2004).

290. Lackner, J. M. Type, rather than number, of mental physical comorbidities increases the severity of symptoms in patients with irritable bowel syndrome. Clin. Gastroenterol. Hepatol. 11, 1147-1157 (2013).

291. Diagnostic and Statistical Manual of Mental Disorders. $5^{\text {th }}$ edition. Washington, DC: American Psychiatric Association (2013).

292. The ICD-10 Classification of Mental and Behavioural Disorders: Diagnostic Criteria for Research. World Health Organization, Geneva (1993).

293. Jylhä, P. \& Isometsä, E. The relationship of neuroticism and extraversion to symptoms of anxiety and depression in the general population. Depress. Anxiety 23, 281-289 (2006).

294. Klein, D. N. et al. Personality and depression: Explanatory models and review of the evidence. Annu. Rev. Clin. Psychol. 7, 269-295 (2011).

295. Lai, H. H. et al. Painful bladder filling and painful urgency are distinct characteristics in men and women with urological chronic pelvic pain syndromes: A MAPP research network study. J. Urol. 194, 1634-1641 (2015).

296. Koh, J. S. et al. The association of personality trait on treatment outcomes in patients with chronic prostatitis/chronic pelvic pain syndrome: An exploratory study. J. Psychosom. Res. 76, 127-133 (2014).

297. Koh, J. S. et al. The impact of depression and somatic symptoms on treatment outcomes in patients with chronic prostatitis/chronic pelvic pain syndrome: A preliminary study in a naturalistic treatment setting. Int. J. Clin. Pract. 68, 478-485 (2014).

298. Tayama, J. et al. Effects of personality traits on the manifestations of irritable bowel syndrome. Bio. Psycho. Soc. Med. 6, 20 (2012).

299. Goldstein, B. L. \& Klein D. N. A review of selected candidate endophenotypes for depression. Clin. Psychol. Rev. 34, 417-27 (2014).

300. Ormel, J. et al. Neuroticism and common mental disorders: Meaning and utility of a complex relationship. Clin. Psychol. Rev. 33, 686-697 (2013).

301. Muscatello, M. R. et al. Role of negative affects in pathophysiology and clinical expression of irritable bowel syndrome. World J. Gastroenterol. 20, 7570-7586 (2014).

302. den Boeft, M. et al. The association between medically unexplained physical symptoms and health care use over two years and the influence of depressive and anxiety disorders and personality traits: A longitudinal study. BMC Health Serv. Res. doi: 10.1186/s12913-016-1332-7 (2016).

303. Kroenke, K. \& Spitzer, R. L. Gender differences in the reporting of physical and somatoform symptoms. Psychosom. Med. 60, 150-155 (1998).

304. Goodwin, R. D. \& Gotlib, I. H. Gender differences in depression: The role of personality factors. Psychiatry Res. 126, 135-142 (2004).

305. Koenig, H. G. Depression in hospitalized older patients with congestive heart failure. Gen. Hosp. Psychiatry 20, 29-43 (1998).

306. Strik, J. J. et al. One-year cumulative incidence of depression following myocardial infarction and impact on cardiac outcome. J. Psychosom. Res. 56, 59-66 (2004).

307. Fishbain, D. A. et al. Chronic pain-associated depression: Antecedent or consequence of chronic pain? A review. Clin. J. Pain 13, 116-137 (1997).

308. Bair, M. J. et al. Anxiety but not Social Stressors Predict 12-Month Depression and Pain Severity. Clin. J. Pain 29, 95-101 (2013).

309. Gerrits, M. M. et al. Pain and the onset of depressive and anxiety disorders. Pain 155, 53-59 (2014).

310. Kroenke, K, et al. Reciprocal Relationship between Pain and Depression: A 12-month longitudinal analysis in Primary Care. J. Pain 12, 964-973 (2011).

311. Koloski, N. A. et al. The brain-gut pathway in functional gastrointestinal disorders is bidirectional: A $12-$ year prospective population-based study. Gut 61, 1284-1290 (2012).

312. Pinto-Sanchez, M. I. et al. Anxiety and depression increase in a stepwise manner in parallel with multiple FGIDs and symptom severity and frequency. Am. J. Gastroenterol. 110, 1038-1048 (2015). 
313. Lai, H. et al. Correlation between psychological stress levels and the severity of overactive bladder symptoms. BMC Urology 15, 14 (2015).

314. Yunus, M. B. Fibromyalgia and overlapping disorders: The unifying concept of central sensitivity syndromes. Semin. Arthritis Rheum. 36, 339-356 (2007).

315. Drossman, D. A. et al. Severity in irritable bowel syndrome: A Rome foundation working team report. Am J. Gastroenterol. 106, 1749-59 (2011).

316. Drossman, D. A. Do psychosocial factors define symptom severity and patient status in irritable bowel syndrome? Am. J. Med. 107, 41S-50 (1999).

317. Surdea-Blaga, T. et al. Psychosocial determinants of irritable bowel syndrome. World J. Gastroenterol. 18, 616-626 (2012).

318. Costa, P. T. \& McCrae, R. R. NEO PI-R professional manual. Odessa, FL. Psychological Assessment Resources, Inc. (1992)

319. Hazlett-Stevens, H. et al. Prevalence of irritable bowel syndrome among university students: The roles of worry, neuroticism, anxiety sensitivity and visceral anxiety. J. Psychosom. Res. 55, 501-505 (2003).

320. Farnam, A. et al. Personality factors and profiles in variants of irritable bowel syndrome. World J. Gastroenterol. 13, 6414-6418 (2007).

321. van Tilburg, M. A. et al. Which psychological factors exacerbate irritable bowel syndrome? Development of a comprehensive model. J. Psychosom. Res. 74, 486-492 (2013).

322. Sullivan, M. J. et al. Theoretical perspectives on the relation between catastrophizing and pain. Clin. J. Pain 17, 52-64 (2001).

323. Lackner, J. M. et al. Depression and abdominal pain in IBS patients: the mediating role of catastrophizing. Psychosom. Med. 66, 435-441 (2004).

324. Lackner, J. M. et al. Patient reported outcomes for irritable bowel syndrome are associated with patients' severity ratings of gastrointestinal symptoms and psychological factors. Clin. Gastroenterol. Hepatol. 9, 957-964 (2011).

325. Spiller, R. Postinfectious functional dyspepsia and postinfectious irritable bowel syndrome: Different symptoms but similar risk factors. Gastroenterology 138, 1660-1663 (2010).

326. Rudick, C. N. et al. O-antigen modulates infection-induced pain states. PLoS One 7, e1273 (2012).

327. O’Brien, V. P. et al. A mucosal imprint left by prior Escherichia coli bladder infection sensitizes to recurrent disease. Nat. Microbiol. 2: 16196 (2016).

328. Rosen, J. M. \& Klumpp, D. J. Mechanisms of pain from urinary tract infection. Int. J. Urol. 21 (Suppl. 1), 26-32 (2014).

329. Janssens, K. A. et al. Mood and anxiety disorders in chronic fatigue syndrome, fibromyalgia, and irritable bowel syndrome: Results from the LifeLines cohort study. Psychosom. Med. 77, 449-457 (2015).

330. Thijssen, A. Y. et al. Dysfunctional cognitions, anxiety and depression in irritable bowel syndrome. J. Clin. Gastroenterol. 44, e236-241 (2010).

331. El-Serag, H. B. et al. Systematic review: Natural history of irritable bowel syndrome. Aliment. Pharmacol. Ther. 19, 861-870 (2004).

332. Giarensis, I. \& Cardozo L. Management of refractory overactive bladder. Minerva Ginecol. 65, 41-52 (2013).

333. Marshall, J. K. et al. Eight year prognosis of post infectious irritablebowel syndrome following waterborne bacterial dysentery. Gut 59, 605-611 (2010).

334. Berghmans, B. et al. Prevalence and triage of first-contact complaints on pelvic floor dysfunctions in male patients referred to a Pelvic Care Centre. Neurourol. Urodyn. 35, 487-491 (2016).

335. Filipetto, F. A. et al. The patient perspective on overactive bladder: A mixed-methods needs assessment. BMC Fam. Pract. doi: 10.1186/1471-2296-15-96 (2014).

336. Bosch, P. C. \& Bosch, D. C. Treating interstitial cystitis/bladder pain syndrome as a chronic disease. Rev. Urol. 16, 83-87 (2014).

337. Mujagic, Z. et al. Systematic review: instruments to assess abdominal pain in irritable bowle syndrome. Aliment. Pharmacol. Ther. 42, 1064-1081 (2015).

338. Myin-Germeys, I. Experience sampling research in psychopathology: Opening the black box of daily life. Psychol. Med. 39, 1533-1547 (2009).

339. van Os, J. et al. Testing an mHealth momentary assessment Routine Outcome Monitoring application: A focus on restoration of daily life positive mood states. PloS One 9:e115254 (2014). 
340. Wichers, M. et al. Momentary assessment technology as a tool to help patients with depression help themselves. Acta Psychiatr. Scand. 124, 262-272 (2011).

341. Myin-Germeys, I. et al. Emotional reactivity to daily life stress in psychosis and affective disorder: An experience sampling study. Acta Psychiatr. Scand. 107, 124-131 (2003).

342. van Os, J. et al. The experience sampling method as an mHealth tool to support self-monitoring, selfinsight, and personalized care in clinical practice. Depress Anxiety doi: 10.1002/da.22647 (2017).

343. Häuser, W. et al. Conctruct validity and clinical utility of current research criteria of DSM-5 somatic symptom disorder diagnosis in patients with fibromyalgia syndrome. J. Psychosom. Res. 78, 546-552 (2015).

344. Levenson, J. L. Essentials of Psychosomatic Medicine. American Psychiatric Press Inc. ISBN 978-1-58562246-247 (2006).

345. Wise, T. N. Update on consultation-liaison psychiatry (psychosomatic medicine). Curr. Opin. Psychiatry 21,196-200 (2008).

346. Nusslock, R. \& Miller, G. E. Early-life adversity and physical and emotional health across the lifespan: A neuroimmune network hypothesis. Biol. Psychiatry Pii:S0006-3223(15)00466-7 (2015).

347. Barnes, J. et al. Gentic contributions of inflammation to depression. Neuropsychopharmacology 42, 8198 (2017).

348. Plummer, J. T. et al. The genetic intersection of neurodevelopmental disorders and shared medical comorbidities - relations that translate from bench to bedside. Front. Psychiatry 7, 142 (2016).

349. Simons, L. et al. Psychological processing in chronic pain: A neural systems approach. Neurosci. Biobehav. Rev. 39, 61-78 (2014).

350. Miller, B. J. \& Goldsmith, D. R. Towards an immunophenotype of schizophrenia: Progress, potential mechanisms, and future directions. Neuropsychopharmacology 42, 299-317 (2017).

351. Shefer, G. et al. Diagnostic overshadowing and other challenges involved in the diagnostic process of patients with mental illness who present in emergency departments with physical symptoms - a qualitative study. PLoS One 9, e111682 (2014).

352. Shefer, G. et al. Improving the diagnosis of physical illness in patients with mental illness who present in emergency departments: consensus study. J. Psychosom. Res. 78, 346-351 (2015).

353. Burian, R. et al. Crossing the bridge - a prospective comparative study of the effect of communication between a hospital based consultation-liaison service and primary care on general practitioners' concordance with consultation-liaison psychiatrists' recommendations. J. Psychosom. Res. 86, 53-59 (2016).

354. Lücke, C. et al. A comparison of two psychiatric service approaches: findings from the Consultation vs. Liaison Psychiatry-Study. BMC Psychiatry 17: 8 (2017).

355. Brownell, A. K. et al. Clinical practitioners' view on the management of patients with medically unexplained physical symptoms (MUPS): A qualitative study. BMJ Open 6: e012379 (2016).

356. Sharpe, M. A collaborative care approach delivering treatment to patients with depression comorbid with diabetes or cardiovascular disease achieves significant but small improvements over usual care in depression and patient satisfaction. Comment in Evid. Based Ment. Health 18, 122 (2015).

357. Barnett, K. et al. Epidemiology of multimorbidity and implications for health care, research, and medical education: A cross-sectional study. Lancet 380, 37-43 (2012).

358. Naylor, C. et al. Long-term conditions and mental health: The cost of co-morbidities. London: The King's Fund (2012).

359. Kendler, K. S. Causal inference in psychiatric epidemiology. JAMA Psychiatry 74, 561-562 (2017).

360. Mujagic, Z. et al. The Experience Sampling Method - a new digital tool for momentary symptom assessment in IBS: an exploratory study. Neurogastroenterol. Motil. 27, 1295-1302 (2015).

361. Katon, W. J. \& Unützer, J. Health reform and the Affordable Care Act: the importance of mental health treatment to achieving the triple aim. J. Psychosom. Res. 74, 533-537 (2013).

362. Kessler, R. C. et al. Lifetime prevalence and age-of-onset distributions of DSM-IV disorders in the national comorbidity survey replication. Arch. Gen. Psychiatry 62, 593-602 (2005).

363. Lovell RM, Ford AC. Global prevalence of and risk factors for irritable bowel syndrome: A meta-analysis. Clin. Gastroenterol. Hepatol. 10, 712-721 (2012).

364. Milsom, I. et al. Global prevalence and economic burden of urgency urinary incontinence: A systematic review. Eur. Urol. 65, 79-95 (2014). 
365. Lackner, J. M. et al. The impact of physical complaints, social environment, and physical functioning on IBS patients' health perception: looking beyond GI symptom severity. Am. J. Gastroenterol. 109, 224-233 (2014).

366. Kessler, R. C. et al. Lifetime prevalence and age-of-onset distributions of DSM-IV disorders in the national comorbidity survey replication. Arch. Gen. Psychiatry 62, 593-602 (2005).

367. Cole J. A. et al. Migraine, fibromyalgia and depression among people with IBS: A prevalence study. BMC Gastroenterology 6, 26 (2006).

368. Clemens, J. Q. et al. Temporal ordering of interstitial cystitis/bladder pain syndrome and non-bladder conditions. Urology. 80, 1227-1231 (2012).

369. Nickel, J. C. et al. Interstitial cystitis/painful bladder syndrome and associated medical conditions with an emphasis on irritable bowel syndrome, fibromyalgia and chronic fatigue syndrome. J. Urol. 184, 13581363 (2010).

370. Lutgendorf, S. K. et al. Stress and symptomatology in patients with interstitial cystitis: A laboratory stress model. J. Urol. 164, 1265-1269 (2000).

371. Nickel, J. C. et al. Clinical and psychological parameters associated with pain pattern phenotypes in women with interstitial cystitis/bladder pain syndrome. J. Urol. 193, 138-144 (2015).

372. Chung, K. H. et al. Bladder pain syndrome/interstitial cystitis is associated with anxiety disorder. Neurourol. Uroldyn. 33, 101-105 (2014).

373. Clemens, J. Q. et al. Temporal ordering of interstitial cystitis/bladder pain syndrome and non-bladder conditions. Urology. 80, 1227-1231 (2012).

374. Nickel, J. C. et al. Interstitial cystitis/painful bladder syndrome and associated medical conditions with an emphasis on irritable bowel syndrome, fibromyalgia and chronic fatigue syndrome. J. Urol. 184, 13581363 (2010).

375. Leue, C. Organisch begründete psychische Erkrankungen - am Beispiel des Delirs. Uexküll: Psychosomatische Medizin - Theoretische Modelle und klinische Praxis. 8. Auflage. Kapitel 99;1123-1130 (2017).

376. Hoogervorst-Schilp, J. Excess length of stay and economic consequences of adverse events in Dutch hopsital patients. BMC Health Serv. Res. 15:531 (2015).

377. Rivara, F. P. et al. The effects of alcohol abuse on readmission for trauma. JAMA 270, 1962-1964 (1993).

378. Hannan, E. L. et al. Predictors of readmission for complications of coronary artery bypass graft surgery. JAMA 290, 773-780 (2003).

379. Heslin, K. C. et al. Hospitalizations involving mental and substance use disorders among adults, 2012. Healthcare cost and utilization project (HCUP), Statistical brief 191, (2015).

380. Marks, E. Complexity science and the readmission dilemma. JAMA Intern. Med. 173, 629-631 (2013).

381. Steiner, C. A. et al. Trends and projections in hospital stays for adults with multiple chronical conditions, 2003-2014. Healthcare cost and utilization project (HCUP), Statistical brief 183, (2014).

382. Barrett, M. L. et al. All-cause readmissions by payer and age, 2009-2013. Healthcare cost and utilization project (HCUP), Statistical brief 199, (2015).

383. Heslin, K. C. \& Weiss, A. J. Hospital readmissions involving psychiatric disorders, 2012. Healthcare cost and utilization project (HCUP), Statistical brief 189, (2015).

384. Vigod, S. N. et al. Transitional interventions to reduce early psychiatric readmissions in adults: Systematic review. Br. J. Psychiatry 202, 187-194 (2013).

385. Vaduganathan, M. et al. Thirty-day readmissions: The clock is ticking. JAMA 309, 345-346 (2013).

386. Donzé, J. et al. Causes and patterns of readmissions in patients with common comorbidities: Retrospective cohort study. BMJ 347, f7171 (2013).

387. Fabbian, F. et al. The crucial factor of hospital readmissions: A retrospective cohort study of patients evaluated in the emergency department and admitted to the department of medicine of a general hospital in Italy. Eur. J. Med. Res. 20, 6 (2015).

388. Scott, I. A. et al. Quality of care factors associated with unplanned readmissions of older medical patients: A case-control study. Intern. Med. J. 44, 161-170 (2014).

389. Kangovi, S. \& Grande, D. Hospital readmissions - not just a measure of qulity. JAMA 306, 1796-1797 (2011).

390. Strunin, L. et al. Understanding rehospitalization risk. J. Hosp. Med. 2, 297-304 (2007). 
391. Medicaid Access Study Group. Access of Medicaid recipients to outpatient care. N. Eng. J. Med. 330, 1426-1430 (1994).

392. Williams, M. V. A requirement to reduce readmissions: Take care of the patient, not just the disease. JAMA 309, 394-396 (2013).

393. Milstein, A. \& Shortell, S. Innovations in care delivery to slow groth of US health spending. JAMA 308, 1439-1440 (2012).

394. Jenq, G. \& Tinetti M. E. The journey across the health care (dis) continuum for vulnerable patients. JAMA 307, 2157-2158 (2012).

396. McCarthy, D. et al. Recasting readmissions by placing the hospital role in community context. JAMA 309, 351-352 (2013).

397. Calvillo-King, L. et al. Impact of social factors on risk of readmission or mortality in pneumonia and heart failure. J. Gen. Intren. Med. 28, 269-282 (2013).

397. Goekoop, R. \& Goekoop, J. G. A network view on psychiatric disorders: Network clusters of symptoms as elementary syndromes of psychopathology. PLoS One 9, e112734 (2014).

398. van Rooijen, G. et al. A state-independent network of depressive, negative and positive symptoms in male patients with schizophrenia spectrum disorders. Schizophr. Res. pii: S0920-9964(17)30446-2 (2017).

399. Grupp, H. et al. Excess costs from functional somatic syndromes in Germany - an analysis using entropy balancing. J. Psychosom. Res. 97, 52-57 (2017).

400. Baumeister, H. et al. Direct and indirect costs with chronic back pain and comorbid mental disorders - a systematic review. J. Psychosom. Res. 73, 79-85 (2012).

401. Gawronski, K. A. et al. Potentially traumatic events and serious life stressors are prospectively associated with frequency of doctor visits and overnight hospital visits. J. Psychosom. Res. 77, 90-96 (2014).

402. van Gils, A. et al. Individual variation in the temporal relationships between stress and functional somatic symptoms. J. Psychosom. Res. 77, 34-39 (2014).

403. Sousa, N. The dynamics of the stress neuromatrix. Mol. Psychiatry 21, 302-312 (2016).

404. Williams, L. J. et al. Pain and the relationship with mood and anxiety disorders and psychological symptoms. J. Psychosom. Res. 72, 452-456 (2012).

405. Jaracz, J. et al. Unexplained painful physical symptoms in patients with major depressive disorder: Prevalence, pathophysiology and management. CNS Drugs 30, 293-304 (2016).

406. Dijkstra-Kersten, S. M. et al. Longitudinal associations of multiple physical symptoms with recurrence of depressive and anxiety disorders. J. Psychosom. Res. 97, 96-101 (2017).

407. Huijbregts, K. M. et al. Negative association of concomitant physical symptoms with teh course of major depressive disorder: A systematic review. J. Psychosom. Res. 68, 511-519 (2010).

408. Auerbach, A. D. et al. Preventability and causes of readmissions in a national cohort of general medicine patients. JAMA Intern. Med. 176, 484-493 (2016).

409. van Galen, L. S. et al. Patients' and providers' perception of the preventability of hopsital readmissions: A prospective, observational study in four European countries. BMJ Qual. Saf. pii: bmjqs-2017-006645 (2017).

410. McAndrew, L. M. et al. High healthcare utilization near the onset of medically unexplained symptoms. J. Psychosom. Res. 98, 98-105 (2017).

411. de Jong, M. J. et al. Telemedicine for management of inflammatory bowel disease (mylBDcoach): A pragmatic, multicentre, randomised controlled trial. Lancet 390, 959-968 (2017).

412. van Os, J. et al. The experience sampling method as an mHealth tool to support self-monitoring, selfinsight, and personalized health care in clinical practice. Depress. Anxiety 34, 481-493 (2017). 


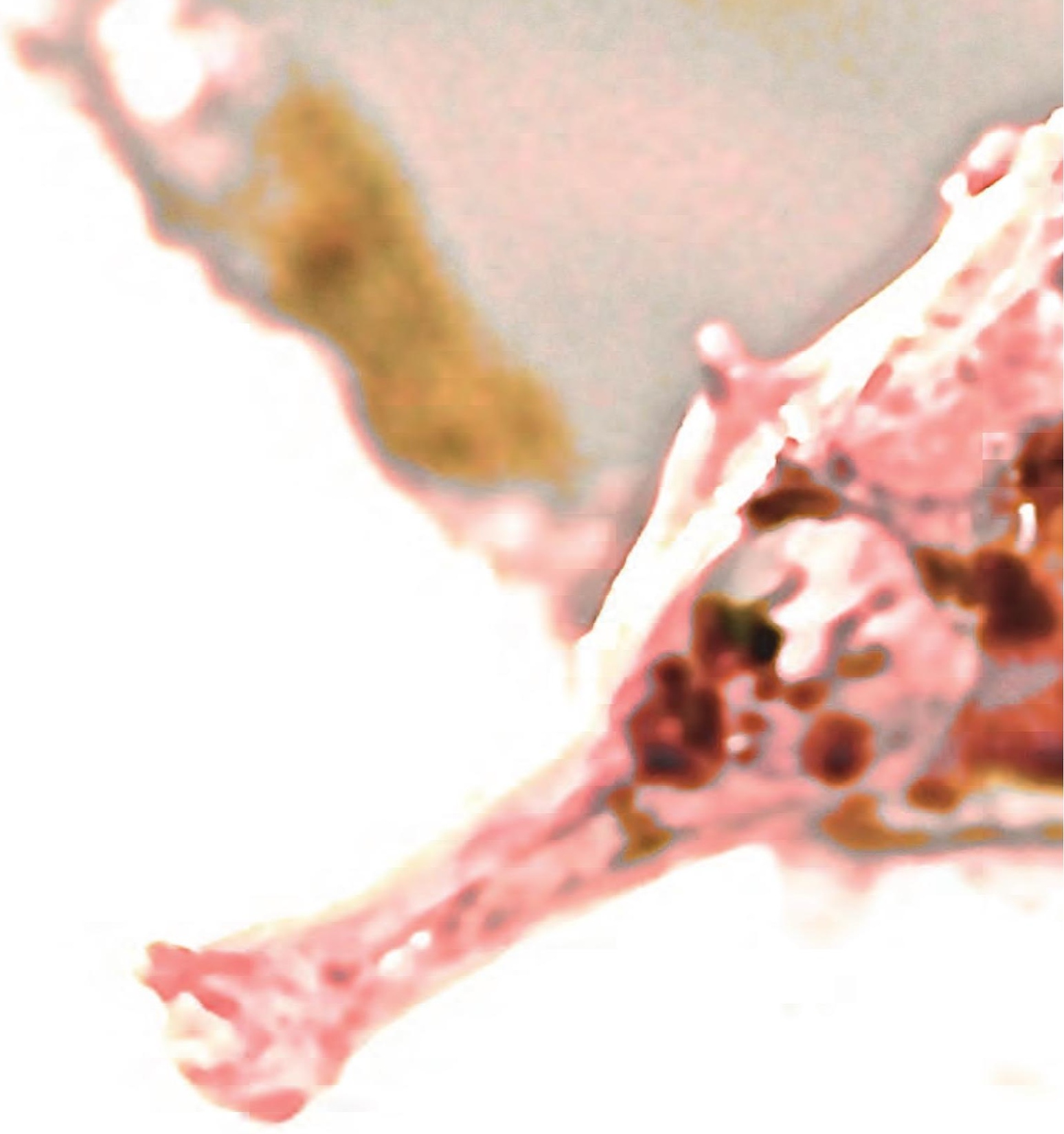




\section{Appendix}

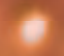

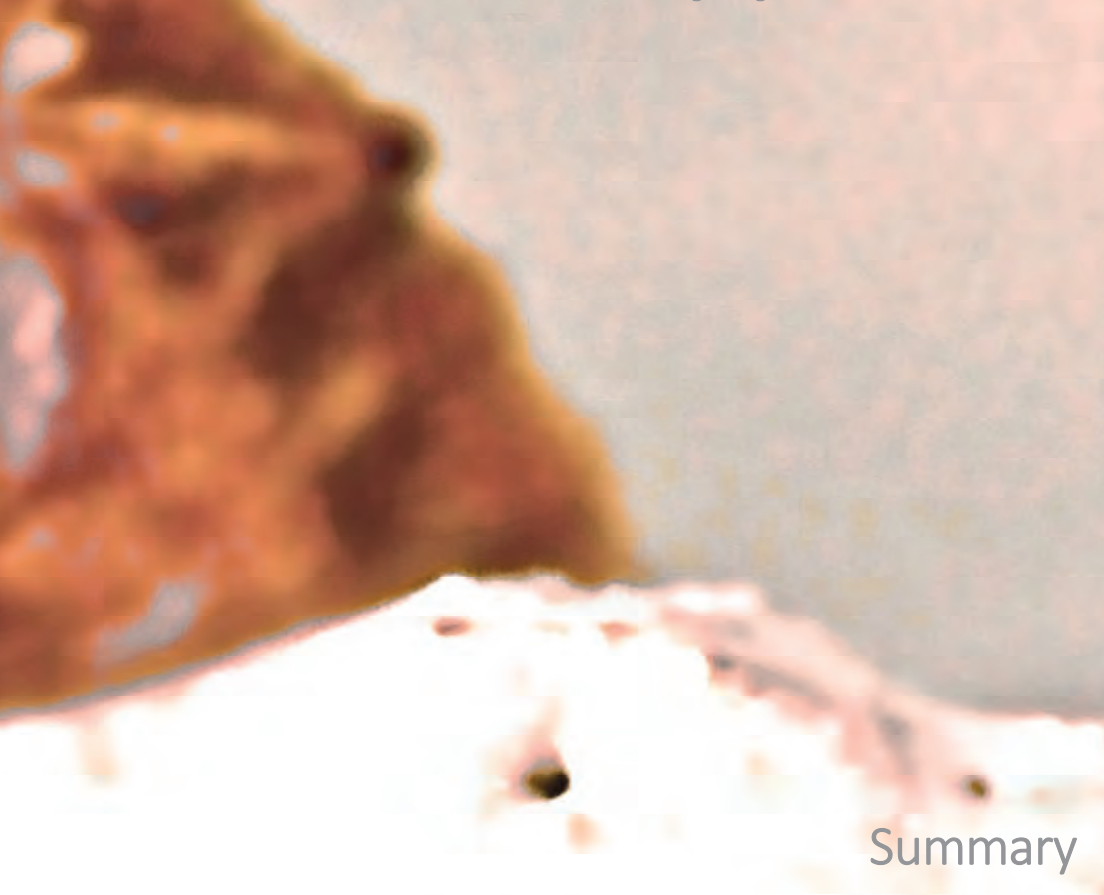

Samenvatting

Dankwoord

Publications

Curriculum vitae

Abbreviations 



\section{Summary}

From the perspective of a psychosomaticist (e.g., hospital psychiatrist, medical psychologist, GP, gastroenterologist, urologist, ENT physician and other specialty consultants with an interest in multi-morbidity or functional complaints), the primary aim of this thesis was to gain deeper insight into meaningful possibilities for integrated care in psychosomatic medicine. Clinical research was conducted against a background of different hospital (i.e., inpatients and outpatients) and primary health care levels and related medical specialties. The secondary aims of this thesis were i) to introduce an overlapping and bridging model of integrated care across settings in order to connect medical specialties in multi-conditional patients and to avoid the disadvantages of mono-disciplinary care; ii) to contribute new insights into etiopathological pathways of multi-morbidity; and iii) to support real-life symptom assessment in psychosomatic medicine, which may help to identify how pleiotropic symptoms cluster to form syndromes, eventually leading to a better conceptualization of multi-morbidity, turning our gaze away from symptom-based diagnostic constructs toward an etiopathogenetic concept of psychosomatic conditions.

This PhD-thesis is divided in six parts, each of which contributed to the abovementioned aims. While chapter 1 delivers as introduction an extensive overview on existing integrated care across various settings, the first part of the thesis describes and investigated our own integrated psychosomatic care in the inpatient hospital setting. Chapter 2 explored the advantages and the costs of admission to a Med-Psych-Unit (MPU). The findings suggest that patients at the interface of psychiatric and somatic morbidity are diagnosed and treated adequately at the MPU, leading to a decrease in medical service use and an appropriate increase in exposure to psychiatric interventions. The results are specifically generalizable to MPUs with a focus on psychosomatic conditions, for instance, somatoform disorders or affective disorders with comorbid somatic diseases. However, failure to show cost savings in general due to longer length of stay (LOS) compared to medical wards, which outweighs cost-benefit derived from lower medical service use, suggests that MPU activities may gain in (cost-) effectiveness if shifted earlier to day-care, outpatient and transitional psychosomatic care solutions. Thus, given that defragmentation of health care is cost effective in general, defragmentation by applying clinically integrated health care for the most complex patients that are sensitive to inpatient interventions should be embedded in transitional psychosomatic hospital outpatient care programs, offering ambulatory solutions when possible that guide patients back to primary care, in order to shorten hospital stay and to prevent unintended readmission.

Hence, the second part of the PhD-thesis examines examples of integrated hospital outpatient care in psychosomatic medicine, which are bundled together with the inpatients MPU and the CLS in the hospital-based Med-Psych-Centre (MPC). Chapter 3 describes the possibility to merge with an integrated outpatient approach in dealing with 
medically unexplained otorhinolaryngological symptoms (MUORLS). Despite MUORLS are common in tertiary care settings, the findings from the present cohort study suggest that the majority of patients with MUORLS suffer from under- or undiagnosed psychiatric morbidity. In order to prevent treatment refractoriness, patients suffering from MUORLS deserve easy access to integrated interdisciplinary care (ORL and psychiatry) followed by detailed psycho-education about the pathogenesis of their complaints and the future integrated psychosomatic approach accordingly. Chapter 4 reports on integrated medical-psychiatric outpatient care in functional gastrointestinal disorders (FGIDs) with regard to patient-related outcomes. The present prospective, open study on the efficacy of routine care applied through an integrated medicalpsychiatric outpatient care model for complex FGID patients still is the first study to report on a joint multidisciplinary approach for complex FGIDs with psychiatric comorbidity in an outpatient setting. We observed that as much as $80 \%$ of patients with complex FGIDs had not been diagnosed or treated for their psychiatric comorbid disorder up to the point of joint assessment. A beneficial effect of the integrated care approach was shown, with a significant improvement of gastrointestinal and psychiatric symptoms, and quality of life after 6 months. Except for the improvement in gastrointestinal symptoms, this improvement was sustained after 1 year, emphasizing the need of appropriate integrated follow-up management of gastrointestinal symptoms associated with psychiatric comorbidity. Eventually, the results show that screening for psychiatric comorbidities in FGID patients is necessary, and that collaboration between gastroenterologists and psychiatrists may lead to a better treatment of complex FGID patients. Chapter 5 considers the prevalence of affective symptoms and their association with pelvic floor dysfunctions (PFDs) at an outpatient-based integrated Pelvic-CareCentre (PCC). In the present study, anxiety and depression, suggestive for affective conditions, were prevalent (30.9\% and $20.3 \%$, respectively) in a cohort of tertiary patients with PFDs. In our final regression model, the explained variance of depression and anxiety, measured with the hospital anxiety and depression scale (HADS) was 12\% and $7.4 \%$, respectively, meaning that, corrected for other contributing factors, $12 \%$ of depression, and $7.4 \%$ of anxiety was directly related to PFDs. Whether depression or anxiety may lead to PFDs was not part of this investigation. We advocate a multidisciplinary approach including psychological and psychiatric assessment, for example, using the HADS questionnaire, at least in complex PFD patients.

The third part of the thesis is devoted to the primary care setting. Chapter 6 describes what happens in the absence of integrated guidance by medical staff concerning chronic pain treatment in the Dutch outpatient and primary care setting. In the presence of a nationwide population-based routine dispensing database, we examined the hypothesis that chronic pharmacological pain treatment of hospital outpatients and patients in primary care would show escalation of analgesics in association with level of urbanisation and neighbourhood index of deprivation. These hypotheses were formulated because of the well-known epidemiological association 
between urban environment and mental health supported by work showing that urban living is associated with increased amygdala activity, a key region in the regulation of stress, affective experience and pain. Pain as the natural comorbid mental experience of somatic conditions is strongly influenced by comorbid common mental disorders particularly affective disorders. Hence, it was further predicted that the prescriptions of psychotropic medication (e.g., antidepressants, antipsychotics, mood stabilisers, etc) would be associated with escalation of analgesics in patients prescribed chronic analgesic treatment. Indeed, escalation of chronic analgesic treatment in persistent pain states is associated with urban environments and deprived neighbourhoods and occurs in a context of psychotropic medication, suggesting that persistent pain outcomes are associated with area influences affecting mental health. Broadening the WHO analgesic ladder to an understanding which includes mental health perspectives will enhance the understanding of central pain sensitisation and could minimise negative classical pain treatment outcomes, for example failed back surgery or negative opioid-associated consequences, especially in patients with undetected mental disorders. From a public health and clinical perspective, a more effective treatment of persistent pain, including treatment of psychiatric comorbidity, may save costs. A new focus in populations with persistent pain states on early recognition and treatment of mental health problems may be more (cost-) effective and also represents an area of unmet clinical need.

The fourth part of this thesis describes how shifting integrated care from the university hospital back to general practitioners may contribute to a successful transition of care. Chapter 7 investigated in a naturalistic pre-post comparison the positive impact of transitional integrated care on costs in multi-conditional patients. The present study still is the first to evaluate cost changes with regard to the referral of patients with somatic and psychiatric multi-morbidity to a transitional Med-Psych-Net. This psychosomatic network consists of the Maastricht University Hospital's (MUMC) psychosomatic inpatient (i.e, MPU and consultation liaison services (CLS)) and outpatient care activities (e.g., PCC, Pain Centre, etc.), which are bundled in the hospital-based MPC, and the staff guided transitional care part that is delivered by community mental health service's (CMHS) case managers (CM) bridging the gap between the MUMC and primary care. Costs concerning LOS, hospital related medical service use and transitional mental health care (MHC) were measured before and after referral to the MPN. The results of the present study confirmed the hypotheses, in that medical service use data revealed a significant cost benefit after MPN referral. Similar results were obtained concerning LOS. In contrast, costs related to psychiatric or psychological interventions increased with MPN treatment. Given the fact that almost each referred patient (95\%) was diagnosed with a psychiatric disorder, while somatic conditions were less (but still high) prevalent (80\%), the increase in costs related to $\mathrm{MHC}$ reflects a desirable and appropriate rise of interventions in psychosomatic conditions. Even though cost associated with $\mathrm{MHC}$ increased, there was a decrease in overall costs, which is estimated as 1000, - $€$ cost benefit per patient and year, as far as care related costs associated with 
the integrated MPN-approach are concerned. These cost developments have emerged without changing of hospital psychiatry or medical psychology related FTEs, since CMHS's care givers started to join primary care during that period. Noteworthy, these care givers were colocated in the MUMC and at GPs and not additionally employed. Thus, cost savings were achieved by care delivery using pre-existing services. Neither the hospital CLS of psychiatry and medical psychology nor the CMHS had to hire extra staff. At both sides of the MPN (MUMC and CMHS), caregivers' fulltime equivalents (FTES) remained the same, reflecting cost-effectiveness from a societal or health system perspective. Patients, if identified at all, would usually have been referred directly to CMHS. Due to the MPN, better case detection as well as medical staff guidance took place. Given the fact that patients adhered to treatment, and therefore, were not lost in the transition, hospital discharge was managed effectively without compromising FTEs. The MPN approach clearly closes gaps between different care providers in psychosomatic conditions, both inside and outside the medical hospital, and is translatable to every care situation with specialty care at different levels. It may even be used as a blue print for transitional complexity interventions in multi-conditional patients without psychiatric comorbidity, since the level of comorbidity care and the quality of illness management is relevant in avoiding every kind of hospital readmission.

The fifth part of the thesis focuses on possible influences that integrated care could have on the conceptual understanding of etiologic pathways in psychosomatic medicine. Chapter 8 describes how affective states may amplify the severity of patients' subjective experience in oropharyngeal dysphagia (OD). Affective symptoms were observed in almost half of the patients with OD. Clinically relevant symptoms of anxiety were significantly associated with some FEES variables (piecemeal deglutition and postswallow vallecular pooling). However, the more severe the score on piecemeal deglutition and postswallow vallecular pooling, the less important the affective complaints were. Thus, anxiety seems to play a role in OD, particularly in the early stages of neurological or oncological disorders or in medically unexplained OD complaints, indicating that affective states might be amplifiers of subjectively experienced symptoms. Chapter 9 reviews the evidence on affective symptoms in patients with OD. The current literature revealed that affective symptoms in patients with OD are common. Screening for affective symptoms in patients with $O D$ is clinically relevant and may help caregivers who are not aware of psychiatric comorbidity to detect cases. Although the likelihood of psychiatric comorbidity in OD patients seems obvious, therapy-effect studies to document effect of integrated care are scarce. At least in complex patients with affective comorbidity, the management of OD requires a multidimensional approach to enable patients to adhere to swallowing rehabilitation. For that reason, psychological or psychiatric expertise should be integrated in multidimensional OD approaches, which might better contribute to treatment satisfaction. Chapter 10 (written in German) demonstrates that transitional integrated care may contribute to better treatment and outcome in severely disturbed delirious patients since delirium still remains frequently 
undetected and untreated, both inside the hospital and after discharge. Chapter 11 (written in Dutch) elucidates the interrelationship between chronic pain and affective disorders with regard to neurobiological and immunological similarities and its implications for integrated care. Chapter 12 makes strides toward a clear definition of what is associated with overactive bladder from a mental health care perspective. This contributes to a better understanding of what might influence functional disorders with regard to stress-related experiences as most research investigates psychosocial aspects aspecifically, without taking psychiatric comorbidity into account. The results of this systematic review revealed a positive association between the co-occurrence of $O A B$ and affective symptoms. Furthermore, there is evidence for new onset of $O A B$ in depressive subjects, but further research is necessary to examine the strength of the effect. Hence, affective dysregulation may occasionally worsen OAB symptoms and poorer treatment outcome. Results also indicate that mental health professionals would do well to take into account urological symptoms in patients with affective complaints. The precise relationship of OAB with affective conditions, is difficult to establish, but may involve a mechanism in the "bladder-brain-axis". Further research is needed in order to determine the direction of the association and the underlying pathophysiological pathways. Then, chapter 13 presents a sensitization and alarm-falsification concept of functional abdominal and urological disorders. Functional urological and gastrointestinal conditions are interrelated and characterized by a chronic course and considerable treatment resistance. Urological disorders associated with a sizeable functional effect include overactive bladder (OAB), interstitial cystitis/bladder pain syndrome (IC/BPS), and chronic prostatitis/chronic pelvic pain syndrome (CP/CPPS). Poor treatment outcomes might in part be attributable to untreated underlying psychiatric disorders, as the co-occurrence of functional urological and gastrointestinal disorders with mood and anxiety disorders is common. The 'bladder-gut-brain axis' (BGBA) is a useful framework under which this interaction can be studied, suggesting that functional disorders represent a sensitized response to earlier threats resulting in perceived emotional and bodily distress - the symptoms of functional disorders. Beside psychological stressors, physical threat - either from external sources or internal sources such as infections might contribute to alarm falsification by influencing the body-brain crosstalk on homeostasis and, therefore, affecting mood, cognition, and behaviour. Psychological and physical stress pathways might contribute to such alarm falsification, and neuroticism could be a vulnerability risk factor for the co-occurrence of functional disorders and affective conditions. Future multidisciplinary research and an integrated care approach is, therefore, required to further elucidate and remediate functional urological and gastrointestinal polymorphic phenotypes. Part of that research should focus on better symptom assessment in daily life, in order to describe how environmental factors may influence stress or threat-related pathways.

Thus, the sixth part of the thesis points out how integrated care may contribute to a more informative approach to symptom assessment as a means to develop a better 
understanding of symptom formation and symptom clustering related to real-life contextual influences. Chapter 14 describes the experience sampling method (ESM) as a tool for momentary assessment in irritable bowel syndrome and how ESM may contribute to real-time symptom recording in psychosomatic medicine. Irritable bowel syndrome patients report higher abdominal pain and flatulence scores in end-of-day diaries when compared to day-average scores of momentary symptom assessment by ESM, with a preference of peak-pain reporting at the end of the day. In retrospective symptom questionnaires that cover a 1-week period, the difference of symptom reporting when compared to electronic ESM is even higher for abdominal pain and the other GI symptoms. Additionally, affective comorbidity in IBS leads to higher abdominal pain and bloating scores compared to IBS patients without comorbidity, indicating that affective states are part of an alarm sensitization process. Moreover, chapter 15 demonstrates the reliability of transdiagnostic $m$-health routine outcome monitoring in an outpatient-based psychosomatic health care service using experience-sampling technology. The ESM-based, transdiagnostic mROM tool can be used reliably in clinical settings: it shows adequate psychometric properties, as well as concurrent validity and sensitivity to change over time with respect to relevant ROM constructs. Person-tailored items can be added. In addition, mROM offers added value over standard symptombased ROM, as it provides information on adaptive functioning in the daily environment of patients.

Finally, Chapter 16 provides a general discussion and valorisation section setting the stage on which to present an outlook on how symptoms related to psychosomatic conditions might better be conceptualized and therefore further investigated and treated. In conclusion, functional somatic conditions with comorbid affective disorders are best understood as a sensitized defence response to earlier threats resulting in perceived emotional and bodily distress (i.e., hyperarousal). Beside psychological stressors, physical threat contributes to an alarm falsification in the body-brain crosstalk and, therefore, affecting mood, cognition, and defensive behaviour - the symptoms of affective and functional disorders. In order to further investigate the relation between contextual threat and symptom formation, a feasible and reliable momentary assessment tool for diagnostic purposes exists (i.e., ESM). Moreover, ESM has been shown to be a reliable transdiagnostic $\mathrm{mROM}$ tool, which also can be used to facilitate shared decision making and monitoring of integrated person-tailored treatment in psychosomatic medicine. In this context, the transitional Med-Psych-Net might help to integrate personalized psychosomatic care across all medical settings and specialties. 


\section{Samenvatting}

Vanuit het perspectief van de psychosomaticus (bijvoorbeeld als ziekenhuis-psychiater, medisch psycholoog, huisarts, gastro-enteroloog, functioneel uroloog, KNO-arts, of als medisch specialist met interesse in multi-morbiditeit en functionele somatische klachten) is het primaire doel van dit proefschrift het creëren van betekenisvolle nieuwe mogelijkheden van integrale zorg in de psychosomatische geneeskunde. In dit kader werd (klinisch) onderzoek op verschillende zorgniveaus in samenwerking met betrokken medisch specialismen verricht, van het algemeen ziekenhuis tot aan de huisartspraktijk. Secundaire doelen van dit proefschrift waren i) de introductie van een overlappend en verbindend integraal zorgmodel dat verschillende medisch specialismen ziekenhuisintern rondom complexe patiënten kan verbinden en een brug kan slaan van het ziekenhuis naar de eerste lijn; ii) de introductie van nieuwe inzichten in ethiopathologische paden met betrekking tot multi-morbiditeit; iii) de introductie van metingen met betrekking tot psychosomatische klachten in het dagelijks leven om op deze manier na te gaan hoe complexe symptomen stress afhankelijk clusteren tot syndromen. Het bereiken van genoemde doelstellingen zou kunnen bijdragen tot een etiologische conceptualisatie van muli-morbiditeit die dit proefschrift overstijgt: op weg van een descriptieve symptoom gebaseerde diagnostiek naar een begrijpende etiologische nosologie in de psychosomatiek.

Dit proefschrift bestaat uit zes delen die allen bijdragen aan de beantwoording van boven genoemde doelstellingen. Hoofdstuk 1 levert als introductie een overzicht over bestaande integrale zorgmodellen in verschillende zorg 'settingen'. Het eerste deel van dit proefschrift beschrijft onze eigen ziekenhuisinterne ervaringen met betrekking tot integrale psychosomatische zorg. Hierbij onderzoekt hoofdstuk 2 voordelen en medische kosten van een ziekenhuisopname op een Medisch-Psychiatrische-'Unit' (MPU). Onze bevindingen duiden aan dat patiënten met psychiatrisch-somatische comorbiditeit op een MPU adequaat gediagnostiseerd en behandeld worden, hetgeen leidt tot afname van medische verrichtingen en toename van psychiatrische zorg. De resultaten zijn specifiek geldig voor MPUs met focus op psychosomatische problematiek, zoals somatoforme stoornissen, functionele somatische aandoeningen en somatische ziekten met affectieve comorbiditeit. Aangezien algehele besparingen bij een relatief lange opnameduur niet konden worden bereikt valt aan te bevelen om medisch-psychiatrische zorg indien nodig kort klinisch maar vooral dagklinisch en poliklinisch aan te bieden en te voorzien van een transmurale zorgoverdracht. Omdat defragmentatie van zorg over het algemeen kosteneffectief is zou de klinische opname van meest complexe patiënten daarom moeten worden ingebed in transmurale psychosomatische zorgprogramma's die patiënten terug naar de huisarts geleiden om op deze manier een lange verblijfsduur in het ziekenhuis en ongeplande heropnames te kunnen voorkomen.

Vanuit deze aanbeveling beschrijft het tweede deel van het proefschrift voorbeelden van integrale poliklinische zorg in de psychosomatische geneeskunde die in 
ons ziekenhuis tezamen met de MPU en de ziekenhuis-psychiatrische en medisch psychologische consultatieve dienst zijn samengevat in het Med-Psych-Centre. Hoofdstuk 3 zet uiteen hoe KNO-artsen en psychiaters poliklinisch patiënten met onbegrepen oto-rhino-laryngale klachten (MUORLS) kunnen benaderen. Ofschoon MUORLS patiënten in de tertiaire academische setting vaak voorkomen worden comorbide psychiatrische stoornissen vaak niet herkend en blijft de geboden zorg voor patiënten en dokters onbevredigend. Om zorgsatisfactie te vergroten wordt aanbevolen patiënten met MUORLS integrale psychosomatische zorg aan te bieden. Dit houdt onder andere in dat psycho-educatie wordt gegeven over de pathofysiologie van de klachten en dat patiënten te weten komen welke integrale behandelmogelijkheden hiervoor bestaan. Hoofdstuk 4 bericht over integrale gastroenterologisch-psychiatrische poliklinische zorg bij functionele gastro-intestinale stoornissen (FGIDs) met een focus op directe patiënten gerelateerde uitkomstmaten. Ons open prospectief onderzoek is de eerste studie die een integraal poliklinisch zorgtraject voor patiënten met FGIDs en psychiatrische comorbiditeit evalueert. Wij konden vaststellen dat vooraf aan het multidisciplinair spreekuur ca. $80 \%$ van patiënten met complexe FGIDs noch op hun psychiatrische comorbiditeit werden gedetecteerd noch hiervoor werden behandeld. Het voordeel van integrale zorg blijkt uit de significante verbetering van gastrointestinale en psychiatrische symptomen na een half jaar alsmede de hogere kwaliteit van leven. Deze verbeteringen hielden ook na een jaar stand, op de gastro-intestinale symptomen na. Aangezien poliklinische vervolgcontacten alleen ziekenhuis-psychiatrisch werden verricht valt daarom te overwegen om ook het 'follow-up' management bij gastro-intestinaal-psychiatrische comorbiditeit integraal plaats te laten vinden. De resultaten van ons onderzoek laten zien dat in het ziekenhuis screening op psychiatrische comorbiditeit bij complexe FGIDs aan te bevelen is en dat de collaboratie tussen gastro-enteroloog en ziekenhuis-psychiater tot een betere behandeling van complexe functionele patiënten kan leiden. Hoofdstuk 5 beschouwt de prevalentie van affectieve symptomen en hun associatie met bekkenbodem disfuncties (PFDs) bij poliklinische patiënten van een integraal 'Pelvic-Care-Centre' (PCC). In ons cohort onderzoek waren angst- en depressiesymptomen met $30.9 \%$ en $20.3 \%$ in een cohort van tertiaire PDF patiënten prevalent aanwezig. Ons laatste regressiemodel levert een verklaarde variantie met betrekking tot angst en depressieve symptomen gemeten met de 'hospital anxiety and depression scale' (HADS) van $12 \%$ en $7.4 \%$ op. Dit houdt in dat gecorrigeerd voor andere bijdragende factoren $12 \%$ van depressieve en $7.4 \%$ van angst symptomen direct aan PDFs te relateren waren. Of depressieve of angstklachten tot PDFs hebben geleid was niet onderwerp van het onderzoek. Wij adviseren daarom een integrale multidisciplinaire benadering inclusieve psychologische en psychiatrische zorg voor complexe PDF patiënten.

Het derde deel van het proefschrift is gewijd aan de eerstelijns zorg van complexe patiënten met het vermoeden op ongedetecteerde somatisch psychiatrische comorbiditeit. Hoofdstuk 6 beschrijft wat er Nederland in de eerste lijn en op 
monodisciplinaire poliklinieken gebeurd als integrale medisch specialistische expertise bij de behandeling van chronische pijn patiënten ontbreekt. Uitgaande van een landelijk populatie databestand van medicatievoorschriften werd onderzocht of chronische farmacologische pijnbehandeling van poliklinische en eerstelijns patiënten een escalatie van analgetica geassocieerd met de graad van urbanisatie en het wonen in achterstandswijken laat zien. Deze hypothese werd geformuleerd tegen de achtergrond van de bekende epidemiologische associatie tussen geestelijke gezondheid en stedelijke omgeving. Deze associatie werd recent etiologisch onderbouwd door aan te tonen dat het leven in een stedelijke omgeving gepaard gaat met verhoogde activiteit van de amygdala, een belangrijk gebied van de hersenen voor affectieve ervaringen, stressregulatie en pijnbeleving. Pijn wordt sterk beïnvloed door psychiatrische aandoeningen met name door affectieve stoornissen. Daarom werd verder voorspeld dat het voorschrijven van psychotrope medicatie, zoals antidepressiva, antipsychotica of stemmings-stabilisatoren, geassocieerd is met de escalatie van analgetica bij patiënten met chronisch analgeticagebruik. Daadwerkelijk is de escalatie bij een chronische analgetica-behandeling geassocieerd met stedelijke omgevingen, achterstandswijken en met het gebruik van psychotrope medicatie. Deze bevindingen suggereren dat behandeluitkomsten van persisterende pijn geassocieerd zijn met omgevingsinvloeden die de mentale gezondheid aantasten. Het verbreden van de WHO-analgeticaladder naar een begripsvorming die psychiatrische comorbiditeit bij chronische pijn omvat kan helpen om het aspect van centrale pijnsensitisatie beter te begrijpen en negatieve uitkomsten van chronische pijnbehandelingen te minimeren, bijvoorbeeld 'failed back surgery' of opiaat geïnduceerde hyperalgesie. Dit geldt met name in het geval van niet gedetecteerde psychiatrische comorbiditeit. In het kader van de publieke gezondheidszorg maar ook vanuit klinisch perspectief zou de behandeling van bijvoorbeeld affectieve comorbiditeit bij chronisch pijn kosten kunnen besparen. Een nieuwe focus in populaties met persisterende pijn zou daarom de vroege herkenning en behandeling van GGZ-problematiek moeten zijn.

Het vierde deel van dit proefschrift laat zien dat een overdracht door middel van integrale zorg vanuit het ziekenhuis terug naar de huisarts een succesvolle bijdrage kan leveren aan transmurale zorg. In hoofdstuk 7 wordt door een naturalistische voor en na vergelijking de positieve impact van transmurale integrale zorg op de medische kosten van complexe psychosomatische patiënten beschreven. Onze studie is het eerste onderzoek dat een evaluatie van medische kostenontwikkelingen bij patiënten met somatisch-psychiatrische multi-morbiditeit door transmurale netwerkzorg door middel van een Med-Psych-Net beschrijft. Dit psycho-somatische netwerk bestaat uit integrale multidisciplinaire zorgactiviteiten die klinisch (MPU en CLS) en poliklinisch (pijncenter, PCC, etc.) binnen het MUMC worden verricht en ter herkenbaarheid onder de naam van het ziekenhuis gerelateerde Med-Psych-Center zijn samengevoegd. Het netwerk wordt echter pas compleet door de transmurale betrokkenheid van eerstelijns of 'communitybased' zorgactiviteiten. Deze betrokkenheid wordt vorm gegeven door case managers 
vanuit de RIAGG die patiënten onder supervisie van medisch specialisten (ziekenhuispsychiaters en somatische specialisten) vanuit de ziekenhuis-psychiatrische en medisch psychologische polikliniek transmuraal terug begeleiden naar de eigen huisarts. De kloof tussen het MUMC en de eerste lijn werd op deze manier effectief overbrugt. Dit blijkt uit kosten voor en na verwijzing naar het MPN traject gerelateerd aan de lengte van opnameduur, medische verrichtingen in het ziekenhuis en de transmurale geestelijke gezondheidszorg. De resultaten van onze studie bevestigen de hypothesen dat lengte van opname en het aantal medische verrichtingen significant zouden dalen door transmurale MPN-zorg. Daarentegen stegen de kosten van psychiatrische en psychologische interventies met de integrale MPN-zorg. Gegeven het feit dat bijna 95\% van de naar het MPN verwezen patiënten aan een psychiatrische aandoening leden terwijl iets minder, namelijk $80 \%$ van de verwezen patiënten, door een somatische ziekte betroffen waren, is de GGZ gerelateerde kostenstijging een wenselijke en adequate uitkomst van de psychosomatische interventie. Temeer omdat de algemene medische kosten met ongeveer 1000,- Euro per patiënt en jaar als gevolg van de MPN-interventie gingen dalen. Deze positieve kostenontwikkeling werd bereikt mede omdat ziekenhuispsychiatrische of medisch-psychologische FTEs niet gingen stijgen aangezien RIAGG case managers (gesuperviseerd door specialisten) patiënten in het ziekenhuis hebben ontvangen en deze CMs in de zelfde periode ook met zorg vanuit de eerste lijn waren gestart. Vermeldenswardig is dat de RIAGG CMs in het MUMC en bij de huisartsen uit bestaande contracten werden geplaatst en niet additioneel in dienst werden genomen. Het ziekenhuis hoefde geen extra personeel in dienst te nemen en kostenbesparingen konden uit geherstructureerde RIAGG dienstverlening worden bereikt. Aan beide kanten van het MPN (MUMC en RIAGG) bleef het aantal FTEs gelijk wat de kostenbalans positief en maatschappelijk relevant maakt. Inhoudelijk hebben de betere detectie van psychosomatische problematiek, de staf supervisie en de warme overdracht tussen ziekenhuis en huisartsenzorg bijgedragen tot het MPN-resultaat. Patiënten toonden grotere compliantie en adherentie vergeleken met eerdere niet integrale transmurale trajecten. Dus, het Med-Psych-Net overbrugt de kloof tussen ziekenhuis en eerstelijns zorg met betrekking tot psychosomatische problematiek aangezien het niveau van comorbiditeitszorg en de kwaliteit van ziekte- of case-managment door de integrale transmurale benadering werd verbeterd.

Het vijfde deel van dit proefschrift focust op mogelijke invloeden die integrale zorg op de conceptuele begripsvorming omtrent etiologische 'pathways' in de psychosomatiek kan ontwikkelen. Hoofdstuk 8 beschrijft dat de affectieve situatie van patiënten de ernst van subjectief beleefde oro-faryngale dysfagie (OD) kan versterken. Affectieve klachten werden in bijna de helft van OD patiënten geobserveerd. Klinisch relevante symptomen van angst waren significant geassocieerd met FEES ('fiberoptic endoscopic evaluation of swallowing') variabelen. Echter, hoe ernstiger de objectieve FEES-score werd (mate van structurele schade) hoe minder belangrijk affectieve symptomen met betrekking tot subjectieve dysfagiebeleving werden. Angst speelt dus in 
het bijzonder een rol in vroege fasen van OD bij neurologische of oncologische ziekten of bij medisch onbegrepen OD. Het lijkt erop dat de affectieve staat waarin een OD-patiënt verkeerd een versterkend effect op subjectief beleefde OD kan hebben. Hoofdstuk 9 geeft een systematisch overzicht over het bestaan van affectieve symptomen bij patiënten met OD. De huidige literatuur geeft aan dat affectieve klachten bij patiënten met OD vaak voorkomen. Het screenen van affectieve symptomen bij patiënten met OD is klinisch relevant en kan somatische hulpverleners helpen bij de detectie van psychiatrische comorbiditeit. Ook al is het voorkomen van psychiatrische comorbiditeit bij complexe OD patiënten eerder de regel dan een uitzondering bestaan er geen effectstudies op een integrale behandeling. Hoofdstuk 10 (Duitstalig) beschrijft dat een transmuraal integraal zorgtraject ook kansen biedt om de uitkomst van ernstig delirante patiënten te verbeteren aangezien een delirium nog steeds vaak onopgemerkt en derhalve onbehandeld blijft. Hoofdstuk 11 (Nederlandstalig) verheldert de relatie tussen chronische pijn en affectieve stoornissen middels een overzicht met focus op neurobiologische en immunologische gelijkenissen. Ook bij chronische pijn biedt integrale zorg een beter op de complexe patiënt toegesneden behandeloptie dan monodisciplinaire zorg. Hoofdstuk 12 maakt stappen naar een heldere definitie van wat er uit psychiatrisch perspectief met een overactieve blaas (OAB) geassocieerd is. Tot kort geleden waren psychosomatische verklaringen vrij diffuus en gingen vaag uit van psychosociale aspecten die een bijdrage leveren aan de klachtenontwikkeling van functionele urologische syndromen. Dit overzicht onderzoekt specifiek en systematisch de mogelijke associatie tussen $O A B$ en affectieve symptomen. De resultaten van onze systematische review tonen aan dat er een positieve associatie tussen $O A B$ en affectieve symptomen bestaat. Verder is er voldoende evidentie dat OAB kan ontstaan vanuit een preexistente depressieve staat. Een affectieve ontregeling kan OAB verergeren en, mits niet gedetecteerd en onbehandeld gebleven, de behandeling van $O A B$ ten ongunste beïnvloeden. De resultaten geven echter ook te kennen dat GGZ hulpverleners functionele urologische klachten bij affectieve stoornissen in overweging moeten nemen. De precieze relatie tussen functionele urologische syndromen en affectieve stoornissen laat zich het best verhelderen langs mechanismen van de 'bladder-brainaxis'. Hiervoor is verder onderzoek vereist om de directie van de associatie en onderliggende pathofysiologische paden te bepalen. Hoofdstuk 13 introduceert vervolgens een sensitisatie en alarmfalsificatie concept voor functionele urologische en gastro-intestinale aandoeningen en hun psychiatrische comorbiditeiten. Functionele stoornissen van de darm en de blaas komen vaak samen voor en zijn gekarakteriseerd door een chronisch beloop en een aanzienlijke behandelresistentie. Voorbeelden van functionele urologische aandoeningen zijn naast $O A B$ ook het interstitiële cystitis/blaaspijn syndroom (IC/BPS) en het chronische prostatitis/chronisch bekkenpijn syndroom (CP/CPPS). Een gedeelte van de slechte behandeluitkomsten ligt gegrond bij de onderdetectie en -behandeling van comorbide psychiatrische aandoeningen terwijl het gelijktijdige optreden van bijvoorbeeld affectieve stoornissen en functionele 
urologische en gastro-intestinale aandoeningen vaak voorkomt. De 'bladder-gut-brain axis' is een nuttig raamwerk waarin de onderlinge interacties kunnen worden bestudeerd. Functionele stoornissen representeren hierin een sensitisatie respons op eerdere bedreigingen die uiteindelijk resulteren in een emotionele en lijfelijke ontregeling - de symptomen van functionele en affectieve stoornissen. Naast psychosociale stressoren draagt ook fysieke bedreiging, bijvoorbeeld ongelukken of infecties, bij tot alarmfalsificatie door beïnvloeding van de 'body-brain crosstalk' met gevolgen voor emotieregulatie, cognitie en gedrag. Psychologische en fysieke stressantwoorden maken deel uit van de alarmfalsificatie en de hierbij horende afweerreactie. Neurotische persoonskenmerken zijn hierbij een risicofactor voor kwetsbaarheid betreffende het gelijktijdige voorkomen van functionele somatische aandoeningen en affectieve stoornissen. Toekomstig multidisciplinair onderzoek en integrale zorg zijn nodig om functionele urologische en gastro-intestinale polymorfe fenotypen te verhelderen en te herstellen. Een deel van dit onderzoek zou zich moeten richten op betere symptoom metingen in het dagelijks leven om daarmee te kunnen vatten hoe omgevingsfactoren pathofysiologische stress- en afweerpaden activeren.

Daarom richt het zesde deel van dit proefschrift zich op de bijdrage die integrale psychosomatische zorg kan leveren aan informatievere manieren van symptoom metingen die rekening houden met contextuele invloeden vanuit het dagelijks leven. Hoofdstuk 14 beschrijft de 'experience sampling method' (ESM) als instrument voor momentane beoordelingen van prikkelbare darm syndroom (PDS) klachten en hoe de ESM kan bijdragen tot een real-time beoordeling van klachten in de psychosomatiek. Prikkelbare darm patiënten rapporteren hogere pijn en flatulentie scores in 'end-of-day' dagboeken vergeleken met momentane symptoom metingen middels ESM, met een piek voor het einde van de dag. In retrospectieve vragenlijsten die een periode van een hele week afdekken zijn de verschillen in de symptoom rapportage vergeleken met de ESM zelfs nog groter. Dit geldt voor pijn en andere gastro-intestinale symptomen. Bovendien leidt affectieve problematiek tot hogere pijn en opgeblazen gevoel scores bij PDS, vergeleken met prikkelbare darm zonder affectieve klachten. Dit duidt aan dat affectieve en pijnsymptomen deel uitmaken van hetzelfde alarm sensitisatie proces. Hoofdstuk 15 demonstreert de betrouwbaarheid van trans- diagnostische 'm-health' routine uitkomstmetingen bij patiënten op een ziekenhuis- psychiatrische of psychosomatische polikliniek middels de 'experience-sampling' technologie. De ESM toont als mROM instrument adequate psychometrische eigenschappen en gelijktijdig geldigheid en gevoeligheid met het oog op relevante ROM constructen voor veranderingen gedurende de tijd. Bovendien kunnen specifieke op personen toegesneden items worden toegevoegd. Al met al biedt mROM middels ESM een toegevoegde waarde vergeleken met standaard symptoom gebaseerde routine uitkomst metingen omdat deze methode gepersonaliseerde informatie levert over het adaptieve functioneren van patiënten in hun dagelijkse leefomgeving. 
Tenslotte, in hoofdstuk 16 wordt in een algemene discussie en een valorisatie paragraaf geschetst welke vooruitzichten er zijn met betrekking tot een betere conceptualisatie van complexe psychosomatische syndromen - wil zeggen pathofysiologisch inhoudelijk in plaats van puur descriptief en symptoom gebaseerd - en hoe deze in de toekomst in het dagelijks leven wetenschappelijk kunnen worden onderzocht en integraal kunnen worden behandeld. Samengevat zijn complexe (functionele) somatische aandoeningen met bijbehorende affectieve en/of somatoforme problematiek het best te begrijpen als een gesensitiseerde en gecontinueerde afweerrespons op een eerder ervaren bedreiging. Deze sensitisatie of alarmfalsificatie wordt beleeft als emotionele en somatische distress of hyperarousal. Naast fysieke bedreigingen dragen ook psychologische stressoren bij tot een alarmfalsificatie van de 'body-brain crosstalk', hetgeen invloed heeft op stemming, cognities en gedrag - de symptomen van affectieve en functionele stoornissen. Om de relatie tussen contextuele bedreiging en symptoomformatie verder te kunnen onderzoeken bestaat een uitvoerbaar en betrouwbaar instrument voor momentane metingen, de 'experience sampling method'. De ESM heeft zich kunnen bewijzen als trans-diagnostisch mROM instrument dat gebruikt kan worden voor 'shared decision making' en gepersonaliseerde zorg. In dit kader schept het Med-Psych-Net een transmuraal kader over medische disciplines en settingen heen voor integrale en gepersonaliseerde psychosomatische behandelingen. 



\section{Dankwoord}

De totstandkoming, verrichting en uitwerking van klinisch wetenschappelijk onderzoek heeft veel gemeen met het beloop van het dagelijks leven. Er komt niets van terecht zonder een goede samenwerking. Dit is te meer waar als het om complexe zorg bij 'multi-conditional' patiënten gaat. Daarom wil ik alle collegae danken die op welke manier dan ook de patiëntenzorg in ons netwerk-approach een warm hart hebben toegedragen.

Met nadruk geldt dit voor mijn promotieteam. Hooggeleerde Van Os, promotor, beste Jim - hartelijk dank voor de gelegenheid om in een voor de psychiater onoverzichtelijke patiëntenpopulatie klinische zorg wetenschappelijk te hebben mogen evalueren. Je geduld, je gunst en je briljant inzicht hebben mij door de afgelopen jaren gedragen en mijn begrip van goede zorg gevormd. Hooggeleerde Masclee, 2 promotor, beste $\mathrm{Ad}$ - je stond mede aan het begin van dit netwerk. De eerste integrale zorgstappen werden mede vanuit de afdeling MDL gezet. Dank voor je bevlogenheid met betrekking tot het ontwikkelen van nieuwe concepten in de functionele somatisch-psychiatrische problematiek. Weledelzeergeleerde Strik en weledelzeergeleerde Kruimel, copromotores, beste Jacqueline en Joanna - zonder medestanders die met een gedeelde visie diep in de klinische modder staan was dit boekwerk niet te voltooien geweest. Dit dankwoord is te kort om mijn waardering in deze beperkte regels genoeg tot uitdrukking te laten komen.

Het zorgnetwerk werd op geleide van groeiend inzicht door de jaren heen uitgebreid. Graag had ik gezien dat er meer mensen lid van het promotieteam waren. Dit geldt zeker voor de bevlogen (functionele) urologen en de KNO-arts met oog voor de psychosomatiek. Hooggeleerde Van Koeveringe, beste Gommert, en weledelzeergeleerde Vrijens en Baijens, beste Daisy en Laura - het enthousiasme waarmee ook snijdende specialisten een uitweg zoeken uit therapieresistentie gekoppeld aan onopgemerkte psychiatrische comorbiditeit werkt aanstekelijk en heeft me verrast. Ik hoop nog veel voor de ziekenhuis-psychiatrie uit chirurgische zorgvisies af te kunnen leiden.

De hoeveelheid patiënten die in het ziekenhuis met psychosomatische aspecten kampen is alleen enigszins te bolwerken door en groep bevlogen collegae en medestanders. Weledelzeergeleerde Tijssen, beste Marijn - voor jou geldt hetzelfde als voor Jacqueline en Joanna! Dank voor je collegialiteit en de momenten die wij met elkaar konden delen onder het wegen van klinische, strategische en minder zorgrelevante vraagstukken. Hooggeleerde Verhey en Peeters, beste Frans en Frenk; weledelzeergeleerde Leentjens, Arts en Schieveld, beste Albert, Baer en Jan psychiaters, zonder collegiaal afdelingsklimaat is wetenschappelijk onderzoek in een universitair ziekenhuis niet mogelijk; dank voor jullie steun, adviezen en collegiale belangstelling! Beste collegae van de medische psychologie (in het bijzonder 
weledelzeergeleerde Severeijns, beste Ruud), beste collega's van de verpleegafdeling en de dagbehandeling - het mag duidelijk zijn: zonder teamwork geen goede zorg, zeer bedankt! Beste medestanders van de polikliniek en het secretariaat - zorg begint en eindigt niet met het zorginhoudelijke patiëntencontact, dank voor jullie 'holding'.

Zorg en wetenschap omtrent patiënten met somatisch-psychiatrische comorbiditeit is onmogelijk zonder toegewijde collegae uit somatische vakken. Met name het enthousiasme en de belangstelling uit bestaande multidisciplinaire samenwerkingsverbanden heeft zeer ondersteunend gewerkt. Plaatsvervangend voor het hele Pelvic Care Centre: hooggeleerde Van Kerrebroek, beste Philip, en weledelzeergeleerde Berghmans en Link, beste Bary en Gerold - hartelijk dank voor de samenwerking. Hetzelfde geldt voor het multidisciplinaire pijn team: hooggeleerde Van Kleef, beste Maarten, en weledelgeleerde Brouwer, beste Brigitte - met dank voor de goede samenwerking en jullie aanstekelijke nieuwsgierigheid en onomwonden kritiek.

Inmiddels tonen veel MUMC hulpverleners die met comorbiditeit in aanraking komen belangstelling voor integrale zorg. Ik kan hier helaas niet iedereen noemen. Daarom vergeef mij a.u.b. als ik me niet expliciet exemplarisch verheug op een toekomstige samenwerking: Annelies en Sebastian - het kleine project waar wij mee bezig zijn is leuk om te doen. Nelleke - ons pijnproject maken wij zeker af. Zlatan, Daniel, Jamie - de intellectuele uitwisseling over onderliggende aspecten van comorbiditeit had ik niet willen missen. Joost, Iris, Raymond en Erwin - alarmfalsificatie en de integrale zorg hiervoor blijft een gedeelde belangstelling.

Wetenschappelijk onderzoek bedrijven is niet alleen klinisch teamwork. Ger, Servaas en Richel - het uitpluizen van geschikte statistische modellen is even spannend als de dagelijkse kliniek. Zonder jullie methodologische kennis en statistische ondersteuning zat het boekje zo niet in elkaar. Tiny, hartelijk dank voor het 'editing proces'!

Beste huisartsen, HAIOS en PRIMA case managers- hartelijk dank voor jullie interesse in de transmurale samenwerking!

Leden van de manuscriptcommissie, Prof. Stokroos (voorzitter), Prof. Honig, Prof. Ponds, Prof. Smeets en Prof. Söllner, dank voor uw bereidheid om zitting te nemen in de commissie en deze PhD-thesis te beoordelen!

Roger Kathol, Reinhold Stockbrügger and Adriaan Honig - you are my founding fathers of integrated care.

Mijn PhD-thesis was niet af zonder een stabiele en steunende omgeving thuis. Er is altijd een $2^{\mathrm{e}}$ netwerk nodig om klinisch wetenschappelijke uitdagingen aan te kunnen gaan en teleurstellingen vanuit het klinisch netwerk te verteren. Marita, My Love, zonder 
jouw liefde, je geduld, creativiteit en steun was ik niet wie ik ben. Ik heb er geen woorden voor om uit te drukken hoe dankbaar ik ben. Liebe Mutter, lieber Vater - für Euch waren die Kinder das Allerwichtigste. Was das bedeutet spür ich noch heute jeden Tag. Ich bin euch aus tiefstem Herzen dankbar für alles! Liebe Anke, lieber Luca - Ihr beiden seid wirklich mein Netz mit doppeltem Boden. Jedes Mal wenn ich intellektuell ab zu heben drohe, holt ihr mich auf den Boden der Tatsachen zurück; eine Erdung, die ich nicht missen kann. Lieve Irma en Hans, lieve schoonouders, dank voor jullie steun en aanmoedigingen. Hub en Inge, ik weet zeker dat jullie deze dag nog hadden mee willen maken; dank voor alles. 



\section{Publications}

Verhagen S, Berben J, Leue C, Marsman A, Delespaul P, van Os J, Lousberg R. Demonstrating the reliability of transdiagnostic mHealth Routine Outcome Monitoring in mental health services using experience sampling technology. PLoS One 10: e0186294 (2017).

Vrijens D, Marcelissen T, Drossaerts J, Heeringa R, Degaillier S, Leue C, van Koeveringe G. Self-consciouness/awareness and bladder sensations: Comparative study of overactive bladder patients and healthy volunteers. Low. Urin. Tract Symptoms doi: 10.1111/luts.12186 (2017).

Mujagic Z, Jonkers D, Ludidi S, Keszthelyi D, Hesselink M, Weerts Z, Kievit R, Althof J, Leue C, Kruimel J, van Schooten F, Masclee A. Biomarkers for visceral hypersensitivity in patients with irritable bowel syndrome. Neurogastroenterol. Motil. doi: 10.1111/nmo.13137 (2017).

Verdonschot R, Baijens L, Vanbelle S, van de Kolk I, Kremer B, Leue C. Affective symptoms in patients with oropharyngeal dysphagia: A systematic review. J. Psychosom. Res. 97, 102-110 (2017).

van Os J, Verhagen S, Marsman A, Peeters F, Bak M, Marcelis M, Drukker M, Reininghaus U, Jacobs N, Lataster T, Simons C, Lousberg R, Gülöksüz S, Leue C, Groot PC, Viechtbauer $W$, Delespaul P. The experience sampling method as an mHealth tool to support selfmonitoring, self-insight, and personalized health care in clinical practice. Depress. Anxiety 34, 481-493 (2017).

Vrijens D, Berghmans B, Nieman F, van Os J, van Koeveringe G, Leue C. Prevalence of anxiety and depressive symptoms and their association with pelvic floor dysfunctions - a cross sectional cohort study at a Pelvic Care Centre. Neurourol. Urodyn. 36, 1816-1823 (2017).

Mujagic Z, Jonkers D, Hungin A, de Wit N, Wensaas K, Palka M, Leeters V, Kruimel J, Leue C, Masclee A, Muris J. Use of Rome criteria for the diagnosis of irritable bowel syndrome in primary care: A survey among European countries. Eur. J. Gastroenterol. Hepatol. 29, 651-656 (2017).

Vrijens D, Drossaerts J, Rademakers K, Smits M, Wachter S, Leue C, van Koeveringe G. Associations of psychometric affective parameters with urodynamic investigation for urinary frequency. Low Urin. Tract. Symptoms 9, 166-170 (2017). 
Leue C, Kruimel J, Vrijens D, Masclee A, van Os J, van Koeveringe G. Functional urological disorders: A sensitized defence response in the bladder-gut-brain axis. Nat. Rev. Urol. 14, 153-163 (2017).

Verdonschot RJ, Baijens L, Vanbelle S, Florie M, Kremer B, Leue C. The relationship between fiberoptic endoscopic evaluation of swallowing outcome and symtoms of anxiety and depression in dysphagic patients. Laryngoscope 126, E199-207 (2016).

Drossaerts J, Vrijens D, Leue C, Schilders I, Van Kerrebroeck P, van Koeveringe G. Screening for depression and anxiety in patients with storage or voiding dysfunction: $A$ retrospective cohort study predicting outcome of sacral neuromodulation. Neurourol. Urodyn. 35, 1011-1016 (2016).

Berghmans B, Nieman F, Leue C, Weemhoff M, Breukink S, van Koeveringe G. Prevalence and triage of first-contact complaints on pelvic floor dysfunctions in female patients at a Pelvic Care Centre. Neurourol. Urodyn. 35, 503-508 (2016).

Berghmans B, Nieman F, Leue C, Weemhoff M, Breukink S, van Koeveringe G. Prevalence and triage of first contact pelvic floor dysfunction complaints in male patients referred to a Pelvic Care Centre. Neurourol. Urodyn. 35, 487-491 (2016).

Honig A, Verwey B, Kromkamp M, Sierink D, Gordijn M, Leue C, Portier B, Scherders M. Reaction on 'Areas of interest within psychiatry: A gain or a loss?' Tijdschr. Psychiatr. 57, 626 (2015).

Mujagic Z, Leue C, Vork L, Lousberg R, Jonkers D, Keszthelyi D, Hesselink M, van Schagen T, van Os J, Masclee A, Kruimel J. The Experience Sampling Method - a new digital tool for momentary symptom assessment in IBS: An exploratory study. Neurogastroenterol. Motil. 27, 1295-1302 (2015).

Baijens L, Verdonschot R, Vanbelle S, Basic S, Kremer B, van de Berg R, Leue C. Medically unexplained otorhinolaryngological symptoms: Towards integrated psychiatric care. Laryngoscope. 125, 1583-1587 (2015).

Leue C, Kruimel J, Winkens B, Marcus D, Schoon S, Dellink R, van Os J, Masclee A. Integrated medical-psychiatric outpatient care in functional gastrointestinal disorders improves outcome: A pilot study. Eur. J. Gastroenterol. Hepatol. 27, 721-727 (2015).

Vrijens D, Drossaerts J, van Koeveringe G, Van Kerrebroeck P, van Os J, Leue C. Affective symptoms and the overactive bladder - a systematic review. J. Psychosom. Res. 78, 95108 (2015). 
van Steenbergen-Weijenburg K, van der Feltz-Cornelis C, van Benthem T, Horn E, Ploeger R, Brals J, Leue C, Spijker J, Hakkaart-van Roijen L, Rutten F, Beekman A. Collaborative care for co-morbid major depressive disorder in chronically ill outpatients in a general hospital. Tijdschr. Psychiatr. 57, 248-257 (2015).

Hundscheid T, van der Hulst R, Rutten B, Leue C. Body dysmorphic disorder in cosmetic surgery - prevalence, psychiatric comorbidity and outcome. Tijdschr. Psychiatr. 56, 514522 (2014).

Verdonschot R, Baijens L, Serroyen J, Leue C, Kremer B. Symptoms of anxiety and depression assessed with the Hospital Anxiety and Depression Scale in patients with oropharyngeal dysphagia. J. Psychosom. Res. 75, 451-455 (2013).

Keszthelyi D, Troost F, Jonkers D, Kruimel J, Leue C, Masclee A. Decreased levels of kynurenic acid in the intestinal mucosa of IBS patients: Relation to serotonin and psychological state. J. Psychosom. Res. 74, 501-504 (2013).

Weusten L, Severeijns R, Leue C. A profound retrograde amnesia of almost 30 years and the role of organic, intentional and psychogenic factors. Tijdschr. Psychiatr. 55, 281-285 (2013).

Esseveld M, Leroy P, Leue C, Strik J, Tijssen M, van de Riet E, Schieveld J. Catatonia and refractory agitation in an updated flow chart for the evaluation of emotional-behavioural disturbances in severely ill children. Intensive Care Med. 39, 528-529 (2013).

Brouns S, Schieveld J, Leue C. The Kleine-Levin syndrome and the treatment with methylphenidate. Tijdschr. Psychiatr. 54, 747-751 (2012).

Leue C, Buijs S, Strik J, Lousberg R, Smit J, van Kleef M, van Os J. Observational evidence that urbanization and neighbourhood deprivation are associated with escalation in chronic pharmacological pain treatment: A longitudinal population-based study in the Netherlands. BMJ Open 2, pii: e000731 (2012).

Thijssen A, Jonkers D, Leue C, van der Veek P, Vidakovic-Vukic M, van Rood Y, Clemens C, Masclee A. Dysfunctional cognitions, anxiety and depression in irritable bowel syndrome. J. Clin. Gastroenterol. 44, e236-241 (2010).

Janssen D, Spruit M, Leue C, Gijsen C, Hameleers H, Schols J, Wouters E. Symptoms of anxiety and depression in COPD patients entering pulmonary rehabilitation. Chron. Respir. Dis. 7, 147-157 (2010). 
Leue C, Driessen G, Strik J, Drukker M, Stockbrügger R, Kuijpers P, Masclee A, van Os J. Managing complex patients on a medical psychiatric unit: An observational study of university hospital costs associated with medical service use, length of stay, and psychiatric intervention. J. Psychosom. Res. 68, 295-302 (2010).

Vossen H, Monsieur D, van Os J, Leue C. Serotonin-noradrenalin reuptake inhibitors in trhe treatment of non-malignant pain syndromes: A systematic review. Tijdschr. Psychiatr. 51, 831-840 (2009).

Rahnama'i M, Leue C, van Koeveringe G. Wired bladder in a cordless era. BMJ Case Rep. pii: bcr12.2008.1365 (2009) .

van Harten A, Leue C, Verhey F. Should depressive symptoms in patients with subclinical hypothyroidism be treated with thyroid hormone? Tijdschr. Psychiatr. 50, 539-543 (2008).

Wichers M, Kenis G, Leue C, Koek G, Robaeys G, Maes M. Baseline immune activation as a risk factor for the onset of depression during interferon-alpha treatment. Biol. Psychiatry 60, 77-79 (2006).

Leue C, van Os J, Neeleman J, de Graaf R, Vollebergh W, Stockbrügger R. Bidirectional associations between depression/anxiety and bowle disease in a population based cohort. J. Epidemiol. Community Health 59, 434 (2005).

Leue C. Invited comment on Tardieu S, Poirier Y, Micallef J, Blin O. Amphetamine-like stimulant cessation in an abusing patient treated with bupropion. Acta Psychiatr. Scand. 109, 75-78 (2004).

Kierdorf H, Leue C, Arns S. Lactate- or bicarbonate-buffered solutions in continuous extracorporeal renal replacement therapies. Kidney Int. Suppl. 72, S32-36 (1999).

Kierdorf H, Leue C, Heintz B, Riehl J, Melzer H, Sieberth H. Continuous venovenous hemofiltration in acute renal failure: Is a bicarbonate- or lactate-buffered substitution better? Contrib. Nephrol. 116, 38-47 (1995).

\section{Book chapters}

Leue C. Organisch begründete psychische Erkrankungen - am Beispiel des Delirs. Uexküll: Psychosomatische Medizin - Theoretische Modelle und klinische Praxis. 8. Auflage, Kapitel 99: 1123-1130 (2017). 
Leue C. Chronische pijn en psychiatrie - een betoog voorbij de pijn-depressie diade. Handboek Psychiatrie in het Ziekenhuis. 1e Editie, Hoofdstuk 19 (in press).

\section{Awards}

Excellence in pain practice award 2013. Presented by the World Institute of Pain to the University Pain Centre Maastricht for comprehensive multidisciplinary pain practice in recognition of excellence in all areas of pain management, including cutting-edge research.

Best practice award 2012. Presented by the Dutch Association of Gastroenterology to Dr Joanna Kruimel, gastroenterologist, and Dr med. Carsten Leue, hospital psychiatrist, for integrated care in functional gastrointestinal disorders. 



\section{Curriculum vitae}

Carsten Leue was born on January $5^{\text {th }} 1963$ in Recklinghausen, Germany. He attended the 'Neusprachliches Gymnasium Viersen', Germany, where he graduated in 1982. After finishing his mandatory community service in 1985, he started medical school in 1986 at the 'Rheinisch Westfälische Technische Hochschule Aachen (RWTH)', Germany, and obtained his Master of Science degree in Medicine in 1996. He finished his dissertation (Dr med. (i.e., MD)) at the RWTH in 1999 ('Kontinuierliche veno-venöse Hämofiltration im akuten Nierenversagen: Vergleich von bikarbonathaltigen (SH-35-HEP) und laktathaltigen (SH-04) Substitutions-lösungen'). Also in 1999, he started his Psychiatry residency in Maastricht, The Netherlands. Since 2003, Carsten Leue is a hospital and consultation-liaison psychiatrist at the Maastricht University Medical Centre (MUMC). He is part of the psychiatric-somatic comorbidity team at the MUMC's Psychiatry department, delivering inpatient, outpatient and transitional care for multi-conditional patients. Dr Leue introduced the Medical Psychiatric Network, which is a transitional integrated care approach in order to bundle different collaborative care solutions, form primary to tertiary care. Consecutively, he is involved in scientific research regarding (cost-) effectiveness of integrated care in complex patients with multi-conditional complaints. Part of this work was awarded by the Dutch Association of Gastroenterology (Best Practice Award 2012) and the World Institute of Pain (WIP Excellence in Pain Practice Award for Comprehensive Multidisciplinary Pain Practice 2013). He has been chairman of the MUMC's Pelvic Care Centre from 2012 to 2014. Carsten Leue is further concerned in education of medical students and (upcoming) psychiatrists and medical consultants, as well as general practitioners. From 2009 to 2012, he was board member of the former European Association of Consultation Liaison Psychiatry and Psychosomatics (EACLPP). Since 2013, he is board member of the European Association of Psychosomatic Medicine (EAPM) and since 2014 board member of the Consultation and Liaison Psychiatry/General Hospital Psychiatry section of the Dutch Psychiatric Association. In 2016, he was elected secretary of the EAPM. Carsten Leue will publicly defend his PhD-thesis (Hyperarousal in the hospital and what to do about it: The MedPsych-Net - a transitional network approach fostering personalized care in psychosomatic medicine) on Thursday, December $14^{\text {th }} 2017$ at Maastricht University. 



\section{Abbreviations}

\begin{tabular}{|c|c|}
\hline $5-\mathrm{HT}$ & Serotonin \\
\hline ACC & Anterior Cingulate Cortex \\
\hline ACE & Angiotensin-Converting-Enzyme \\
\hline ACTH & Adrenocorticothropic Hormone \\
\hline$A D$ & Antidepressant \\
\hline AP & Abdominal Pain \\
\hline APA & American Psychiatric Association \\
\hline ASB & Asymptomatic Bacteriuria \\
\hline ATP & Adenosine Triphosphate \\
\hline $\mathrm{BDI}$ & Beck Depression Inventory \\
\hline BGBA & Bladder-Gut-Brain Axis \\
\hline $\mathrm{BMI}$ & Body Mass Index \\
\hline BPPV & Benign Paroxysmal Positional Vertigo \\
\hline CAM & Confusion Assessment Method \\
\hline CBS & Central Bureau for Statistics \\
\hline CBT & Cognitive Behavioural Therapy (CGT in Dutch) \\
\hline $\mathrm{CC}$ & Collaborative Care \\
\hline CEBM & Centre for Evidence-Based Medicine \\
\hline CES-D & Centre of Epidemiologic Studies - Depression Scale \\
\hline $\mathrm{Cl}$ & Confidence Interval \\
\hline CIDI-SF & Composite International Diagnostic Interview - Short Form \\
\hline CIWA & Clinical Institute Withdrawal Assessment Scale \\
\hline $\mathrm{CL}$ & Consultation-Liaison \\
\hline CLS & Consultation-Liaison Service \\
\hline $\mathrm{CM}$ & Case Manager, Case Management \\
\hline CMHS & Community Mental Health Service \\
\hline CNS & Central Nervous System \\
\hline COPD & Chronic Obstructive Pulmonary Disease \\
\hline CP/CPPS & Chronic Prostatitis/Chronic Pelvic Pain Syndrome \\
\hline CPPS & Chronic Pelvic Pain Syndrome \\
\hline CRF & Corticotropin-Releasing Factor \\
\hline CS & Constipation Syndrome \\
\hline CS-FBD & Cognitive Scale for Functional Bowel Disorders \\
\hline $\mathrm{CT}$ & Computed Tomography \\
\hline $\mathrm{CTI}$ & Care Transition Intervention \\
\hline DAMP & Damage-Associated Molecular Pattern \\
\hline DM & Diabetes Mellitus \\
\hline DMN & Default Mode Network \\
\hline DMSS & Delirium Motor Subtype Scale \\
\hline
\end{tabular}




\begin{tabular}{|c|c|}
\hline DNA & Deoxyribonucleic Acid \\
\hline DRS & Delirium Rating Scale \\
\hline DS & Diarrhea Syndrome \\
\hline DSM & Diagnostic and Statistical Manual of Mental Disorders \\
\hline DSS & Dysphagia Severity Scale \\
\hline EAPM & European Association of Psychosomatic Medicine \\
\hline ECT & Electro-Convulsive Therapy \\
\hline ED & Emergency Department \\
\hline EEG & Electroencephalography \\
\hline e-Health & Electronic Health \\
\hline EMA & Ecological Momentary Assessment \\
\hline ENT & Ear Nose Throat \\
\hline$E R$ & Emergency Room \\
\hline ESM & Experience Sampling Method \\
\hline FDA & Food and Drug Administration \\
\hline FEES & Fiberoptic Endoscopic Evaluation of Swallowing \\
\hline FGID & Functional Gastrointestinal Disorder \\
\hline $\mathrm{FI}$ & Fecal Incontinence \\
\hline FM & Fibromyalgia \\
\hline FTE & Full Time Equivalent \\
\hline GABA & Gamma Aminobutyric Acid \\
\hline GDS & Geriatric Depression Scale \\
\hline GE & Gastroenterology \\
\hline GERD & Gastroesophageal Reflux Disease \\
\hline GI & Gastrointestinal \\
\hline GP & General Practitioner \\
\hline GPC & General Practitioner Consultation \\
\hline GPU & General Psychiatric Unit \\
\hline GRADE & $\begin{array}{l}\text { Grading of Recommendation Assessment, Development and } \\
\text { Evaluation }\end{array}$ \\
\hline GSRS & Gastrointestinal Symptom Rating Scale \\
\hline HADS & Hospital Anxiety and Depression Scale \\
\hline HADS-A & Hospital Anxiety and Depression Scale - Anxiety subscore \\
\hline HADS-D & Hospital Anxiety and Depression Scale - Depression subscore \\
\hline IBS & Irritable Bowel Syndrome \\
\hline IBS-C & Irritable Bowel Syndrome - Constipation predominant \\
\hline IBS-D & Irritable Bowel Syndrome - Diarrhea predominant \\
\hline IBS-M & Irritable Bowel Syndrome - Mixed type \\
\hline IBD & Inflammatory Bowel Disorder \\
\hline IC & Insula Cortex \\
\hline IC/BPS & Interstitial Cystitis/Bladder Pain Syndrome \\
\hline
\end{tabular}




\begin{tabular}{|c|c|}
\hline ICl-RS & International Consultation on Incontinence-Research Society \\
\hline ICS & International Continence Society \\
\hline ICU & Intensive Care Unit \\
\hline IDO & Indoleamine-Dioxygenase \\
\hline IL-1 $\alpha$ & Interleukin-1 Alpha \\
\hline IL-1 $\beta$ & Interleukin-1 Beta \\
\hline IL-6 & Interleukin-6 \\
\hline IL-10 & Interleukin-10 \\
\hline LOS & Length Of (hospital) Stay \\
\hline LPS & Lipopolysaccharide \\
\hline LUTS & Lower Urinary Tract Symptoms \\
\hline MA & Momentary Assessment \\
\hline MAO & Monoamine Oxidase \\
\hline MBT & Mindfulness-Based Therapy \\
\hline MCS & Mental Composite Score \\
\hline MMSE & Mini Mental State Examination \\
\hline Med-Psych-Centre & Medical Psychiatric Centre \\
\hline Med-Psych-Net & Medical Psychiatric Network \\
\hline Med-Psych-Unit & Medical Psychiatric Unit \\
\hline MDC & Multidisciplinary Consultation \\
\hline $\mathrm{MHC}$ & Mental Health Care \\
\hline MHCS & Mental Health Care Service \\
\hline $\mathrm{MHCS}$ & Mental Health Care Specialist \\
\hline MHS & Mental Health Service \\
\hline m-Health & Mobile Health \\
\hline MPC & Med-Psych-Centre \\
\hline MPN & Med-Psych-Net \\
\hline MPU & Med-Psych-Unit \\
\hline MRI & Magnetic Resonance Imaging (MRT in German) \\
\hline $\mathrm{mROM}$ & Mobile Routine Outcome Monitoring \\
\hline MUI & Mixed Urinary Incontinence \\
\hline MUMC & Maastricht University Medical Centre \\
\hline MUORLS & Medically Unexplained Otorhinolaryngological Symptoms \\
\hline MUPS & Medically Unexplained Physical Symptoms \\
\hline NA & Negative Affect \\
\hline NAC & Nucleus Accumbens \\
\hline NCGC & National Clinical Guideline Centre \\
\hline NDI & Neighbourhood Deprivation Index \\
\hline NEECHAM & Neelon Champagne Confusion Scale \\
\hline$N F-K B$ & Nuclear Factor Kappa B \\
\hline NICE & National Institute for Health and Care Excellence \\
\hline
\end{tabular}


NIVEL

NMDA

nSONS

NTS

nVNS

$O A B$

OAB-dry

OAB-wet

OD

OR

ORL

PA

PAG

PAMP

PCA

PCC

PCR

PCS

PFC

PFD

$\mathrm{PHCS}$

POP

PRISMA

PROM

PT

PTSD

QUADAS

QoL

$R$

RAND-36

RCT

ROM

RVM

RWTH

SD

SE

SF-36

SNRI

SPECT

SSRI

STAI
Netherlands Institute for Research in Healthcare

N-Methyl-D-Aspartate

Nervus Supra-Orbitalis Neuro-Stimulation

Nucleus Tractus Solitarius

Nervus Vagus Neuro-Stimulation

Overactive Bladder

Overactive Bladder, without urinary incontinence

Overactive Bladder, with urinary incontinence

Oropharyngeal Dysphagia

Odds Ratio

Otorhinolaryngological

Positive Affect

Periaqueductal Gray

Pathogen-Associated Molecular Pattern

Principal Component Analysis

Pelvic Care Centre

Psychiatric Case Register

Physical Composite Score

Prefrontal Cortex

Pelvic Floor Dysfunction

Physical Health Care Specialist

Pelvic Organ Prolapse

Preferred Reporting Items for Systematic Reviews and Meta-Analyses

Patient-Reported Outcome Measures

Psychotherapy

Posttraumatic Stress Disorder

Quality Assessment of Diagnostic Accuracy Studies

Quality of Life

Referral

Research and Development - 36

Randomized-Controlled Trial

Routine Outcome Monitoring

Rostral Ventromedial Medulla

Rheinisch Westfälische Technische Hochschule

Standard Deviation

Standard Error

Short Form - 36 (RAND-36)

Selective Noradrenaline-Serotonin Reuptake Inhibitor

Single Photon Emission Computed Tomography

Selective Serotonin Reuptake Inhibitor

State-Trait Anxiety Inventory 
STAI-S

STAI-T

SUI

TCA

TNF- $\alpha$

TLR

TRPV1

UI

UMC

UPEC

UTI

UUI

VAS

VFSS

VTA

WHO
State-Trait Anxiety Inventory - State subscore

State-Trait Anxiety Inventory - Trait subscore

Stress Urinary Incontinence

Tricyclic Antidepressant

Tumor Necrosis Factor-Alpha

Toll-Like Receptor

Transient Receptor Potential Vanilloid Type 1

Urinary Incontinence

University Medical Centre

Uropathogenic Escherichia Coli

Urinary Tract Infection

Urgency Urinary Incontinence

Visual Analogue Scale

Videofluoroscopic Swallowing Study

Ventral Tegmental Area

World Health Organization 


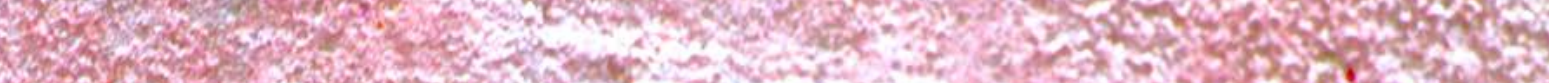

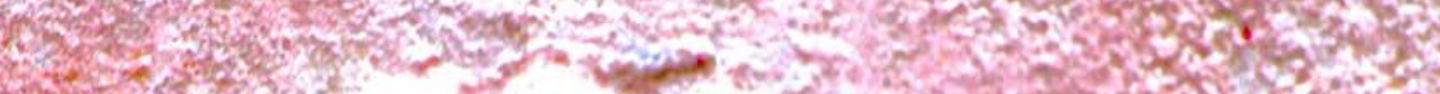

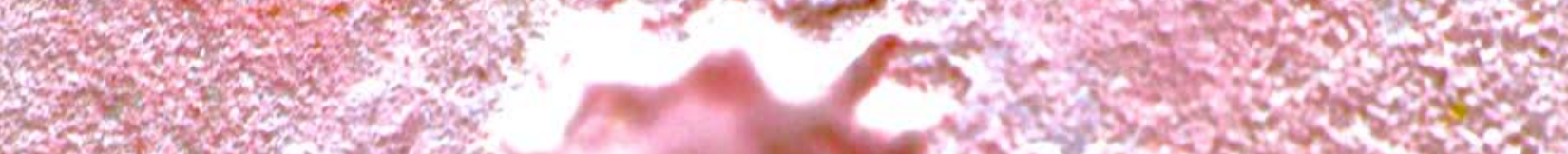

